\title{
A redox-neutral Fe-catalyzed cycloisomerization of enyne acetates
}

\author{
Johannes Teske, and Bernd Plietker*
}

Institut für Organische Chemie, Universität Stuttgart, Pfaffenwaldring 55, DE-70569 Stuttgart, Germany. bernd.plietker@oc.uni-stuttgart.de

\section{Table of contents}

1 General Remarks $\quad$ S2

2 General Optimizations $\quad$ S3

3 Preparation of Complexes s1, 6, 7 and $8 \quad S 4$

4 Preparation of 1,6-Enyne Acetates $\quad$ S7

5 Fe-catalyzed cycloisomerizations $\quad$ S67

6 Control experiments $\quad 593$

7 References $\quad 599$

8 Copies of EPR, ${ }^{1} \mathrm{H}$ and ${ }^{13} \mathrm{C}$ NMR $\quad \mathrm{S} 100$

9 Chiral HPLC chromatograms $\quad \mathrm{S160}$ 


\section{General Remarks}

All reactions and manipulations which are sensitive towards air or moisture were performed under dry nitrogen by using standard Schlenk techniques. All solvents were purified prior to use. All chemicals were purchased from Acros Organics, Sigma Aldrich, Alfa Aesar, TCI or ChemPUR. NMR spectra were recorded on a Bruker Avance 300 spectrometer at $300 \mathrm{MHz}$ ( ${ }^{1} \mathrm{H}$ NMR), $75 \mathrm{MHz}\left({ }^{13} \mathrm{C}\right.$ NMR), a Bruker Ascend 400 spectrometer at $400 \mathrm{MHz}\left({ }^{1} \mathrm{H} \mathrm{NMR}\right)$, $376 \mathrm{MHz}\left({ }^{19} \mathrm{~F} \mathrm{NMR}\right), 101 \mathrm{MHz}\left({ }^{13} \mathrm{C} \mathrm{NMR}\right)$ or a Bruker Avance 500 spectrometer at $500 \mathrm{MHz}\left({ }^{1} \mathrm{H}\right.$ NMR), $126 \mathrm{MHz}\left({ }^{13} \mathrm{C}\right.$ NMR), $202 \mathrm{MHz}\left({ }^{31} \mathrm{P} \mathrm{NMR}\right)$. Chemical shifts were reported in ppm down field using tetramethylsilane as an internal standard. IR spectra were measured on a FT-IR spectrometer in an ATR mode. Mass spectra were measured using electrospray ionization on a Bruker micrOTOF-Q. High performance liquid chromatography (HPLC) was performed using a Knauer K-501 pump, Knauer RI-detector K 2400 and a Macherey-Nagel VP250/21 Nucleodur 100-5 column. Semi-preparative chiral HPLC was performed using a Knauer K-501 pump, Knauer RI-detector K 2400 and a Grom Chiralpak OD-CSP 250x20 mm column. Analytical chiral HPLC was performed using a HP Series 1050 HPLC module and a Deicel ChiraCel OD 250x4.6 mm or a Deicel Chiralpak AD-H 250x4.6mm column. Specific rotation values were measured on a Perkin-Elmer 241 polarimeter at $25{ }^{\circ} \mathrm{C}$ using the sodium lamp. ESR spectra were recorded on a Bruker EMX $\mathrm{X}$-band spectrometer at $110 \mathrm{~K}$ without solvent in a NMR-tube under a dry nitrogen atmosphere. X-ray structures were measured on a Bruker Kappa APEXII Duo spectrometer. The carbonylation reaction was performed in a $100 \mathrm{~mL}$ stainless steel autoclave (Parr Instrument Company) that was suitable for pressure up to 140 bar. Melting points were recorded on a Stuart SMP10 melting point apparatus and are uncorrected. 


\section{General Optimizations}<smiles>CC(=O)OC(C)(C)C#CCN([12CH3])C/C=C\c1ccccc1</smiles>

\begin{tabular}{|c|c|c|c|c|}
\hline entry $^{\text {a) }}$ & catalyst & temperature & reaction time & yield $^{b)} 10: 36: 37$ \\
\hline 1 & $6[5 \mathrm{~mol} \%]$ & $40^{\circ} \mathrm{C}$ & $3 \mathrm{~d}$ & $49 \%^{\mathrm{c})}: 0: 0$ \\
\hline 2 & $6[2.5 \mathrm{~mol} \%]$ & $40^{\circ} \mathrm{C}$ & $22 \mathrm{~h}$ & 10\%:0:0 \\
\hline 3 & $6[1 \mathrm{~mol} \%]$ & $50^{\circ} \mathrm{C}$ & $22 \mathrm{~h}$ & traces:0:0 \\
\hline 4 & $6[2.5 \mathrm{~mol} \%]$ & $50{ }^{\circ} \mathrm{C}$ & $22 \mathrm{~h}$ & 18\%:0:0 \\
\hline 5 & $6[5 \mathrm{~mol} \%]$ & $50^{\circ} \mathrm{C}$ & $22 \mathrm{~h}$ & $44 \%: 0: 0$ \\
\hline 6 & $6[10 \mathrm{~mol} \%]$ & $50{ }^{\circ} \mathrm{C}$ & $22 \mathrm{~h}$ & $67 \%: 0: 0$ \\
\hline 7 & $\mathrm{AuCl}_{3}[10 \mathrm{~mol} \%]$ & $50{ }^{\circ} \mathrm{C}$ & $22 \mathrm{~h}$ & $0: 0: 81 \%^{\mathrm{c})}$ \\
\hline 8 & 39 [10 mol\%] & $50{ }^{\circ} \mathrm{C}$ & $22 \mathrm{~h}$ & 0:0:quant. ${ }^{\mathrm{c}}$ \\
\hline 9 & $\mathrm{PtCl}_{2}$ & $50^{\circ} \mathrm{C}$ & $22 \mathrm{~h}$ & $0: 78 \%^{\mathrm{c})}: 0$ \\
\hline
\end{tabular}

a) All reactions were performed according to GP-4. b) Yields were determined by ${ }^{1} \mathrm{H}$ NMR in $\mathrm{CDCl}_{3}$ using mesitylene ( $\left.28 \mu \mathrm{L}, 0.2 \mathrm{mmol}\right)$ as an internal standard. c) Isolated yield. 


\section{Preparation of Complexes s1, 6, 7 and 8}

\subsection{Preparation of $\mathrm{HFe}(\mathrm{CO})(\mathrm{NO})\left(\mathrm{PPh}_{3}\right)_{2} \mathrm{s1}$}<smiles>O=[N+]([O-])[P-](P)(P)P</smiles>

To a $\mathrm{N}_{2}$-degassed solution of sodium nitrite $(2.6 \mathrm{~g}, 37.7 \mathrm{mmol})$ and sodium methoxide $(4.7 \mathrm{~g}$, $87 \mathrm{mmol})$ in methanol $(200 \mathrm{~mL})$ was added iron pentacarbonyl (>99.99\% Fe, $5 \mathrm{~mL}$, $37.2 \mathrm{mmol}$ ). After stirring the reaction at $65^{\circ} \mathrm{C}$ for 3 hours the solvent was removed at $30{ }^{\circ} \mathrm{C}$ under reduced pressure using an ether bridge connected to a liquid $\mathrm{N}_{2}$ cooled trap. The obtained solid was dried under high vacuum for at least 24 hours, dissolved in $\mathrm{N}_{2}$-degassed diethyl ether $(500 \mathrm{~mL})$ and filtered under nitrogen. To this solution was added $\mathrm{PPh}_{3}(39 \mathrm{~g}$, $149 \mathrm{mmol})$ and trifluoroacetic acid $(4.3 \mathrm{~mL}, 56 \mathrm{mmol})$ at $0{ }^{\circ} \mathrm{C}$ followed by stirring for 2 hours at $0{ }^{\circ} \mathrm{C}$. The precipitate was isolated with an inert gas filter, washed with $\mathrm{N}_{2}$-degassed diethyl ether $(50 \mathrm{~mL})$, dried under high vacuum for at least 24 hours and dissolved in $\mathrm{N}_{2}$-degassed benzene $(250 \mathrm{~mL})$. This solution was concentrated at $30{ }^{\circ} \mathrm{C}$ under reduced pressure using an ether bridge connected to a liquid $\mathrm{N}_{2}$ cooled trap to yield the title complex $\mathbf{s} \mathbf{1}$ which was dried under high vacuum for at least 24 hours. The spectral data are in good agreement with previous reports. ${ }^{[1]}$

Yield: $14.84 \mathrm{~g}(23.21 \mathrm{mmol}, 62 \%)$.

Physical State: orange solid.

${ }^{1} \mathbf{H}$ NMR (Avance $\left.500 \mathrm{MHz}, \mathrm{THF}_{-} \mathrm{d}_{8}\right) \delta 7.55$ (s, 12H), $7.27(\mathrm{~s}, 18 \mathrm{H}),-4.99(\mathrm{t}, J=78.1 \mathrm{~Hz}$, 1H) $\mathrm{ppm}$.

${ }^{13}$ C NMR (Avance $\left.126 \mathrm{MHz}, \mathrm{THF}_{-} \mathrm{d}_{8}\right) \delta 222.2(\mathrm{t}, J=21.8 \mathrm{~Hz}), 138.7(\mathrm{t}, J=23.7 \mathrm{~Hz}), 133.9$ (t, $J=5.4 \mathrm{~Hz}), 130.4,128.9,128.8(\mathrm{t}, J=4.4 \mathrm{~Hz}) \mathrm{ppm}$.

${ }^{31} \mathbf{P}$ NMR (Avance $202 \mathrm{MHz}, \mathrm{THF}-\mathrm{d}_{8}$ ) $\delta 77.3(\mathrm{~d}, J=78.0 \mathrm{~Hz}) \mathrm{ppm}$.

IR (ATR, in THF-d 8 ) v 3054 (w), 1902 (s), 1673 (s), 1478 (m), 1432 (s), 1091 (m) cm cm $^{-1}$

HRMS (ESI, m/z) calcd. for $\mathrm{C}_{37} \mathrm{H}_{31} \mathrm{FeNO}_{2} \mathrm{P}_{2} \mathrm{Na}^{+}$: 662.1072, found: 662.1097. 


\subsection{Preparation of $\left[\mathrm{Fe}(\mathrm{CO})(\mathrm{NO})\left(\mathrm{PPh}_{3}\right)_{2}\right]\left[\mathrm{BF}_{4}\right] 6$}

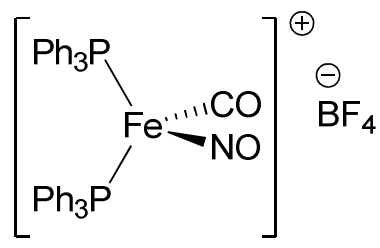

To a solution of complex S1 $(2.0 \mathrm{~g}, 3.13 \mathrm{mmol})$ in THF $(40 \mathrm{~mL})$ was added $\mathrm{HBF}_{4} * \mathrm{OEt}_{2}$ $(1.3 \mathrm{~mL}, 9.38 \mathrm{mmol})$ dropwise at $0{ }^{\circ} \mathrm{C}$ under argon and the solution was stirred for 3 hours at $0{ }^{\circ} \mathrm{C}$. The formed precipitate was triturated with $0{ }^{\circ} \mathrm{C}$ cold THF $(9 \mathrm{x} 10 \mathrm{~mL})$. The residual THF is removed at $30{ }^{\circ} \mathrm{C}$ under reduced pressure using an ether bridge connected to a liquid $\mathrm{N}_{2}$ cooled trap to yield the title complex $\mathbf{6}$ which was dried under high vacuum for at least 24 hours. The spectral data are in good agreement with previous reports. ${ }^{[2]}$

Yield: $1.43 \mathrm{~g}(1.97 \mathrm{mmol}, 63 \%)$.

Physical State: yellow solid.

EPR $(110 \mathrm{~K}$, neat) $g=1.922,2.033,4.240$.

\subsection{Preparation of $\left[\mathrm{Fe}(\mathrm{CO})_{2}(\mathrm{NO})\left(\mathrm{PPh}_{3}\right)_{2}\right]\left[\mathrm{BF}_{4}\right] 7$}

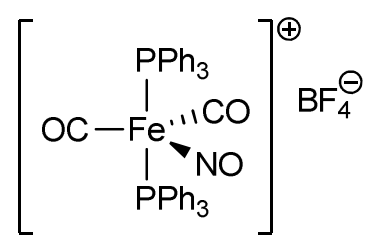

A solution of complex $6(132.0 \mathrm{mg}, 0.182 \mathrm{mmol})$ in $\mathrm{CH}_{2} \mathrm{Cl}_{2}(10 \mathrm{~mL})$ was stirred in an autoclave at room temperature under an atmosphere of CO (5 bar) for $22 \mathrm{~h}$. The resulting solution was transferred to a round bottom flask and the solvent was removed under reduced pressure. The yellow powder was dried under high vacuum for at least 24 hours to yield the title complex 7. The spectral data are in good agreement with previous reports. ${ }^{[2]}$

Yield: $136.8 \mathrm{mg}(0.182 \mathrm{mmol}$, quant.).

Physical State: yellow solid.

${ }^{1} \mathbf{H}$ NMR (Avance $500 \mathrm{MHz}, \mathrm{CDCl}_{3}$ ) $\delta 7.63-7.66(\mathrm{~m}, 18 \mathrm{H}), 7.43(\mathrm{~s}, 12 \mathrm{H}) \mathrm{ppm}$.

${ }^{13}$ C NMR (Avance $126 \mathrm{MHz}, \mathrm{CDCl}_{3}$ ) $\delta 205.7(\mathrm{t}, J=26.1 \mathrm{~Hz}), 133.2(\mathrm{~s}), 133.0(\mathrm{t}, J=5.2 \mathrm{~Hz}$ ), $130.2(\mathrm{t}, J=5.6 \mathrm{~Hz}), 128.4-129.3(\mathrm{~m}) \mathrm{ppm}$. 
${ }^{31} \mathbf{P}$ NMR (Avance $202 \mathrm{MHz}, \mathrm{CDCl}_{3}$ ) $\delta 59.0 \mathrm{ppm}$.

IR (ATR, in $\mathrm{CDCl}_{3}$ ) v 3062 (w), 2048 (m), 1985 (s), 1782 (s), 1482 (w), 1436 (m), 1312 (w), 1285 (w), 1189 (w), 1165 (w), 1094 (m), 1055 (s) $\mathrm{cm}^{-1}$.

HRMS (ESI, m/z) calcd. for $\mathrm{C}_{38} \mathrm{H}_{30} \mathrm{FeNO}_{3} \mathrm{P}_{2}^{+}$: 666.1046, found: 666.1029.

X-Ray: X-Ray structure of $\mathbf{7}$ is deposited at Cambridge Crystallographic Data Centre (www.ccdc.cam.ac.uk) under CCDC 1440735.

\subsection{Preparation of $\left[\left(\mathrm{H}_{3} \mathrm{CCN}\right) \mathrm{Fe}(\mathrm{CO})(\mathrm{NO})\left(\mathrm{PPh}_{3}\right)_{2}\right]\left[\mathrm{BF}_{4}\right] 8$}

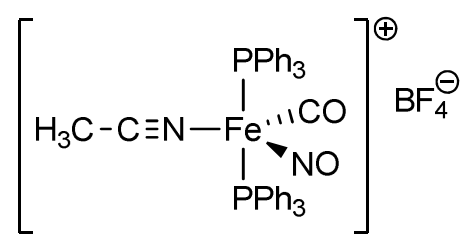

A solution of complex $6(200 \mathrm{mg}, 0.28 \mathrm{mmol})$ in acetonitrile $(2.5 \mathrm{~mL})$ was stirred for 14 hours at room temperature followed by addition of diethyl ether $(5 \mathrm{~mL})$. The formed precipitate was isolated and dried under high vacuum for at least 24 hours to yield the title complex $\mathbf{8}$. The spectral data are in good agreement with previous reports. ${ }^{[2]}$

Yield: $89.3 \mathrm{mg}(0.12 \mathrm{mmol}, 42 \%)$.

Physical State: orange solid.

${ }^{1} \mathbf{H}$ NMR (Avance $500 \mathrm{MHz}, \mathrm{CD}_{2} \mathrm{Cl}_{2}$ ) $\delta 7.62(\mathrm{~s}, 30 \mathrm{H}), 1.61(\mathrm{~s}, 3 \mathrm{H}) \mathrm{ppm}$.

${ }^{13} \mathbf{C}$ NMR (Avance $126 \mathrm{MHz}, \mathrm{CD}_{2} \mathrm{Cl}_{2}$ ) $\delta 213.4(\mathrm{t}, J=33.3 \mathrm{~Hz}$ ), 137.3, 133.8, 132.5, 129.9, $2.8 \mathrm{ppm}$.

${ }^{31}$ P NMR (Avance $\left.202 \mathrm{MHz}, \mathrm{CD}_{2} \mathrm{Cl}_{2}\right) \delta 60.1 \mathrm{ppm}$.

IR (ATR, in $\left.\mathrm{CD}_{2} \mathrm{Cl}_{2}\right) \vee 3063$ (w), 2862 (w), 1949 (m), 1739 (m), 1481 (w), 1436 (m), $1096(\mathrm{~m}), 1056(\mathrm{~m}) \mathrm{cm}^{-1}$.

X-Ray: X-Ray structure of $\mathbf{8}$ is deposited at Cambridge Crystallographic Data Centre (www.ccdc.cam.ac.uk) under CCDC 1440736. 


\section{Preparation of 1,6-Enyne Acetates}

\section{General Procedure 1 (GP-1)}

To a stirred solution of the alkyne $(4 \mathrm{mmol})$ in THF $(40 \mathrm{~mL})$ was added $n$-Butyllithium (1.6 $\mathrm{M}$ in hexane, $2.5 \mathrm{~mL}, 4 \mathrm{mmol}$ ) at $-78^{\circ} \mathrm{C}$. After stirring for 1 hour at $-78{ }^{\circ} \mathrm{C}$, the carbonyl compound (4.6 mmol, a solution in THF for solids) was slowly added and the reaction mixture was stirred at room temperature for 3 hours. The reaction was hydrolyzed by addition of sat. $\mathrm{NH}_{4} \mathrm{Cl}(25 \mathrm{~mL})$ and extracted with diethyl ether $(3 \mathrm{x} 30 \mathrm{~mL})$. The combined organic layers were dried over anhydrous $\mathrm{Na}_{2} \mathrm{SO}_{4}$ and concentrated under reduced pressure. The residue was purified by column chromatography on silica gel to yield the propargylic alcohol.

\section{General Procedure 2 (GP-2)}

To a stirred solution of the alcohol $(3 \mathrm{mmol})$ in $\mathrm{CH}_{2} \mathrm{Cl}_{2}(3 \mathrm{~mL})$ was added DMAP (18.3 $\mathrm{mg}$, $0.15 \mathrm{mmol})$, acetic anhydride $(0.85 \mathrm{~mL}, 9 \mathrm{mmol})$ and triethyl amine $(0.62 \mathrm{~mL}, 4.5 \mathrm{mmol})$ at room temperature. After stirring overnight at room temperature sat. $\mathrm{NH}_{4} \mathrm{Cl}(10 \mathrm{~mL})$ was added followed by extraction with $\mathrm{CH}_{2} \mathrm{Cl}_{2}(3 \times 15 \mathrm{~mL})$. The combined organic layers were dried over anhydrous $\mathrm{Na}_{2} \mathrm{SO}_{4}$ and concentrated under reduced pressure. The residue was purified by column chromatography on silica gel to yield the propargylic acetate.

\section{General Procedure 3 (GP-3)}

To a stirred solution of the amine $(10 \mathrm{mmol})$ in THF $(40 \mathrm{~mL})$ was added the alcohol $(10.5 \mathrm{mmol}), \mathrm{PPh}_{3}(2.6 \mathrm{~g}, 10 \mathrm{mmol})$ and DIAD $(2 \mathrm{~mL}, 10 \mathrm{mmol})$ at $0{ }^{\circ} \mathrm{C}$. After stirring overnight at room temperature the reaction mixture was concentrated under reduced pressure. The residue was purified by column chromatography on silica gel to yield the enyne. 


\subsection{Preparation of (E)-5-(N-cinnamyl-4-methylphenylsulfonamido)-2-methylpent-3-}

yn-2-yl acetate 9
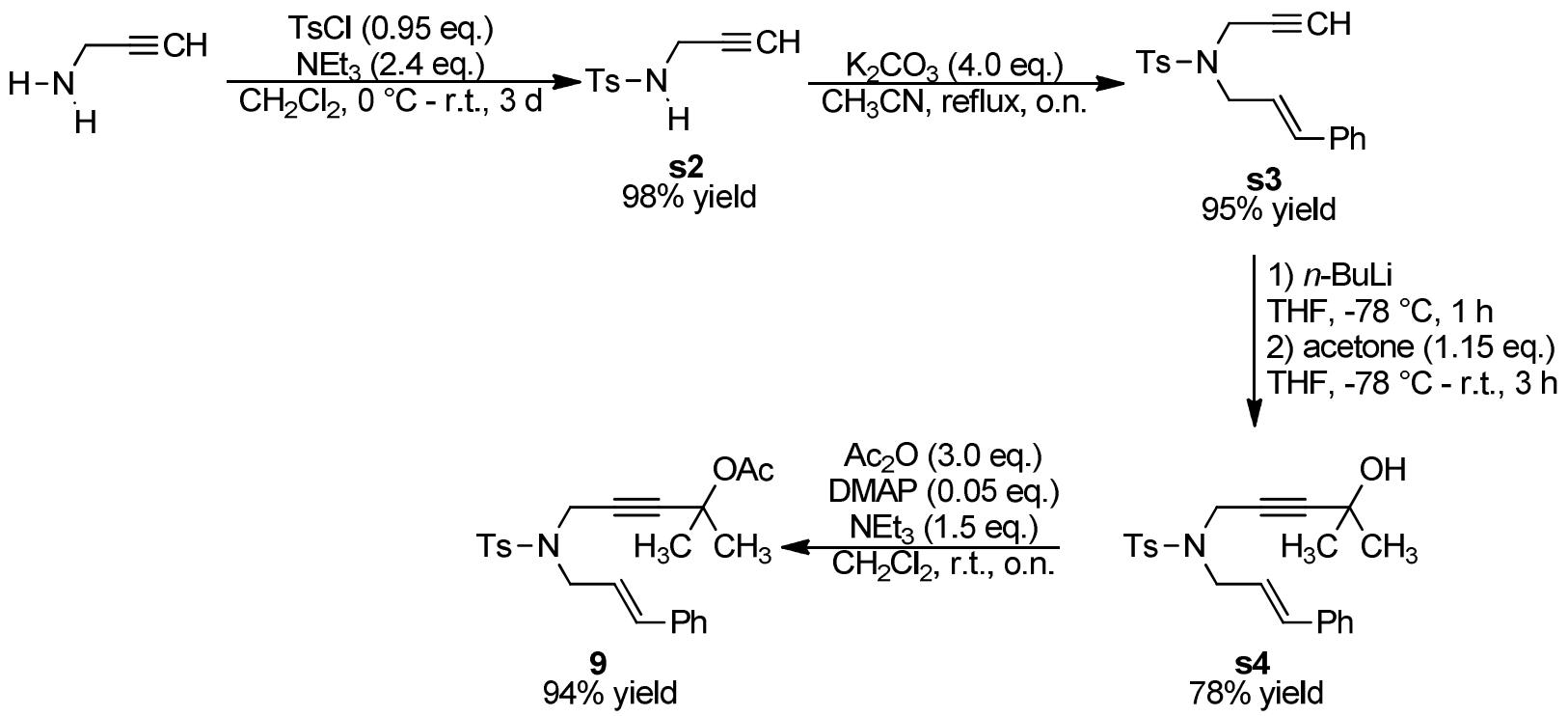

4-methyl- $N$-(prop-2-ynyl)benzenesulfonamide s2<smiles>[3H]NCC#C</smiles>

To a stirred solution of propargylamine $(7.7 \mathrm{~mL}, 120 \mathrm{mmol})$ in $\mathrm{CH}_{2} \mathrm{Cl}_{2}(285 \mathrm{~mL})$ was added triethylamine $(40 \mathrm{~mL}, 285 \mathrm{mmol})$ and 4-toluenesulfonyl chloride $(21.8 \mathrm{~g}, 114 \mathrm{mmol})$ at $0{ }^{\circ} \mathrm{C}$. After stirring for 3 days at room temperature diethyl ether $(1.15 \mathrm{~L})$ was added, the reaction mixture was washed with aqueous $\mathrm{HCl}(1 \mathrm{~N}, 500 \mathrm{~mL})$, sat. $\mathrm{NH}_{4} \mathrm{Cl}(500 \mathrm{~mL})$ and brine $(500 \mathrm{~mL})$. The organic phase was dried over anhydrous $\mathrm{Na}_{2} \mathrm{SO}_{4}$ and concentrated under reduced pressure to yield $\mathbf{s} \mathbf{2}$. The spectral data are in good agreement with previous reports. ${ }^{[3]}$ Yield: $23.46 \mathrm{~g}(112.13 \mathrm{mmol}, 98 \%)$.

Physical State: white solid.

$\mathbf{R}_{\mathbf{f}}$ Value: 0.13 (petroleum ether/EtOAc - 5/1).

${ }^{1} \mathbf{H}$ NMR (Avance $300 \mathrm{MHz}, \mathrm{CDCl}_{3}$ ) $\delta 7.77(\mathrm{~d}, J=8.2 \mathrm{~Hz}, 2 \mathrm{H}), 7.29(\mathrm{~d}, J=8.1 \mathrm{~Hz}, 2 \mathrm{H}), 5.07$ $(\mathrm{t}, J=5.7 \mathrm{~Hz}, 1 \mathrm{H}), 3.80(\mathrm{dd}, J=2.5,6.0 \mathrm{~Hz}, 2 \mathrm{H}), 2.41(\mathrm{~s}, 3 \mathrm{H}), 2.08(\mathrm{t}, J=2.5 \mathrm{~Hz}, 1 \mathrm{H}) \mathrm{ppm}$.

${ }^{13}$ C NMR (Avance $75 \mathrm{MHz}, \mathrm{CDCl}_{3}$ ) $\delta$ 143.9, 136.6, 129.8, 127.5, 78.1, 73.0, 32.9, 21.6 ppm.

IR (ATR, in $\left.\mathrm{CDCl}_{3}\right) \vee 3254$ (s), 2933 (w), 2860 (w), 2118 (w), 1925 (w), 1598 (w), 1495 (w), 1433 (m), 1324 (s), 1304 (m), 1293 (m), 1244 (w), 1158 (s), 1092 (m), 1059 (s) cm cm $^{-1}$ 
HRMS (ESI, m/z) calcd. for $\mathrm{C}_{10} \mathrm{H}_{11} \mathrm{NO}_{2} \mathrm{SNa}^{+}$: 232.0403, found: 232.0391 .

\section{$N$-cinnamyl-4-methyl- $N$-(prop-2-ynyl)benzenesulfonamide s3}

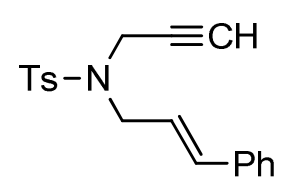

To a stirred slurry of $\mathbf{s} 2(6.28 \mathrm{~g}, 30 \mathrm{mmol})$ and potassium carbonate $(16.6 \mathrm{~g}, 120 \mathrm{mmol})$ in acetonitrile $(300 \mathrm{~mL})$ was added cinnamyl bromide $(8.9 \mathrm{~mL}, 60 \mathrm{mmol})$ dropwise. The reaction mixture was refluxed for $18 \mathrm{~h}$. After cooling to room temperature. the solvent was removed under reduced pressure. The residue was dissolved in a sat. $\mathrm{NaHCO}_{3}(400 \mathrm{~mL})$ and extracted with diethyl ether $(3 \mathrm{x} 200 \mathrm{~mL})$. The combined organic phases were dried over anhydrous $\mathrm{Na}_{2} \mathrm{SO}_{4}$ and concentrated. The residue was purified by column chromatography on silica gel (petroleum ether/EtOAc $-30 / 1 \rightarrow 5 / 1$ ). The crude product was recrystallised from boiling $n$-hexane ( $100 \mathrm{~mL}$ for every $1.5 \mathrm{~g}$ of crude product) to yield the title compound $\mathbf{s} 3$. The spectral data are in good agreement with previous reports. ${ }^{[4]}$

Yield: $9.3 \mathrm{~g}(28.58 \mathrm{mmol}, 95 \%)$.

Physical State: white solid.

$\mathbf{R}_{\mathbf{f}}$ Value: 0.26 (petroleum ether/EtOAc - 10/1).

${ }^{1} \mathbf{H}$ NMR (Avance $300 \mathrm{MHz}, \mathrm{CDCl}_{3}$ ) $\delta 7.75-7.79(\mathrm{~m}, 2 \mathrm{H}), 7.25-7.35(\mathrm{~m}, 7 \mathrm{H}), 6.57$ (dt, $J=1.3,15.8 \mathrm{~Hz}, 1 \mathrm{H}), 6.08(\mathrm{dt}, J=6.9,15.7 \mathrm{~Hz}, 1 \mathrm{H}), 4.13(\mathrm{~d}, J=2.4 \mathrm{~Hz}, 2 \mathrm{H}), 3.99$ (dd, $J=1.1,6.8 \mathrm{~Hz}, 2 \mathrm{H}), 2.44(\mathrm{~s}, 3 \mathrm{H}), 2.05(\mathrm{t}, J=2.4 \mathrm{~Hz}, 1 \mathrm{H}) \mathrm{ppm}$.

${ }^{13}$ C NMR (Avance $75 \mathrm{MHz}, \mathrm{CDCl}_{3}$ ) $\delta$ 143.8, 136.2, 136.2, 135.1, 129.7, 128.8, 128.2, 128.0, 126.7, 123.1, 74.0, 48.7, 36.0, 21.7 ppm. 1 carbon was hidden under $\mathrm{CDCl}_{3}$.

${ }^{13}$ C NMR (Avance 75 MHz, Acetone-d $\mathrm{d}_{6}$ ) $\delta$ 144.6, 137.6, 137.4, 135.2, 130.5, 129.5, 128.8, $128.6,127.4,124.2,77.8,75.5,49.5,36.8,21.5 \mathrm{ppm}$.

IR (ATR, in $\mathrm{CDCl}_{3}$ ) v 3290 (w), 3027 (w), 2979 (w), 2922 (w), 2856 (w), 2119 (w), 1734 (w), 1671 (w), 1597 (w), 1495 (w), 1447 (w), 1347 (m), 1326 (m), 1241 (w), 1159 (s), $1091(\mathrm{~m}) \mathrm{cm}^{-1}$.

HRMS (ESI, m/z) calcd. for $\mathrm{C}_{19} \mathrm{H}_{19} \mathrm{NO}_{2} \mathrm{SNa}^{+}: 348.1029$, found: 348.1019 . 


\section{$N$-cinnamyl- $N$-(4-hydroxy-4-methyl-pent-2-ynyl)-4-methylbenzenesulfonamide s4}

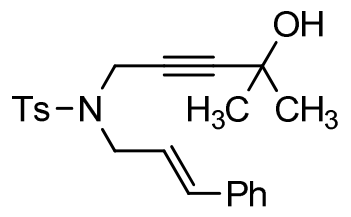

According to GP-1 s3 (5.0 g, $15.4 \mathrm{mmol})$ was dissolved in THF (150 mL), treated with $n$-BuLi (1.6 M in hexanes, $9.6 \mathrm{~mL}, 15.4 \mathrm{mmol})$ and acetone $(1.3 \mathrm{~mL}, 17.7 \mathrm{mmol})$ to yield $\mathbf{s} 4$ after column chromatography on silica gel (petroleum ether/EtOAc $-5 / 1 \rightarrow 2 / 1$ ). The spectral data are in good agreement with previous reports. ${ }^{[5]}$

Yield: $4.57 \mathrm{~g}(11.91 \mathrm{mmol}, 78 \%)$.

Physical State: yellow solid.

$\mathbf{R}_{\mathbf{f}}$ Value: 0.25 (petroleum ether/EtOAc - 3/1).

${ }^{1} \mathbf{H}$ NMR (Avance $\left.300 \mathrm{MHz}, \mathrm{CDCl}_{3}\right) \delta 7.76-7.79(\mathrm{~m}, 2 \mathrm{H}), 7.22$ - $7.35(\mathrm{~m}, 7 \mathrm{H}), 6.57$ (d, $J=15.8 \mathrm{~Hz}, 1 \mathrm{H}), 6.10(\mathrm{dt}, J=6.8,15.8 \mathrm{~Hz}, 1 \mathrm{H}), 4.13(\mathrm{~s}, 2 \mathrm{H}), 3.99(\mathrm{dd}, J=0.8,6.8 \mathrm{~Hz}, 2 \mathrm{H})$, $2.44(\mathrm{~s}, 3 \mathrm{H}), 1.59(\mathrm{~s}, 1 \mathrm{H}), 1.28(\mathrm{~s}, 6 \mathrm{H}) \mathrm{ppm}$.

${ }^{13}$ C NMR (Avance $75 \mathrm{MHz}, \mathrm{CDCl}_{3}$ ) $\delta$ 143.7, 136.4, 136.2, 134.9, 129.7, 128.8, 128.2, 128.1, $126.7,123.1,90.7,74.9,64.9,48.9,36.3,31.2,21.6$ ppm.

IR (ATR, neat) v 3510 (m), 3058 (w), 3030 (w), 3001 (w), 2981 (w), 2930 (w), 2913 (w), 2852 (w), 1597 (w), 1494 (w), 1453 (w), 1435 (w), 1390 (w), 1369 (w), 1358 (w), 1339 (m), 1321 (m), 1305 (m), 1233 (w), 1156 (s), 1138 (s), 1096 (m), 1085 (m), 1061 (w) cm cm $^{-1}$

HRMS (ESI, m/z) calcd. for $\mathrm{C}_{22} \mathrm{H}_{25} \mathrm{NO}_{3} \mathrm{SNa}^{+}$: 406.1447, found: 406.1448 .

\section{(E)-5-(N-cinnamyl-4-methylphenylsulfonamido)-2-methylpent-3-yn-2-yl acetate 9}

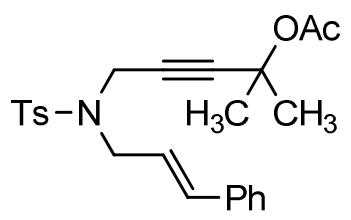

According to GP-2 s4 (4.0 g, $10.4 \mathrm{mmol})$ was dissolved in $\mathrm{CH}_{2} \mathrm{Cl}_{2}(10 \mathrm{~mL})$, treated with acetic anhydride $(3.0 \mathrm{~mL}, 31.3 \mathrm{mmol})$, DMAP $(63.7 \mathrm{mg}, 0.52 \mathrm{mmol})$ and triethylamine (2.2 $\mathrm{mL}, 15.6 \mathrm{mmol})$ to yield 9 after column chromatography on silica gel ( $n$-pentane/Et ${ }_{2} \mathrm{O}-$ $2 / 1)$. The spectral data are in good agreement with previous reports. ${ }^{[6]}$

Yield: $4.19 \mathrm{~g}(9.85 \mathrm{mmol}, 94 \%)$. 
Physical State: white solid.

Melting Point: $112^{\circ} \mathrm{C}$.

$\mathbf{R}_{\mathbf{f}}$ Value: $0.42\left(n\right.$-pentane/ $\left.\mathrm{Et}_{2} \mathrm{O}-2 / 1\right)$.

${ }^{1} \mathbf{H}$ NMR (Avance $300 \mathrm{MHz}, \mathrm{CDCl}_{3}$ ) $\delta 7.74-7.78(\mathrm{~m}, 2 \mathrm{H}), 7.21-7.37$ (m, 7H), $6.64(\mathrm{~d}$, $J=15.8 \mathrm{~Hz}, 1 \mathrm{H}), 6.08(\mathrm{dt}, J=6.9,15.8 \mathrm{~Hz}, 1 \mathrm{H}), 4.16(\mathrm{~s}, 2 \mathrm{H}), 4.00(\mathrm{dd}, J=0.8,6.9 \mathrm{~Hz}, 2 \mathrm{H})$, $2.43(\mathrm{~s}, 3 \mathrm{H}), 1.97(\mathrm{~s}, 3 \mathrm{H}), 1.41(\mathrm{~s}, 6 \mathrm{H}) \mathrm{ppm}$.

${ }^{13}$ C NMR (Avance $75 \mathrm{MHz}, \mathrm{CDCl}_{3}$ ) $\delta$ 169.2, 143.5, 136.5, 136.4, 135.2, 129.7, 128.7, 128.1, 127.9, 126.7, 123.0, 87.1, 77.0, 71.5, 48.6, 36.4, 28.8, 22.0, 21.7 ppm.

IR (ATR, neat) v 3016 (w), 2986 (w), 2937 (w), 2858 (w), 1730 (s), 1656 (w), 1596 (w), 1493 (w), 1448 (w), 1368 (w), 1347 (m), 1328 (m), 1262 (m), 1243 (s), 1163 (s), 1115 (m), $1089(\mathrm{~m}), 1036(\mathrm{w}), 1016(\mathrm{w}) \mathrm{cm}^{-1}$.

HRMS (ESI, m/z) calcd. for $\mathrm{C}_{24} \mathrm{H}_{27} \mathrm{NO}_{4} \mathrm{SNa}^{+}: 448.1553$, found: 448.1555 .

X-Ray: X-Ray structure of $\mathbf{9}$ is deposited at Cambridge Crystallographic Data Centre (www.ccdc.cam.ac.uk) under CCDC 1440737.

\subsection{Preparation of $(E)-4-(N$-cinnamyl-4-methylphenylsulfonamido)-1-phenylbut-2-yn-} 1-yl acetate 40

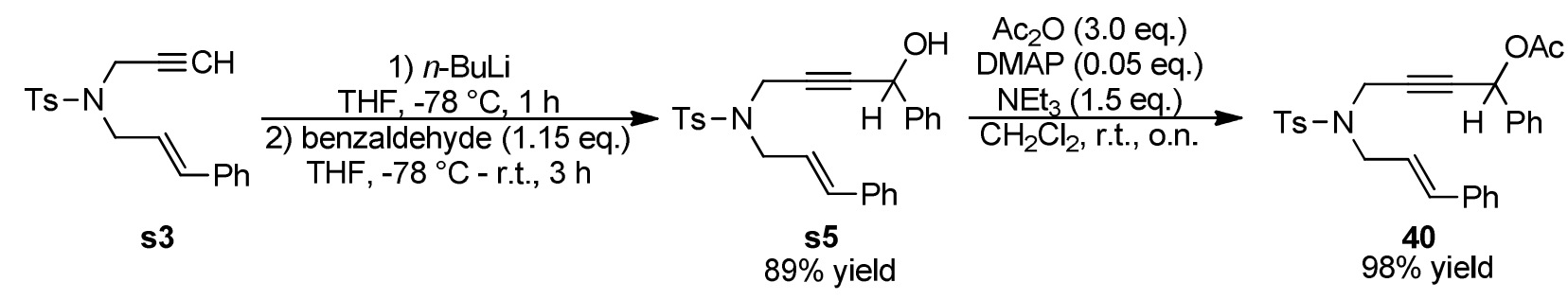

$N$-cinnamyl- $N$-(4-hydroxy-4-phenylbut-2-yn-1-yl)-4-methylbenzenesulfonamide s5<smiles>[13CH3]N(CC#CC(O)(c1ccccc1)c1ccccc1)C/C=C\c1ccccc1</smiles> 
According to GP-1 s3 (1.63 g, $5 \mathrm{mmol})$ was dissolved in THF (50 mL), treated with $n$-BuLi (1.6 $\mathrm{M}$ in hexanes, $3.1 \mathrm{~mL}, 5 \mathrm{mmol})$ and benzaldehyde $(0.58 \mathrm{~mL}, 5.8 \mathrm{mmol})$ to yield $\mathbf{s 5}$ after column chromatography on silica gel (petroleum ether/EtOAc $-5 / 1 \rightarrow 3 / 1$ ).

Yield: $1.92 \mathrm{~g}(4.45 \mathrm{mmol}, 89 \%)$.

Physical State: yellow solid.

$\mathbf{R}_{\mathbf{f}}$ Value: 0.29 (petroleum ether/EtOAc - 3/1).

${ }^{1} \mathbf{H}$ NMR (Avance $400 \mathrm{MHz}, \mathrm{CDCl}_{3}$ ) $\delta 7.74-7.76(\mathrm{~m}, 2 \mathrm{H}), 7.24-7.36(\mathrm{~m}, 10 \mathrm{H}), 7.20$ - 7.22 (m, 2H), $6.49(\mathrm{~d}, J=15.8 \mathrm{~Hz}, 1 \mathrm{H}), 6.07(\mathrm{dt}, J=6.9,15.7 \mathrm{~Hz}, 1 \mathrm{H}), 5.19(\mathrm{~s}, 1 \mathrm{H}), 4.15-4.26$ (m, 2H), $3.92-4.02(\mathrm{~m}, 2 \mathrm{H}), 2.36(\mathrm{~s}, 3 \mathrm{H}), 1.95(\mathrm{~s}, 1 \mathrm{H}) \mathrm{ppm}$.

${ }^{13}$ C NMR (Avance $101 \mathrm{MHz}, \mathrm{CDCl}_{3}$ ) $\delta$ 143.8, 140.2, 136.1, 135.1, 129.6, 128.7, 128.7, $128.6,128.2,128.0,126.7,126.5,123.0,85.7,79.6,64.4,49.0,36.4,21.6$ ppm. 1 carbon was not observed due to incidental equivalence.

IR (ATR, in $\left.\mathrm{CDCl}_{3}\right)$ v 3488 (w, broad), 3029 (w), 2920 (w), 1597 (w), 1494 (w), 1449 (w), 1399 (w), 1342 (m), 1327 (m), 1306 (w), 1242 (w), 1154 (s), 1118 (w), 1091 (m), 1056 (w), $1017(\mathrm{w}) \mathrm{cm}^{-1}$.

HRMS (ESI, m/z) calcd. for $\mathrm{C}_{26} \mathrm{H}_{25} \mathrm{NO}_{3} \mathrm{SNa}^{+}$: 454.1447, found: 454.1442.

\section{(E)-4-(N-cinnamyl-4-methylphenylsulfonamido)-1-phenylbut-2-yn-1-yl acetate 40}

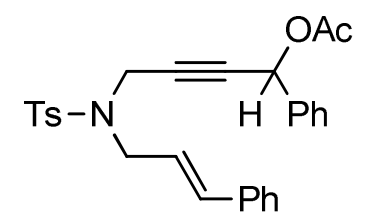

According to GP-2 s5 (1.73 g, $4 \mathrm{mmol})$ was dissolved in $\mathrm{CH}_{2} \mathrm{Cl}_{2}(4 \mathrm{~mL})$, treated with acetic anhydride $(1.1 \mathrm{~mL}, 12 \mathrm{mmol})$, DMAP $(24.4 \mathrm{mg}, 0.2 \mathrm{mmol})$ and triethylamine $(0.83 \mathrm{~mL}$, $6 \mathrm{mmol}$ ) to yield 40 after column chromatography on silica gel (petroleum ether/EtOAc $5 / 1)$

Yield: $1.85 \mathrm{~g}(3.90 \mathrm{mmol}, 98 \%)$.

Physical State: yellow oil.

$\mathbf{R}_{\mathbf{f}}$ Value: 0.33 (petroleum ether/EtOAc - 5/1). 
${ }^{1} \mathbf{H}$ NMR (Avance $\left.400 \mathrm{MHz}, \mathrm{CDCl}_{3}\right) \delta 7.71-7.73(\mathrm{~m}, 2 \mathrm{H}), 7.33-7.38(\mathrm{~m}, 3 \mathrm{H}), 7.23-7.30$ (m, 7H), $7.18-7.20(\mathrm{~m}, 2 \mathrm{H}), 6.50(\mathrm{~d}, J=15.8 \mathrm{~Hz}, 1 \mathrm{H}), 6.18(\mathrm{~s}, 1 \mathrm{H}), 6.07(\mathrm{dt}, J=6.9$, $15.7 \mathrm{~Hz}, 1 \mathrm{H}), 4.17-4.29(\mathrm{~m}, 2 \mathrm{H}), 3.89-4.00(\mathrm{~m}, 2 \mathrm{H}), 2.35$ (s, 3H), 2.06 (s, 3H) ppm.

${ }^{13}$ C NMR (Avance $101 \mathrm{MHz}, \mathrm{CDCl}_{3}$ ) $\delta$ 169.7, 143.7, 136.6, 136.2, 135.9, 135.2, 129.7, $129.2,128.8,128.7,128.2,127.9,127.7,126.7,122.8,82.6,80.2,65.4,49.0,36.4,21.7,21.1$ ppm.

IR (ATR, in $\mathrm{CDCl}_{3}$ ) v 3031 (w), 2922 (w), 1737 (m), 1598 (w), 1495 (w), 1450 (w), 1368 (w), 1347 (m), 1306 (w), 1223 (s), 1184 (w), 1159 (s), 1120 (w), 1092 (w), 1074 (w), $1055(\mathrm{w}), 1016(\mathrm{w}) \mathrm{cm}^{-1}$.

HRMS (ESI, m/z) calcd. for $\mathrm{C}_{28} \mathrm{H}_{27} \mathrm{NO}_{4} \mathrm{SNa}^{+}$: 496.1553, found: 496.1545.

\subsection{Preparation of $(E)-5-(N$-cinnamyl-4-methylphenylsulfonamido)-2-phenylpent-3-} yn-2-yl acetate s7

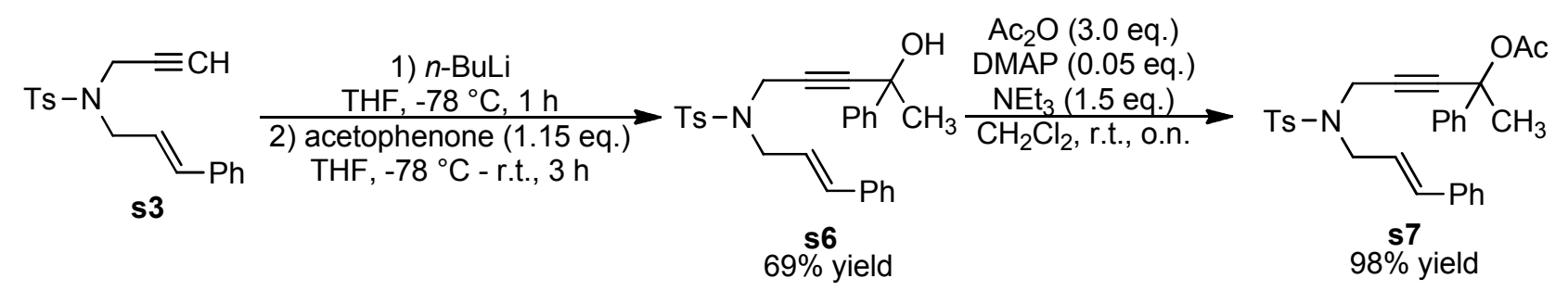

$N$-cinnamyl- $N$-(4-hydroxy-4-phenylpent-2-yn-1-yl)-4-methylbenzenesulfonamide s6

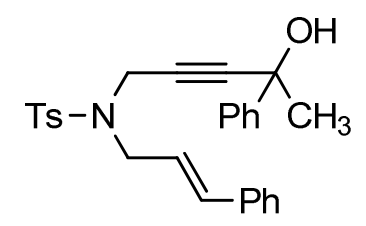

According to GP-1 s3 (1.3 g, $4 \mathrm{mmol})$ was dissolved in THF (40 mL), treated with $n$-BuLi (1.6 $\mathrm{M}$ in hexanes, $2.5 \mathrm{~mL}, 4 \mathrm{mmol})$ and acetophenone $(0.54 \mathrm{~mL}, 4.6 \mathrm{mmol})$ to yield $\mathbf{s 6}$ after column chromatography on silica gel (petroleum ether/EtOAc $-5 / 1 \rightarrow 3 / 1$ ).

Yield: $1.23 \mathrm{~g}$ (2.77 mmol, 69\%).

Physical State: pale yellow oil.

$\mathbf{R}_{\mathbf{f}}$ Value: 0.20 (petroleum ether/EtOAc - 5/1). 
${ }^{1} \mathbf{H}$ NMR (Avance $400 \mathrm{MHz}, \mathrm{CDCl}_{3}$ ) $\delta 7.74-7.76(\mathrm{~m}, 2 \mathrm{H}), 7.38-7.42(\mathrm{~m}, 2 \mathrm{H}), 7.23-7.34$ (m, 8H), $7.19-7.21(\mathrm{~m}, 2 \mathrm{H}), 6.53(\mathrm{~d}, J=15.8 \mathrm{~Hz}, 1 \mathrm{H}), 6.10(\mathrm{dt}, J=6.9,15.8 \mathrm{~Hz}, 1 \mathrm{H})$, 4.16 - $4.28(\mathrm{~m}, 2 \mathrm{H}), 3.94-4.05(\mathrm{~m}, 2 \mathrm{H}), 2.34(\mathrm{~s}, 3 \mathrm{H}), 2.01(\mathrm{~s}, 1 \mathrm{H}), 1.53(\mathrm{~s}, 3 \mathrm{H}) \mathrm{ppm}$.

${ }^{13}$ C NMR (Avance $101 \mathrm{MHz}, \mathrm{CDCl}_{3}$ ) $\delta$ 145.2, 143.8, 136.2, 136.1, 135.0, 129.7, 128.7, $128.4,128.2$, 128.0, 127.9, 126.6, 124.8, 123.0, 89.5, 77.6, 69.7, 49.0, 36.4, 32.9, 21.6 ppm.

IR (ATR, in $\mathrm{CDCl}_{3}$ ) v 3496 (w), 3059 (w), 3027 (w), 2982 (w), 2925 (w), 1598 (w), 1494 (w), 1447 (w), 1344 (m), 1327 (m), 1306 (w), 1232 (w), 1155 (s), 1118 (w), 1092 (m), $1070(\mathrm{w}), 1027(\mathrm{w}), 1017(\mathrm{w}) \mathrm{cm}^{-1}$.

HRMS (ESI, m/z) calcd. for $\mathrm{C}_{27} \mathrm{H}_{27} \mathrm{NO}_{3} \mathrm{SNa}^{+}$: 468.1604, found: 468.1613.

\section{(E)-5-(N-cinnamyl-4-methylphenylsulfonamido)-2-phenylpent-3-yn-2-yl acetate s7}

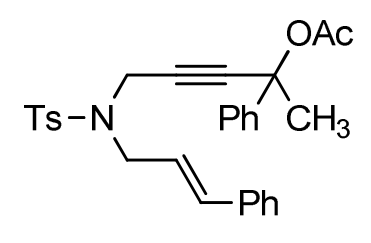

According to GP-2 s6 (1.20 g, $2.68 \mathrm{mmol})$ was dissolved in $\mathrm{CH}_{2} \mathrm{Cl}_{2}(3 \mathrm{~mL})$, treated with acetic anhydride $(0.76 \mathrm{~mL}, 8.1 \mathrm{mmol})$, DMAP $(16.4 \mathrm{mg}, 0.13 \mathrm{mmol})$ and triethylamine $(0.56 \mathrm{~mL}, 4.0 \mathrm{mmol})$ to yield $\mathbf{s} 7$ after column chromatography on silica gel (petroleum ether/EtOAc - 4/1).

Yield: $1.28 \mathrm{~g}(2.62 \mathrm{mmol}, 98 \%)$.

Physical State: white solid.

Melting Point: $108^{\circ} \mathrm{C}$.

$\mathbf{R}_{\mathbf{f}}$ Value: 0.41 (petroleum ether/EtOAc - 4/1).

${ }^{1} \mathbf{H}$ NMR (Avance $400 \mathrm{MHz}, \mathrm{CDCl}_{3}$ ) $\delta 7.78-7.80(\mathrm{~m}, 2 \mathrm{H}), 7.21-7.33(\mathrm{~m}, 12 \mathrm{H}), 6.65(\mathrm{~d}$, $J=15.8 \mathrm{~Hz}, 1 \mathrm{H}), 6.10(\mathrm{dt}, J=7.0,15.7 \mathrm{~Hz}, 1 \mathrm{H}), 4.30(\mathrm{~s}, 2 \mathrm{H}), 4.04(\mathrm{~d}, J=6.7 \mathrm{~Hz}, 2 \mathrm{H}), 2.34$ (s, 3H), 1.99 (s, 3H), 1.63 (s, 3H) ppm.

${ }^{13}$ C NMR (Avance $101 \mathrm{MHz}, \mathrm{CDCl}_{3}$ ) $\delta$ 168.3, 143.5, 142.2, 136.3, 136.2, 135.3, 129.7, 128.6, 128.3, 128.0, 127.9, 127.8, 126.6, 124.7, 122.7, 85.4, 80.1, 75.2, 48.6, 36.4, 31.7, 21.7, $21.5 \mathrm{ppm}$. 
IR (ATR, in $\mathrm{CDCl}_{3}$ ) v 3028 (w), 2990 (w), 2932 (w), 1745 (m), 1598 (w), 1494 (w), 1448 (w), 1365 (w), 1347 (m), 1306 (w), 1292 (w), 1235 (m), 1186 (w), 1160 (s), 1119 (w), $1091(\mathrm{w}), 1060(\mathrm{~m}) \mathrm{cm}^{-1}$.

HRMS (ESI, m/z) calcd. for $\mathrm{C}_{29} \mathrm{H}_{29} \mathrm{NO}_{4} \mathrm{SNa}^{+}:$510.1710, found: 510.1704 .

\subsection{Preparation of $(E)-4-(N$-cinnamyl-4-methylphenylsulfonamido)-1,1-diphenylbut-2-} ynyl acetate 34

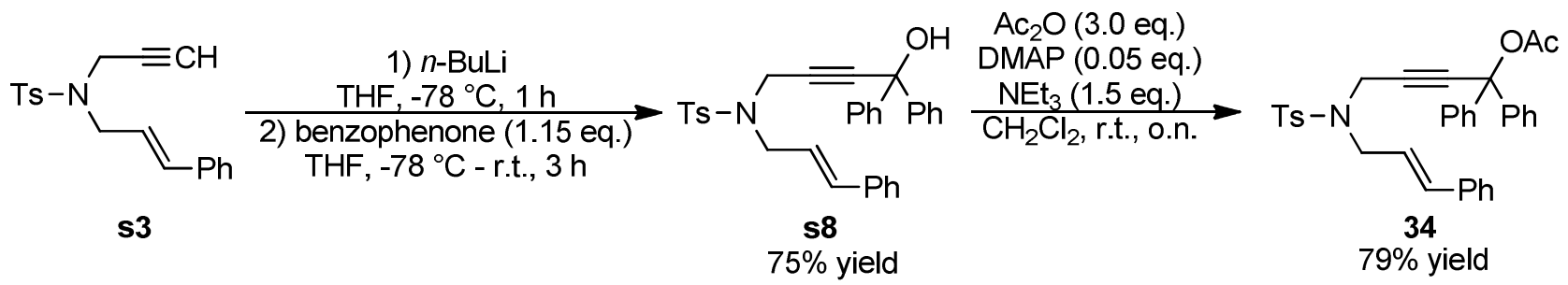

$N$-cinnamyl- $N$-(4-hydroxy-4,4-diphenylbut-2-ynyl)-4-methylbenzenesulfonamide s8

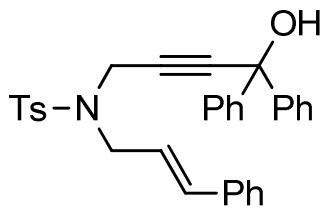

According to GP-1 s3 (1.0 g, $3.1 \mathrm{mmol})$ was dissolved in THF (30 mL), treated with $n$-BuLi (1.6 $\mathrm{M}$ in hexanes, $1.9 \mathrm{~mL}, 3.1 \mathrm{mmol})$ and benzophenone $(0.64 \mathrm{~g}, 3.5 \mathrm{mmol})$ in THF $(3 \mathrm{~mL})$ to yield s8 after column chromatography on silica gel (petroleum ether/EtOAc - 7/1).

Yield: $1.08 \mathrm{~g}(2.13 \mathrm{mmol}, 75 \%)$.

Physical State: pale yellow oil.

$\mathbf{R}_{\mathbf{f}}$ Value: 0.17 (petroleum ether/EtOAc - 7/1).

${ }^{1} \mathbf{H}$ NMR (Avance $300 \mathrm{MHz}, \mathrm{CDCl}_{3}$ ) $\delta 7.73$ - $7.75(\mathrm{~m}, 2 \mathrm{H}), 7.34-7.37$ (m, 4H), 7.24 - 7.31 (m, 11H), $7.11-7.14(\mathrm{~m}, 2 \mathrm{H}), 6.43(\mathrm{~d}, J=15.8 \mathrm{~Hz}, 1 \mathrm{H}), 6.08(\mathrm{dt}, J=6.9,15.8 \mathrm{~Hz}, 1 \mathrm{H}), 4.29$ (s, 2H), $3.98(\mathrm{~d}, J=6.8 \mathrm{~Hz}, 2 \mathrm{H}), 2.31(\mathrm{~s}, 3 \mathrm{H}), 2.22(\mathrm{~s}, 1 \mathrm{H}) \mathrm{ppm}$.

${ }^{13}$ C NMR (Avance $75 \mathrm{MHz}, \mathrm{CDCl}_{3}$ ) $\delta$ 144.4, 143.7, 136.1, 136.0, 135.1, 129.6, 128.6, 128.2, $128.1,127.9,127.8,126.6,125.9,122.8,88.7,80.1,74.1,49.0,36.4,21.5$ ppm. 
IR (ATR, in $\left.\mathrm{CDCl}_{3}\right)$ v 3483 (w, broad), 3059 (w), 3027 (w), 2920 (w), 1597 (w), 1491 (w), $1448(\mathrm{~m}), 1335(\mathrm{~m}), 1157$ (s), $1092(\mathrm{~m}) \mathrm{cm}^{-1}$.

HRMS (ESI, m/z) calcd. for $\mathrm{C}_{32} \mathrm{H}_{29} \mathrm{NO}_{3} \mathrm{SNa}^{+}:$530.1760, found: 530.1754 .

\section{(E)-4-(N-cinnamyl-4-methylphenylsulfonamido)-1,1-diphenylbut-2-ynyl acetate 34}

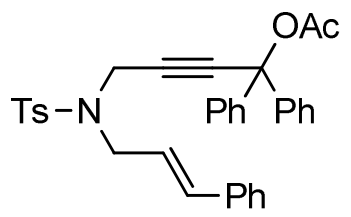

According to GP-2 s8 $(0.9 \mathrm{~g}, 1.8 \mathrm{mmol})$ was dissolved in $\mathrm{CH}_{2} \mathrm{Cl}_{2}(2 \mathrm{~mL})$, treated with acetic anhydride $(0.5 \mathrm{~mL}, 5.3 \mathrm{mmol})$, DMAP $(10.8 \mathrm{mg}, 0.09 \mathrm{mmol})$ and triethylamine $(0.46 \mathrm{~mL}$, $2.7 \mathrm{mmol}$ ) to yield 34 after column chromatography on silica gel ( $n$-pentane/ $\operatorname{Et}_{2} \mathrm{O}-2 / 1$ ).

Yield: $0.75 \mathrm{~g}(1.37 \mathrm{mmol}, 79 \%)$.

Physical State: white solid.

Melting Point: $148^{\circ} \mathrm{C}$.

$\mathbf{R}_{\mathbf{f}}$ Value: $0.27\left(n\right.$-pentane/ $\left.\mathrm{Et}_{2} \mathrm{O}-2 / 1\right)$.

${ }^{1} \mathbf{H}$ NMR (Avance $300 \mathrm{MHz}, \mathrm{CDCl}_{3}$ ) $\delta 7.74-7.76(\mathrm{~m}, 2 \mathrm{H}), 7.18-7.30$ (m, 17H), 6.53 (dt, $J=1.1 \mathrm{~Hz}, 15.9 \mathrm{~Hz}, 1 \mathrm{H}), 6.06(\mathrm{dt}, J=7.0,15.7 \mathrm{~Hz}, 1 \mathrm{H}), 4.33(\mathrm{~s}, 2 \mathrm{H}), 3.96(\mathrm{dd}, J=0.5$, $6.9 \mathrm{~Hz}, 2 \mathrm{H}), 2.35$ (s, 3H), $2.08(\mathrm{~s}, 3 \mathrm{H}) \mathrm{ppm}$.

${ }^{13}$ C NMR (Avance $75 \mathrm{MHz}, \mathrm{CDCl}_{3}$ ) $\delta 167.9,143.5,142.2,136.3,136.3,135.4,129.8,128.7$, $128.3,128.1,128.0,127.8,126.7,126.2$, 122.7, 85.0, 82.9, 79.1, 48.7, 36.5, 21.9, 21.7 ppm.

IR (ATR, in $\mathrm{CDCl}_{3}$ ) v 3027 (w), 2924 (w), 1752 (m), 1598 (w), 1493 (w), 1450 (w), 1349 (m), 1227 (m), 1163 (s), $1095(\mathrm{w}) \mathrm{cm}^{-1}$.

HRMS (ESI, m/z) calcd. for $\mathrm{C}_{34} \mathrm{H}_{31} \mathrm{NO}_{4} \mathrm{SNa}^{+}$: 572.1866, found: 572.1874.

X-Ray: X-Ray structure of $\mathbf{3 4}$ is deposited at Cambridge Crystallographic Data Centre (www.ccdc.cam.ac.uk) under CCDC 1440739. 
4.5 Preparation of $(E)-4-(N$-cinnamyl-4-methylphenylsulfonamido)-1-(4-fluorophenyl)but-2-yn-1-yl acetate s10
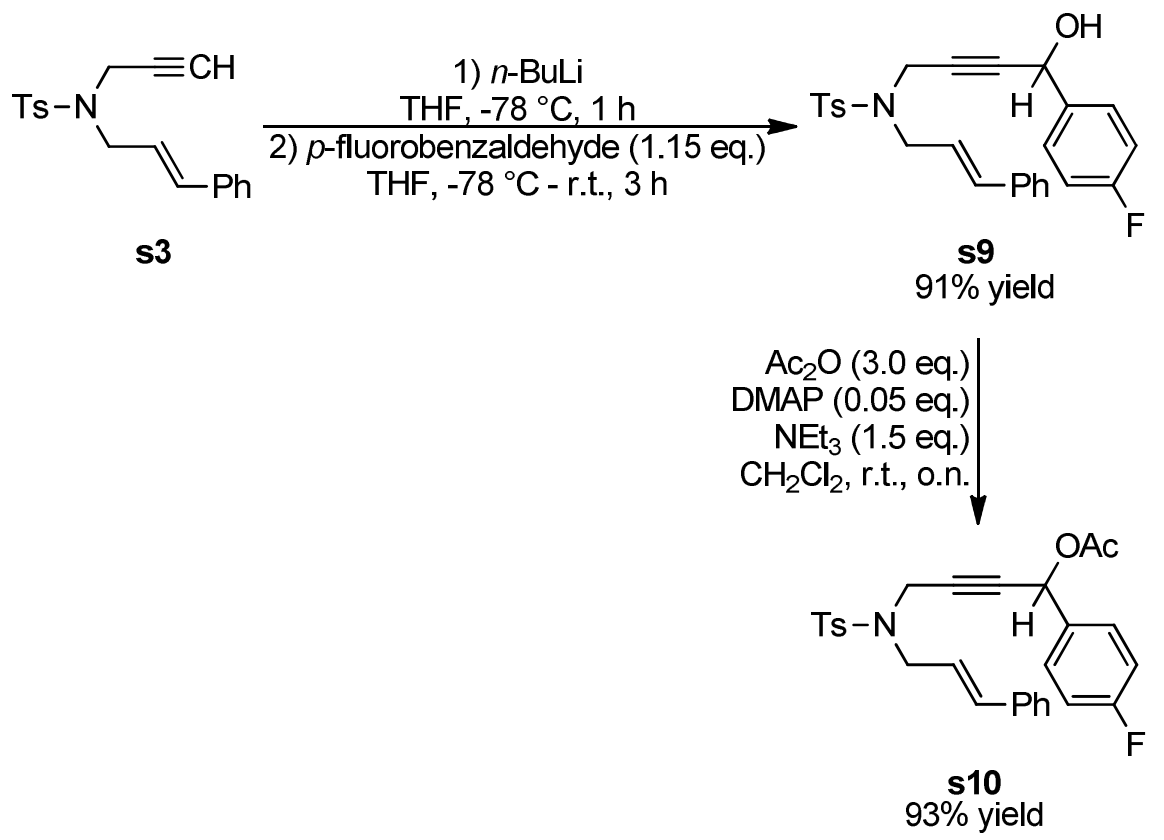

$N$-cinnamyl- $N$-(4-(4-fluorophenyl)-4-hydroxybut-2-yn-1-yl)-4-methylbenzenesulfonamide $\mathbf{s} 9$

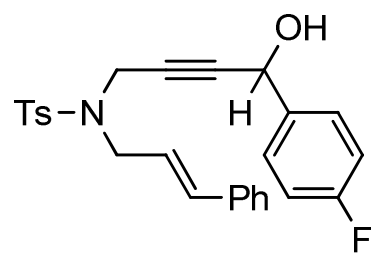

According to GP-1 s3 (1.30 g, $4 \mathrm{mmol})$ was dissolved in THF (40 mL), treated with $n$-BuLi (1.6 $\mathrm{M}$ in hexanes, $2.5 \mathrm{~mL}, 4 \mathrm{mmol})$ and $p$-fluorobenzaldehyde $(0.49 \mathrm{~mL}, 4.6 \mathrm{mmol})$ to yield s9 after column chromatography on silica gel (petroleum ether/EtOAc - 3/1).

Yield: $1.63 \mathrm{~g}$ (3.62 mmol, 91\%).

Physical State: yellow solid.

$\mathbf{R}_{\mathbf{f}}$ Value: 0.26 (petroleum ether/EtOAc - 3/1).

${ }^{1} \mathbf{H}$ NMR (Avance $400 \mathrm{MHz}, \mathrm{CDCl}_{3}$ ) $\delta 7.71$ - $7.73(\mathrm{~m}, 2 \mathrm{H}), 7.21$ - 7.30 (m, 7H), 7.19 - 7.21 $(\mathrm{m}, 2 \mathrm{H}), 6.96-7.00(\mathrm{~m}, 2 \mathrm{H}), 6.48(\mathrm{~d}, J=15.8 \mathrm{~Hz}, 1 \mathrm{H}), 6.04(\mathrm{dt}, J=6.9,15.6 \mathrm{~Hz}, 1 \mathrm{H}), 5.18$ (s, 1H), $4.17(\mathrm{~s}, 2 \mathrm{H}), 3.95(\mathrm{~d}, J=6.8 \mathrm{~Hz}, 2 \mathrm{H}), 2.50(\mathrm{~s}, 1 \mathrm{H}), 2.43(\mathrm{~s}, 3 \mathrm{H}) \mathrm{ppm}$.

${ }^{19}$ F NMR (Avance $376 \mathrm{MHz}, \mathrm{CDCl}_{3}$ ) $\delta-113.6$ ppm. 
${ }^{13}$ C NMR (Avance $\left.101 \mathrm{MHz}, \mathrm{CDCl}_{3}\right) \delta 162.6(\mathrm{~d}, J=246.9 \mathrm{~Hz}), 143.8,136.2(\mathrm{~d}, J=4.5 \mathrm{~Hz})$, 136.0, 135.0, 129.6, 128.7, $128.3(\mathrm{~d}, J=8.2 \mathrm{~Hz}), 128.2,127.9,126.6,122.9,115.4(\mathrm{~d}$, $J=21.3 \mathrm{~Hz}), 85.5,79.7,63.5,49.1,36.4,21.5 \mathrm{ppm}$. 1 carbon was not observed due to incidental equivalence.

IR (ATR, in $\left.\mathrm{CDCl}_{3}\right)$ v 3490 (w, broad), 3028 (w), 2922 (w), 1602 (w), 1507 (m), 1448 (w), 1343 (m), 1327 (m), 1306 (w), 1222 (m), 1155 (s), 1118 (w), 1092 (m), 1016 (w) cm $\mathrm{cm}^{-1}$.

HRMS (ESI, m/z) calcd. for $\mathrm{C}_{26} \mathrm{H}_{24} \mathrm{NO}_{3} \mathrm{SFNa}^{+}$: 472.1353, found: 472.1348 .

(E)-4-(N-cinnamyl-4-methylphenylsulfonamido)-1-(4-fluorophenyl)but-2-yn-1-yl acetate s10

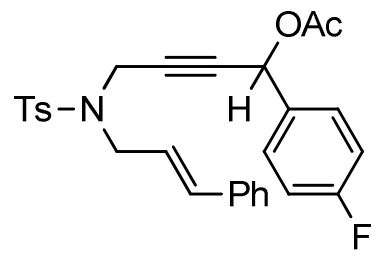

According to GP-2 s9 (1.35 g, $3 \mathrm{mmol})$ was dissolved in $\mathrm{CH}_{2} \mathrm{Cl}_{2}(3 \mathrm{~mL})$, treated with acetic anhydride $(0.85 \mathrm{~mL}, 9 \mathrm{mmol})$, DMAP $(18.3 \mathrm{mg}, 0.15 \mathrm{mmol})$ and triethylamine $(0.62 \mathrm{~mL}$, $4.5 \mathrm{mmol}$ ) to yield $\mathbf{s 1 0}$ after column chromatography on silica gel (petroleum ether/EtOAc $5 / 1)$.

Yield: $1.38 \mathrm{~g}(2.80 \mathrm{mmol}, 93 \%)$.

Physical State: yellow oil.

$\mathbf{R}_{\mathbf{f}}$ Value: 0.29 (petroleum ether/EtOAc - 5/1).

${ }^{1} \mathbf{H}$ NMR (Avance $400 \mathrm{MHz}, \mathrm{CDCl}_{3}$ ) $\delta 7.72$ - $7.74(\mathrm{~m}, 2 \mathrm{H}), 7.22$ - $7.30(\mathrm{~m}, 7 \mathrm{H}), 7.19-7.21$ $(\mathrm{m}, 2 \mathrm{H}), 6.99-7.03(\mathrm{~m}, 2 \mathrm{H}), 6.51(\mathrm{~d}, J=15.8 \mathrm{~Hz}, 1 \mathrm{H}), 6.18(\mathrm{~s}, 1 \mathrm{H}), 6.06(\mathrm{dt}, J=6.9$, $15.8 \mathrm{~Hz}, 1 \mathrm{H}), 4.17-4.28(\mathrm{~m}, 2 \mathrm{H}), 3.91-4.01(\mathrm{~m}, 2 \mathrm{H}), 2.35$ (s, 3H), 2.04 (s, 3H) ppm.

${ }^{19}$ F NMR (Avance $376 \mathrm{MHz}, \mathrm{CDCl}_{3}$ ) $\delta-112.1 \mathrm{ppm}$.

${ }^{13}$ C NMR (Avance $101 \mathrm{MHz}, \mathrm{CDCl}_{3}$ ) $\delta$ 169.5, $163.0(\mathrm{~d}, J=248.6 \mathrm{~Hz}$ ), 143.6, 136.1, 136.0, 135.0, 132.5 (d, $J=3.1 \mathrm{~Hz}), 129.6$ (d, $J=8.2 \mathrm{~Hz}), 129.6,128.7,128.1,127.8,126.6,122.8$, $115.6,82.2,80.5,64.6,49.0,36.4,21.5,21.0 \mathrm{ppm}$.

IR (ATR, in $\left.\mathrm{CDCl}_{3}\right)$ v 3029 (w), 1738 (m), 1605 (w), 1510 (m), 1448 (w), 1369 (w), 1348 (m), 1222 (s), 1159 (s), 1094 (m), 1055 (w), 1015 (m) $\mathrm{cm}^{-1}$. 
HRMS (ESI, m/z) calcd. for $\mathrm{C}_{28} \mathrm{H}_{26} \mathrm{NO}_{4} \mathrm{SFNa}^{+}$: 514.1459, found: 514.1463.

\subsection{Preparation of $(E)-4-(N$-cinnamyl-4-methylphenylsulfonamido)-1-(4-chloro- phenyl)but-2-yn-1-yl acetate s12}
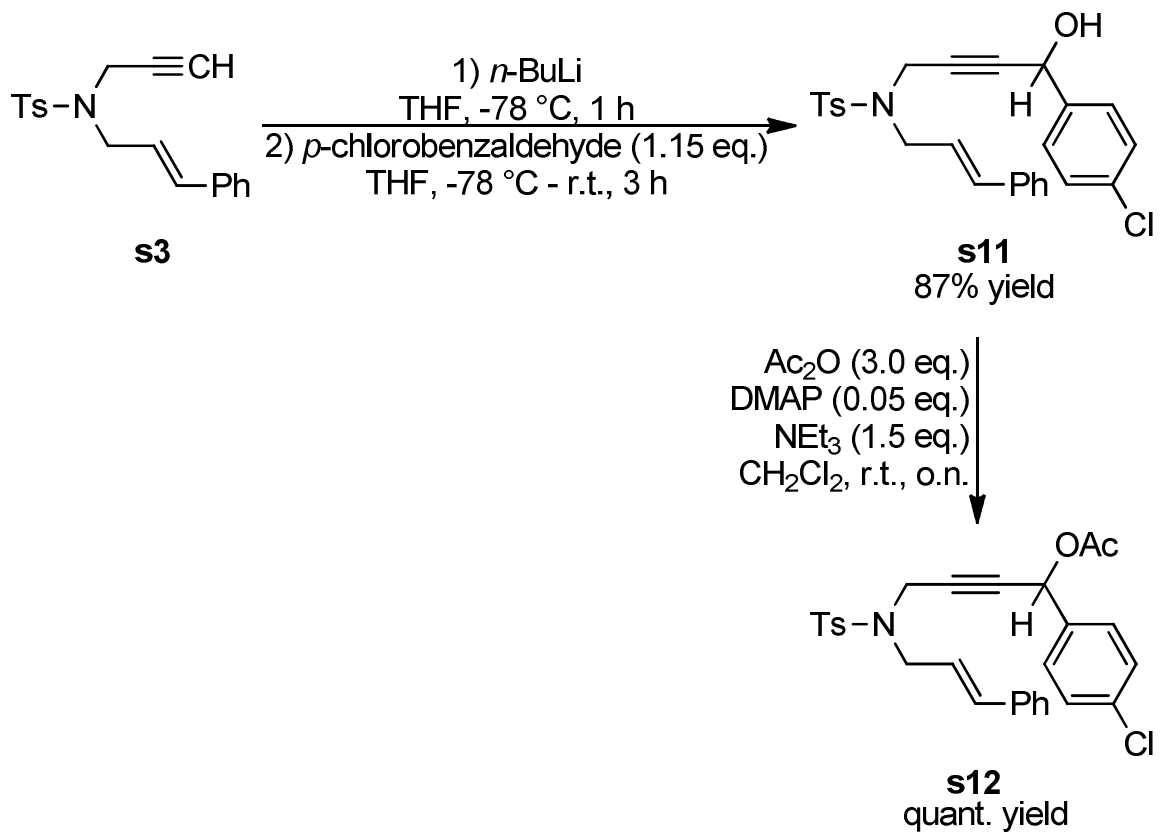

$N$-cinnamyl- $N$-(4-(4-chlorophenyl)-4-hydroxybut-2-yn-1-yl)-4-methylbenzenesulfonamide s11

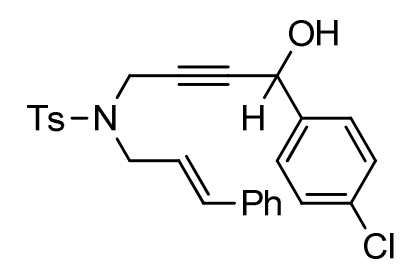

According to GP-1 s3 (1.30 g, 4 mmol) was dissolved in THF (40 mL), treated with $n$-BuLi (1.6 $\mathrm{M}$ in hexanes, $2.5 \mathrm{~mL}, 4 \mathrm{mmol})$ and $p$-chlorobenzaldehyde $(0.65 \mathrm{~g}, 4.6 \mathrm{mmol})$ in $\mathrm{THF}$ (3 mL) to yield s11 after column chromatography on silica gel (petroleum ether/EtOAc - 3/1).

Yield: $1.62 \mathrm{~g}(3.47 \mathrm{mmol}, 87 \%)$.

Physical State: yellow solid.

$\mathbf{R}_{\mathbf{f}}$ Value: 0.29 (petroleum ether/EtOAc - 3/1). 
${ }^{1} \mathbf{H}$ NMR (Avance $400 \mathrm{MHz}, \mathrm{CDCl}_{3}$ ) $\delta 7.71$ - $7.73(\mathrm{~m}, 2 \mathrm{H}), 7.24-7.31(\mathrm{~m}, 7 \mathrm{H}), 7.20$ - 7.24 (m, 4H), $6.48(\mathrm{~d}, J=15.8 \mathrm{~Hz}, 1 \mathrm{H}), 6.04(\mathrm{dt}, J=6.8,15.7 \mathrm{~Hz}, 1 \mathrm{H}), 5.17(\mathrm{~s}, 1 \mathrm{H}), 4.17$ (d, $J=1.5 \mathrm{~Hz}, 2 \mathrm{H}), 3.90-3.99(\mathrm{~m}, 2 \mathrm{H}), 2.35(\mathrm{~s}, 4 \mathrm{H}) \mathrm{ppm}$.

${ }^{13} \mathbf{C}$ NMR (Avance $101 \mathrm{MHz}, \mathrm{CDCl}_{3}$ ) $\delta$ 143.9, 138.7, 136.0, 135.0, 134.2, 129.6, 128.7, 128.2, 127.9, 127.9, 126.6, 122.8, 85.3, 79.9, 63.5, 49.1, 36.4, 21.6 ppm. 2 carbons were not observed due to incidental equivalence.

IR (ATR, in $\left.\mathrm{CDCl}_{3}\right)$ v 3474 (w, broad), 3028 (w), 2919 (w), 1654 (w), 1597 (w), 1578 (w), 1489 (m), 1447 (w), 1402 (w), 1343 (m), 1327 (m), 1306 (w), 1155 (s), 1118 (m), 1089 (s), $1056(\mathrm{w}), 1013(\mathrm{w}) \mathrm{cm}^{-1}$.

HRMS (ESI, m/z) calcd. for $\mathrm{C}_{26} \mathrm{H}_{24} \mathrm{NO}_{3} \mathrm{ClSNa}^{+}$: 488.1058, found: 488.1036 .

(E)-4-(N-cinnamyl-4-methylphenylsulfonamido)-1-(4-chlorophenyl)but-2-yn-1-yl acetate s12

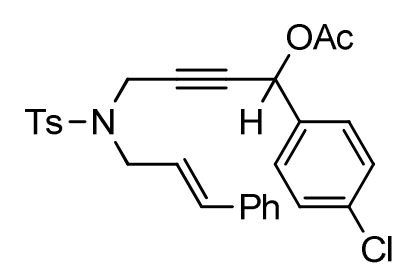

According to GP-2 s11 (1.17 g, $2.5 \mathrm{mmol})$ was dissolved in $\mathrm{CH}_{2} \mathrm{Cl}_{2}(3 \mathrm{~mL})$, treated with acetic anhydride $(0.71 \mathrm{~mL}, 7.5 \mathrm{mmol})$, DMAP $(15.3 \mathrm{mg}, 0.13 \mathrm{mmol})$ and triethylamine $(0.52 \mathrm{~mL}, 3.8 \mathrm{mmol})$ to yield $\mathbf{s 1 2}$ after column chromatography on silica gel (petroleum ether/EtOAc - 5/1).

Yield: $1.27 \mathrm{~g}$ (2.5 mmol, quant.).

Physical State: yellow oil.

$\mathbf{R}_{\mathbf{f}}$ Value: 0.31 (petroleum ether/EtOAc - 5/1).

${ }^{1} \mathbf{H}$ NMR (Avance $400 \mathrm{MHz}, \mathrm{CDCl}_{3}$ ) $\delta 7.71$ - $7.73(\mathrm{~m}, 2 \mathrm{H}), 7.25$ - $7.29(\mathrm{~m}, 6 \mathrm{H}), 7.17$ - 7.24 $(\mathrm{m}, 5 \mathrm{H}), 6.50(\mathrm{~d}, J=15.8 \mathrm{~Hz}, 1 \mathrm{H}), 6.17(\mathrm{~s}, 1 \mathrm{H}), 6.04(\mathrm{dt}, J=6.9,15.7 \mathrm{~Hz}, 1 \mathrm{H}), 4.16-4.26$ $(\mathrm{m}, 2 \mathrm{H}), 3.90-4.00(\mathrm{~m}, 2 \mathrm{H}), 2.32(\mathrm{~s}, 3 \mathrm{H}), 2.02(\mathrm{~s}, 3 \mathrm{H}) \mathrm{ppm}$.

${ }^{13}$ C NMR (Avance $101 \mathrm{MHz}, \mathrm{CDCl}_{3}$ ) $\delta$ 169.2, 143.5, 135.9, 135.8, 135.0, 134.9, 134.7, $129.4,128.9,128.7,128.5,128.0,127.6,126.4,122.6,81.9,80.5,64.4,48.9,36.3,21.4,20.8$ ppm. 
IR (ATR, in $\mathrm{CDCl}_{3}$ ) v 3029 (w), 1740 (m), 1598 (w), 1492 (w), 1448 (w), 1369 (w), 1348 (m), 1223 (s), 1160 (s), 1091 (m), 1056 (w), 1015 (m) $\mathrm{cm}^{-1}$.

HRMS (ESI, m/z) calcd. for $\mathrm{C}_{28} \mathrm{H}_{26} \mathrm{NO}_{4} \mathrm{ClSNa}^{+}$: 530.1163, found: 530.1167 .

\subsection{Preparation of $(E)-4-(N$-cinnamyl-4-methylphenylsulfonamido)-1-(4-bromo-} phenyl)but-2-yn-1-yl acetate s14
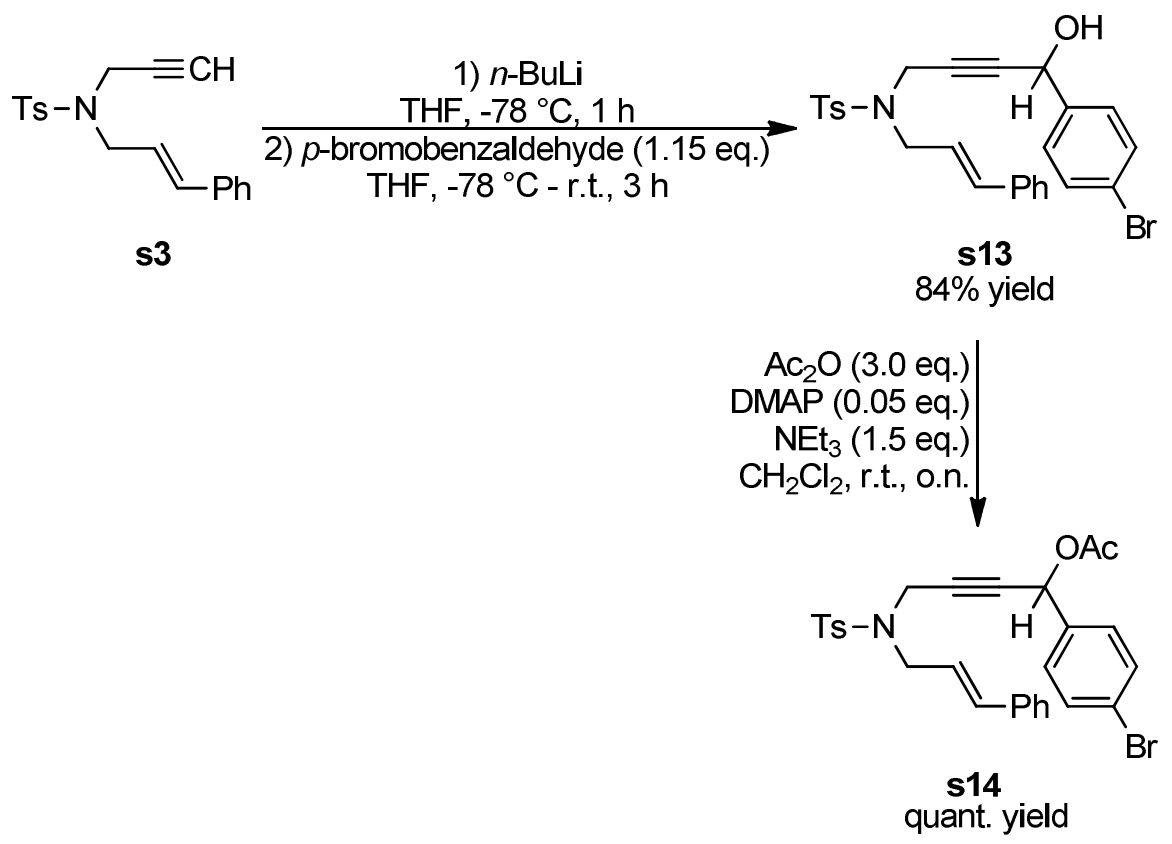

$N$-cinnamyl- $N$-(4-(4-bromophenyl)-4-hydroxybut-2-yn-1-yl)-4-methylbenzenesulfonamide s13

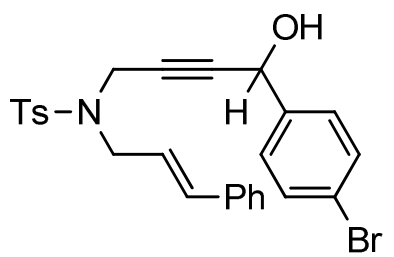

According to GP-1 s3 (1.30 g, 4 mmol) was dissolved in THF (40 mL), treated with $n$-BuLi (1.6 $\mathrm{M}$ in hexanes, $2.5 \mathrm{~mL}, 4 \mathrm{mmol})$ and $p$-bromobenzaldehyde $(0.85 \mathrm{~g}, 4.6 \mathrm{mmol})$ in THF (3 mL) to yield s13 after column chromatography on silica gel (petroleum ether/EtOAc - 3/1).

Yield: $1.71 \mathrm{~g}(3.35 \mathrm{mmol}, 84 \%)$.

Physical State: yellow/orange solid. 
$\mathbf{R}_{\mathbf{f}}$ Value: 0.29 (petroleum ether/EtOAc - 3/1).

${ }^{1} \mathbf{H}$ NMR (Avance $400 \mathrm{MHz}, \mathrm{CDCl}_{3}$ ) $\delta 7.71$ - $7.73(\mathrm{~m}, 2 \mathrm{H}), 7.42-7.44(\mathrm{~m}, 2 \mathrm{H}), 7.24-7.31$ (m, 5H), $7.20-7.22(\mathrm{~m}, 2 \mathrm{H}), 7.15-7.17(\mathrm{~m}, 2 \mathrm{H}), 6.48(\mathrm{~d}, J=15.8 \mathrm{~Hz}, 1 \mathrm{H}), 6.05(\mathrm{dt}, J=6.9$, $15.7 \mathrm{~Hz}, 1 \mathrm{H}), 5.16(\mathrm{~s}, 1 \mathrm{H}), 4.18(\mathrm{~d}, J=1.1 \mathrm{~Hz}, 2 \mathrm{H}), 3.90-3.40(\mathrm{~m}, 2 \mathrm{H}), 2.36(\mathrm{~s}, 3 \mathrm{H}), 2.28$ (s, 1H) $\mathrm{ppm}$.

${ }^{13}$ C NMR (Avance $101 \mathrm{MHz}, \mathrm{CDCl}_{3}$ ) $\delta$ 143.9, 139.3, 136.1, 136.1, 135.0, 131.7, 129.7, $128.7,128.2,128.2,127.9,126.6,122.9,122.4,85.2,80.0,63.6,49.1,36.4,21.6$ ppm.

IR (ATR, in $\mathrm{CDCl}_{3}$ ) v 3477 (w, broad), 3027 (w), 2920 (w), 1596 (w), 1485 (w), 1447 (w), 1400 (w), 1343 (m), 1327 (m), 1306 (w), 1242 (w), 1155 (s), 1118 (w), 1092 (m), 1069 (m), $1009(\mathrm{~m}) \mathrm{cm}^{-1}$.

HRMS (ESI, m/z) calcd. for $\mathrm{C}_{26} \mathrm{H}_{24} \mathrm{NO}_{3} \mathrm{BrSNa}^{+}$: 532.0552, found: 532.0558 .

(E)-4-(N-cinnamyl-4-methylphenylsulfonamido)-1-(4-bromophenyl)but-2-yn-1-yl acetate s14

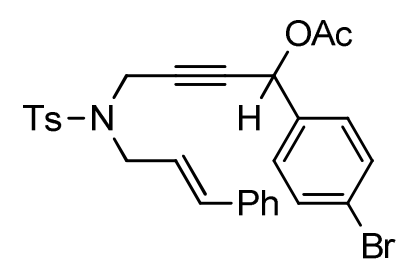

According to GP-2 s13 (1.53 g, $3.0 \mathrm{mmol})$ was dissolved in $\mathrm{CH}_{2} \mathrm{Cl}_{2}(3 \mathrm{~mL})$, treated with acetic anhydride $(0.85 \mathrm{~mL}, 9.0 \mathrm{mmol})$, DMAP $(18.3 \mathrm{mg}, 0.15 \mathrm{mmol})$ and triethylamine $(0.62 \mathrm{~mL}, 4.5 \mathrm{mmol})$ to yield $\mathbf{s 1 4}$ after column chromatography on silica gel (petroleum ether/EtOAc - 5/1).

Yield: $1.71 \mathrm{~g}$ (2.5 mmol, quant.).

Physical State: yellow oil.

$\mathbf{R}_{\mathbf{f}}$ Value: 0.30 (petroleum ether/EtOAc - 5/1).

${ }^{1} \mathbf{H}$ NMR (Avance $400 \mathrm{MHz}, \mathrm{CDCl}_{3}$ ) $\delta 7.71-7.73(\mathrm{~m}, 2 \mathrm{H}), 7.42-7.46(\mathrm{~m}, 2 \mathrm{H}), 7.26-7.31$ (m, 4H), $7.21-7.25(\mathrm{~m}, 1 \mathrm{H}), 7.18-7.20(\mathrm{~m}, 2 \mathrm{H}), 7.13-7.16(\mathrm{~m}, 2 \mathrm{H}), 6.50$ (d, J=15.8 Hz, $1 \mathrm{H}), 6.15(\mathrm{~s}, 1 \mathrm{H}), 6.05(\mathrm{dt}, J=6.9,15.7 \mathrm{~Hz}, 1 \mathrm{H}), 4.16-4.27(\mathrm{~m}, 2 \mathrm{H}), 3.90-4.00(\mathrm{~m}, 2 \mathrm{H})$, 2.34 (s, 3H), 2.04 (s, 3H) ppm. 
${ }^{13}$ C NMR (Avance $101 \mathrm{MHz}, \mathrm{CDCl}_{3}$ ) $\delta$ 169.3, 143.6, 136.0, 135.9, 135.5, 135.0, 131.7, $129.5,129.2,128.6,128.1,127.7,126.5,123.1,122.7,81.8,80.1,64.5,49.0,36.3,21.5,20.9$ ppm.

IR (ATR, in $\mathrm{CDCl}_{3}$ ) v 3029 (w), 1739 (m), 1596 (w), 1487 (w), 1448 (w), 1407 (w), 1368 (w), 1348 (m), 1306 (w), 1222 (s), 1159 (s), 1093 (m), 1071 (m), 1011 (m) cm cm $^{-1}$

HRMS (ESI, m/z) calcd. for $\mathrm{C}_{28} \mathrm{H}_{26} \mathrm{NO}_{4} \mathrm{BrSNa}^{+}$: 574.0658, found: 574.0665 .

4.8 Preparation of (E)-methyl 4-(1-acetoxy-4-( $N$-cinnamyl-4-methylphenylsulfonamido)but-2-yn-1-yl)benzoate s16

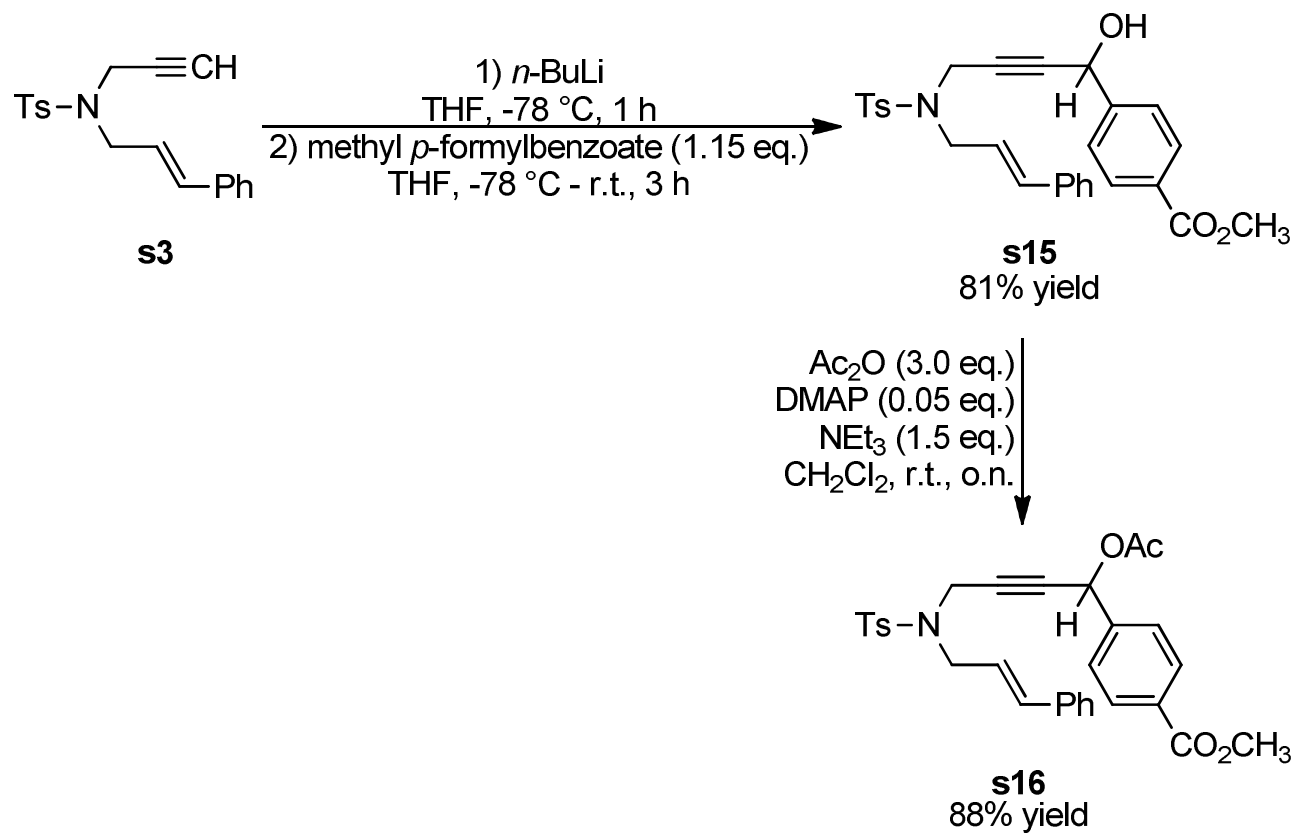

(E)-methyl 4-(4-(N-cinnamyl-4-methylphenylsulfonamido)-1-hydroxybut-2-yn-1-yl)benzoate $\mathbf{s 1 5}$

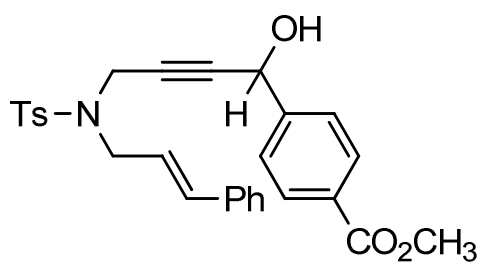

According to GP-1 s3 (1.30 g, $4 \mathrm{mmol})$ was dissolved in THF (40 mL), treated with $n$-BuLi (1.6 $\mathrm{M}$ in hexanes, $2.5 \mathrm{~mL}, 4 \mathrm{mmol})$ and methyl $p$-formylbenzoate $(0.76 \mathrm{~g}, 4.6 \mathrm{mmol})$ in $\mathrm{THF}$ 
(3 $\mathrm{mL})$ to yield $\mathbf{s 1 5}$ after column chromatography on silica gel (petroleum ether/EtOAc $2 / 1 \rightarrow 1.5 / 1)$.

Yield: $1.60 \mathrm{~g}(3.26 \mathrm{mmol}, 81 \%)$.

Physical State: yellow solid.

$\mathbf{R}_{\mathbf{f}}$ Value: 0.23 (petroleum ether/EtOAc - 2/1).

${ }^{1} \mathbf{H}$ NMR (Avance $400 \mathrm{MHz}, \mathrm{CDCl}_{3}$ ) $\delta 7.95$ - $7.97(\mathrm{~m}, 2 \mathrm{H}), 7.70$ - $7.72(\mathrm{~m}, 2 \mathrm{H}), 7.34$ - 7.36 $(\mathrm{m}, 2 \mathrm{H}), 7.22-7.29(\mathrm{~m}, 5 \mathrm{H}), 7.18-7.20(\mathrm{~m}, 2 \mathrm{H}), 6.45(\mathrm{~d}, J=15.8 \mathrm{~Hz}, 1 \mathrm{H}), 6.02(\mathrm{dt}, J=7.3$, $14.6 \mathrm{~Hz}, 1 \mathrm{H}), 5.26(\mathrm{~s}, 1 \mathrm{H}), 4.16(\mathrm{~s}, 2 \mathrm{H}), 3.87-3.94(\mathrm{~m}, 5 \mathrm{H}), 3.07$ (s, 1H), 2.33 (s, 3H) ppm.

${ }^{13} \mathrm{C}$ NMR (Avance $101 \mathrm{MHz}, \mathrm{CDCl}_{3}$ ) $\delta$ 166.8, 145.2, 143.8, 136.0, 135.9, 134.9, 129.8, 129.6, 128.6, 128.1, 127.8, 126.5, 126.3, 122.7, 85.2, 79.9, 63.6, 52.2, 49.0, 36.3, 21.5 ppm. 1 carbon was not observed due to incidental equivalence.

IR (ATR, in $\left.\mathrm{CDCl}_{3}\right) \vee 3476$ (w, broad), 3028 (w), 2952 (w), 1717 (m), $1611(\mathrm{w}), 1598$ (w), 1578 (w), 1495 (w), 1436 (w), 1412 (w), 1345 (w), 1308 (w), 1277 (s), 1190 (w), 1157 (s), $1102(\mathrm{~m}), 1017(\mathrm{w}) \mathrm{cm}^{-1}$.

HRMS (ESI, m/z) calcd. for $\mathrm{C}_{28} \mathrm{H}_{27} \mathrm{NO}_{5} \mathrm{SNa}^{+}:$512.1502, found: 512.1520.

(E)-methyl 4-(1-acetoxy-4-(N-cinnamyl-4-methylphenylsulfonamido)but-2-yn-1-yl)benzoate s16

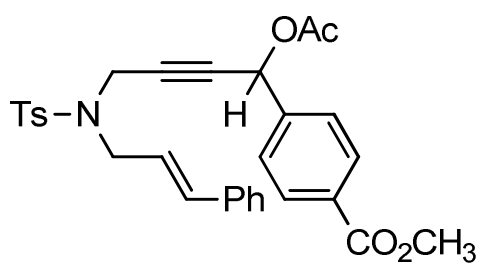

According to GP-2 s15 (0.98 g, $2.0 \mathrm{mmol})$ was dissolved in $\mathrm{CH}_{2} \mathrm{Cl}_{2}(2 \mathrm{~mL})$, treated with acetic anhydride $(0.57 \mathrm{~mL}, 6.0 \mathrm{mmol})$, DMAP $(12.2 \mathrm{mg}, 0.1 \mathrm{mmol})$ and triethylamine $(0.42 \mathrm{~mL}, 3.0 \mathrm{mmol})$ to yield $\mathbf{s 1 6}$ after column chromatography on silica gel (petroleum ether/EtOAc - 3/1).

Yield: $0.93 \mathrm{~g}(1.75 \mathrm{mmol}, 88 \%)$.

Physical State: yellow oil.

$\mathbf{R}_{\mathbf{f}}$ Value: 0.38 (petroleum ether/EtOAc - 5/1). 
${ }^{1} \mathbf{H}$ NMR (Avance $400 \mathrm{MHz}, \mathrm{CDCl}_{3}$ ) $\delta 8.00-8.02(\mathrm{~m}, 2 \mathrm{H}), 7.72-7.74(\mathrm{~m}, 2 \mathrm{H}), 7.33-7.35$ (m, 2H), $7.26-7.29(\mathrm{~m}, 4 \mathrm{H}), 7.23-7.25(\mathrm{~m}, 1 \mathrm{H}), 7.19-7.21(\mathrm{~m}, 2 \mathrm{H}), 6.49(\mathrm{~d}, J=15.8 \mathrm{~Hz}$, 1H), $6.23(\mathrm{~s}, 1 \mathrm{H}), 6.06(\mathrm{dt}, J=6.9,15.7 \mathrm{~Hz}, 1 \mathrm{H}), 4.18-4.28(\mathrm{~m}, 2 \mathrm{H}), 3.92-3.96(\mathrm{~m}, 5 \mathrm{H})$, $2.34(\mathrm{~s}, 3 \mathrm{H}), 2.07$ (s, 3H) ppm.

${ }^{13} \mathrm{C}$ NMR (Avance $101 \mathrm{MHz}, \mathrm{CDCl}_{3}$ ) $\delta$ 169.3, 166.4, 143.6, 141.2, 136.0, 135.9, 135.0, $130.6,129.9,129.6,128.6,128.1,127.7,127.3,126.5,122.7,81.8,80.8,64.6,52.3,49.0$, $36.3,21.5,20.8 \mathrm{ppm}$.

IR (ATR, in $\left.\mathrm{CDCl}_{3}\right)$ v 2953 (w), 1720 (s), 1613 (w), 1598 (w), 1579 (w), 1495 (w), 1436 (w), 1369 (w), 1348 (w), 1307 (w), 1279 (s), 1222 (s), 1160 (s), 1101 (m), 1017 (m) cm cm $^{-1}$

HRMS (ESI, m/z) calcd. for $\mathrm{C}_{30} \mathrm{H}_{29} \mathrm{NO}_{6} \mathrm{SNa}^{+}$: 554.1608, found: 554.1598.

\subsection{Preparation of (E)-4-(N-cinnamyl-4-methylphenylsulfonamido)-1-(3-methoxy-} phenyl)but-2-yn-1-yl acetate s18

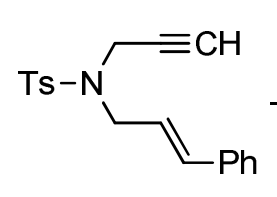

s3
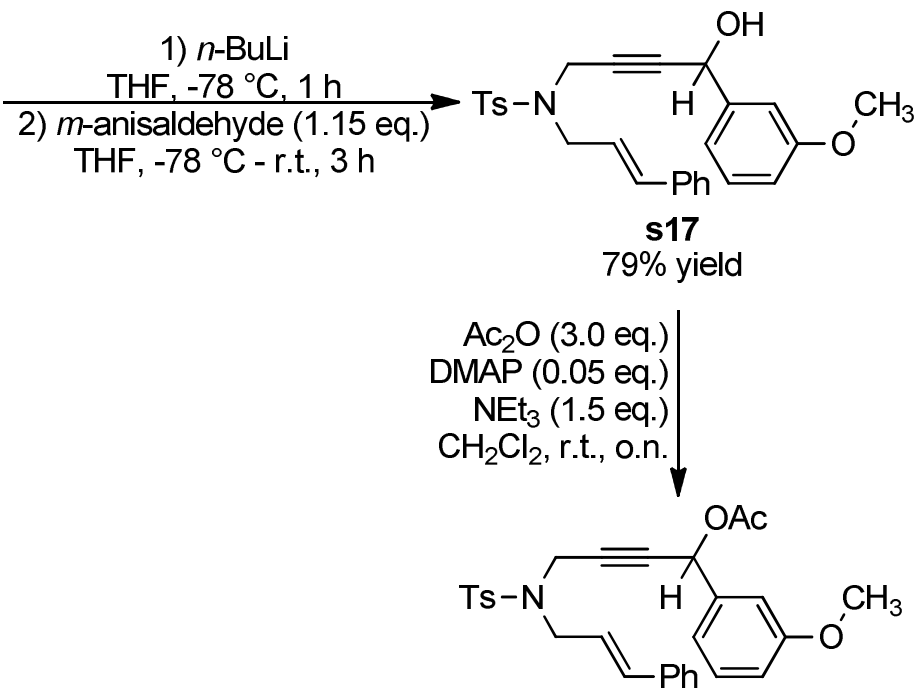

s18

94\% yield 

sulfonamide $\mathbf{s 1 7}$

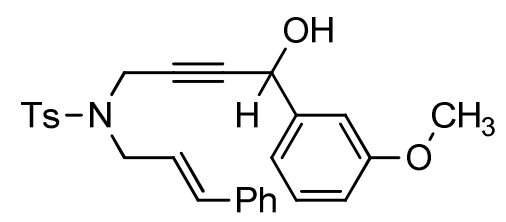

According to GP-1 s3 (1.30 g, 4 mmol) was dissolved in THF (40 mL), treated with $n$-BuLi (1.6 $\mathrm{M}$ in hexanes, $2.5 \mathrm{~mL}, 4 \mathrm{mmol})$ and $m$-anisaldehyde $(0.56 \mathrm{~mL}, 4.6 \mathrm{mmol})$ to yield $\mathbf{s} 17$ after column chromatography on silica gel (petroleum ether/EtOAc - 3/1).

Yield: $1.46 \mathrm{~g}(3.17 \mathrm{mmol}, 79 \%)$.

Physical State: orange oil.

$\mathbf{R}_{\mathbf{f}}$ Value: 0.23 (petroleum ether/EtOAc - 3/1).

${ }^{1} \mathbf{H}$ NMR (Avance $400 \mathrm{MHz}, \mathrm{CDCl}_{3}$ ) $\delta 7.70$ - $7.72(\mathrm{~m}, 2 \mathrm{H}), 7.20$ - 7.27 (m, 6H), 7.17 - 7.19 $(\mathrm{m}, 2 \mathrm{H}), 6.82-6.90(\mathrm{~m}, 3 \mathrm{H}), 6.47(\mathrm{~d}, J=15.8 \mathrm{~Hz}, 1 \mathrm{H}), 6.03(\mathrm{dt}, J=6.9,15.7 \mathrm{~Hz}, 1 \mathrm{H}), 5.15$ (s, 1H), $4.16(\mathrm{~s}, 2 \mathrm{H}), 3.94(\mathrm{~d}, J=6.8 \mathrm{~Hz}, 2 \mathrm{H}), 3.76(\mathrm{~s}, 3 \mathrm{H}), 2.48(\mathrm{~s}, 1 \mathrm{H}), 2.33$ (s, 3H) ppm.

${ }^{13} \mathrm{C}$ NMR (Avance $101 \mathrm{MHz}, \mathrm{CDCl}_{3}$ ) $\delta$ 159.7, 143.7, 141.9, 136.1, 135.9, 135.0, 129.6, 129.6, 128.6, 128.1, 127.8, 126.6, 122.8, 118.7, 113.7, 112.1, 85.7, 79.3, 64.1, 55.3, 48.9, $36.3,21.5 \mathrm{ppm}$.

IR (ATR, in $\left.\mathrm{CDCl}_{3}\right)$ v 3486 (w, broad), 3028 (w), 2920 (w), 2836 (w), 1599 (m), 1488 (m), 1451 (m), 1435 (m), 1343 (m), 1326 (m), 1259 (m), 1155 (s), 1118 (m), 1091 (m), 1041 (m), $1018(\mathrm{w}) \mathrm{cm}^{-1}$.

HRMS (ESI, m/z) calcd. for $\mathrm{C}_{27} \mathrm{H}_{27} \mathrm{NO}_{4} \mathrm{SNa}^{+}$: 484.1553, found: 484.1543.

\section{(E)-4-(N-cinnamyl-4-methylphenylsulfonamido)-1-(3-methoxyphenyl)but-2-yn-1-yl} acetate s18

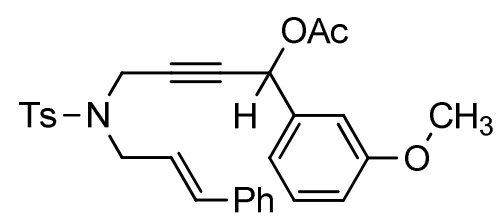

According to GP-2 s17 (1.38 g, $3.0 \mathrm{mmol})$ was dissolved in $\mathrm{CH}_{2} \mathrm{Cl}_{2}(3 \mathrm{~mL})$, treated with acetic anhydride $(0.85 \mathrm{~mL}, 9.0 \mathrm{mmol})$, DMAP $(18.3 \mathrm{mg}, 0.15 \mathrm{mmol})$ and triethylamine 
(0.62 $\mathrm{mL}, 4.5 \mathrm{mmol})$ to yield $\mathbf{s 1 8}$ after column chromatography on silica gel (petroleum ether/EtOAc - 4/1).

Yield: $1.42 \mathrm{~g}(2.82 \mathrm{mmol}, 94 \%)$.

Physical State: yellow oil.

$\mathbf{R}_{\mathbf{f}}$ Value: 0.34 (petroleum ether/EtOAc - 4/1).

${ }^{1}$ H NMR (Avance $400 \mathrm{MHz}, \mathrm{CDCl}_{3}$ ) $\delta 7.71-7.73(\mathrm{~m}, 2 \mathrm{H}), 7.26-7.30(\mathrm{~m}, 4 \mathrm{H}), 7.21-7.26$ (m, 2H), $7.17-7.19(\mathrm{~m}, 2 \mathrm{H}), 6.86-6.90(\mathrm{~m}, 3 \mathrm{H}), 6.50(\mathrm{~d}, J=15.8 \mathrm{~Hz}, 1 \mathrm{H}), 6.15(\mathrm{~s}, 1 \mathrm{H})$, $6.05(\mathrm{dt}, J=6.7,15.8 \mathrm{~Hz}, 1 \mathrm{H}), 4.16-4.26(\mathrm{~m}, 2 \mathrm{H}), 3.89-4.00(\mathrm{~m}, 2 \mathrm{H}), 3.78(\mathrm{~s}, 3 \mathrm{H}), 2.34(\mathrm{~s}$, $3 \mathrm{H}), 2.04(\mathrm{~s}, 3 \mathrm{H}) \mathrm{ppm}$.

${ }^{13}$ C NMR (Avance $101 \mathrm{MHz}, \mathrm{CDCl}_{3}$ ) $\delta$ 169.4, 159.7, 143.6, 137.9, 136.1, 135.8, 135.0, $129.7,129.5,128.6,128.0,127.7,126.5,122.7,119.7,114.2,113.3,82.4,80.0,65.1,55.2$, $48.8,36.3,21.5,20.9$ ppm.

IR (ATR, in $\left.\mathrm{CDCl}_{3}\right) \vee 2939$ (w), 1738 (m), 1600 (m), 1491 (m), 1437 (w), 1368 (w), 1347 (m), 1286 (m), 1223 (s), 1159 (s), 1092 (m), 1047 (m), 1016 (m) cm².

HRMS (ESI, m/z) calcd. for $\mathrm{C}_{29} \mathrm{H}_{29} \mathrm{NO}_{5} \mathrm{SNa}^{+}$: 526.1659, found: 526.1541 . 
4.10 Preparation of (E)-1-(3-acetamidophenyl)-4-( $N$-cinnamyl-4-methylphenylsulfonamido)but-2-yn-1-yl acetate s22

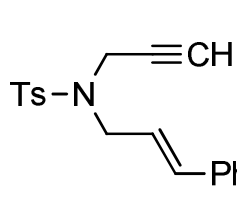

s3

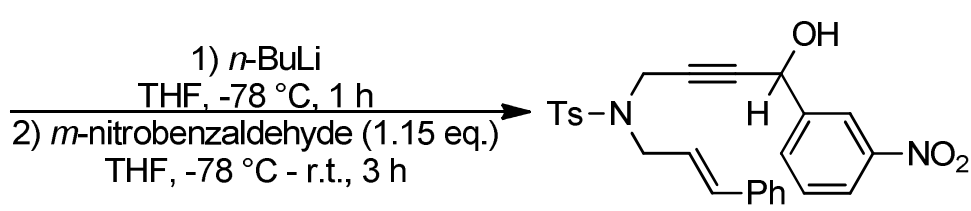

s19

$93 \%$ yield

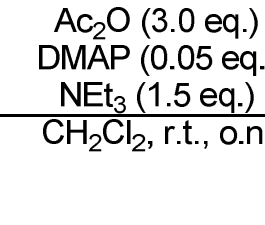

NEt $(1.5$ eq)

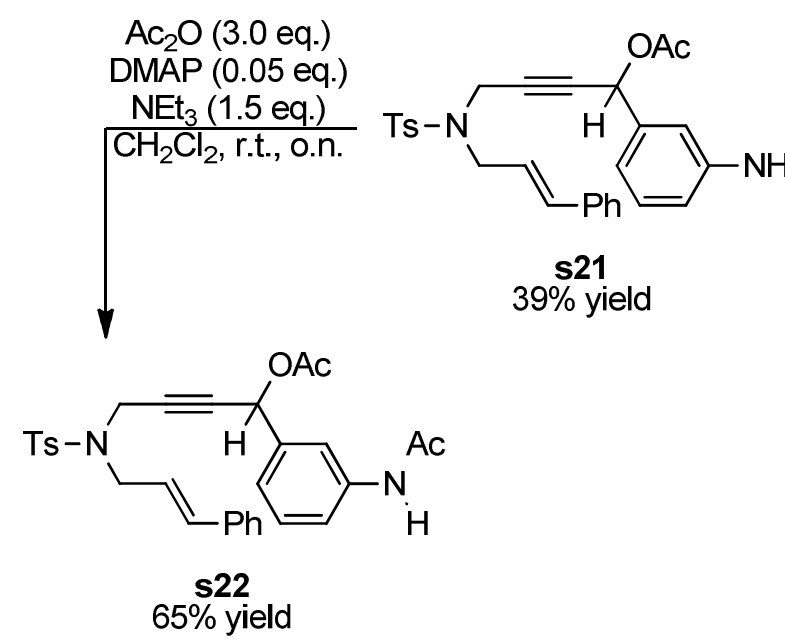

s20 quant. yield

$65 \%$ yield

$N$-cinnamyl- $N$-(4-hydroxy-4-(3-nitrophenyl)but-2-yn-1-yl)-4-methylbenzenesulfonamide s19<smiles>O=[N+]([O-])c1cccc(P/C=C\CN([13S])CC#CCO)c1</smiles>

According to GP-1 s3 (1.63 g, $5 \mathrm{mmol})$ was dissolved in THF (40 mL), treated with $n$-BuLi (1.6 $\mathrm{M}$ in hexanes, $3.2 \mathrm{~mL}, 5 \mathrm{mmol})$ and $m$-nitrobenzaldehyde $(0.87 \mathrm{~g}, 5.8 \mathrm{mmol})$ to yield $\mathbf{s 1 9}$ after column chromatography on silica gel (petroleum ether/EtOAc - 2/1).

Yield: $2.21 \mathrm{~g}(4.64 \mathrm{mmol}, 93 \%)$.

Physical State: orange solid.

$\mathbf{R}_{\mathbf{f}}$ Value: 0.34 (petroleum ether/EtOAc - 2/1).

${ }^{1}$ H NMR (Avance $400 \mathrm{MHz}$, acetone-d $\mathrm{d}_{6}$ ) $\delta 8.25$ (s, 1H), $8.18-8.20(\mathrm{~m}, 1 \mathrm{H}), 7.76-7.79(\mathrm{~m}$, $3 \mathrm{H}), 7.63-7.67(\mathrm{~m}, 1 \mathrm{H}), 7.28-7.37(\mathrm{~m}, 6 \mathrm{H}), 7.23-7.26(\mathrm{~m}, 1 \mathrm{H}), 6.59(\mathrm{~d}, J=15.8 \mathrm{~Hz}, 1 \mathrm{H})$, 
$6.15(\mathrm{dt}, J=6.8,15.8 \mathrm{~Hz}, 1 \mathrm{H}), 5.47(\mathrm{~s}, 1 \mathrm{H}), 5.33(\mathrm{~d}, J=5.4 \mathrm{~Hz}, 1 \mathrm{H}), 4.26(\mathrm{~s}, 2 \mathrm{H}), 4.01(\mathrm{~d}$, $J=6.7 \mathrm{~Hz}, 2 \mathrm{H}), 2.34(\mathrm{~s}, 3 \mathrm{H}) \mathrm{ppm}$.

${ }^{13}$ C NMR (Avance $101 \mathrm{MHz}$, acetone-d $\mathrm{d}_{6}$ ) $\delta$ 149.1, 144.9, 144.5, 137.3, 137.2, 135.2, 133.6, $130.5,130.4$, 129.4, 128.7, 128.6, 127.3, 124.1, 123.4, 121.8, 86.5, 80.1, 63.2, 49.7, 37.1, $21.4 \mathrm{ppm}$.

IR (ATR, in acetone-d d $_{6}$ v 3474 (w, broad), 3028 (w), 2922 (w), 1694 (m), 1597 (w), 1527 (s), 1495 (w), 1447 (w), 1347 (s), 1247 (m), 1197 (w), 1155 (s), 1119 (w), 1090 (m) cm c $^{-1}$.

HRMS (ESI, m/z) calcd. for $\mathrm{C}_{26} \mathrm{H}_{24} \mathrm{~N}_{2} \mathrm{O}_{5} \mathrm{SNa}^{+}$: 499.1298, found: 499.1279 .

(E)-4-(N-cinnamyl-4-methylphenylsulfonamido)-1-(3-nitrophenyl)but-2-yn-1-yl acetate s20

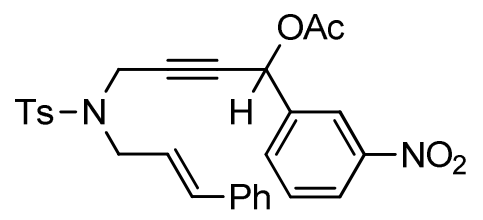

According to GP-2 s19 (2.21 g, $4.6 \mathrm{mmol})$ was dissolved in $\mathrm{CH}_{2} \mathrm{Cl}_{2}(5 \mathrm{~mL})$, treated with acetic anhydride $(1.32 \mathrm{~mL}, 13.9 \mathrm{mmol})$, DMAP $(28.3 \mathrm{mg}, 0.23 \mathrm{mmol})$ and triethylamine $(0.96 \mathrm{~mL}, 7.0 \mathrm{mmol})$ to yield $\mathbf{s 2 0}$ after column chromatography on silica gel (petroleum ether/EtOAc - 3/1).

Yield: $2.40 \mathrm{~g}$ (4.6 mmol, quant.).

Physical State: orange oil.

$\mathbf{R}_{\mathbf{f}}$ Value: 0.40 (petroleum ether/EtOAc - 3/1).

${ }^{1} \mathbf{H}$ NMR (Avance $400 \mathrm{MHz}, \mathrm{CDCl}_{3}$ ) 8.18 - 8.21 (m, 1H), 8.16 - $8.18(\mathrm{~m}, 1 \mathrm{H}), 7.72$ - 7.74 $(\mathrm{m}, 2 \mathrm{H}), 7.63-7.64(\mathrm{~m}, 1 \mathrm{H}), 7.50-7.54(\mathrm{~m}, 1 \mathrm{H}), 7.28-7.31(\mathrm{~m}, 4 \mathrm{H}), 7.24-7.27(\mathrm{~m}, 1 \mathrm{H})$, $7.21-7.22(\mathrm{~m}, 2 \mathrm{H}), 6.54(\mathrm{~d}, J=15.8 \mathrm{~Hz}, 1 \mathrm{H}), 6.27(\mathrm{~s}, 1 \mathrm{H}), 6.07(\mathrm{dt}, J=6.8,15.8 \mathrm{~Hz}, 1 \mathrm{H})$, $4.23(\mathrm{~d}, J=1.6 \mathrm{~Hz}, 2 \mathrm{H}), 3.99(\mathrm{~d}, J=6.6 \mathrm{~Hz}, 2 \mathrm{H}), 2.33(\mathrm{~s}, 3 \mathrm{H}), 2.09$ (s, 3H) ppm.

${ }^{13}$ C NMR (Avance $101 \mathrm{MHz}, \mathrm{CDCl}_{3}$ ) $\delta$ 169.2, 148.2, 143.6, 138.5, 136.0, 135.8, 134.9, $133.4,129.7,129.5,128.6,128.1,127.7,126.5,123.8,122.7,122.3,81.5,81.1,64.0,49.1$, $36.3,21.4,20.8 \mathrm{ppm}$.

IR (ATR, in $\left.\mathrm{CDCl}_{3}\right)$ v 3028 (w), 1743 (m), 1598 (w), 1531 (m), 1495 (w), 1447 (w), 1348 (s), 1306 (w), 1219 (s), 1159 (s), 1092 (m), 1054 (w), 1017 (m) cm ${ }^{-1}$. 
HRMS (ESI, m/z) calcd. for $\mathrm{C}_{28} \mathrm{H}_{26} \mathrm{~N}_{2} \mathrm{O}_{6} \mathrm{SNa}^{+}$: 541.1404, found: 541.1424.

(E)-1-(3-aminophenyl)-4-( $N$-cinnamyl-4-methylphenylsulfonamido)but-2-yn-1-yl acetate s21

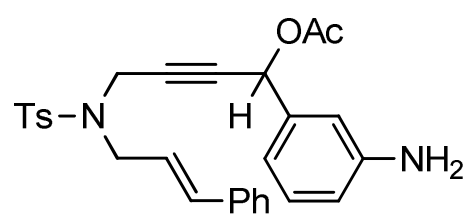

A $50 \mathrm{~mL}$ Schlenk tube was dried under high vacuum with a heat gun for 10 minutes. After cooling to room temperature $\mathbf{s 2 0}(2.41 \mathrm{~g}, 4.6 \mathrm{mmol})$ was dissolved in 1,4-dioxane $(10 \mathrm{~mL})$. Under a stream of $\mathrm{N}_{2} \mathrm{KOH}(65.1 \mathrm{mg}, 1.16 \mathrm{mmol})$, Zn-powder (1.0 g, $\left.15.3 \mathrm{mmol}\right)$, $\mathrm{RuCl}_{2}\left(\mathrm{PPh}_{3}\right)_{3}(222.4 \mathrm{mg}, 0.12 \mathrm{mmol})$ and $\mathrm{H}_{2} \mathrm{O}(0.67 \mathrm{~mL}, 37.1 \mathrm{mmol})$ were added The Schlenk tube was sealed and the reaction mixture was stirred for two days at room temperature. The reaction mixture was filtered through a pad of silica gel (solvent: EtOAc), evaporated and submitted to column chromatography on silica gel (petroleum ether/EtOAc 2/1) to yield the corresponding amine s21. For more information, see Lit [7].

Yield: $0.88 \mathrm{~g}(1.8 \mathrm{mmol}, 39 \%)$.

Physical State: brown oil.

$\mathbf{R}_{\mathbf{f}}$ Value: 0.34 (petroleum ether/EtOAc - 2/1).

${ }^{1} \mathbf{H}$ NMR (Avance $\left.400 \mathrm{MHz}, \mathrm{CDCl}_{3}\right) 7.71$ - $7.73(\mathrm{~m}, 2 \mathrm{H}), 7.26$ - 7.30 (m, 4H), 7.23 - 7.26 (m, 1H), $7.19-7.21(\mathrm{~m}, 2 \mathrm{H}), 7.08-7.12(\mathrm{~m}, 1 \mathrm{H}), 6.62-6.66(\mathrm{~m}, 3 \mathrm{H}), 6.50(\mathrm{~d}, J=15.8 \mathrm{~Hz}$, $1 \mathrm{H}), 6.10(\mathrm{~s}, 1 \mathrm{H}), 6.05(\mathrm{dt}, J=6.9,15.9 \mathrm{~Hz}, 1 \mathrm{H}), 4.15-4.27(\mathrm{~m}, 2 \mathrm{H}), 3.89-4.00(\mathrm{~m}, 2 \mathrm{H})$, $3.74(\mathrm{~s}, 2 \mathrm{H}), 2.36(\mathrm{~s}, 3 \mathrm{H}), 2.04(\mathrm{~s}, 3 \mathrm{H}) \mathrm{ppm}$.

${ }^{13} \mathrm{C}$ NMR (Avance $101 \mathrm{MHz}, \mathrm{CDCl}_{3}$ ) $\delta$ 169.6, 146.9, 143.7, 137.6, 136.2, 135.8, 135.1, 129.6, 128.7, 128.1, 127.8, 126.6, 122.8, 117.5, 115.6, 114.0, 82.7, 79.9, 65.3, 48.9, 36.4, 21.6, $21.1 \mathrm{ppm} .1$ carbon was not observed due to incidental equivalence.

IR (ATR, in $\left.\mathrm{CDCl}_{3}\right)$ v 3468 (w, broad), 3380 (w, broad), 3081 (w), 3027 (w), 2979 (w), 2923 (w), 2861 (w), 1736 (m), 1622 (m), 1609 (m), 1598 (m), 1530 (w), 1494 (m), 1463 (w), 1448 (w), 1369 (m), 1345 (m), 1306 (m), 1227 (s), 1160 (s), $1119(\mathrm{w}), 1092$ (w), 1017 (w) cm $\mathrm{cm}^{-1}$.

HRMS (ESI, m/z) calcd. for $\mathrm{C}_{28} \mathrm{H}_{28} \mathrm{~N}_{2} \mathrm{O}_{4} \mathrm{SNa}^{+}$: 511.1662, found: 511.1655. 

acetate s22

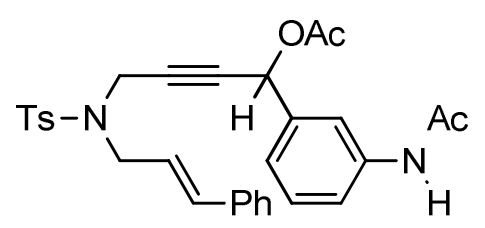

According to GP-2 s21 (0.57 g, $1.2 \mathrm{mmol})$ was dissolved in $\mathrm{CH}_{2} \mathrm{Cl}_{2}(5 \mathrm{~mL})$, treated with acetic anhydride $(0.33 \mathrm{~mL}, 3.5 \mathrm{mmol})$, DMAP $(7.1 \mathrm{mg}, 0.06 \mathrm{mmol})$ and triethylamine $(0.24 \mathrm{~mL}, 1.7 \mathrm{mmol})$ to yield $\mathbf{s 2 2}$ after column chromatography on silica gel (petroleum ether/EtOAc $-2 / 1 \rightarrow 1 / 1)$.

Yield: $0.40 \mathrm{~g}(0.75 \mathrm{mmol}, 65 \%)$.

Physical State: pale yellow oil.

$\mathbf{R}_{\mathbf{f}}$ Value: 0.29 (petroleum ether/EtOAc - 1/1).

${ }^{1} \mathbf{H}$ NMR (Avance $400 \mathrm{MHz}, \mathrm{CDCl}_{3}$ ) $8.00(\mathrm{~s}, 1 \mathrm{H}), 7.71-7.73(\mathrm{~m}, 2 \mathrm{H}), 7.70(\mathrm{~s}, 1 \mathrm{H})$, $7.58-7.60(\mathrm{~m}, 1 \mathrm{H}), 7.19-7.28(\mathrm{~m}, 8 \mathrm{H}), 6.99-7.01(\mathrm{~m}, 1 \mathrm{H}), 6.51(\mathrm{~d}, J=15.8 \mathrm{~Hz}, 1 \mathrm{H}), 6.15$ (s, 1H), 6.02 (dt, J=6.9, $15.7 \mathrm{~Hz}, 1 \mathrm{H}), 4.14-4.25(\mathrm{~m}, 2 \mathrm{H}), 3.91-4.00(\mathrm{~m}, 2 \mathrm{H}), 2.35$ (s, 3H), $2.15(\mathrm{~s}, 3 \mathrm{H}), 2.03(\mathrm{~s}, 3 \mathrm{H}) \mathrm{ppm}$.

${ }^{13}$ C NMR (Avance $101 \mathrm{MHz}, \mathrm{CDCl}_{3}$ ) $\delta$ 169.6, 168.8, 143.9, 138.7, 137.3, 136.1, 135.7, 135.1, 129.7, 129.2, 128.6, 128.1, 127.7, 126.6, 123.0, 122.6, 120.3, 118.9, 82.4, 80.4, 65.1, $49.0,36.4,24.5,21.5,21.0 \mathrm{ppm}$.

IR (ATR, in $\left.\mathrm{CDCl}_{3}\right) \vee 3344$ (w, broad), 3150 (w), 3028 (w), 2978 (w), 2925 (w), 2860 (w), 1737 (m), 1672 (m), 1597 (m), 1548 (m), 1490 (m), 1440 (m), 1369 (m), 1345 (m), 1327 (m), 1306 (m), 1222 (s), 1157 (s), $1120(\mathrm{w}), 1091(\mathrm{~m}), 1016(\mathrm{~m}) \mathrm{cm}^{-1}$.

HRMS (ESI, m/z) calcd. for $\mathrm{C}_{30} \mathrm{H}_{30} \mathrm{~N}_{2} \mathrm{O}_{5} \mathrm{SNa}^{+}:$553.1768, found: 553.1754. 
4.11 Preparation of $(E)$-4-( $N$-cinnamyl-4-methylphenylsulfonamido)-1,1-bis(4fluorophenyl)but-2-yn-1-yl acetate s24

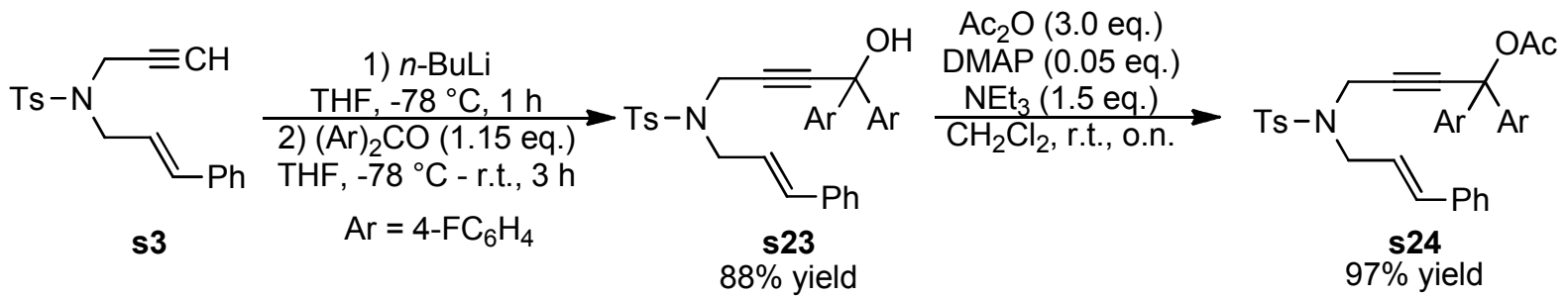

$N$-(4,4-bis(4-fluorophenyl)-4-hydroxybut-2-yn-1-yl)-N-cinnamyl-4methylbenzenesulfonamide $\mathbf{s 2 3}$

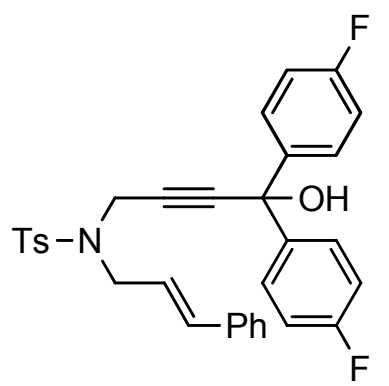

According to GP-1 s3 (0.98 g, $3 \mathrm{mmol})$ was dissolved in THF (50 mL), treated with $n$-BuLi (1.6 $\mathrm{M}$ in hexanes, $1.9 \mathrm{~mL}, 3 \mathrm{mmol})$ and 4,4'-difluorobenzophenone (0.75 g, $3.5 \mathrm{mmol})$ in THF ( $5 \mathrm{~mL}$ ) to yield $\mathbf{s 2 3}$ after column chromatography on silica gel (petroleum ether/EtOAc $5 / 1 \rightarrow 3 / 1)$.

Yield: $1.43 \mathrm{~g}(2.63 \mathrm{mmol}, 88 \%)$.

Physical State: pale yellow oil.

$\mathbf{R}_{\mathbf{f}}$ Value: 0.34 (petroleum ether/EtOAc - 5/1).

${ }^{1} \mathbf{H}$ NMR (Avance $400 \mathrm{MHz}, \mathrm{CDCl}_{3}$ ) $\delta 7.71$ - $7.73(\mathrm{~m}, 2 \mathrm{H}), 7.27$ - $7.32(\mathrm{~m}, 6 \mathrm{H}), 7.22$ - 7.26 (m, 3H), $7.14-7.16(\mathrm{~m}, 2 \mathrm{H}), 6.93-6.98(\mathrm{~m}, 4 \mathrm{H}), 6.42(\mathrm{~d}, J=15.8 \mathrm{~Hz}, 1 \mathrm{H}), 6.06(\mathrm{dt}, J=6.8$, $15.8 \mathrm{~Hz}, 1 \mathrm{H}), 4.26(\mathrm{~s}, 2 \mathrm{H}), 3.95(\mathrm{~d}, J=6.8 \mathrm{~Hz}, 2 \mathrm{H}), 2.48-2.50(\mathrm{~m}, 1 \mathrm{H}), 2.32(\mathrm{~s}, 3 \mathrm{H}) \mathrm{ppm}$.

${ }^{19}$ F NMR (Avance $376 \mathrm{MHz}, \mathrm{CDCl}_{3}$ ) $\delta-114.3 \mathrm{ppm}$.

${ }^{13} \mathrm{C}$ NMR (Avance $\left.101 \mathrm{MHz}, \mathrm{CDCl}_{3}\right) \delta 162.3(\mathrm{~d}, J=247 \mathrm{~Hz}), 143.9,140.3(\mathrm{~d}$, $J=3 \mathrm{~Hz}), 136.2,136.0,135.2,129.7,128.8,128.3,128.0,127.8$ (d, $J=8 \mathrm{~Hz}), 126.6,122.8$, $115.2(\mathrm{~d}, J=21 \mathrm{~Hz}), 88.2,80.8,73.3,49.3,36.5,21.6 \mathrm{ppm}$. 
IR (ATR, in $\left.\mathrm{CDCl}_{3}\right) \vee 3471$ (w, broad), 3029 (w), 1601 (m), 1504 (m), 1345 (m), 1224 (m), 1155 (s), $1094(\mathrm{~m}), 1013(\mathrm{~m}) \mathrm{cm}^{-1}$.

HRMS (ESI, m/z) calcd. for $\mathrm{C}_{32} \mathrm{H}_{27} \mathrm{~F}_{2} \mathrm{NO}_{3} \mathrm{SNa}^{+}$: 566.1534, found: 566.1527.

\section{(E)-4-(N-cinnamyl-4-methylphenylsulfonamido)-1,1-bis(4-fluorophenyl)but-2-yn-1-yl} acetate $\mathbf{s 2 4}$

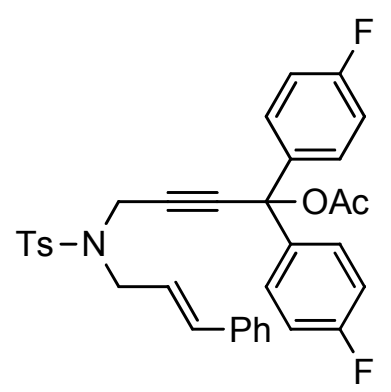

According to GP-2 s23 (1.38 g, $2.5 \mathrm{mmol})$ was dissolved in $\mathrm{CH}_{2} \mathrm{Cl}_{2}(3 \mathrm{~mL})$, treated with acetic anhydride $(0.72 \mathrm{~mL}, 7.6 \mathrm{mmol})$, DMAP $(15.5 \mathrm{mg}, 0.13 \mathrm{mmol})$ and triethylamine $(0.53 \mathrm{~mL}, 3.8 \mathrm{mmol})$ to yield $\mathbf{s} \mathbf{2 4}$ after column chromatography on silica gel (petroleum ether/EtOAc $-7 / 1 \rightarrow 5 / 1)$.

Yield: $1.45 \mathrm{~g}(2.47 \mathrm{mmol}, 97 \%)$.

Physical State: white solid.

Melting Point: $125^{\circ} \mathrm{C}$.

$\mathbf{R}_{\mathbf{f}}$ Value: 0.33 (petroleum ether/EtOAc - 5/1).

${ }^{1} \mathbf{H}$ NMR (Avance $400 \mathrm{MHz}, \mathrm{CDCl}_{3}$ ) $\delta 7.74-7.76(\mathrm{~m}, 2 \mathrm{H}), 7.19-7.30(\mathrm{~m}, 11 \mathrm{H}), 6.92-6.97$ (m, 4H), $6.53(\mathrm{~d}, J=15.8 \mathrm{~Hz}, 1 \mathrm{H}), 6.05(\mathrm{dt}, J=7.3,14.7 \mathrm{~Hz}, 1 \mathrm{H}), 4.32(\mathrm{~s}, 2 \mathrm{H}), 3.97(\mathrm{~d}$, $J=6.8 \mathrm{~Hz}, 2 \mathrm{H}), 2.37$ (s, 3H), $2.08(\mathrm{~s}, 3 \mathrm{H}) \mathrm{ppm}$.

${ }^{19}$ F NMR (Avance $376 \mathrm{MHz}, \mathrm{CDCl}_{3}$ ) $\delta-113.8 \mathrm{ppm}$.

${ }^{13}$ C NMR (Avance $\left.101 \mathrm{MHz}, \mathrm{CDCl}_{3}\right) \delta 167.9,162.3(\mathrm{~d}, J=248 \mathrm{~Hz}$ ), 143.6, 137.9 (d, $J=4 \mathrm{~Hz}), 136.3,136.2,135.4,129.8,128.7,128.2,128.1$ (d, $J=8 \mathrm{~Hz}), 127.8,126.7,122.7$, $115.3(\mathrm{~d}, J=22 \mathrm{~Hz}), 84.4,83.5,78.1,48.8,36.5,21.8,21.7 \mathrm{ppm}$.

IR (ATR, in $\left.\mathrm{CDCl}_{3}\right)$ v 3028 (w), 1755 (m), 1602 (w), 1505 (m), 1349 (m), 1223 (s), 1160 (s), $1097(\mathrm{~m}), 1016(\mathrm{w}) \mathrm{cm}^{-1}$.

HRMS (ESI, m/z) calcd. for $\mathrm{C}_{34} \mathrm{H}_{29} \mathrm{~F}_{2} \mathrm{NO}_{4} \mathrm{SNa}^{+}$: 608.1678, found: 608.1659. 
4.12 Preparation of $\quad(E)-6-(N$-cinnamyl-4-methylphenylsulfonamido)-2,2,3trimethylhex-4-yn-3-yl acetate s26

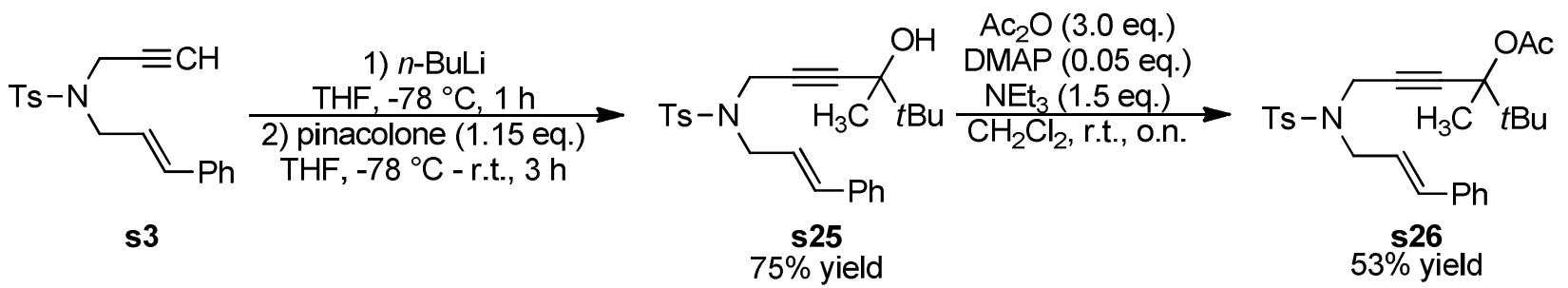

$N$-cinnamyl- $N$-(4-hydroxy-4,5,5-trimethylhex-2-ynyl)-4-methylbenzenesulfonamide s25

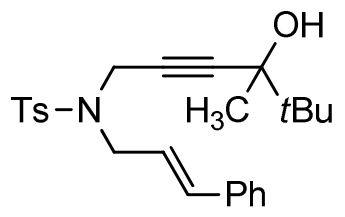

According to GP-1 s3 (1.0 g, $3.1 \mathrm{mmol})$ was dissolved in THF (30 mL), treated with $n$-BuLi (1.6 $\mathrm{M}$ in hexanes, $1.9 \mathrm{~mL}, 3.1 \mathrm{mmol})$ and pinacolone $(0.44 \mathrm{~mL}, 3.5 \mathrm{mmol})$ to yield $\mathbf{s} 25$ after column chromatography on silica gel ( $n$-pentane/Et $\left.{ }_{2} \mathrm{O}-2 / 1\right)$.

Yield: $0.90 \mathrm{~g}(2.12 \mathrm{mmol}, 75 \%)$.

Physical State: white solid.

$\mathbf{R}_{\mathbf{f}}$ Value: 0.31 (n-pentane/ $\left.\mathrm{Et}_{2} \mathrm{O}-2 / 1\right)$.

${ }^{1} \mathbf{H}$ NMR (Avance $\left.300 \mathrm{MHz}, \mathrm{CDCl}_{3}\right) \delta 7.76-7.78(\mathrm{~m}, 2 \mathrm{H}), 7.23-7.33(\mathrm{~m}, 7 \mathrm{H}), 6.58(\mathrm{~d}$, $J=15.8 \mathrm{~Hz}, 1 \mathrm{H}), 6.11(\mathrm{dt}, J=6.9,15.8 \mathrm{~Hz}, 1 \mathrm{H}), 4.18(\mathrm{~s}, 2 \mathrm{H}), 4.01(\mathrm{~d}, J=6.7 \mathrm{~Hz}, 2 \mathrm{H}), 2.43$ (s, 3H), $1.41(\mathrm{~s}, 1 \mathrm{H}), 1.21(\mathrm{~s}, 3 \mathrm{H}), 0.88(\mathrm{~s}, 9 \mathrm{H}) \mathrm{ppm}$.

${ }^{13}$ C NMR (Avance $75 \mathrm{MHz}, \mathrm{CDCl}_{3}$ ) $\delta$ 143.7, 136.5, 136.2, 135.0, 129.8, 128.8, 128.3, 127.9, 126.7, 123.1, 89.6, 76.8, 73.8, 48.9, 38.1, 36.3, 25.1, 24.7, 21.7 ppm.

IR (ATR, in $\left.\mathrm{CDCl}_{3}\right) \vee 3529$ (w, broad), 3027 (w), 2968 (w), 2872 (w), 1598 (w), 1495 (w), $1448(\mathrm{w}), 1346(\mathrm{~m}), 1158(\mathrm{~s}), 1092(\mathrm{~m}) \mathrm{cm}^{-1}$.

HRMS (ESI, m/z) calcd. for $\mathrm{C}_{25} \mathrm{H}_{31} \mathrm{NO}_{3} \mathrm{SNa}^{+}$: 448.1917, found: 448.1942. 
(E)-6-(N-cinnamyl-4-methylphenylsulfonamido)-2,2,3-trimethylhex-4-yn-3-yl acetate s26

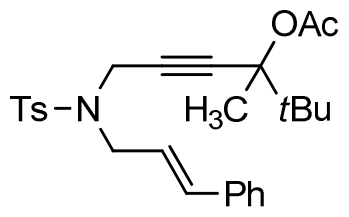

According to GP-2 s25 (0.84 g, $2.0 \mathrm{mmol})$ was dissolved in $\mathrm{CH}_{2} \mathrm{Cl}_{2}(2 \mathrm{~mL})$, treated with acetic anhydride $(0.56 \mathrm{~mL}, 5.9 \mathrm{mmol})$, DMAP $(12.0 \mathrm{mg}, 0.10 \mathrm{mmol})$ and triethylamine $(0.51 \mathrm{~mL}, 2.9 \mathrm{mmol})$ to yield $\mathbf{s} \mathbf{2 6}$ after column chromatography on silica gel ( $n$-pentane/Et ${ }_{2} \mathrm{O}$ $-2 / 1)$.

Yield: $0.49 \mathrm{~g}(1.04 \mathrm{mmol}, 53 \%)$.

Physical State: white solid.

Melting Point: $100{ }^{\circ} \mathrm{C}$.

$\mathbf{R}_{\mathbf{f}}$ Value: $0.51\left(n\right.$-pentane/ $\left./ \mathrm{Et}_{2} \mathrm{O}-2 / 1\right)$.

${ }^{1} \mathbf{H}$ NMR (Avance $\left.300 \mathrm{MHz}, \mathrm{CDCl}_{3}\right) \delta 7.74-7.77(\mathrm{~m}, 2 \mathrm{H}), 7.21-7.37$ (m, 7H), $6.68(\mathrm{~d}$, $J=15.8 \mathrm{~Hz}, 1 \mathrm{H}), 6.09(\mathrm{dt}, J=6.9,15.7 \mathrm{~Hz}, 1 \mathrm{H}), 4.21(\mathrm{~d}, J=1.9 \mathrm{~Hz}, 2 \mathrm{H}), 4.07(\mathrm{~d}, J=2.2 \mathrm{~Hz}$, $1 \mathrm{H}), 4.05(\mathrm{~d}, J=3.3 \mathrm{~Hz}, 1 \mathrm{H}), 2.41(\mathrm{~s}, 3 \mathrm{H}), 1.96(\mathrm{~s}, 3 \mathrm{H}), 1.43(\mathrm{~s}, 3 \mathrm{H}), 0.91$ (s, 9H) ppm.

${ }^{13}$ C NMR (Avance $75 \mathrm{MHz}, \mathrm{CDCl}_{3}$ ) $\delta$ 168.9, 143.1, 136.3, 136.3, 134.9, 129.6, 128.5, 127.8, $127.5,126.5,122.9,85.1,80.1,78.7,48.2,38.5,36.0,24.8,21.8,21.4,20.4$ ppm.

IR (ATR, neat) v 2973 (w), 1737 (m), 1598 (w), 1494 (w), 1370 (m), 1345 (m), 1325 (w), 1245 (m), 1189 (w), 1160 (s), 1126 (m), 1080 (m), 1020 (m) $\mathrm{cm}^{-1}$.

HRMS (ESI, m/z) calcd. for $\mathrm{C}_{27} \mathrm{H}_{33} \mathrm{NO}_{4} \mathrm{SNa}^{+}$: 490.2023, found: 490.2034.

4.13 Preparation of (E)-6-( $N$-cinnamyl-4-methylphenylsulfonamido)-3-isopropyl-2methylhex-4-yn-3-yl acetate s28

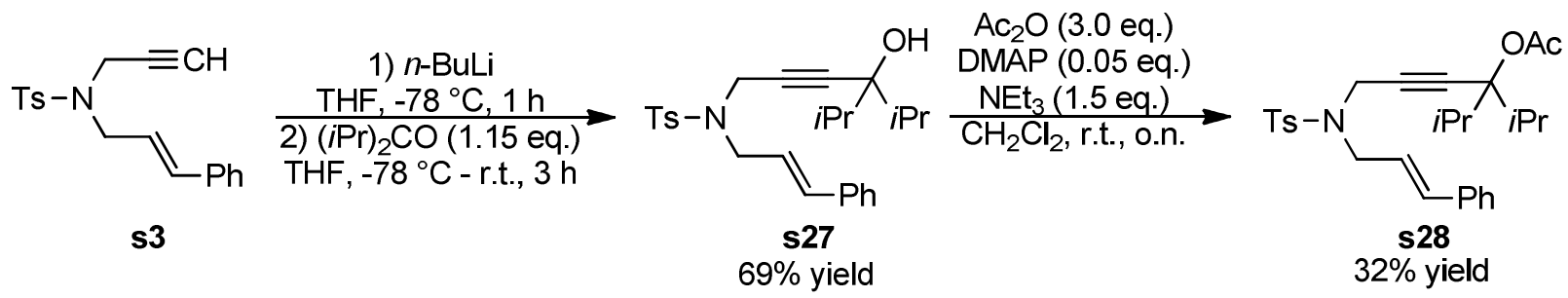



amide $\mathbf{2 7}$

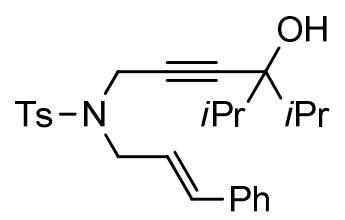

According to GP-1 s3 (2.0 g, $6.1 \mathrm{mmol})$ was dissolved in THF $(60 \mathrm{~mL})$, treated with $n$-BuLi (1.6 $\mathrm{M}$ in hexanes, $3.8 \mathrm{~mL}, 6.1 \mathrm{mmol})$ and diisopropyl ketone $(1.0 \mathrm{~mL}, 7.1 \mathrm{mmol})$ to yield $\mathbf{s} 27$ after column chromatography on silica gel (petroleum ether/EtOAc - 7/1).

Yield: $0.86 \mathrm{~g}(1.56 \mathrm{mmol}, 69 \%)$.

Physical State: pale yellow oil.

$\mathbf{R}_{\mathbf{f}}$ Value: 0.19 (petroleum ether/EtOAc - 7/1).

${ }^{1} \mathbf{H}$ NMR (Avance $300 \mathrm{MHz}, \mathrm{CDCl}_{3}$ ) $\delta 7.75$ - $7.77(\mathrm{~m}, 2 \mathrm{H}), 7.23$ - 7.35 (m, 7H), $6.58(\mathrm{~d}$, $J=15.8 \mathrm{~Hz}, 1 \mathrm{H}), 6.11(\mathrm{dt}, J=6.8,15.8 \mathrm{~Hz}, 1 \mathrm{H}), 4.03(\mathrm{dd}, J=0.8,6.8 \mathrm{~Hz}, 2 \mathrm{H}), 4.21(\mathrm{~s}, 2 \mathrm{H})$, 1.74 (septet, $J=6.7 \mathrm{~Hz}, 2 \mathrm{H}), 2.43$ (s, 3H), 1.30 (s, 1H), 0.85 (d, $J=6.7 \mathrm{~Hz}, 6 \mathrm{H}), 0.82$ (d, $J=6.8 \mathrm{~Hz}, 6 \mathrm{H}) \mathrm{ppm}$.

${ }^{13}$ C NMR (Avance $75 \mathrm{MHz}, \mathrm{CDCl}_{3}$ ) $\delta$ 143.7, 136.6, 136.2, 135.0, 129.8, 128.8, 128.3, 127.8, $126.7,123.2,87.1,78.3,77.4,48.7,36.2,34.2,21.6,18.1,16.3$ ppm. 1 carbon was not observed due to incidental equivalence.

IR (ATR, in $\left.\mathrm{CDCl}_{3}\right)$ v 3532 (m, broad), 3028 (w), 2967 (w), 2875 (w), 1598 (w), 1494 (w), 1467 (w), 1449 (w), 1347 (m), 1159 (s), 1094 (m) cm².

HRMS (ESI, m/z) calcd. for $\mathrm{C}_{26} \mathrm{H}_{33} \mathrm{NO}_{3} \mathrm{SNa}^{+}$: 462.2073, found: 462.2093 .

\section{(E)-6-(N-cinnamyl-4-methylphenylsulfonamido)-3-isopropyl-2-methylhex-4-yn-3-yl} acetate $\mathbf{s 2 8}$

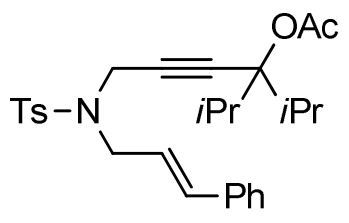

According to GP-2 s27 $(1.7 \mathrm{~g}, 3.9 \mathrm{mmol})$ was dissolved in $\mathrm{CH}_{2} \mathrm{Cl}_{2}(4 \mathrm{~mL})$, treated with acetic anhydride $(1.1 \mathrm{~mL}, 11.6 \mathrm{mmol})$, DMAP $(23.2 \mathrm{mg}, 0.19 \mathrm{mmol})$ and triethylamine $(0.8 \mathrm{~mL}$, 
$5.8 \mathrm{mmol}$ ) to yield $\mathbf{s} \mathbf{2 8}$ after column chromatography on silica gel ( $n$-pentane/ $\left.\mathrm{Et}_{2} \mathrm{O}-3 / 1\right)$. The spectral data are in good agreement with previous reports. ${ }^{[6]}$

Yield: $0.59 \mathrm{~g}(1.22 \mathrm{mmol}, 32 \%)$.

Physical State: white solid.

Melting Point: $79^{\circ} \mathrm{C}$.

$\mathbf{R}_{\mathbf{f}}$ Value: 0.43 (n-pentane/Et $\left.2 \mathrm{O}-3 / 1\right)$.

${ }^{1} \mathbf{H}$ NMR (Avance $\left.300 \mathrm{MHz}, \mathrm{CDCl}_{3}\right) \delta 7.74-7.77(\mathrm{~m}, 2 \mathrm{H}), 7.21-7.36(\mathrm{~m}, 7 \mathrm{H}), 6.68(\mathrm{~d}$, $J=15.8 \mathrm{~Hz}, 1 \mathrm{H}), 6.10(\mathrm{dt}, J=6.9,15.8 \mathrm{~Hz}, 1 \mathrm{H}), 4.26(\mathrm{~s}, 2 \mathrm{H}), 4.10(\mathrm{dd}, J=0.8,6.9 \mathrm{~Hz}, 2 \mathrm{H})$, 2.40 (s, 3H), 2.05 (septet, $J=7.1 \mathrm{~Hz}, 2 \mathrm{H}), 1.98$ (s, 3H), 0.87 (d, $J=6.8 \mathrm{~Hz}, 6 \mathrm{H}), 0.86$ (d, $J=6.8 \mathrm{~Hz}, 6 \mathrm{H}) \mathrm{ppm}$.

${ }^{13}$ C NMR (Avance $75 \mathrm{MHz}, \mathrm{CDCl}_{3}$ ) $\delta$ 168.8, 143.1, 136.7, 136.4, 135.0, 129.7, 128.6, 127.9, 127.6, 126.6, 123.1, 85.3, 83.0, 81.1, 48.3, 36.2, 34.8, 21.9, 21.5, 18.4, 17.5 ppm. 1 carbon was not observed due to incidental equivalence.

IR (ATR, in $\mathrm{CDCl}_{3}$ ) v 3027 (w), 2972 (w), 2936 (w), 2877 (w), 1742 (m), 1598 (w), 1495 (w), 1449 (w), 1349 (m), 1235 (m), 1161 (s), 1096 (w), 1018 (w) cm ${ }^{-1}$.

HRMS (ESI, m/z) calcd. for $\mathrm{C}_{28} \mathrm{H}_{35} \mathrm{NO}_{4} \mathrm{SNa}^{+}$: 504.2179, found: 504.2184.

\subsection{Preparation of $(E)-5-(N$-cinnamyl-4-methylphenylsulfonamido)-2-methyl-1-} phenylpent-3-yn-2-yl acetate s30

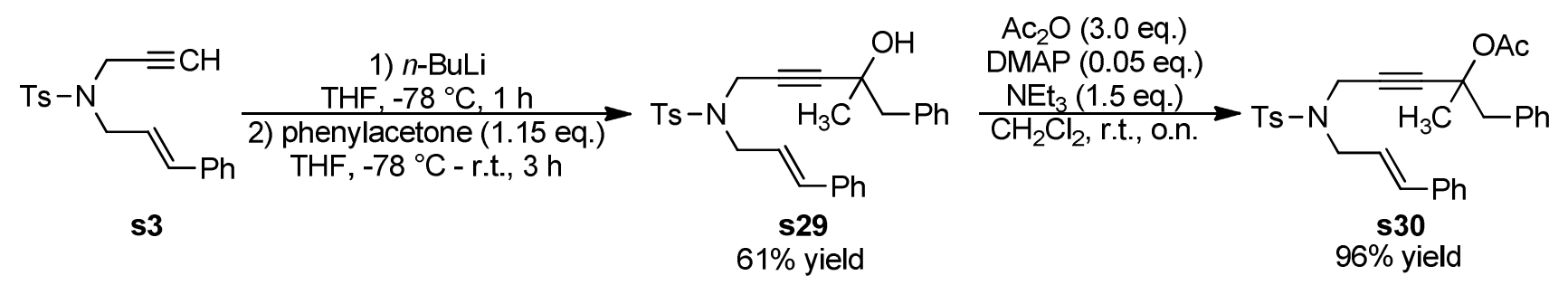

$N$-cinnamyl- $N$-(4-hydroxy-4-methyl-5-phenylpent-2-yn-1-yl)-4-methylbenzenesulfonamide s29 


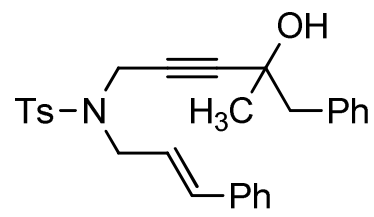

According to GP-1 s3 (1.63 g, $5.0 \mathrm{mmol})$ was dissolved in THF (50 mL), treated with $n$-BuLi (1.6 $\mathrm{M}$ in hexanes, $3.1 \mathrm{~mL}, 5.0 \mathrm{mmol})$ and phenylacetone $(0.76 \mathrm{~mL}, 5.8 \mathrm{mmol})$ to yield $\mathbf{s 2 9}$ after column chromatography on silica gel (petroleum ether/EtOAc $-5 / 1 \rightarrow 3 / 1$ ).

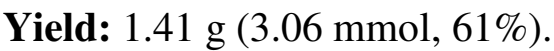

Physical State: pale yellow oil.

$\mathbf{R}_{\mathbf{f}}$ Value: 0.31 (petroleum ether/EtOAc - 3/1).

${ }^{1} \mathbf{H}$ NMR (Avance $\left.400 \mathrm{MHz}, \mathrm{CDCl}_{3}\right) \delta 7.68$ - $7.70(\mathrm{~m}, 2 \mathrm{H}), 7.28$ - $7.34(\mathrm{~m}, 7 \mathrm{H}), 7.23$ - 7.27 $(\mathrm{m}, 1 \mathrm{H}), 7.20-7.22(\mathrm{~m}, 2 \mathrm{H}), 7.15-7.17(\mathrm{~m}, 2 \mathrm{H}), 6.44(\mathrm{~d}, J=15.9 \mathrm{~Hz}, 1 \mathrm{H}), 6.05$ (dd, $J=6.8,15.8 \mathrm{~Hz}, 1 \mathrm{H}), 4.05-4.18(\mathrm{~m}, 2 \mathrm{H}), 3.84-3.97(\mathrm{~m}, 2 \mathrm{H}), 2.73$ (s, 2H), 2.39 (s, 3H), $1.61(\mathrm{~s}, 1 \mathrm{H}), 1.31(\mathrm{~s}, 3 \mathrm{H}) \mathrm{ppm}$.

${ }^{13}$ C NMR (Avance $101 \mathrm{MHz}, \mathrm{CDCl}_{3}$ ) $\delta 143.5,136.2,136.1,136.0,134.7,130.6,129.5$, 128.6, 128.2, 128.1, 127.9, 127.1, 126.5, 123.0, 88.9, 77.2, 67.7, 49.1, 48.7, 36.1, 29.4, 21.5 ppm.

IR (ATR, in $\mathrm{CDCl}_{3}$ ) v 3507 (m, broad), 3083 (w), 3060 (w), 3028 (w), 2980 (w), 2923 (w), 2861 (w), 1598 (w), 1495 (w), 1449 (w), 1345 (s), 1158 (s), 1119 (m), 1091 (s), 1069 (w), $1030(\mathrm{w}), 1018(\mathrm{w}) \mathrm{cm}^{-1}$.

HRMS (ESI, m/z) calcd. for $\mathrm{C}_{28} \mathrm{H}_{29} \mathrm{NO}_{3} \mathrm{SNa}^{+}$: 482.1753, found: 482.1743 .

\section{(E)-5-(N-cinnamyl-4-methylphenylsulfonamido)-2-methyl-1-phenylpent-3-yn-2-yl} acetate s30

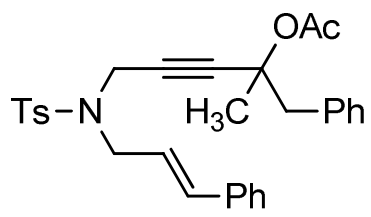

According to GP-2 s29 $(1.38 \mathrm{~g}, 3.0 \mathrm{mmol})$ was dissolved in $\mathrm{CH}_{2} \mathrm{Cl}_{2}(3 \mathrm{~mL})$, treated with acetic anhydride $(0.85 \mathrm{~mL}, 9.0 \mathrm{mmol})$, DMAP $(18.3 \mathrm{mg}, 0.15 \mathrm{mmol})$ and triethylamine $(0.62 \mathrm{~mL}, 4.5 \mathrm{mmol})$ to yield $\mathbf{s 3 0}$ after column chromatography on silica gel (petroleum ether/EtOAc - 5/1). 
Yield: $1.44 \mathrm{~g}(2.88 \mathrm{mmol}, 96 \%)$.

Physical State: yellow oil.

$\mathbf{R}_{\mathbf{f}}$ Value: 0.33 (petroleum ether/EtOAc - 5/1).

${ }^{1} \mathbf{H}$ NMR (Avance $400 \mathrm{MHz}, \mathrm{CDCl}_{3}$ ) $\delta 7.71-7.73(\mathrm{~m}, 2 \mathrm{H}), 7.27-7.33(\mathrm{~m}, 7 \mathrm{H}), 7.25-7.26$ (m, 1H), $7.22-7.24(\mathrm{~m}, 2 \mathrm{H}), 7.13-7.15(\mathrm{~m}, 2 \mathrm{H}), 6.56(\mathrm{~d}, J=15.8 \mathrm{~Hz}, 1 \mathrm{H}), 6.05$ (dd, $J=6.9,15.7 \mathrm{~Hz}, 1 \mathrm{H}), 4.12-4.22(\mathrm{~m}, 2 \mathrm{H}), 3.91-4.01(\mathrm{~m}, 2 \mathrm{H}), 2.95(\mathrm{~s}, 2 \mathrm{H}), 2.38$ (s, 3H), $1.96(\mathrm{~s}, 3 \mathrm{H}), 1.37$ (s, 3H) ppm.

${ }^{13}$ C NMR (Avance $101 \mathrm{MHz}, \mathrm{CDCl}_{3}$ ) $\delta$ 169.1, 143.4, 136.4, 136.4, 135.5, 135.2, 130.9, 129.7, 128.7, 128.1, 128.1, 127.9, 127.2, 126.7, 123.0, 85.9, 79.1, 74.4, 48.6, 46.7, 36.3, 26.1, 22.0, $21.6 \mathrm{ppm}$.

IR (ATR, in $\mathrm{CDCl}_{3}$ ) v 3028 (w), 2925 (w), 1737 (m), 1598 (w), 1495 (w), 1450 (w), 1451 (w), 1367 (w), 1347 (m), 1234 (m), 1159 (s), 1086 (m), 1061 (w), 1016 (w) cm . $^{-1}$

HRMS (ESI, m/z) calcd. for $\mathrm{C}_{30} \mathrm{H}_{31} \mathrm{NO}_{4} \mathrm{SNa}^{+}$: 524.1858, found: 524.1846.

4.15 Preparation of 4-(N-cinnamyl-4-methylphenylsulfonamido)-1-(4-( $(E)$-styryl)phenyl)but-2-yn-1-yl acetate s32

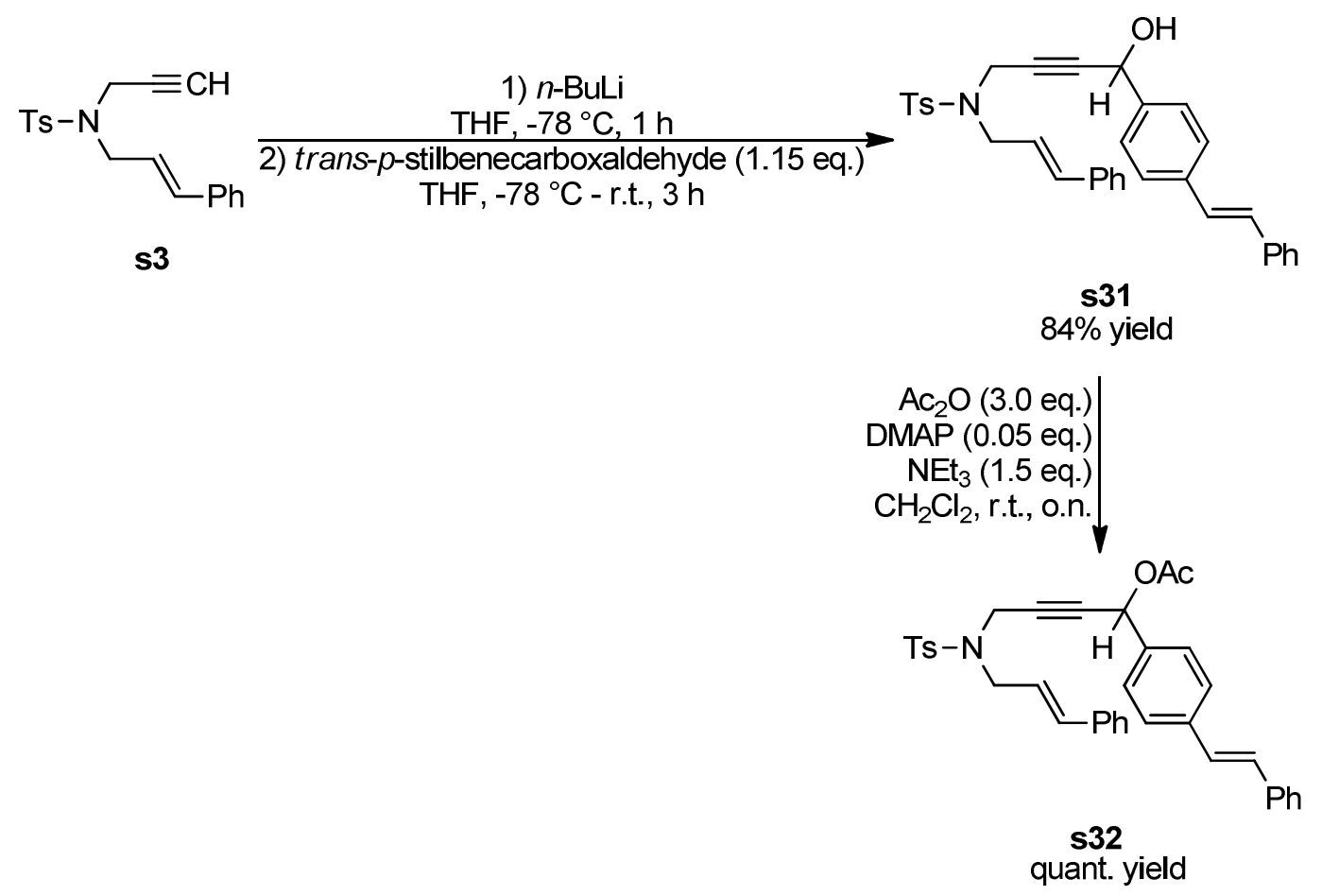



sulfonamide s31

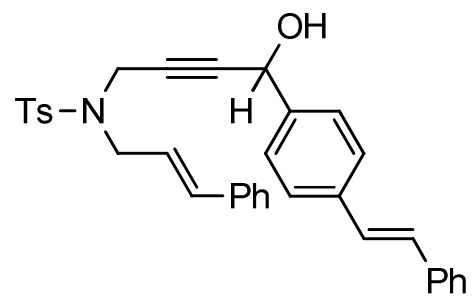

According to GP-1 s3 (1.30 g, $4 \mathrm{mmol})$ was dissolved in THF (40 mL), treated with $n$-BuLi (1.6 $\mathrm{M}$ in hexanes, $2.5 \mathrm{~mL}, 4 \mathrm{mmol})$ and trans-p-stilbenecarboxaldehyde $(0.96 \mathrm{~g}, 4.6 \mathrm{mmol})$ in THF (3 $\mathrm{mL})$ to yield $\mathbf{s 3 1}$ after column chromatography on silica gel (petroleum ether/EtOAc $-3 / 1 \rightarrow 2 / 1)$.

Yield: $1.79 \mathrm{~g}(3.35 \mathrm{mmol}, 84 \%)$.

Physical State: yellow solid.

$\mathbf{R}_{\mathbf{f}}$ Value: 0.24 (petroleum ether/EtOAc - 3/1).

${ }^{1} \mathbf{H}$ NMR (Avance $400 \mathrm{MHz}, \mathrm{CDCl}_{3}$ ) $\delta 7.75$ - $7.77(\mathrm{~m}, 2 \mathrm{H}), 7.52$ - $7.54(\mathrm{~m}, 2 \mathrm{H}), 7.47$ - 7.49 (m, 2H), $7.36-7.39(\mathrm{~m}, 2 \mathrm{H}), 7.29-7.31(\mathrm{~m}, 7 \mathrm{H}), 7.21-7.26(\mathrm{~m}, 3 \mathrm{H}), 7.11-7.12(\mathrm{~m}, 2 \mathrm{H})$, $6.51(\mathrm{~d}, J=15.8 \mathrm{~Hz}, 1 \mathrm{H}), 6.08(\mathrm{dt}, J=6.8,15.7 \mathrm{~Hz}, 1 \mathrm{H}), 5.20(\mathrm{~s}, 1 \mathrm{H}), 4.17-4.27(\mathrm{~m}, 2 \mathrm{H})$, $3.94-4.04(\mathrm{~m}, 2 \mathrm{H}), 2.36(\mathrm{~s}, 3 \mathrm{H}), 1.86-1.98(\mathrm{~m}, 1 \mathrm{H}) \mathrm{ppm}$.

${ }^{13}$ C NMR (Avance $101 \mathrm{MHz}, \mathrm{CDCl}_{3}$ ) $\delta$ 143.8, 139.4, 137.7, 137.2, 136.2, 136.2, 135.1, 129.7, 129.5, 128.9, 128.8, 128.2, 128.0, 128.0, 126.9, 126.8, 126.7, 123.0, 85.6, 79.8, 64.2, 49.1, 36.5, 21.7 ppm. 2 carbons were not observed due to incidental equivalence.

IR (ATR, in $\left.\mathrm{CDCl}_{3}\right)$ v 3484 (w, broad), 3026 (w), 2920 (w), 1597 (w), 1509 (w), 1494 (w), 1448 (w), 1343 (m), 1327 (m), 1305 (w), 1155 (s), 1091 (m), $1071(\mathrm{w}), 1056(\mathrm{w}) \mathrm{cm}^{-1}$.

HRMS (ESI, m/z) calcd. for $\mathrm{C}_{34} \mathrm{H}_{31} \mathrm{NO}_{3} \mathrm{SNa}^{+}$: 556.1917, found: 556.2024.

\section{4-(N-cinnamyl-4-methylphenylsulfonamido)-1-(4-((E)-styryl)phenyl)but-2-yn-1-yl} acetate $\mathbf{s 3 2}$

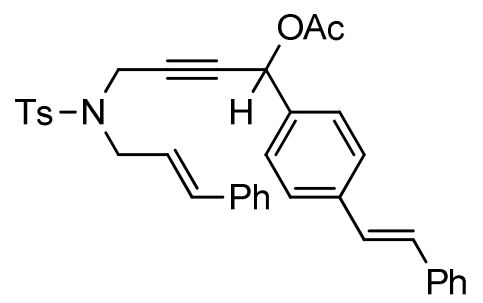


According to GP-2 s31 (1.33 g, $2.5 \mathrm{mmol})$ was dissolved in $\mathrm{CH}_{2} \mathrm{Cl}_{2}$ (3 mL), treated with acetic anhydride $(0.71 \mathrm{~mL}, 7.5 \mathrm{mmol})$, DMAP $(15.3 \mathrm{mg}, 0.13 \mathrm{mmol})$ and triethylamine $(0.52 \mathrm{~mL}, 3.8 \mathrm{mmol})$ to yield $\mathbf{s 3 2}$ after column chromatography on silica gel (petroleum ether/EtOAc - 4/1).

Yield: $1.44 \mathrm{~g}$ (2.5 mmol, quant.).

Physical State: yellow oil.

$\mathbf{R}_{\mathbf{f}}$ Value: 0.36 (petroleum ether/EtOAc - 4/1).

${ }^{1} \mathbf{H}$ NMR (Avance $400 \mathrm{MHz}, \mathrm{CDCl}_{3}$ ) $\delta 7.72$ - $7.74(\mathrm{~m}, 2 \mathrm{H}), 7.51-7.53(\mathrm{~m}, 2 \mathrm{H}), 7.46-7.48$ $(\mathrm{m}, 2 \mathrm{H}), 7.34-7.38(\mathrm{~m}, 2 \mathrm{H}), 7.27-7.29(\mathrm{~m}, 7 \mathrm{H}), 7.21-7.25(\mathrm{~m}, 1 \mathrm{H}), 7.16-7.19(\mathrm{~m}, 2 \mathrm{H})$, $7.11-7.12(\mathrm{~m}, 2 \mathrm{H}), 6.51(\mathrm{~d}, J=15.8 \mathrm{~Hz}, 1 \mathrm{H}), 6.19(\mathrm{~s}, 1 \mathrm{H}), 6.06(\mathrm{dt}, J=6.9,15.7 \mathrm{~Hz}, 1 \mathrm{H})$, $4.17-4.28(\mathrm{~m}, 2 \mathrm{H}), 3.91-4.01(\mathrm{~m}, 2 \mathrm{H}), 2.33(\mathrm{~s}, 3 \mathrm{H}), 2.05(\mathrm{~s}, 3 \mathrm{H}) \mathrm{ppm}$.

${ }^{13}$ C NMR (Avance $101 \mathrm{MHz}, \mathrm{CDCl}_{3}$ ) $\delta$ 169.5, 143.6, 138.2, 137.0, 136.1, 135.9, 135.6, 135.1, 129.9, 129.6, 128.8, 128.7, 128.1, 128.0, 127.8, 127.7, 126.7, 126.7, 126.6, 122.8, 82.4, $80.3,65.1,49.0,36.4,21.6,21.0 \mathrm{ppm}$. 1 carbon was not observed due to incidental equivalence.

IR (ATR, in $\left.\mathrm{CDCl}_{3}\right)$ v 3028 (w), 1736 (m), 1598 (w), 1578 (w), 1510 (w), 1494 (w), 1448 (w), 1368 (w), 1347 (m), 1306 (w), 1223 (s), 1182 (w), 1159 (s), 1092 (m), 1071 (w), 1055 (w), $1015(\mathrm{~m}) \mathrm{cm}^{-1}$.

HRMS (ESI, m/z) calcd. for $\mathrm{C}_{36} \mathrm{H}_{33} \mathrm{NO}_{4} \mathrm{SNa}^{+}$: 598.2023, found: 598.2042. 
4.16 Preparation of $(E)-6$-( $N$-cinnamyl-4-methylphenylsulfonamido)-1-phenylhex-1-en4-yn-3-yl acetate s34

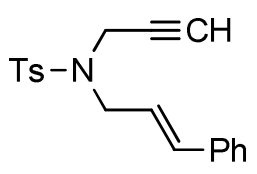

s3
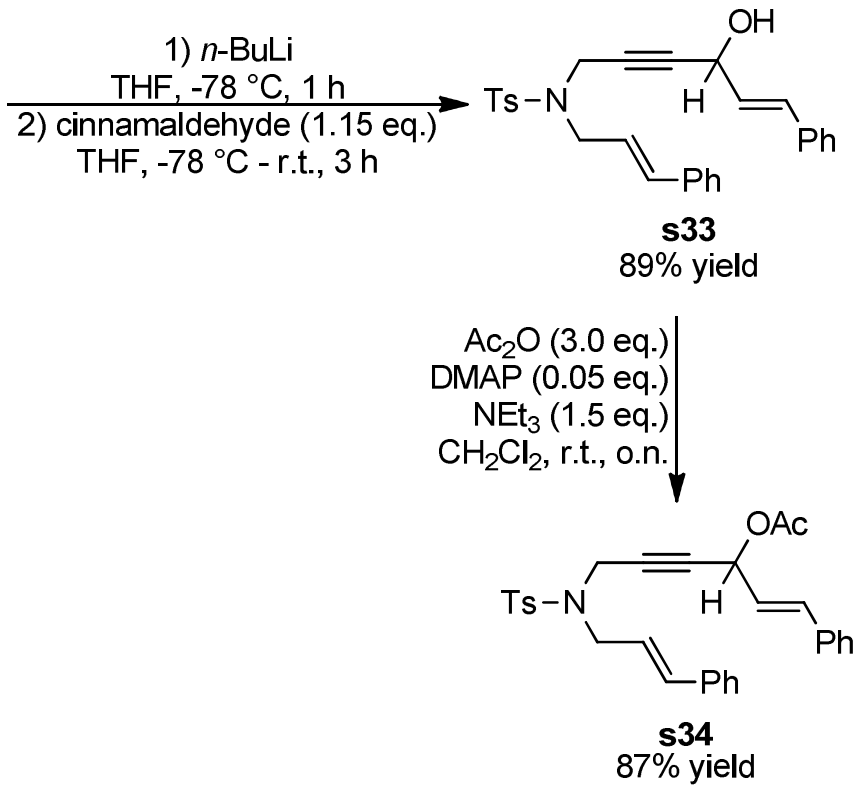

$N$-cinnamyl- $N$-((E)-4-hydroxy-6-phenylhex-5-en-2-yn-1-yl)-4-methylbenzenesulfonamide s33

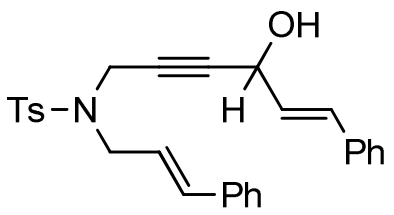

According to GP-1 s3 (1.30 g, 4 mmol) was dissolved in THF (40 mL), treated with $n$-BuLi (1.6 $\mathrm{M}$ in hexanes, $2.5 \mathrm{~mL}, 4 \mathrm{mmol})$ and cinnamaldehyde $(0.58 \mathrm{~mL}, 4.6 \mathrm{mmol})$ to yield $\mathbf{s 3 3}$ after column chromatography on silica gel (petroleum ether/EtOAc $-4 / 1 \rightarrow 3 / 1$ ).

Yield: $1.63 \mathrm{~g}(3.55 \mathrm{mmol}, 89 \%)$.

Physical State: yellow oil.

$\mathbf{R}_{\mathbf{f}}$ Value: 0.29 (petroleum ether/EtOAc - 3/1).

${ }^{1} \mathbf{H}$ NMR (Avance $400 \mathrm{MHz}, \mathrm{CDCl}_{3}$ ) $\delta 7.77$ - $7.79(\mathrm{~m}, 2 \mathrm{H}), 7.34$ - 7.35 (m, 4H), 7.24 - 7.30 $(\mathrm{m}, 8 \mathrm{H}), 6.55-6.59(\mathrm{~m}, 2 \mathrm{H}), 6.05-6.13(\mathrm{~m}, 2 \mathrm{H}), 4.80(\mathrm{~s}, 1 \mathrm{H}), 4.21(\mathrm{~d}, J=2.0 \mathrm{~Hz}, 2 \mathrm{H}), 4.00$ $(\mathrm{d}, J=6.8 \mathrm{~Hz}, 2 \mathrm{H}), 2.32(\mathrm{~s}, 3 \mathrm{H}), 1.73(\mathrm{~d}, J=6.1 \mathrm{~Hz}, 1 \mathrm{H}) \mathrm{ppm}$.

${ }^{13}$ C NMR (Avance $101 \mathrm{MHz}, \mathrm{CDCl}_{3}$ ) $\delta$ 143.8, 136.2, 136.1, 135.9, 135.0, 132.1, 129.7, $128.8,128.8,128.4,128.2,128.0,127.5,126.9,126.7,123.0,84.8,79.5,62.7,49.1,36.4,21.6$ ppm. 
IR (ATR, in $\left.\mathrm{CDCl}_{3}\right)$ v 3483 (w, broad), 3026 (w), 2920 (w), 1597 (w), 1578 (w), 1495 (w), 1448 (w), 1399 (w), 1342 (m), 1327 (m), 1306 (w), 1207 (w), 1154 (s), 1119 (w), 1090 (m), $1069(\mathrm{~m}), 1018(\mathrm{w}) \mathrm{cm}^{-1}$.

HRMS (ESI, m/z) calcd. for $\mathrm{C}_{28} \mathrm{H}_{27} \mathrm{NO}_{3} \mathrm{SNa}^{+}: 480.1604$, found: 480.1587 .

(E)-6-( $N$-cinnamyl-4-methylphenylsulfonamido)-1-phenylhex-1-en-4-yn-3-yl acetate s34

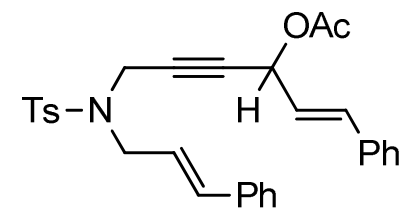

According to GP-2 s33 (1.60 g, $3.5 \mathrm{mmol})$ was dissolved in $\mathrm{CH}_{2} \mathrm{Cl}_{2}(4 \mathrm{~mL})$, treated with acetic anhydride $(0.99 \mathrm{~mL}, 10.5 \mathrm{mmol})$, DMAP $(21.4 \mathrm{mg}, 0.18 \mathrm{mmol})$ and triethylamine $(0.73 \mathrm{~mL}, 5.3 \mathrm{mmol})$ to yield $\mathbf{s 3 4}$ after column chromatography on silica gel (petroleum ether/EtOAc - 5/1).

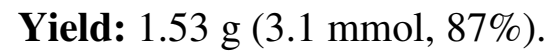

Physical State: orange oil.

$\mathbf{R}_{\mathbf{f}}$ Value: 0.31 (petroleum ether/EtOAc - 5/1).

${ }^{1} \mathbf{H}$ NMR (Avance $400 \mathrm{MHz}, \mathrm{CDCl}_{3}$ ) $\delta 7.75-7.77(\mathrm{~m}, 2 \mathrm{H}), 7.27-7.34(\mathrm{~m}, 9 \mathrm{H}), 7.22-7.25$ $(\mathrm{m}, 3 \mathrm{H}), 6.56-6.64(\mathrm{~m}, 2 \mathrm{H}), 5.99-6.12(\mathrm{~m}, 2 \mathrm{H}), 5.79-5.81(\mathrm{~m}, 1 \mathrm{H}), 4.23(\mathrm{~s}, 2 \mathrm{H})$, $3.94-4.04$ (m, 2H), 2.27 (s, 3H), 2.06 (s, 3H) ppm.

${ }^{13}$ C NMR (Avance $101 \mathrm{MHz}, \mathrm{CDCl}_{3}$ ) $\delta$ 169.5, 143.7, 136.0, 135.8, 135.4, 135.0, 134.5, 129.6, 128.7, 128.6, 128.6, 128.1, 127.8, 126.9, 126.6, 123.2, 122.8, 81.5, 80.1, 63.9, 48.9, $36.4,21.4,21.0 \mathrm{ppm}$.

IR (ATR, in $\mathrm{CDCl}_{3}$ ) v 3028 (w), 1737 (m), 1653 (w), 1598 (w), 1495 (w), 1449 (w), 1347 (m), 1224 (s), 1159 (s), 1092 (m), 1070 (w), 1015 (w) $\mathrm{cm}^{-1}$.

HRMS (ESI, m/z) calcd. for $\mathrm{C}_{30} \mathrm{H}_{29} \mathrm{NO}_{4} \mathrm{SNa}^{+}$: 522.1710, found: 522.1715 . 


\subsection{Preparation of $(E)-5-($ cinnamyloxy)-2-methylpent-3-yn-2-yl acetate s37}
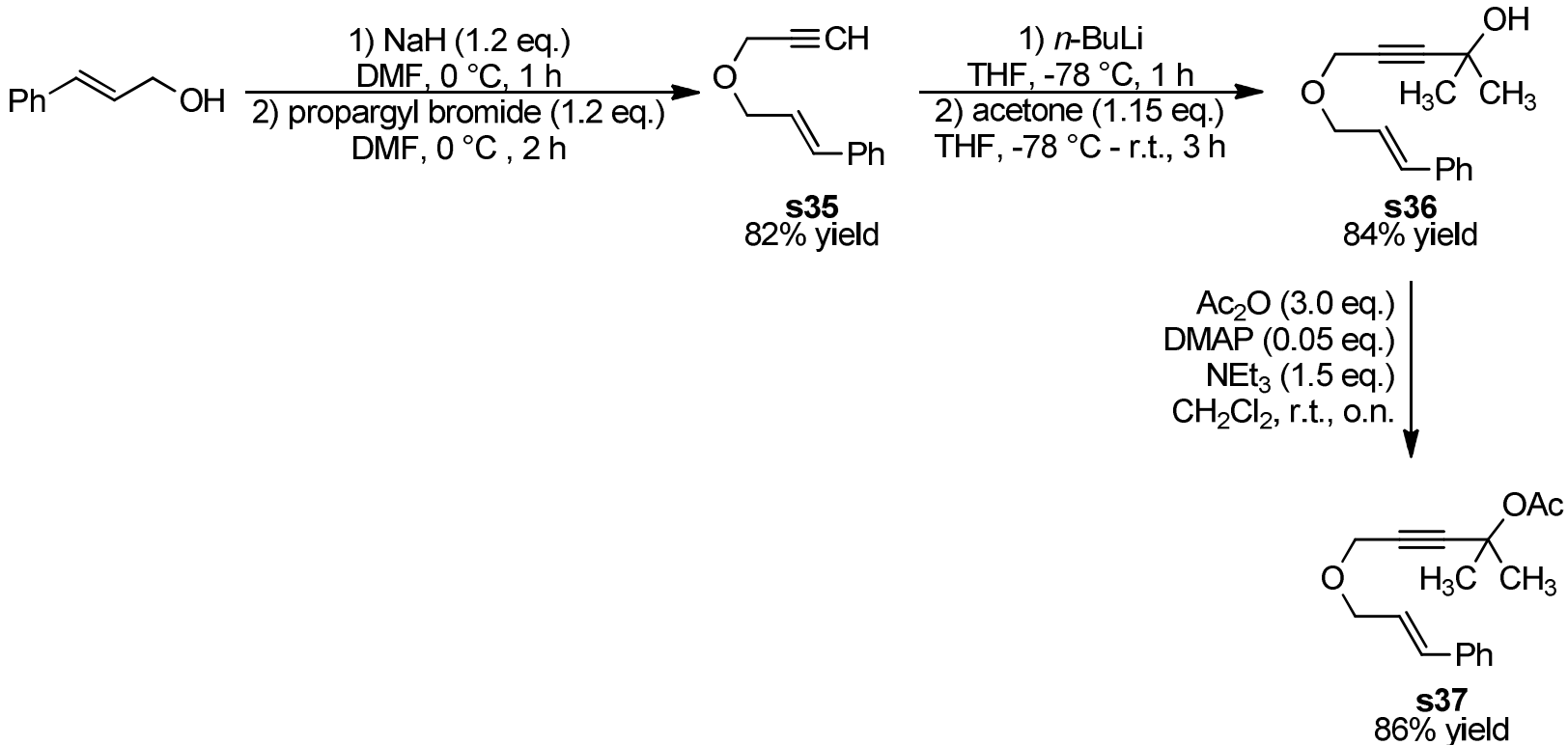

(E)-(3-(prop-2-yn-1-yloxy)prop-1-en-1-yl)benzene s35

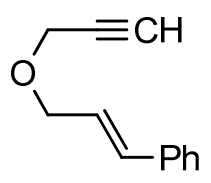

To a solution of cinnamyl alcohol $(1.94 \mathrm{~mL}, 15 \mathrm{mmol})$ in DMF $(25 \mathrm{~mL})$ was added $\mathrm{NaH}$ $(60 \%$ in mineral oil, $0.72 \mathrm{~g}, 18 \mathrm{mmol})$ at $0{ }^{\circ} \mathrm{C}$. After stirring for 1 hour at $0{ }^{\circ} \mathrm{C}$ propargyl bromide ( $80 \%$ in toluene, $1.94 \mathrm{~mL}, 18 \mathrm{mmol}$ ) was added dropwise and the mixture was stirred for additional 2 hours at room temperature. The solution was hydrolysed with sat. $\mathrm{NH}_{4} \mathrm{Cl}$ $(250 \mathrm{~mL})$ and extracted with diethyl ether $(5 \times 100 \mathrm{~mL})$. The combined organic layers were washed with brine $(200 \mathrm{~mL})$, dried over anhydrous $\mathrm{MgSO}_{4}$ and concentrated. The residue was purified by column chromatography on silica gel ( $n$-pentane/Et ${ }_{2} \mathrm{O}-100 / 0 \rightarrow 97 / 3$ ) to yield the title compound $\mathbf{s 3 5}$. The spectral data are in good agreement with previous reports. ${ }^{[8]}$

Yield: $2.13 \mathrm{~g}(12.37 \mathrm{mmol}, 82 \%)$.

Physical State: pale yellow liquid.

$\mathbf{R}_{\mathbf{f}}$ Value: $0.29(n$-pentane/Et $2 \mathrm{O}-97 / 3)$.

${ }^{1} \mathbf{H}$ NMR (Avance $\left.300 \mathrm{MHz}, \mathrm{CDCl}_{3}\right) \delta 7.22-7.42(\mathrm{~m}, 5 \mathrm{H}), 6.65(\mathrm{dt}, J=1.3,16.0 \mathrm{~Hz}, 1 \mathrm{H})$, $6.28(\mathrm{dt}, J=6.2,16.0 \mathrm{~Hz}, 1 \mathrm{H}), 4.25(\mathrm{dd}, J=1.3,6.2 \mathrm{~Hz}, 2 \mathrm{H}), 4.21(\mathrm{~d}, J=2.3 \mathrm{~Hz}, 2 \mathrm{H}), 2.46$ (t, $J=2.3 \mathrm{~Hz}, 1 \mathrm{H}) \mathrm{ppm}$. 
${ }^{13}$ C NMR (Avance $75 \mathrm{MHz}, \mathrm{CDCl}_{3}$ ) $\delta 136.7,133.6,128.7,128.0,126.7,125.2,79.8,74.7$, $70.4,57.2 \mathrm{ppm}$.

IR (ATR, in $\mathrm{CDCl}_{3}$ ) v 3297 (w), 3026 (w), 2850 (w), 1657 (w), 1599 (w), 1495 (w), 1449 (w), 1388 (w), 1355 (w), 1261 (w), 1118 (m), 1075 (s), 1028 (w) cm $\mathrm{cm}^{-1}$

HRMS (EI) calcd. for $\mathrm{C}_{12} \mathrm{H}_{12} \mathrm{O}: 172.0888$, found: 172.0863 .

\section{5-(cinnamyloxy)-2-methylpent-3-yn-2-ol s36}

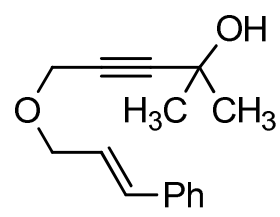

According to GP-1 s35 (1.03 g, $6 \mathrm{mmol})$ was dissolved in THF (60 mL), treated with $n$-BuLi (1.6 $\mathrm{M}$ in hexanes, $3.8 \mathrm{~mL}, 6 \mathrm{mmol})$ and acetone $(0.63 \mathrm{~mL}, 6.9 \mathrm{mmol})$ to yield $\mathbf{s 3 6}$ after column chromatography on silica gel (n-pentane/Et $\left.{ }_{2} \mathrm{O}-20 / 1\right)$. The spectral data are in good agreement with previous reports. ${ }^{[8]}$

Yield: $1.16 \mathrm{~g}(5.04 \mathrm{mmol}, 84 \%)$.

Physical State: pale yellow liquid.

$\mathbf{R}_{\mathbf{f}}$ Value: 0.43 (n-pentane/Et $\left.2 \mathrm{O}-20 / 1\right)$.

${ }^{1} \mathbf{H}$ NMR (Avance $300 \mathrm{MHz}, \mathrm{CDCl}_{3}$ ) $\delta 7.38$ - $7.41(\mathrm{~m}, 2 \mathrm{H}), 7.30$ - $7.35(\mathrm{~m}, 2 \mathrm{H}), 7.24-7.28$ (m, 1H), $6.62(\mathrm{~d}, J=16.0 \mathrm{~Hz}, 1 \mathrm{H}), 6.27(\mathrm{dt}, J=6.5,15.9 \mathrm{~Hz}, 1 \mathrm{H}), 4.21-4.23(\mathrm{~m}, 4 \mathrm{H}), 1.93$ $(\mathrm{s}, 1 \mathrm{H}), 1.54(\mathrm{~s}, 6 \mathrm{H}) \mathrm{ppm}$.

${ }^{13}$ C NMR (Avance $75 \mathrm{MHz}, \mathrm{CDCl}_{3}$ ) $\delta 136.6,133.4,128.6,127.9,126.6,125.2,91.4,77.9$, 70.3, 65.1, 57.4, $31.4 \mathrm{ppm}$.

IR (ATR, in $\left.\mathrm{CDCl}_{3}\right)$ v 3399 (w, broad), 3026 (w), 2980 (w), 2932 (w), 2852 (w), 1655 (w), 1599 (w), 1578 (w), 1492 (w), 1449 (w), 1356 (m), 1233 (w), 1166 (m), 1116 (m), 1074 (s), $1041(\mathrm{~m}), 1016(\mathrm{w}) \mathrm{cm}^{-1}$.

HRMS (EI) calcd. for $\mathrm{C}_{15} \mathrm{H}_{18} \mathrm{O}_{2}: 230.1307$, found: 230.1303 . 


\section{(E)-5-(cinnamyloxy)-2-methylpent-3-yn-2-yl acetate s37}

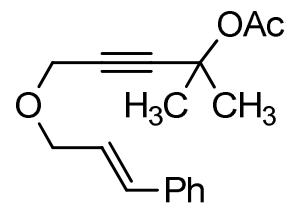

According to GP-2 s36 (0.69 g, $3 \mathrm{mmol})$ was dissolved in $\mathrm{CH}_{2} \mathrm{Cl}_{2}(3 \mathrm{~mL})$, treated with acetic anhydride $(0.85 \mathrm{~mL}, 9 \mathrm{mmol})$, DMAP $(18.3 \mathrm{mg}, 0.15 \mathrm{mmol})$ and triethylamine $(0.78 \mathrm{~mL}$, $4.5 \mathrm{mmol}$ ) to yield $\mathbf{s 3 7}$ after column chromatography on silica gel ( $n$-pentane/Et ${ }_{2} \mathrm{O}-5 / 1$ ). The spectral data are in good agreement with previous reports. ${ }^{[6]}$

Yield: $0.66 \mathrm{~g}(1.72 \mathrm{mmol}, 86 \%)$.

Physical State: pale yellow liquid.

$\mathbf{R}_{\mathbf{f}}$ Value: $0.34(n$-pentane/Et $2 \mathrm{O}-5 / 1)$.

${ }^{1} \mathbf{H}$ NMR (Avance $300 \mathrm{MHz}, \mathrm{CDCl}_{3}$ ) $\delta 7.38$ - $7.40(\mathrm{~m}, 2 \mathrm{H}), 7.28$ - $7.33(\mathrm{~m}, 2 \mathrm{H}), 7.21$ - 7.26 (m, 1H), $6.64(\mathrm{~d}, J=16.0 \mathrm{~Hz}, 1 \mathrm{H}), 6.27(\mathrm{dt}, J=6.2,15.9 \mathrm{~Hz}, 1 \mathrm{H}), 4.21-4.23(\mathrm{~m}, 4 \mathrm{H}), 2.02$ (s, 3H), 1.67 (s, 6H) ppm.

${ }^{13}$ C NMR (Avance $75 \mathrm{MHz}, \mathrm{CDCl}_{3}$ ) $\delta$ 169.5, 136.8, 133.5, 128.7, 127.9, 126.7, 125.4, 87.8, $80.2,72.0,70.2,57.4,29.1,22.1 \mathrm{ppm}$.

IR (ATR, in $\left.\mathrm{CDCl}_{3}\right)$ v 3082 (w), 3059 (w), 3026 (w), 2987 (w), 2938 (w), 2849 (w), 1739 (s), 1599 (w), 1495 (w), 1449 (w), 1364 (m), 1261 (m), 1238 (s), 1194 (w), 1133 (s), 1082 (m), $1044(\mathrm{~m}), 1014(\mathrm{~m}) \mathrm{cm}^{-1}$.

HRMS (ESI, m/z) calcd. for $\mathrm{C}_{17} \mathrm{H}_{20} \mathrm{O}_{2} \mathrm{Na}^{+}$: 295.1305, found: 295.1306.

\subsection{Preparation of $(E)-4-($ cinnamyloxy)-1,1-diphenylbut-2-yn-1-yl acetate s39}

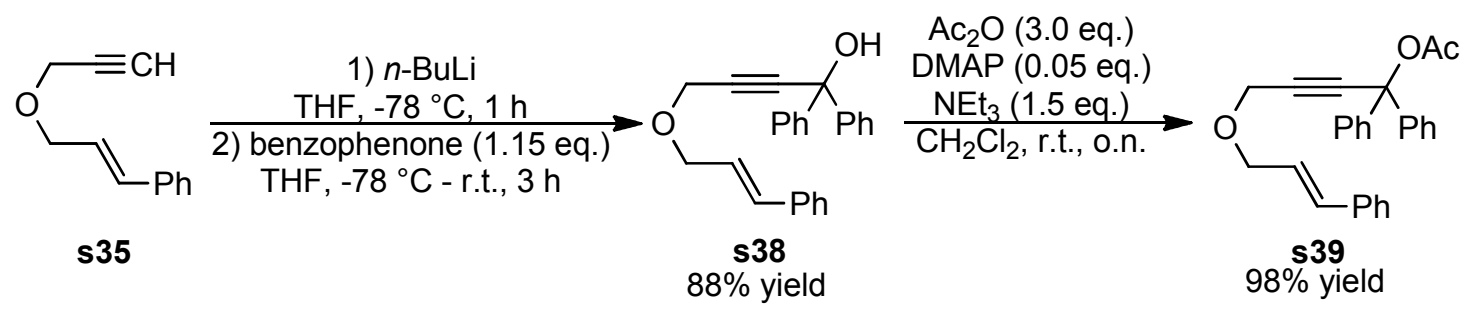




\section{4-(cinnamyloxy)-1,1-diphenylbut-2-yn-1-ol s38}

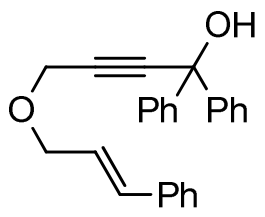

According to GP-1 s35 (0.86 g, $5 \mathrm{mmol})$ was dissolved in THF (50 mL), treated with $n$-BuLi (1.6 $\mathrm{M}$ in hexanes, $3.1 \mathrm{~mL}, 5 \mathrm{mmol})$ and benzophenone $(1.05 \mathrm{~g}, 5.8 \mathrm{mmol})$ in THF $(5 \mathrm{~mL})$ to yield s38 after column chromatography on silica gel (petroleum ether/EtOAc - 7/1).

Yield: $1.55 \mathrm{~g}(4.38 \mathrm{mmol}, 88 \%)$.

Physical State: white solid.

$\mathbf{R}_{\mathbf{f}}$ Value: 0.31 (petroleum ether/EtOAc - 7/1).

${ }^{1} \mathbf{H}$ NMR (Avance $400 \mathrm{MHz}, \mathrm{CDCl}_{3}$ ) $\delta 7.52$ - $7.53(\mathrm{~m}, 4 \mathrm{H}), 7.15$ - $7.29(\mathrm{~m}, 11 \mathrm{H}), 6.52(\mathrm{~d}$, $J=15.9 \mathrm{~Hz}, 1 \mathrm{H}), 6.19(\mathrm{dt}, J=6.2,15.9 \mathrm{~Hz}, 1 \mathrm{H}), 4.26(\mathrm{~s}, 2 \mathrm{H}), 4.17(\mathrm{~d}, J=6.2 \mathrm{~Hz}, 2 \mathrm{H}), 2.85$ $(\mathrm{s}, 1 \mathrm{H}) \mathrm{ppm}$.

${ }^{13}$ C NMR (Avance $101 \mathrm{MHz}, \mathrm{CDCl}_{3}$ ) $\delta$ 144.9, 136.6, 133.7, 128.7, 128.4, 128.0, 127.9, $126.7,126.1,125.2,89.3,83.5,74.5,70.5,57.5$ ppm.

IR (ATR, in $\left.\mathrm{CDCl}_{3}\right)$ v 3388 (w, broad), 3058 (w), 3026 (w), 2850 (w), 1598 (w), 1490 (w), 1448 (m), 1353 (w), 1202 (w), 1179 (w), 1107 (w), $1074(w), 1031(w), 1011(\mathrm{~m}) \mathrm{cm}^{-1}$.

HRMS (ESI, m/z) calcd. for $\mathrm{C}_{25} \mathrm{H}_{22} \mathrm{O}_{2} \mathrm{Na}^{+}: 377.1512$, found: 377.1498 .

\section{(E)-4-(cinnamyloxy)-1,1-diphenylbut-2-yn-1-yl acetate s39}

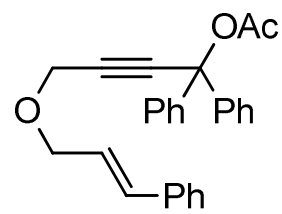

According to GP-2 s38 (1.06 g, $3 \mathrm{mmol})$ was dissolved in $\mathrm{CH}_{2} \mathrm{Cl}_{2}(3 \mathrm{~mL})$, treated with acetic anhydride $(0.85 \mathrm{~mL}, 9 \mathrm{mmol})$, DMAP $(18.3 \mathrm{mg}, 0.15 \mathrm{mmol})$ and triethylamine $(0.78 \mathrm{~mL}$, $4.5 \mathrm{mmol}$ ) to yield $\mathbf{s 3 9}$ after column chromatography on silica gel (petroleum ether/EtOAc $10 / 1 \rightarrow 7 / 1)$

Yield: $1.17 \mathrm{~g}(2.96 \mathrm{mmol}, 98 \%)$.

Physical State: pale yellow oil. 
$\mathbf{R}_{\mathbf{f}}$ Value: 0.31 (petroleum ether/EtOAc - 7/1).

${ }^{1} \mathbf{H}$ NMR (Avance $\left.400 \mathrm{MHz}, \mathrm{CDCl}_{3}\right) \delta 7.54-7.57(\mathrm{~m}, 4 \mathrm{H}), 7.26-7.42(\mathrm{~m}, 11 \mathrm{H}), 6.64(\mathrm{~d}$, $J=15.9 \mathrm{~Hz}, 1 \mathrm{H}), 6.30(\mathrm{dt}, J=6.3,15.9 \mathrm{~Hz}, 1 \mathrm{H}), 4.41(\mathrm{~s}, 2 \mathrm{H}), 4.28(\mathrm{dd}, J=1.2,6.3 \mathrm{~Hz}, 2 \mathrm{H})$, $2.20(\mathrm{~s}, 3 \mathrm{H}) \mathrm{ppm}$.

${ }^{13} \mathrm{C}$ NMR (Avance $101 \mathrm{MHz}, \mathrm{CDCl}_{3}$ ) $\delta$ 168.3, 142.5, 136.7, 133.7, 128.7, 128.4, 128.0, $127.9,126.7,126.3,125.2,86.1,85.6,79.3,70.1,57.4,22.0 \mathrm{ppm}$.

IR (ATR, in $\left.\mathrm{CDCl}_{3}\right)$ v 3060 (w), 3026 (w), 2849 (w), 1751 (s), 1598 (w), 1492 (m), 1449 (m), 1365 (m), 1220 (s), 1200 (m), 1129 (m), 1074 (m), 1014 (m) $\mathrm{cm}^{-1}$.

HRMS (ESI, m/z) calcd. for $\mathrm{C}_{27} \mathrm{H}_{24} \mathrm{O}_{3} \mathrm{Na}^{+}:$419.1618, found: 419.1614 .

\subsection{Preparation of (E)-4-( $N$-cinnamylmethylsulfonamido)-1,1-diphenylbut-2-yn-1-yl} acetate $s 43$

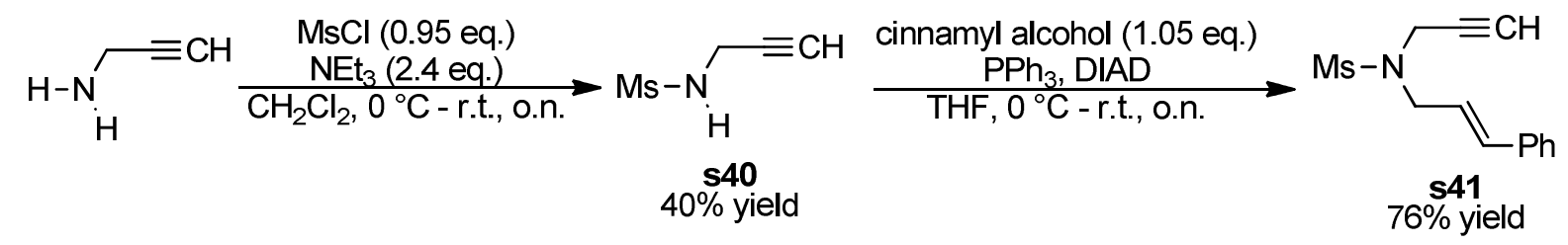

THF, $-78^{\circ} \mathrm{C}, 1 \mathrm{~h}$

2) benzophenone (1.15 eq.)

THF, $-78^{\circ} \mathrm{C}-$ r.t., $3 \mathrm{~h}$

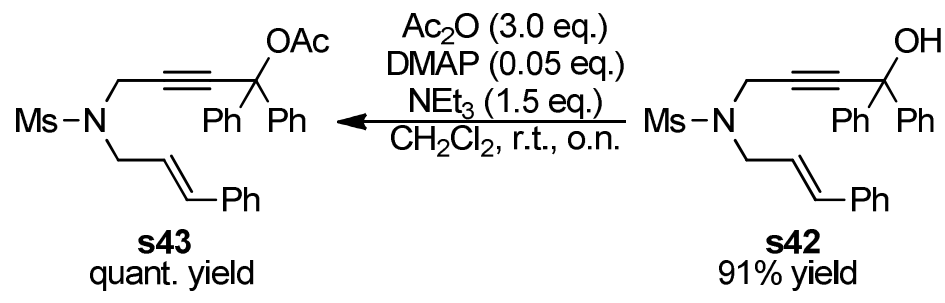

$N$-(prop-2-yn-1-yl)methanesulfonamide s40<smiles>C#CCNC(C)(C)C</smiles>

To a stirred solution of propargylamine $(2.05 \mathrm{~mL}, 32 \mathrm{mmol})$ in $\mathrm{CH}_{2} \mathrm{Cl}_{2}(75 \mathrm{~mL})$ was added triethylamine $(10.6 \mathrm{~mL}, 76 \mathrm{mmol})$ and methanesulfonyl chloride $(2.35 \mathrm{~mL}, 30 \mathrm{mmol})$ at $0{ }^{\circ} \mathrm{C}$. After stirring overnight at room temperature diethyl ether $(300 \mathrm{~mL})$ was added, the reaction 
mixture was washed with aqueous $\mathrm{HCl}(1 \mathrm{~N}, 100 \mathrm{~mL})$, sat. $\mathrm{NH}_{4} \mathrm{Cl}(100 \mathrm{~mL})$ and brine $(100 \mathrm{~mL})$. The organic phase was dried over anhydrous $\mathrm{Na}_{2} \mathrm{SO}_{4}$ and concentrated. Additional column chromatography on silica gel (petroleum ether/EtOAc - 1.5/1) afforded the title compound s40. The spectral data are in good agreement with previous reports. ${ }^{[9]}$

Yield: $1.61 \mathrm{~g}(12.1 \mathrm{mmol}, 40 \%)$.

Physical State: colourless oil.

$\mathbf{R}_{\mathbf{f}}$ value: 0.26 (petroleum ether/EtOAc $-2 / 1$ ).

${ }^{1} \mathbf{H}$ NMR (Avance $\left.400 \mathrm{MHz}, \mathrm{CDCl}_{3}\right) \delta 4.79(\mathrm{~s}, 1 \mathrm{H}), 3.98(\mathrm{dd}, J=2.5,6.2 \mathrm{~Hz}, 2 \mathrm{H}), 3.10$ (s, $3 \mathrm{H}), 2.39(\mathrm{t}, J=2.5 \mathrm{~Hz}, 1 \mathrm{H}) \mathrm{ppm}$.

${ }^{13}$ C NMR (Avance $101 \mathrm{MHz}, \mathrm{CDCl}_{3}$ ) $\delta$ 79.0, 73.5, 41.6, 32.3 ppm.

IR (ATR, in $\left.\mathrm{CDCl}_{3}\right)$ v 3278 (m, broad), 3022 (w), 2933 (w), $1628(\mathrm{w}), 1432$ (m), 1412 (m), 1308 (s), 1140 (s), 1068 (s) $\mathrm{cm}^{-1}$.

HRMS (ESI, m/z) calcd. for $\mathrm{C}_{4} \mathrm{H}_{7} \mathrm{NO}_{2} \mathrm{SNa}^{+}$: 156.0090 , found: 156.0099 .

\section{$N$-cinnamyl- $N$-(prop-2-yn-1-yl)methanesulfonamide s41}

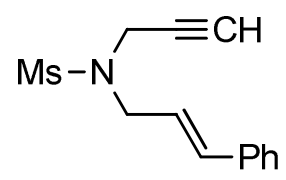

According to GP-3 s40 (1.33 g, $10 \mathrm{mmol}$ ) was dissolved in THF (40 mL), treated with cinnamyl alcohol (1.35 mL, $10.5 \mathrm{mmol}), \mathrm{PPh}_{3}(2.6 \mathrm{~g}, 10 \mathrm{mmol})$ and DIAD (2.1 mL, $10 \mathrm{mmol}$ ) to yield $\mathbf{s 4 1}$ after column chromatography on silica gel (petroleum ether/ $\mathrm{CH}_{2} \mathrm{Cl}_{2}-$ $1 / 3)$.

Yield: $1.89 \mathrm{~g}(7.57 \mathrm{mmol}, 76 \%)$.

Physical State: white solid.

$\mathbf{R}_{\mathbf{f}}$ Value: 0.43 (petroleum ether/ $\mathrm{CH}_{2} \mathrm{Cl}_{2}-1 / 3$ ).

${ }^{1} \mathbf{H}$ NMR (Avance $400 \mathrm{MHz}, \mathrm{CDCl}_{3}$ ) $\delta 7.39$ - $7.41(\mathrm{~m}, 2 \mathrm{H}), 7.32$ - $7.35(\mathrm{~m}, 2 \mathrm{H}), 7.27$ - 7.29 $(\mathrm{m}, 1 \mathrm{H}), 6.66(\mathrm{~d}, J=15.8 \mathrm{~Hz}, 1 \mathrm{H}), 6.17(\mathrm{dt}, J=6.9,15.7 \mathrm{~Hz}, 1 \mathrm{H}), 4.11(\mathrm{~d}, J=2.3 \mathrm{~Hz}, 2 \mathrm{H})$, $4.06(\mathrm{~d}, J=6.8 \mathrm{~Hz}, 2 \mathrm{H}), 2.99(\mathrm{~s}, 3 \mathrm{H}), 2.41(\mathrm{t}, J=2.3 \mathrm{~Hz}, 1 \mathrm{H}) \mathrm{ppm}$.

${ }^{13}$ C NMR (Avance $101 \mathrm{MHz}, \mathrm{CDCl}_{3}$ ) $\delta$ 136.0, 135.2, 128.8, 128.3, 126.7, 122.9, 77.4, 74.6, 48.8, 38.6, $35.8 \mathrm{ppm}$. 
IR (ATR, in $\left.\mathrm{CDCl}_{3}\right)$ v 3251 (m), 3018 (w), 2973 (w), 2933 (w), 2855 (w), 2115 (w), 1597 (w), 1495 (w), 1445 (w), 1430 (w), 1376 (w), 1341 (m), 1319 (s), 1278 (w), $1241(\mathrm{w})$, $1205(\mathrm{w}), 1179(\mathrm{w}), 1138(\mathrm{~s}), 1070(\mathrm{~m}), 1026(\mathrm{w}) \mathrm{cm}^{-1}$.

HRMS (ESI, m/z) calcd. for $\mathrm{C}_{13} \mathrm{H}_{15} \mathrm{NO}_{2} \mathrm{SNa}^{+}$: 272.0716, found: 272.0706.

\section{$N$-cinnamyl- $N$-(4-hydroxy-4,4-diphenylbut-2-yn-1-yl)methanesulfonamide $s 42$}

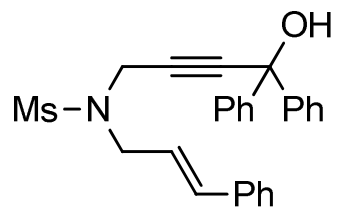

According to GP-1 s41 (0.75 g, 3 mmol) was dissolved in THF (30 mL), treated with $n$-BuLi (1.6 $\mathrm{M}$ in hexanes, $1.9 \mathrm{~mL}, 3 \mathrm{mmol})$ and benzophenone $(0.63 \mathrm{~g}, 3.5 \mathrm{mmol})$ in THF $(3 \mathrm{~mL})$ to yield $\mathbf{s} 42$ after column chromatography on silica gel (petroleum ether/EtOAc - 3/1).

Yield: $1.18 \mathrm{~g} \mathrm{(2.74} \mathrm{mmol,} \mathrm{91 \% ).}$

Physical State: pale yellow oil.

$\mathbf{R}_{\mathbf{f}}$ Value: 0.34 (petroleum ether/EtOAc - 3/1).

${ }^{1}$ H NMR (Avance $\left.400 \mathrm{MHz}, \mathrm{CDCl}_{3}\right) \delta 7.54-7.56(\mathrm{~m}, 4 \mathrm{H}), 7.24-7.36(\mathrm{~m}, 11 \mathrm{H}), 6.58(\mathrm{~d}$, $J=15.8 \mathrm{~Hz}, 1 \mathrm{H}), 6.15(\mathrm{dt}, J=6.9,15.6 \mathrm{~Hz}, 1 \mathrm{H}), 4.23(\mathrm{~s}, 2 \mathrm{H}), 3.98(\mathrm{~d}, J=6.8 \mathrm{~Hz}, 2 \mathrm{H}), 2.93$ (s, 1H), 2.74 (s, 3H) ppm.

${ }^{13}$ C NMR (Avance $101 \mathrm{MHz}, \mathrm{CDCl}_{3}$ ) $\delta 144.5,136.0,135.3,128.8,128.5,128.4,128.2$, $126.7,126.1,122.8,89.4,81.2,74.6,49.3,38.4,36.4$ ppm.

IR (ATR, in $\mathrm{CDCl}_{3}$ ) v 3464 (w, broad), 3059 (w), 3026 (w), 2928 (w), 2854 (w), 1959 (w), 1598 (w), 1401 (w), 1449 (m), 1321 (s), 1204 (w), 1147 (s), 1073 (w), 1032 (w), 1014 (w) $\mathrm{cm}^{-1}$.

HRMS (ESI, m/z) calcd. for $\mathrm{C}_{26} \mathrm{H}_{25} \mathrm{NO}_{3} \mathrm{SNa}^{+}$: 454.1447, found: 454.1424.

\section{(E)-4-(N-cinnamylmethylsulfonamido)-1,1-diphenylbut-2-yn-1-yl acetate $s 43$}

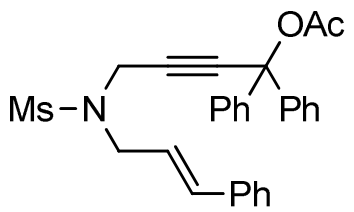


According to GP-2 s42 $(1.1 \mathrm{~g}, 2.6 \mathrm{mmol})$ was dissolved in $\mathrm{CH}_{2} \mathrm{Cl}_{2}(3 \mathrm{~mL})$, treated with acetic anhydride $(0.72 \mathrm{~mL}, 7.7 \mathrm{mmol})$, DMAP $(15.6 \mathrm{mg}, 0.13 \mathrm{mmol})$ and triethylamine $(0.53 \mathrm{~mL}$, $3.8 \mathbf{m m o l}$ ) to yield $\mathbf{s} \mathbf{4 3}$ after column chromatography on silica gel (petroleum ether/EtOAc $5 / 1 \rightarrow 4 / 1)$.

Yield: $1.21 \mathrm{~g}$ (2.55 mmol, quant.).

Physical State: white solid.

Melting Point: $47^{\circ} \mathrm{C}$.

$\mathbf{R}_{\mathbf{f}}$ Value: 0.37 (petroleum ether/EtOAc - 3/1).

${ }^{1} \mathbf{H}$ NMR (Avance $\left.400 \mathrm{MHz}, \mathrm{CDCl}_{3}\right) \delta 7.46$ - $7.48(\mathrm{~m}, 4 \mathrm{H}), 7.25$ - $7.38(\mathrm{~m}, 11 \mathrm{H}), 6.64(\mathrm{~d}$, $J=15.8 \mathrm{~Hz}, 1 \mathrm{H}), 6.17(\mathrm{dt}, J=7.1,15.3 \mathrm{~Hz}, 1 \mathrm{H}), 4.26(\mathrm{~s}, 2 \mathrm{H}), 4.02(\mathrm{~d}, J=6.8 \mathrm{~Hz}, 2 \mathrm{H}), 2.79$ (s, 3H), 2.17 (s, 3H) ppm.

${ }^{13}$ C NMR (Avance $101 \mathrm{MHz}, \mathrm{CDCl}_{3}$ ) $\delta 168.4,142.1,136.1,135.3,128.8,128.6,128.3$, $126.7,126.2,123.0,85.6,83.8,79.2,49.1,38.2,36.5,21.9$ ppm. 1 carbon was not observed due to incidental equivalence.

IR (ATR, in $\left.\mathrm{CDCl}_{3}\right)$ v 3060 (w), 3027 (w), 2931 (w), 2256 (w), 1749 (s), 1598 (w), 1493 (w), 1450 (w), 1367 (w), 1342 (s), 1327 (s), 1224 (s), 1200 (m), 1151 (s), 1072 (w), 1015 (w) cm cm $^{-1}$ HRMS (ESI, m/z) calcd. for $\mathrm{C}_{28} \mathrm{H}_{27} \mathrm{NO}_{4} \mathrm{SNa}^{+}$: 496.1553, found: 496.1565 . 


\subsection{Preparation of $(E)-4-(N$-cinnamylphenylsulfonamido)-1,1-diphenylbut-2-yn-1-yl}

acetate 35
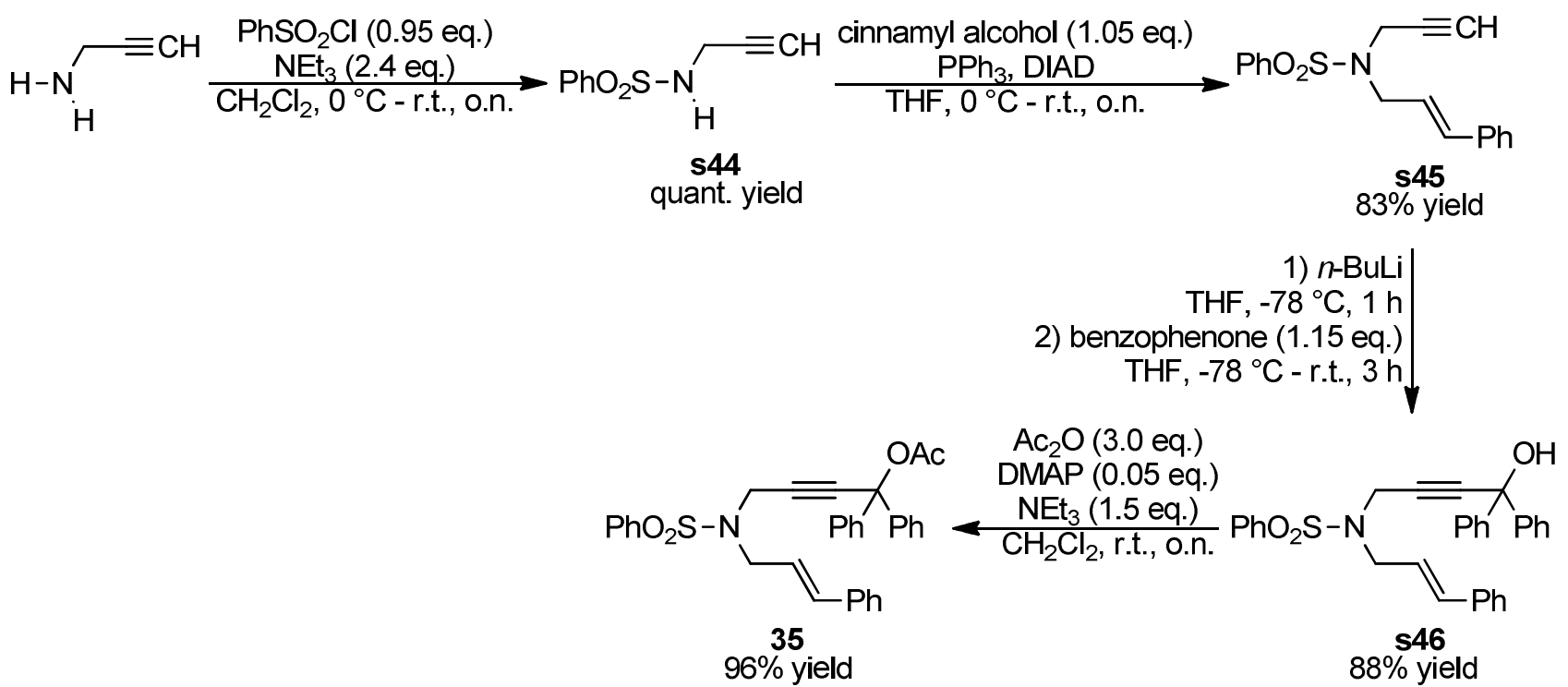

$N$-(prop-2-yn-1-yl)benzenesulfonamide s44<smiles>C#CCNS(=O)(=O)O</smiles>

To a stirred solution of propargylamine $(1.34 \mathrm{~mL}, 21 \mathrm{mmol})$ in $\mathrm{CH}_{2} \mathrm{Cl}_{2}(50 \mathrm{~mL})$ was added triethylamine $(7 \mathrm{~mL}, 50 \mathrm{mmol})$ and benzenesulfonyl chloride $(2.56 \mathrm{~mL}, 20 \mathrm{mmol})$ at $0{ }^{\circ} \mathrm{C}$. After stirring overnight at room temperature diethyl ether $(200 \mathrm{~mL})$ was added, the reaction mixture was washed with aqueous $\mathrm{HCl}(1 \mathrm{~N}, 80 \mathrm{~mL})$, sat. $\mathrm{NH}_{4} \mathrm{Cl}(80 \mathrm{~mL})$ and brine $(80 \mathrm{~mL})$. The organic phase was dried over anhydrous $\mathrm{Na}_{2} \mathrm{SO}_{4}$ and concentrated under reduced pressure to yield s44. The spectral data are in good agreement with previous reports. ${ }^{[10]}$

Yield: $3.98 \mathrm{~g}$ (20.4 mmol, quant.).

Physical State: white solid.

${ }^{1}$ H NMR (Avance $300 \mathrm{MHz}, \mathrm{CDCl}_{3}$ ) $\delta 7.88$ - $7.92(\mathrm{~m}, 2 \mathrm{H}), 7.49-7.62(\mathrm{~m}, 3 \mathrm{H}), 4.95(\mathrm{~s}, 1 \mathrm{H})$, $3.85(\mathrm{dd}, J=2.5,6.1 \mathrm{~Hz}, 2 \mathrm{H}), 2.07(\mathrm{t}, J=2.5 \mathrm{~Hz}, 1 \mathrm{H}) \mathrm{ppm}$.

${ }^{13}$ C NMR (Avance $75 \mathrm{MHz}, \mathrm{CDCl}_{3}$ ) $\delta$ 139.7, 133.1, 129.2, 127.5, 78.0, 73.1, 33.0 ppm.

IR (ATR, in $\left.\mathrm{CDCl}_{3}\right)$ v 3279 (m, broad), 1586 (w), 1480 (w), 1447 (m), 1429 (w), 1321 (s), 1154 (s), 1091 (s), 1068 (s), 1025 (w) $\mathrm{cm}^{-1}$.

HRMS (ESI, m/z) calcd. for $\mathrm{C}_{9} \mathrm{H}_{9} \mathrm{NO}_{2} \mathrm{SNa}^{+}$: 218.0246, found: 218.0264. 


\section{$N$-cinnamyl- $N$-(prop-2-yn-1-yl)benzenesulfonamide s45}

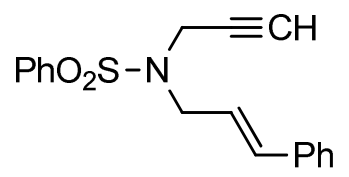

According to GP-3 s44 (1.95 g, $10 \mathrm{mmol}$ ) was dissolved in THF (40 mL), treated with cinnamyl alcohol (1.35 mL, $10.5 \mathrm{mmol}), \mathrm{PPh}_{3}(2.6 \mathrm{~g}, 10 \mathrm{mmol})$ and DIAD (2.1 mL, $10 \mathbf{m m o l}$ ) to yield $\mathbf{s 4 5}$ after column chromatography on silica gel (petroleum ether/EtOAc $7 / 1)$.

Yield: $2.57 \mathrm{~g}(8.26 \mathrm{mmol}, 83 \%)$.

Physical State: white solid.

$\mathbf{R}_{\mathbf{f}}$ Value: 0.29 (petroleum ether/EtOAc - 7/1).

${ }^{1} \mathbf{H}$ NMR (Avance $400 \mathrm{MHz}, \mathrm{CDCl}_{3}$ ) $\delta 7.80$ - $7.82(\mathrm{~m}, 2 \mathrm{H}), 7.49$ - $7.53(\mathrm{~m}, 1 \mathrm{H}), 7.42$ - 7.46 (m, 2H), $7.23-7.27(\mathrm{~m}, 4 \mathrm{H}), 7.16-7.21(\mathrm{~m}, 1 \mathrm{H}), 6.50(\mathrm{~d}, J=15.8 \mathrm{~Hz}, 1 \mathrm{H}), 6.00(\mathrm{dt}, J=6.9$, $15.8 \mathrm{~Hz}, 1 \mathrm{H}), 4.06(\mathrm{~d}, J=2.4 \mathrm{~Hz}, 2 \mathrm{H}), 3.94(\mathrm{~d}, J=6.4 \mathrm{~Hz}, 2 \mathrm{H}), 1.94(\mathrm{t}, J=2.4 \mathrm{~Hz}, 1 \mathrm{H}) \mathrm{ppm}$.

${ }^{13}$ C NMR (Avance $101 \mathrm{MHz}, \mathrm{CDCl}_{3}$ ) $\delta 139.1,136.1,135.2,133.0,129.0,128.7,128.2$, $127.8,126.7,122.8,76.5,74.0,48.7,36.0 \mathrm{ppm}$.

IR (ATR, in $\mathrm{CDCl}_{3}$ ) v 3284 (w), 3061 (w), 3027 (w), 2917 (w), 1598 (w), 1496 (w), 1479 (w), 1446 (w), 1346 (m), 1329 (m), 1157 (s), 1093 (m), 1071 (w), 1025 (w) $\mathrm{cm}^{-1}$.

HRMS (ESI, m/z) calcd. for $\mathrm{C}_{18} \mathrm{H}_{17} \mathrm{NO}_{2} \mathrm{SNa}^{+}$: 334.0872, found: 334.0871.

\section{$N$-cinnamyl- $N$-(4-hydroxy-4,4-diphenylbut-2-yn-1-yl)benzenesulfonamide s46}

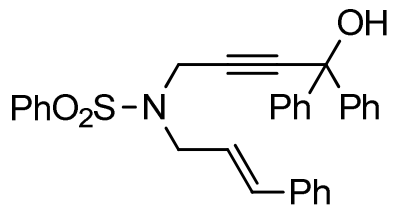

According to GP-1 s45 (2.49 g, $8 \mathrm{mmol})$ was dissolved in THF (100 mL), treated with $n$-BuLi (1.6 M in hexanes, $5 \mathrm{~mL}, 3 \mathrm{mmol})$ and benzophenone $(1.68 \mathrm{~g}, 9.2 \mathrm{mmol})$ in $\mathrm{THF}$ $(10 \mathrm{~mL})$ to yield $\mathbf{s 4 6}$ after column chromatography on silica gel (petroleum ether/EtOAc $5 / 1)$.

Yield: $3.34 \mathrm{~g}(7.04 \mathrm{mmol}, 88 \%)$.

Physical State: white solid. 
$\mathbf{R}_{\mathbf{f}}$ Value: 0.23 (petroleum ether/EtOAc - 5/1).

${ }^{1} \mathbf{H}$ NMR (Avance $400 \mathrm{MHz}, \mathrm{CDCl}_{3}$ ) $\delta 7.83$ - $7.85(\mathrm{~m}, 2 \mathrm{H}), 7.44-7.47(\mathrm{~m}, 1 \mathrm{H}), 7.32-7.36$ $(\mathrm{m}, 6 \mathrm{H}), 7.22-7.29(\mathrm{~m}, 11 \mathrm{H}), 6.42(\mathrm{~d}, J=15.8 \mathrm{~Hz}, 1 \mathrm{H}), 6.05(\mathrm{dt}, J=6.9,15.7 \mathrm{~Hz}, 1 \mathrm{H}), 4.28$ (s, 2H), $3.97(\mathrm{~d}, J=6.8 \mathrm{~Hz}, 2 \mathrm{H}), 2.45(\mathrm{~s}, 1 \mathrm{H}) \mathrm{ppm}$.

${ }^{13} \mathbf{C}$ NMR (Avance $101 \mathrm{MHz}, \mathrm{CDCl}_{3}$ ) $\delta$ 144.4, 139.0, 136.0, 135.3, 132.8, 129.1, 128.7, $128.3,128.2,127.9,127.8,126.6,126.0,122.7,88.8,80.1,74.2,49.1,36.5$ ppm.

IR (ATR, in $\left.\mathrm{CDCl}_{3}\right)$ v 3482 (w, broad), 3062 (w), 1598 (w), 1491 (w), 1448 (w), 1347 (w), $1329(\mathrm{w}), 1162(\mathrm{~m}), 1095(\mathrm{w}), 1010(\mathrm{w}) \mathrm{cm}^{-1}$.

HRMS (ESI, m/z) calcd. for $\mathrm{C}_{31} \mathrm{H}_{27} \mathrm{NO}_{3} \mathrm{SNa}^{+}:$516.1604, found: 516.1603.

\section{(E)-4-(N-cinnamylphenylsulfonamido)-1,1-diphenylbut-2-yn-1-yl acetate 35}

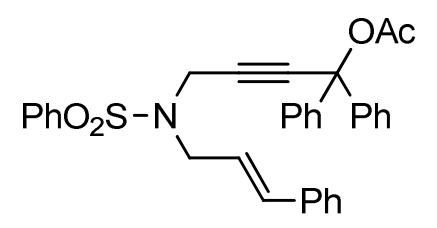

According to GP-2 s46 (1.0 g, $2 \mathrm{mmol})$ was dissolved in $\mathrm{CH}_{2} \mathrm{Cl}_{2}(2 \mathrm{~mL})$, treated with acetic anhydride $(0.57 \mathrm{~mL}, 6 \mathrm{mmol})$, DMAP $(12.2 \mathrm{mg}, 0.12 \mathrm{mmol})$ and triethylamine $(0.42 \mathrm{~mL}$, $3 \mathrm{mmol}$ ) to yield 35 after column chromatography on silica gel ( $n$-pentane/ $\mathrm{Et}_{2} \mathrm{O}-2 / 1$ ).

Yield: $1.03 \mathrm{~g}(1.93 \mathrm{mmol}, 96 \%)$.

Physical State: white solid.

Melting Point: $137^{\circ} \mathrm{C}$.

$\mathbf{R}_{\mathbf{f}}$ Value: $0.31\left(n\right.$-pentane/ $\left.\mathrm{Et}_{2} \mathrm{O}-2 / 1\right)$.

${ }^{1} \mathbf{H}$ NMR (Avance $400 \mathrm{MHz}, \mathrm{CDCl}_{3}$ ) $\delta 7.87$ - $7.89(\mathrm{~m}, 2 \mathrm{H}), 7.51-7.55(\mathrm{~m}, 1 \mathrm{H}), 7.41-7.45$ $(\mathrm{m}, 2 \mathrm{H}), 7.23-7.30(\mathrm{~m}, 15 \mathrm{H}), 6.52(\mathrm{~d}, J=15.8 \mathrm{~Hz}, 1 \mathrm{H}), 6.06(\mathrm{dt}, J=7.0,15.6 \mathrm{~Hz}, 1 \mathrm{H}), 4.35$ (s, 2H), $3.98(\mathrm{~d}, J=6.9 \mathrm{~Hz}, 2 \mathrm{H}), 2.08(\mathrm{~s}, 3 \mathrm{H}) \mathrm{ppm}$.

${ }^{13} \mathbf{C}$ NMR (Avance $101 \mathrm{MHz}, \mathrm{CDCl}_{3}$ ) $\delta$ 168.0, 142.2, 139.3, 136.3, 135.5, 132.8, 129.2, $128.7,128.4,128.1,128.1,127.7,126.7,126.2,122.6,85.0,82.8,79.0,48.7,36.5,22.0$ ppm.

IR (ATR, in $\mathrm{CDCl}_{3}$ ) v 3062 (w), 3028 (w), 1750 (m), 1598 (w), 1492 (w), 1448 (w),

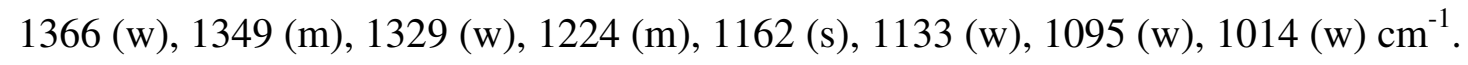

HRMS (ESI, m/z) calcd. for $\mathrm{C}_{33} \mathrm{H}_{29} \mathrm{NO}_{4} \mathrm{SNa}^{+}:$558.1710, found: 558.1735. 


\section{$D_{3}-(E)-4-(N$-cinnamylphenylsulfonamido)-1,1-diphenylbut-2-yn-1-yl acetate 35-D}

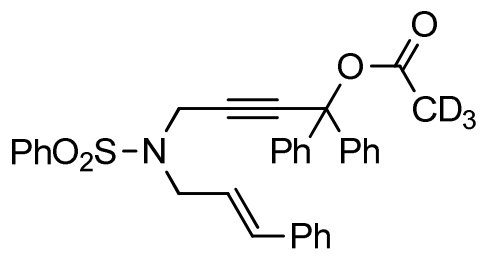

According to GP-2 s46 (0.62 g, $1.25 \mathrm{mmol})$ was dissolved in $\mathrm{CH}_{2} \mathrm{Cl}_{2}(1.5 \mathrm{~mL})$, treated with acetic anhydride- $\mathrm{d}_{6}(0.35 \mathrm{~mL}, 3.75 \mathrm{mmol})$, DMAP $(7.7 \mathrm{mg}, 0.06 \mathrm{mmol})$ and triethylamine $(0.26 \mathrm{~mL}, 1.87 \mathrm{mmol})$ to yield $\mathbf{3 5}-\mathbf{D}_{\mathbf{3}}$ after column chromatography on silica gel (n-pentane/ $\left.\mathrm{Et}_{2} \mathrm{O}-2 / 1\right)$.

Yield: $0.66 \mathrm{~g}(1.23 \mathrm{mmol}, 99 \%)$.

Physical State: white solid.

$\mathbf{R}_{\mathbf{f}}$ Value: 0.34 (n-pentane/Et $\left.2 \mathrm{O}-2 / 1\right)$.

${ }^{1} \mathbf{H}$ NMR (Avance $400 \mathrm{MHz}, \mathrm{CDCl}_{3}$ ) $\delta 7.86$ - $7.88(\mathrm{~m}, 2 \mathrm{H}), 7.51-7.54(\mathrm{~m}, 1 \mathrm{H}), 7.41-7.45$ (m, 2H), $7.25-7.29(\mathrm{~m}, 15 \mathrm{H}), 6.52(\mathrm{~d}, J=15.8 \mathrm{~Hz}, 1 \mathrm{H}), 6.05(\mathrm{dt}, J=7.4,14.7 \mathrm{~Hz}, 1 \mathrm{H}), 4.35$ (s, 2H), $3.98(\mathrm{~d}, J=6.8 \mathrm{~Hz}, 2 \mathrm{H}), 2.05(\mathrm{~s}, 0.14 \mathrm{H}) \mathrm{ppm}$.

${ }^{13}$ C NMR (Avance $101 \mathrm{MHz}, \mathrm{CDCl}_{3}$ ) $\delta$ 168.0, 142.1, 139.2, 136.3, 135.5, 132.8, 129.1, $128.7,128.3,128.1,128.0,127.7,126.7,126.1,122.6,85.0,82.8,79.0,48.7,36.5$ ppm. $\mathrm{CD}_{3}$ was not observed.

IR (ATR, in $\mathrm{CDCl}_{3}$ ) v 3060 (w), 3027 (w), 2918 (w), 1747 (m), 1598 (w), 1492 (w), 1447 (w), 1349 (m), 1329 (w), 1238 (m), 1200 (w), 1162 (s), 1134 (w), 1094 (w), 1067 (m), $1031(\mathrm{w}) \mathrm{cm}^{-1}$.

HRMS (ESI, m/z) calcd. for $\mathrm{C}_{33} \mathrm{H}_{26} \mathrm{D}_{3} \mathrm{NO}_{4} \mathrm{SNa}^{+}$: 561.1898, found: 561.1873. 


\subsection{Preparation of 4-(4-methyl- $N$-(3-methylbut-2-en-1-yl)phenylsulfonamido)-1-}

phenylbut-2-yn-1-yl acetate 38
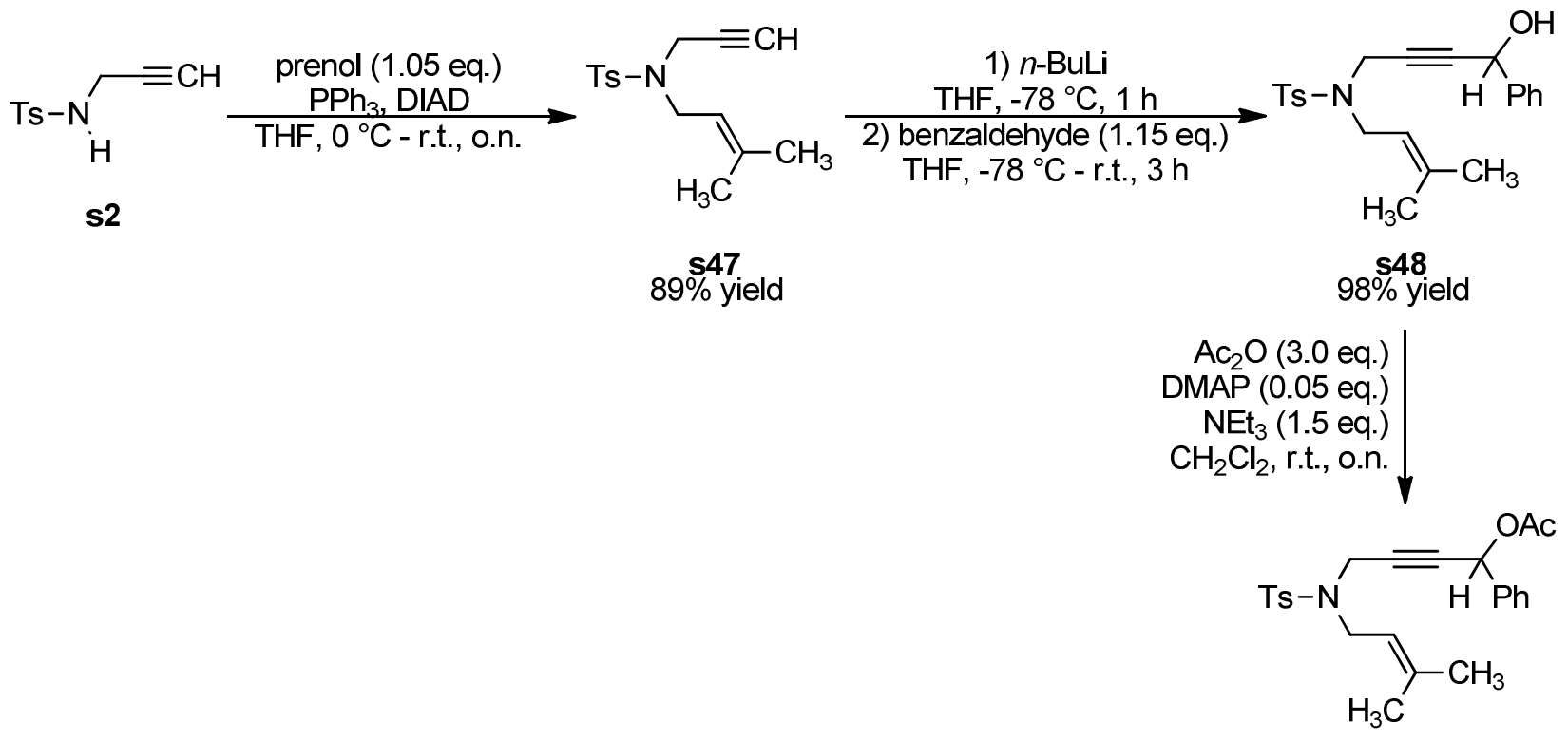

38

$93 \%$ yield

\section{4-methyl- $N$-(3-methylbut-2-enyl)- $N$-(prop-2-ynyl)benzenesulfonamide s47}<smiles>C#CCN([Hg])CC=C(C)C</smiles>

According to GP-3 s2 (2.1 g, $10 \mathrm{mmol})$ was dissolved in THF (40 mL), treated with prenol (1.1 mL, $10.5 \mathrm{mmol}), \mathrm{PPh}_{3}(2.6 \mathrm{~g}, 10 \mathrm{mmol})$ and DIAD $(2.1 \mathrm{~mL}, 10 \mathrm{mmol})$ to yield $\mathbf{s} 47 \mathrm{after}$ column chromatography on silica gel (petroleum ether/EtOAc - 20/1). The spectral data are in good agreement with previous reports. ${ }^{[11]}$

Yield: $2.48 \mathrm{~g}(8.93 \mathrm{mmol}, 89 \%)$.

Physical State: pale yellow liquid.

$\mathbf{R}_{\mathbf{f}}$ Value: 0.29 (petroleum ether/EtOAc - 20/1).

${ }^{1} \mathbf{H}$ NMR (Avance $400 \mathrm{MHz}, \mathrm{CDCl}_{3}$ ) $\delta 7.73$ - $7.75(\mathrm{~m}, 2 \mathrm{H}), 7.28-7.30(\mathrm{~m}, 2 \mathrm{H}), 5.08-5.12$ $(\mathrm{m}, 1 \mathrm{H}), 4.07(\mathrm{~d}, J=2.4 \mathrm{~Hz}, 2 \mathrm{H}), 3.81(\mathrm{~d}, J=7.3 \mathrm{~Hz}, 2 \mathrm{H}), 2.43(\mathrm{~s}, 3 \mathrm{H}), 1.98$ (t, $J=2.5 \mathrm{~Hz}$, $1 \mathrm{H}), 1.72(\mathrm{~s}, 3 \mathrm{H}), 1.70$ (s, 3H) ppm. 
${ }^{1} \mathbf{H}$ NMR (Avance $\left.400 \mathrm{MHz}, \mathrm{C}_{6} \mathrm{D}_{6}\right) \delta 7.77-7.79(\mathrm{~m}, 2 \mathrm{H}), 6.80-6.82(\mathrm{~m}, 2 \mathrm{H}), 5.03-5.07$ (m, 1H), 3.98 (d, $J=2.4 \mathrm{~Hz}, 2 \mathrm{H}), 3.87$ (d, $J=7.3 \mathrm{~Hz}, 2 \mathrm{H}), 1.92(\mathrm{~s}, 3 \mathrm{H}), 1.53$ (t, $J=2.4 \mathrm{~Hz}$, $1 \mathrm{H}), 1.46(\mathrm{~s}, 6 \mathrm{H}) \mathrm{ppm}$.

${ }^{13}$ C NMR (Avance $101 \mathrm{MHz}, \mathrm{CDCl}_{3}$ ) $\delta$ 143.4, 139.1, 136.1, 129.4, 127.8, 117.9, 73.4, 43.9, 35.4, 25.9, 21.6, 17.9 ppm. 1 carbon was hidden under $\mathrm{CDCl}_{3}$.

${ }^{13}$ C NMR (Avance $101 \mathrm{MHz}, \mathrm{C}_{6} \mathrm{D}_{6}$ ) $\delta$ 142.9, 138.5, 137.6, 129.5, 128.3, 118.8, 77.6, 73.4, $44.2,35.6,25.7,21.2,17.7 \mathrm{ppm}$.

IR (ATR, in $\mathrm{CDCl}_{3}$ ) v 3276 (w), 2973 (w), 2921 (w), 1673 (w), 1598 (w), 1494 (w), 1444 (w), 1342 (m), 1157 (s), 1092 (m), 1070 (w) $\mathrm{cm}^{-1}$.

HRMS (ESI, m/z) calcd. for $\mathrm{C}_{15} \mathrm{H}_{19} \mathrm{NO}_{2} \mathrm{SNa}^{+}: 300.1029$, found: 300.1030 .

\section{$N$-(4-hydroxy-4-phenylbut-2-yn-1-yl)-4-methyl- $N$-(3-methylbut-2-en-1-}

yl)benzenesulfonamide $\mathrm{s} 48$

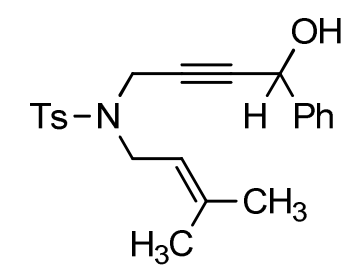

According to GP-1 s47 (1.1 g, 4 mmol) was dissolved in THF (40 mL), treated with $n$-BuLi (1.6 $\mathrm{M}$ in hexanes, $2.5 \mathrm{~mL}, 4 \mathrm{mmol})$ and benzaldehyde $(0.47 \mathrm{~mL}, 4.6 \mathrm{mmol})$ to yield $\mathbf{s} 48$ after column chromatography on silica gel (petroleum ether/EtOAc $-4 / 1 \rightarrow 3 / 1$ ).

Yield: $1.51 \mathrm{~g}(3.94 \mathrm{mmol}, 98 \%)$.

Physical State: pale yellow oil.

$\mathbf{R}_{\mathbf{f}}$ Value: 0.23 (petroleum ether/EtOAc - 4/1).

${ }^{1} \mathbf{H}$ NMR (Avance $400 \mathrm{MHz}, \mathrm{CDCl}_{3}$ ) $\delta 7.70$ - $7.72(\mathrm{~m}, 2 \mathrm{H}), 7.28$ - $7.33(\mathrm{~m}, 3 \mathrm{H}), 7.25$ - 7.27 $(\mathrm{m}, 2 \mathrm{H}), 7.18-7.20(\mathrm{~m}, 2 \mathrm{H}), 5.15-5.16(\mathrm{~m}, 1 \mathrm{H}), 5.07-5.11(\mathrm{~m}, 1 \mathrm{H}), 4.08-4.18(\mathrm{~m}, 2 \mathrm{H})$, $3.74-3.83(\mathrm{~m}, 2 \mathrm{H}), 2.33$ (s, 3H), 2.13 (s, 1H), 1.69 (s, 3H), 1.57 (s, 3H) ppm.

${ }^{13}$ C NMR (Avance $101 \mathrm{MHz}, \mathrm{CDCl}_{3}$ ) $\delta$ 143.5, 140.2, 139.2, 136.1, 129.5, 128.6, 128.4, $128.0,126.4,117.9,85.2,79.8,64.3,44.2,35.8,25.9,21.6,17.9$ ppm. 
IR (ATR, in $\mathrm{CDCl}_{3}$ ) v 3488 (w, broad), 3031 (w), 2972 (w), 2917 (w), 1672 (w), 1598 (w), 1494 (w), 1451 (w), 1378 (w), 1338 (m), 1306 (w), 1259 (w), 1186 (w), 1155 (s), 1091 (m), $1068(\mathrm{w}), 1017(\mathrm{w}) \mathrm{cm}^{-1}$.

HRMS (ESI, m/z) calcd. for $\mathrm{C}_{22} \mathrm{H}_{25} \mathrm{NO}_{3} \mathrm{SNa}^{+}:$406.1447, found: 406.1438 .

\subsection{4-(4-methyl- $N$-(3-methylbut-2-en-1-yl)phenylsulfonamido)-1-phenylbut-2-yn-1-yl acetate 38}

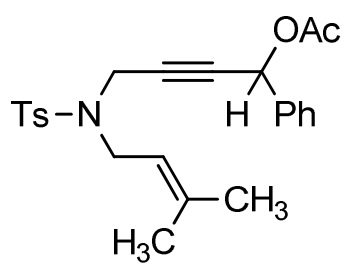

According to GP-2 s48 (1.46 g, $3.8 \mathrm{mmol})$ was dissolved in $\mathrm{CH}_{2} \mathrm{Cl}_{2}(4 \mathrm{~mL})$, treated with acetic anhydride $(1.1 \mathrm{~mL}, 11.4 \mathrm{mmol})$, DMAP $(23.2 \mathrm{mg}, 0.19 \mathrm{mmol})$ and triethylamine $(0.79 \mathrm{~mL}, 5.7 \mathrm{mmol})$ to yield 38 after column chromatography on silica gel ( $n$-pentane/Et ${ }_{2} \mathrm{O}-$ $4 / 1)$.

Yield: $1.51 \mathrm{~g}(3.54 \mathrm{mmol}, 93 \%)$.

Physical State: pale yellow oil.

$\mathbf{R}_{\mathbf{f}}$ Value: $0.26\left(n\right.$-pentane/ $\left.\mathrm{Et}_{2} \mathrm{O}-4 / 1\right)$.

${ }^{1}$ H NMR (Avance $\left.400 \mathrm{MHz}, \mathrm{CDCl}_{3}\right) \delta 7.69$ - $7.71(\mathrm{~m}, 2 \mathrm{H}), 7.30$ - $7.35(\mathrm{~m}, 3 \mathrm{H}), 7.23$ - 7.27 $(\mathrm{m}, 2 \mathrm{H}), 7.16-7.18(\mathrm{~m}, 2 \mathrm{H}), 6.16(\mathrm{t}, J=1.6 \mathrm{~Hz}, 1 \mathrm{H}), 5.07-5.11(\mathrm{~m}, 1 \mathrm{H}), 4.11-4.23(\mathrm{~m}$, 2H), $3.71-3.83(\mathrm{~m}, 2 \mathrm{H}), 2.34(\mathrm{~s}, 3 \mathrm{H}), 2.04(\mathrm{~s}, 3 \mathrm{H}), 1.70$ (s, 3H), 1.57 (s, 3H) ppm.

${ }^{13}$ C NMR (Avance $101 \mathrm{MHz}, \mathrm{CDCl}_{3}$ ) $\delta$ 169.6, 143.4, 139.2, 136.6, 136.0, 129.5, 129.0, $128.7,127.8,127.6,117.9,82.0,80.6,65.3,44.2,35.8,25.9,21.6,21.0,17.8$ ppm.

IR (ATR, in $\left.\mathrm{CDCl}_{3}\right) \vee 2925$ (w), 1739 (m), 1598 (w), 1495 (w), 1454 (w), 1370 (w), 1346 (m), 1226 (m), 1160 (s), 1092 (m), 1069 (w), 1017 (w) $\mathrm{cm}^{-1}$.

HRMS (ESI, m/z) calcd. for $\mathrm{C}_{24} \mathrm{H}_{27} \mathrm{NO}_{4} \mathrm{SNa}^{+}:$448.1553, found: 448.1554 . 


\subsection{Preparation of 4-(4-methyl- $N$-(3-methylbut-2-enyl)phenylsulfonamido)-1,1-}

diphenylbut-2-ynyl acetate s50
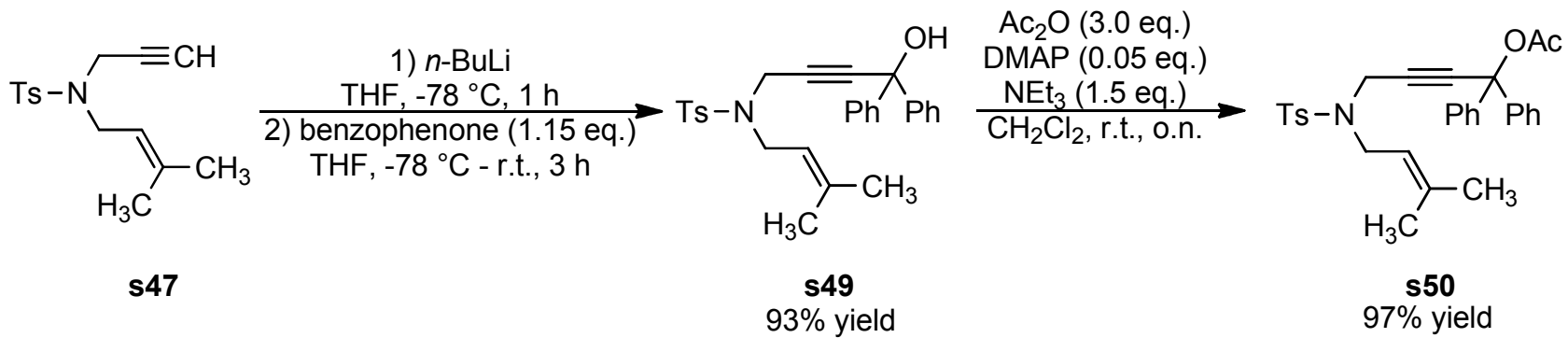

\section{$N$-(4-hydroxy-4,4-diphenylbut-2-ynyl)-4-methyl- $N$-(3-methylbut-2-}

enyl)benzenesulfonamide $\mathbf{s} 49$<smiles>CC(C)=CCN([Hg])CC#CC(O)(c1ccccc1)c1ccccc1</smiles>

According to GP-1 s47 (1.1 g, 4 mmol) was dissolved in THF (40 mL), treated with $n$-BuLi (1.6 $\mathrm{M}$ in hexanes, $2.5 \mathrm{~mL}, 4 \mathrm{mmol})$ and benzophenone $(0.84 \mathrm{~g}, 3.5 \mathrm{mmol})$ in THF $(4 \mathrm{~mL})$ to yield $\mathbf{s 9 9}$ after column chromatography on silica gel (petroleum ether/EtOAc - 5/1).

Yield: $1.71 \mathrm{~g}(3.72 \mathrm{mmol}, 93 \%)$.

Physical State: white solid.

$\mathbf{R}_{\mathbf{f}}$ Value: 0.31 (petroleum ether/EtOAc - 5/1).

${ }^{1} \mathbf{H}$ NMR (Avance $400 \mathrm{MHz}, \mathrm{CDCl}_{3}$ ) $\delta 7.69$ - $7.71(\mathrm{~m}, 2 \mathrm{H}), 7.29$ - $7.33(\mathrm{~m}, 4 \mathrm{H}), 7.23$ - 7.28 $(\mathrm{m}, 6 \mathrm{H}), 7.06-7.08(\mathrm{~m}, 2 \mathrm{H}), 5.08-5.13(\mathrm{~m}, 1 \mathrm{H}), 4.22(\mathrm{~s}, 2 \mathrm{H}), 3.80(\mathrm{~d}, J=7.2 \mathrm{~Hz}, 2 \mathrm{H})$, $2.35-2.36(\mathrm{~m}, 1 \mathrm{H}), 2.26(\mathrm{~s}, 3 \mathrm{H}), 1.68(\mathrm{~s}, 3 \mathrm{H}), 1.51$ (s, 3H) ppm.

${ }^{13} \mathrm{C}$ NMR (Avance $101 \mathrm{MHz}, \mathrm{CDCl}_{3}$ ) $\delta$ 144.4, 143.5, 139.2, 136.3, 129.5, 128.2, 127.9, $127.8,125.9,117.9,88.1,80.4,74.1,44.2,35.8,25.9,21.5,17.8 \mathrm{ppm}$.

IR (ATR, in $\mathrm{CDCl}_{3}$ ) v 3482 (w, broad), 3060 (w), 3028 (w), 2972 (w), 2917 (w), 1672 (w), 1598 (w), 1491 (w), 1449 (w), 1341 (m), 1156 (s), 1069 (m), 1015 (m) cm ${ }^{-1}$.

HRMS (ESI, m/z) calcd. for $\mathrm{C}_{28} \mathrm{H}_{29} \mathrm{NO}_{3} \mathrm{SNa}^{+}$: 482.1760, found: 482.1752. 

s50

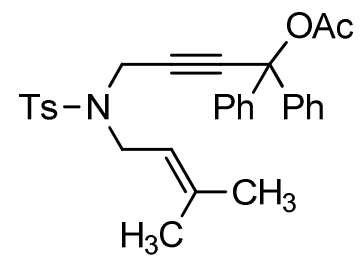

According to GP-2 s49 (1.37 g, $3.0 \mathrm{mmol})$ was dissolved in $\mathrm{CH}_{2} \mathrm{Cl}_{2}(3 \mathrm{~mL})$, treated with acetic anhydride $(0.85 \mathrm{~mL}, 9 \mathrm{mmol})$, DMAP $(18.3 \mathrm{mg}, 0.15 \mathrm{mmol})$ and triethylamine $(0.62 \mathrm{~mL}, 4.5 \mathrm{mmol})$ to yield $\mathbf{5 5 0}$ after column chromatography on silica gel (petroleum ether/EtOAc - 5/1).

Yield: $1.45 \mathrm{~g}$ (2.90 mmol, 97\%).

Physical State: white solid.

Melting Point: $139^{\circ} \mathrm{C}$.

$\mathbf{R}_{\mathbf{f}}$ Value: 0.29 (petroleum ether/EtOAc - 5/1).

${ }^{1} \mathbf{H}$ NMR (Avance $300 \mathrm{MHz}, \mathrm{CDCl}_{3}$ ) $\delta 7.70$ - $7.73(\mathrm{~m}, 2 \mathrm{H}), 7.25(\mathrm{~s}, 10 \mathrm{H}), 7.12$ - $7.15(\mathrm{~m}$, 2H), $5.08-5.13(\mathrm{~m}, 1 \mathrm{H}), 4.29(\mathrm{~s}, 2 \mathrm{H}), 3.77(\mathrm{~d}, J=7.2 \mathrm{~Hz}, 2 \mathrm{H}), 2.32$ (s, 3H), 2.09 (s, 3H), $1.68(\mathrm{~s}, 3 \mathrm{H}), 1.53(\mathrm{~s}, 3 \mathrm{H}) \mathrm{ppm}$.

${ }^{13}$ C NMR (Avance $75 \mathrm{MHz}, \mathrm{CDCl}_{3}$ ) $\delta$ 167.9, 143.2, 142.2, 139.0, 136.7, 129.7, 128.3, 128.0, $127.7,126.2,118.2,84.2,83.6,79.2,44.1,36.1,25.9,21.8,21.7,17.8$ ppm.

IR (ATR, in $\mathrm{CDCl}_{3}$ ) v 3061 (w), 2972 (w), 2918 (w), 1752 (m), 1598 (w), 1492 (w), 1450 (w), 1345 (m), 1222 (s), 1158 (s), 1093 (m) $\mathrm{cm}^{-1}$.

HRMS (ESI, m/z) calcd. for $\mathrm{C}_{30} \mathrm{H}_{31} \mathrm{NO}_{4} \mathrm{SNa}^{+}$: 524.1886, found: 524.1881. 


\subsection{Preparation of (E)-4-(4-methyl- $N$-(3-(naphthalen-2-yl)allyl)phenylsulfonamido)-}

\section{1,1-diphenylbut-2-yn-1-yl acetate s55}<smiles>O=Cc1ccc2ccccc2c1</smiles>
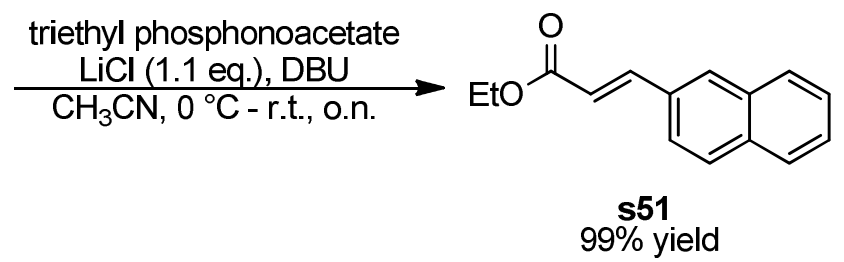

1) DIBAL-H (2.3 eq.)

THF, $-78^{\circ} \mathrm{C}, 2 \mathrm{~h}$

2) $0^{\circ} \mathrm{C}, 30 \mathrm{~min}$

99\% yie

1) $n$-BuLi

2) benzophenone (1.15 eq.)

THF, $-78{ }^{\circ} \mathrm{C}$ - r.t., $3 \mathrm{~h}$

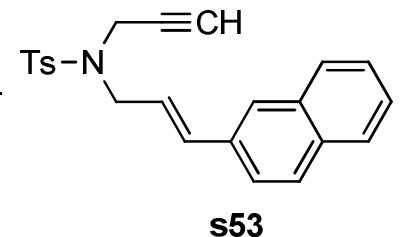

$95 \%$ yield

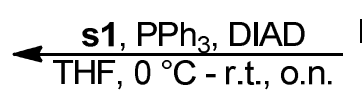<smiles>OC/C=C/c1ccc2ccccc2c1</smiles>

s52 quant. yield

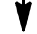

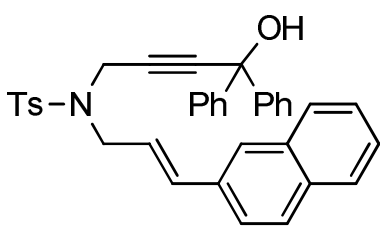

s54

$72 \%$ yield
$\mathrm{Ac}_{2} \mathrm{O}$ (3.0 eq.) DMAP (0.05 eq.)

$\frac{\mathrm{NEt}_{3} \text { (1.5 eq.) }}{\mathrm{CH}_{2} \mathrm{Cl}_{2} \text {, r.t., o.n. }}$

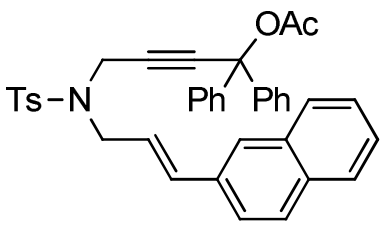

s55

$86 \%$ yield

(E)-ethyl-3-(naphthalen-2-yl)acrylate s51 ${ }^{[12]}$<smiles>CCOC(=O)/C=C/c1ccc2ccccc2c1</smiles>

To a stirred slurry of $\mathrm{LiCl}(0.85 \mathrm{~g}, 20 \mathrm{mmol})$ in acetonitrile $(60 \mathrm{~mL})$ was added triethyl phosphonoacetate $(3.6 \mathrm{~mL}, 18 \mathrm{mmol})$ and 2-naphthaldehyde $(2.81 \mathrm{~g}, 18 \mathrm{mmol})$ at $0{ }^{\circ} \mathrm{C}$. After stirring for 5 minutes at $0{ }^{\circ} \mathrm{C} \mathrm{DBU}(2.7 \mathrm{~mL}, 18 \mathrm{mmol})$ was added and the reaction was stirred overnight at room temperature. The resulting slurry was diluted with diethyl ether $(200 \mathrm{~mL})$, washed with sat. $\mathrm{NH}_{4} \mathrm{Cl}(100 \mathrm{~mL})$, brine $(100 \mathrm{~mL})$, dried over anhydrous $\mathrm{MgSO}_{4}$, filtered through celite (diethyl ether) and concentrated. The residue was purified by column chromatography on silica gel (petroleum ether/EtOAc - 20/1) to yield the title compound s51. The spectral data are in good agreement with previous reports. ${ }^{[12]}$

Yield: $4.04 \mathrm{~g}(17.85 \mathrm{mmol}, 99 \%)$.

Physical State: white solid.

$\mathbf{R}_{\mathbf{f}}$ Value: 0.31 (petroleum ether/EtOAc - 20/1). 
${ }^{1} \mathbf{H}$ NMR (Avance $400 \mathrm{MHz}, \mathrm{CDCl}_{3}$ ) $\delta 7.93(\mathrm{~s}, 1 \mathrm{H}), 7.82-7.87(\mathrm{~m}, 4 \mathrm{H}), 7.66-7.68(\mathrm{~m}, 1 \mathrm{H})$, $7.49-7.54(\mathrm{~m}, 2 \mathrm{H}), 6.56(\mathrm{~d}, J=16.0 \mathrm{~Hz}, 1 \mathrm{H}), 4.30(\mathrm{q}, J=7.1 \mathrm{~Hz}, 2 \mathrm{H}), 1.37(\mathrm{t}, J=7.1 \mathrm{~Hz}$, 3H) ppm.

${ }^{13}$ C NMR (Avance $101 \mathrm{MHz}, \mathrm{CDCl}_{3}$ ) $\delta$ 167.1, 144.6, 134.2, 133.3, 131.9, 129.9, 128.7, $128.5,127.8,127.2,126.7,123.5,118.4,60.5,14.4 \mathrm{ppm}$.

IR (ATR, in $\left.\mathrm{CDCl}_{3}\right)$ v 3057 (w), 2979 (w), 2936 (w), 2902 (w), 1705 (s), 1633 (s), 1367 (m), 1307 (m), 1292 (m), 1257 (s), 1169 (s), 1037 (m) cm $\mathrm{cm}^{-1}$.

HRMS (ESI, m/z) calcd. for $\mathrm{C}_{15} \mathrm{H}_{14} \mathrm{O}_{2} \mathrm{Na}^{+}$: 249.0886, found: 249.0882.

\section{(E)-3-(naphthalen-2-yl)prop-2-en-1-ol s52 ${ }^{[13]}$}

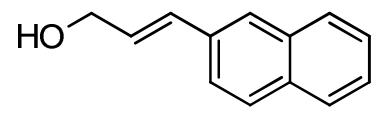

To a stirred solution of $\mathbf{s 5 1}(3.4 \mathrm{~g}, 15 \mathrm{mmol})$ in THF $(60 \mathrm{~mL})$ was added DIBAL-H $(34.5 \mathrm{~mL}$, $34.5 \mathrm{mmol}$ ) at $-78{ }^{\circ} \mathrm{C}$. After stirring the solution for 2 hours at $-78{ }^{\circ} \mathrm{C}$ and 30 minutes at $0{ }^{\circ} \mathrm{C}$ sat. $\mathrm{NH}_{4} \mathrm{Cl}(25 \mathrm{~mL})$ was added. Sat. aqueous Rochelle's salt solution $(75 \mathrm{~mL})$ and EtOAc $(75 \mathrm{~mL})$ were added and stirred until a phase separation was observed followed by extraction of the aqueous phase with EtOAc $(3 \times 100 \mathrm{~mL})$. The combined organic layers were washed with brine $(200 \mathrm{~mL})$, dried over $\mathrm{MgSO}_{4}$, filtered through a silica pad (diethyl ether) and concentrated to yield the title compound s52. The spectral data are in good agreement with previous reports. ${ }^{[13]}$

Yield: $2.80 \mathrm{~g}$ (15.00 mmol, quant).

Physical State: white solid.

$\mathbf{R}_{\mathbf{f}}$ Value: 0.17 (petroleum ether/EtOAc - 5/1).

${ }^{1} \mathbf{H}$ NMR (Avance $400 \mathrm{MHz}, \mathrm{CDCl}_{3}$ ) $\delta 7.71-7.77(\mathrm{~m}, 3 \mathrm{H}), 7.66(\mathrm{~s}, 1 \mathrm{H}), 7.53-7.55(\mathrm{~m}, 1 \mathrm{H})$, $7.41-7.43(\mathrm{~m}, 2 \mathrm{H}), 6.71(\mathrm{~d}, J=15.9 \mathrm{~Hz}, 1 \mathrm{H}), 6.43(\mathrm{dt}, J=15.9,5.7 \mathrm{~Hz}, 1 \mathrm{H}), 4.32(\mathrm{~d}, J=5.3$ $\mathrm{Hz}, 2 \mathrm{H}), 2.15$ (s, 1H) ppm.

${ }^{13}$ C NMR (Avance $101 \mathrm{MHz}, \mathrm{CDCl}_{3}$ ) $\delta$ 134.2, 133.6, 133.1, 131.2, 129.0, 128.3, 128.1, $127.7,126.6,126.3,126.0,123.6,63.7 \mathrm{ppm}$.

IR (ATR, in $\left.\mathrm{CDCl}_{3}\right) \vee 3316$ (m, broad), 3053 (w), 2920 (w), 2859 (w), 1703 (w), 1333 (w), $1368(\mathrm{w}), 1175(\mathrm{w}), 1092(\mathrm{w}), 1010(\mathrm{~m}) \mathrm{cm}^{-1}$. 
HRMS (ESI, m/z) calcd. for $\mathrm{C}_{13} \mathrm{H}_{12} \mathrm{ONa}^{+}$: 207.0770, found: 207.0765.

(E)-4-methyl- $N$-(3-(naphthalen-2-yl)allyl)- $N$-(prop-2-yn-1-yl)benzenesulfonamide s38

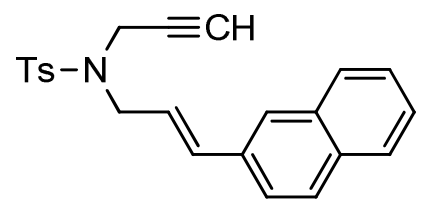

According to GP-3 s2 (1.46 g, $7 \mathrm{mmol})$ was dissolved in THF (30 mL), treated with s52 (1.35 g, $7.35 \mathrm{mmol}), \mathrm{PPh}_{3}(1.84 \mathrm{~g}, 7 \mathrm{mmol})$ and DIAD $(1.5 \mathrm{~mL}, 7 \mathrm{mmol})$ to yield $\mathbf{s 5 3}$ after column chromatography on silica gel (petroleum ether/EtOAc - 7/1). The spectral data are in good agreement with previous reports. ${ }^{[14]}$

Yield: $2.51 \mathrm{~g}(6.68 \mathrm{mmol}, 95 \%)$.

Physical State: white solid.

$\mathbf{R}_{\mathbf{f}}$ Value: 0.26 (petroleum ether/EtOAc - 7/1).

${ }^{1} \mathbf{H}$ NMR (Avance $400 \mathrm{MHz}, \mathrm{CDCl}_{3}$ ) $\delta 7.77$ - $7.83(\mathrm{~m}, 5 \mathrm{H}), 7.70(\mathrm{~s}, 1 \mathrm{H}), 7.53-7.55(\mathrm{~m}, 1 \mathrm{H})$, $7.43-7.50(\mathrm{~m}, 2 \mathrm{H}), 7.31-7.33(\mathrm{~m}, 2 \mathrm{H}), 6.74(\mathrm{~d}, J=15.8 \mathrm{~Hz}, 1 \mathrm{H}), 6.21(\mathrm{dt}, J=6.8,15.8 \mathrm{~Hz}$, $1 \mathrm{H}), 4.17(\mathrm{~d}, J=2.3 \mathrm{~Hz}, 2 \mathrm{H}), 4.06(\mathrm{~d}, J=6.7 \mathrm{~Hz}, 2 \mathrm{H}), 2.44(\mathrm{~s}, 3 \mathrm{H}), 2.08(\mathrm{t}, J=2.4 \mathrm{~Hz}, 1 \mathrm{H})$ ppm.

${ }^{13}$ C NMR (Avance $101 \mathrm{MHz}, \mathrm{CDCl}_{3}$ ) $\delta$ 143.8, 136.1, 135.1, 133.7, 133.6, 133.3, 129.7, 128.4, 128.1, 127.9, 127.8, 126.9, 126.5, 126.3, 123.5, 123.4, 76.7, 74.0, 48.8, 36.1, 21.7 ppm.

IR (ATR, in $\mathrm{CDCl}_{3}$ ) v 3288 (w), 3055 (w), 2978 (w), 2921 (w), 2858 (w), 1597 (w), 1437 (w), 1345 (m), 1330 (m), 1158 (s), 1094 (m) cm $\mathrm{cm}^{-1}$.

HRMS (ESI, m/z) calcd. for $\mathrm{C}_{23} \mathrm{H}_{21} \mathrm{NO}_{2} \mathrm{SNa}^{+}$: 398.1178, found: 398.1169.

(E)-N-(4-hydroxy-4,4-diphenylbut-2-yn-1-yl)-4-methyl- $N$-(3-(naphthalen-2yl)allyl)benzenesulfonamide 554

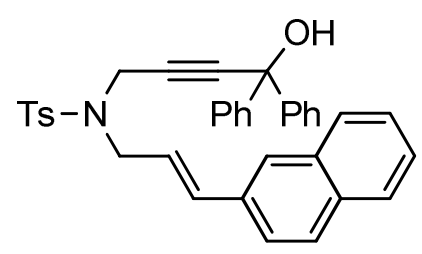


According to GP-1 s53 (1.1 g, 3 mmol) was dissolved in THF (30 mL), treated with $n$-BuLi (1.6 $\mathrm{M}$ in hexanes, $1.9 \mathrm{~mL}, 3 \mathrm{mmol})$ and benzophenone $(0.63 \mathrm{~g}, 3.5 \mathrm{mmol})$ in THF $(4 \mathrm{~mL})$ to yield s54 after column chromatography on silica gel (petroleum ether/EtOAc - 5/1).

Yield: $1.21 \mathrm{~g}(2.16 \mathrm{mmol}, 72 \%)$.

Physical State: pale yellow oil.

$\mathbf{R}_{\mathbf{f}}$ Value: 0.27 (petroleum ether/EtOAc $-5 / 1$ ).

${ }^{1}$ H NMR (Avance $400 \mathrm{MHz}, \mathrm{CDCl}_{3}$ ) $\delta 7.74-7.80$ (m, 5H), 7.57 (s, 1H), 7.43 - $7.48(\mathrm{~m}, 3 \mathrm{H})$, $7.36-7.39$ (m, 4H), $7.28-7.32(\mathrm{~m}, 6 \mathrm{H}), 7.12-7.14(\mathrm{~m}, 2 \mathrm{H}), 6.59$ (d, J = $15.8 \mathrm{~Hz}, 1 \mathrm{H}), 6.20$ $(\mathrm{dt}, J=6.9,15.6 \mathrm{~Hz}, 1 \mathrm{H}), 4.32(\mathrm{~s}, 2 \mathrm{H}), 4.03(\mathrm{~d}, J=6.8 \mathrm{~Hz}, 2 \mathrm{H}), 2.30(\mathrm{~s}, 3 \mathrm{H}), 2.29(\mathrm{~s}, 1 \mathrm{H})$ ppm.

${ }^{13}$ C NMR (Avance $101 \mathrm{MHz}, \mathrm{CDCl}_{3}$ ) $\delta$ 144.5, 143.8, 136.2, 135.4, 133.5, 133.3, 129.8, $128.5,128.4,128.1,128.0,128.0,127.8,127.0,126.5,126.3,126.0,123.5,123.2,88.9,80.2$, $74.3,49.2,36.6,21.6 \mathrm{ppm} .1$ carbon was not observed due to incidental equivalence.

IR (ATR, in $\left.\mathrm{CDCl}_{3}\right)$ v 3477 (w, broad), 3058 (w), 1730 (w), 1597 (w), 1490 (w), 1345 (m), $1158(\mathrm{~m}), 1093(\mathrm{~m}) \mathrm{cm}^{-1}$.

HRMS (ESI, m/z) calcd. for $\mathrm{C}_{36} \mathrm{H}_{31} \mathrm{NO}_{3} \mathrm{SNa}^{+}:$580.1917, found: 580.1903 .

(E)-4-(4-methyl- $N$-(3-(naphthalen-2-yl)allyl)phenylsulfonamido)-1,1-diphenylbut-2-yn-1yl acetate 555

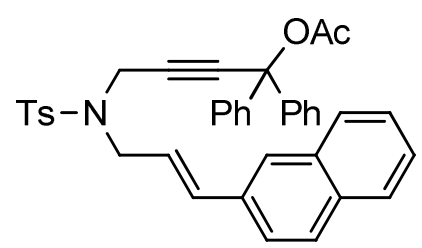

According to GP-2 s54 (1.74 g, $1.7 \mathrm{mmol})$ was dissolved in $\mathrm{CH}_{2} \mathrm{Cl}_{2}(5 \mathrm{~mL})$, treated with acetic anhydride $(0.49 \mathrm{~mL}, 5.2 \mathrm{mmol})$, DMAP $(10.6 \mathrm{mg}, 0.087 \mathrm{mmol})$ and triethylamine $(0.36 \mathrm{~mL}, 2.6 \mathrm{mmol})$ to yield $\mathbf{s 5 5}$ after column chromatography on silica gel (petroleum ether/EtOAc - 5/1).

Yield: $0.90 \mathrm{~g}(1.50 \mathrm{mmol}, 86 \%)$.

Physical State: white solid.

Melting Point: $139^{\circ} \mathrm{C}$. 
$\mathbf{R}_{\mathbf{f}}$ Value: 0.29 (petroleum ether/EtOAc - 5/1).

${ }^{1} \mathbf{H}$ NMR (Avance $400 \mathrm{MHz}, \mathrm{CDCl}_{3}$ ) $\delta 7.75-7.80(\mathrm{~m}, 5 \mathrm{H}), 7.63(\mathrm{~s}, 1 \mathrm{H}), 7.42-7.52(\mathrm{~m}, 3 \mathrm{H})$, $7.20-7.27(\mathrm{~m}, 12 \mathrm{H}), 6.72(\mathrm{~d}, J=15.8 \mathrm{~Hz}, 1 \mathrm{H}), 6.19(\mathrm{dt}, J=7.4,14.8 \mathrm{~Hz}, 1 \mathrm{H}), 4.37$ (s, 2H), $4.03(\mathrm{~d}, J=6.9 \mathrm{~Hz}, 2 \mathrm{H}), 2.36(\mathrm{~s}, 3 \mathrm{H}), 2.10(\mathrm{~s}, 3 \mathrm{H}) \mathrm{ppm}$.

${ }^{13}$ C NMR (Avance $101 \mathrm{MHz}, \mathrm{CDCl}_{3}$ ) $\delta$ 168.0, 143.5, 142.2, 136.2, 135.6, 133.8, 133.5, 133.2, 129.8, 128.3, 128.1, 128.1, 127.8, 127.8, 127.0, 126.4, 126.2, 123.6, 123.0, 85.0, 82.8, 79.1, 48.8, 36.6, 21.9, 21.7 ppm. 2 carbons were not observed due to incidental equivalence.

IR (ATR, in $\mathrm{CDCl}_{3}$ ) v 3059 (w), 1749 (m), 1597 (w), 1492 (w), 1450 (w), 1347 (m), $1225(\mathrm{~m}), 1160(\mathrm{~s}), 1094(\mathrm{~m}) \mathrm{cm}^{-1}$.

HRMS (ESI, m/z) calcd. for $\mathrm{C}_{38} \mathrm{H}_{33} \mathrm{NO}_{4} \mathrm{SNa}^{+}$: 622.2023, found: 622.2001.

\subsection{Preparation of $(E)-5$-( $N$-cinnamyl-4-methylphenylsulfonamido)-2-methylpent-3- yn-2-yl benzoate s56}

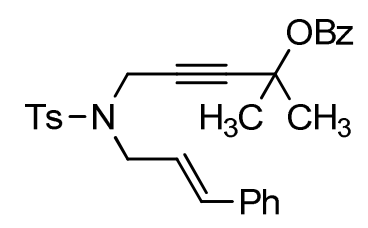

To a stirred solution of $\mathbf{s} 4(0.84 \mathrm{~g}, 1.97 \mathrm{mmol})$ in THF $(20 \mathrm{~mL})$ was added $n$-BuLi $(1.6 \mathrm{M}$ in hexanes, $1.25 \mathrm{~mL}, 2.0 \mathrm{mmol}$ ) dropwise at $-78{ }^{\circ} \mathrm{C}$. After stirring for 1 hour at $-78{ }^{\circ} \mathrm{C}$ benzoyl chloride $(0.25 \mathrm{~mL}, 2.2 \mathrm{mmol})$ in THF $(4 \mathrm{~mL})$ was added, followed by warming up to room temperature and stirring overnight. The solution was hydrolysed by addition of sat. $\mathrm{NH}_{4} \mathrm{Cl}$ $(15 \mathrm{~mL})$ and extracted with $\mathrm{CH}_{2} \mathrm{Cl}_{2}(3 \times 20 \mathrm{~mL})$. The combined organic layers were dried over anhydrous $\mathrm{Na}_{2} \mathrm{SO}_{4}$ and concentrated. The residue was purified by column chromatography on silica gel ( $n$-pentane/Et $\left.{ }_{2} \mathrm{O}-2 / 1\right)$ to yield the title compound $\mathbf{s 5 6}$.

Yield: $0.58 \mathrm{~g}(1.19 \mathrm{mmol}, 60 \%)$.

Physical State: white solid.

Melting Point: $118^{\circ} \mathrm{C}$.

$\mathbf{R}_{\mathbf{f}}$ Value: $0.26\left(n\right.$-pentane/Et $\left.{ }_{2} \mathrm{O}-2 / 1\right)$. 
${ }^{\mathbf{1}} \mathbf{H}$ NMR (Avance $300 \mathrm{MHz}, \mathrm{CDCl}_{3}$ ) $\delta 7.97-8.00(\mathrm{~m}, 2 \mathrm{H}), 7.73-7.76(\mathrm{~m}, 2 \mathrm{H}), 7.55-7.61$ $(\mathrm{m}, 1 \mathrm{H}), 7.43-7.48(\mathrm{~m}, 2 \mathrm{H}), 7.20-7.24(\mathrm{~m}, 7 \mathrm{H}), 6.64$ (d, $J=15.8 \mathrm{~Hz}, 1 \mathrm{H}), 6.08$ (dt, $J=7.0$, $15.8 \mathrm{~Hz}, 1 \mathrm{H}), 4.17(\mathrm{~s}, 2 \mathrm{H}), 4.05(\mathrm{dd}, J=0.8,6.9 \mathrm{~Hz}, 2 \mathrm{H}), 2.32(\mathrm{~s}, 3 \mathrm{H}), 1.55$ (s, 6H) ppm.

${ }^{13}$ C NMR (Avance $75 \mathrm{MHz}, \mathrm{CDCl}_{3}$ ) $\delta$ 164.7, 143.5, 136.4, 136.3, 135.4, 133.1, 131.0, 129.7, 128.6, 128.5, 128.0, 127.9, 126.7, 123.0, 87.2, 77.2, 72.2, 48.6, 36.3, 28.9, 21.6 ppm. 1 carbon was not observed due to incidental equivalence.

IR (ATR, in $\mathrm{CDCl}_{3}$ ) v 2986 (w), 2922 (w), 1720 (s), 1599 (w), 1494 (w), 1450 (w), 1349 (m), $1281(\mathrm{~s}), 1259(\mathrm{~m}), 1162(\mathrm{~s}), 1141(\mathrm{~m}), 1098(\mathrm{~m}) \mathrm{cm}^{-1}$.

HRMS (ESI, m/z) calcd. for $\mathrm{C}_{29} \mathrm{H}_{29} \mathrm{NO}_{4} \mathrm{SNa}^{+}:$510.1710, found: 510.1704.

X-Ray: X-Ray structure of $\mathbf{5 5 6}$ is deposited at Cambridge Crystallographic Data Centre (www.ccdc.cam.ac.uk) under CCDC 1440741. 


\section{Fe-catalyzed cycloisomerizations}

\section{General Procedure 4 (GP-4)}

A $15 \mathrm{~mL}$ Schlenk tube with a screwcap sealing was dried under high vacuum with a heat gun for at least 10 minutes. After cooling to room temperature the enyne ester $(0.2 \mathrm{mmol})$ and $\left[\mathrm{Fe}(\mathrm{CO})(\mathrm{NO})\left(\mathrm{PPh}_{3}\right)_{2}\right]\left[\mathrm{BF}_{4}\right](14.5 \mathrm{mg}, 0.02 \mathrm{mmol})$ were added under an atmosphere of $\mathrm{N}_{2}$. The Schlenk tube was carefully evacuated again, refilled with an atmosphere of $\mathrm{N}_{2}$, and $\mathrm{CH}_{2} \mathrm{Cl}_{2}(1 \mathrm{~mL})$ was added. The sealed tube was heated to $50{ }^{\circ} \mathrm{C}$ for 22 hours. The reaction mixture was cooled to room temperature and directly submitted to column chromatography on silica gel to yield the corresponding allenyl pyrrolidine.

\section{General Procedure 5 (GP-5)}

A $15 \mathrm{~mL}$ Schlenk tube with a screwcap sealing was dried under high vacuum with a heat gun for at least 10 minutes. After cooling to room temperature the enyne ester $(0.2 \mathrm{mmol})$ was dissolved in $\mathrm{CH}_{2} \mathrm{Cl}_{2}(1 \mathrm{~mL})$ and $\left[\mathrm{Fe}(\mathrm{CO})(\mathrm{NO})\left(\mathrm{PPh}_{3}\right)_{2}\right]\left[\mathrm{BF}_{4}\right](14.5 \mathrm{mg}, 0.02 \mathrm{mmol})$ was added under a stream of $\mathrm{N}_{2}$. The sealed tube was heated to $50{ }^{\circ} \mathrm{C}$ for 22 hours. The reaction mixture was cooled to room temperature and directly submitted to column chromatography on silica gel to yield the corresponding allenyl pyrrolidine. 


\section{1 (4-(2-methylprop-1-en-1-ylidene)-1-tosylpyrrolidin-3-yl)(phenyl)methyl acetate 10}<smiles>CC(=O)O[C@H](c1ccccc1)[C@H]1CN([As])C/C1=C\C(C)C</smiles>

According to GP-4 $9(85.1 \mathrm{mg}, 0.2 \mathrm{mmol})$ and $\left[\mathrm{Fe}(\mathrm{CO})(\mathrm{NO})\left(\mathrm{PPh}_{3}\right)_{2}\right]\left[\mathrm{BF}_{4}\right](14.5 \mathrm{mg}$, $0.02 \mathrm{mmol})$ were dissolved in $\mathrm{CH}_{2} \mathrm{Cl}_{2}(1 \mathrm{~mL})$ and stirred at $50{ }^{\circ} \mathrm{C}$ for 22 hours to yield the title compound $\mathbf{1 0}$ after column chromatography on silica gel (n-pentane/Et ${ }_{2} \mathrm{O}-2 / 1$ ).

Yield: $56.4 \mathrm{mg}(0.132 \mathrm{mmol}, 66 \%)$.

Physical State: white solid.

Melting Point: $120{ }^{\circ} \mathrm{C}$ (decomposition).

$\mathbf{R}_{\mathbf{f}}$ Value: $0.31(n$-pentane/Et $2 \mathrm{O}-2 / 1)$.

2.0 mmol scale: A $50 \mathrm{~mL}$ Schlenk flask was dried under high vacuum with a heat gun for 15 minutes. After cooling to room temperature $9(0.85 \mathrm{~g}, 2.0 \mathrm{mmol})$ and $\left[\mathrm{Fe}(\mathrm{CO})(\mathrm{NO})\left(\mathrm{PPh}_{3}\right)_{2}\right]\left[\mathrm{BF}_{4}\right](145 \mathrm{mg}, 0.2 \mathrm{mmol})$ were added under an atmosphere of $\mathrm{N}_{2}$. The Schlenk flask was carefully evacuated again, refilled with an atmosphere of $\mathrm{N}_{2}$, and $\mathrm{CH}_{2} \mathrm{Cl}_{2}$ $(10 \mathrm{~mL})$ was added. The sealed flask was heated to $50{ }^{\circ} \mathrm{C}$ for 22 hours. The reaction mixture was cooled to room temperature, filtered through a pad of silica gel (solvent: $\mathrm{Et}_{2} \mathrm{O}$ ), evaporated and submitted to column chromatography on silica gel (petroleum ether/EtOAc 5/1) to yield $\mathbf{1 0 .}$

Yield: $0.50 \mathrm{~g}(1.18 \mathrm{mmol}, 59 \%)$.

Physical State: white solid.

$\mathbf{R}_{\mathbf{f}}$ Value: 0.29 (petroleum ether/EtOAc - 5/1).

${ }^{1} \mathbf{H}$ NMR (Avance $\left.300 \mathrm{MHz}, \mathrm{CDCl}_{3}\right) \delta 7.69-7.72(\mathrm{~m}, 2 \mathrm{H}), 7.20-7.36(\mathrm{~m}, 7 \mathrm{H}), 5.63(\mathrm{~d}$, $J=6.8 \mathrm{~Hz}, 1 \mathrm{H}), 3.83(\mathrm{~d}, J=12.7 \mathrm{~Hz}, 1 \mathrm{H}), 3.68(\mathrm{dd}, J=1.0,12.7 \mathrm{~Hz}, 1 \mathrm{H}), 3.43-3.51(\mathrm{~m}$, $1 \mathrm{H}), 3.20-3.28(\mathrm{~m}, 2 \mathrm{H}), 2.45(\mathrm{~s}, 3 \mathrm{H}), 1.98(\mathrm{~s}, 3 \mathrm{H}), 1.40$ (s, 3H), 1.31 (s, 3H) ppm.

${ }^{13}$ C NMR (Avance $75 \mathrm{MHz}, \mathrm{CDCl}_{3}$ ) $\delta$ 194.9, 169.8, 143.8, 138.6, 133.1, 129.8, 128.4, 128.2, $127.9,127.2,101.8,96.1,75.9,50.2,50.1,46.2,21.7,21.1,20.5,20.3$ ppm. 
IR (ATR, in $\mathrm{CDCl}_{3}$ ) v 3034 (w), 2961 (w), 2923 (w), 2902 (w), 2845 (w), 1743 (m), 1596 (w), 1495 (w), 1456 (w), 1374 (w), 1342 (m), 1217 (s), 1156 (s), 1109 (w), 1090 (m), $1051(\mathrm{~m}), 1009(\mathrm{w}) \mathrm{cm}^{-1}$.

HRMS (ESI, m/z) calcd. for $\mathrm{C}_{24} \mathrm{H}_{27} \mathrm{NO}_{4} \mathrm{SNa}^{+}$: 448.1553, found: 448.1559 .

X-Ray: X-Ray structure of $\mathbf{1 0}$ is deposited at Cambridge Crystallographic Data Centre (www.ccdc.cam.ac.uk) under CCDC 1440738.

\section{2 phenyl(4-(2-phenylvinylidene)-1-tosylpyrrolidin-3-yl)methyl acetate 11}

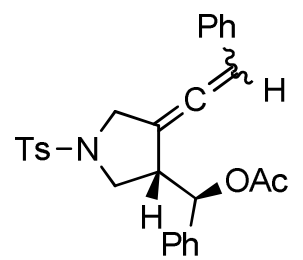

According to GP-5 40 (94.7 mg, $0.2 \mathrm{mmol})$ was dissolved in $\mathrm{CH}_{2} \mathrm{Cl}_{2}(1 \mathrm{~mL})$, treated with $\left[\mathrm{Fe}(\mathrm{CO})(\mathrm{NO})\left(\mathrm{PPh}_{3}\right)_{2}\right]\left[\mathrm{BF}_{4}\right](14.5 \mathrm{mg}, 0.02 \mathrm{mmol})$ and stirred at $50{ }^{\circ} \mathrm{C}$ for 22 hours to yield the title compound 11 after column chromatography on silica gel (n-pentane/ $\left.\mathrm{Et}_{2} \mathrm{O}-1.5 / 1\right)$ as a 1:1 mixture of inseparable allene isomers.

Yield: $88.4 \mathrm{mg}(0.187 \mathrm{mmol}, 93 \%)$.

Physical State: pale yellow oil.

$\mathbf{R}_{\mathbf{f}}$ Value: $0.30(n$-pentane/Et $2 \mathrm{O}-1.5 / 1)$.

${ }^{1} \mathbf{H}$ NMR (Avance $400 \mathrm{MHz}, \mathrm{CDCl}_{3}$ ) $\delta 7.73$ - $7.74(\mathrm{~m}, 2 \mathrm{H}), 7.71-7.72(\mathrm{~m}, 2 \mathrm{H}), 7.38-7.38$ $(\mathrm{m}, 2 \mathrm{H}), 7.36-7.36(\mathrm{~m}, 2 \mathrm{H}), 7.14-7.25(\mathrm{~m}, 16 \mathrm{H}), 6.99-7.01(\mathrm{~m}, 2 \mathrm{H}), 6.94-6.96(\mathrm{~m}, 2 \mathrm{H})$, $6.06-6.09(\mathrm{~m}, 1 \mathrm{H}), 5.85-5.88(\mathrm{~m}, 1 \mathrm{H}), 5.71-5.73(\mathrm{~m}, 2 \mathrm{H}), 3.83-4.05(\mathrm{~m}, 4 \mathrm{H})$, $3.50-3.56(\mathrm{~m}, 2 \mathrm{H}), 3.35-3.43(\mathrm{~m}, 4 \mathrm{H}), 2.47(\mathrm{~s}, 6 \mathrm{H}), 2.01(\mathrm{~s}, 3 \mathrm{H}), 1.90(\mathrm{~s}, 3 \mathrm{H}) \mathrm{ppm}$.

${ }^{13}$ C NMR (Avance $101 \mathrm{MHz}, \mathrm{CDCl}_{3}$ ) $\delta$ 198.3, 198.0, 169.7, 169.6, 144.1, 144.0, 138.3, 138.2 $133.6,133.5,133.0,132.8,129.9,128.6,128.5,128.5,128.4,128.3,128.0,127.9,127.6$, $127.5,127.2,127.1,126.9,126.9,102.5,102.3,99.8,99.6,75.7,75.5,50.1,50.1,49.9,47.4$, 47.0, 21.7, 21.1, $21.1 \mathrm{ppm} .4$ carbons were not observed due to incidental equivalence.

IR (ATR, in $\left.\mathrm{CDCl}_{3}\right) \vee 3032$ (w), 1963 (w), 1741 (m), 1598 (w), 1495 (w), 1457 (w), 1372 (w), 1346 (m), 1306 (w), 1290 (w), 1225 (s), 1161 (s), 1091 (m), 1029 (m), 1015 (m) $\mathrm{cm}^{-1}$. 
HRMS (ESI, m/z) calcd. for $\mathrm{C}_{28} \mathrm{H}_{27} \mathrm{NO}_{4} \mathrm{SNa}^{+}$: 496.1553, found: 496.1551.

\section{3 phenyl(4-(2-phenylprop-1-en-1-ylidene)-1-tosylpyrrolidin-3-yl)methyl acetate 12}<smiles>CC(=O)O[C@H](c1ccccc1)[C@@H]1CN([12F])C/C1=C\C(C)c1ccccc1</smiles>

According to GP-4 s7 (97.5 mg, $0.2 \mathrm{mmol})$ and $\left[\mathrm{Fe}(\mathrm{CO})(\mathrm{NO})\left(\mathrm{PPh}_{3}\right)_{2}\right]\left[\mathrm{BF}_{4}\right](14.5 \mathrm{mg}$, $0.02 \mathrm{mmol})$ were dissolved in $\mathrm{CH}_{2} \mathrm{Cl}_{2}(1 \mathrm{~mL})$ and stirred at $50{ }^{\circ} \mathrm{C}$ for 22 hours to yield the title compound 12 after column chromatography on silica gel (petroleum ether/EtOAc - 5/1) as a 1:1 mixture of allene isomers which could be separated using HPLC (petroleum ether/EtOAc - 5/1).

Yield: $73.7 \mathrm{mg}(0.151 \mathrm{mmol}, 76 \%)$.

Physical State: pale yellow oil.

$\mathbf{R}_{\mathbf{f}}$ Value: 0.37 (petroleum ether/EtOAc - 5/1).

\section{HPLC fraction 1}

${ }^{1} \mathbf{H}$ NMR (Avance $400 \mathrm{MHz}, \mathrm{CDCl}_{3}$ ) $\delta 7.75$ - $7.77(\mathrm{~m}, 2 \mathrm{H}), 7.39-7.41(\mathrm{~m}, 2 \mathrm{H}), 7.21-7.28$ $(\mathrm{m}, 3 \mathrm{H}), 7.14-7.20(\mathrm{~m}, 5 \mathrm{H}), 7.06-7.09(\mathrm{~m}, 2 \mathrm{H}), 5.66(\mathrm{~d}, J=7.2 \mathrm{~Hz}, 1 \mathrm{H}), 4.06(\mathrm{~d}$, $J=13.0 \mathrm{~Hz}, 1 \mathrm{H}), 3.88(\mathrm{dd}, J=1.2,12.9 \mathrm{~Hz}, 1 \mathrm{H}), 3.65(\mathrm{dd}, J=7.5,9.6 \mathrm{~Hz}, 1 \mathrm{H}), 3.42-3.48$ (m, 1H), 3.29 (dd, $J=8.3,9.5 \mathrm{~Hz}, 1 \mathrm{H}), 2.51$ (s, 3H), 1.87 (s, 3H), 1.80 (s, 3H) ppm.

${ }^{13}$ C NMR (Avance $101 \mathrm{MHz}, \mathrm{CDCl}_{3}$ ) $\delta$ 196.6, 169.7, 144.0, 138.3, 136.5, 133.1, 130.0, $128.4,128.3,128.0,127.3,127.0,126.1,106.8,100.3,75.7,50.5,50.2,46.9,21.8,21.0,16.9$ ppm. 1 carbon was not observed due to incidental equivalence.

IR (ATR, in $\left.\mathrm{CDCl}_{3}\right)$ v 3032 (w), 2947 (w), 1741 (m), 1597 (w), 1493 (w), 1346 (m), 1227 (s), $1161(\mathrm{~s}), 1092(\mathrm{~m}), 1017(\mathrm{~m}) \mathrm{cm}^{-1}$.

HRMS (ESI, m/z) calcd. for $\mathrm{C}_{29} \mathrm{H}_{29} \mathrm{NO}_{4} \mathrm{SNa}^{+}$: 510.1710, found: 510.1711.

\section{HPLC fraction 2}

${ }^{\mathbf{1}} \mathbf{H}$ NMR (Avance $400 \mathrm{MHz}, \mathrm{CDCl}_{3}$ ) $\delta 7.75$ - $7.77(\mathrm{~m}, 2 \mathrm{H}), 7.39-7.41(\mathrm{~m}, 2 \mathrm{H}), 7.19-7.28$ (m, 8H), $7.10-7.12(\mathrm{~m}, 2 \mathrm{H}), 5.73(\mathrm{~d}, J=7.0 \mathrm{~Hz}, 1 \mathrm{H}), 4.02(\mathrm{~d}, J=13.0 \mathrm{~Hz}, 1 \mathrm{H}), 3.87$ (dd, 
$J=1.1,13.0 \mathrm{~Hz}, 1 \mathrm{H}), 3.58(\mathrm{dd}, J=6.8,9.2 \mathrm{~Hz}, 1 \mathrm{H}), 3.41-3.46(\mathrm{~m}, 1 \mathrm{H}), 3.38(\mathrm{dd}, J=6.3$, $9.2 \mathrm{~Hz}, 1 \mathrm{H}), 2.50(\mathrm{~s}, 3 \mathrm{H}), 2.04(\mathrm{~s}, 3 \mathrm{H}), 1.72(\mathrm{~s}, 3 \mathrm{H}) \mathrm{ppm}$.

${ }^{13} \mathbf{C}$ NMR (Avance $101 \mathrm{MHz}, \mathrm{CDCl}_{3}$ ) $\delta$ 197.7, 169.8, 144.0, 138.4, 136.4, 133.0, 129.9, $128.6,128.4$, 128.4, 128.0, 127.3, 127.1, 126.0, 106.5, 100.2, 75.9, 50.3, 49.9, 46.6, 21.8, 21.2, 16.9 ppm.

IR (ATR, in $\left.\mathrm{CDCl}_{3}\right)$ v 3031 (w), 2947 (w), 1740 (m), 1597 (w), 1493 (w), 1346 (m), 1226 (s), $1160(\mathrm{~s}), 1091(\mathrm{~m}), 1016(\mathrm{~m}) \mathrm{cm}^{-1}$.

HRMS (ESI, m/z) calcd. for $\mathrm{C}_{29} \mathrm{H}_{29} \mathrm{NO}_{4} \mathrm{SNa}^{+}$: 510.1710, found: 510.1707.

\section{4 (4-(2,2-diphenylvinylidene)-1-tosylpyrrolidin-3-yl)(phenyl)methyl acetate 13}

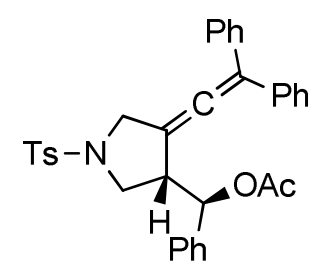

According to GP-4 $34(109.9 \mathrm{mg}, 0.2 \mathrm{mmol})$ and $\left[\mathrm{Fe}(\mathrm{CO})(\mathrm{NO})\left(\mathrm{PPh}_{3}\right)_{2}\right]\left[\mathrm{BF}_{4}\right](14.5 \mathrm{mg}$, $0.02 \mathrm{mmol})$ were dissolved in $\mathrm{CH}_{2} \mathrm{Cl}_{2}(1 \mathrm{~mL})$ and stirred at $50{ }^{\circ} \mathrm{C}$ for 22 hours to yield the title compound 13 after column chromatography on silica gel ( $n$-pentane/ $\left.\mathrm{Et}_{2} \mathrm{O}-2 / 1\right)$.

Yield: $87.1 \mathrm{mg}(0.158 \mathrm{mmol}, 79 \%)$.

Physical State: pale yellow oil.

$\mathbf{R}_{\mathbf{f}}$ Value: $0.19\left(n\right.$-pentane/ $\left.\mathrm{Et}_{2} \mathrm{O}-2 / 1\right)$.

${ }^{1} \mathbf{H}$ NMR (Avance $300 \mathrm{MHz}, \mathrm{CDCl}_{3}$ ) $\delta 7.71$ - $7.73(\mathrm{~m}, 2 \mathrm{H}), 7.34-7.36(\mathrm{~m}, 2 \mathrm{H}), 7.23$ - 7.27 $(\mathrm{m}, 6 \mathrm{H}), 7.11(\mathrm{~s}, 5 \mathrm{H}), 6.99-7.02(\mathrm{~m}, 2 \mathrm{H}), 6.93-6.96(\mathrm{~m}, 2 \mathrm{H}), 5.69(\mathrm{~d}, J=6.8 \mathrm{~Hz}, 1 \mathrm{H}), 4.09$ $(\mathrm{d}, J=13.1 \mathrm{~Hz}, 1 \mathrm{H}), 3.96(\mathrm{dd}, J=1.3,13.2 \mathrm{~Hz}, 1 \mathrm{H}), 3.59(\mathrm{dd}, J=8.3,8.3 \mathrm{~Hz}, 1 \mathrm{H}), 3.51$ (ddd, $J=7.2,7.2,7.2 \mathrm{~Hz}, 1 \mathrm{H}), 3.31(\mathrm{dd}, J=7.0,9.2 \mathrm{~Hz}, 1 \mathrm{H}), 2.47(\mathrm{~s}, 3 \mathrm{H}), 1.81(\mathrm{~s}, 3 \mathrm{H}) \mathrm{ppm}$.

${ }^{13}$ C NMR (Avance $75 \mathrm{MHz}, \mathrm{CDCl}_{3}$ ) $\delta$ 198.3, 169.7, 144.0, 138.2, 136.3, 136.3, 133.0, 130.0, $128.7,128.6,128.5,128.5,128.4,128.0,127.8,127.0,115.3,101.6,75.7,50.6,50.5,47.3$, 21.8, 20.9 ppm. 2 carbons were not observed due to incidental equivalence.

IR (ATR, in $\left.\mathrm{CDCl}_{3}\right)$ v 3060 (w), 3031 (w), 2925 (w), 2254 (w), 1952 (w), 1740 (s), 1598 (m), $1493(\mathrm{~m}), 1453(\mathrm{~m}), 1371(\mathrm{~s}), 1226(\mathrm{~s}), 1163(\mathrm{~s}) \mathrm{cm}^{-1}$.

HRMS (ESI, m/z) calcd. for $\mathrm{C}_{34} \mathrm{H}_{31} \mathrm{NO}_{4} \mathrm{SNa}^{+}:$572.1866, found: 572.1852. 


\subsection{4-(2-(4-fluorophenyl)vinylidene)-1-tosylpyrrolidin-3-yl)(phenyl)methyl acetate 14}

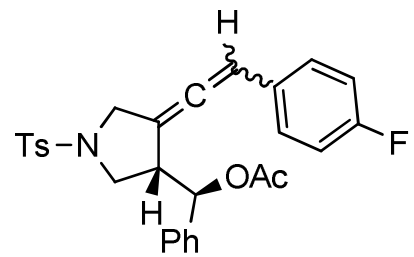

According to GP-5 s10 (98.3 mg, $0.2 \mathrm{mmol})$ was dissolved in $\mathrm{CH}_{2} \mathrm{Cl}_{2}(1 \mathrm{~mL})$, treated with $\left[\mathrm{Fe}(\mathrm{CO})(\mathrm{NO})\left(\mathrm{PPh}_{3}\right)_{2}\right]\left[\mathrm{BF}_{4}\right](14.5 \mathrm{mg}, 0.02 \mathrm{mmol})$ and stirred at $50{ }^{\circ} \mathrm{C}$ for 22 hours to yield the title compound $\mathbf{1 4}$ after column chromatography on silica gel (petroleum ether/EtOAc 4/1) as a 1:1 mixture of allene isomers which could be separated using HPLC (petroleum ether/EtOAc - 4/1).

Yield: $98.3 \mathrm{mg}$ (0.2 mmol, quant.).

Physical State: pale yellow oil.

$\mathbf{R}_{\mathbf{f}}$ Value: 0.29 (petroleum ether/EtOAc - 4/1).

\section{HPLC fraction 1}

${ }^{1}$ H NMR (Avance $400 \mathrm{MHz}, \mathrm{CDCl}_{3}$ ) $\delta 7.71-7.74(\mathrm{~m}, 2 \mathrm{H}), 7.36-7.38(\mathrm{~m}, 2 \mathrm{H}), 7.13-7.19$ (m, 5H), $6.89-6.90(\mathrm{~m}, 2 \mathrm{H}), 6.88(\mathrm{~s}, 2 \mathrm{H}), 6.02-6.05(\mathrm{~m}, 1 \mathrm{H}), 5.70-5.71(\mathrm{~m}, 1 \mathrm{H}), 3.98(\mathrm{dd}$, $J=3.6,13.1 \mathrm{~Hz}, 1 \mathrm{H}), 3.92(\mathrm{ddd}, J=1.1,4.0,13.1 \mathrm{~Hz}, 1 \mathrm{H}), 3.49-3.55(\mathrm{~m}, 1 \mathrm{H}), 3.36-3.44$ (m, 2H), 2.47 (s, 3H), $1.92(\mathrm{~s}, 3 \mathrm{H}) \mathrm{ppm}$.

${ }^{19}$ F NMR (Avance $376 \mathrm{MHz}, \mathrm{CDCl}_{3}$ ) $\delta-112.1 \mathrm{ppm}$.

${ }^{13}$ C NMR (Avance $\left.101 \mathrm{MHz}, \mathrm{CDCl}_{3}\right) \delta 197.9(\mathrm{~d}, J=2.4 \mathrm{~Hz}), 169.6,162.2(\mathrm{~d}, J=247.0 \mathrm{~Hz})$, 144.1, 138.1, 133.0, 129.9, 129.5 (d, $J=3.4 \mathrm{~Hz}), 128.7$ (d, $J=8.1 \mathrm{~Hz}), 128.5,128.0,127.0$, $115.5(\mathrm{~d}, J=21.8 \mathrm{~Hz}), 102.7,98.8,75.6,50.2,50.0,47.4,21.7,21.1 \mathrm{ppm} .1$ carbon was not observed due to incidental equivalence.

IR (ATR, in $\mathrm{CDCl}_{3}$ ) v 3034 (w), 1965 (w), 1740 (m), 1599 (w), 1508 (m), 1474 (w), 1455 (w), 1372 (w), 1346 (m), 1305 (w), 1221 (s), 1155 (s), 1091 (m), 1030 (m), 1015 (m) cm cm $^{-1}$.

HRMS (ESI, m/z) calcd. for $\mathrm{C}_{28} \mathrm{H}_{26} \mathrm{FNO}_{4} \mathrm{SNa}^{+}$: 514.1459, found: 514.1452.

\section{HPLC fraction 2}

${ }^{1} \mathbf{H}$ NMR (Avance $400 \mathrm{MHz}, \mathrm{CDCl}_{3}$ ) $\delta 7.72$ - $7.74(\mathrm{~m}, 2 \mathrm{H}), 7.36$ - 7.38 (m, 2H), 7.15 - 7.26 $(\mathrm{m}, 5 \mathrm{H}), 6.88-6.99(\mathrm{~m}, 4 \mathrm{H}), 5.81-5.84(\mathrm{~m}, 1 \mathrm{H}), 5.71-5.73(\mathrm{~m}, 1 \mathrm{H}), 4.03(\mathrm{dd}, J=3.7$, 
13.2 Hz, 1H), 3.83 (ddd, $J=1.4,4.1,13.2 \mathrm{~Hz}, 1 \mathrm{H}), 3.55$ (dd, $J=7.3,9.6 \mathrm{~Hz}, 1 \mathrm{H}), 3.38-3.45$ (m, 1H), $3.34(\mathrm{dd}, J=6.4,9.7 \mathrm{~Hz}, 1 \mathrm{H}), 2.47$ (s, 3H), 2.01 (s, 3H) ppm.

${ }^{19}$ F NMR (Avance $376 \mathrm{MHz}, \mathrm{CDCl}_{3}$ ) $\delta-114.2 \mathrm{ppm}$.

${ }^{13}$ C NMR (Avance $101 \mathrm{MHz}, \mathrm{CDCl}_{3}$ ) $\delta 198.1(\mathrm{~d}, J=2.3 \mathrm{~Hz}), 169.7,162.2(\mathrm{~d}, J=247.1 \mathrm{~Hz}$ ), 144.1, 138.3, 132.9, 129.9, 129.5 (d, $J=3.4 \mathrm{~Hz}), 128.6$ (d, $J=8.1 \mathrm{~Hz}), 128.5,128.4,128.0$, 127.0, $115.6(\mathrm{~d}, J=21.9 \mathrm{~Hz}), 102.7,98.7,75.7,50.2,50.0,47.0,21.7,21.1 \mathrm{ppm}$.

IR (ATR, in $\left.\mathrm{CDCl}_{3}\right)$ v 3034 (w), 1741 (m), 1599 (m), 1508 (m), 1474 (w), 1455 (w), 1372 (w), 1346 (m), 1305 (w), 1222 (s), 1184 (w), 1155 (s), 1091 (m), 1030 (m), 1015 (m) cm cm $^{-1}$.

HRMS (ESI, m/z) calcd. for $\mathrm{C}_{28} \mathrm{H}_{26} \mathrm{FNO}_{4} \mathrm{SNa}^{+}$: 514.1459, found: 514.1445.

\subsection{4-(2-(4-chlorophenyl)vinylidene)-1-tosylpyrrolidin-3-yl)(phenyl)methyl acetate 15}

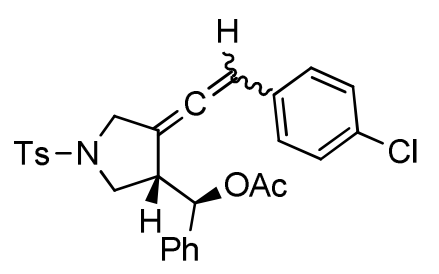

According to GP-5 s12 (101.6 mg, $0.2 \mathrm{mmol}$ ) was dissolved in $\mathrm{CH}_{2} \mathrm{Cl}_{2}(1 \mathrm{~mL})$, treated with $\left[\mathrm{Fe}(\mathrm{CO})(\mathrm{NO})\left(\mathrm{PPh}_{3}\right)_{2}\right]\left[\mathrm{BF}_{4}\right](14.5 \mathrm{mg}, 0.02 \mathrm{mmol})$ and stirred at $50{ }^{\circ} \mathrm{C}$ for 22 hours to yield the title compound $\mathbf{1 5}$ after column chromatography on silica gel (petroleum ether/EtOAc 5/1) as a 1:1 mixture of allene isomers which could be separated using HPLC (petroleum ether/EtOAc - 4/1).

Yield: $101.6 \mathrm{mg}$ (0.2 mmol, quant.).

Physical State: pale yellow oil.

$\mathbf{R}_{\mathbf{f}}$ Value: 0.17 (petroleum ether/EtOAc - 5/1).

\section{HPLC fraction 1}

${ }^{1} \mathbf{H}$ NMR (Avance $400 \mathrm{MHz}, \mathrm{CDCl}_{3}$ ) $\delta 7.72$ - $7.74(\mathrm{~m}, 2 \mathrm{H}), 7.36-7.38(\mathrm{~m}, 2 \mathrm{H}), 7.14-7.19$ $(\mathrm{m}, 7 \mathrm{H}), 6.82-6.86(\mathrm{~m}, 2 \mathrm{H}), 6.00-6.03(\mathrm{~m}, 1 \mathrm{H}), 5.70(\mathrm{~d}, J=6.8 \mathrm{~Hz}, 1 \mathrm{H}), 3.98(\mathrm{dd}, J=3.6$, $13.1 \mathrm{~Hz}, 1 \mathrm{H}), 3.89-3.94(\mathrm{~m}, 1 \mathrm{H}), 3.53(\mathrm{dd}, J=6.6,8.8 \mathrm{~Hz}, 1 \mathrm{H}), 3.36-3.45(\mathrm{~m}, 2 \mathrm{H}), 2.48$ (s, 3H), 1.93 (s, 3H) ppm. 
${ }^{13}$ C NMR (Avance $101 \mathrm{MHz}, \mathrm{CDCl}_{3}$ ) $\delta$ 198.2, 169.6, 144.1, 138.1, 133.2, 133.1, 132.1, 130.0, 128.7, 128.6, 128.6, 128.4, 128.0, 127.1, 103.0, 98.8, 75.7, 50.3, 50.0, 47.5, 21.7, 21.1 ppm.

IR (ATR, in $\left.\mathrm{CDCl}_{3}\right)$ v 3033 (w), 2927 (w), 1741 (s), 1597 (m), 1492 (m), 1455 (w), 1434 (w), 1371 (w), 1346 (s), 1305 (w), 1225 (s), 1162 (s), 1090 (s), 1031 (m), 1014 (s) cm cm $^{-1}$

HRMS (ESI, m/z) calcd. for $\mathrm{C}_{28} \mathrm{H}_{26} \mathrm{ClNO}_{4} \mathrm{SNa}^{+}$: 530.1163, found: 530.1158.

\section{HPLC fraction 2}

${ }^{1} \mathbf{H}$ NMR (Avance $400 \mathrm{MHz}, \mathrm{CDCl}_{3}$ ) $\delta 7.72-7.74(\mathrm{~m}, 2 \mathrm{H}), 7.37-7.39(\mathrm{~m}, 2 \mathrm{H}), 7.17-7.23$ (m, 7H), $6.90-6.92(\mathrm{~m}, 2 \mathrm{H}), 5.78-5.81(\mathrm{~m}, 1 \mathrm{H}), 5.71(\mathrm{~d}, J=7.1 \mathrm{~Hz}, 1 \mathrm{H}), 4.03(\mathrm{dd}, J=3.7$, $13.3 \mathrm{~Hz}, 1 \mathrm{H}), 3.83$ (ddd, $J=1.4,4.1,13.3 \mathrm{~Hz}, 1 \mathrm{H}), 3.56(\mathrm{dd}, J=7.4,9.7 \mathrm{~Hz}, 1 \mathrm{H}), 3.40-3.46$ (m, 1H), 3.33 (dd, $J=6.4,9.8 \mathrm{~Hz}, 1 \mathrm{H}), 2.48(\mathrm{~s}, 3 \mathrm{H}), 2.01(\mathrm{~s}, 3 \mathrm{H}) \mathrm{ppm}$.

${ }^{13}$ C NMR (Avance $101 \mathrm{MHz}, \mathrm{CDCl}_{3}$ ) $\delta$ 198.4, 169.8, 144.1, 138.3, 133.2, 132.9, 132.2, 130.0, 128.8, 128.5, 128.5, 128.3, 128.0, 127.1, 102.9, 98.8, 75.8, 50.3, 50.0, 47.0, 21.8, 21.1 ppm.

IR (ATR, in $\left.\mathrm{CDCl}_{3}\right)$ v 3033 (w), 2930 (w), 1740 (m), 1597 (w), 1491 (m), 1454 (w), 1434 (w), 1371 (w), 1346 (m), 1305 (w), 1225 (s), 1161 (s), 1089 (s), 1031 (m), 1013 (m) cm ${ }^{-1}$. HRMS (ESI, m/z) calcd. for $\mathrm{C}_{28} \mathrm{H}_{26} \mathrm{ClNO}_{4} \mathrm{SNa}^{+}$: 530.1163, found: 530.1156.

\subsection{4-(2-(4-bromophenyl)vinylidene)-1-tosylpyrrolidin-3-yl)(phenyl)methyl acetate 16}

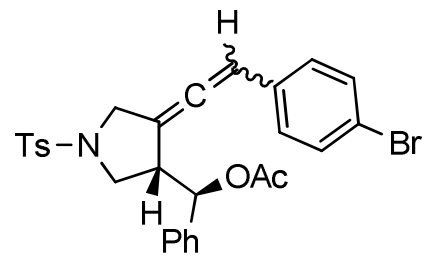

According to GP-5 s14 (110.5 mg, $0.2 \mathrm{mmol})$ was dissolved in $\mathrm{CH}_{2} \mathrm{Cl}_{2}(1 \mathrm{~mL})$, treated with $\left[\mathrm{Fe}(\mathrm{CO})(\mathrm{NO})\left(\mathrm{PPh}_{3}\right)_{2}\right]\left[\mathrm{BF}_{4}\right](14.5 \mathrm{mg}, 0.02 \mathrm{mmol})$ and stirred at $50{ }^{\circ} \mathrm{C}$ for 22 hours to yield the title compound $\mathbf{1 6}$ after column chromatography on silica gel (petroleum ether/EtOAc 4/1) as a 1:1 mixture of allene isomers which could be separated using HPLC (petroleum ether/EtOAc - 4/1).

Yield: $110.5 \mathrm{mg}(0.2 \mathrm{mmol}$, quant.).

Physical State: pale yellow solid. 


\section{Melting Point: $72{ }^{\circ} \mathrm{C}$.}

$\mathbf{R}_{\mathbf{f}}$ Value: 0.31 (petroleum ether/EtOAc - 4/1).

\section{HPLC fraction 1}

${ }^{1} \mathbf{H}$ NMR (Avance $400 \mathrm{MHz}, \mathrm{CDCl}_{3}$ ) $\delta 7.71$ - $7.73(\mathrm{~m}, 2 \mathrm{H}), 7.35$ - 7.37 (m, 2H), 7.30 - 7.32 $(\mathrm{m}, 2 \mathrm{H}), 7.14-7.19(\mathrm{~m}, 5 \mathrm{H}), 6.76-6.78(\mathrm{~m}, 2 \mathrm{H}), 5.99-6.02(\mathrm{~m}, 1 \mathrm{H}), 5.70(\mathrm{~d}, J=6.7 \mathrm{~Hz}$, $1 \mathrm{H}), 3.98(\mathrm{dd}, J=3.6,13.1 \mathrm{~Hz}, 1 \mathrm{H}), 3.92(\mathrm{dd}, J=3.5,13.2 \mathrm{~Hz}, 1 \mathrm{H}), 3.53(\mathrm{dd}, J=6.4,8.6 \mathrm{~Hz}$, $1 \mathrm{H}), 3.36-3.45(\mathrm{~m}, 2 \mathrm{H}), 2.47(\mathrm{~s}, 3 \mathrm{H}), 1.92(\mathrm{~s}, 3 \mathrm{H}) \mathrm{ppm}$.

${ }^{13}$ C NMR (Avance $101 \mathrm{MHz}, \mathrm{CDCl}_{3}$ ) $\delta$ 198.2, 169.5, 144.1, 138.0, 133.0, 132.5, 131.6, $129.9,128.7,128.6,128.5,127.9,127.0,121.3,103.0,98.9,75.6,50.2,50.0,47.4,21.7,21.1$ ppm.

IR (ATR, in $\left.\mathrm{CDCl}_{3}\right) \vee 3033$ (w), 1739 (m), 1597 (w), 1488 (m), 1455 (w), 1371 (w), 1346 (m), 1225 (s), 1160 (s), 1091 (m), 1069 (m), 1030 (m), 1010 (m) $\mathrm{cm}^{-1}$.

HRMS (ESI, m/z) calcd. for $\mathrm{C}_{28} \mathrm{H}_{26} \mathrm{BrNO}_{4} \mathrm{SNa}^{+}$: 574.0658, found: 574.0677.

\section{HPLC fraction 2}

${ }^{1}$ H NMR (Avance $400 \mathrm{MHz}, \mathrm{CDCl}_{3}$ ) $\delta 7.72-7.74(\mathrm{~m}, 2 \mathrm{H}), 7.36-7.38(\mathrm{~m}, 2 \mathrm{H}), 7.31-7.36$ $(\mathrm{m}, 2 \mathrm{H}), 7.18-7.22(\mathrm{~m}, 5 \mathrm{H}), 6.83-6.85(\mathrm{~m}, 2 \mathrm{H}), 5.77-5.79(\mathrm{~m}, 1 \mathrm{H}), 5.70(\mathrm{~d}, J=7.2 \mathrm{~Hz}$, $1 \mathrm{H}), 4.03(\mathrm{dd}, J=3.7,13.3 \mathrm{~Hz}, 1 \mathrm{H}), 3.84(\mathrm{ddd}, J=1.3,4.0,13.3 \mathrm{~Hz}, 1 \mathrm{H}), 3.56(\mathrm{dd}, J=7.4$, $9.7 \mathrm{~Hz}, 1 \mathrm{H}), 3.40-3.46(\mathrm{~m}, 1 \mathrm{H}), 3.33$ (dd, $J=6.4,9.7 \mathrm{~Hz}, 1 \mathrm{H}), 2.47$ (s, 3H), 2.01 (s, 3H) ppm.

${ }^{13}$ C NMR (Avance $101 \mathrm{MHz}, \mathrm{CDCl}_{3}$ ) $\delta$ 198.4, 169.7, 144.1, 138.2, 132.8, 132.6, 131.7, $129.9,128.6,128.5,128.4,128.0,127.1,121.2,102.9,98.8,75.7,50.2,49.9,46.9,21.7,21.1$ ppm.

IR (ATR, in $\mathrm{CDCl}_{3}$ ) v 3033 (w), 2927 (w), 1741 (m), 1597 (w), 1488 (m), 1454 (w), 1433 (w), 1372 (w), 1347 (m), 1226 (s), 1162 (s), 1092 (m), 1069 (m), 1031 (m), 1010 (m) cm cm $^{-1}$.

HRMS (ESI, m/z) calcd. for $\mathrm{C}_{28} \mathrm{H}_{26} \mathrm{BrNO}_{4} \mathrm{SNa}^{+}:$574.0658, found: 574.0655 . 
5.8 methyl 4-(2-(4-(acetoxy(phenyl)methyl)-1-tosylpyrrolidin-3-ylidene)vinyl)benzoate 17<smiles>[3H]N1C[C@H]2[C@H](OC(C)=O)c3ccccc3N([B])C/C(=C\Cc3ccc(C(=O)OC)cc3)[C@@H]2C1</smiles>

According to GP-5 s16 (106.3 mg, $0.2 \mathrm{mmol}$ ) was dissolved in $\mathrm{CH}_{2} \mathrm{Cl}_{2}$ (1 mL), treated with $\left[\mathrm{Fe}(\mathrm{CO})(\mathrm{NO})\left(\mathrm{PPh}_{3}\right)_{2}\right]\left[\mathrm{BF}_{4}\right](14.5 \mathrm{mg}, 0.02 \mathrm{mmol})$ and stirred at $50{ }^{\circ} \mathrm{C}$ for 22 hours to yield the title compound $\mathbf{1 7}$ after column chromatography on silica gel (petroleum ether/EtOAc 3/1) as an inseparable 1:1 mixture of allene isomers.

Yield: $56.7 \mathrm{mg}(0.107 \mathrm{mmol}, 53 \%)$.

Yield of recovered starting material: $33.2 \mathrm{mg}(0.063 \mathrm{mmol}, 31 \%)$.

Yield based on recovered starting material: $78 \%$.

Physical State: pale yellow oil.

$\mathbf{R}_{\mathbf{f}}$ Value: 0.33 (petroleum ether/EtOAc - 3/1).

${ }^{1} \mathbf{H}$ NMR (Avance $400 \mathrm{MHz}, \mathrm{CDCl}_{3}$ ) $\delta 7.86$ - $7.78(\mathrm{~m}, 4 \mathrm{H}), 7.72$ - $7.75(\mathrm{~m}, 4 \mathrm{H}), 7.37$ - 7.39 $(\mathrm{m}, 4 \mathrm{H}), 7.16-7.22(\mathrm{~m}, 7 \mathrm{H}), 7.12-7.15(\mathrm{~m}, 3 \mathrm{H}), 7.01-7.03(\mathrm{~m}, 2 \mathrm{H}), 6.96-6.98(\mathrm{~m}, 2 \mathrm{H})$, $6.08-6.11(\mathrm{~m}, 1 \mathrm{H}), 5.84-5.87(\mathrm{~m}, 1 \mathrm{H}), 5.72(\mathrm{~d}, J=7.3 \mathrm{~Hz}, 1 \mathrm{H}), 5.71(\mathrm{~d}, J=6.9 \mathrm{~Hz}, 1 \mathrm{H})$, $3.85-4.08(\mathrm{~m}, 10 \mathrm{H}), 3.54-3.60(\mathrm{~m}, 2 \mathrm{H}), 3.43-3.50(\mathrm{~m}, 2 \mathrm{H}), 3.34-3.40(\mathrm{~m}, 2 \mathrm{H}), 2.48(\mathrm{~s}$, $6 \mathrm{H}), 2.02(\mathrm{~s}, 3 \mathrm{H}), 1.92(\mathrm{~s}, 3 \mathrm{H}) \mathrm{ppm}$.

${ }^{13}$ C NMR (Avance $101 \mathrm{MHz}, \mathrm{CDCl}_{3}$ ) $\delta$ 199.3, 199.1, 169.7, 169.5, 166.8, 144.2, 144.1, $138.5,138.4,138.1,138.0,132.9,132.8,130.0,129.9$, 129.8, 129.0, 129.0, 128.6, 128.5, 128.5, 128.4, 128.0, 128.0, 127.1, 127.0, 127.0, 126.9, 103.0, 102.9, 99.2, 99.0, 75.7, 75.5, $52.2,50.3,50.3,50.0,49.9,47.4,47.0,21.7,21.1 \mathrm{ppm} .5$ carbons were not observed due to incidental equivalence.

IR (ATR, in $\left.\mathrm{CDCl}_{3}\right)$ v 2952 (w), 1716 (s), 1606 (m), 1571 (w), 1494 (w), 1435 (m), 1372 (w), 1347 (m), 1276 (s), 1225 (s), 1161 (s), 1108 (s), 1092 (s), 1031 (m), 1016 (s) cm cm$^{-1}$

HRMS (ESI, m/z) calcd. for $\mathrm{C}_{30} \mathrm{H}_{29} \mathrm{NO}_{6} \mathrm{SNa}^{+}:$554.1608, found: 554.1591. 
5.9 (4-(2-(3-methoxyphenyl)vinylidene)-1-tosylpyrrolidin-3-yl)(phenyl)methyl acetate 18<smiles>COc1cccc(C=C=C2CN([As])C[C@H]2[C@H](OC(C)=O)c2ccccc2)c1</smiles>

According to GP-5 s18 (100.7 mg, $0.2 \mathrm{mmol})$ was dissolved in $\mathrm{CH}_{2} \mathrm{Cl}_{2}$ (1 mL), treated with $\left[\mathrm{Fe}(\mathrm{CO})(\mathrm{NO})\left(\mathrm{PPh}_{3}\right)_{2}\right]\left[\mathrm{BF}_{4}\right](14.5 \mathrm{mg}, 0.02 \mathrm{mmol})$ and stirred at $50{ }^{\circ} \mathrm{C}$ for 22 hours to yield the title compound $\mathbf{1 8}$ after column chromatography on silica gel (petroleum ether/EtOAc 3/1) as an inseparable 1:1 mixture of allene isomers.

Yield: $67.7 \mathrm{mg}(0.134 \mathrm{mmol}, 67 \%)$.

Physical State: colourless oil.

$\mathbf{R}_{\mathbf{f}}$ Value: 0.34 (petroleum ether/EtOAc - 3/1).

${ }^{1} \mathbf{H}$ NMR (Avance $400 \mathrm{MHz}, \mathrm{CDCl}_{3}$ ) $\delta 7.70$ - $7.74(\mathrm{~m}, 4 \mathrm{H}), 7.36-7.38(\mathrm{~m}, 4 \mathrm{H}), 7.11-7.25$ $(\mathrm{m}, 12 \mathrm{H}), 6.71-6.75(\mathrm{~m}, 2 \mathrm{H}), 6.55-6.61(\mathrm{~m}, 4 \mathrm{H}), 6.05-6.08(\mathrm{~m}, 1 \mathrm{H}), 5.82-5.85(\mathrm{~m}, 1 \mathrm{H})$, $5.72(\mathrm{~d}, J=6.1 \mathrm{~Hz}, 2 \mathrm{H}), 3.98-4.04(\mathrm{~m}, 2 \mathrm{H}), 3.84-3.93(\mathrm{~m}, 2 \mathrm{H}), 3.76(\mathrm{~s}, 3 \mathrm{H}), 3.75(\mathrm{~s}, 3 \mathrm{H})$, $3.48-3.55(\mathrm{~m}, 2 \mathrm{H}), 3.33-3.41(\mathrm{~m}, 4 \mathrm{H}), 2.46(\mathrm{~s}, 6 \mathrm{H}), 2.01(\mathrm{~s}, 3 \mathrm{H}), 1.91(\mathrm{~s}, 3 \mathrm{H}) \mathrm{ppm}$.

${ }^{13}$ C NMR (Avance $101 \mathrm{MHz}, \mathrm{CDCl}_{3}$ ) $\delta$ 198.4, 198.0, 169.7, 169.6, 159.8, 159.8, 144.1, $144.0,138.3,138.2$, 135.0, 135.0, 133.0, 132.8, 129.9, 129.9, 129.6, 129.5, 128.5, 128.4, 128.3, 127.9, 127.9, 126.9, 126.8, 119.9, 119.8, 113.0, 112.8, 112.7, 102.7, 102.4, 99.8, 99.5, 75.7, 75.4, 55.3, 50.1, 50.0, 49.9, 49.9, 47.5, 47.1, 21.7, 21.0, 21.0 ppm. 4 carbons were not observed due to incidental equivalence.

IR (ATR, in $\left.\mathrm{CDCl}_{3}\right) \vee 2941$ (w), 2837 (w), 1741 (m), 1597 (m), 1492 (m), 1454 (w), 1408 (w), 1372 (w), 1346 (m), 1288 (w), 1259 (m), 1225 (s), 1157 (s), 1091 (m), 1036 (m), $1015(\mathrm{~m}) \mathrm{cm}^{-1}$.

HRMS (ESI, m/z) calcd. for $\mathrm{C}_{29} \mathrm{H}_{29} \mathrm{NO}_{5} \mathrm{SNa}^{+}:$526.1659, found: 526.1668 . 


\subsection{0 (4-(2-(3-acetamidophenyl)vinylidene)-1-tosylpyrrolidin-3-yl)(phenyl)methyl}

acetate 19

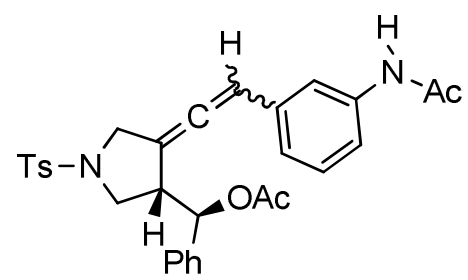

According to GP-5 s22 $(106.1 \mathrm{mg}, 0.2 \mathrm{mmol})$ was dissolved in $\mathrm{CH}_{2} \mathrm{Cl}_{2}(1 \mathrm{~mL})$, treated with $\left[\mathrm{Fe}(\mathrm{CO})(\mathrm{NO})\left(\mathrm{PPh}_{3}\right)_{2}\right]\left[\mathrm{BF}_{4}\right](14.5 \mathrm{mg}, 0.02 \mathrm{mmol})$ and stirred at $50{ }^{\circ} \mathrm{C}$ for 22 hours to yield the title compound 19 after column chromatography on silica gel (petroleum ether/EtOAc 1/1) as an inseparable 1.5:1 mixture of allene isomers.

Yield: $53.8 \mathrm{mg}(0.101 \mathrm{mmol}, 51 \%)$.

Yield of recovered starting material: $15.1 \mathrm{mg}(0.028 \mathrm{mmol}, 14 \%)$.

Yield based on recovered starting material: $59 \%$.

Physical State: yellow oil.

$\mathbf{R}_{\mathbf{f}}$ Value: 0.34 (petroleum ether/EtOAc - 1/1.5).

${ }^{1} \mathbf{H}$ NMR (Avance $400 \mathrm{MHz}, \mathrm{CDCl}_{3}$ ) $\delta 7.58-7.79(\mathrm{~m}, 7 \mathrm{H}), 7.36-7.42(\mathrm{~m}, 5 \mathrm{H}), 7.05-7.22$ (m, 14H), $6.73-6.74(\mathrm{~m}, 2 \mathrm{H}), 5.68-6.14(\mathrm{~m}, 4 \mathrm{H}), 3.77-4.04(\mathrm{~m}, 4 \mathrm{H}), 3.28-3.55(\mathrm{~m}, 6 \mathrm{H})$, $2.46-2.47(\mathrm{~m}, 6 \mathrm{H}), 2.14(\mathrm{~s}, 6 \mathrm{H}), 1.98-2.00(\mathrm{~m}, 6 \mathrm{H}) \mathrm{ppm}$.

${ }^{13}$ C NMR (Avance $101 \mathrm{MHz}, \mathrm{CDCl}_{3}$ ) $\delta 198.5,198.3,170.4,169.7,168.6,144.2,144.1$, $138.7,138.5,138.2$, 138.1, 134.4, 134.1, 133.1, 132.7, 130.0, 129.9, 129.3, 129.2, 128.7, 128.5, 128.5, 128.4, 128.0, 127.9, 127.0, 126.5, 122.9, 119.1, 118.3, 117.7, 103.3, 102.5, 99.8, 99.5, 75.8, 50.2, 50.1, 50.0, 49.3, 47.7, 47.0, 24.6, 21.7, 21.7, 21.2, 21.1 ppm. 5 carbons were not observed due to incidental equivalence.

IR (ATR, in $\left.\mathrm{CDCl}_{3}\right)$ v 3359 (w, broad), 3033 (w), 1739 (m), 1672 (m), 1607 (m), 1592 (m), 1546 (m), 1491 (m), 1455 (m), 1371 (m), 1343 (m), 1305 (m), 1226 (s), 1158 (s), 1091 (m), $1030(\mathrm{~m}), 1015(\mathrm{~m}) \mathrm{cm}^{-1}$.

HRMS (ESI, m/z) calcd. for $\mathrm{C}_{30} \mathrm{H}_{30} \mathrm{~N}_{2} \mathrm{O}_{5} \mathrm{SNa}^{+}:$553.1768, found: 553.1759 . 


\subsection{1 (4-(2,2-bis(4-fluorophenyl)vinylidene)-1-tosylpyrrolidin-3-yl)(phenyl)methyl}

acetate 20

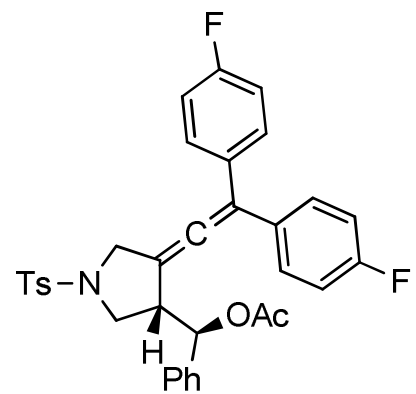

According to GP-4 s24 (117.1 mg, $0.2 \mathrm{mmol})$ and $\left[\mathrm{Fe}(\mathrm{CO})(\mathrm{NO})\left(\mathrm{PPh}_{3}\right)_{2}\right]\left[\mathrm{BF}_{4}\right](14.5 \mathrm{mg}$, $0.02 \mathrm{mmol})$ were dissolved in $\mathrm{CH}_{2} \mathrm{Cl}_{2}(1 \mathrm{~mL})$ and stirred at $50{ }^{\circ} \mathrm{C}$ for 22 hours to yield the title compound 20 after column chromatography on silica gel (petroleum ether/EtOAc - 5/1).

Yield: $95.9 \mathrm{mg}(0.164 \mathrm{mmol}, 82 \%)$.

Physical State: white solid.

Melting Point: $87^{\circ} \mathrm{C}$.

$\mathbf{R}_{\mathbf{f}}$ Value: 0.24 (petroleum ether/EtOAc - 5/1).

${ }^{1}$ H NMR (Avance $\left.400 \mathrm{MHz}, \mathrm{CDCl}_{3}\right) \delta 7.72$ - $7.74(\mathrm{~m}, 2 \mathrm{H}), 7.35$ - 7.37 (m, 2H), $7.11(\mathrm{~s}, 5 \mathrm{H})$, $6.90-6.96(\mathrm{~m}, 6 \mathrm{H}), 6.83-6.86(\mathrm{~m}, 2 \mathrm{H}), 5.65(\mathrm{~d}, J=7.7 \mathrm{~Hz}, 1 \mathrm{H}), 4.07(\mathrm{~d}, J=13.2 \mathrm{~Hz}, 1 \mathrm{H})$, $3.94(\mathrm{~d}, J=13.2 \mathrm{~Hz}, 1 \mathrm{H}), 3.60-3.64(\mathrm{~m}, 1 \mathrm{H}), 3.54(\mathrm{dd}, J=7.4,14.8 \mathrm{~Hz}, 1 \mathrm{H}), 3.27-3.32$ (m, 1H), $2.48(\mathrm{~s}, 3 \mathrm{H}), 1.85(\mathrm{~s}, 3 \mathrm{H}) \mathrm{ppm}$.

${ }^{19}$ F NMR (Avance $376 \mathrm{MHz}, \mathrm{CDCl}_{3}$ ) $\delta-113.9,-114.0 \mathrm{ppm}$.

${ }^{13}$ C NMR (Avance $101 \mathrm{MHz}, \mathrm{CDCl}_{3}$ ) $\delta$ 198.0, 169.6, 163.7 (d, $\left.J=2 \mathrm{~Hz}\right), 161.2(\mathrm{~d}, J=2 \mathrm{~Hz}$ ), 144.1, 138.1, 132.8, 132.1 (d, $J=3 \mathrm{~Hz}), 132.0$ (d, $J=3 \mathrm{~Hz}), 130.2$ (d, $J=8 \mathrm{~Hz}), 130.1$ (d, $J=8 \mathrm{~Hz}), 130.0,128.5,128.5,128.0,127.2,115.4(\mathrm{~d}, J=22 \mathrm{~Hz}), 113.4,101.9,75.7,50.7$, 50.5, 47.2, 21.7, 20.9 ppm. 1 carbon was no observed due to incidental equivalence.

IR (ATR, in $\left.\mathrm{CDCl}_{3}\right) \vee 2927$ (w), 1740 (m), 1599 (m), 1505 (s), 1347 (m), 1220 (s) 1156 (s), 1092 (m), 1014 (m) $\mathrm{cm}^{-1}$.

HRMS (ESI, m/z) calcd. for $\mathrm{C}_{34} \mathrm{H}_{29} \mathrm{~F}_{2} \mathrm{NO}_{4} \mathrm{SNa}^{+}$: 608.1678, found: 608.1667. 
5.12 phenyl(1-tosyl-4-(2,3,3-trimethylbut-1-en-1-ylidene)pyrrolidin-3-yl)methyl acetate 21<smiles>CC(=O)O[C@H](c1ccccc1)[C@H]1CN([13CH3])C/C1=C\C(C)(C)C</smiles>

According to GP-4 s26 $(93.5 \mathrm{mg}, 0.2 \mathrm{mmol})$ and $\left[\mathrm{Fe}(\mathrm{CO})(\mathrm{NO})\left(\mathrm{PPh}_{3}\right)_{2}\right]\left[\mathrm{BF}_{4}\right](14.5 \mathrm{mg}$, $0.02 \mathrm{mmol})$ were dissolved in $\mathrm{CH}_{2} \mathrm{Cl}_{2}(1 \mathrm{~mL})$ and stirred at $50{ }^{\circ} \mathrm{C}$ for 22 hours to yield the title compound 21 after column chromatography on silica gel (petroleum ether/EtOAc - 5/1) as a 1.5:1 mixture of allene isomers which could be separated using HPLC (petroleum ether/EtOAc - 5/1).

Yield: $69.0 \mathrm{mg}(0.148 \mathrm{mmol}, 74 \%)$.

Physical State: pale yellow oil.

$\mathbf{R}_{\mathbf{f}}$ Value: 0.37 (petroleum ether/EtOAc - 5/1).

\section{HPLC fraction 1}

${ }^{1} \mathbf{H}$ NMR (Avance $400 \mathrm{MHz}, \mathrm{CDCl}_{3}$ ) $\delta 7.72$ - $7.74(\mathrm{~m}, 2 \mathrm{H}), 7.36$ - $7.38(\mathrm{~m}, 2 \mathrm{H}), 7.23$ - 7.34 (m, 5H), $5.66-5.70(\mathrm{~m}, 1 \mathrm{H}), 3.96(\mathrm{~d}, J=12.6 \mathrm{~Hz}, 1 \mathrm{H}), 3.72(\mathrm{~d}, J=12.5 \mathrm{~Hz}, 1 \mathrm{H}), 3.49-3.56$ (m, 1H), $3.23-3.31(\mathrm{~m}, 2 \mathrm{H}), 2.47$ (s, 3H), 1.99 (s, 3H), 1.46 (s, 3H), 0.86 (s, 9H) ppm.

${ }^{13}$ C NMR (Avance $101 \mathrm{MHz}, \mathrm{CDCl}_{3}$ ) $\delta$ 193.0, 169.9, 143.8, 138.7, 133.3, 129.8, 128.6, 128.3, 127.9, 126.9, 115.6, 98.3, 76.1, 51.0, 50.0, 46.1, 34.0, 28.8, 21.7, 21.2, 15.0 ppm.

IR (ATR, in $\mathrm{CDCl}_{3}$ ) v 3033 (w), 2961 (w), 2867 (w), 1742 (m), 1598 (w), 1494 (w), 1346 (m), 1306 (w), 1226 (s), 1159 (s), 1092 (m) $\mathrm{cm}^{-1}$.

HRMS (ESI, m/z) calcd. for $\mathrm{C}_{27} \mathrm{H}_{33} \mathrm{NO}_{4} \mathrm{SNa}^{+}$: 490.2023, found: 490.2023.

\section{HPLC fraction 2}

${ }^{1} \mathbf{H}$ NMR (Avance $400 \mathrm{MHz}, \mathrm{CDCl}_{3}$ ) $\delta 7.72$ - $7.74(\mathrm{~m}, 2 \mathrm{H}), 7.36-7.38(\mathrm{~m}, 2 \mathrm{H}), 7.22-7.32$ $(\mathrm{m}, 5 \mathrm{H}), 5.60(\mathrm{~d}, J=8.0 \mathrm{~Hz}, 1 \mathrm{H}), 3.87(\mathrm{~d}, J=12.7 \mathrm{~Hz}, 1 \mathrm{H}), 3.71(\mathrm{dd}, J=1.5,12.7 \mathrm{~Hz}, 1 \mathrm{H})$, 3.58 (dd, $J=7.4,9.8 \mathrm{~Hz}, 1 \mathrm{H}), 3.29-3.35$ (m, 1H), 3.17 (dd, $J=7.3,9.8 \mathrm{~Hz}, 1 \mathrm{H}), 2.47$ (s, $3 \mathrm{H}), 2.00(\mathrm{~s}, 3 \mathrm{H}), 1.31$ (s, 3H), 0.79 (s, 9H) ppm.

${ }^{13}$ C NMR (Avance $101 \mathrm{MHz}, \mathrm{CDCl}_{3}$ ) $\delta$ 193.6, 169.7, 143.8, 138.6, 132.8, 129.8, 128.4, $128.3,127.9,127.4,115.0,97.3,76.3,50.6,50.5,46.2,34.1,28.8,21.6,21.1,14.9$ ppm. 
IR (ATR, in $\left.\mathrm{CDCl}_{3}\right)$ v 3033 (w), 2961 (w), 2866 (w), 1742 (m), 1598 (w), 1494 (w), 1348 (m), 1228 (s), 1116 (s), 1093 (w), 1044 (m), 1016 (m) cm ${ }^{-1}$.

HRMS (ESI, m/z) calcd. for $\mathrm{C}_{27} \mathrm{H}_{33} \mathrm{NO}_{4} \mathrm{SNa}^{+}$: 490.2023, found: 490.2031 .

\subsection{3 (4-(2-isopropyl-3-methylbut-1-en-1-ylidene)-1-tosylpyrrolidin-3-yl)(phenyl)methyl} acetate 22

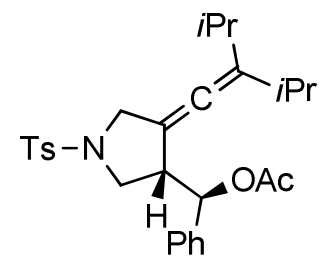

According to GP-5 s28 $(96.3 \mathrm{mg}, 0.2 \mathrm{mmol})$ was dissolved in $\mathrm{CH}_{2} \mathrm{Cl}_{2}(1 \mathrm{~mL})$, treated with $\left[\mathrm{Fe}(\mathrm{CO})(\mathrm{NO})\left(\mathrm{PPh}_{3}\right)_{2}\right]\left[\mathrm{BF}_{4}\right](14.5 \mathrm{mg}, 0.02 \mathrm{mmol})$ and stirred at $50{ }^{\circ} \mathrm{C}$ for 22 hours to yield the title compound 22 after column chromatography on silica gel ( $n$-pentane/ $\mathrm{Et}_{2} \mathrm{O}-2 / 1$ ).

Yield: $45.7 \mathrm{mg}(0.095 \mathrm{mmol}, 47 \%)$.

Phyiscal State: pale yellow oil.

$\mathbf{R}_{\mathbf{f}}$ Value: 0.34 (n-pentane/Et $\left.2 \mathrm{O}-2 / 1\right)$.

${ }^{1} \mathbf{H}$ NMR (Avance $400 \mathrm{MHz}, \mathrm{CDCl}_{3}$ ) $\delta 7.72$ - $7.74(\mathrm{~m}, 2 \mathrm{H}), 7.36$ - $7.38(\mathrm{~m}, 2 \mathrm{H}), 7.25$ - 7.33 (m, 3H), $7.21-7.23(\mathrm{~m}, 2 \mathrm{H}), 5.65(\mathrm{~d}, J=5.6 \mathrm{~Hz}, 1 \mathrm{H}), 3.95(\mathrm{~d}, J=12.7 \mathrm{~Hz}, 1 \mathrm{H}), 3.76(\mathrm{dd}$, $J=1.1,12.6 \mathrm{~Hz}, 1 \mathrm{H}), 3.51-3.57(\mathrm{~m}, 1 \mathrm{H}), 3.24-3.31$ (m, 2H), 2.47 (s, 3H), $2.00-2.09$ (m, $2 \mathrm{H}), 1.98(\mathrm{~s}, 3 \mathrm{H}), 0.84(\mathrm{~d}, J=6.7 \mathrm{~Hz}, 3 \mathrm{H}), 0.82(\mathrm{~d}, J=6.5 \mathrm{~Hz}, 3 \mathrm{H}), 0.81(\mathrm{~d}, J=6.4 \mathrm{~Hz}, 3 \mathrm{H})$, $0.73(\mathrm{~d}, J=6.8 \mathrm{~Hz}, 3 \mathrm{H}) \mathrm{ppm}$.

${ }^{13}$ C NMR (Avance $101 \mathrm{MHz}, \mathrm{CDCl}_{3}$ ) $\delta$ 192.0, 169.8, 143.8, 138.8, 133.2, 129.8, 128.6, $128.3,127.9,126.9,125.1,102.1,76.1,51.0,50.1,46.7,30.1,30.0,22.4,22.3,22.0,21.8$, 21.7, $21.2 \mathrm{ppm}$.

IR (ATR, in $\left.\mathrm{CDCl}_{3}\right) \vee 3033$ (w), 2960 (w), 2928 (w), 2870 (w), 1744 (m), 1598 (w), 1494 (w), 1456 (w), 1348 (s), 1230 (s), 1162 (s), 1092 (m), 1031 (m) cm $\mathrm{cm}^{-1}$

HRMS (ESI, m/z) calcd. for $\mathrm{C}_{28} \mathrm{H}_{35} \mathrm{NO}_{4} \mathrm{SNa}^{+}$: 504.2179, found: 504.2179. 


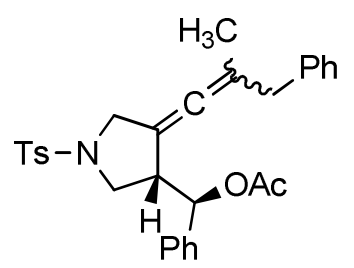

According to GP-5 s30 (100.3 mg, $0.2 \mathrm{mmol}$ ) was dissolved in $\mathrm{CH}_{2} \mathrm{Cl}_{2}(1 \mathrm{~mL})$, treated with $\left[\mathrm{Fe}(\mathrm{CO})(\mathrm{NO})\left(\mathrm{PPh}_{3}\right)_{2}\right]\left[\mathrm{BF}_{4}\right](14.5 \mathrm{mg}, 0.02 \mathrm{mmol})$ and stirred at $50{ }^{\circ} \mathrm{C}$ for 22 hours to yield the title compound $\mathbf{2 3}$ after column chromatography on silica gel (petroleum ether/EtOAc 5/1) as a 1.2:1 mixture of allene isomers which could be separated using HPLC (petroleum ether/EtOAc - 5/1).

Yield: $67.3 \mathrm{mg}(0.134 \mathrm{mmol}, 67 \%)$.

Physical State: white oil.

$\mathbf{R}_{\mathbf{f}}$ Value: 0.31 (petroleum ether/EtOAc - 5/1).

\section{HPLC fraction 1}

${ }^{1} \mathbf{H}$ NMR (Avance $400 \mathrm{MHz}, \mathrm{CDCl}_{3}$ ) $\delta 7.70-7.72(\mathrm{~m}, 2 \mathrm{H}), 7.34-7.36(\mathrm{~m}, 2 \mathrm{H}), 7.28-7.32$ (m, 2H), $7.17-7.27(\mathrm{~m}, 5 \mathrm{H}), 7.11-7.15(\mathrm{~m}, 1 \mathrm{H}), 7.00-7.03(\mathrm{~m}, 2 \mathrm{H}), 5.56(\mathrm{~d}, J=7.6 \mathrm{~Hz}$, $1 \mathrm{H}), 3.89(\mathrm{~d}, J=12.7 \mathrm{~Hz}, 1 \mathrm{H}), 3.68(\mathrm{dd}, J=1.4,12.6 \mathrm{~Hz}, 1 \mathrm{H}), 3.49(\mathrm{dd}, J=7.3,9.6 \mathrm{~Hz}, 1 \mathrm{H})$, $3.24-3.30(\mathrm{~m}, 1 \mathrm{H}), 3.11(\mathrm{dd}, J=7.1,9.6 \mathrm{~Hz}, 1 \mathrm{H}), 2.94(\mathrm{~d}, J=14.6 \mathrm{~Hz}, 1 \mathrm{H}), 2.85(\mathrm{~d}$, $J=14.7 \mathrm{~Hz}, 1 \mathrm{H}), 2.45$ (s, 3H), 1.97 (s, 3H), 1.32 (s, 3H) ppm.

${ }^{13}$ C NMR (Avance $101 \mathrm{MHz}, \mathrm{CDCl}_{3}$ ) $\delta$ 195.5, 169.7, 143.8, 138.9, 138.6, 133.3, 129.8, 128.9, 128.5, 128.3, 127.9, 127.3, 126.4, 105.6, 97.5, 75.9, 50.4, 50.1, 46.2, 41.1, 21.7, 21.1, $18.1 \mathrm{ppm} .1$ carbon was not observed due to incidental equivalence.

IR (ATR, in $\mathrm{CDCl}_{3}$ ) v 3062 (w), 3029 (w), 2924 (w), 1739 (m), 1598 (w), 1494 (w), 1453 (w), 1407 (w), 1370 (w), 1345 (m), 1305 (w), 1290 (w), 1225 (s), 1159 (s), 1092 (m), $1042(\mathrm{~m}), 1016(\mathrm{~m}) \mathrm{cm}^{-1}$.

HRMS (ESI, m/z) calcd. for $\mathrm{C}_{30} \mathrm{H}_{31} \mathrm{NO}_{4} \mathrm{SNa}^{+}$: 524.1866, found: 524.1880.

\section{HPLC fraction 2}

${ }^{1} \mathbf{H}$ NMR (Avance $400 \mathrm{MHz}, \mathrm{CDCl}_{3}$ ) $\delta 7.66$ - $7.68(\mathrm{~m}, 2 \mathrm{H}), 7.33-7.35(\mathrm{~m}, 2 \mathrm{H}), 7.24-7.30$ $(\mathrm{m}, 3 \mathrm{H}), 7.14-7.21(\mathrm{~m}, 4 \mathrm{H}), 7.09-7.13(\mathrm{~m}, 1 \mathrm{H}), 6.91-6.93(\mathrm{~m}, 2 \mathrm{H}), 5.57(\mathrm{~d}, J=7.8 \mathrm{~Hz}$, $1 \mathrm{H}), 3.70(\mathrm{dd}, J=1.4,12.8 \mathrm{~Hz}, 1 \mathrm{H}), 3.63(\mathrm{~d}, J=12.9 \mathrm{~Hz}, 1 \mathrm{H}), 3.29(\mathrm{dd}, J=7.5,10.0 \mathrm{~Hz}$, 
1H), $3.22(\mathrm{dd}, J=6.1,9.9 \mathrm{~Hz}, 1 \mathrm{H}), 3.01-3.06(\mathrm{~m}, 1 \mathrm{H}), 3.98(\mathrm{~d}, J=5.2 \mathrm{~Hz}, 2 \mathrm{H}), 2.44(\mathrm{~s}$, $3 \mathrm{H}), 1.96$ (s, 3H), 1.28 (s, 3H) ppm.

${ }^{13} \mathbf{C}$ NMR (Avance $101 \mathrm{MHz}, \mathrm{CDCl}_{3}$ ) $\delta$ 196.1, 169.7, 143.8, 139.0, 138.6, 133.0, 129.8, $128.8,128.4,128.3,128.2$, 127.9, 127.3, 126.3, 106.2 , 97.8, 75.9, 50.2, 49.8, 46.4, 41.0, 21.7, $21.1,18.5 \mathrm{ppm}$.

IR (ATR, in $\mathrm{CDCl}_{3}$ ) v 3026 (w), 3028 (w), 2900 (w), 1741 (m), 1598 (w), 1494 (w), $1474(\mathrm{w}), 1453$ (w), 1408 (w), 1370 (w), 1346 (m), 1306 (w), 1290 (w), 1225 (s), 1159 (s), $1092(\mathrm{~m}), 1041(\mathrm{~m}), 1016(\mathrm{~m}) \mathrm{cm}^{-1}$.

HRMS (ESI, m/z) calcd. for $\mathrm{C}_{30} \mathrm{H}_{31} \mathrm{NO}_{4} \mathrm{SNa}^{+}$: 524.1866, found: 524.1898.

\subsection{5 (E)-phenyl(4-(2-(4-styrylphenyl)vinylidene)-1-tosylpyrrolidin-3-yl)methyl acetate} 24

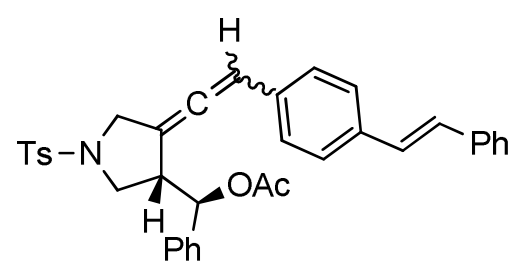

According to GP-5 s32 (115.1 mg, $0.2 \mathrm{mmol})$ was dissolved in $\mathrm{CH}_{2} \mathrm{Cl}_{2}(1 \mathrm{~mL})$, treated with $\left[\mathrm{Fe}(\mathrm{CO})(\mathrm{NO})\left(\mathrm{PPh}_{3}\right)_{2}\right]\left[\mathrm{BF}_{4}\right](14.5 \mathrm{mg}, 0.02 \mathrm{mmol})$ and stirred at $50{ }^{\circ} \mathrm{C}$ for 22 hours to yield the title compound $\mathbf{2 4}$ after column chromatography on silica gel (petroleum ether/EtOAc 4/1) as a 2.3:1 mixture of allene isomers which could be separated using HPLC (petroleum ether/EtOAc - 4/1).

Yield: $96.9 \mathrm{mg}(0.168 \mathrm{mmol}, 84 \%)$.

Physical State: orange oil.

$\mathbf{R}_{\mathbf{f}}$ Value: 0.26 (petroleum ether/EtOAc - 4/1).

\section{HPLC fraction 1}

${ }^{1} \mathbf{H}$ NMR (Avance $400 \mathrm{MHz}, \mathrm{CDCl}_{3}$ ) $\delta 7.72$ - $7.74(\mathrm{~m}, 2 \mathrm{H}), 7.50-7.52(\mathrm{~m}, 2 \mathrm{H}), 7.34-7.38$ (m, 6H), $7.24-7.27(\mathrm{~m}, 1 \mathrm{H}), 7.15-7.21(\mathrm{~m}, 5 \mathrm{H}), 7.07-7.08(\mathrm{~m}, 2 \mathrm{H}), 6.93-6.95(\mathrm{~m}, 2 \mathrm{H})$, $6.07-6.09(\mathrm{~m}, 1 \mathrm{H}), 5.73(\mathrm{~d}, J=6.5 \mathrm{~Hz}, 1 \mathrm{H}), 4.00(\mathrm{dd}, J=3.6,13.1 \mathrm{~Hz}, 1 \mathrm{H}), 3.93(\mathrm{dd}$, $J=4.0,13.1 \mathrm{~Hz}, 1 \mathrm{H}), 3.51-3.57(\mathrm{~m}, 1 \mathrm{H}), 3.36-3.45(\mathrm{~m}, 2 \mathrm{H}), 2.48(\mathrm{~s}, 3 \mathrm{H}), 1.93(\mathrm{~s}, 3 \mathrm{H})$ ppm. 
${ }^{13}$ C NMR (Avance $101 \mathrm{MHz}, \mathrm{CDCl}_{3}$ ) $\delta$ 198.3, 169.7, 144.0, 138.2, 137.3, 136.8, 133.1, 132.8, 129.9, 128.8, 128.5, 128.5, 128.3, 128.0, 127.8, 127.6, 127.0, 126.7, 126.6, 102.6, 99.7, 75.7, 50.2, 50.2, 47.5, 21.7, 21.1 ppm. 1 carbon was not observed due to incidental equivalence.

IR (ATR, in $\mathrm{CDCl}_{3}$ ) v 3027 (w), 1739 (m), 1597 (w), 1512 (w), 1494 (w), 1474 (w), 1449 (w), 1398 (w), 1371 (w), 1345 (m), 1305 (w), 1290 (w), 1225 (s), 1161 (s), 1091 (m), $1029(\mathrm{~m}), 1015(\mathrm{~m}) \mathrm{cm}^{-1}$.

HRMS (ESI, m/z) calcd. for $\mathrm{C}_{36} \mathrm{H}_{33} \mathrm{NO}_{4} \mathrm{SNa}^{+}$: 598.2023, found: 598.2013.

\section{HPLC fraction 2}

${ }^{1} \mathbf{H}$ NMR (Avance $400 \mathrm{MHz}, \mathrm{CDCl}_{3}$ ) $\delta 7.73$ - $7.75(\mathrm{~m}, 2 \mathrm{H}), 7.49$ - $7.51(\mathrm{~m}, 2 \mathrm{H}), 7.34-7.39$ (m, 6H), $7.19-7.27(\mathrm{~m}, 6 \mathrm{H}), 7.06-7.07(\mathrm{~m}, 2 \mathrm{H}), 6.98-7.00(\mathrm{~m}, 2 \mathrm{H}), 5.84-5.88(\mathrm{~m}, 1 \mathrm{H})$, $5.73(\mathrm{~d}, J=6.9 \mathrm{~Hz}, 1 \mathrm{H}), 4.05(\mathrm{dd}, J=3.7,13.2 \mathrm{~Hz}, 1 \mathrm{H}), 3.85(\mathrm{ddd}, J=1.3,4.0,13.2 \mathrm{~Hz}, 1 \mathrm{H})$, $3.56(\mathrm{~d}, J=7.2,9.5 \mathrm{~Hz}, 1 \mathrm{H}), 3.40-3.46(\mathrm{~m}, 1 \mathrm{H}), 3.36(\mathrm{dd}, J=6.3,9.6 \mathrm{~Hz}, 1 \mathrm{H}), 2.49$ (s, 3H), $2.02(\mathrm{~s}, 3 \mathrm{H}) \mathrm{ppm}$.

${ }^{13}$ C NMR (Avance $101 \mathrm{MHz}, \mathrm{CDCl}_{3}$ ) $\delta$ 198.7, 169.8, 144.1, 138.4, 137.3, 136.8, 133.0, 132.9, 130.0, 128.8, 128.6, 128.4, 128.3, 128.1, 127.9, 127.5, 127.0, 126.8, 126.6, 102.5, 99.5, $75.8,50.2,50.0,47.1,21.8,21.1$ ppm. 1 carbon was not observed due to incidental equivalence.

IR (ATR, in $\left.\mathrm{CDCl}_{3}\right) \vee 3028$ (w), 1741 (m), 1597 (w), 1512 (w), 1494 (w), 1473 (w), 1450 (w), 1398 (w), 1371 (w), 1347 (m), 1305 (w), 1290 (w), 1226 (s), 1162 (s), 1092 (m), $1030(\mathrm{~m}), 1016(\mathrm{~m}) \mathrm{cm}^{-1}$.

HRMS (ESI, m/z) calcd. for $\mathrm{C}_{36} \mathrm{H}_{33} \mathrm{NO}_{4} \mathrm{SNa}^{+}$: 598.2023, found: 598.2026.

\subsection{6 (E)-phenyl(4-(4-phenylbuta-1,3-dien-1-ylidene)-1-tosylpyrrolidin-3-yl)methyl} acetate 25

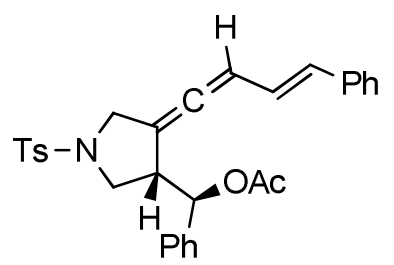

According to GP-5 s34 (99.9 mg, $0.2 \mathrm{mmol})$ was dissolved in $\mathrm{CH}_{2} \mathrm{Cl}_{2}(1 \mathrm{~mL})$, treated with $\left[\mathrm{Fe}(\mathrm{CO})(\mathrm{NO})\left(\mathrm{PPh}_{3}\right)_{2}\right]\left[\mathrm{BF}_{4}\right](14.5 \mathrm{mg}, 0.02 \mathrm{mmol})$ and stirred at $50{ }^{\circ} \mathrm{C}$ for 22 hours to yield 
the title compound $\mathbf{2 5}$ after column chromatography on silica gel (petroleum ether/EtOAc $5 / 1)$ as a single allene isomer.

Yield: $50.0 \mathrm{mg}(0.100 \mathrm{mmol}, 50 \%)$.

Physical State: yellow oil.

$\mathbf{R}_{\mathbf{f}}$ Value: 0.23 (petroleum ether/EtOAc - 5/1).

${ }^{1} \mathbf{H}$ NMR (Avance $400 \mathrm{MHz}, \mathrm{CDCl}_{3}$ ) $\delta 7.71$ - $7.73(\mathrm{~m}, 2 \mathrm{H}), 7.35$ - 7.37 (m, 2H), 7.24 - 7.31 $(\mathrm{m}, 8 \mathrm{H}), 7.18-7.22(\mathrm{~m}, 2 \mathrm{H}), 6.31(\mathrm{~d}, J=14.6 \mathrm{~Hz}, 1 \mathrm{H}), 5.85-5.92(\mathrm{~m}, 2 \mathrm{H}), 5.67(\mathrm{~d}, J=7.9$ $\mathrm{Hz}, 1 \mathrm{H}), 3.83-3.90(\mathrm{~m}, 2 \mathrm{H}), 3.39-3.46(\mathrm{~m}, 2 \mathrm{H}), 3.31-3.35(\mathrm{~m}, 1 \mathrm{H}), 2.46(\mathrm{~s}, 3 \mathrm{H}), 2.01$ (s, 3H) ppm.

${ }^{13} \mathrm{C}$ NMR (Avance $101 \mathrm{MHz}, \mathrm{CDCl}_{3}$ ) $\delta$ 201.4, 169.7, 144.1, 138.3, 137.0, 133.0, 131.9, $129.9,128.7,128.5,128.5,128.0,127.8,127.5,126.5,123.6,99.8,99.4,76.1,50.4,49.9$, 47.5, 21.7, $21.2 \mathrm{ppm}$.

IR (ATR, in $\left.\mathrm{CDCl}_{3}\right) \vee 3030$ (w), 1741 (m), 1597 (w), 1494 (w), 1474 (w), 1452 (w), 1372 (w), 1346 (m), 1305 (w), 1291 (w), 1223 (s), 1162 (s), 1091 (m), 1030 (m), 1016 (m) cm . $^{-1}$.

HRMS (ESI, m/z) calcd. for $\mathrm{C}_{30} \mathrm{H}_{29} \mathrm{NO}_{4} \mathrm{SNa}^{+}$: 522.1710, found: 522.1705.

\subsection{7 (4-(2-methylprop-1-en-1-ylidene)tetrahydrofuran-3-yl)(phenyl)methyl acetate 26}

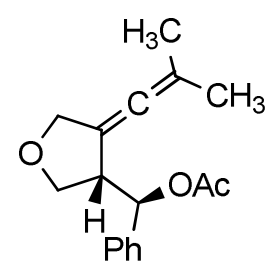

According to GP-5 s37 (54.5 mg, $0.2 \mathrm{mmol}$ ) was dissolved in $\mathrm{CH}_{2} \mathrm{Cl}_{2}(1 \mathrm{~mL})$, treated with $\left[\mathrm{Fe}(\mathrm{CO})(\mathrm{NO})\left(\mathrm{PPh}_{3}\right)_{2}\right]\left[\mathrm{BF}_{4}\right](14.5 \mathrm{mg}, 0.02 \mathrm{mmol})$ and stirred at $50{ }^{\circ} \mathrm{C}$ for 22 hours to yield the title compound 26 after column chromatography on silica gel ( $n$-pentane/ $\operatorname{Et}_{2} \mathrm{O}-5 / 1$ ).

Yield: $14.3 \mathrm{mg}(0.053 \mathrm{mmol}, 26 \%)$.

Physical State: colourless liquid.

$\mathbf{R}_{\mathbf{f}}$ Value: 0.31 (n-pentane/Et $\left.2 \mathrm{O}-5 / 1\right)$. 
${ }^{1} \mathbf{H}$ NMR (Avance $300 \mathrm{MHz}, \mathrm{CDCl}_{3}$ ) $\delta 7.32$ - $7.34(\mathrm{~m}, 4 \mathrm{H}), 7.27-7.30(\mathrm{~m}, 1 \mathrm{H}), 5.78(\mathrm{~d}$, $J=8.0 \mathrm{~Hz}, 1 \mathrm{H}), 4.27(\mathrm{~s}, 2 \mathrm{H}), 3.97(\mathrm{dd}, J=6.7,8.9 \mathrm{~Hz}, 1 \mathrm{H}), 3.89(\mathrm{dd}, J=5.8,8.9 \mathrm{~Hz}, 1 \mathrm{H})$, $3.30-3.36(\mathrm{~m}, 1 \mathrm{H}), 2.05(\mathrm{~s}, 3 \mathrm{H}), 1.48(\mathrm{~s}, 3 \mathrm{H}), 1.30(\mathrm{~s}, 3 \mathrm{H}) \mathrm{ppm}$.

${ }^{13}$ C NMR (Avance $75 \mathrm{MHz}, \mathrm{CDCl}_{3}$ ) $\delta$ 193.4, 170.1, 139.1, 128.4, 128.1, 127.6, 100.8, 98.6, $76.3,71.1,69.6,47.7,21.3,20.6,20.5$ ppm.

IR (ATR, in $\left.\mathrm{CDCl}_{3}\right)$ v 3033 (w), 2980 (w), 2935 (w), 2910 (w), 2852 (w), 1739 (s), 1496 (w), 1454 (w), 1401 (w), 1367 (m), 1232 (s), 1137 (w), 1051 (m), 1019 (m) cm $\mathrm{cm}^{-1}$

HRMS (ESI, m/z) calcd. for $\mathrm{C}_{17} \mathrm{H}_{20} \mathrm{O}_{3} \mathrm{Na}^{+}:$295.1305, found: 295.1300 .

\subsection{8 (4-(2,2-diphenylvinylidene)tetrahydrofuran-3-yl)(phenyl)methyl acetate 27}

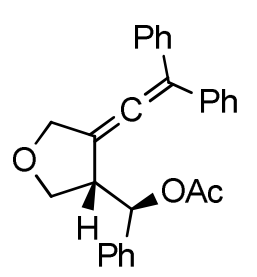

According to GP-5 s39 $(79.3 \mathrm{mg}, 0.2 \mathrm{mmol})$ was dissolved in $\mathrm{CH}_{2} \mathrm{Cl}_{2}(1 \mathrm{~mL})$, treated with $\left[\mathrm{Fe}(\mathrm{CO})(\mathrm{NO})\left(\mathrm{PPh}_{3}\right)_{2}\right]\left[\mathrm{BF}_{4}\right](14.5 \mathrm{mg}, 0.02 \mathrm{mmol})$ and stirred at $50{ }^{\circ} \mathrm{C}$ for 22 hours to yield the title compound 27 after column chromatography on silica gel (petroleum ether/EtOAc $7 / 1)$.

Yield: $29.3 \mathrm{mg}(0.074 \mathrm{mmol}, 37 \%)$.

Physical State: brown oil.

$\mathbf{R}_{\mathbf{f}}$ Value: 0.34 (petroleum ether/EtOAc - 7/1).

${ }^{1} \mathbf{H}$ NMR (Avance $\left.400 \mathrm{MHz}, \mathrm{CDCl}_{3}\right) \delta 7.24$ - $7.31(\mathrm{~m}, 6 \mathrm{H}), 7.19-7.21(\mathrm{~m}, 2 \mathrm{H}), 7.09$ - 7.10 $(\mathrm{m}, 2 \mathrm{H}), 7.05-7.07(\mathrm{~m}, 5 \mathrm{H}), 5.82(\mathrm{~d}, J=8.1 \mathrm{~Hz}, 1 \mathrm{H}), 4.99-4.55(\mathrm{~m}, 2 \mathrm{H}), 4.10(\mathrm{dd}, J=6.9$, $8.9 \mathrm{~Hz}, 1 \mathrm{H}), 3.96(\mathrm{dd}, J=6.7,8.8 \mathrm{~Hz}, 1 \mathrm{H}), 3.68(\mathrm{ddd}, J=7.3,7.3,7.3 \mathrm{~Hz}, 1 \mathrm{H}), 1.92(\mathrm{~s}, 3 \mathrm{H})$ ppm.

${ }^{13} \mathbf{C}$ NMR (Avance $101 \mathrm{MHz}, \mathrm{CDCl}_{3}$ ) $\delta$ 197.0, 169.9, 138.6, 136.9, 136.8, 128.8, 128.6, 128.4, 128.3, 128.3, 127.5, 127.4, 114.6, 103.9, 76.1, 71.6, 70.0, 49.2, 21.1 ppm. 2 carbons were not observed due to incidental equivalence.

IR (ATR, in $\mathrm{CDCl}_{3}$ ) v 3058 (w), 3029 (w), 2930 (w), 2852 (w), 1953 (w), 1747 (m), 1597 (w), 1492 (w), 1453 (w), 1443 (w), 1370 (w), 1228 (s), $1136(w), 1053(w), 1019$ (w) cm . $^{-1}$ 
HRMS (ESI, m/z) calcd. for $\mathrm{C}_{27} \mathrm{H}_{24} \mathrm{O}_{3} \mathrm{Na}^{+}$: 419.1618, found: 419.1636 .

\subsection{9 (4-(2,2-diphenylvinylidene)-1-(methylsulfonyl)pyrrolidin-3-yl)(phenyl)methyl} acetate 28

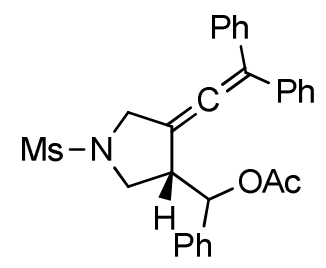

According to GP-4 s43 $(94.7 \mathrm{mg}, 0.2 \mathrm{mmol})$ and $\left[\mathrm{Fe}(\mathrm{CO})(\mathrm{NO})\left(\mathrm{PPh}_{3}\right)_{2}\right]\left[\mathrm{BF}_{4}\right](14.5 \mathrm{mg}$, $0.02 \mathrm{mmol})$ were dissolved in $\mathrm{CH}_{2} \mathrm{Cl}_{2}(1 \mathrm{~mL})$ and stirred at $50{ }^{\circ} \mathrm{C}$ for 22 hours to yield the title compound 28 after column chromatography on silica gel (petroleum ether/EtOAc - 4/1).

Yield: $51.5 \mathrm{mg}(0.109 \mathrm{mmol}, 54 \%)$.

Physical State: colourless oil.

$\mathbf{R}_{\mathbf{f}}$ Value: 0.13 (petroleum ether/EtOAc - 4/1).

${ }^{1} \mathbf{H}$ NMR (Avance $400 \mathrm{MHz}, \mathrm{CDCl}_{3}$ ) $\delta 7.28$ - $7.32(\mathrm{~m}, 8 \mathrm{H}), 7.16$ - 7.17 (m, 3H), 7.11 - 7.13 (m, 2H), $7.05-7.07(\mathrm{~m}, 2 \mathrm{H}), 5.85(\mathrm{~d}, J=6.1 \mathrm{~Hz}, 1 \mathrm{H}), 4.22(\mathrm{~d}, J=13.2 \mathrm{~Hz}, 1 \mathrm{H}), 4.12(\mathrm{~d}$, $J=13.2 \mathrm{~Hz}, 1 \mathrm{H}), 3.65-3.72(\mathrm{~m}, 2 \mathrm{H}), 3.44-3.50(\mathrm{~m}, 1 \mathrm{H}), 2.85(\mathrm{~s}, 3 \mathrm{H}), 1.84(\mathrm{~s}, 3 \mathrm{H}) \mathrm{ppm}$.

${ }^{13}$ C NMR (Avance $101 \mathrm{MHz}, \mathrm{CDCl}_{3}$ ) $\delta$ 198.4, 169.8, 138.1, 136.3, 136.2, 128.8, 128.6, 128.6, 128.6, 128.5, 128.5, 127.9, 126.9, 115.6, 101.5, 75.4, 50.3, 50.2, 47.8, 35.8, 20.9 ppm. 1 carbon was not observed due to incidental equivalence.

IR (ATR, in $\mathrm{CDCl}_{3}$ ) v 3059 (w), 3029 (w), 2928 (w), 1957 (w), 1737 (m), 1598 (w), 1493 (w), 1453 (w), 1443 (w), 1371 (w), 1336 (s), 1226 (s), 1152 (s), 1044 (m), 1027 (m) cm cm $^{-1}$.

HRMS (ESI, m/z) calcd. for $\mathrm{C}_{28} \mathrm{H}_{27} \mathrm{NO}_{4} \mathrm{SNa}^{+}$: 496.1553, found: 496.1572.

\subsection{0 (4-(2,2-diphenylvinylidene)-1-(phenylsulfonyl)pyrrolidin-3-yl)(phenyl)methyl} acetate 29

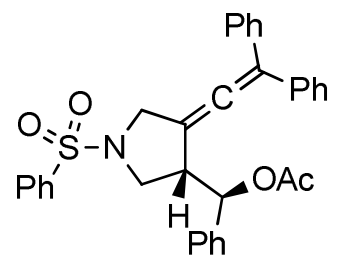


According to GP-4 $35(107.1 \mathrm{mg}, 0.2 \mathrm{mmol})$ and $\left[\mathrm{Fe}(\mathrm{CO})(\mathrm{NO})\left(\mathrm{PPh}_{3}\right)_{2}\right]\left[\mathrm{BF}_{4}\right](14.5 \mathrm{mg}$, $0.02 \mathrm{mmol})$ were dissolved in $\mathrm{CH}_{2} \mathrm{Cl}_{2}(1 \mathrm{~mL})$ and stirred at $50{ }^{\circ} \mathrm{C}$ for 22 hours to yield the title compound 29 after column chromatography on silica gel (n-pentane/ $\left.\mathrm{Et}_{2} \mathrm{O}-2 / 1\right)$.

Yield: $77.0 \mathrm{mg}(0.144 \mathrm{mmol}, 72 \%)$.

Physical State: pale yellow oil.

$\mathbf{R}_{\mathbf{f}}$ Value: 0.23 (petroleum ether/EtOAc - 2/1).

${ }^{1} \mathbf{H}$ NMR (Avance $400 \mathrm{MHz}, \mathrm{CDCl}_{3}$ ) $\delta 7.83-7.85(\mathrm{~m}, 2 \mathrm{H}), 7.65-7.68(\mathrm{~m}, 1 \mathrm{H}), 7.55-7.58$ (m, 2H), $7.24-7.27(\mathrm{~m}, 6 \mathrm{H}), 7.12(\mathrm{~s}, 5 \mathrm{H}), 6.98-7.01(\mathrm{~m}, 2 \mathrm{H}), 6.94-6.96(\mathrm{~m}, 2 \mathrm{H}), 5.69(\mathrm{~d}$, $J=7.1 \mathrm{~Hz}, 1 \mathrm{H}), 4.11(\mathrm{~d}, J=13.2 \mathrm{~Hz}, 1 \mathrm{H}), 3.98(\mathrm{dd}, J=1.2,13.2 \mathrm{~Hz}, 1 \mathrm{H}), 3.61(\mathrm{dd}, J=7.5$, $9.7 \mathrm{~Hz}, 1 \mathrm{H}), 3.51(\mathrm{ddd}, J=7.3,7.3,7.3 \mathrm{~Hz}, 1 \mathrm{H}), 3.35(\mathrm{dd}, J=7.4,9.7 \mathrm{~Hz}, 1 \mathrm{H}), 1.80(\mathrm{~s}, 3 \mathrm{H})$ ppm

${ }^{13}$ C NMR (Avance $101 \mathrm{MHz}, \mathrm{CDCl}_{3}$ ) $\delta$ 198.3, 169.7, 138.2, 136.2, 136.2, 136.0, 133.2, $129.4,128.7,128.6,128.6,128.5,128.4,127.9,127.8,126.9,115.4,101.5,75.6,50.5,50.5$, 47.4, 20.9 ppm. 2 carbons were not observed due to incidental equivalence.

IR (ATR, in $\mathrm{CDCl}_{3}$ ) v 3059 (w), 3030 (w), 2933 (w), 1956 (w), 1739 (m), 1598 (w), 1492 (w), 1445 (w), 1371 (w), 1348 (m), 1309 (w), 1225 (s), 1165 (s), 1091 (m), 1073 (w), $1019(\mathrm{~m}) \mathrm{cm}^{-1}$.

HRMS (ESI, m/z) calcd. for $\mathrm{C}_{33} \mathrm{H}_{29} \mathrm{NO}_{4} \mathrm{SNa}^{+}$: 558.1710, found: 558.1713 .

\section{$5.21 \mathrm{D}_{3}$-(4-(2,2-diphenylvinylidene)-1-(phenylsulfonyl)pyrrolidin-3-yl)(phenyl)methyl} acetate $29-D_{3}$<smiles>CC(=O)OC(c1ccccc1)[C@H]1CN(S(=O)(=O)c2ccccc2)C/C1=C\c1ccccc1</smiles>

According to GP-4 35-D $3(107.7 \mathrm{mg}, 0.2 \mathrm{mmol})$ and $\left[\mathrm{Fe}(\mathrm{CO})(\mathrm{NO})\left(\mathrm{PPh}_{3}\right)_{2}\right]\left[\mathrm{BF}_{4}\right](14.5 \mathrm{mg}$, $0.02 \mathrm{mmol})$ were dissolved in $\mathrm{CH}_{2} \mathrm{Cl}_{2}(1 \mathrm{~mL})$ and stirred at $50{ }^{\circ} \mathrm{C}$ for 22 hours to yield the

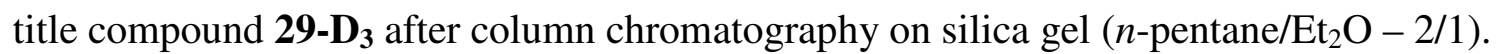

Yield: $75.6 \mathrm{mg}(0.140 \mathrm{mmol}, 70 \%)$.

Physical State: pale yellow oil. 
$\mathbf{R}_{\mathbf{f}}$ Value: 0.20 (petroleum ether/EtOAc - 2/1).

${ }^{1} \mathbf{H}$ NMR (Avance $400 \mathrm{MHz}, \mathrm{CDCl}_{3}$ ) $\delta 7.83$ - $7.85(\mathrm{~m}, 2 \mathrm{H}), 7.63-7.67(\mathrm{~m}, 1 \mathrm{H}), 7.53-7.57$ $(\mathrm{m}, 2 \mathrm{H}), 7.23-7.26(\mathrm{~m}, 6 \mathrm{H}), 7.11(\mathrm{~s}, 5 \mathrm{H}), 6.98-7.01(\mathrm{~m}, 2 \mathrm{H}), 6.94-6.96(\mathrm{~m}, 2 \mathrm{H}), 5.70(\mathrm{~d}$, $J=7.1 \mathrm{~Hz}, 1 \mathrm{H}), 4.11(\mathrm{~d}, J=13.2 \mathrm{~Hz}, 1 \mathrm{H}), 3.98(\mathrm{~d}, J=13.2 \mathrm{~Hz}, 1 \mathrm{H}), 3.61(\mathrm{dd}, J=7.7$, $9.4 \mathrm{~Hz}, 1 \mathrm{H}), 3.51$ (ddd, $J=7.3,7.3,7.3 \mathrm{~Hz}, 1 \mathrm{H}), 3.45$ (dd, $J=7.5,9.6 \mathrm{~Hz}, 1 \mathrm{H}), 1.76$ (s, $0.17 \mathrm{H}) \mathrm{ppm}$.

${ }^{13}$ C NMR (Avance $101 \mathrm{MHz}, \mathrm{CDCl}_{3}$ ) $\delta$ 198.2, 169.6, 138.1, 136.2, 136.1, 136.0, 133.2, $129.3,128.7,128.6,128.5,128.4,127.8,127.8,126.9,115.3,101.4,75.5,50.5,50.4,47.3$ ppm. 3 carbons were not observed due to incidental equivalence. $\mathrm{CD}_{3}$ was not observed.

IR (ATR, in $\left.\mathrm{CDCl}_{3}\right)$ v 3060 (w), 3030 (w), 2927 (w), 1951 (w), 1737 (m), 1598 (w), 1492 (w), 1445 (w), 1348 (m), 1309 (w), 1239 (m), 1165 (s), 1091 (m), 1071 (m), 1030 (m) cm c $^{-1}$

HRMS (ESI, m/z) calcd. for $\mathrm{C}_{33} \mathrm{H}_{26} \mathrm{D}_{3} \mathrm{NO}_{4} \mathrm{SNa}^{+}$: 561.1898, found: 561.1881.

\subsection{2-(4-(2-phenylvinylidene)-1-tosylpyrrolidin-3-yl)propan-2-yl acetate 30}

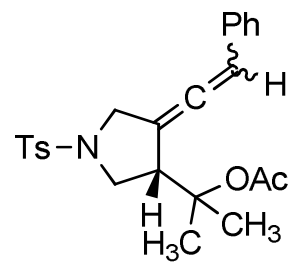

According to GP-5 $38(85.1 \mathrm{mg}, 0.2 \mathrm{mmol})$ was dissolved in $\mathrm{CH}_{2} \mathrm{Cl}_{2}(1 \mathrm{~mL})$, treated with $\left[\mathrm{Fe}(\mathrm{CO})(\mathrm{NO})\left(\mathrm{PPh}_{3}\right)_{2}\right]\left[\mathrm{BF}_{4}\right](14.5 \mathrm{mg}, 0.02 \mathrm{mmol})$ and stirred at $50{ }^{\circ} \mathrm{C}$ for 22 hours to yield the title compound 30 after column chromatography on silica gel (n-pentane/ $\left.\mathrm{Et}_{2} \mathrm{O}-2 / 1\right)$ as a 1.5:1 mixture of inseparable allene isomers.

Yield: $58.5 \mathrm{mg}(0.138 \mathrm{mmol}, 69 \%)$.

Phyiscal State: pale yellow oil.

$\mathbf{R}_{\mathbf{f}}$ Value: $0.31(n$-pentane/Et $2 \mathrm{O}-2 / 1)$.

${ }^{1} \mathbf{H}$ NMR (Avance $400 \mathrm{MHz}, \mathrm{CDCl}_{3}$ ) $\delta 7.72$ - $7.75(\mathrm{~m}, 4 \mathrm{H}), 7.36$ - $7.39(\mathrm{~m}, 4 \mathrm{H}), 7.13$ - 7.32 $(\mathrm{m}, 10 \mathrm{H}), 6.24-6.29(\mathrm{~m}, 2 \mathrm{H}), 3.82-4.06(\mathrm{~m}, 4 \mathrm{H}), 3.64-3.71(\mathrm{~m}, 2 \mathrm{H}), 3.30-3.50(\mathrm{~m}, 4 \mathrm{H})$, $2.47(\mathrm{~s}, 3.6 \mathrm{H}), 2.46(\mathrm{~s}, 2.4 \mathrm{H}), 1.83(\mathrm{~s}, 3.6 \mathrm{H}), 1.69(\mathrm{~s}, 2.4 \mathrm{H}), 1.44-1.50(\mathrm{~m}, 12 \mathrm{H}) \mathrm{ppm}$.

${ }^{13}$ C NMR (Avance $101 \mathrm{MHz}, \mathrm{CDCl}_{3}$ ) $\delta$ 199.4, 199.2, 170.6, 170.4, 144.1, 144.1, 133.8, $133.7,132.6,132.2,130.0,129.9,128.9,128.8,128.1$, 128.0, 127.7, 127.7, 127.2, 127.1, 
$102.7,102.5,98.9,98.5,83.3,83.2,50.8,50.8,49.9,49.8,49.5,49.3,24.1,23.9,23.7,23.3$, 22.4, 22.3, 21.7, $21.7 \mathrm{ppm}$.

IR (ATR, in $\mathrm{CDCl}_{3}$ ) v 2983 (w), 2938 (w), 1960 (w), 1736 (m), 1598 (w), 1495 (w), 1459 (w), 1387 (w), 1367 (w), 1345 (m), 1306 (w), 1289 (w), 1247 (m), 1162 (s), 1091 (m), $1016(\mathrm{~m}) \mathrm{cm}^{-1}$.

HRMS (ESI, m/z) calcd. for $\mathrm{C}_{24} \mathrm{H}_{27} \mathrm{NO}_{4} \mathrm{SNa}^{+}$: 448.1564, found: 448.1561.

\subsection{2-(4-(2,2-diphenylvinylidene)-1-tosylpyrrolidin-3-yl)propan-2-yl acetate 31}

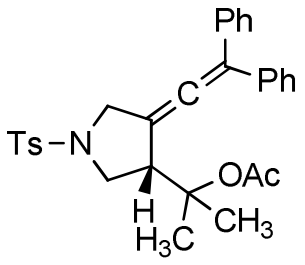

According to GP-4 s50 (100.3 mg, $0.2 \mathrm{mmol})$ and $\left[\mathrm{Fe}(\mathrm{CO})(\mathrm{NO})\left(\mathrm{PPh}_{3}\right)_{2}\right]\left[\mathrm{BF}_{4}\right](14.5 \mathrm{mg}$, $0.02 \mathrm{mmol})$ were dissolved in $\mathrm{CH}_{2} \mathrm{Cl}_{2}(1 \mathrm{~mL})$ and stirred at $50{ }^{\circ} \mathrm{C}$ for 22 hours to yield the title compound $\mathbf{3 1}$ after column chromatography on silica gel (petroleum ether/EtOAc - 5/1).

Yield: $30.8 \mathrm{mg}(0.061 \mathrm{mmol}, 31 \%)$.

Phyiscal State: pale yellow oil.

$\mathbf{R}_{\mathbf{f}}$ Value: 0.31 (petroleum ether/EtOAc - 5/1).

${ }^{1} \mathbf{H}$ NMR (Avance $400 \mathrm{MHz}, \mathrm{CDCl}_{3}$ ) $\delta 7.70$ - $7.72(\mathrm{~m}, 2 \mathrm{H}), 7.21$ - 7.34 (m, 12H), 4.09 (dd, $J=1.2,13.0 \mathrm{~Hz}, 1 \mathrm{H}), 3.93(\mathrm{~d}, J=13.0 \mathrm{~Hz}, 1 \mathrm{H}), 3.79-3.83(\mathrm{~m}, 1 \mathrm{H}), 3.32-3.39(\mathrm{~m}, 2 \mathrm{H})$, 2.45 (s, 3H), 1.70 (s, 3H), 1.41 (s, 3H), 1.38 (s, 3H) ppm.

${ }^{13}$ C NMR (Avance $101 \mathrm{MHz}, \mathrm{CDCl}_{3}$ ) $\delta$ 199.4, 170.6, 144.1, 136.3, 136.3, 132.3, 130.0, 128.7, 128.6, 128.5, 128.1, 127.9, 127.8, 113.9, 101.7, 83.3, 51.1, 50.0, 49.4, 23.9, 23.7, 22.3, 21.7 ppm. 1 carbon was not observed due to incidental equivalence.

IR (ATR, in $\mathrm{CDCl}_{3}$ ) v 3058 (w), 3027 (w), 2982 (w), 2939 (w), 1954 (w), 1728 (m), 1597 (w), 1492 (w), 1453 (w), 1367 (w), 1347 (m), 1248 (m), 1164 (s), 1092 (m), 1017 (m) $\mathrm{cm}^{-1}$.

HRMS (ESI, m/z) calcd. for $\mathrm{C}_{30} \mathrm{H}_{31} \mathrm{NO}_{4} \mathrm{SNa}^{+}$: 524.1866, found: 524.1846. 

32

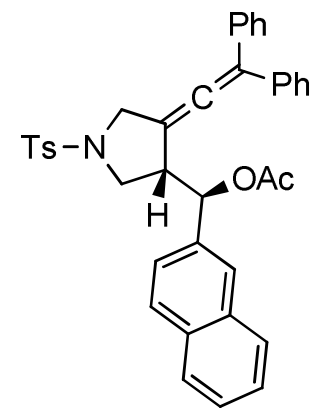

According to GP-4 s55 (119.9 mg, $0.2 \mathrm{mmol})$ and $\left[\mathrm{Fe}(\mathrm{CO})(\mathrm{NO})\left(\mathrm{PPh}_{3}\right)_{2}\right]\left[\mathrm{BF}_{4}\right](14.5 \mathrm{mg}$, $0.02 \mathrm{mmol})$ were dissolved in $\mathrm{CH}_{2} \mathrm{Cl}_{2}(1 \mathrm{~mL})$ and stirred at $50{ }^{\circ} \mathrm{C}$ for 22 hours to yield the title compound 32 after column chromatography on silica gel (petroleum ether/EtOAc - 5/1).

Yield: $39.4 \mathrm{mg}(0.066 \mathrm{mmol}, 33 \%)$.

Phyiscal State: pale yellow oil.

$\mathbf{R}_{\mathbf{f}}$ Value: 0.24 (petroleum ether/EtOAc - 5/1).

${ }^{1} \mathbf{H}$ NMR (Avance $400 \mathrm{MHz}, \mathrm{CDCl}_{3}$ ) $\delta 7.67$ - $7.73(\mathrm{~m}, 3 \mathrm{H}), 7.59-7.63(\mathrm{~m}, 2 \mathrm{H}), 7.53$ - 7.55 $(\mathrm{m}, 1 \mathrm{H}), 7.36-7.42(\mathrm{~m}, 2 \mathrm{H}), 7.32-7.35(\mathrm{~m}, 2 \mathrm{H}), 7.27-7.30(\mathrm{~m}, 1 \mathrm{H}), 7.08-7.19(\mathrm{~m}, 6 \mathrm{H})$, $6.80-6.83(\mathrm{~m}, 4 \mathrm{H}), 5.84-5.88(\mathrm{~m}, 1 \mathrm{H}), 4.09(\mathrm{~d}, J=13.2 \mathrm{~Hz}, 1 \mathrm{H}), 3.99(\mathrm{dd}, J=0.7$, $13.1 \mathrm{~Hz}, 1 \mathrm{H}), 3.60-3.68(\mathrm{~m}, 2 \mathrm{H}), 3.36-3.42(\mathrm{~m}, 1 \mathrm{H}), 2.47$ (s, 3H), 1.86 (s, 3H) ppm.

${ }^{13}$ C NMR (Avance $101 \mathrm{MHz}, \mathrm{CDCl}_{3}$ ) $\delta$ 198.4, 169.7, 144.0, 136.1, 135.9, 135.4, 133.3, $133.0,133.0,130.0,128.5,128.4,128.3,128.2$, 128.0, 127.7, 127.7, 127.7, 127.0, 126.3, $126.3,124.4,115.1,101.4,76.0,50.8,50.5,47.1,21.8,21.0$ ppm. 2 carbons were not observed due to incidental equivalence.

IR (ATR, in $\mathrm{CDCl}_{3}$ ) v 3056 (w), 2923 (w), 2851 (w), 1952 (w), 1741 (m), 1598 (w), 1509 (w), 1492 (w), 1453 (w), 1443 (w), 1346 (m), 1305 (w), 1225 (s), 1162 (s), 1125 (w), $1091(\mathrm{~m}), 1016(\mathrm{~m}) \mathrm{cm}^{-1}$.

HRMS (ESI, m/z) calcd. for $\mathrm{C}_{38} \mathrm{H}_{33} \mathrm{NO}_{4} \mathrm{SNa}^{+}:$622.2023, found: 622.2049 . 
5.25 (4-(2-methylprop-1-en-1-ylidene)-1-tosylpyrrolidin-3-yl)(phenyl)methyl benzoate 33<smiles>CC(C)=C=C1CN([As])C[C@]1(c1ccccc1)[C@H](OC(=O)c1ccccc1)c1ccccc1</smiles>

According to GP-4 s56 $(97.5 \mathrm{mg}, 0.2 \mathrm{mmol})$ and $\left[\mathrm{Fe}(\mathrm{CO})(\mathrm{NO})\left(\mathrm{PPh}_{3}\right)_{2}\right]\left[\mathrm{BF}_{4}\right](14.5 \mathrm{mg}$, $0.02 \mathrm{mmol})$ were dissolved in $\mathrm{CH}_{2} \mathrm{Cl}_{2}(1 \mathrm{~mL})$ and stirred at $50{ }^{\circ} \mathrm{C}$ for 22 hours to yield the title compound 33 after column chromatography on silica gel (n-pentane/ $\mathrm{Et}_{2} \mathrm{O}-2 / 1$ ).

Yield: $60.1 \mathrm{mg}(0.123 \mathrm{mmol}, 62 \%)$.

Physical State: white solid.

$\mathbf{R}_{\mathbf{f}}$ Value: $0.34(n$-pentane/Et $2 \mathrm{O}-2 / 1)$.

${ }^{1} \mathbf{H}$ NMR (Avance $300 \mathrm{MHz}, \mathrm{CDCl}_{3}$ ) $\delta 7.92$ - $7.94(\mathrm{~m}, 2 \mathrm{H}), 7.70-7.73(\mathrm{~m}, 2 \mathrm{H}), 7.53-7.59$ (m, 1H), $7.23-7.43(\mathrm{~m}, 9 \mathrm{H}), 5.92(\mathrm{~d}, J=5.7 \mathrm{~Hz}, 1 \mathrm{H}), 3.88(\mathrm{~d}, J=12.7 \mathrm{~Hz}, 1 \mathrm{H}), 3.69$ (dd, $J=0.8,12.6 \mathrm{~Hz}, 1 \mathrm{H}), 3.46-3.54(\mathrm{~m}, 1 \mathrm{H}), 3.31-3.42(\mathrm{~m}, 2 \mathrm{H}), 2.46(\mathrm{~s}, 3 \mathrm{H}), 1.48(\mathrm{~s}, 3 \mathrm{H})$, $1.32(\mathrm{~s}, 3 \mathrm{H}) \mathrm{ppm}$.

${ }^{13}$ C NMR (Avance $75 \mathrm{MHz}, \mathrm{CDCl}_{3}$ ) $\delta$ 194.7, 165.3, 143.8, 138.7, 133.3, 132.7, 130.0, 129.8, 129.7, 128.6, 128.6, 128.2, 128.1, 126.7, 102.3, 96.4, 76.1, 50.3, 49.8, 46.7, 21.7, 20.6, 20.4 ppm.

IR (ATR, in $\mathrm{CDCl}_{3}$ ) v 3033 (w), 2982 (w), 2910 (w), 1722 (s), 1599 (w), 1451 (m), 1347 (s), 1268 (s), 1166 (s), 1109 (s), 1094 (s), 1069 (m), 1026 (m) cm . $^{-1}$

HRMS (ESI, m/z) calcd. for $\mathrm{C}_{29} \mathrm{H}_{29} \mathrm{NO}_{4} \mathrm{SNa}^{+}$: 510.1710, found: 510.1739. 


\section{Control experiments}

\section{1 (E)-1-(N-cinnamyl-4-methylphenylsulfonamido)-4-methylpenta-2,3-dien-2-yl}

acetate 36

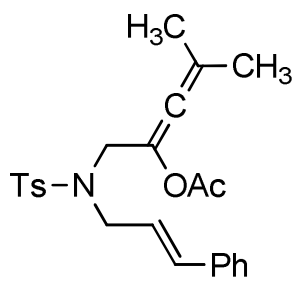

According to GP-4 $9(85.1 \mathrm{mg}, 0.2 \mathrm{mmol})$ and $\mathrm{PtCl}_{2}(5.3 \mathrm{mg}, 0.02 \mathrm{mmol})$ were dissolved in $\mathrm{CH}_{2} \mathrm{Cl}_{2}(1 \mathrm{~mL})$ and stirred at $50{ }^{\circ} \mathrm{C}$ for 22 hours to yield the title compound $\mathbf{3 6}$ as an inseperable mixture with its starting material after column chromatography on silica gel (petroleum ether/EtOAc - 5/1).

Yield: $66.8 \mathrm{mg}(0.157 \mathrm{mmol}, 78 \%)$.

Phyiscal State: yellow oil.

$\mathbf{R}_{\mathbf{f}}$ Value: 0.37 (petroleum ether/EtOAc $-5 / 1$ ).

${ }^{1} \mathbf{H}$ NMR (Avance $\left.400 \mathrm{MHz}, \mathrm{CDCl}_{3}\right) \delta 7.72-7.74(\mathrm{~m}, 2 \mathrm{H}), 7.22-7.36(\mathrm{~m}, 7 \mathrm{H}), 6.42(\mathrm{~d}$, $J=15.8 \mathrm{~Hz}, 1 \mathrm{H}), 5.91(\mathrm{dt}, J=6.9,15.7 \mathrm{~Hz}, 1 \mathrm{H}), 4.07(\mathrm{~d}, J=6.8 \mathrm{~Hz}, 2 \mathrm{H}), 3.99(\mathrm{~s}, 2 \mathrm{H}), 2.41$ (s, 3H), 2.03 (s, 3H), 1.78 (s, 6H) ppm.

${ }^{13}$ C NMR (Avance $101 \mathrm{MHz}, \mathrm{CDCl}_{3}$ ) $\delta$ 193.3, 168.9, 143.2, 138.0, 136.2, 134.3, 129.7, 128.6, 127.9, 127.2, 126.4, 123.4, 114.3, 109.4, 48.6, 47.6, 21.5, 21.3, 21.0 ppm. 1 carbon was not observed due to incidental equivalence.

6.2 2-methyl-1-(6-phenyl-3-tosyl-3-azabicyclo[3.1.0]hexan-1-yl)prop-1-en-1-yl acetate 37<smiles>CC(=O)OC(=C(C)C)[C@@]1(c2ccccc2)CN([Tl])C[C@H]1c1ccccc1</smiles> 
According to GP-4 9 (85.1 mg, $0.2 \mathrm{mmol})$ and Au-catalyst 39 (15.4 mg, $0.02 \mathrm{mmol})$ were dissolved in $\mathrm{CH}_{2} \mathrm{Cl}_{2}(1 \mathrm{~mL})$ and stirred at $50{ }^{\circ} \mathrm{C}$ for 22 hours to yield the title compound 37 after column chromatography on silica gel (petroleum ether/EtOAc - 5/1).

Yield: $85.1 \mathrm{mg}(0.2 \mathrm{mmol}$, quant.).

Phyiscal State: white solid.

Melting Point: $146{ }^{\circ} \mathrm{C}$.

$\mathbf{R}_{\mathbf{f}}$ Value: 0.26 (petroleum ether/EtOAc - 5/1).

${ }^{1} \mathbf{H}$ NMR (Avance $400 \mathrm{MHz}, \mathrm{CDCl}_{3}$ ) $\delta 7.71$ - $7.74(\mathrm{~m}, 2 \mathrm{H}), 7.35$ - $7.37(\mathrm{~m}, 2 \mathrm{H}), 7.12$ - 7.22 $(\mathrm{m}, 3 \mathrm{H}), 6.91-6.92(\mathrm{~m}, 2 \mathrm{H}), 3.88(\mathrm{~d}, J=9.5 \mathrm{~Hz}, 1 \mathrm{H}), 3.71(\mathrm{~d}, J=9.4 \mathrm{~Hz}, 1 \mathrm{H}), 3.54(\mathrm{~d}$, $J=9.4 \mathrm{~Hz}, 1 \mathrm{H}), 3.21(\mathrm{dd}, J=3.7,9.4 \mathrm{~Hz}, 1 \mathrm{H}), 2.45(\mathrm{~s}, 3 \mathrm{H}), 2.28(\mathrm{~d}, J=4.4 \mathrm{~Hz}, 1 \mathrm{H}), 1.97(\mathrm{~s}$, $3 \mathrm{H}), 1.90$ (t, $J=4.1 \mathrm{~Hz}, 1 \mathrm{H}), 1.37$ (s, 3H), 1.33 (s, 3H) ppm.

${ }^{13}$ C NMR (Avance $101 \mathrm{MHz}, \mathrm{CDCl}_{3}$ ) $\delta$ 169.3, 143.6, 137.3, 136.4, 133.7, 129.8, 127.8, $127.6,127.0,126.7,126.1,54.3,50.2,37.6,32.5,29.5,21.7,20.5,18.5,17.7$ ppm.

IR (ATR, in $\mathrm{CDCl}_{3}$ ) v 3033 (w), 2920 (w), 2860 (w), 1744 (m), 1599 (w), 1498 (w), 1455 (w), 1369 (w), 1345 (m), 1306 (w), 1290 (w), 1264 (w), 1214 (s), 1161 (s), 1102 (s), 1087 (m), $1068(\mathrm{w}), 1029(\mathrm{~m}), 1015(\mathrm{w}) \mathrm{cm}^{-1}$.

HRMS (ESI, m/z) calcd. for $\mathrm{C}_{24} \mathrm{H}_{27} \mathrm{NO}_{4} \mathrm{SNa}^{+}$: 448.1553, found: 448.1554 .

X-Ray: X-Ray structure of $\mathbf{3 7}$ is deposited at Cambridge Crystallographic Data Centre (www.ccdc.cam.ac.uk) under CCDC 1440740.

\subsection{Chiral separation of $\mathrm{s5}$}

The racemic enyne alcohol s5 was separated via semi-preparative chiral HPLC into its enantiomers (-)-s5 and (+)-s5.

Column: Grom Chiralpak OD-CSP 250x20 mm.

Solvent: $n$-heptane/i-propanol - 70/30.

Flow: $10 \mathrm{~mL} / \mathrm{min}$. 


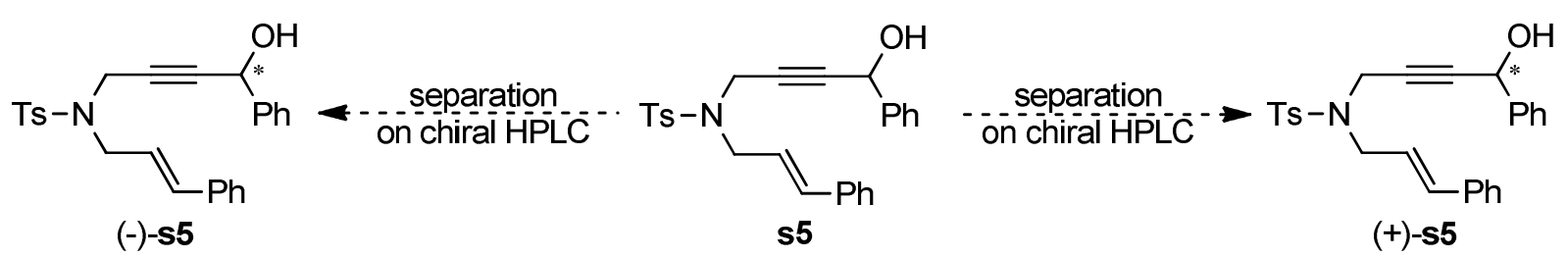

$0.68 \mathrm{~g}, 1.56 \mathrm{mmol}$ ee $>99 \%$

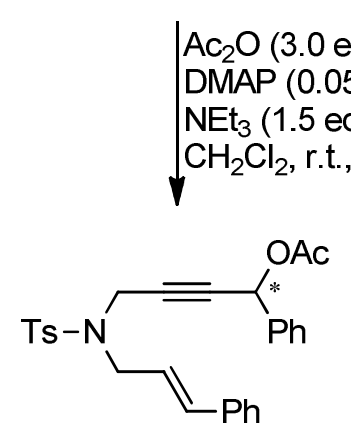

$(-)-40$

$99 \%$ yield ee $>99 \%$

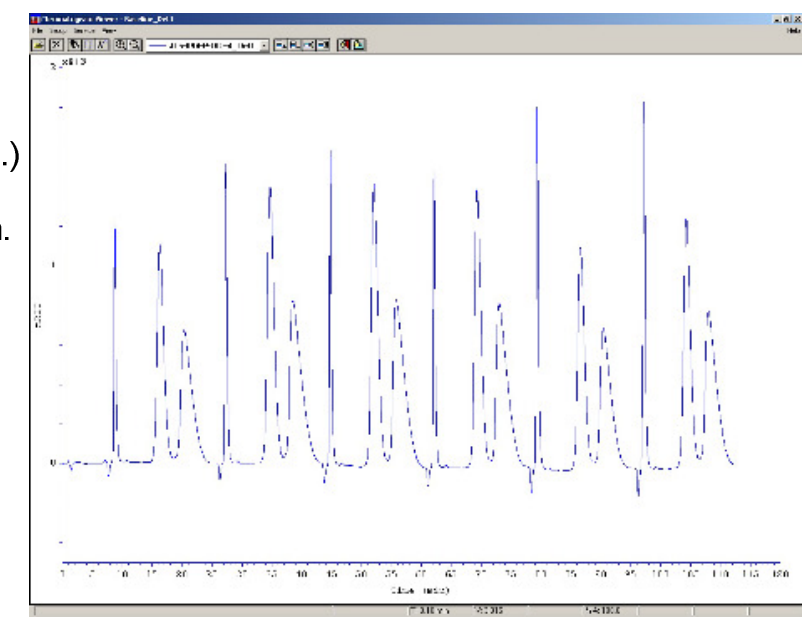

$0.65 \mathrm{~g}, 1.50 \mathrm{mmol}$ ee $>99 \%$

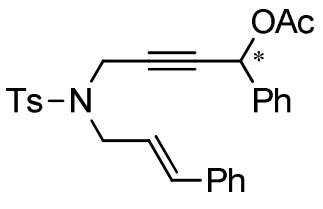

$(+)-40$

quant. yield ee $>99 \%$

\section{4 (-)- $N$-cinnamyl- $N$-(4-hydroxy-4-phenylbut-2-yn-1-yl)-4-methylbenzenesulfon-} amide (-)-s5

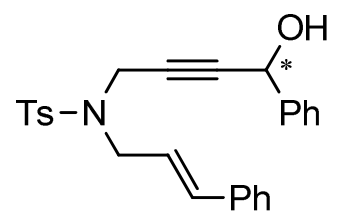

Yield: $0.68 \mathrm{~g}(1.56 \mathrm{mmol})$.

ee $>99 \%$.

$[\boldsymbol{\alpha}]^{25}{ }_{\mathbf{D}}=-22.8\left(\mathrm{c}=10 \mathrm{mg} / \mathrm{mL}\right.$ in $\left.\mathrm{CH}_{2} \mathrm{Cl}_{2}\right)$.

For further analytical data see achiral substances above.

For chiral HPLC chromatograms see below.

$6.5(-)-(E)-4-(N-$ cinnamyl-4-methylphenylsulfonamido)-1-phenylbut-2-yn-1-yl acetate $(-)-40$

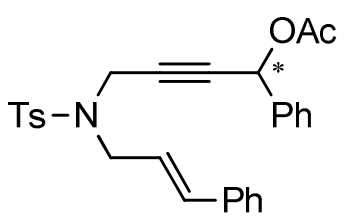


According to GP-2 (-)-s5 (0.68 g, $1.56 \mathrm{mmol})$ was dissolved in $\mathrm{CH}_{2} \mathrm{Cl}_{2}(2 \mathrm{~mL})$, treated with acetic anhydride $(0.44 \mathrm{~mL}, 4.69 \mathrm{mmol})$, DMAP $(9.6 \mathrm{mg}, 0.08 \mathrm{mmol})$ and triethylamine $(0.33 \mathrm{~mL}, 2.35 \mathrm{mmol})$ to yield (-)-40 after column chromatography on silica gel (petroleum ether/EtOAc - 4/1).

Yield: $0.73 \mathrm{~g}(1.54 \mathrm{mmol}, 99 \%)$.

ee $>99 \%$.

$[\boldsymbol{\alpha}]^{25}=-23.1\left(\mathrm{c}=10 \mathrm{mg} / \mathrm{mL}\right.$ in $\left.\mathrm{CH}_{2} \mathrm{Cl}_{2}\right)$.

For further analytical data see achiral substances above.

For chiral HPLC chromatograms see below.

\section{6 (+)- $N$-cinnamyl- $N$-(4-hydroxy-4-phenylbut-2-yn-1-yl)-4-methylbenzenesulfon-} amide (+)-s5

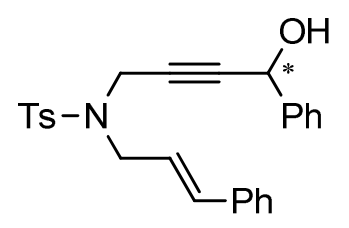

Yield: $0.65 \mathrm{~g}(1.50 \mathrm{mmol})$.

ee $>99 \%$.

$[\boldsymbol{\alpha}]^{25}=+21.7\left(\mathrm{c}=10 \mathrm{mg} / \mathrm{mL}\right.$ in $\left.\mathrm{CH}_{2} \mathrm{Cl}_{2}\right)$.

For further analytical data see achiral substances above.

For chiral HPLC chromatograms see below.

$6.7(+)-(E)-4-(N$-cinnamyl-4-methylphenylsulfonamido)-1-phenylbut-2-yn-1-yl acetate $(+)-40$

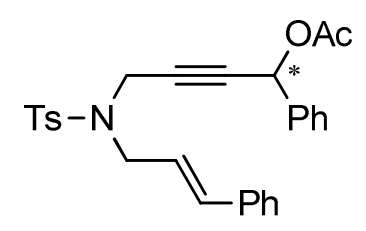

According to GP-2 (+)-s5 (0.65 g, $1.50 \mathrm{mmol})$ was dissolved in $\mathrm{CH}_{2} \mathrm{Cl}_{2}(2 \mathrm{~mL})$, treated with acetic anhydride $(0.43 \mathrm{~mL}, 4.5 \mathrm{mmol})$, DMAP $(9.2 \mathrm{mg}, 0.08 \mathrm{mmol})$ and triethylamine 
$(0.31 \mathrm{~mL}, 2.25 \mathrm{mmol})$ to yield (+)-40 after column chromatography on silica gel (petroleum ether/EtOAc - 4/1).

Yield: $0.71 \mathrm{~g}$ (1.50 mmol, quant.).

ee $>99 \%$.

$[\boldsymbol{\alpha}]^{25}=+24.3\left(\mathrm{c}=10 \mathrm{mg} / \mathrm{mL}\right.$ in $\left.\mathrm{CH}_{2} \mathrm{Cl}_{2}\right)$.

For further analytical data see achiral substances above.

For chiral HPLC chromatograms see below.

\section{8 phenyl(4-(2-phenylvinylidene)-1-tosylpyrrolidin-3-yl)methyl acetate 11}<smiles>CC(=O)O[C@H](c1ccccc1)[C@H]1CN([Hg])C/C1=C\c1ccccc1</smiles>

According to GP-5 (-)-40 or (+)-40 (94.7 mg, $0.2 \mathrm{mmol})$ was dissolved in $\mathrm{CH}_{2} \mathrm{Cl}_{2}(1 \mathrm{~mL})$, treated with $\left[\mathrm{Fe}(\mathrm{CO})(\mathrm{NO})\left(\mathrm{PPh}_{3}\right)_{2}\right]\left[\mathrm{BF}_{4}\right](14.5 \mathrm{mg}, 0.02 \mathrm{mmol})$ and stirred at $50{ }^{\circ} \mathrm{C}$ for 22 hours to yield the title compound $\mathbf{1 1}$ after column chromatography on silica gel (petroleum ether/EtOAc - 4/1) as a mixture of inseparable allene isomers.

Yield: $91.5 \mathrm{mg}$ (0.193 mmol, 97\%) from (-)-40 or $92.8 \mathrm{mg}(0.196 \mathrm{mmol}, \mathbf{9 8 \%})$ from (+)-40.

For further analytical data see above.

For chiral HPLC chromatograms see below.

\section{9 (Z)-2-phenyl-1-(6-phenyl-3-tosyl-3-azabicyclo[3.1.0]hexan-1-yl)vinyl acetate 41}<smiles>[3H]N1C[C@@H](c2ccccc2)C(/C(=C/c2ccccc2)OC(C)=O)(c2ccccc2)C1</smiles>

According to GP-5 (-)-40 or (+)-40 (94.7 mg, $0.2 \mathrm{mmol})$ was dissolved in $\mathrm{CH}_{2} \mathrm{Cl}_{2}(1 \mathrm{~mL})$, treated with $\mathrm{Au}^{\mathrm{I}}$-catalyst $39(15.4 \mathrm{mg}, 0.02 \mathrm{mmol})$ and stirred at $50{ }^{\circ} \mathrm{C}$ for 22 hours to yield 
the title compound $\mathbf{4 1}$ after column chromatography on silica gel (petroleum ether/EtOAc 4/1).

Yield: $54.4 \mathrm{mg}(0.115 \mathrm{mmol}, 57 \%)$ from (-)-40 or $52.9 \mathrm{mg}(0.112 \mathrm{mmol}, 56 \%)$ from (+)-40.

Phyiscal State: pale yellow oil.

$\mathbf{R}_{\mathbf{f}}$ Value: 0.30 (petroleum ether/EtOAc - 4/1).

${ }^{1} \mathbf{H}$ NMR (Avance $400 \mathrm{MHz}, \mathrm{CDCl}_{3}$ ) $\delta 7.73$ - 7.75 (m, 2H), 7.35 - 7.37 (m, 2H), 7.14 - 7.25 (m, 6H), $7.09-7.11(\mathrm{~m}, 2 \mathrm{H}), 7.04-7.06(\mathrm{~m}, 2 \mathrm{H}), 5.78(\mathrm{~s}, 1 \mathrm{H}), 3.91(\mathrm{~d}, J=9.5 \mathrm{~Hz}, 1 \mathrm{H}), 3.77$ (d, $J=9.4 \mathrm{~Hz}, 1 \mathrm{H}), 3.49$ (d, $J=9.5 \mathrm{~Hz}, 1 \mathrm{H}), 3.30(\mathrm{dd}, J=3.8,9.4 \mathrm{~Hz}, 1 \mathrm{H}), 2.45$ (s, 3H), 2.41 (d, $J=4.6 \mathrm{~Hz}, 1 \mathrm{H}), 2.19$ (dd, $J=4.2,4.2 \mathrm{~Hz}, 1 \mathrm{H}), 1.92$ (s, 3H) ppm.

${ }^{13} \mathrm{C}$ NMR (Avance $101 \mathrm{MHz}, \mathrm{CDCl}_{3}$ ) $\delta$ 168.5, 143.8, 143.5, 136.4, 133.8, 133.6, 129.9, 128.4 , 128.3, 128.2, 128.2, 127.7, 127.6, 126.5, 121.4, 54.3, 50.2, 39.4, 31.6, 29.4, 21.7, 20.8 ppm.

IR (ATR, in $\left.\mathrm{CDCl}_{3}\right)$ v 3030 (w), 2863 (w), 1754 (m), 1670 (w), 1599 (w), 1496 (w), 1476 (w), 1448 (w), 1344 (m), 1305 (w), 1290 (w), 1200 (m), 1161 (s), 1102 (m), 1057 (w), $1028(\mathrm{~m}), 1015(\mathrm{~m}) \mathrm{cm}^{-1}$.

HRMS (ESI, m/z) calcd. for $\mathrm{C}_{28} \mathrm{H}_{27} \mathrm{NO}_{4} \mathrm{SNa}^{+}$: 496.1553, found: 496.1562.

For chiral HPLC chromatograms see below. 


\section{References}

[1] Belger, C.; Plietker, B. Chem. Commun. 2012, 48, 5419-5421.

[2] Roustan, J. L. A.; Merour, J. Y.; Forgues, A. J. Organomet. Chem. 1980, 186, C23-C26.

[3] Cambeiro, F.; López, S.; Valera, J. A.; Saá, C. Angew. Chem. Int. Ed. 2014, 53, 59595963.

[4] Dieltiens, N.; Moonens, K.; Stevens, C. V. Chem. Eur. J. 2007, 13, 203-214.

[5] Pawliczek, M.; Jones, P. G.; Werz, D. B. Eur. J. Org. Chem. 2015, 2015, 6278-6288.

[6] Zhang, Z.; Shi, Z. M. Eur. J. Org. Chem. 2011, 2011, 2610-2614.

[7] Schabel, T.; Belger, C.; Plietker, B. Org. Lett. 2013, 15, 2858-2861.

[8] Pawliczek, M.; Schneider, T. F.; Maßß, C.; Stalke, D.; Werz, D. B. Angew. Chem. Int. Ed. 2015, 54, 4119-4123.

[9] Cao, W.; Yu, B. Adv. Synth. Catal. 2011, 353, 1903-1907.

[10] Harmata, M.; Zheng, P.; Huang, C.; Gomes, M. G.; Ying, W.; Ranyanil, K.-O.; Balan, G.; Calkins, N. L. J. Org. Chem. 2007, 72, 683-685.

[11] Teller, H.; Corbet, M.; Mantilli, L.; Gopakumar, G.; Goddard, R.; Thiel, W.; Fürstner, A. J. Am. Chem. Soc. 2012, 134, 15331-15342.

[12] Bouziane, A.; Hélou, M.; Carboni, B.; Carreaux, F.; Demerseman, B.; Bruneau, C.; Renaud, J. L. Chem. Eur. J. 2008, 14, 5630-5637.

[13] Reichl, K. D.; Dunn, N. L.; Fastuca, N. J.; Radosevich, A. T. J. Am. Chem. Soc. 2015, $137,5292-5295$.

[14] Dieckmann, M.; Jang, Y.-S.; Cramer, N. Angew. Chem. Int. Ed. 2015, 54, 12149-12152. 


\section{Copies of EPR, ${ }^{1} \mathrm{H}$ and ${ }^{13} \mathrm{C}$ NMR}

EPR spectrum of $\mathbf{6}$

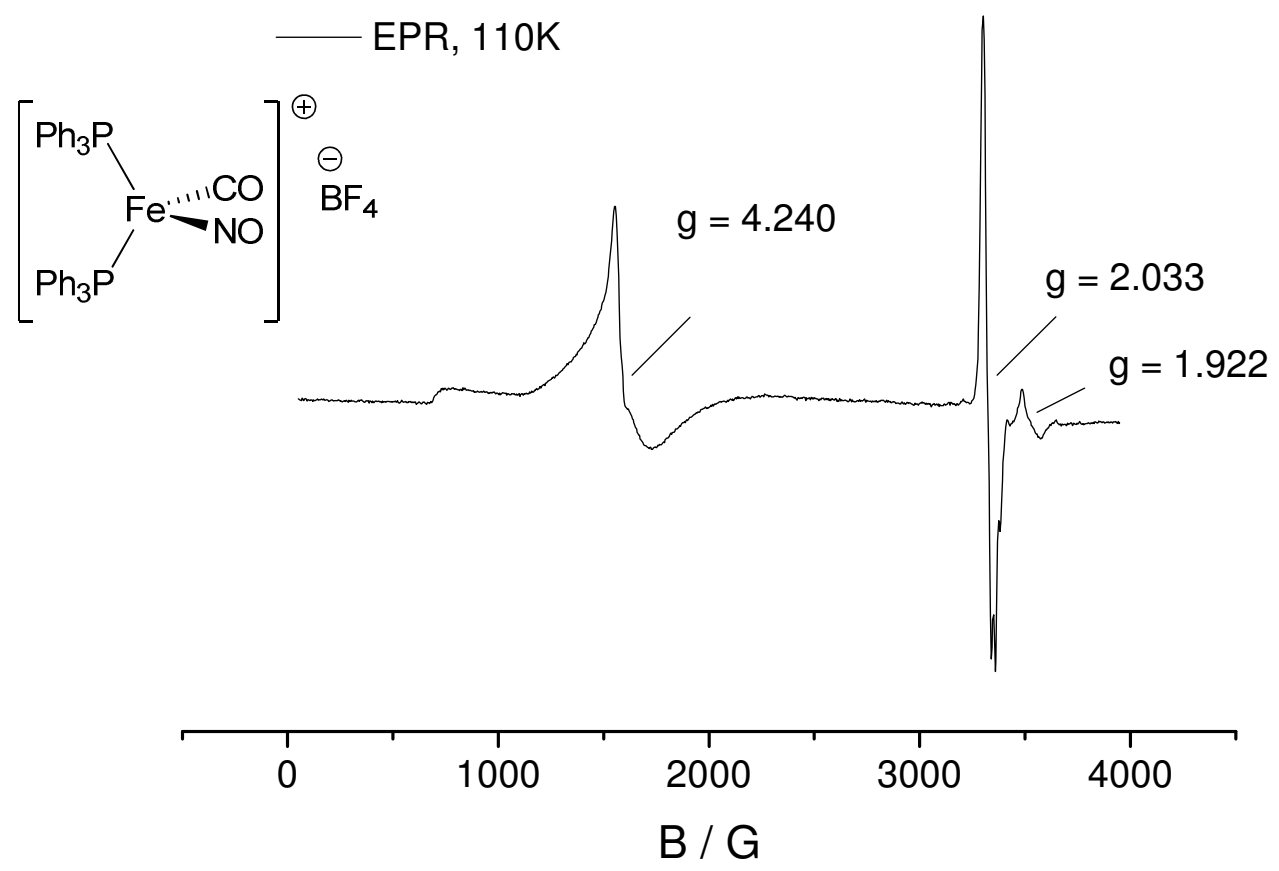


${ }^{1} \mathrm{H}-$ and ${ }^{13} \mathrm{C}$ NMR spectra of 9

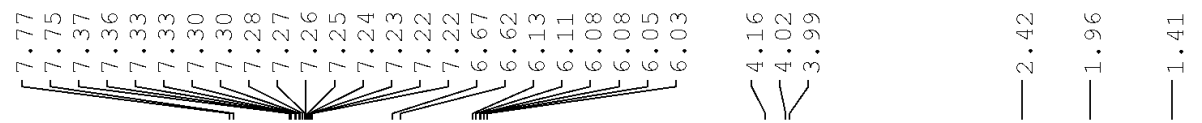<smiles>CC(=O)OC(C)(C)[C@@H](C)/C=C/CN([13CH3])C/C=C\c1ccccc1</smiles>
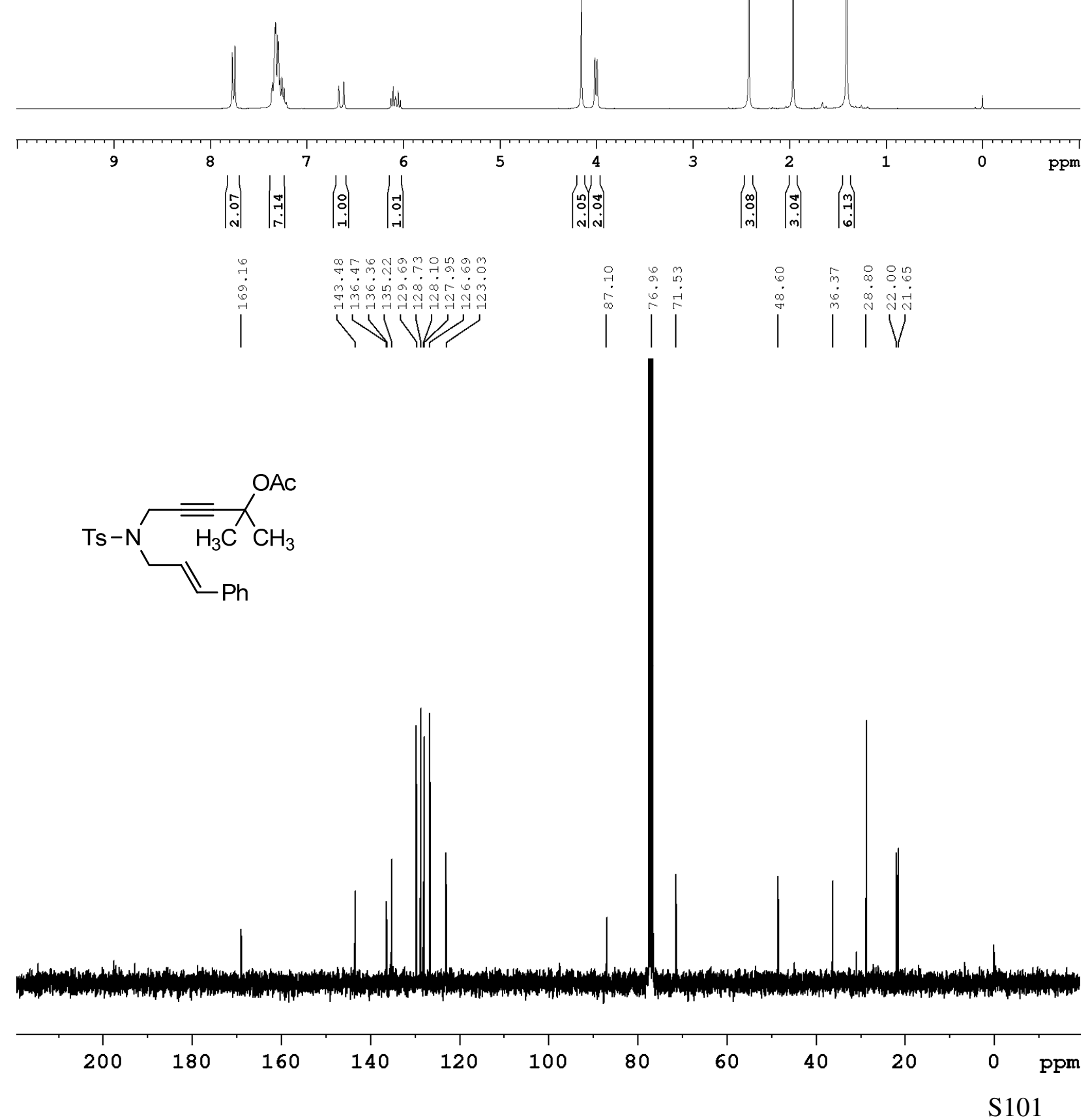
${ }^{1} \mathrm{H}$ - and ${ }^{13} \mathrm{C}$ NMR spectra of $\mathbf{4 0}$
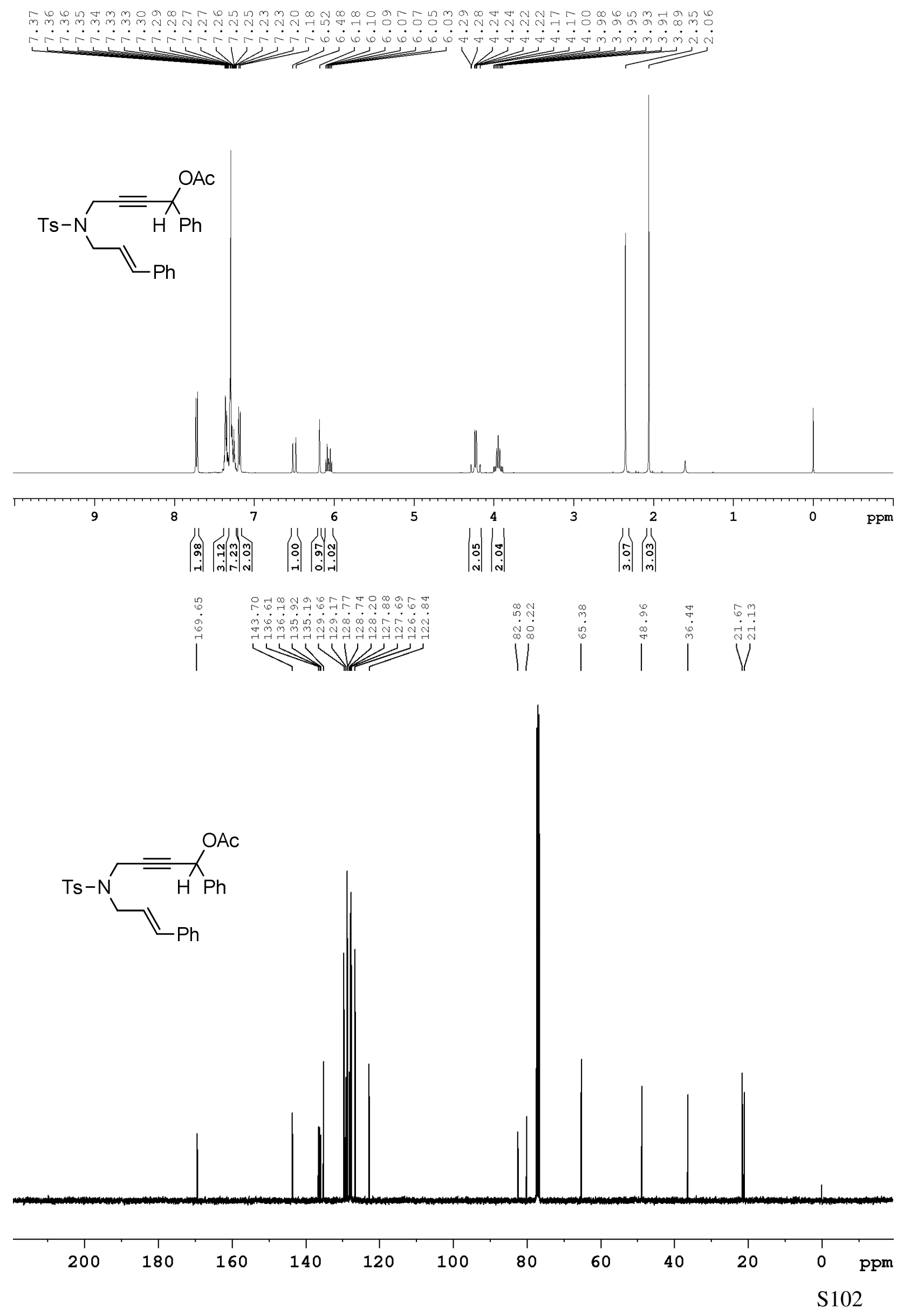


\section{${ }^{1} \mathrm{H}$ - and ${ }^{13} \mathrm{C}$ NMR spectra of $\mathbf{s} 7$}
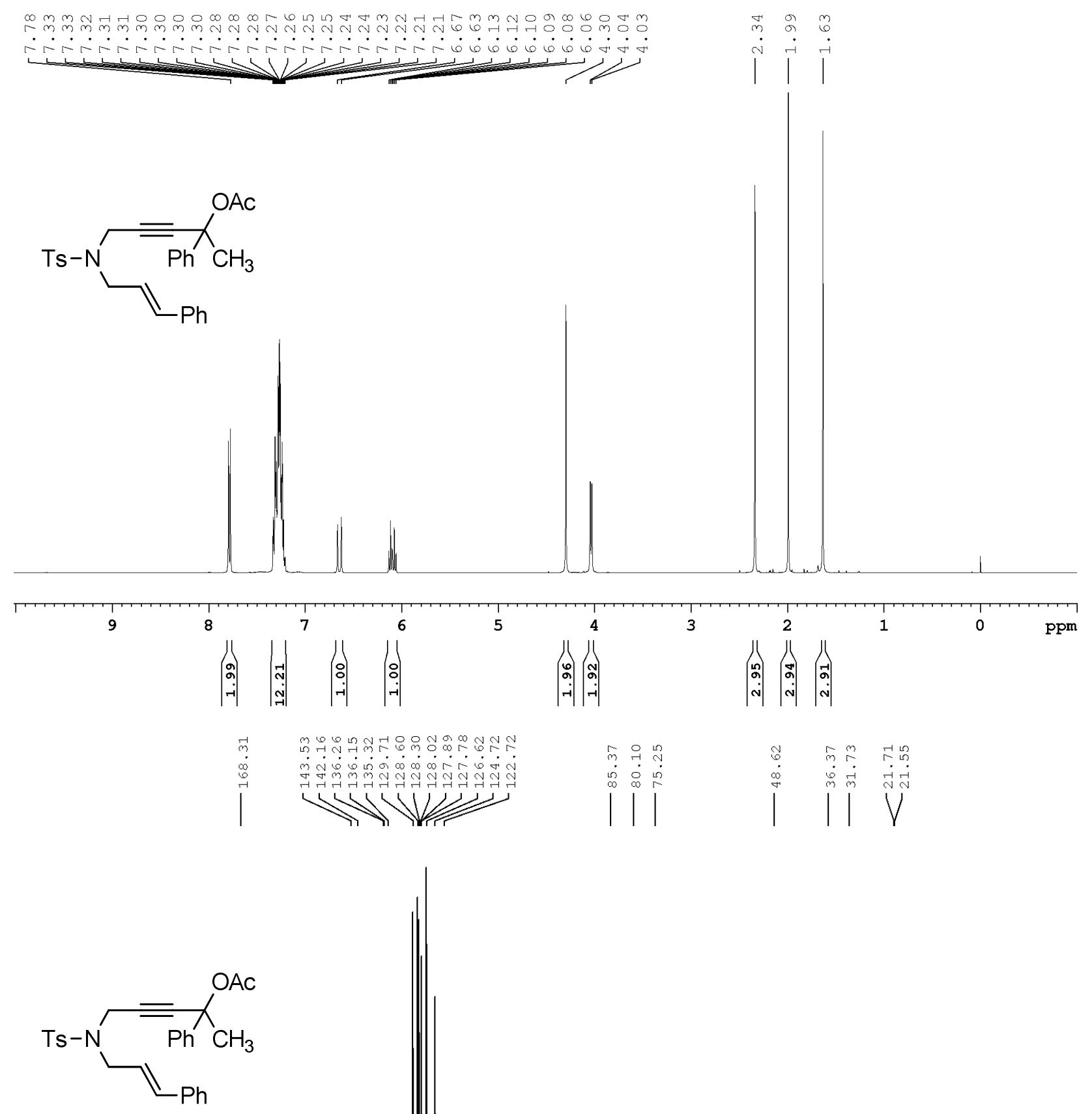
${ }^{1} \mathrm{H}$ - and ${ }^{13} \mathrm{C}$ NMR spectra of $\mathbf{3 4}$
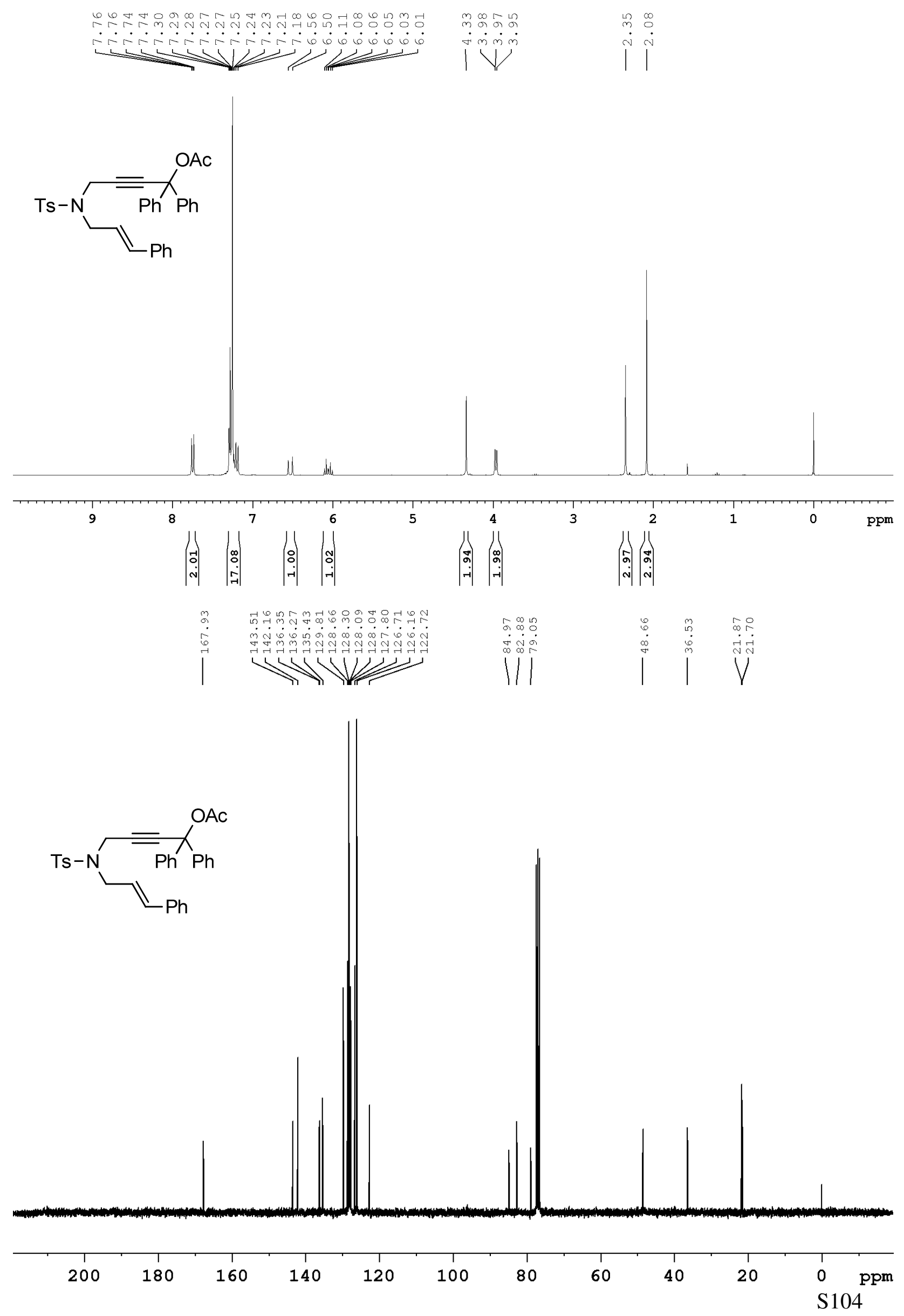
${ }^{1} \mathrm{H}$ - and ${ }^{13} \mathrm{C}$ NMR spectra of $\mathbf{s 1 0}$
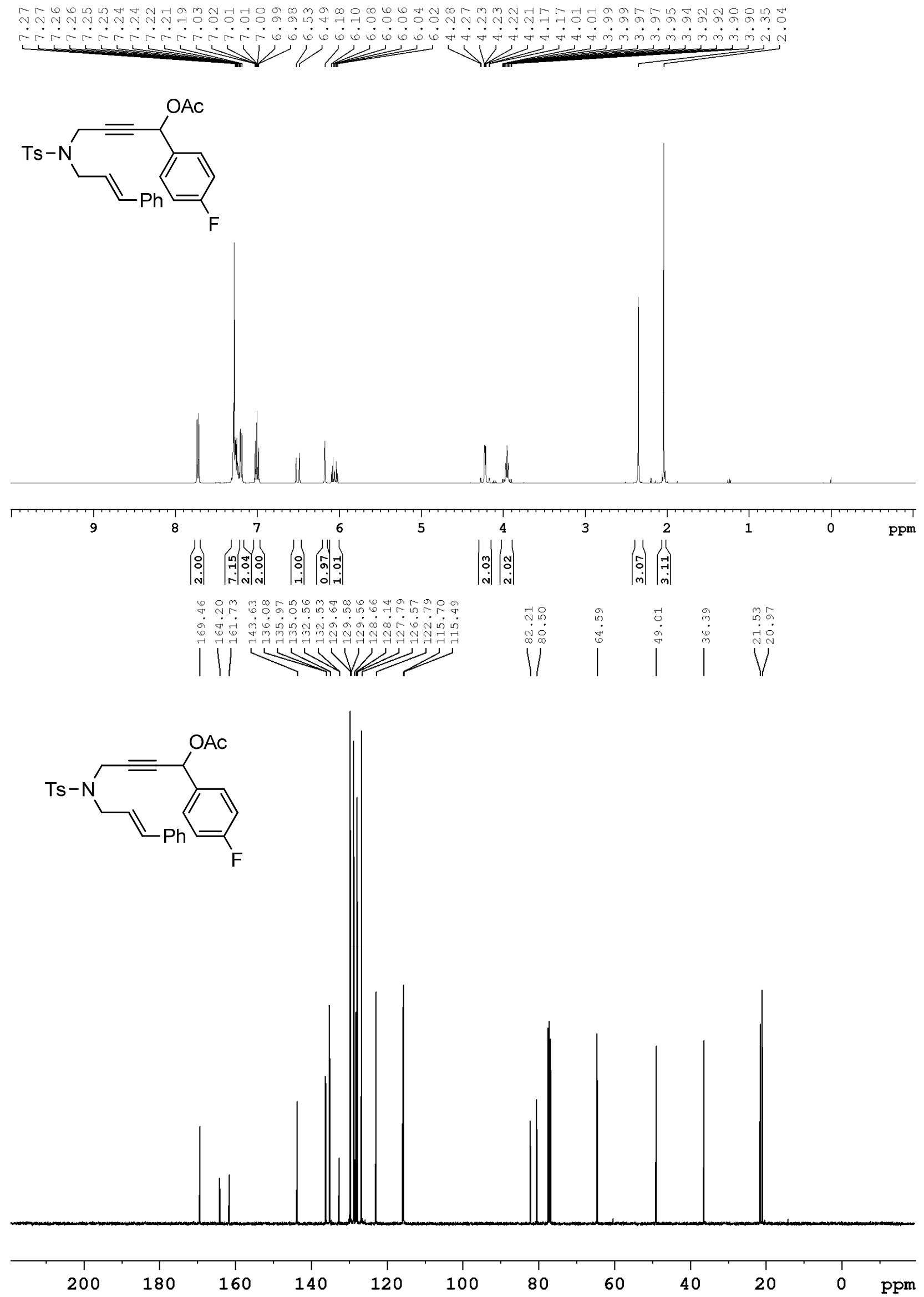
${ }^{1} \mathrm{H}$ - and ${ }^{13} \mathrm{C}$ NMR spectra of $\mathbf{s 1 2}$
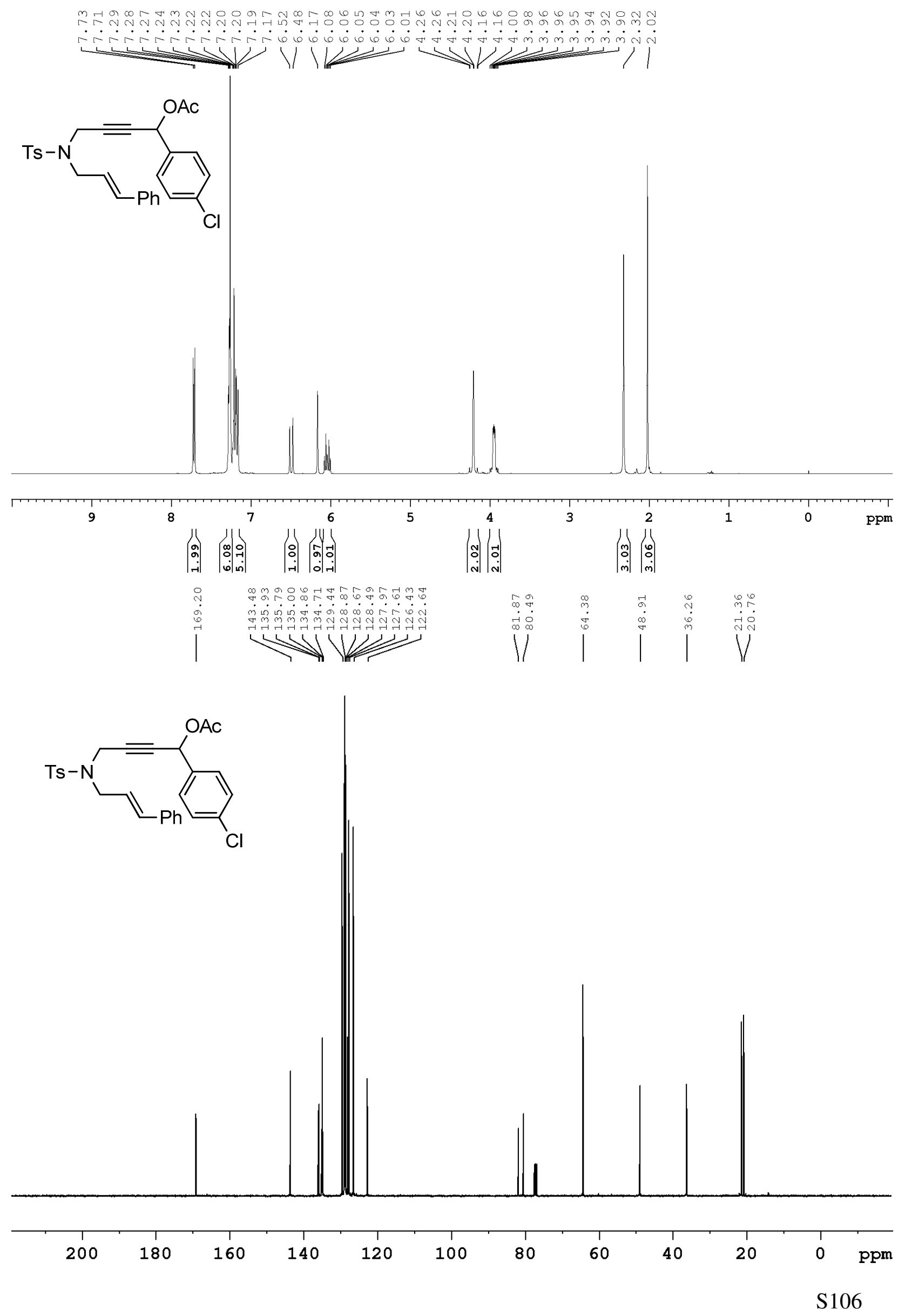
${ }^{1} \mathrm{H}$ - and ${ }^{13} \mathrm{C}$ NMR spectra of $\mathbf{s 1 4}$
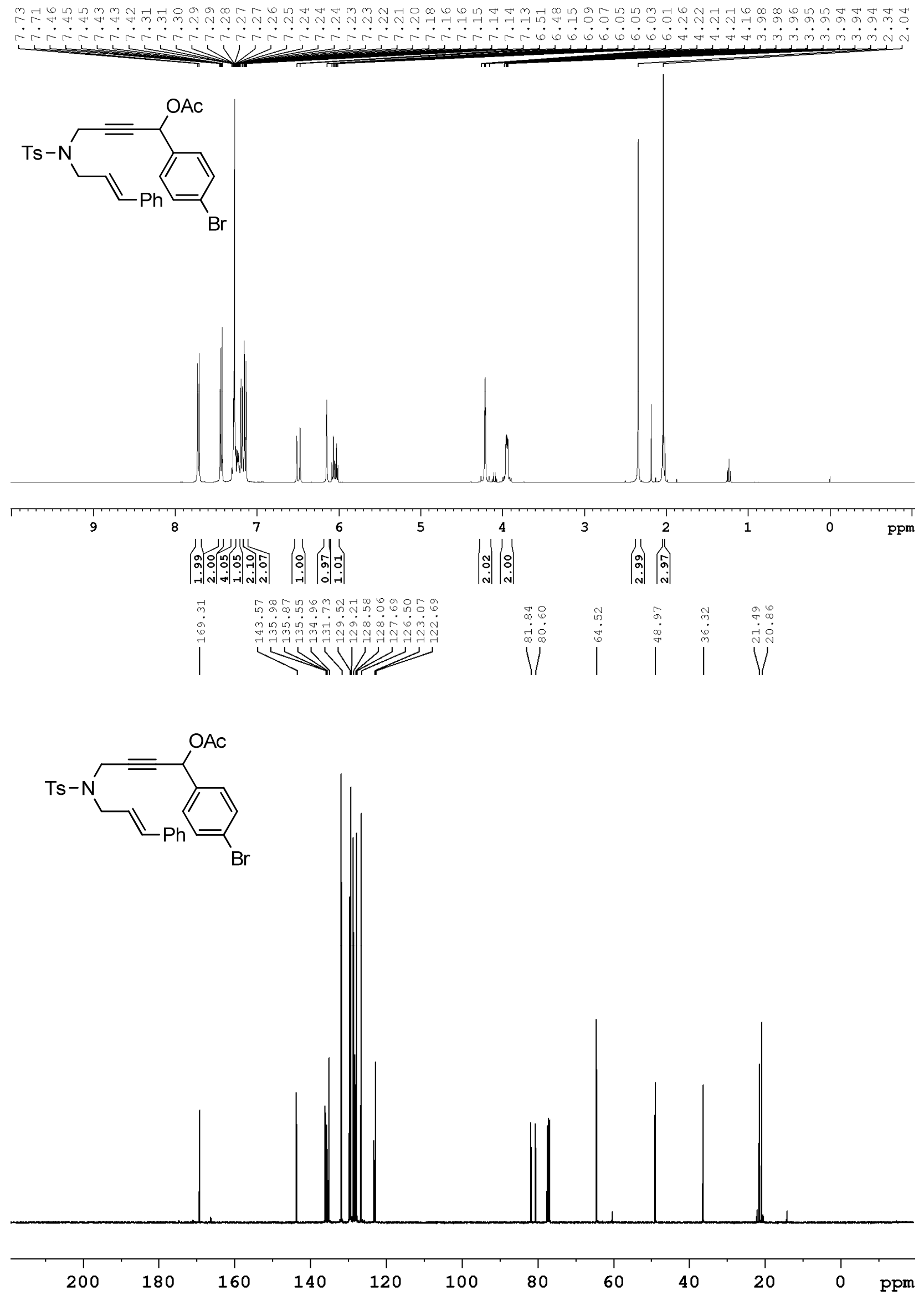
${ }^{1} \mathrm{H}$ - and ${ }^{13} \mathrm{C}$ NMR spectra of s16

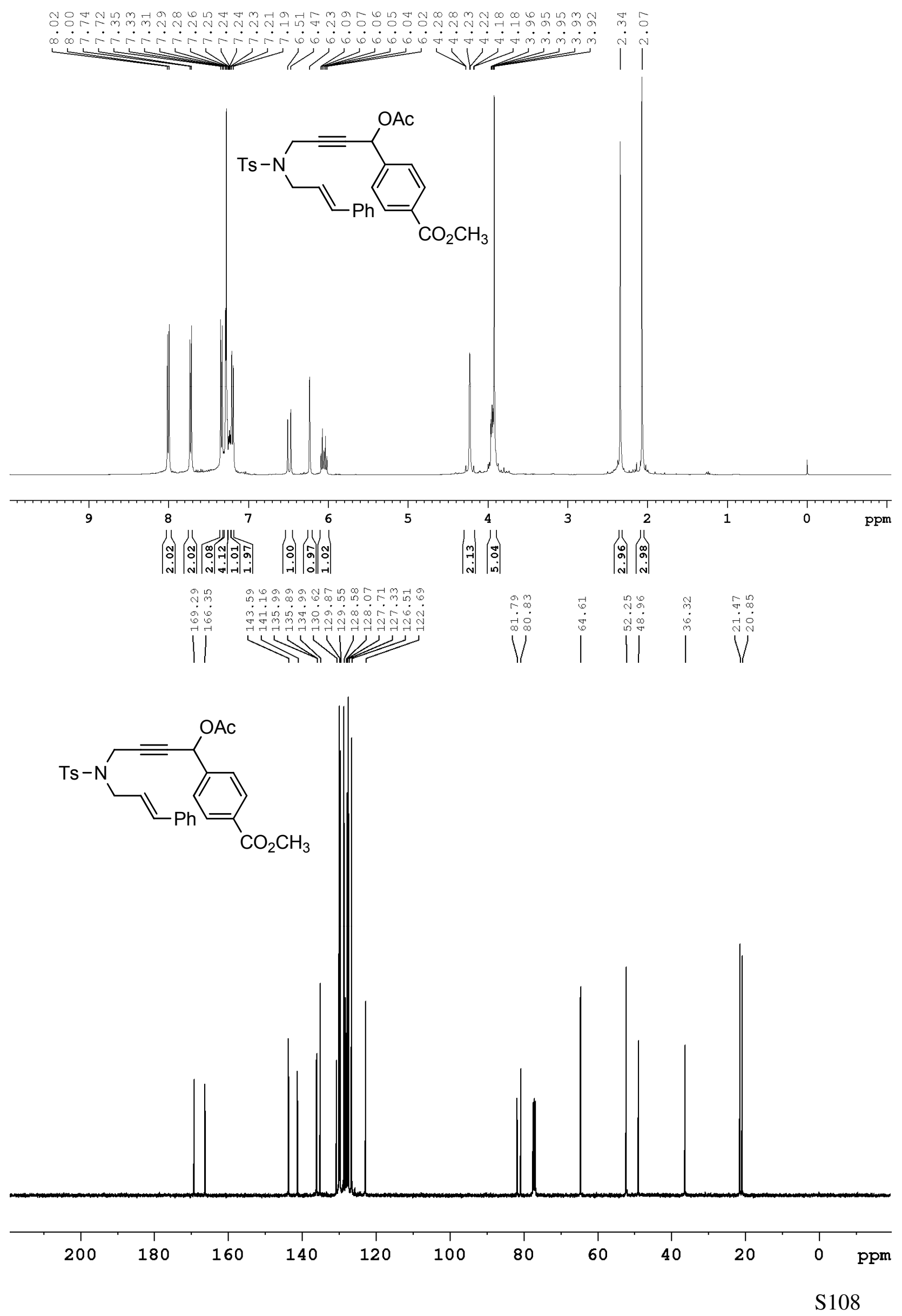


${ }^{1} \mathrm{H}$ - and ${ }^{13} \mathrm{C}$ NMR spectra of s18

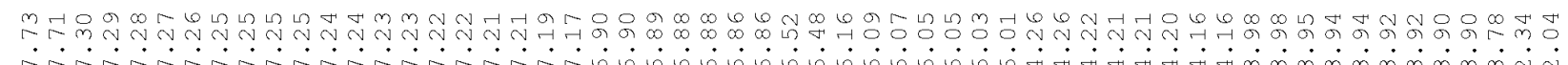
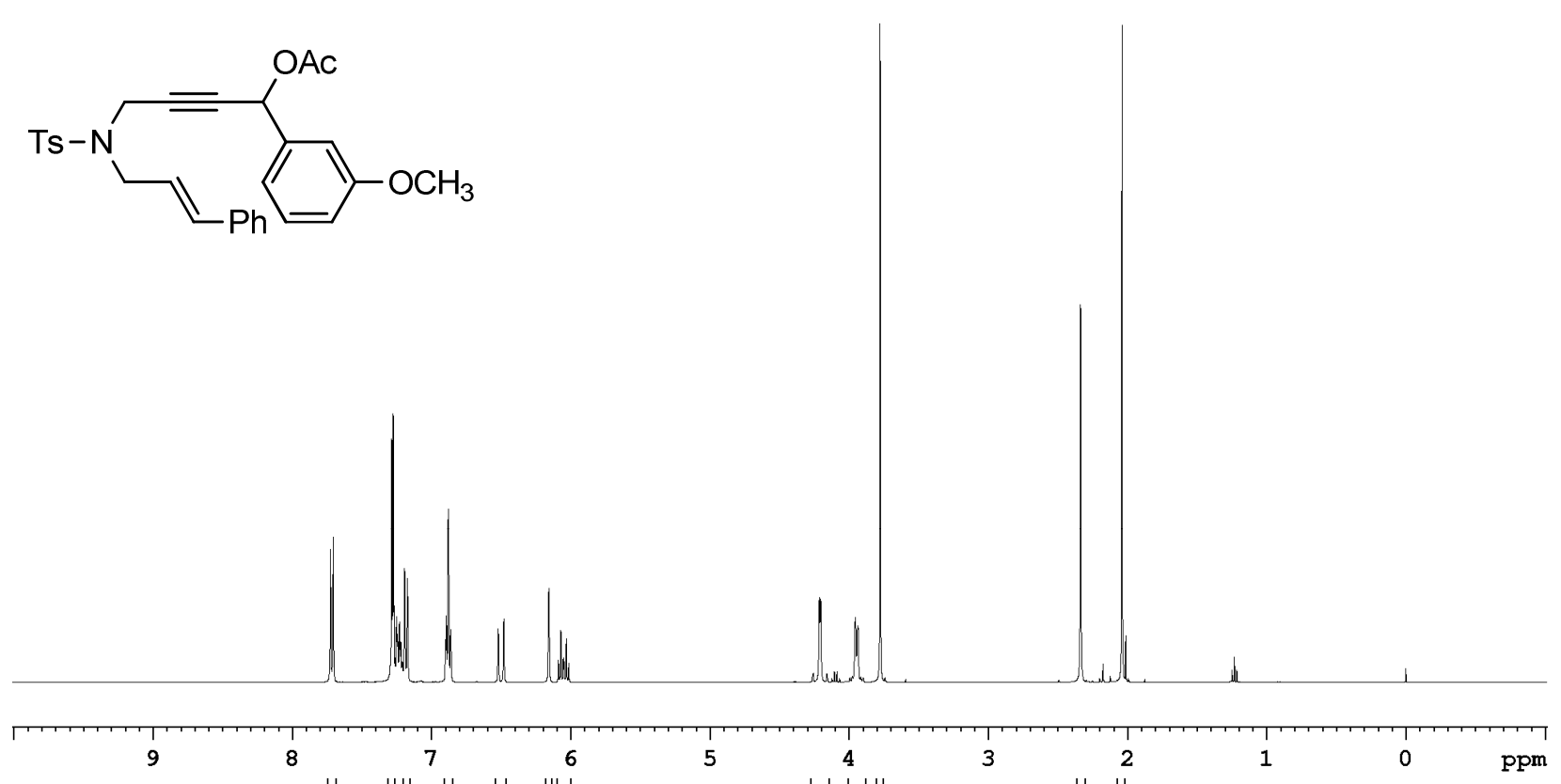

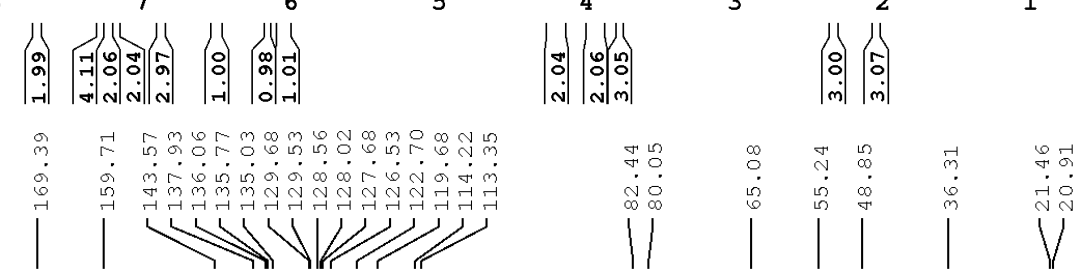<smiles>[B]N(CC#CC(OC(C)=O)c1cccc(OC)c1)C/C=C\P</smiles>
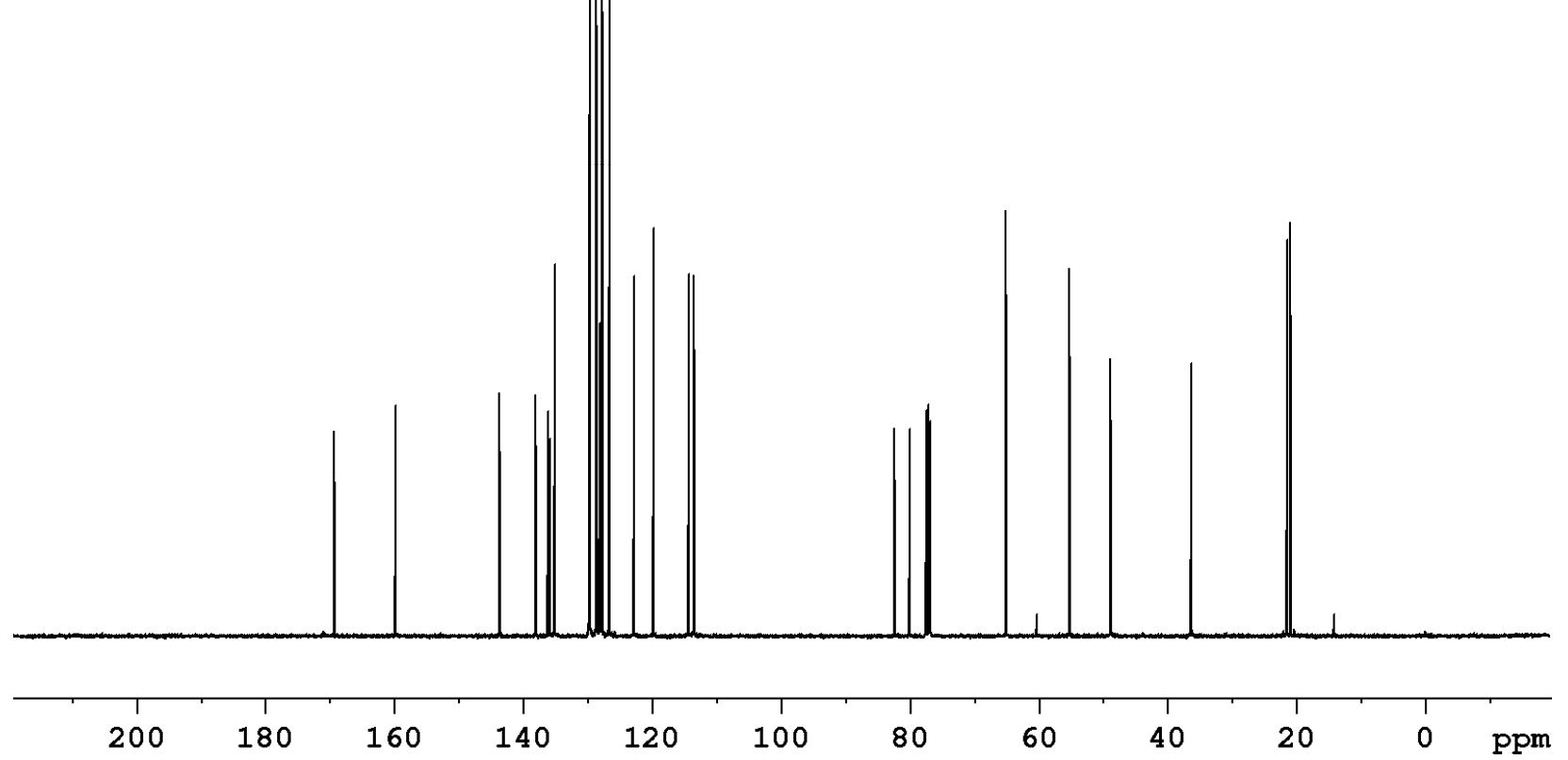
${ }^{1} \mathrm{H}$ - and ${ }^{13} \mathrm{C}$ NMR spectra of $\mathbf{s 2 2}$

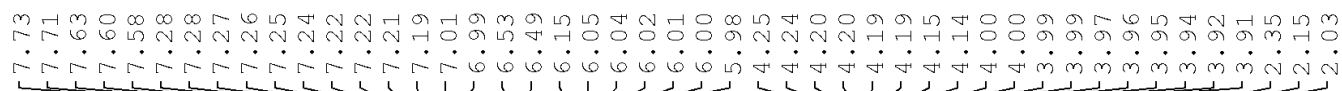
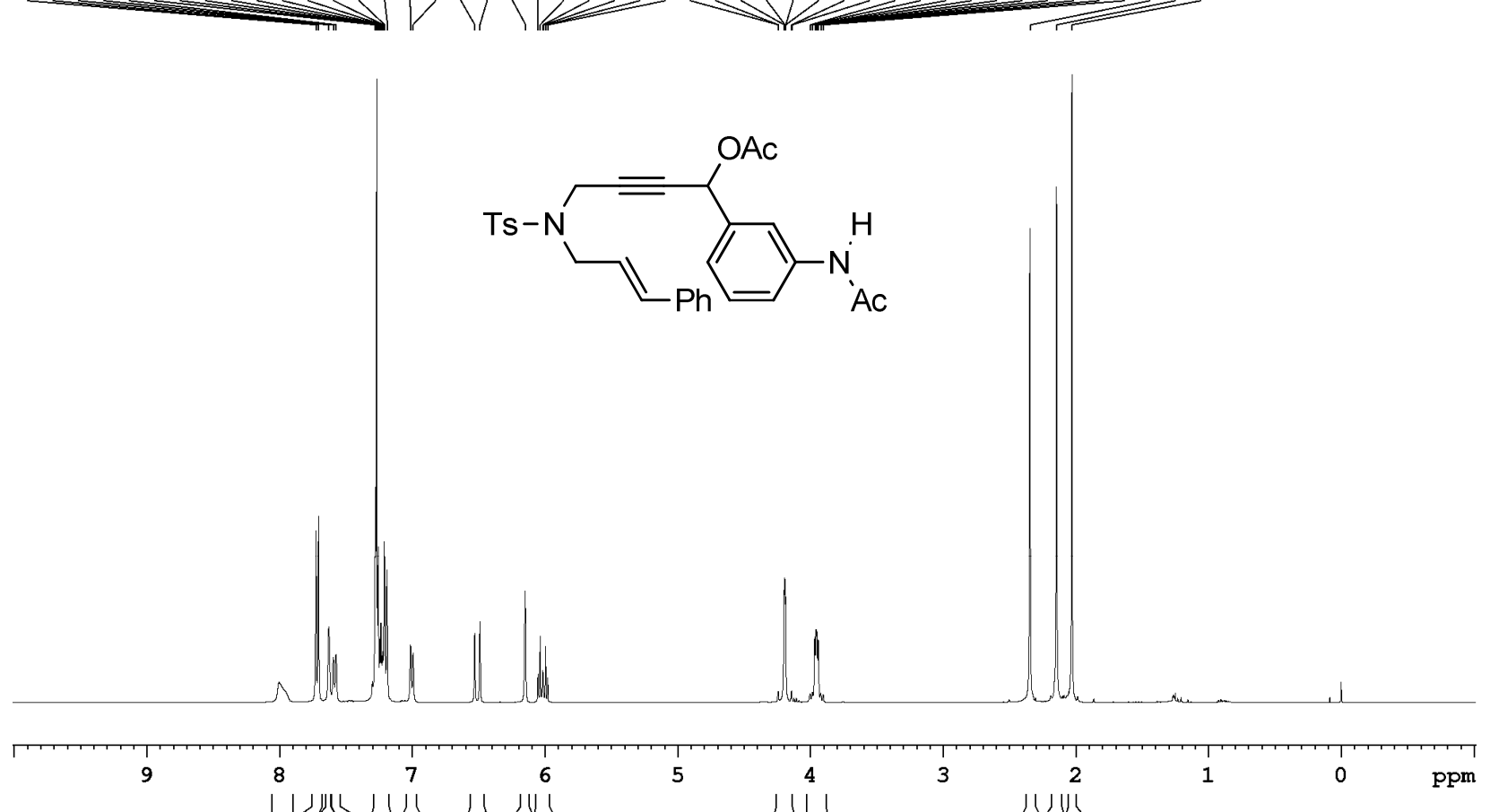

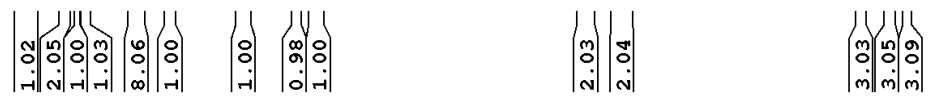

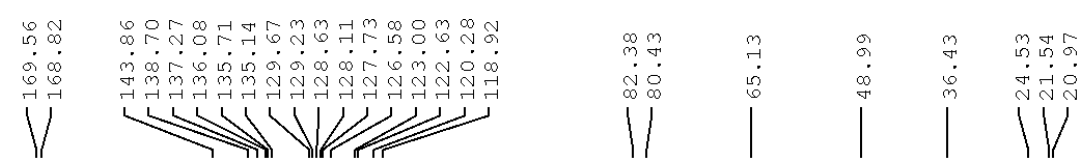
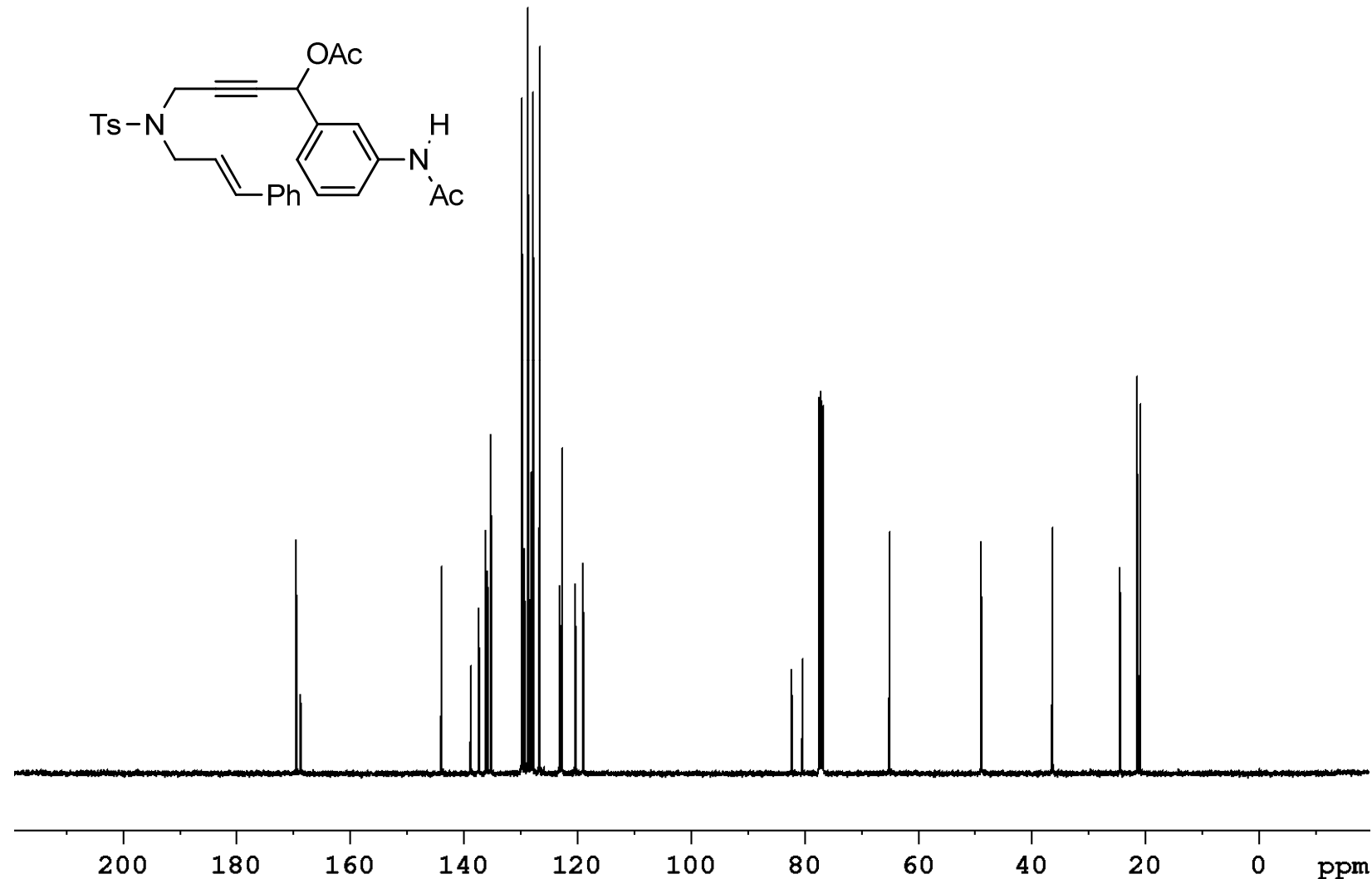
${ }^{1} \mathrm{H}$ - and ${ }^{13} \mathrm{C}$ NMR spectra of $\mathbf{s} 24$

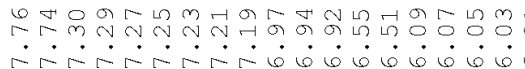

近

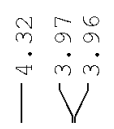

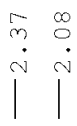<smiles>CC(C)(C#CCN([As])C/C=C\Pc1ccc(F)cc1)c1ccc(F)cc1</smiles>

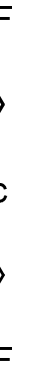
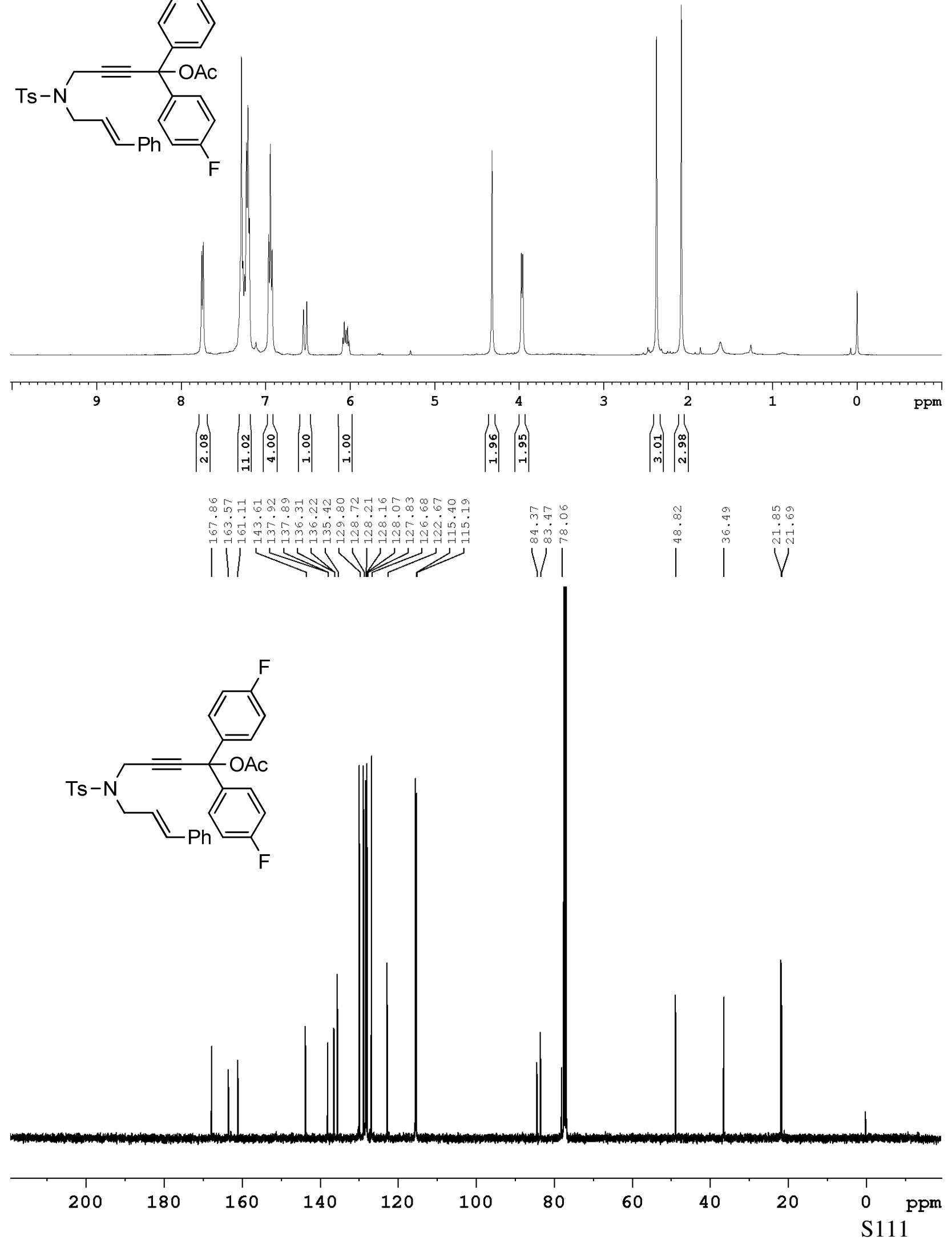
${ }^{1} \mathrm{H}$ - and ${ }^{13} \mathrm{C}$ NMR spectra of $\mathbf{s 2 6}$

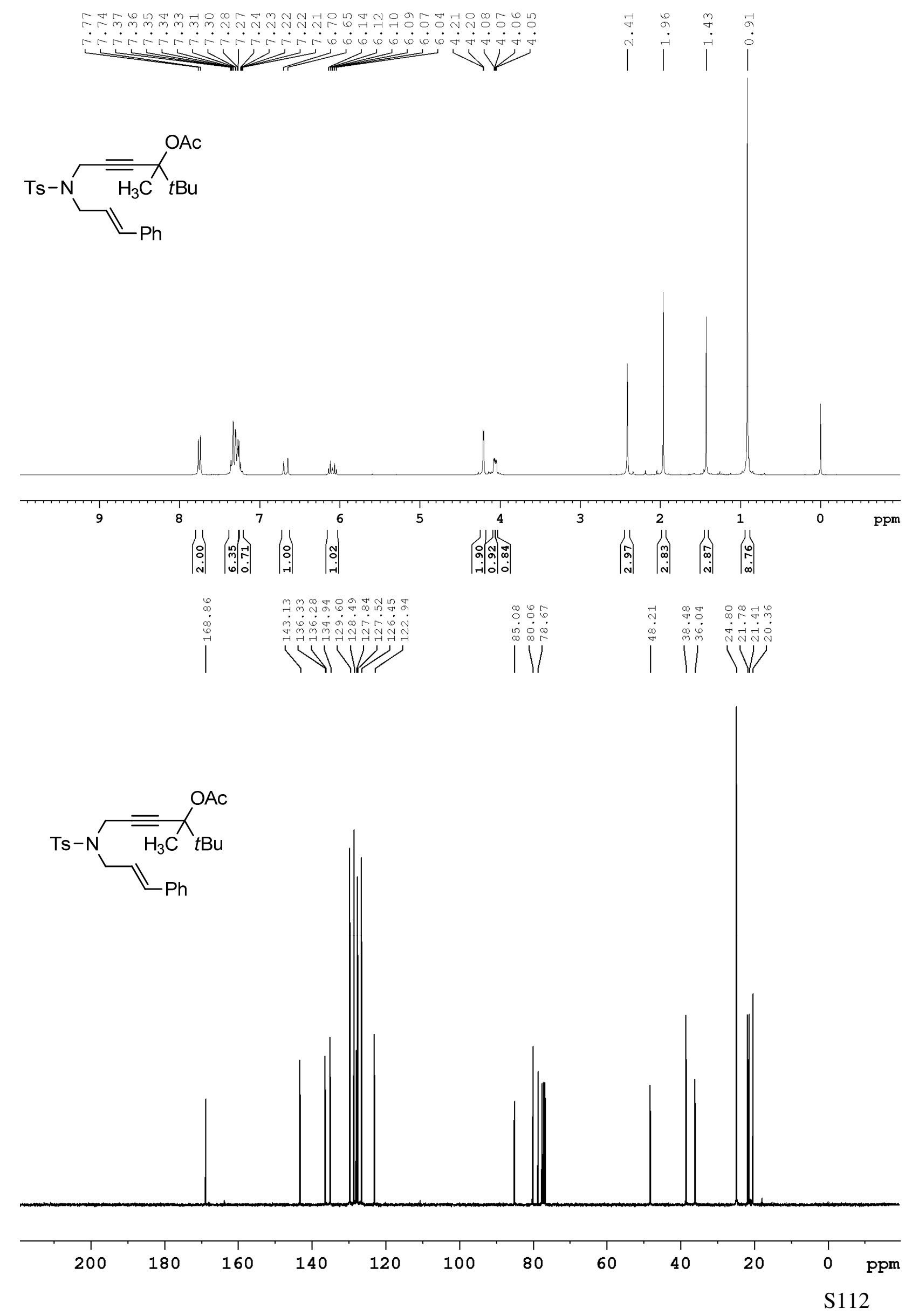


${ }^{1} \mathrm{H}$ - and ${ }^{13} \mathrm{C}$ NMR spectra of $\mathbf{s} 28$
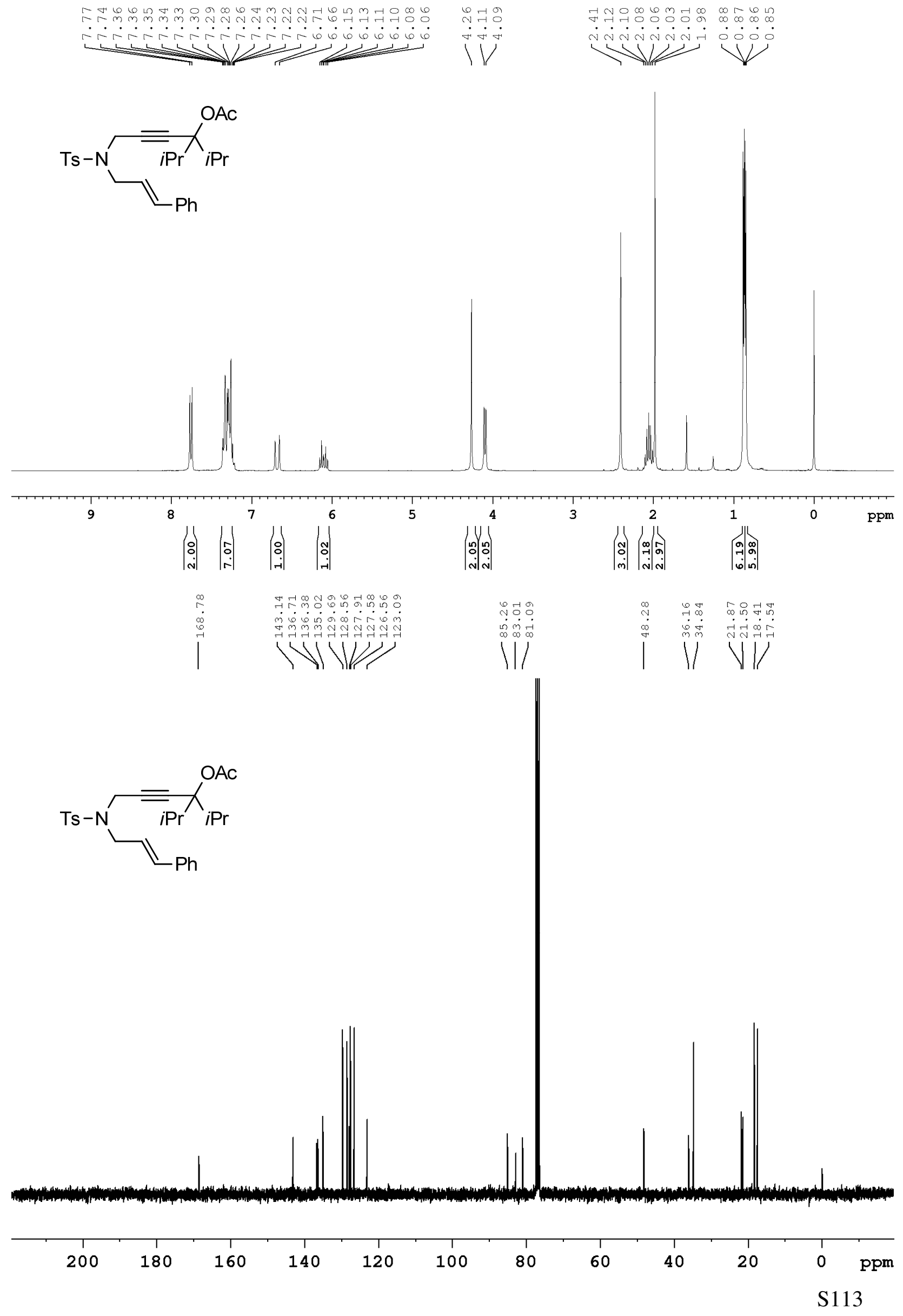
${ }^{1} \mathrm{H}$ - and ${ }^{13} \mathrm{C}$ NMR spectra of $\mathbf{s 3 0}$
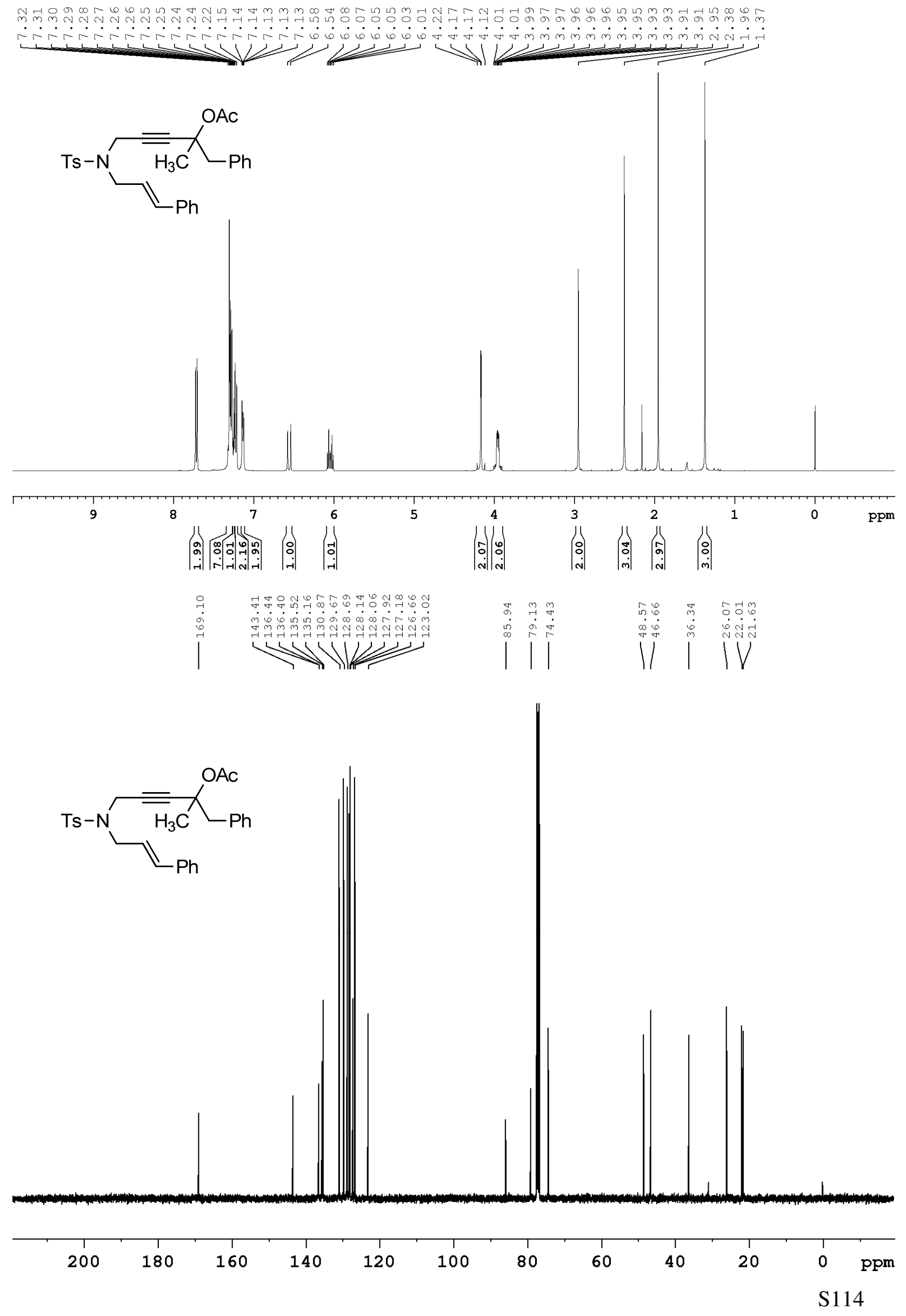
${ }^{1} \mathrm{H}$ - and ${ }^{13} \mathrm{C}$ NMR spectra of $\mathbf{s 3 2}$
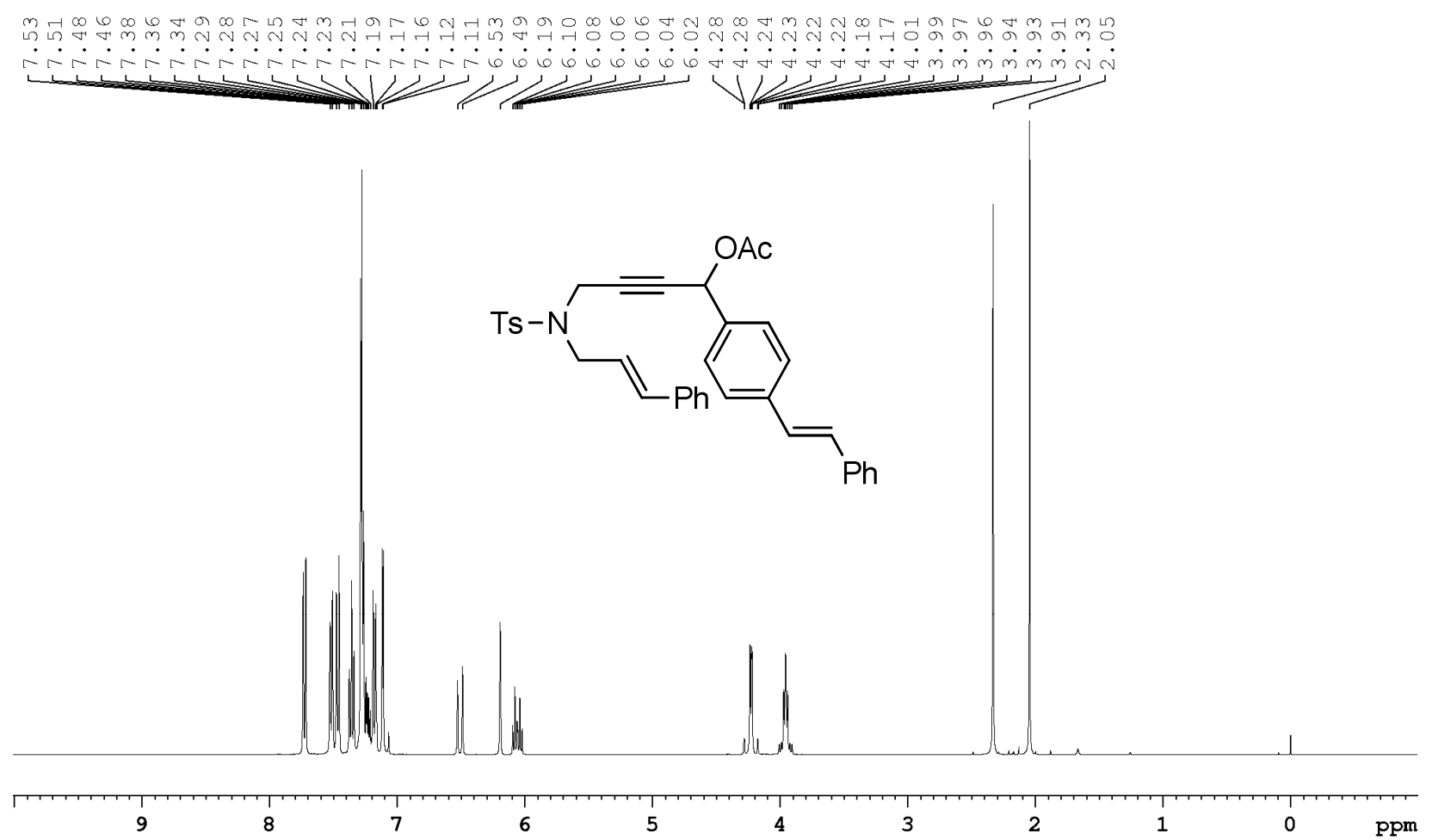

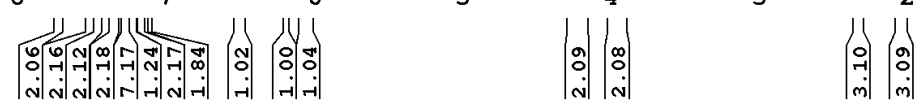

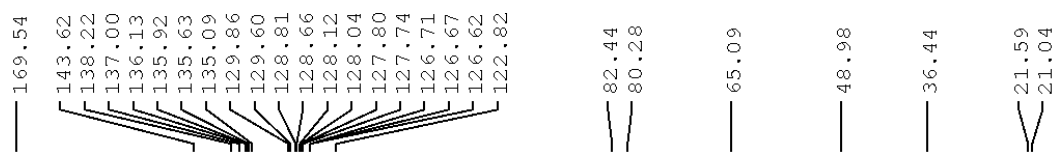

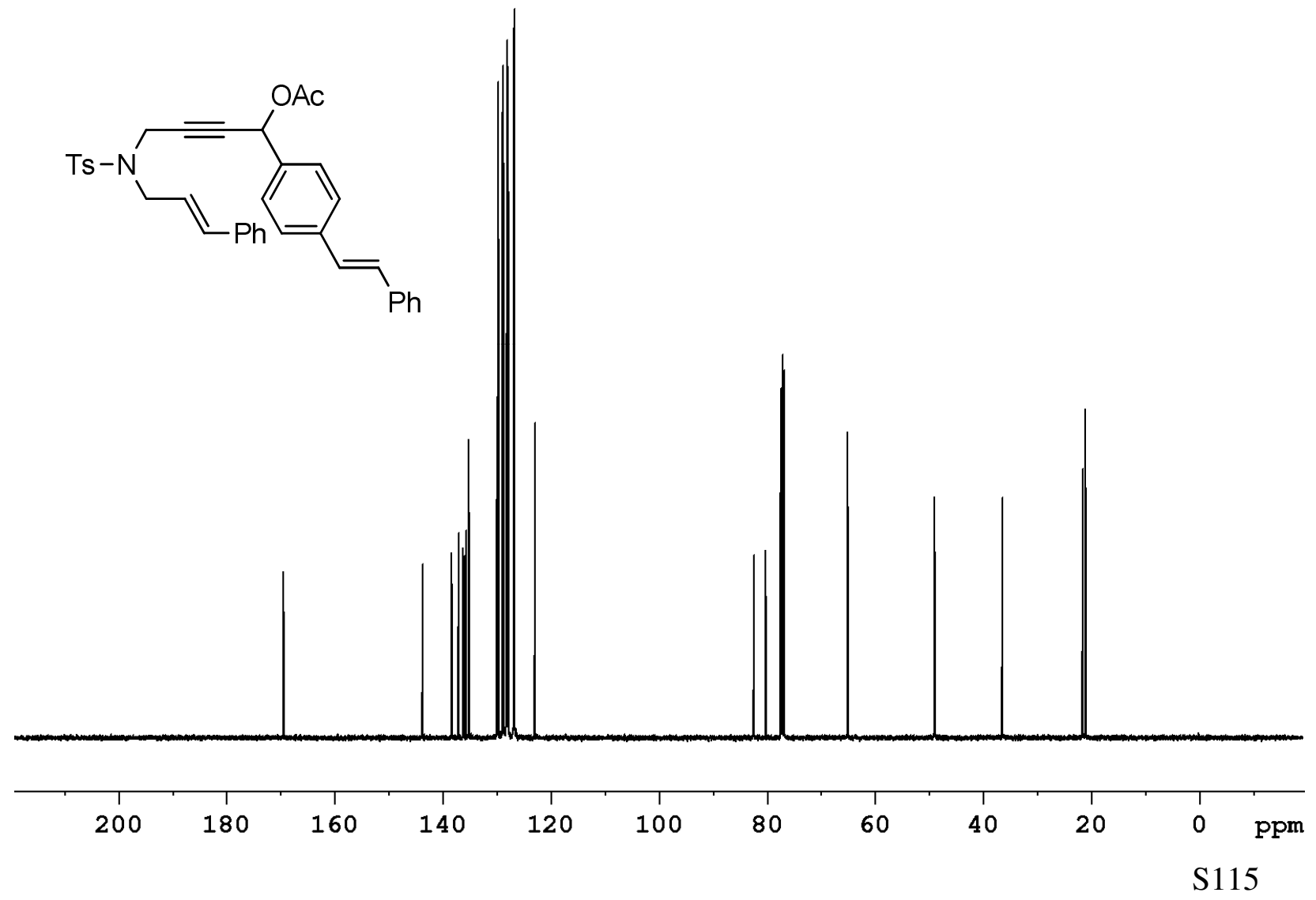


${ }^{1} \mathrm{H}$ - and ${ }^{13} \mathrm{C}$ NMR spectra of $\mathbf{s 3 4}$
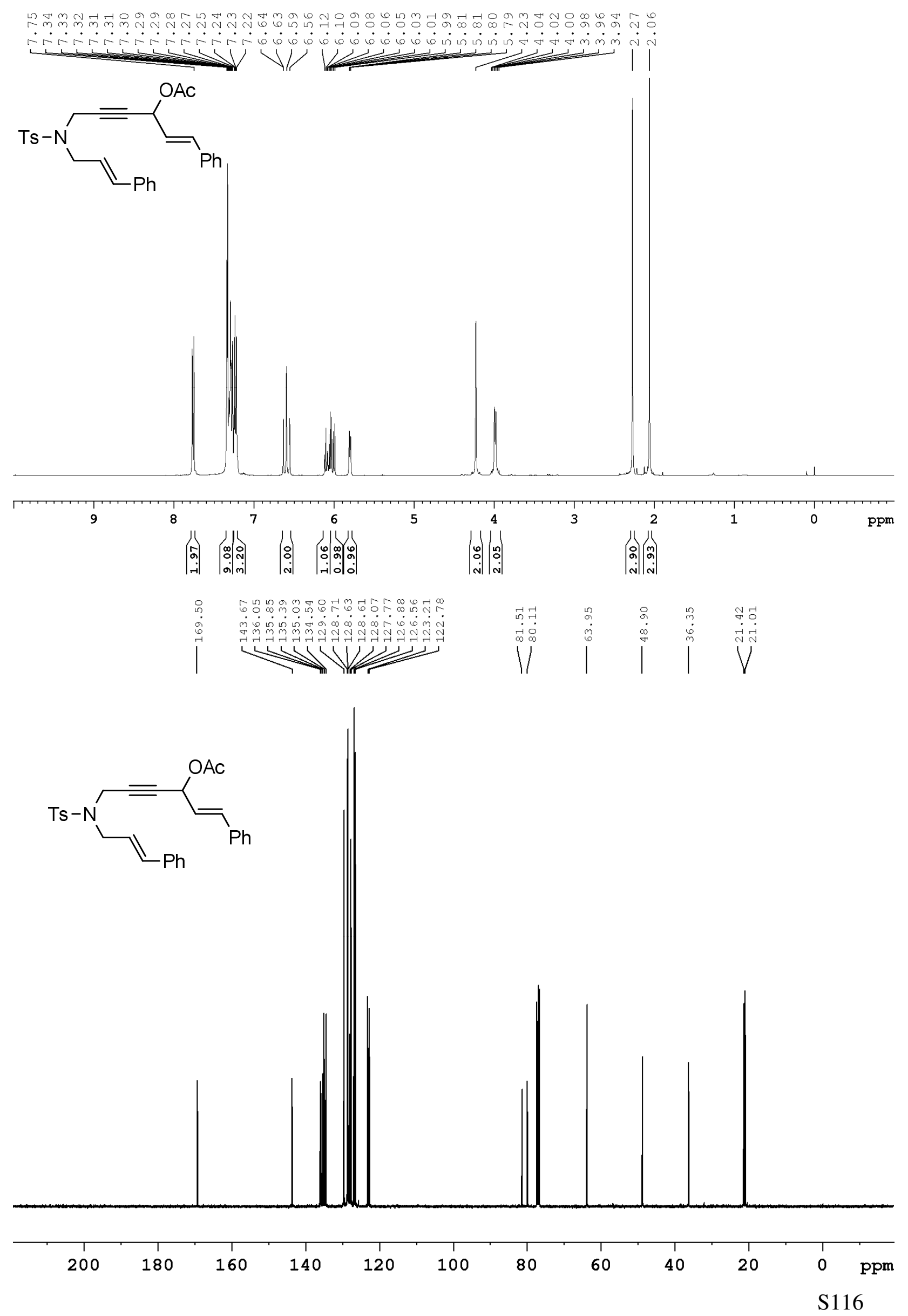
${ }^{1} \mathrm{H}$ - and ${ }^{13} \mathrm{C}$ NMR spectra of $\mathbf{s 3 7}$

$\mathrm{H}_{\mathrm{Ph}}^{\mathrm{OAC}}$
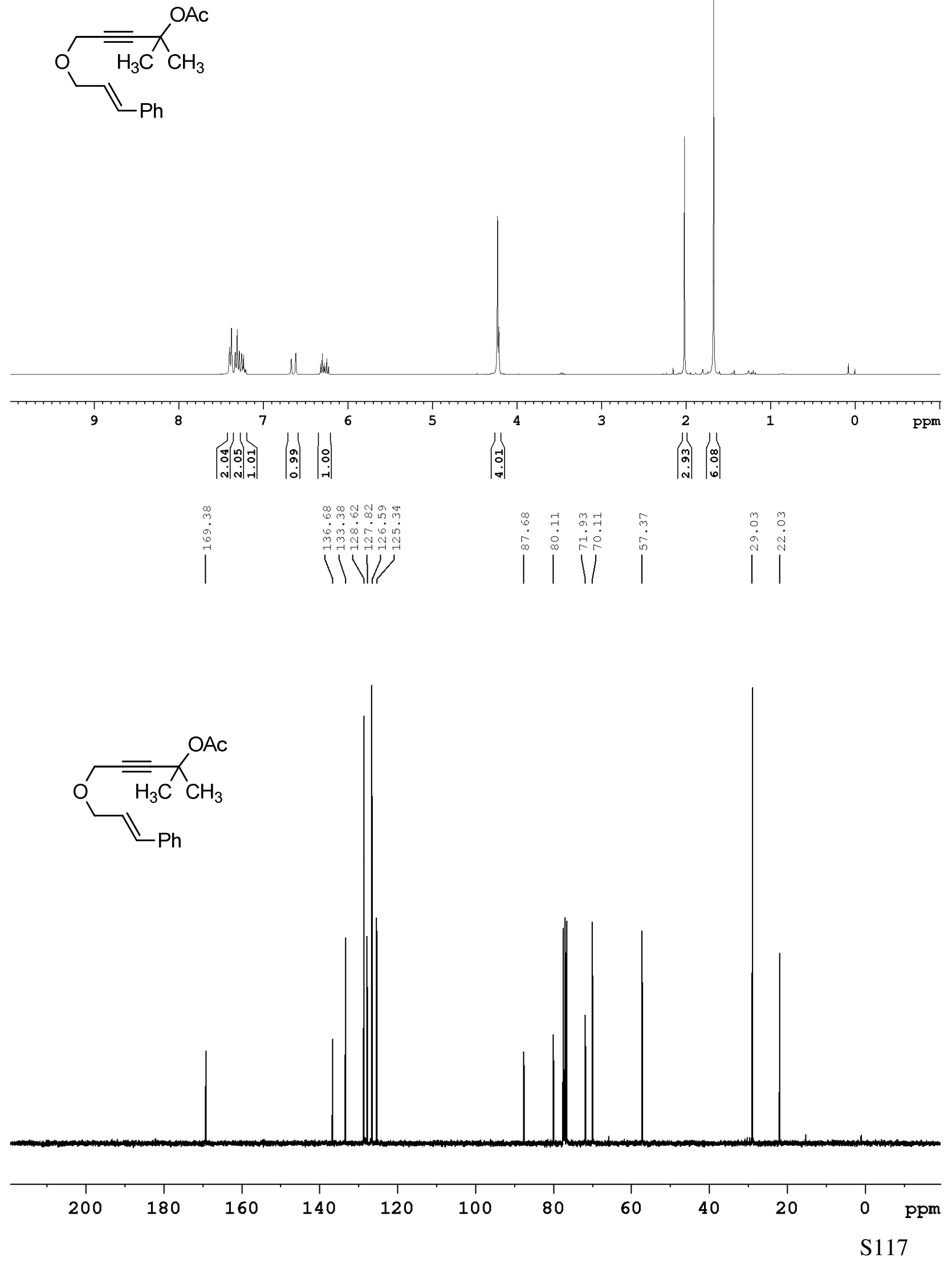
${ }^{1} \mathrm{H}$ - and ${ }^{13} \mathrm{C}$ NMR spectra of $\mathbf{s} 39$
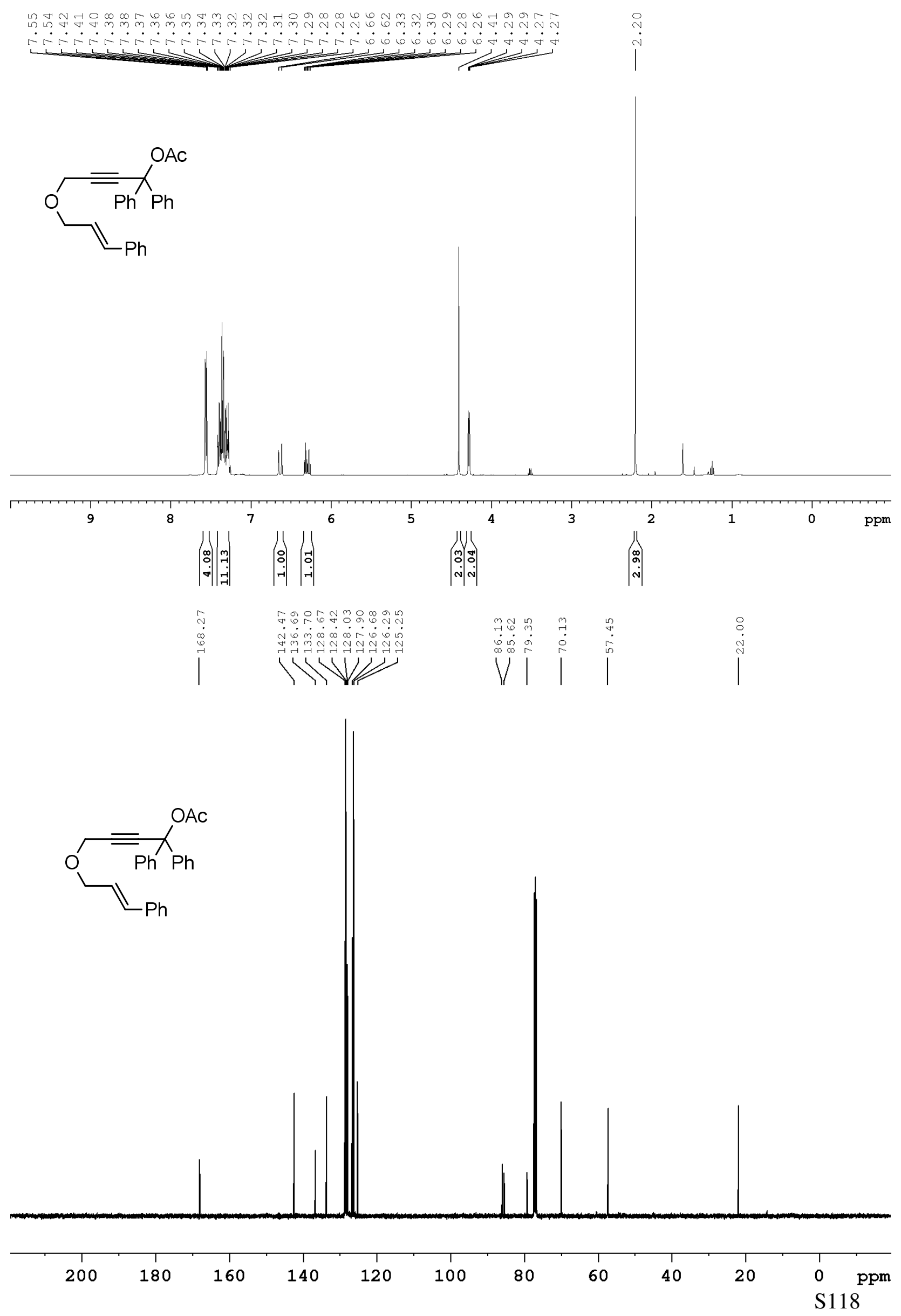
${ }^{1} \mathrm{H}$ - and ${ }^{13} \mathrm{C}$ NMR spectra of $\mathbf{s 4 3}$

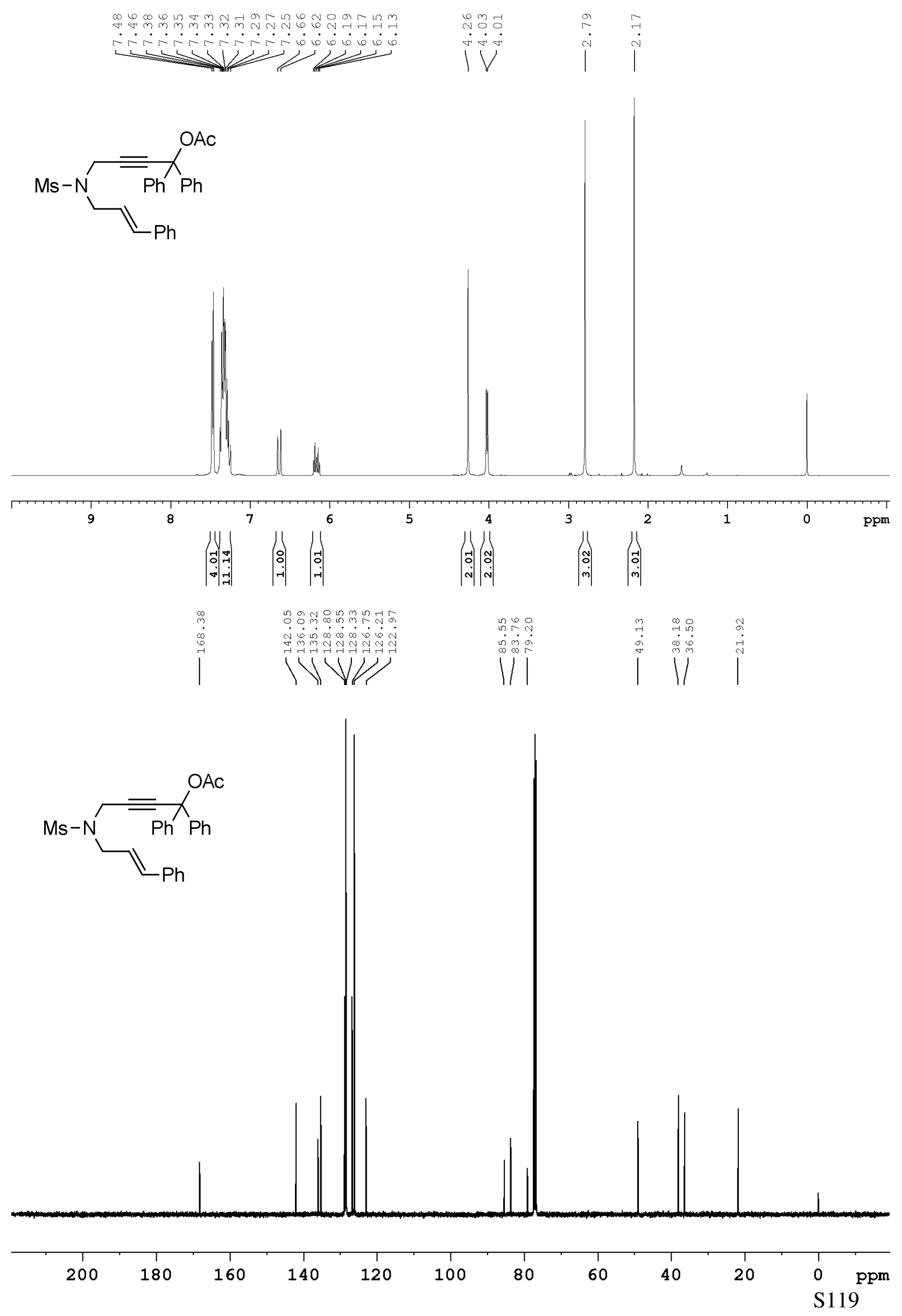


${ }^{1} \mathrm{H}-$ and ${ }^{13} \mathrm{C}$ NMR spectra of $\mathbf{3 5}$
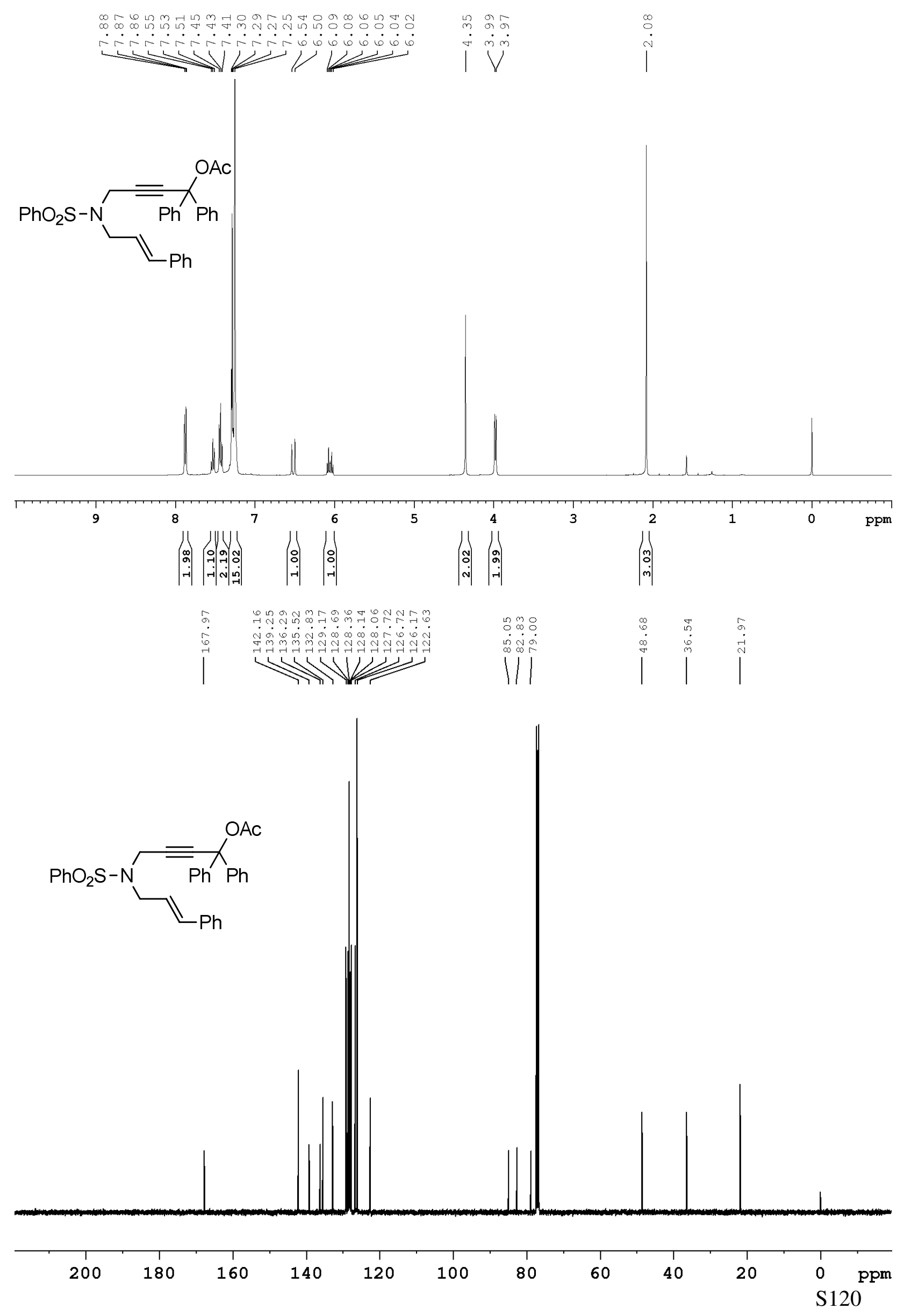
${ }^{1} \mathrm{H}$ - and ${ }^{13} \mathrm{C}$ NMR spectra of $\mathbf{3 5}-\mathbf{D}_{\mathbf{3}}$
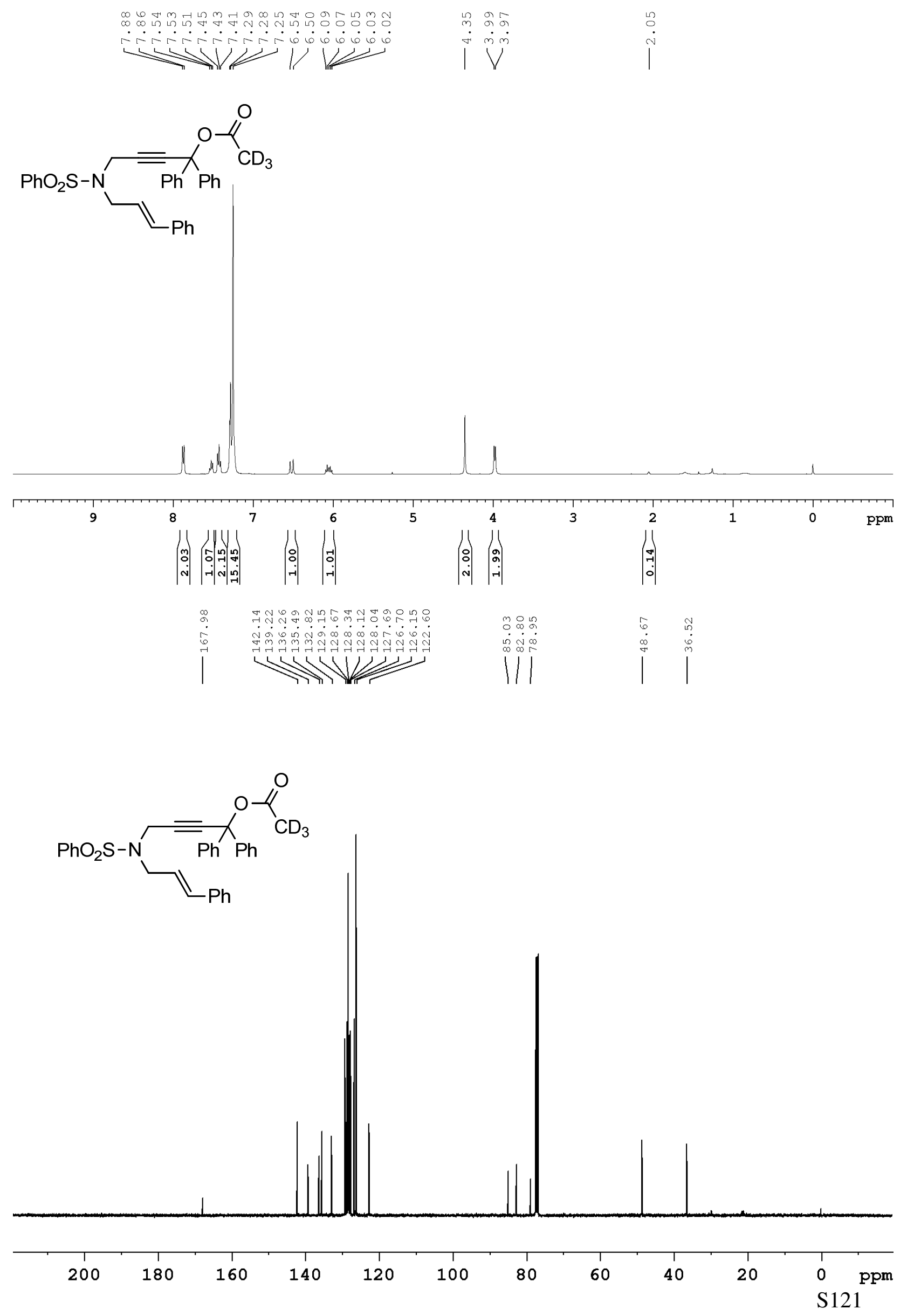
${ }^{1} \mathrm{H}$ - and ${ }^{13} \mathrm{C}$ NMR spectra of $\mathbf{3 8}$
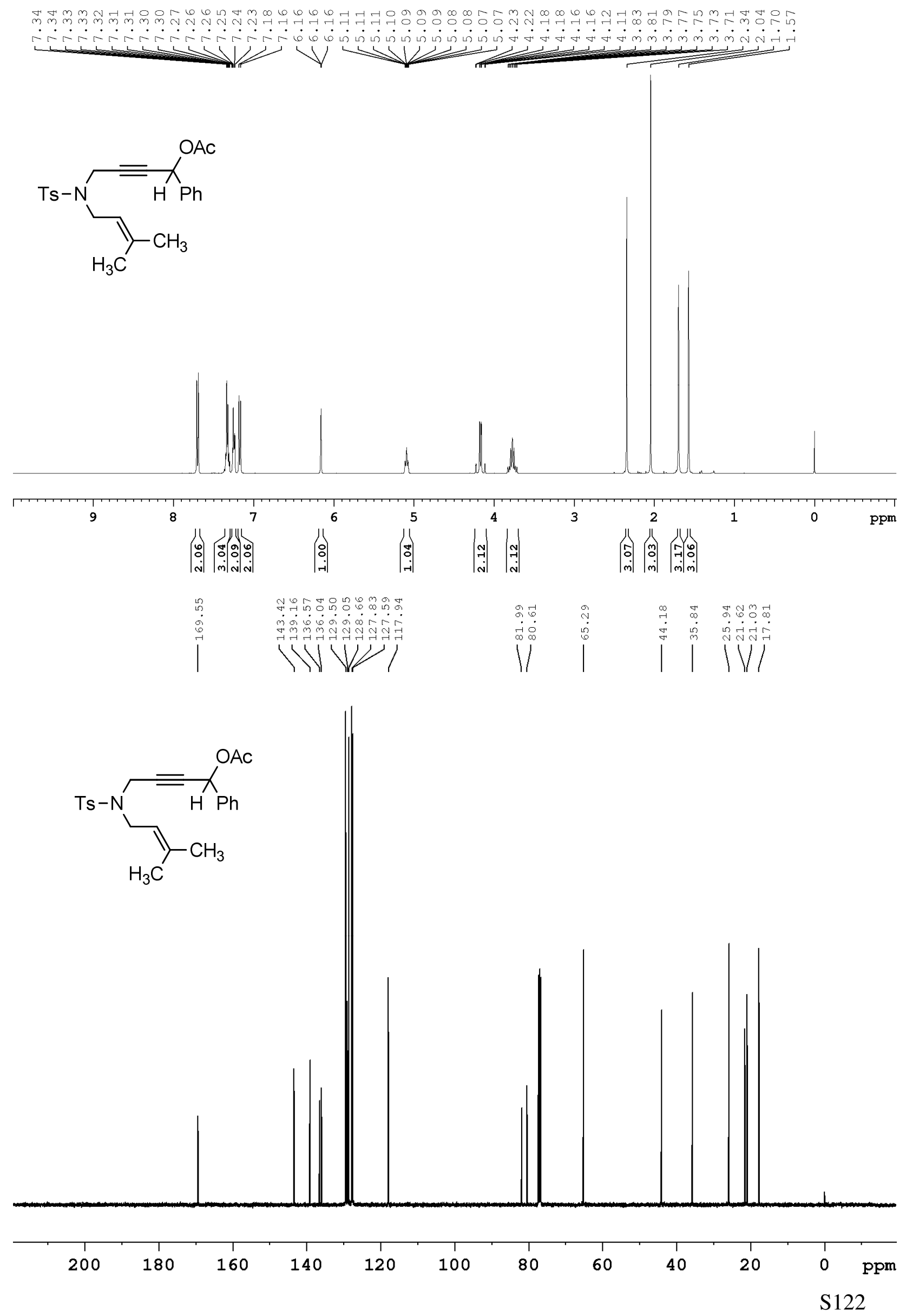
${ }^{1} \mathrm{H}$ - and ${ }^{13} \mathrm{C}$ NMR spectra of $\mathbf{s 5 0}$
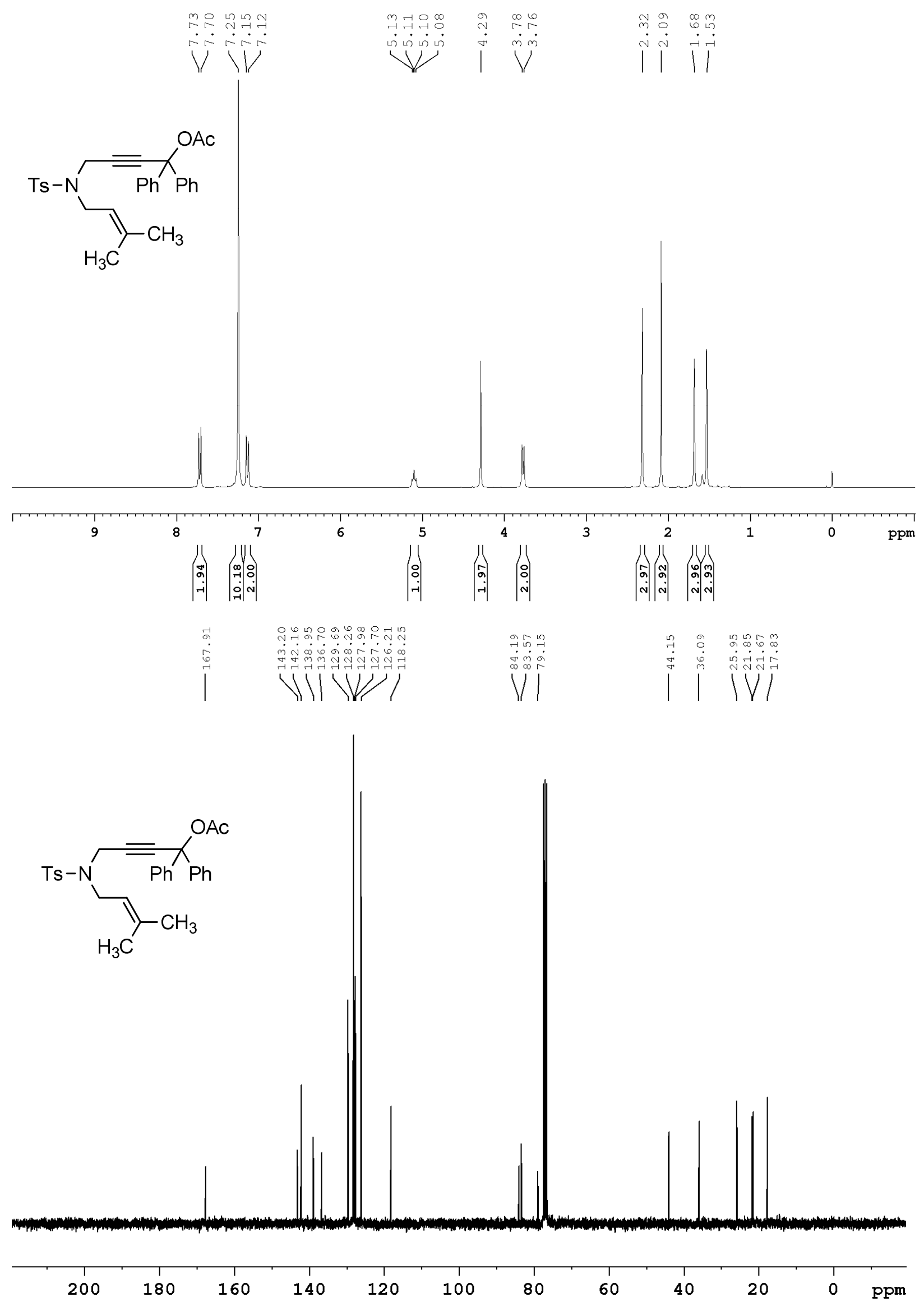
${ }^{1} \mathrm{H}$ - and ${ }^{13} \mathrm{C}$ NMR spectra of $\mathbf{5 5 5}$
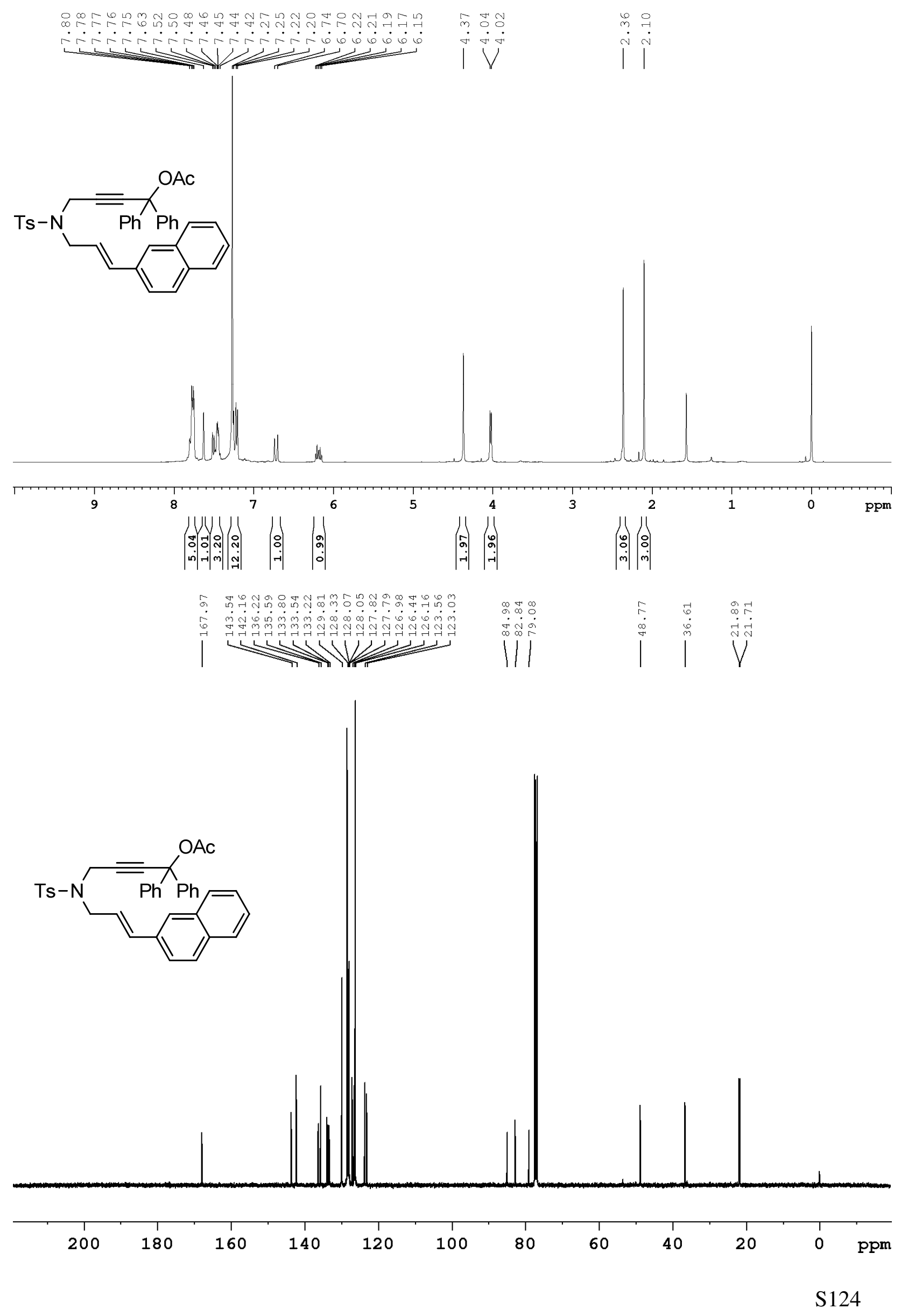
${ }^{1} \mathrm{H}$ - and ${ }^{13} \mathrm{C}$ NMR spectra of $\mathbf{s 5 6}$

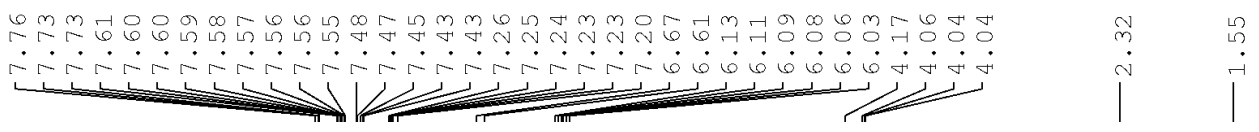

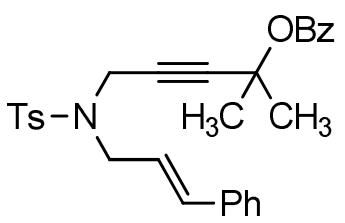
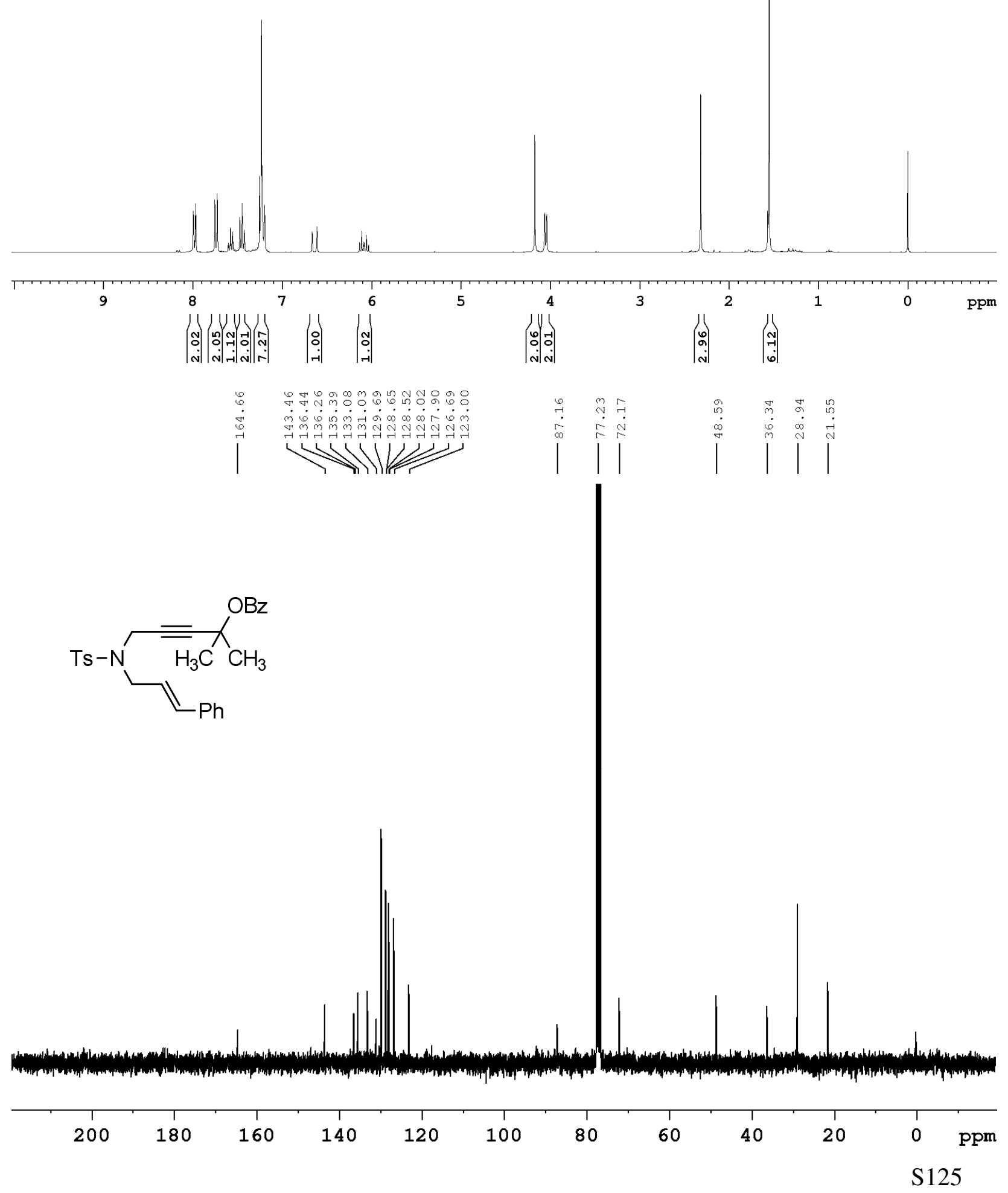
${ }^{1} \mathrm{H}$ - and ${ }^{13} \mathrm{C}$ NMR spectra of $\mathbf{1 0}$
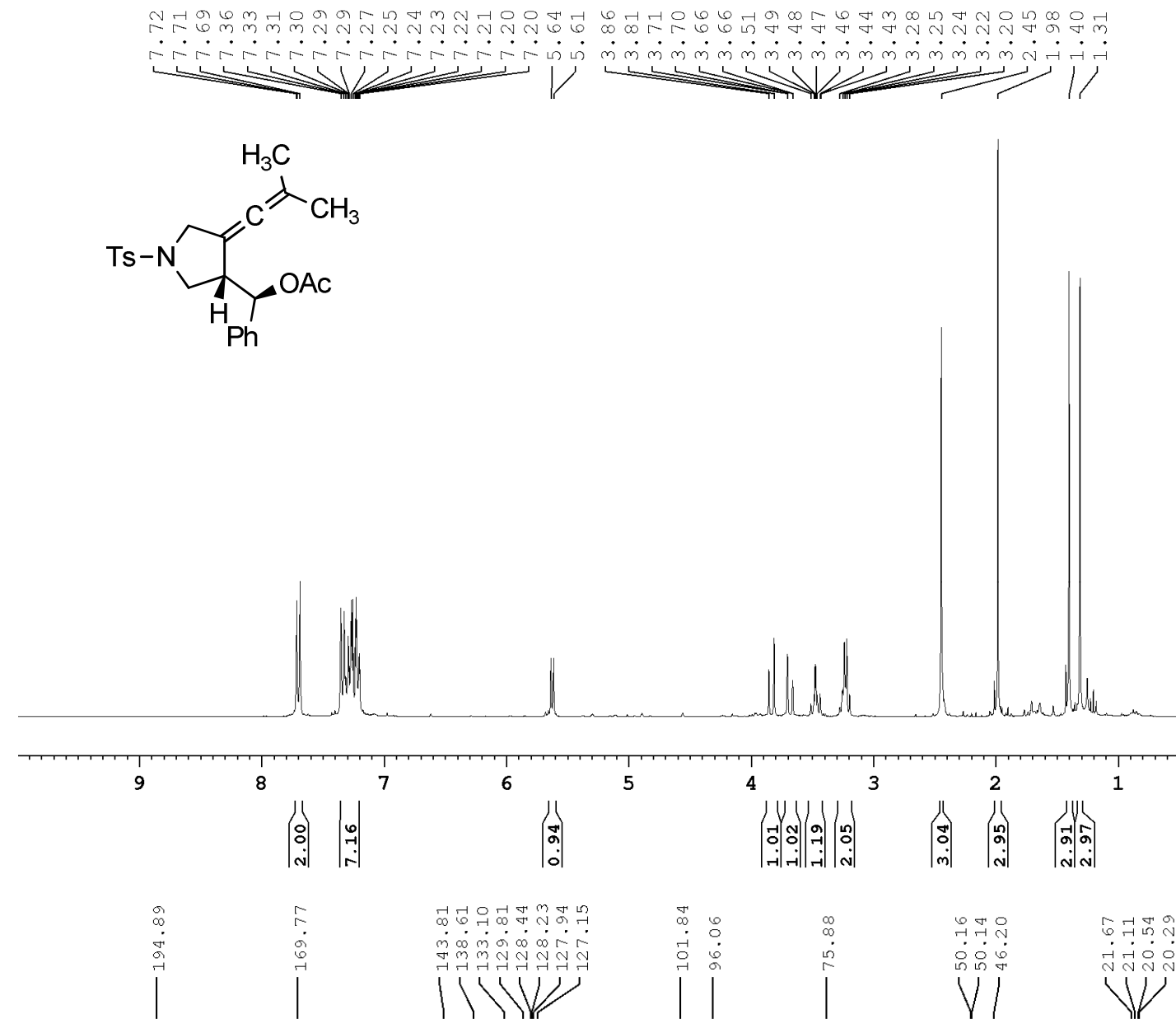<smiles>[B]N1CC(=C=C(C)C)[C@H]([C@H](OC(C)=O)c2ccccc2)C1</smiles>

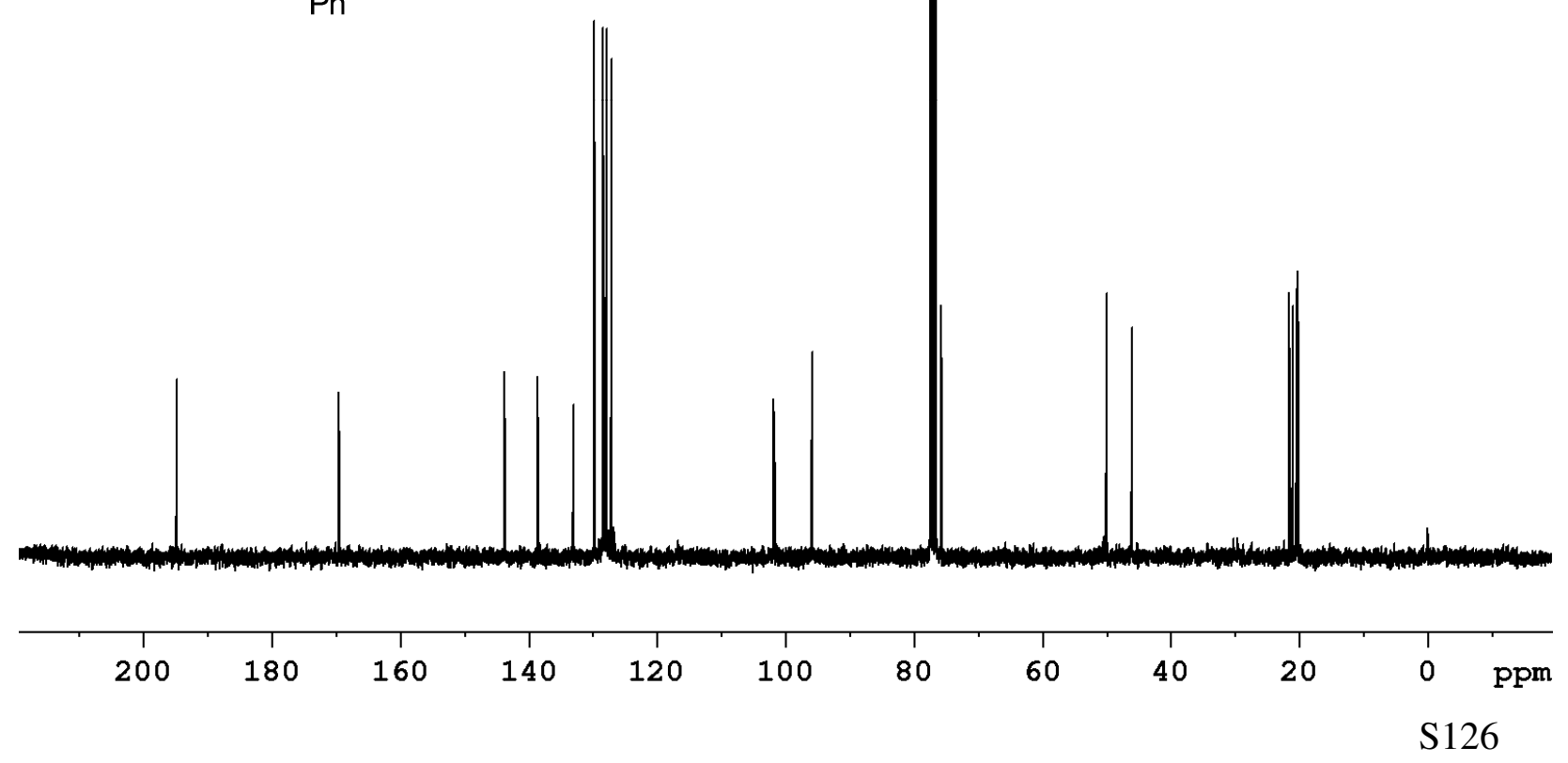


${ }^{1} \mathrm{H}$ - and ${ }^{13} \mathrm{C}$ NMR spectra of $\mathbf{1 1}$
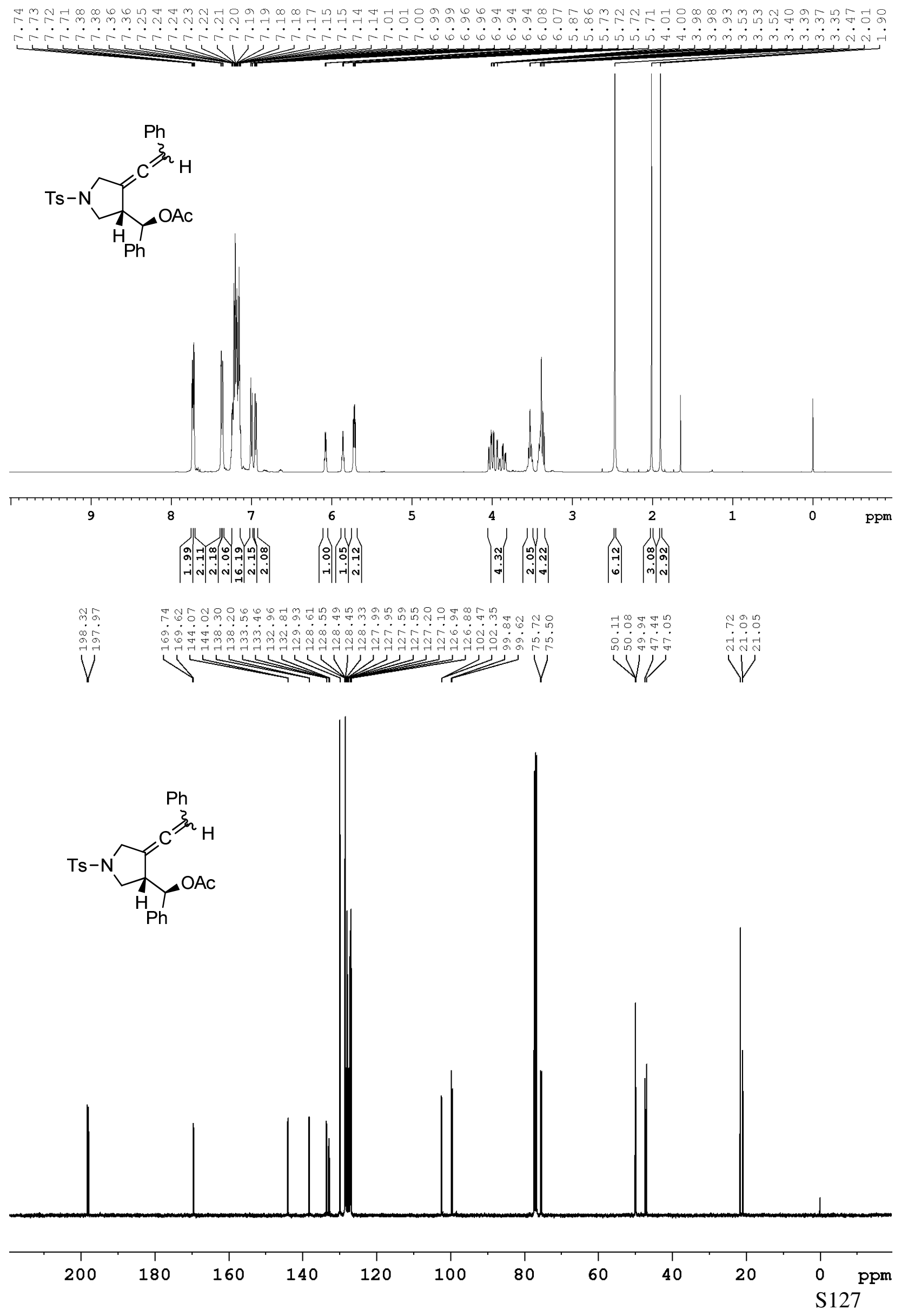
${ }^{1} \mathrm{H}$ - and ${ }^{13} \mathrm{C}$ NMR spectra of $\mathbf{1 2}$ (HPLC fraction 1)

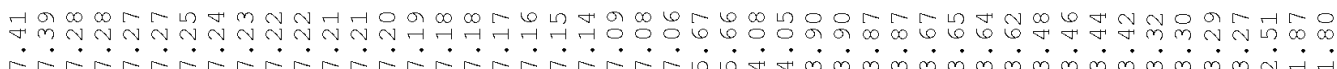

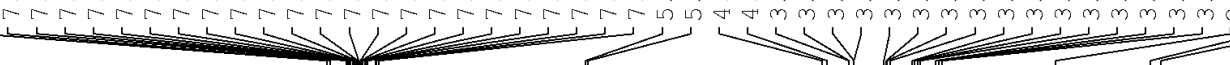<smiles>CC(=O)O[C@H](c1ccccc1)[C@H]1CN([13F])C/C1=C\C(C)c1ccccc1</smiles>

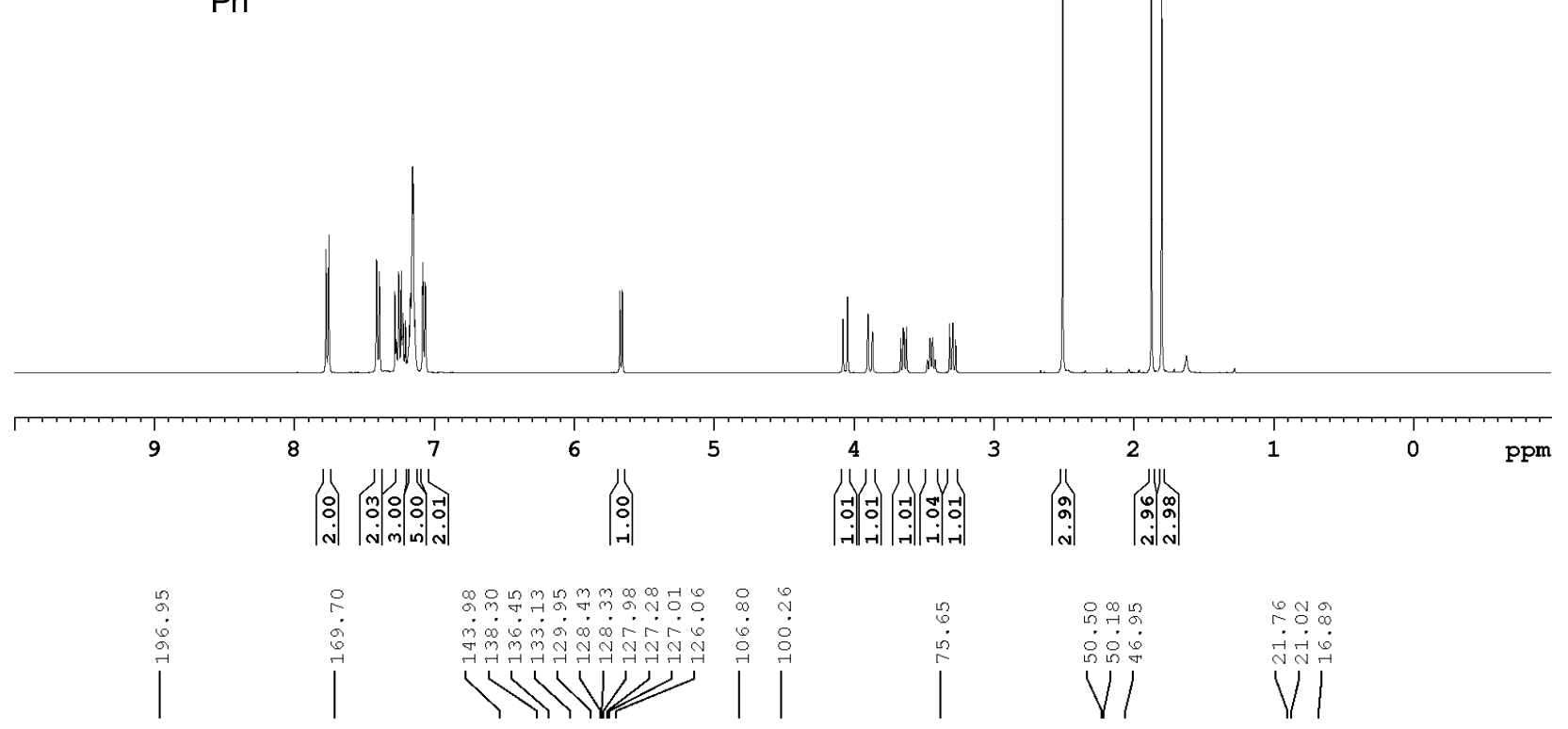<smiles>CC(=O)O[C@H](c1ccccc1)[C@H]1CN([As])C/C1=C\C(C)c1ccccc1</smiles>

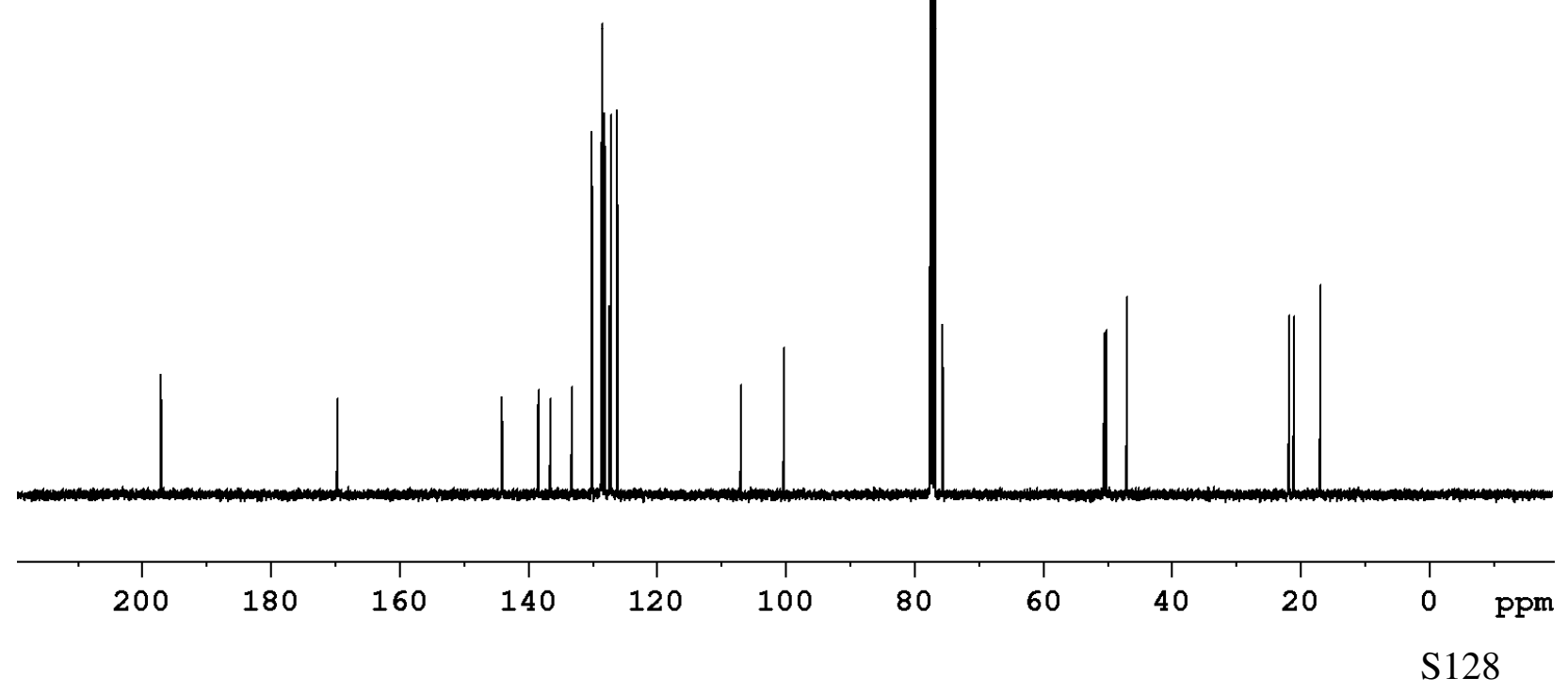


${ }^{1} \mathrm{H}$ - and ${ }^{13} \mathrm{C}$ NMR spectra of 12 (HPLC fraction 2)

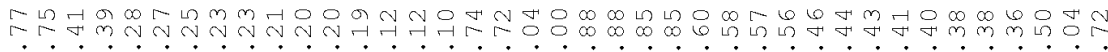

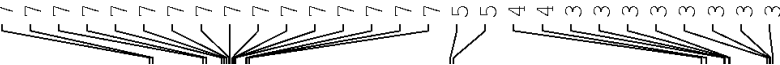

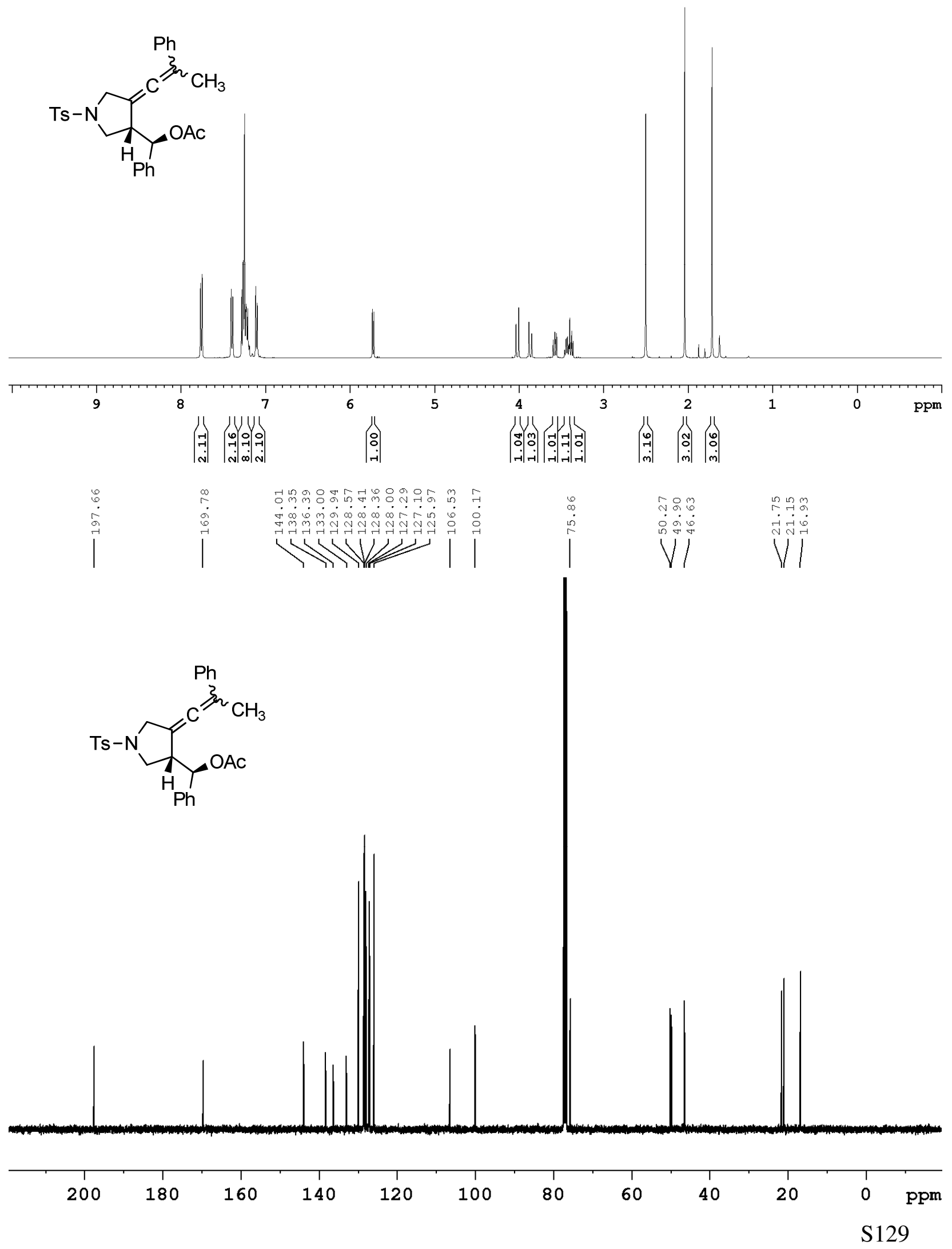


${ }^{1} \mathrm{H}$ - and ${ }^{13} \mathrm{C}$ NMR spectra of $\mathbf{1 3}$

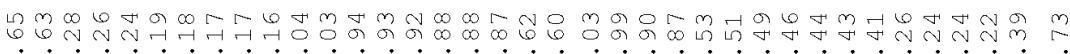

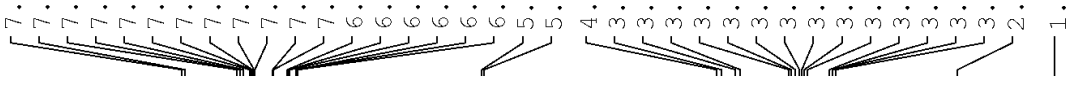<smiles>CC(=O)O[C@H](c1ccccc1)[C@H]1CN([As])C/C1=C\C(c1ccccc1)c1ccccc1</smiles>
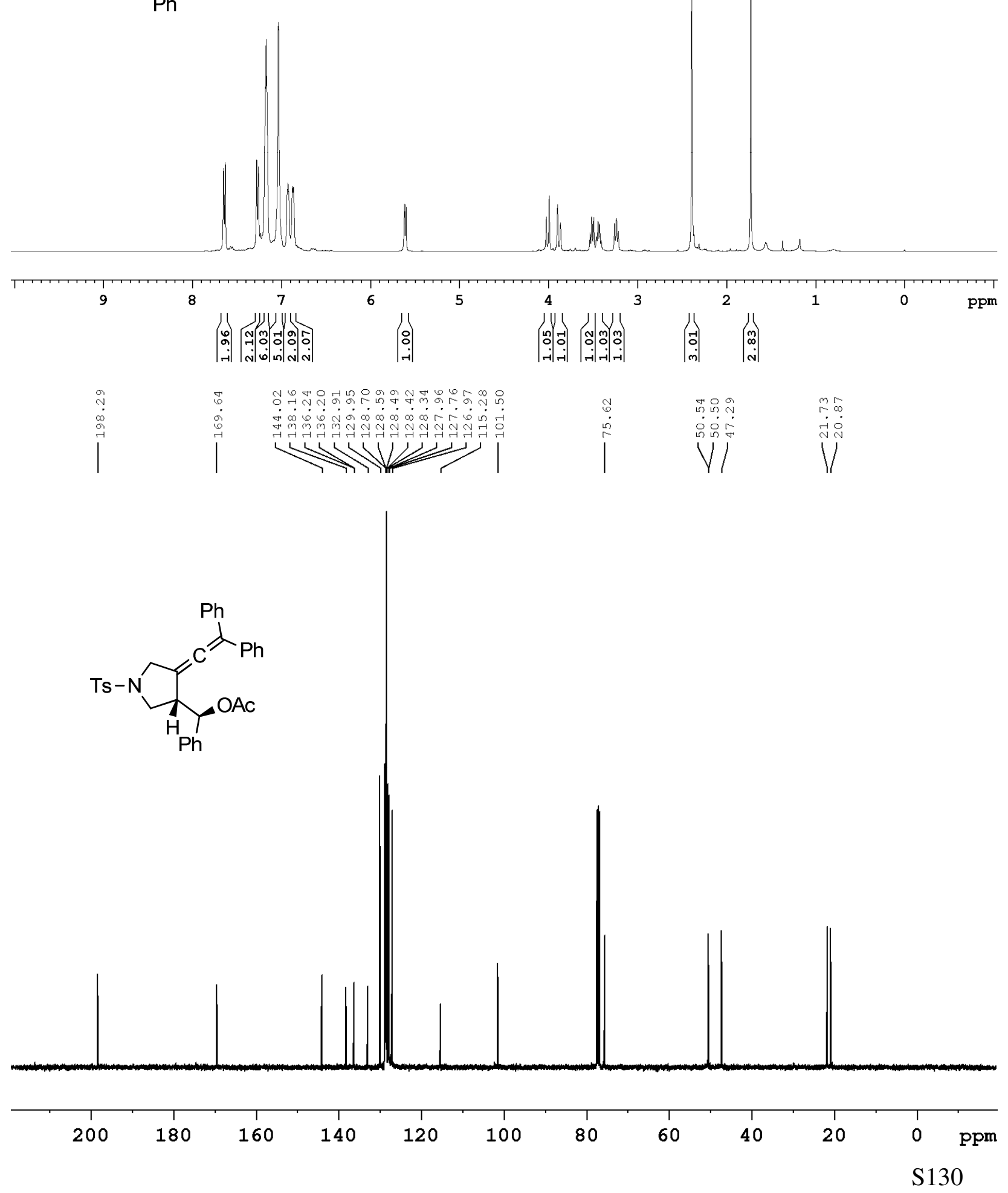
${ }^{1} \mathrm{H}$ - and ${ }^{13} \mathrm{C}$ NMR spectra of $\mathbf{1 4}$ (HPLC fraction 1)

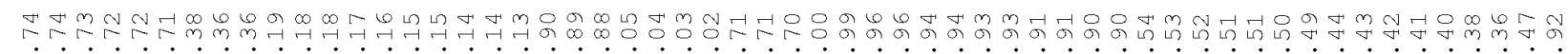
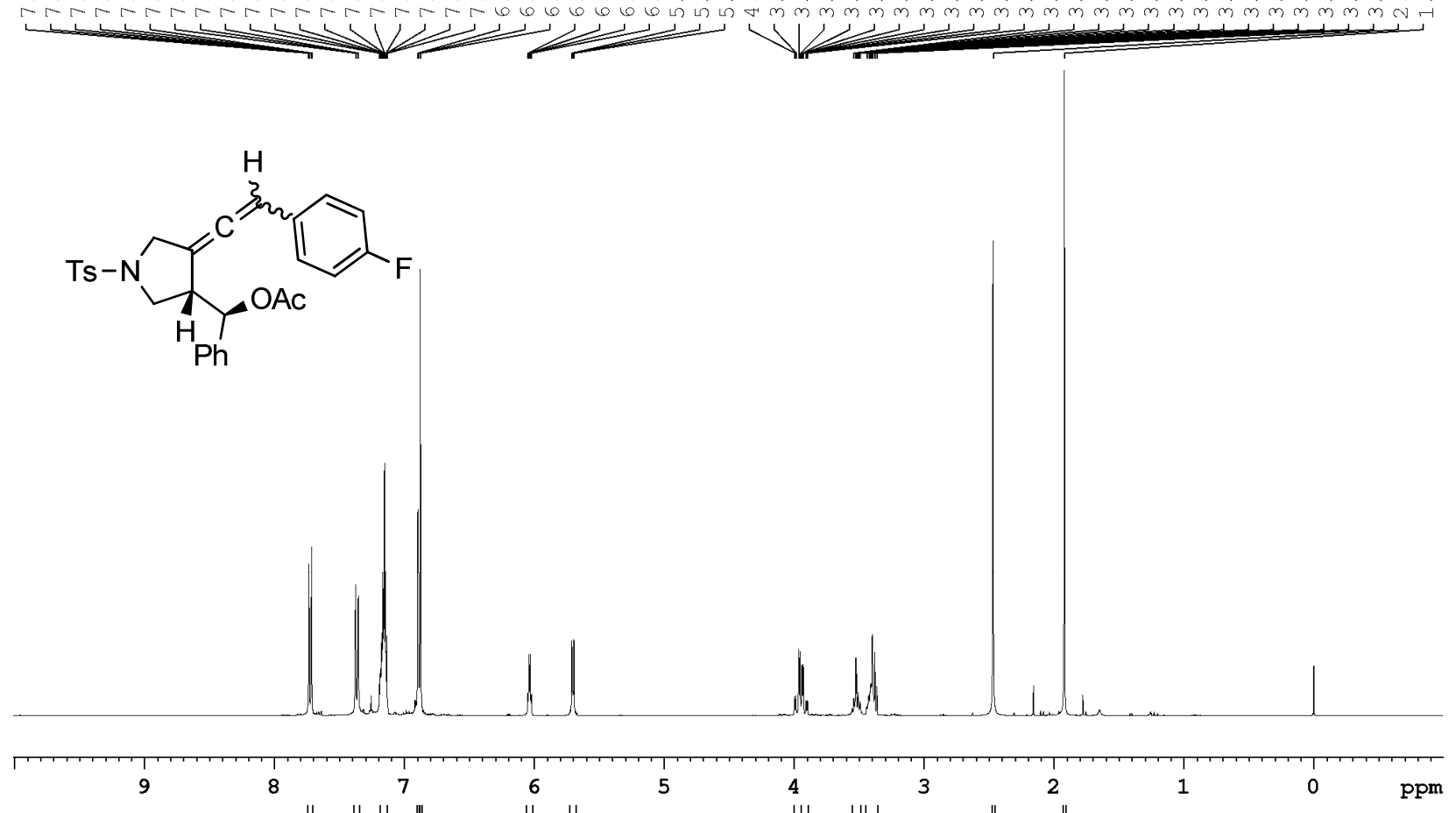

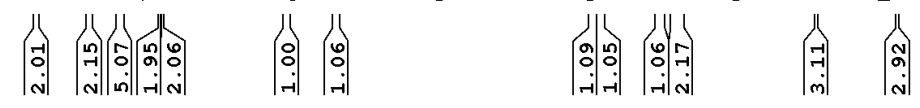

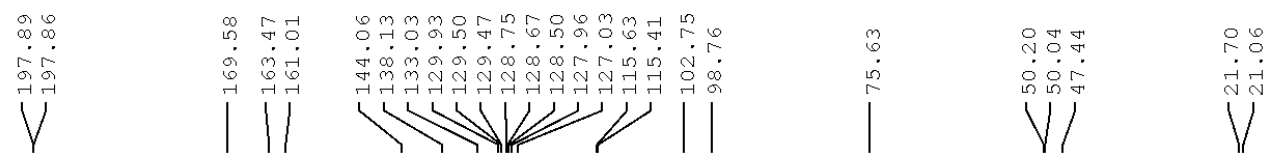

(c)

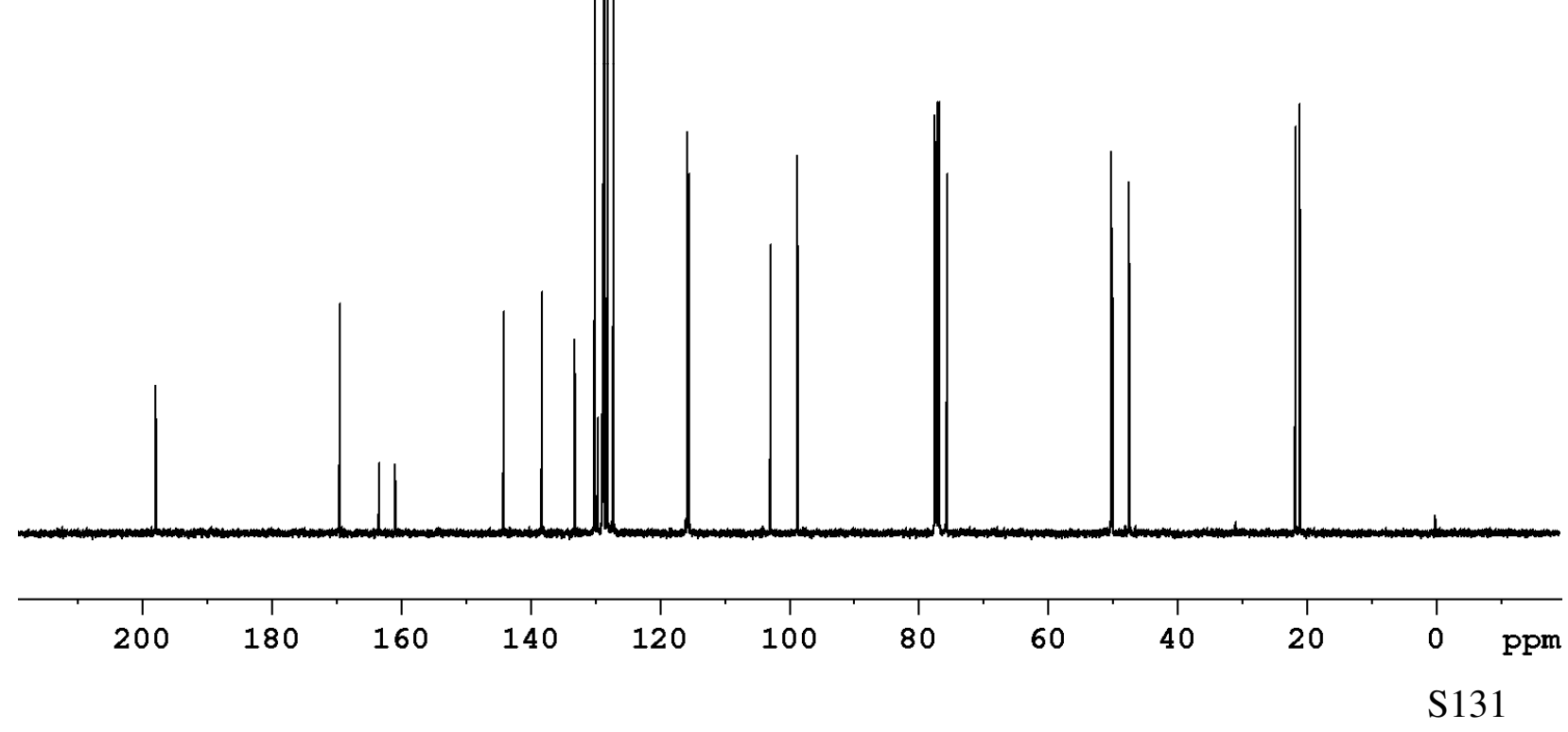


${ }^{1} \mathrm{H}$ - and ${ }^{13} \mathrm{C}$ NMR spectra of 14 (HPLC fraction 2)

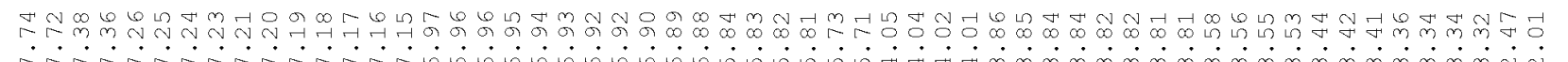

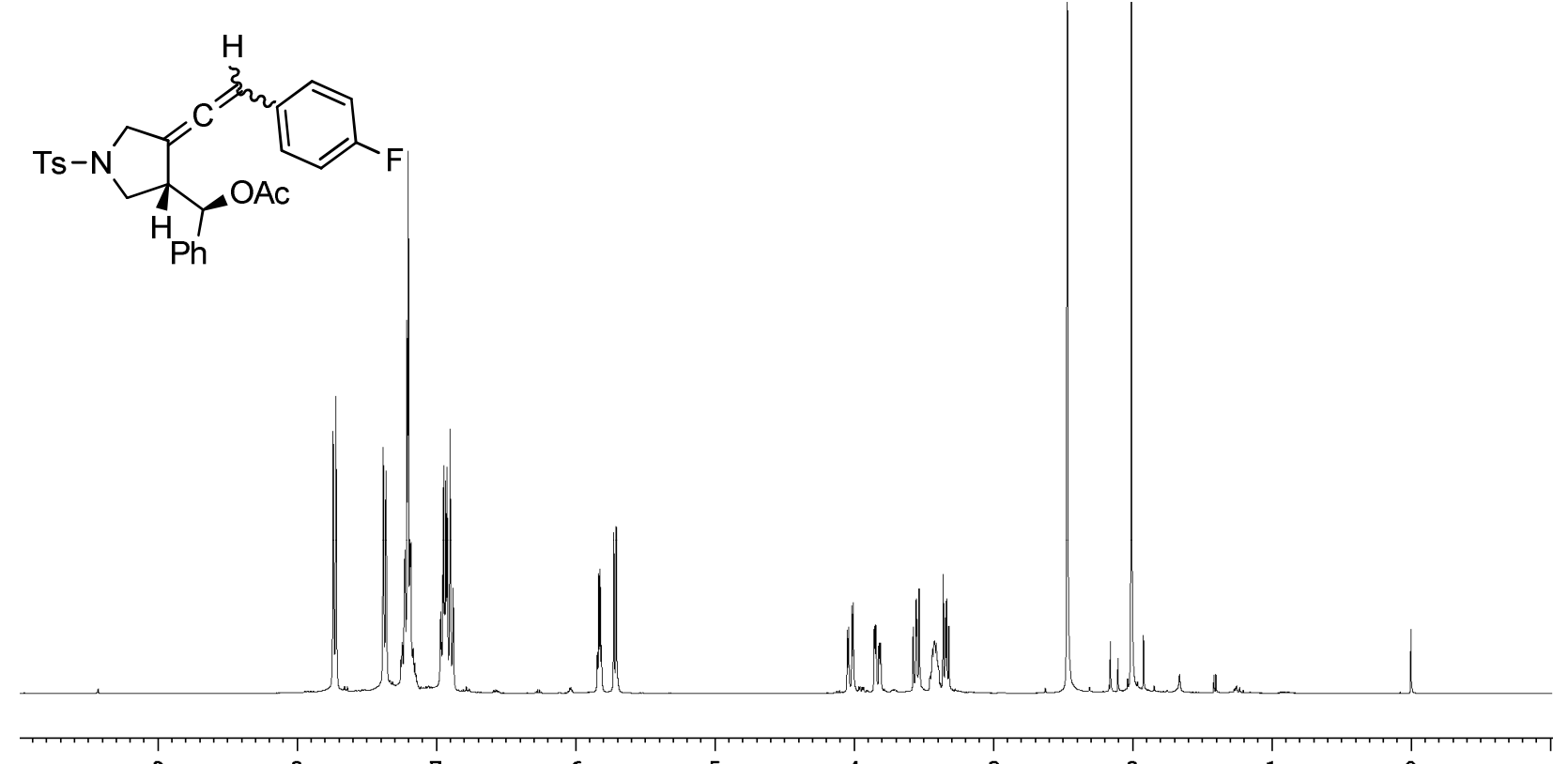

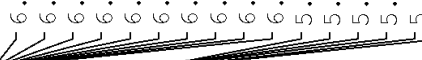

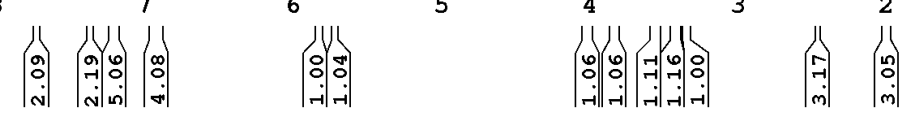

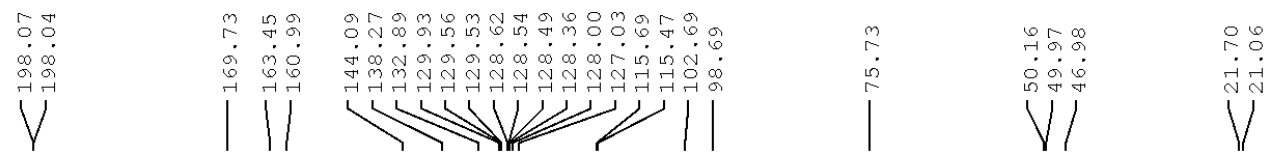

(c)

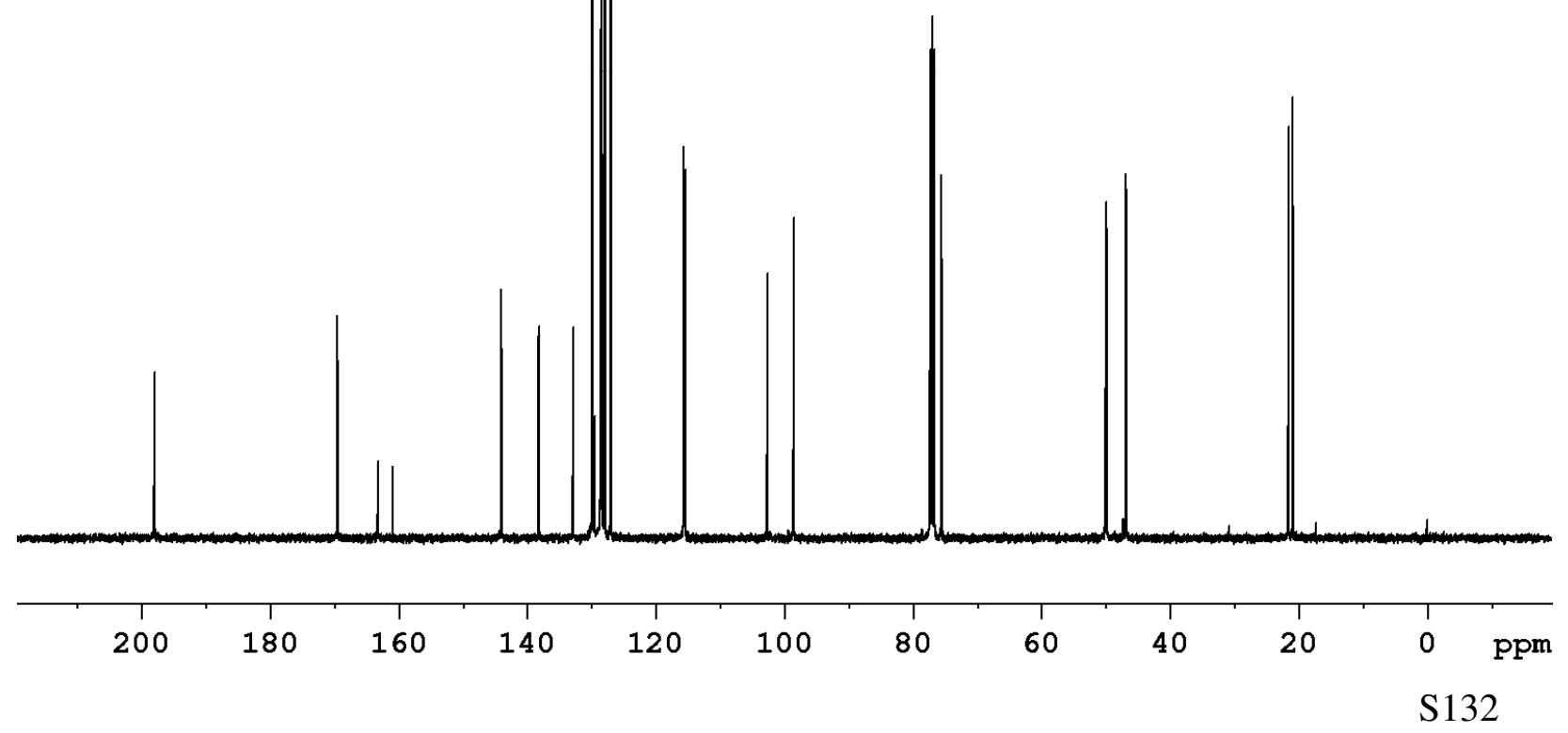


${ }^{1} \mathrm{H}$ - and ${ }^{13} \mathrm{C}$ NMR spectra of 15 (HPLC fraction 1)

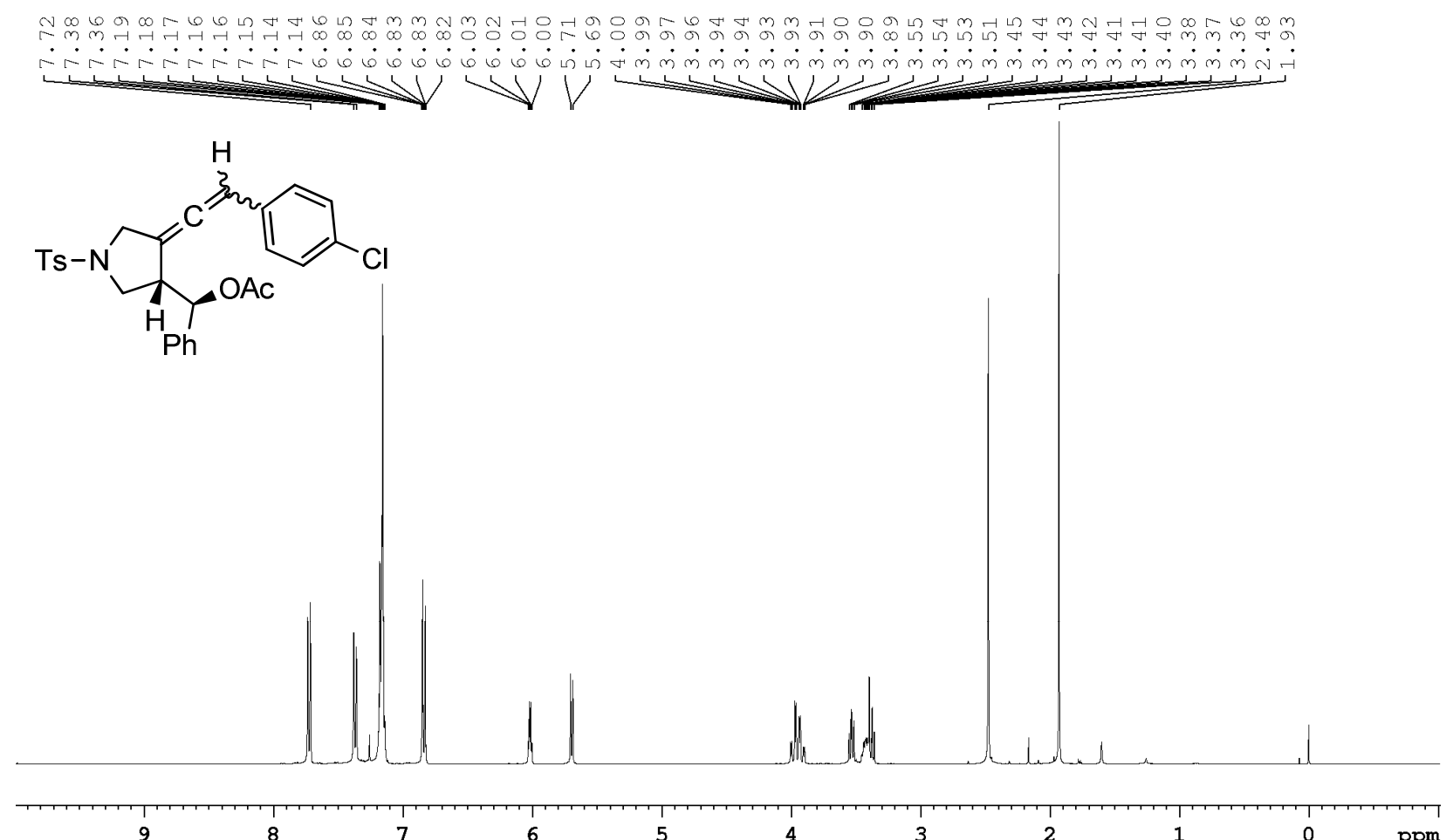

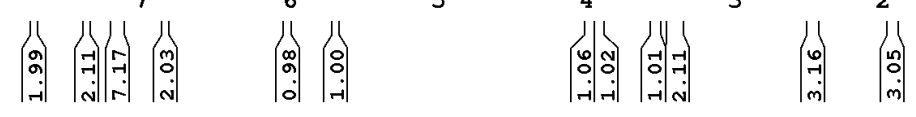

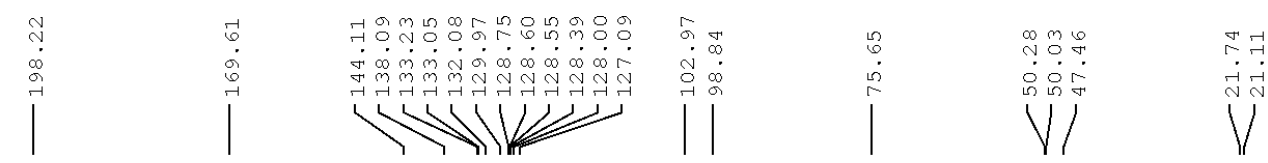

(c)

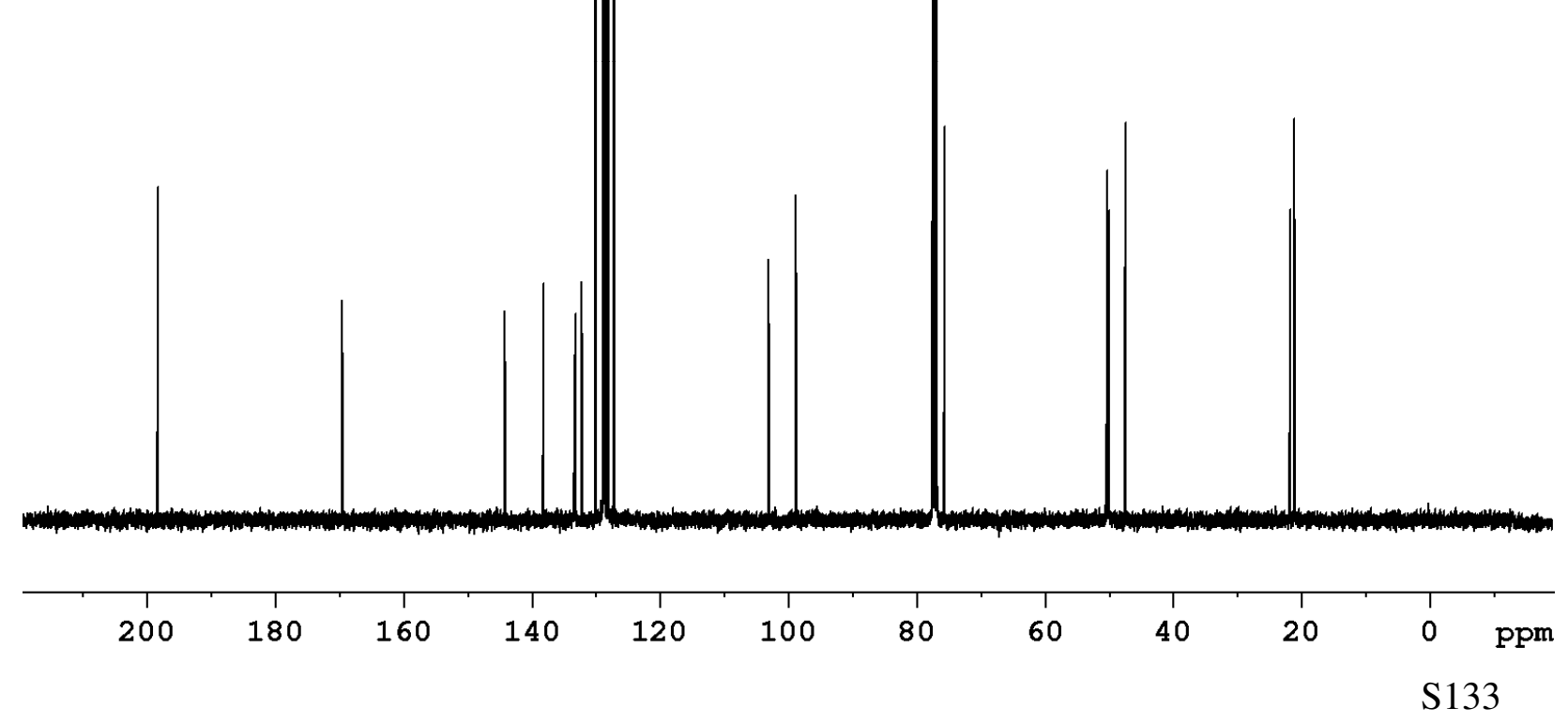


${ }^{1} \mathrm{H}$ - and ${ }^{13} \mathrm{C}$ NMR spectra of 15 (HPLC fraction 2)

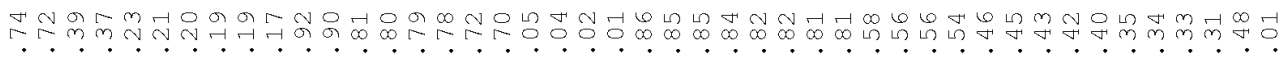

11<smiles>[3H]N1CC(=Cc2ccc(Cl)cc2)C(OC(=O)O)C1c1ccccc1</smiles>

Whlld

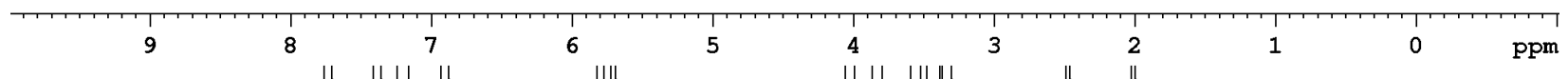

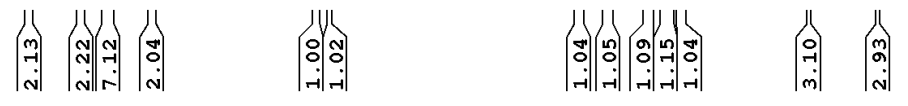

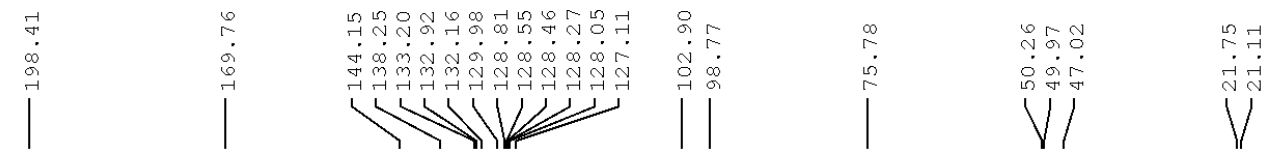<smiles>[3H]N1CC(=Cc2ccc(Cl)cc2)C(OC(=O)c2ccccc2)C1c1ccccc1</smiles>
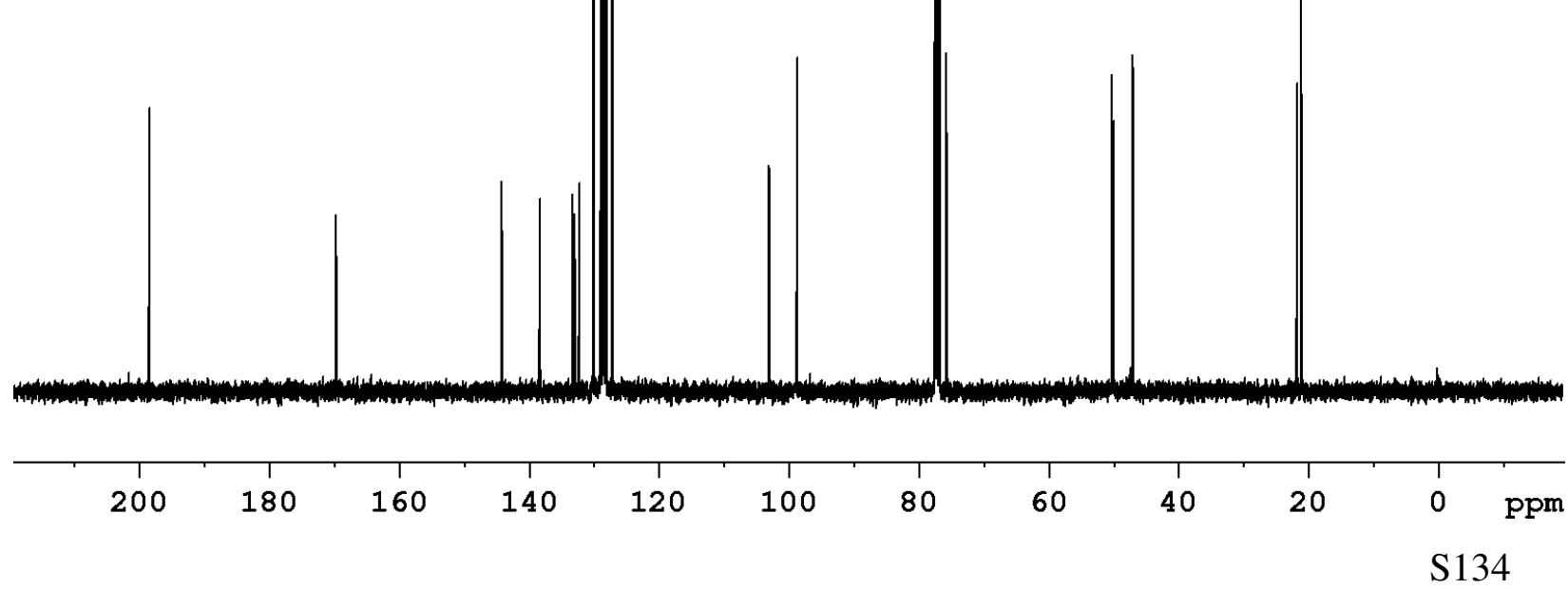
${ }^{1} \mathrm{H}$ - and ${ }^{13} \mathrm{C}$ NMR spectra of $\mathbf{1 6}$ (HPLC fraction 1)

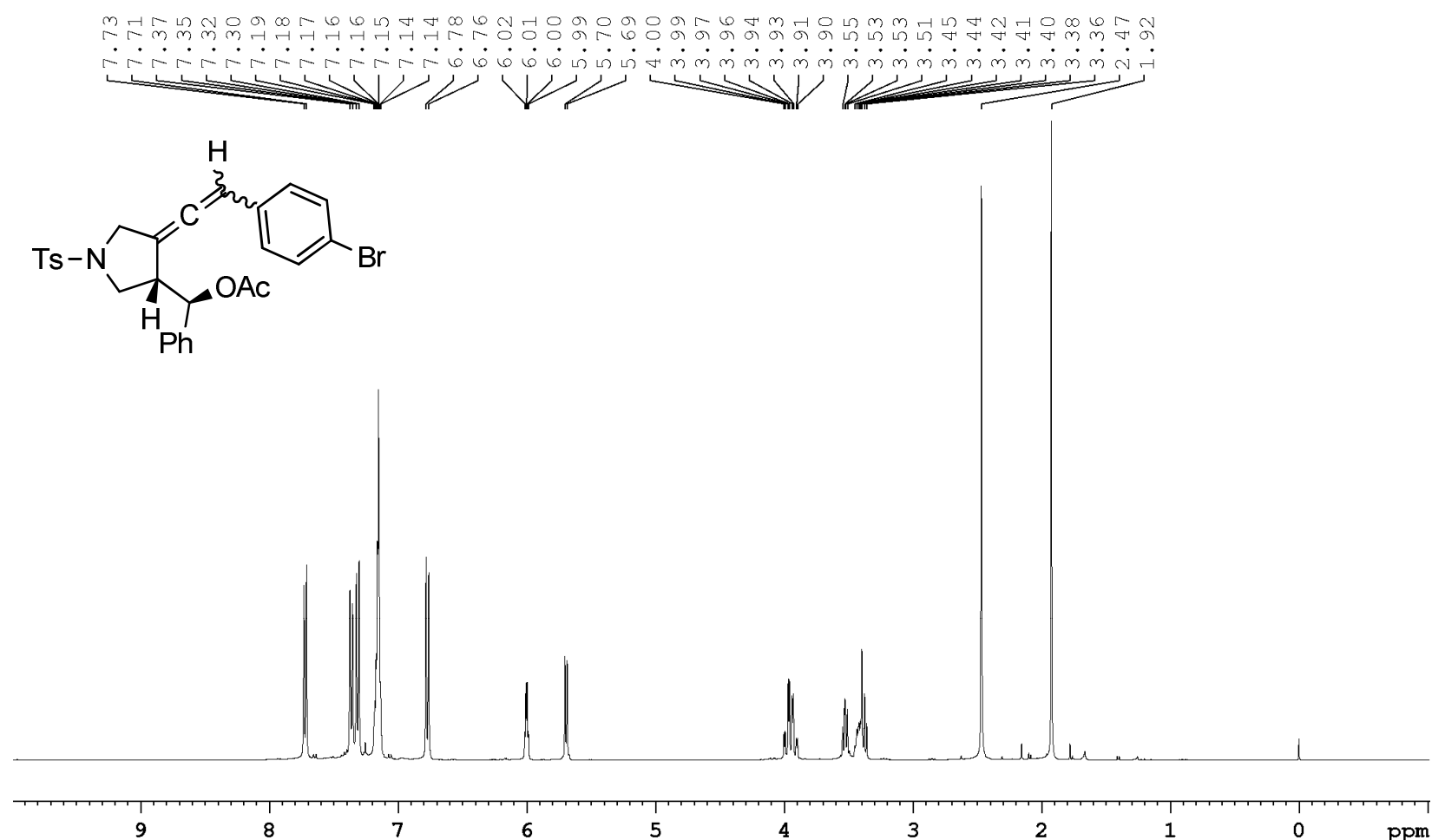

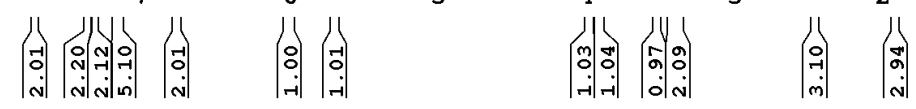

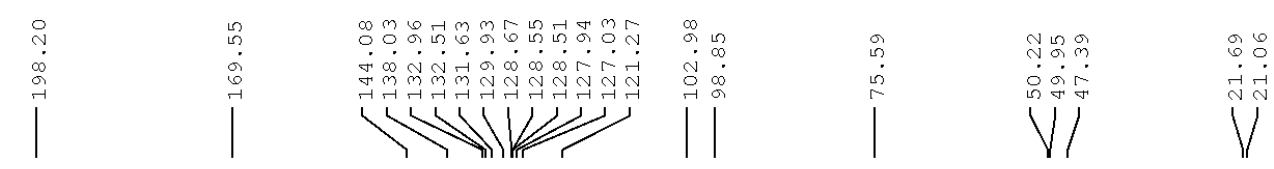

(c)

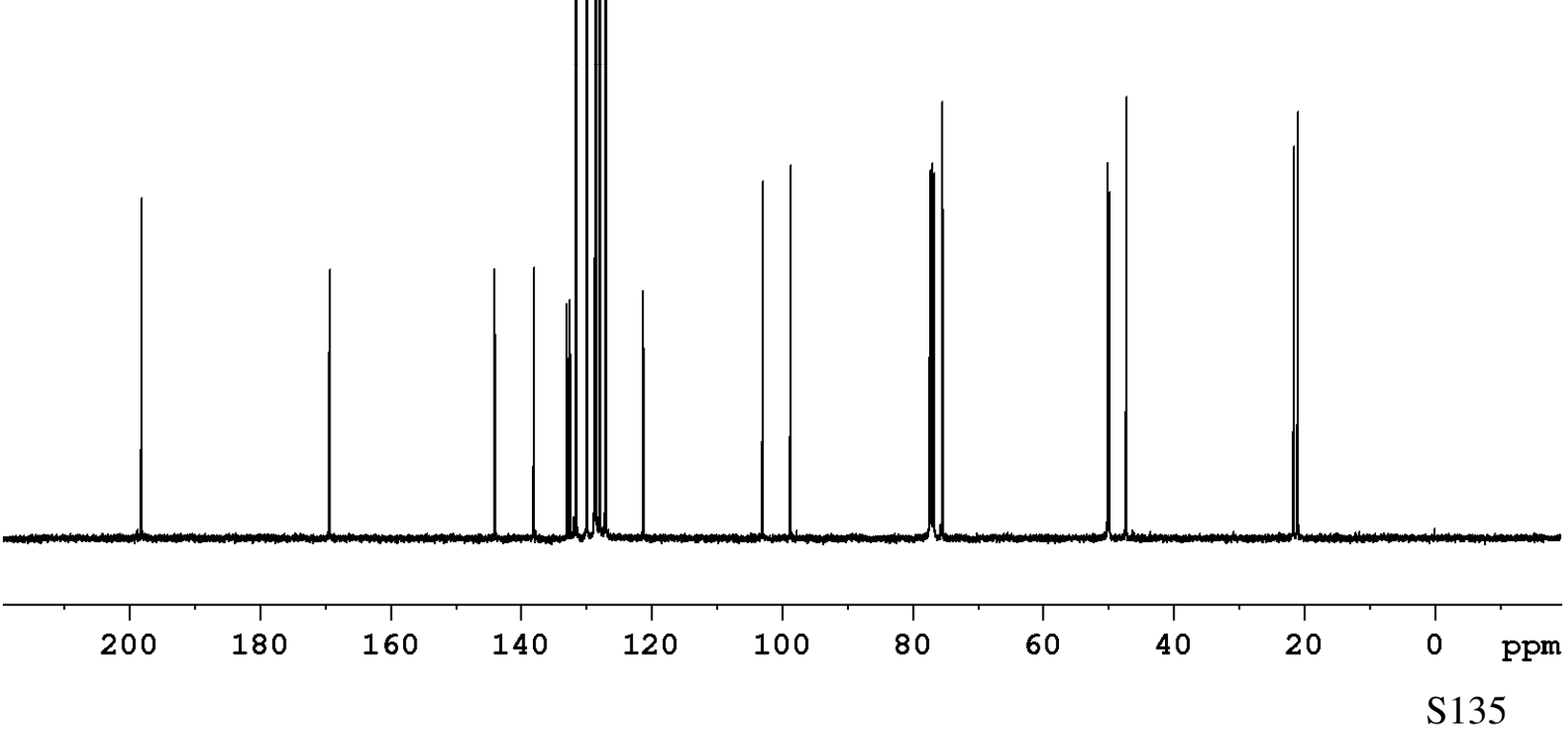


${ }^{1} \mathrm{H}$ - and ${ }^{13} \mathrm{C}$ NMR spectra of $\mathbf{1 6}$ (HPLC fraction 2)

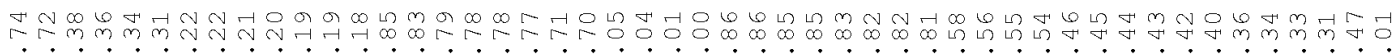

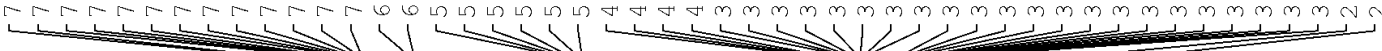

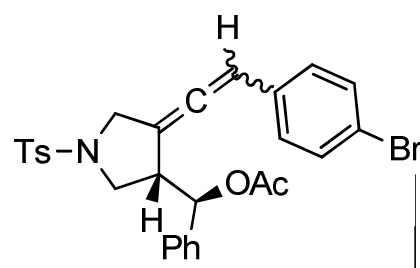

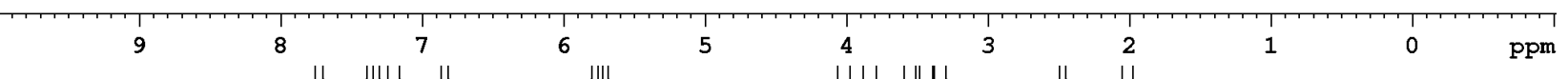

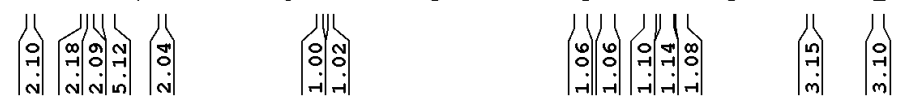
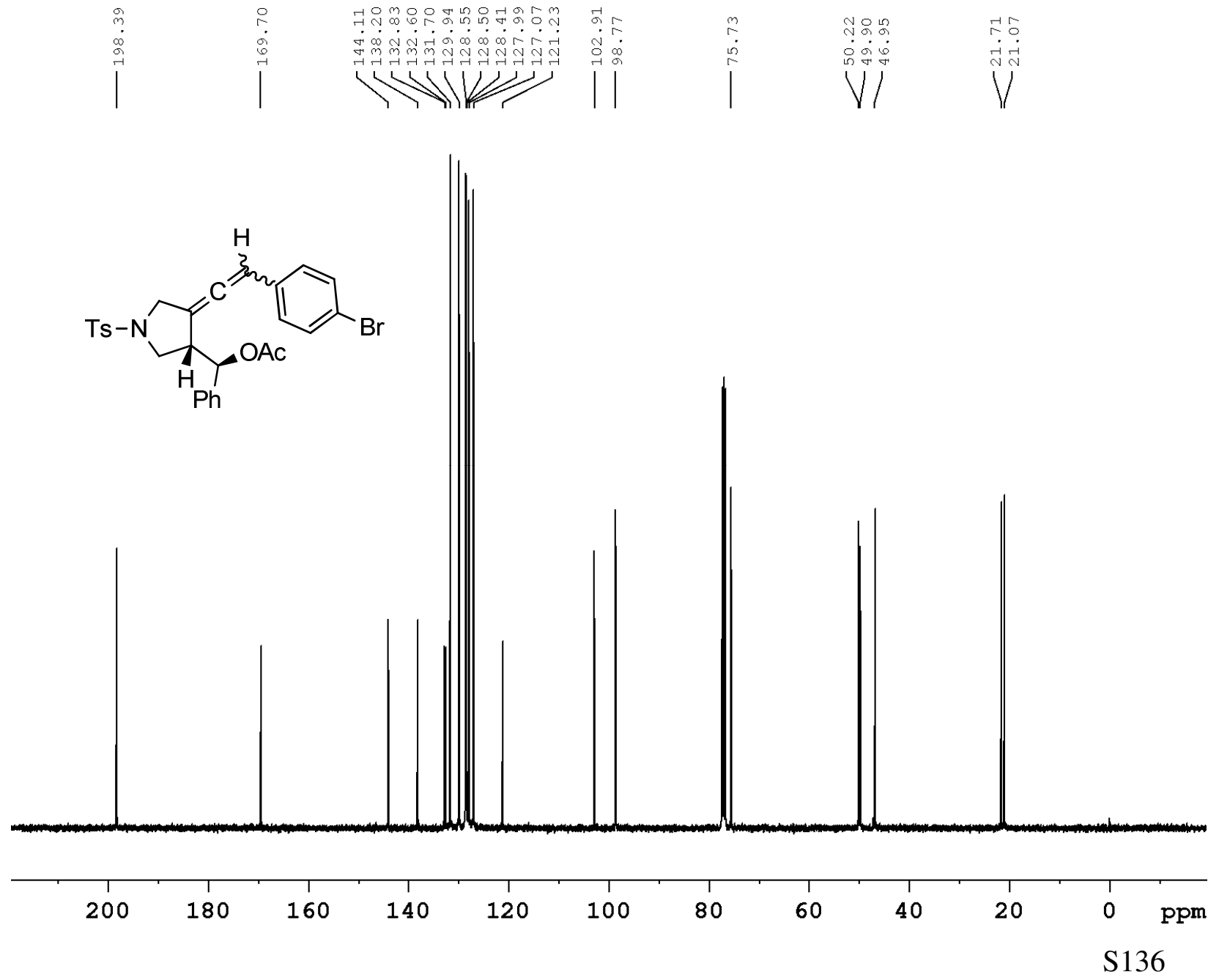
${ }^{1} \mathrm{H}-$ and ${ }^{13} \mathrm{C}$ NMR spectra of $\mathbf{1 7}$

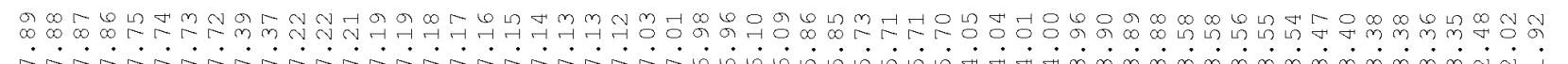

بc

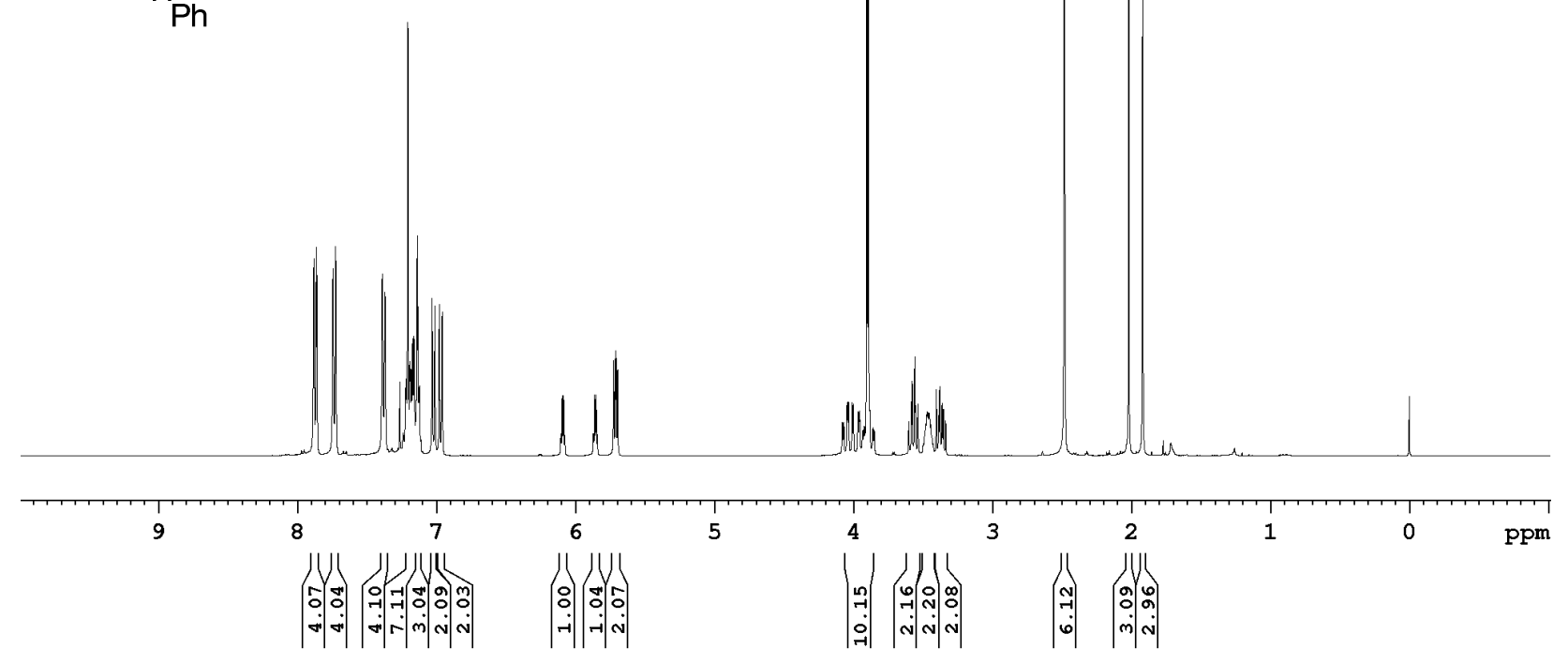

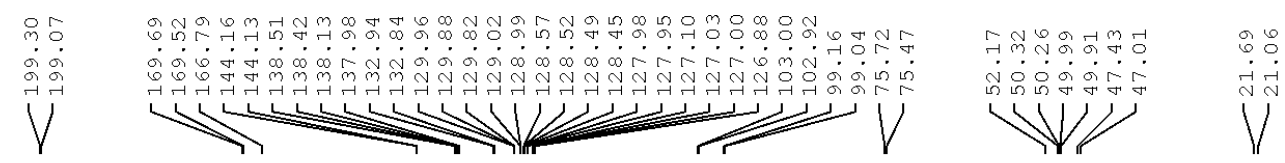

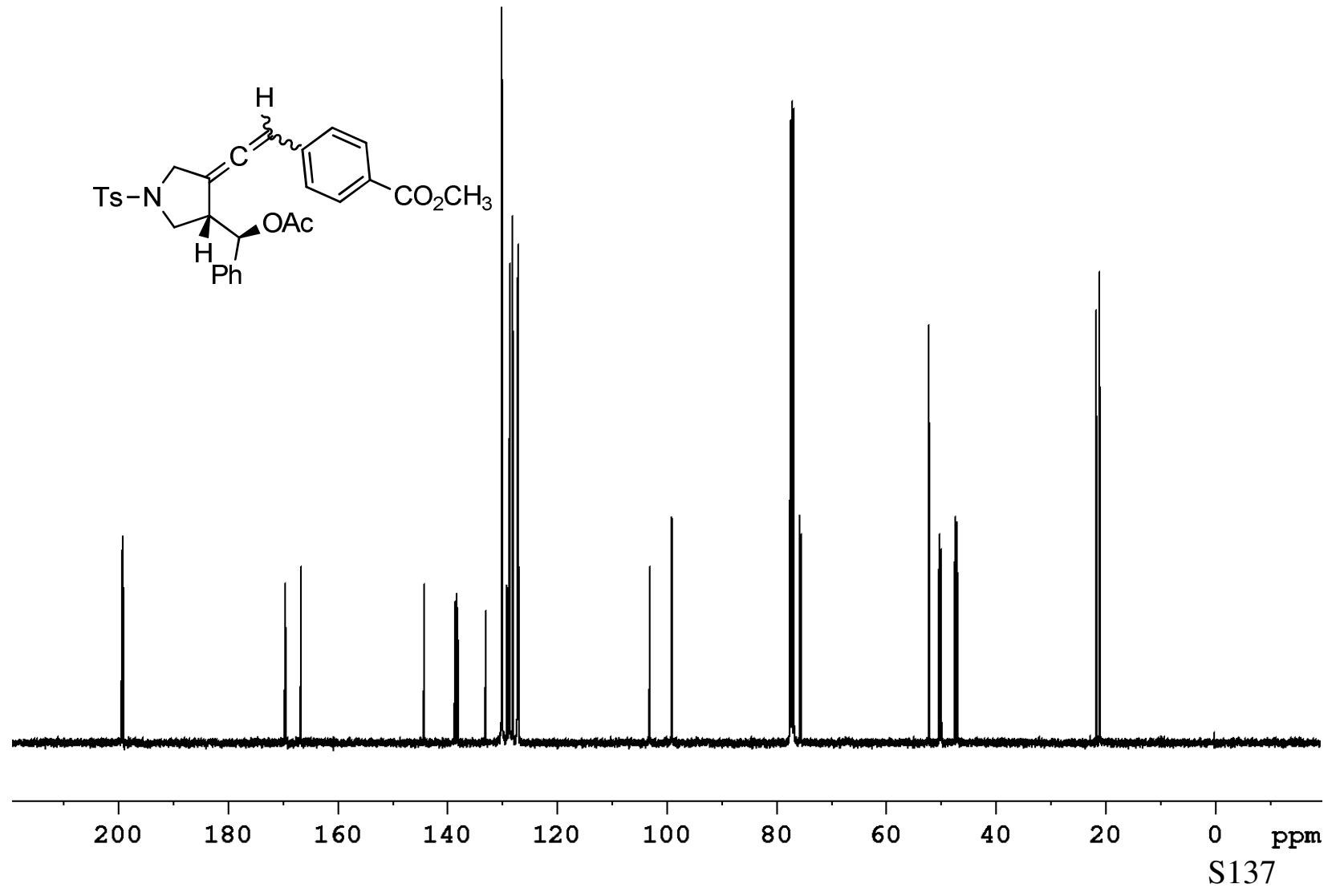


${ }^{1} \mathrm{H}$ - and ${ }^{13} \mathrm{C}$ NMR spectra of $\mathbf{1 8}$

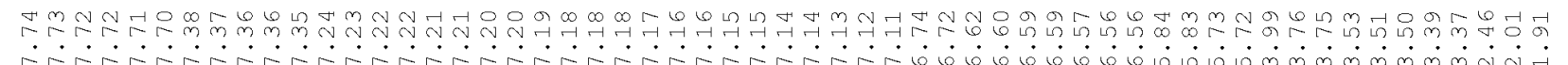

(N)

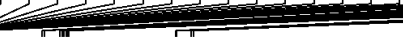
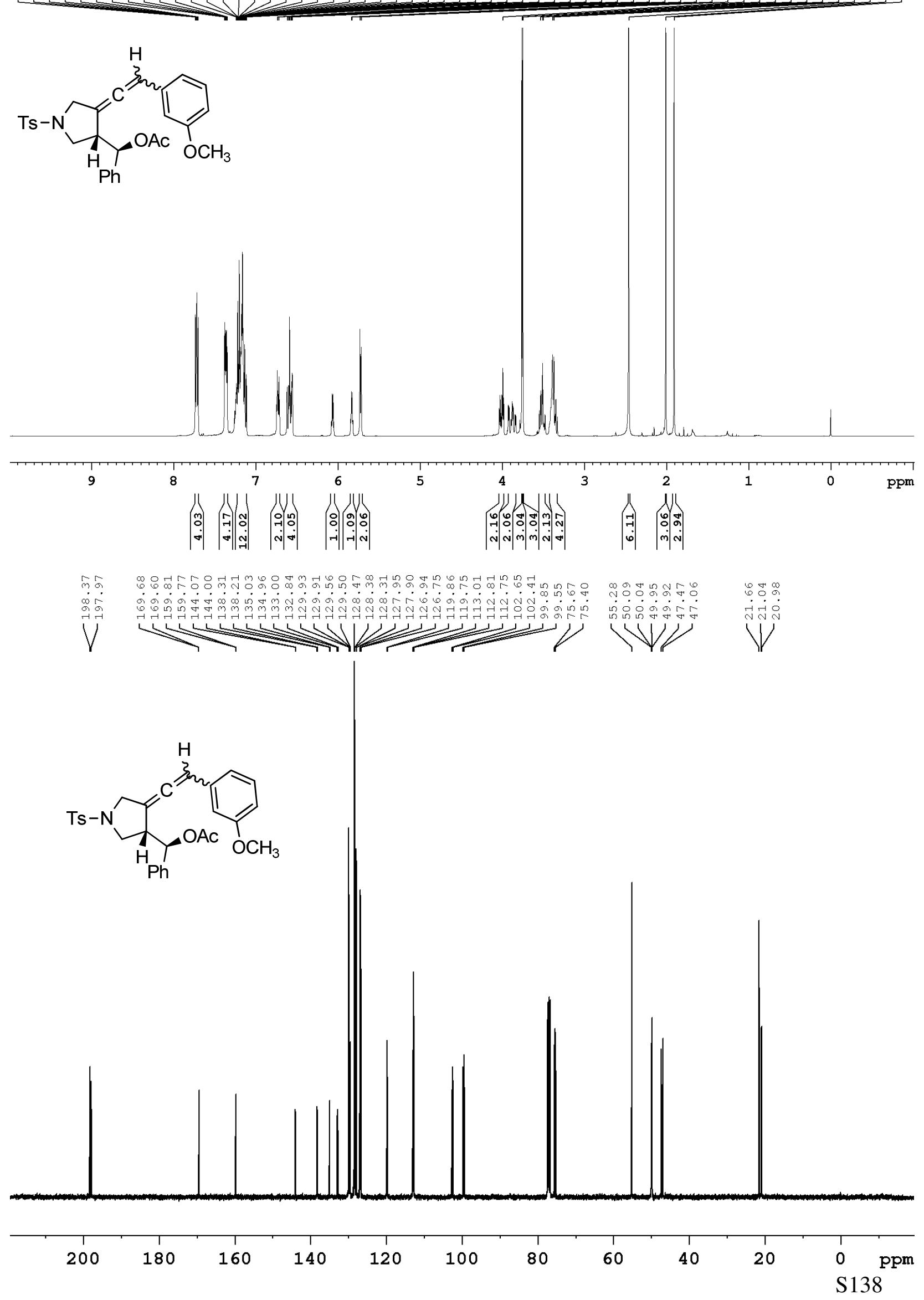
${ }^{1} \mathrm{H}$ - and ${ }^{13} \mathrm{C}$ NMR spectra of $\mathbf{1 9}$

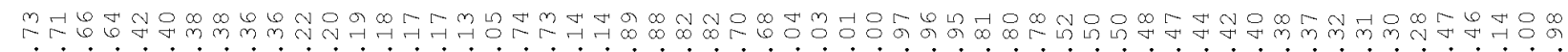
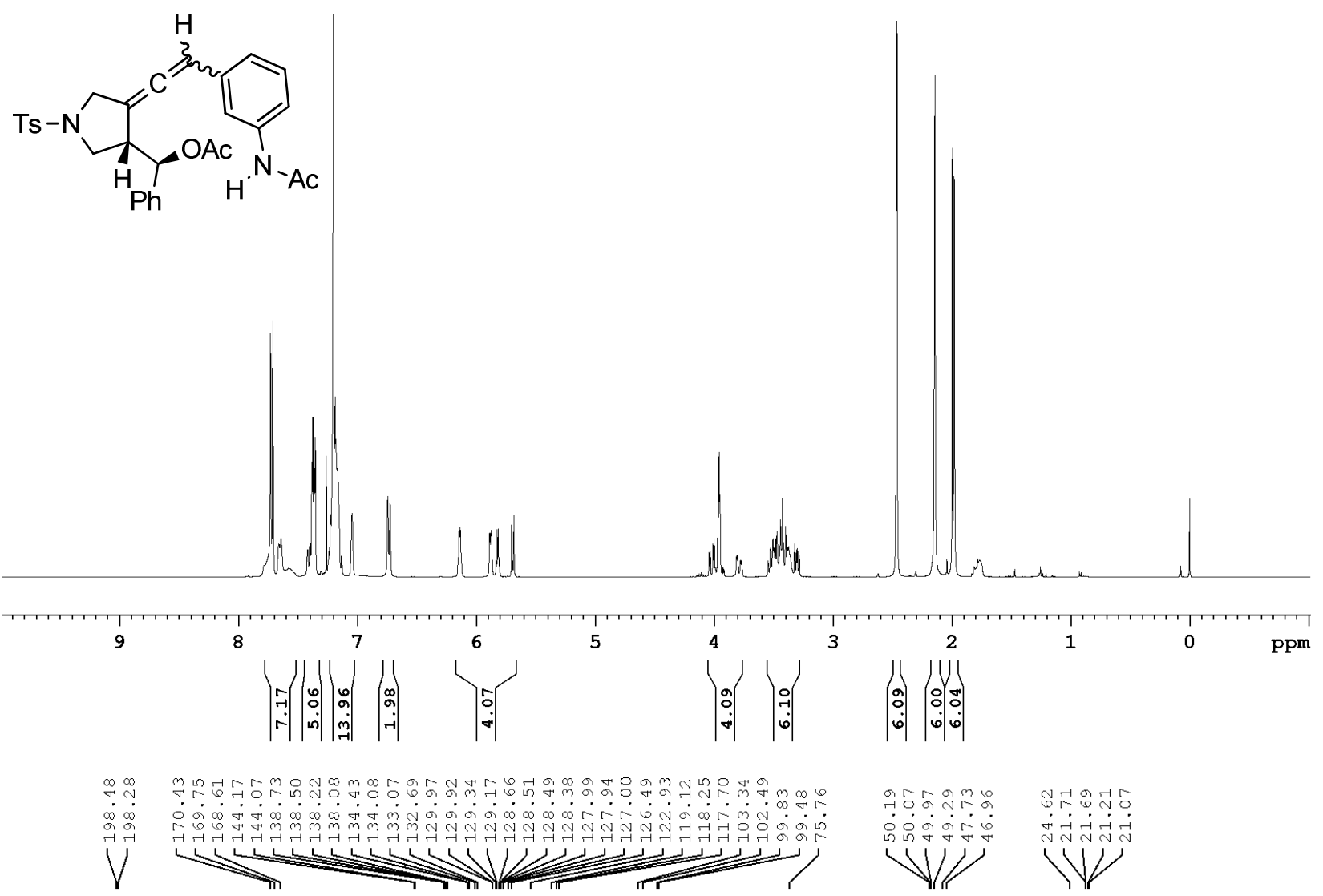

$\underbrace{O A C}_{P h}$

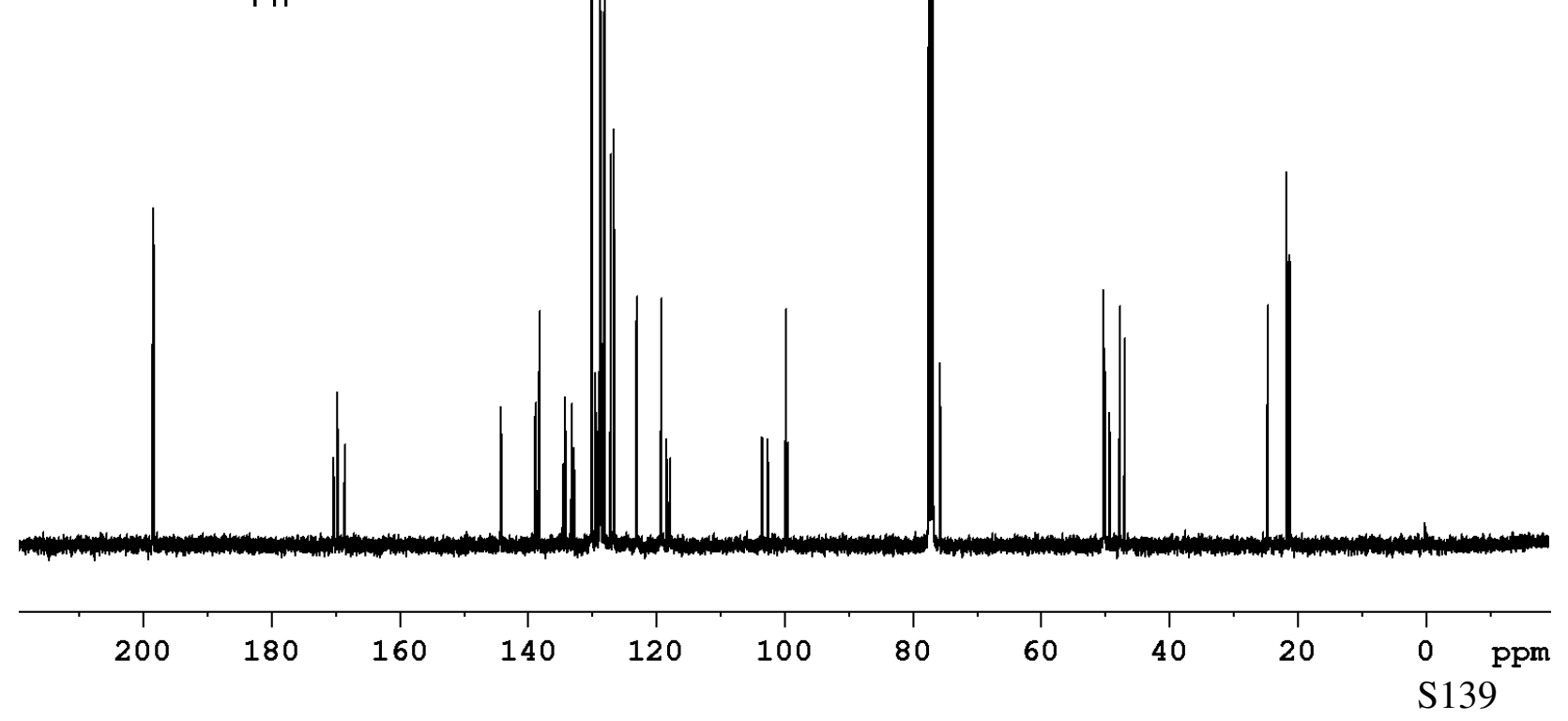


${ }^{1} \mathrm{H}$ - and ${ }^{13} \mathrm{C}$ NMR spectra of $\mathbf{2 0}$

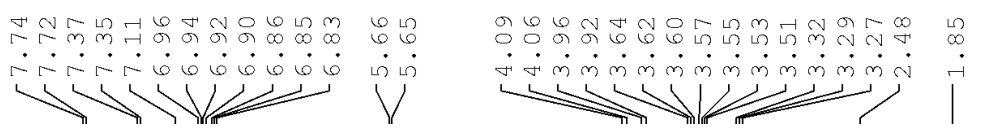

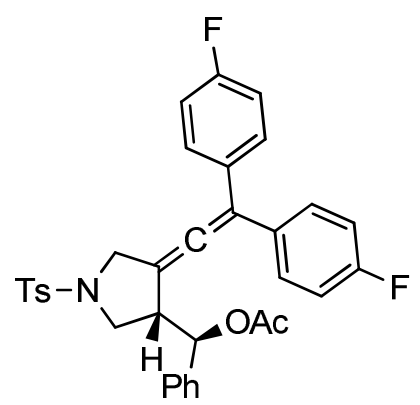

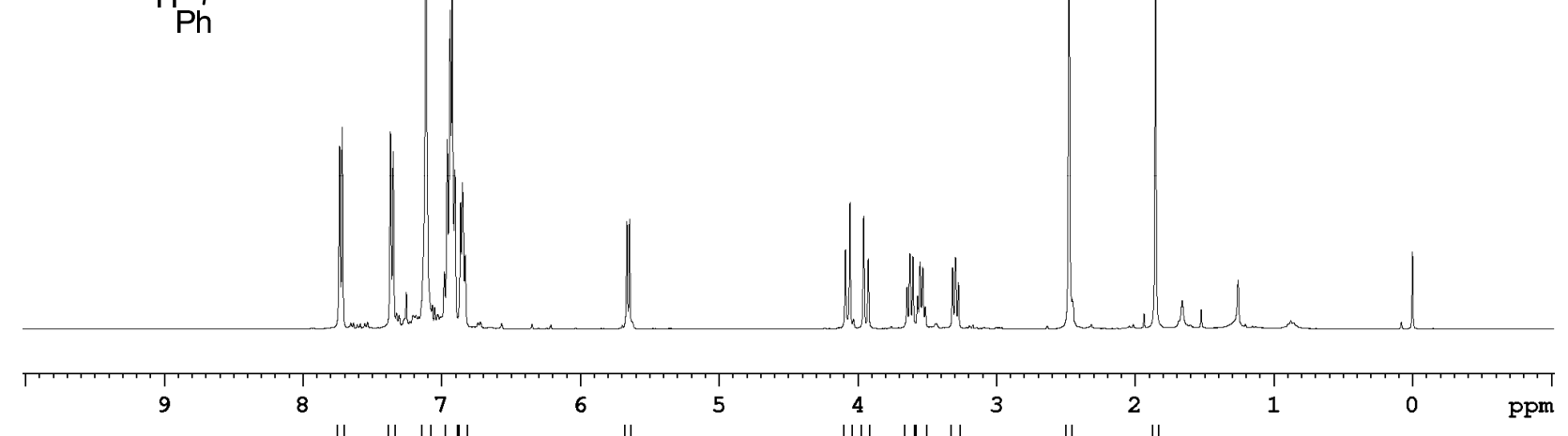

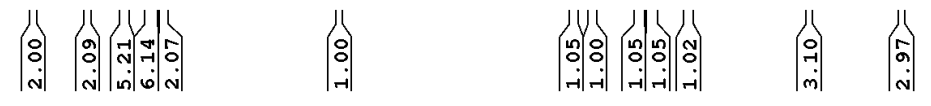

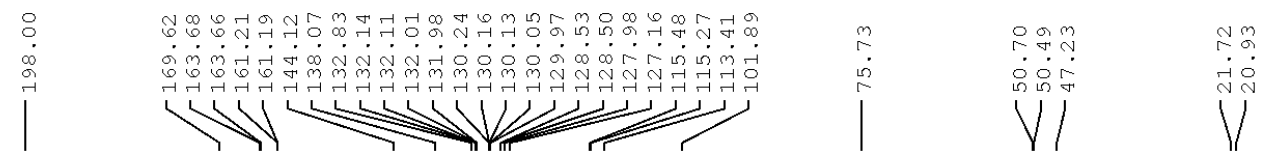

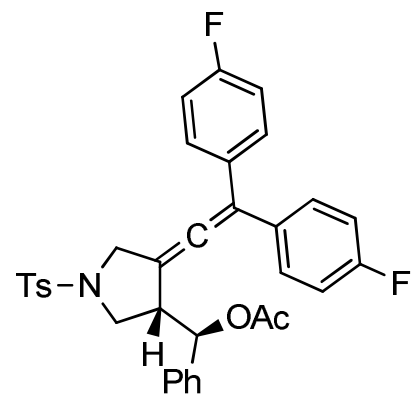

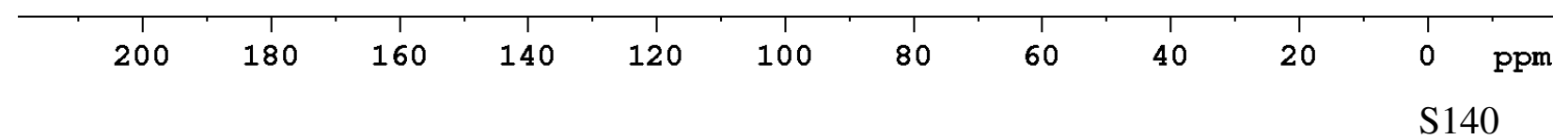


${ }^{1} \mathrm{H}$ - and ${ }^{13} \mathrm{C}$ NMR spectra of 21 (HPLC fraction 1)

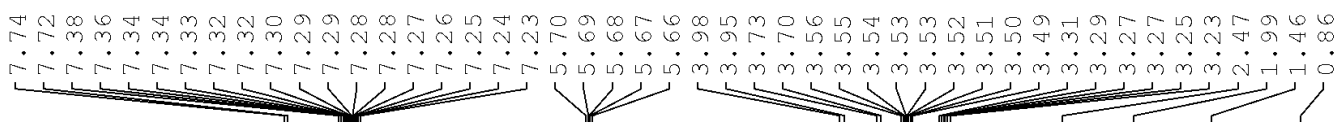

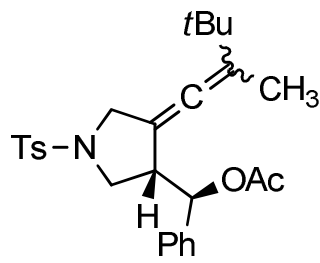
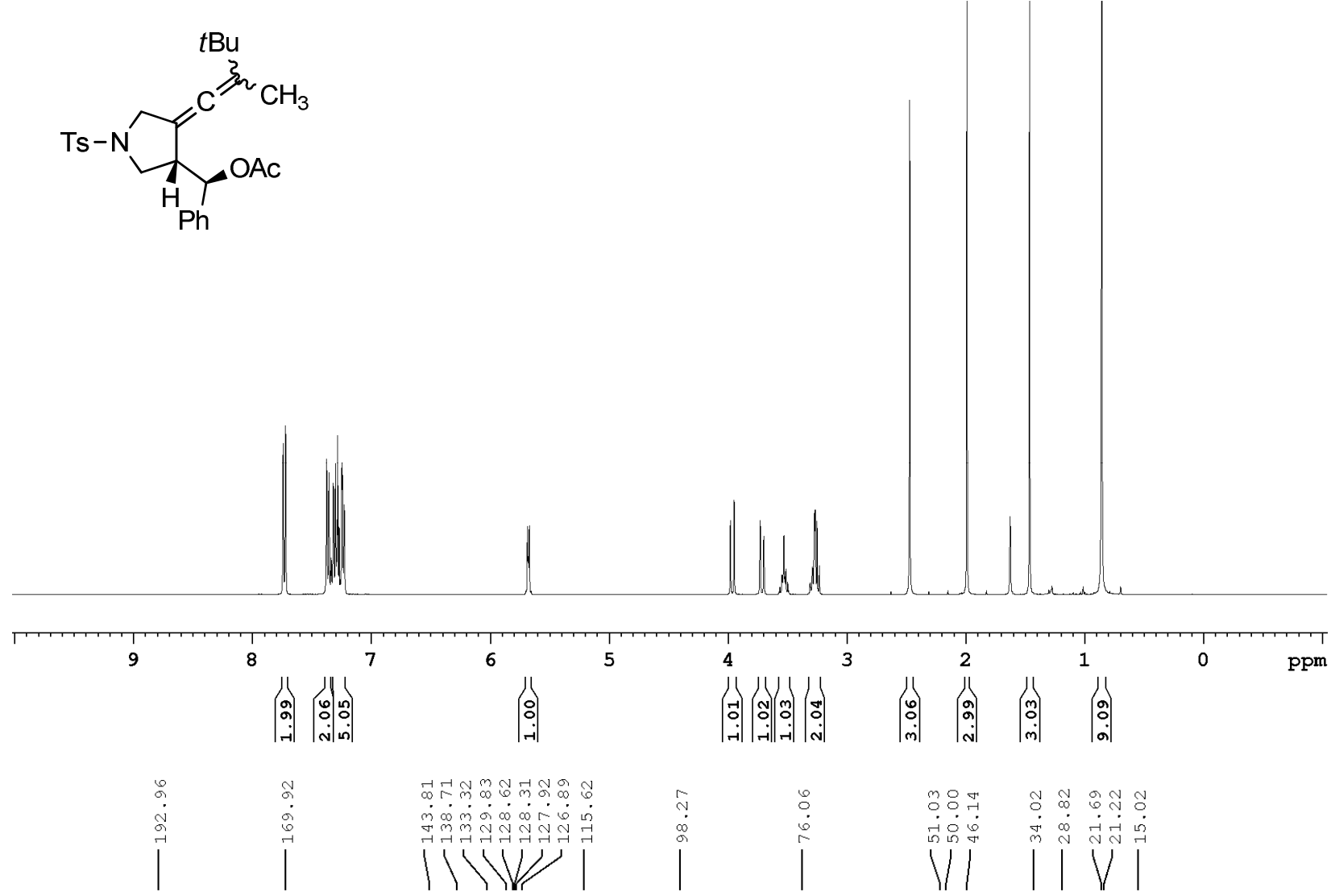

$\mid$

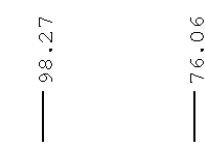

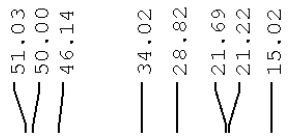<smiles>[B]N1C/C(=C\C(C)(C)C)[C@H]([C@H](OC(C)=O)c2ccccc2)C1</smiles>

$\mathrm{Ph}$

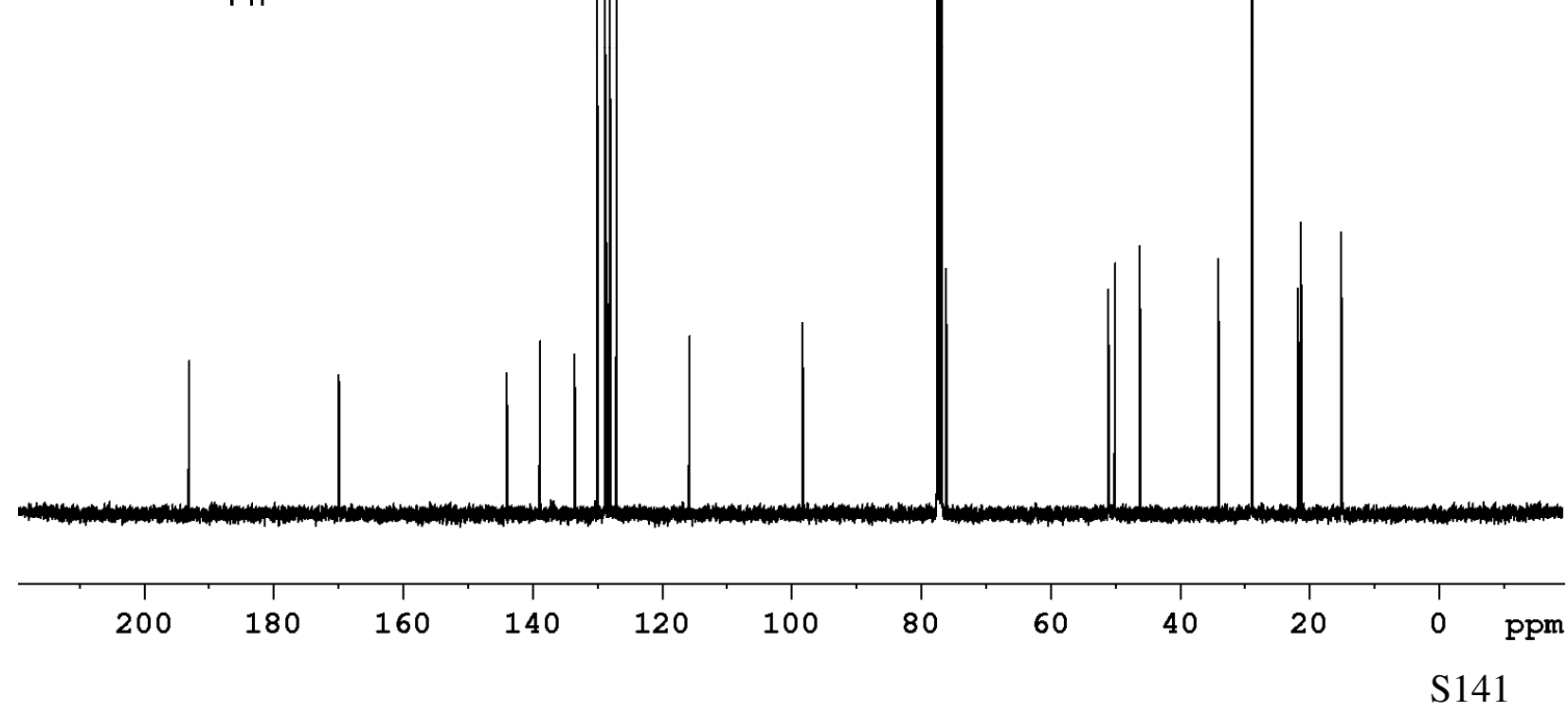


${ }^{1} \mathrm{H}$ - and ${ }^{13} \mathrm{C}$ NMR spectra of 21 (HPLC fraction 2)

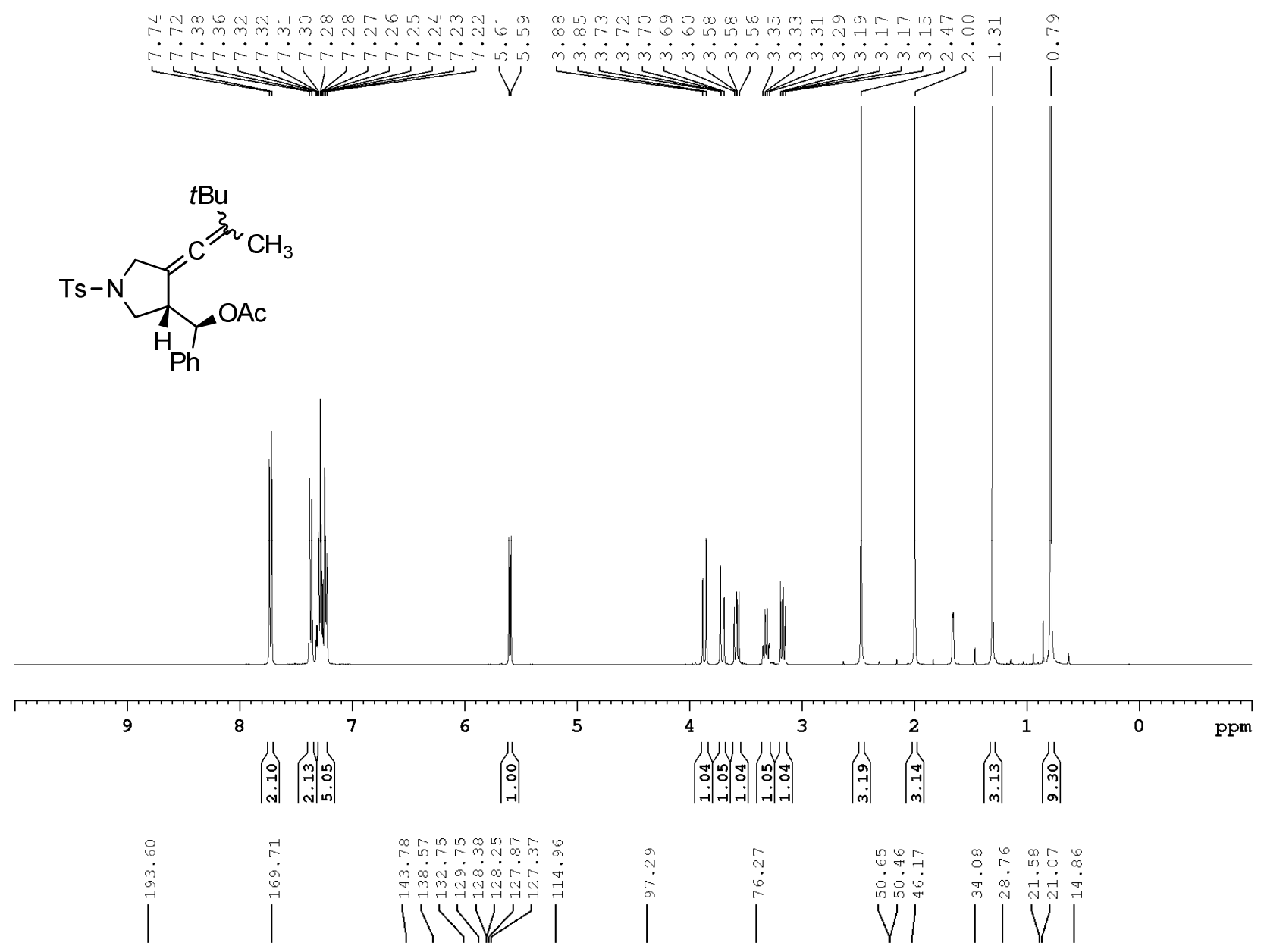<smiles>CC(=O)OC(c1ccccc1)C1CN([As])CC1=CC(C)(Br)Br</smiles>

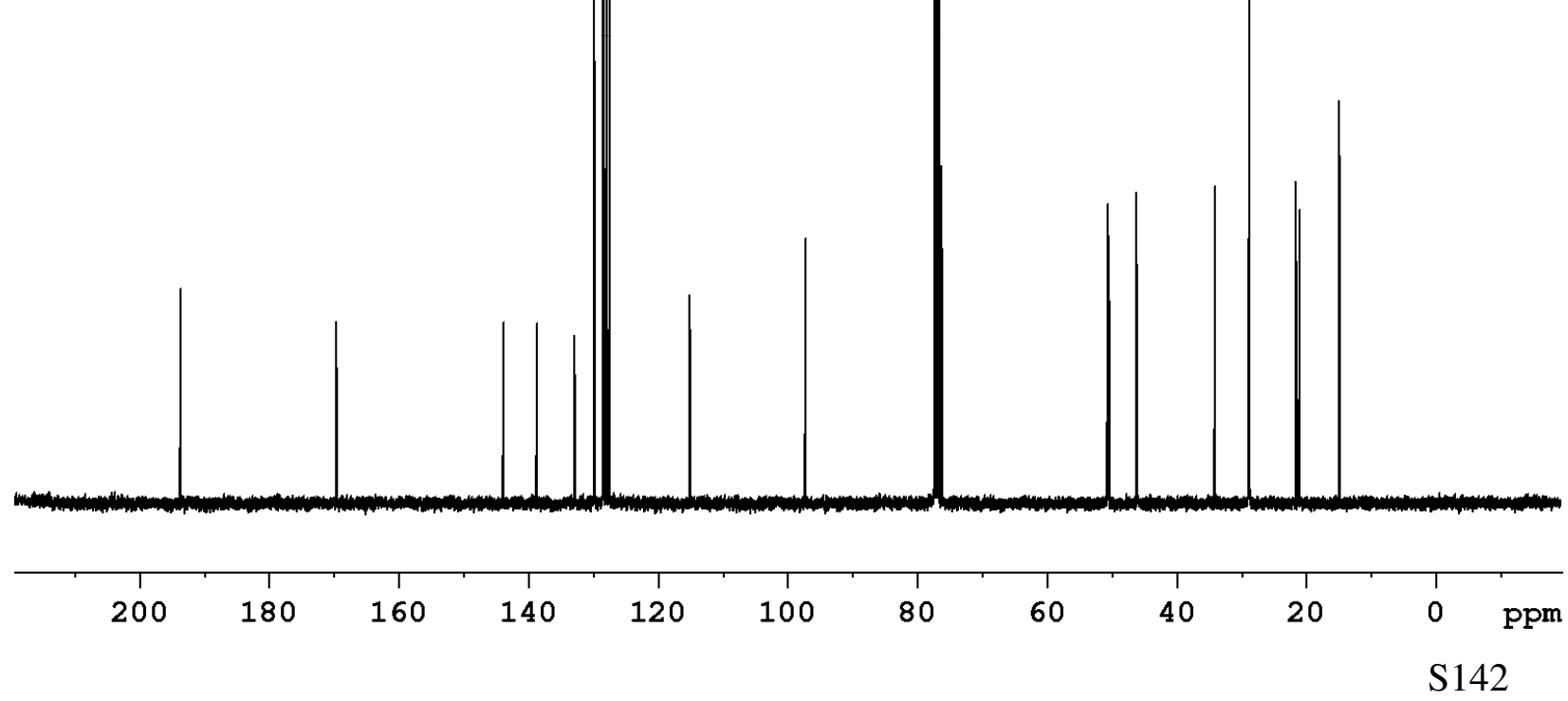


${ }^{1} \mathrm{H}-$ and ${ }^{13} \mathrm{C}$ NMR spectra of 22

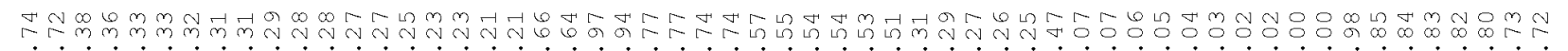

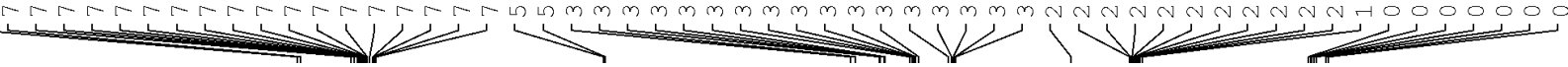<smiles>CCCC(=O)OC(c1ccccc1)C1CN(C)CC1=CC(C)CC</smiles>
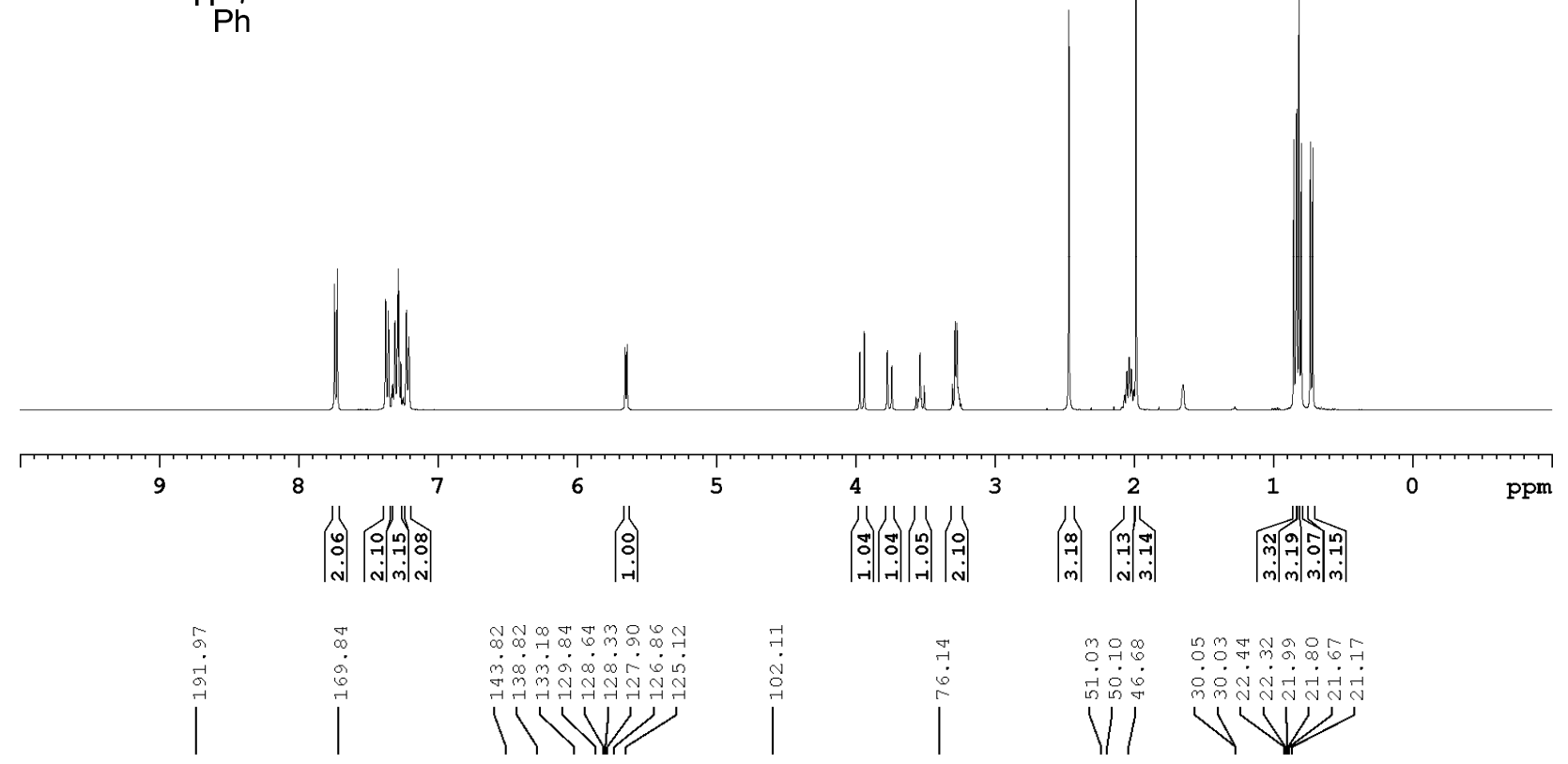

$\underbrace{i P r}_{P A C}$

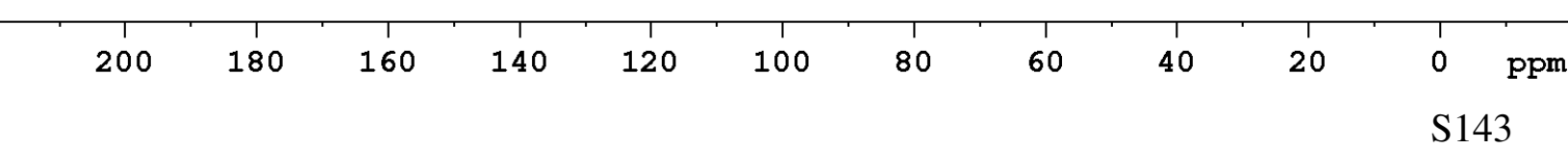


${ }^{1} \mathrm{H}-$ and ${ }^{13} \mathrm{C}$ NMR spectra of $\mathbf{2 3}$ (HPLC fraction 1)

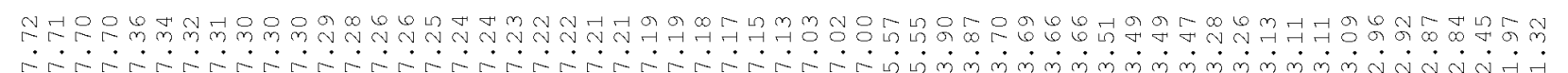
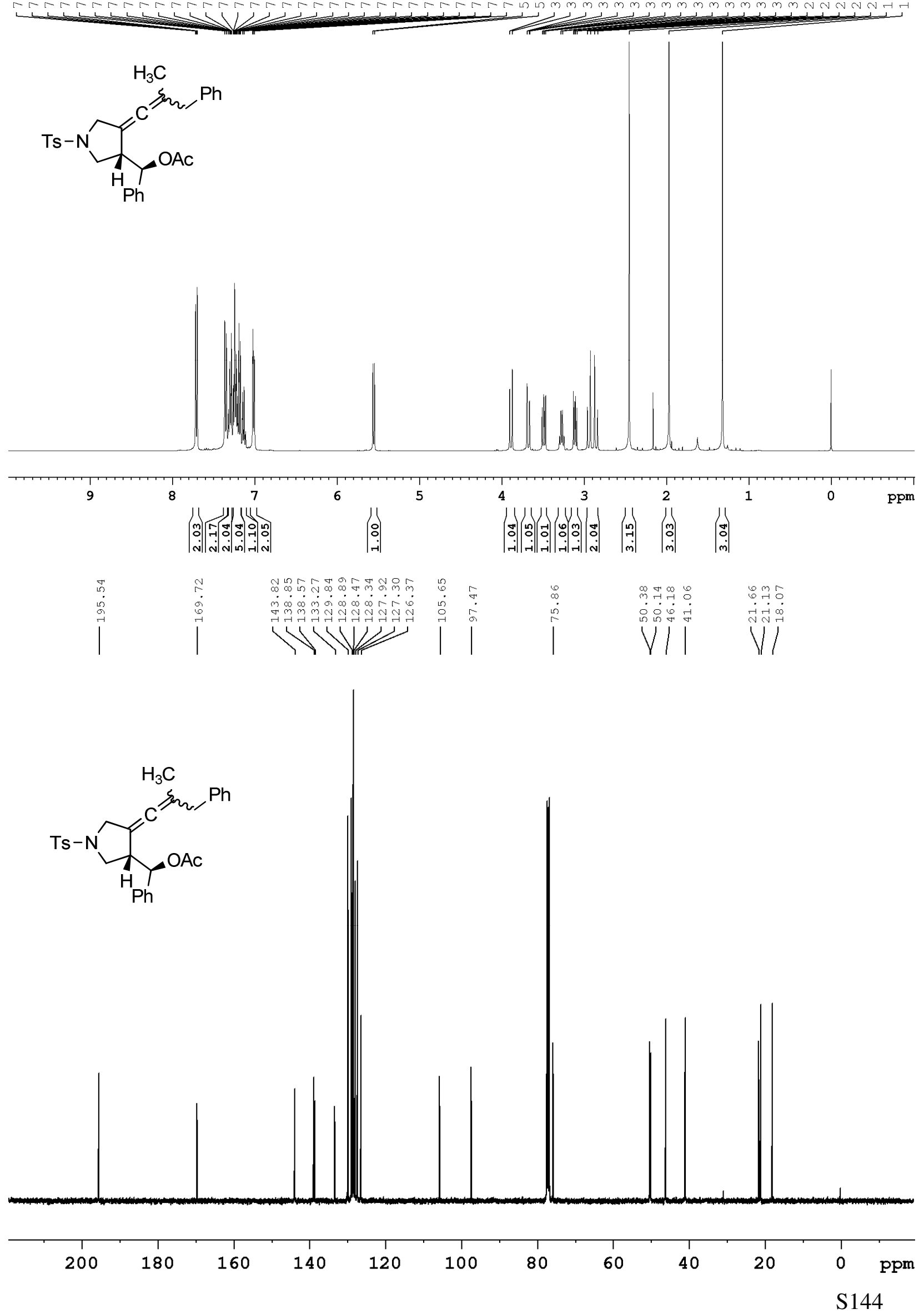
${ }^{1} \mathrm{H}$ - and ${ }^{13} \mathrm{C}$ NMR spectra of 23 (HPLC fraction 2)
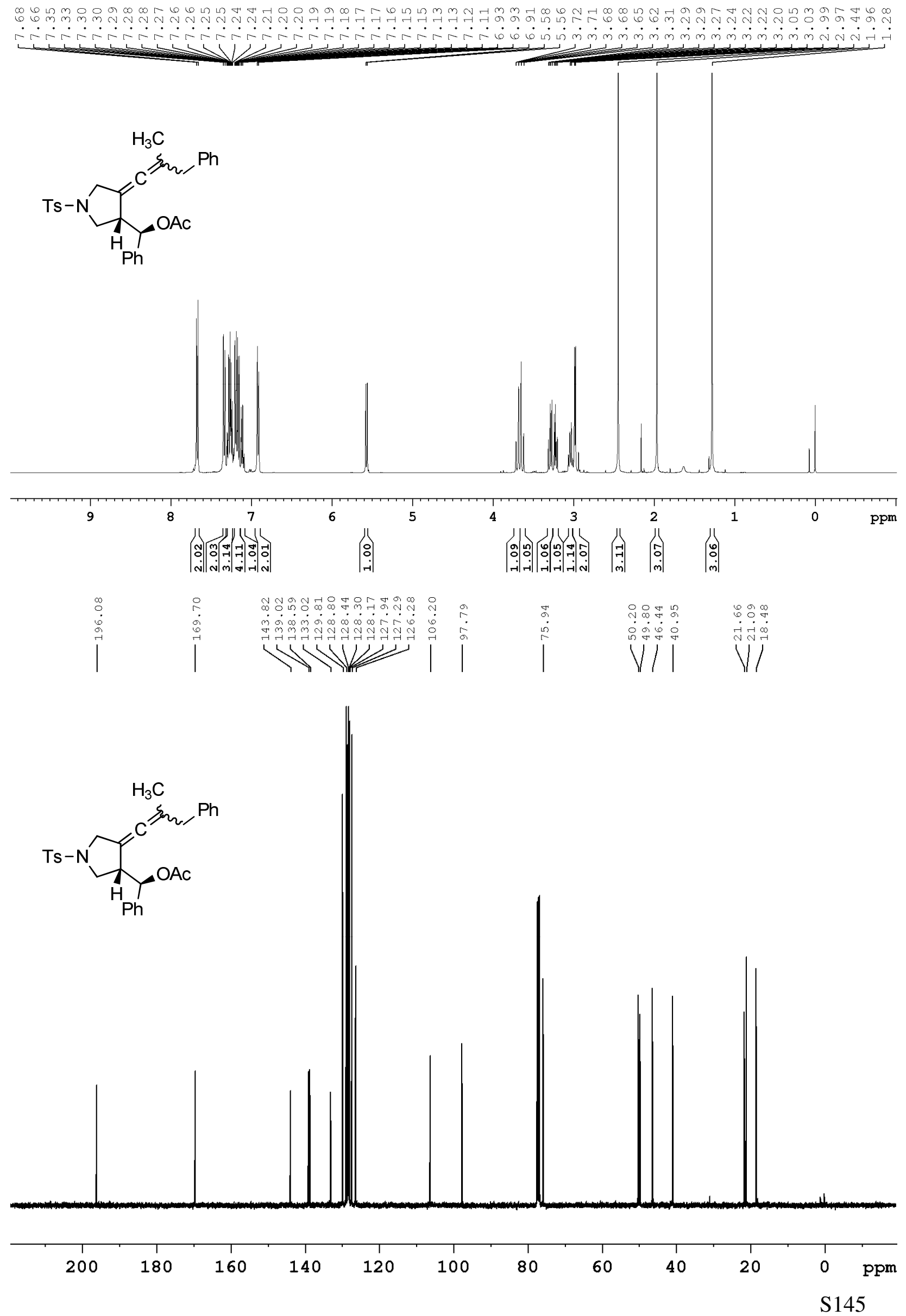
${ }^{1} \mathrm{H}$ - and ${ }^{13} \mathrm{C}$ NMR spectra of $\mathbf{2 4}$ (HPLC fraction 1)

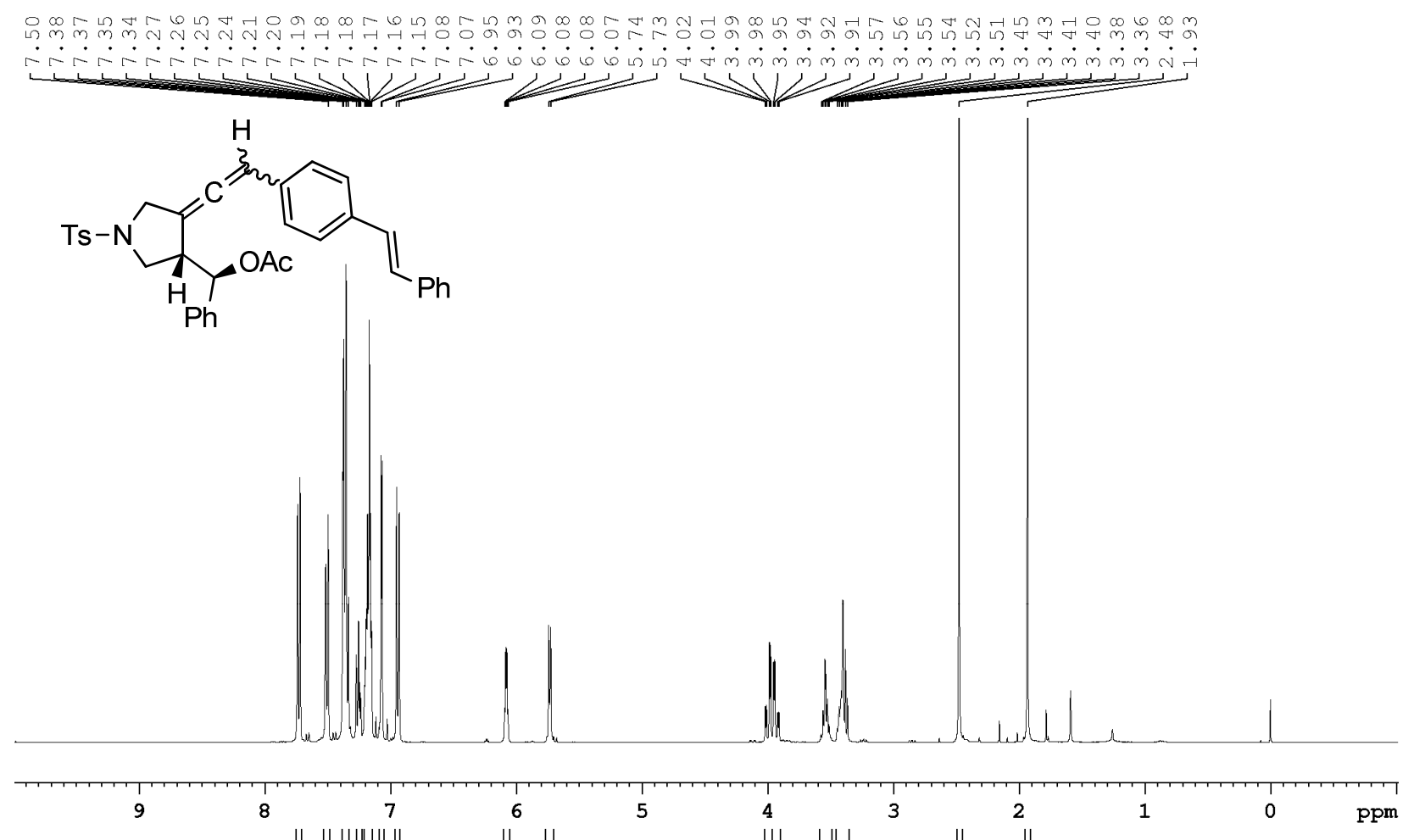

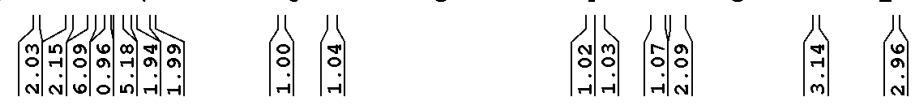

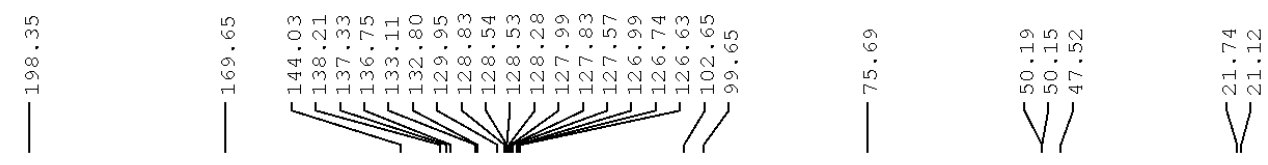

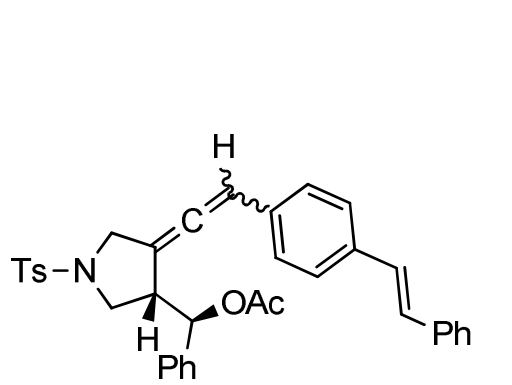

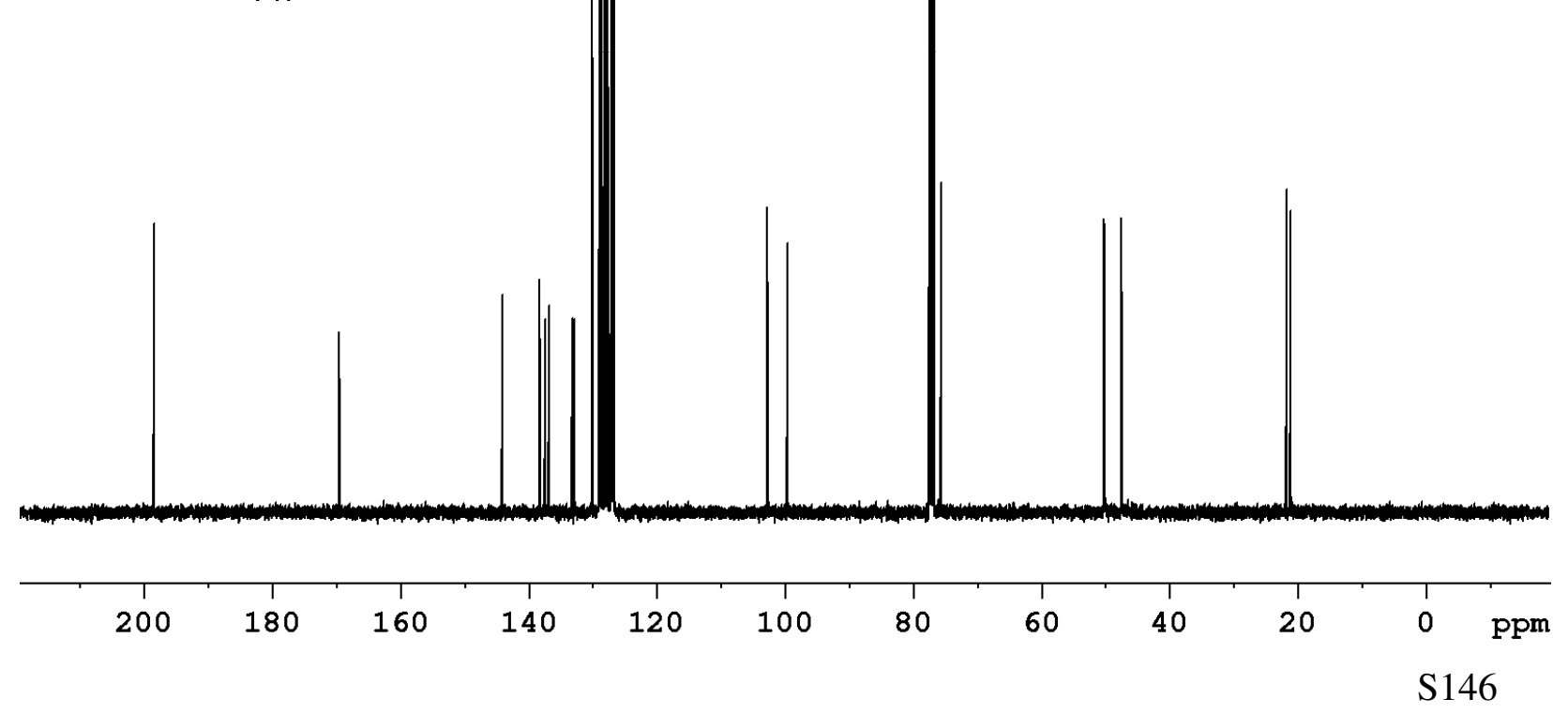


${ }^{1} \mathrm{H}$ - and ${ }^{13} \mathrm{C}$ NMR spectra of $\mathbf{2 4}$ (HPLC fraction 2)
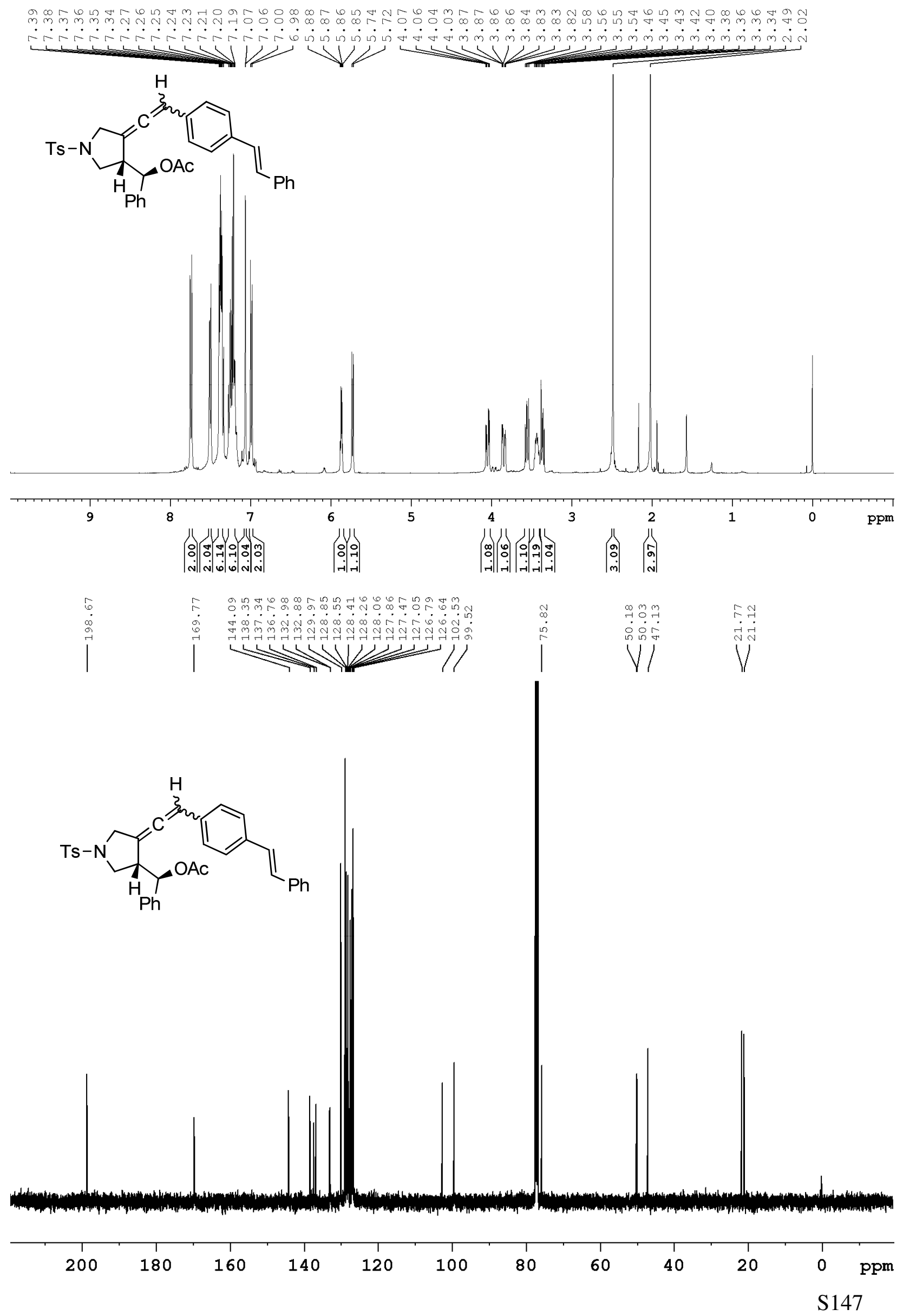
${ }^{1} \mathrm{H}$ - and ${ }^{13} \mathrm{C}$ NMR spectra of $\mathbf{2 5}$
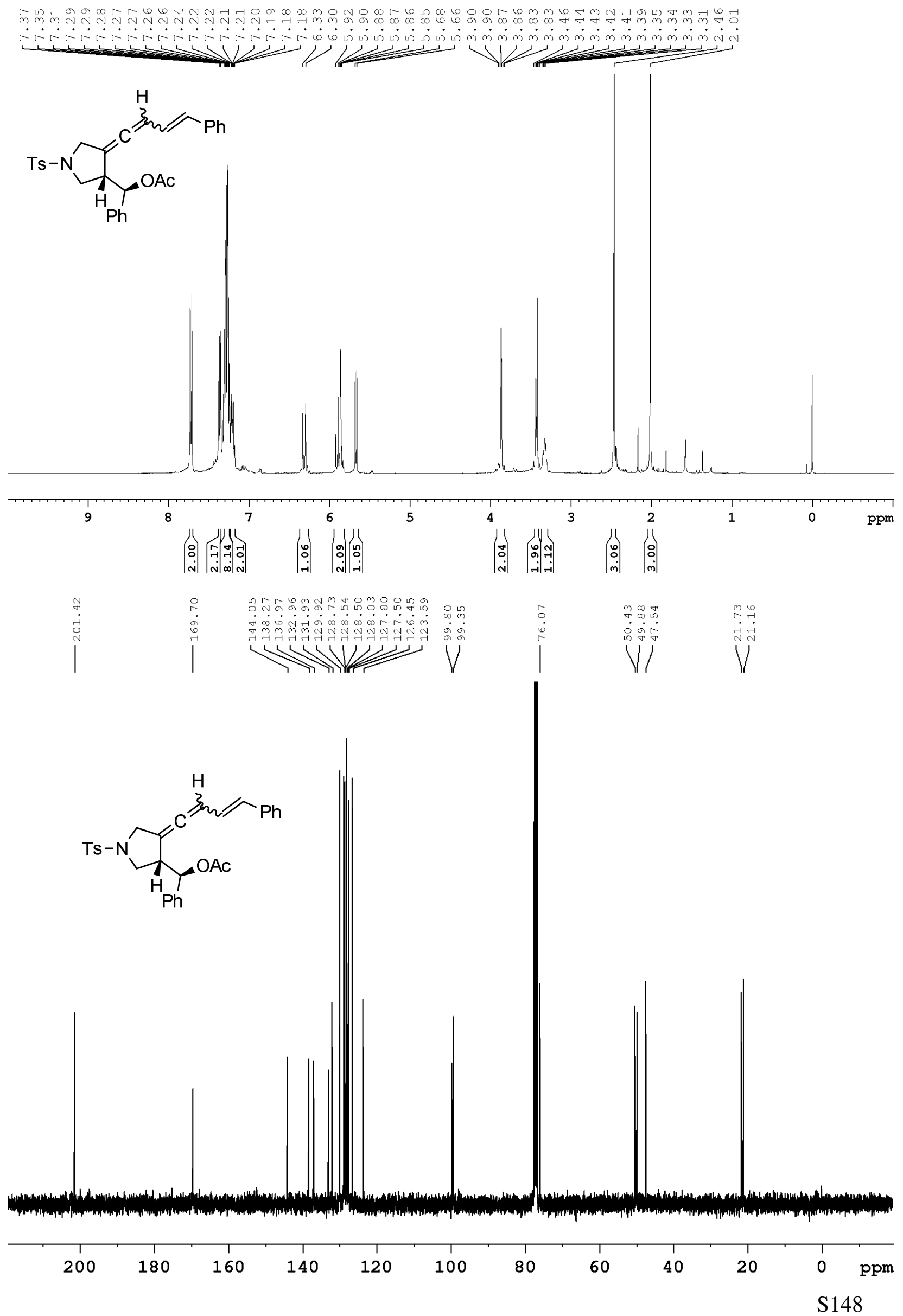
${ }^{1} \mathrm{H}$ - and ${ }^{13} \mathrm{C}$ NMR spectra of $\mathbf{2 6}$
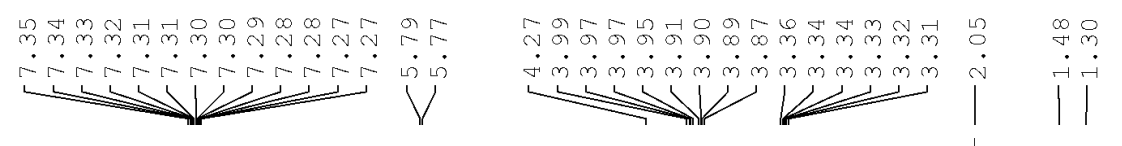<smiles>CC(=O)O[C@H](c1ccccc1)[C@@H]1COCC1=C=C(C)C</smiles>
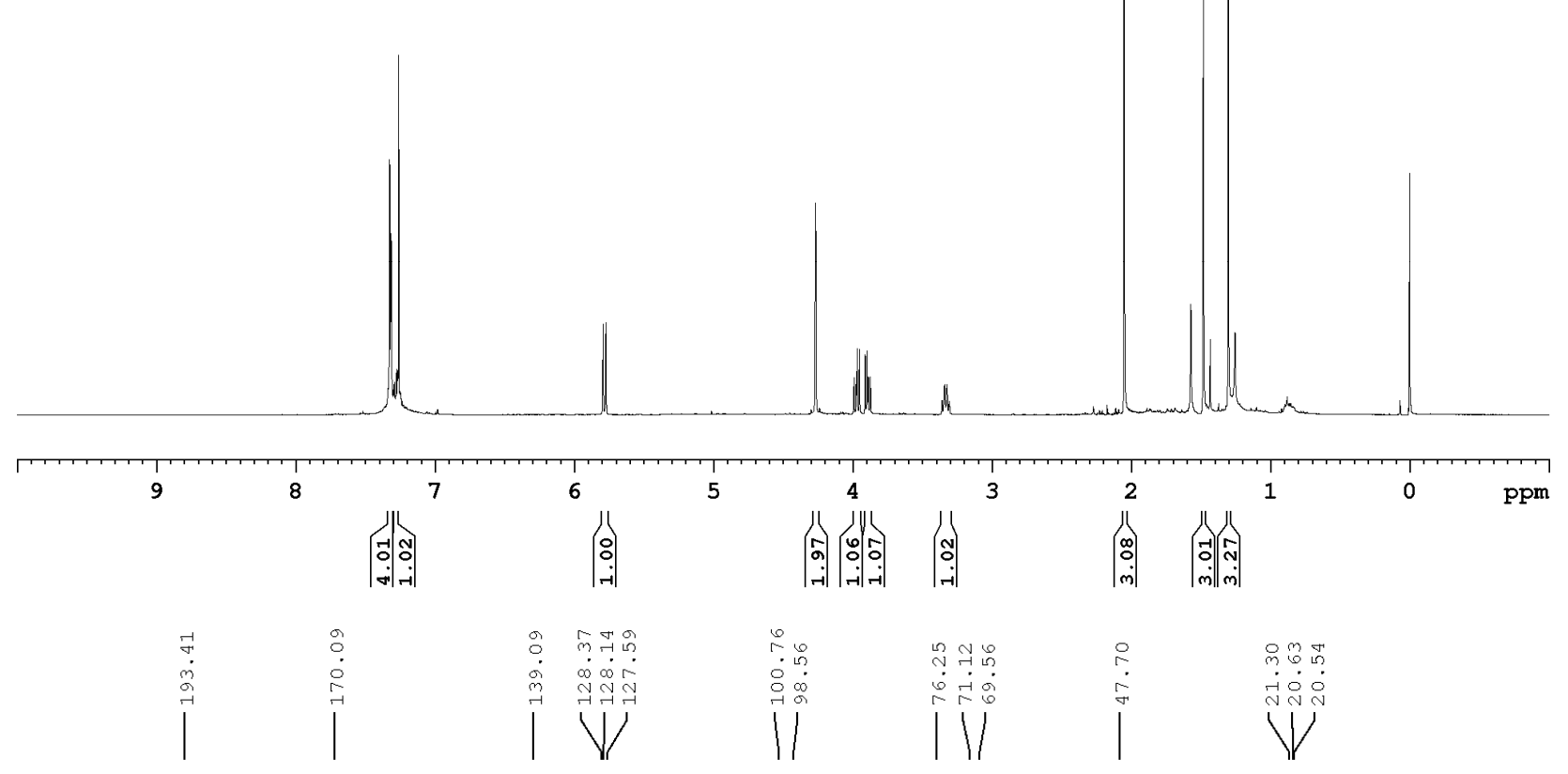<smiles>CC(=O)O[C@H](c1ccccc1)[C@@H]1COCC1=C=C(C)C</smiles>

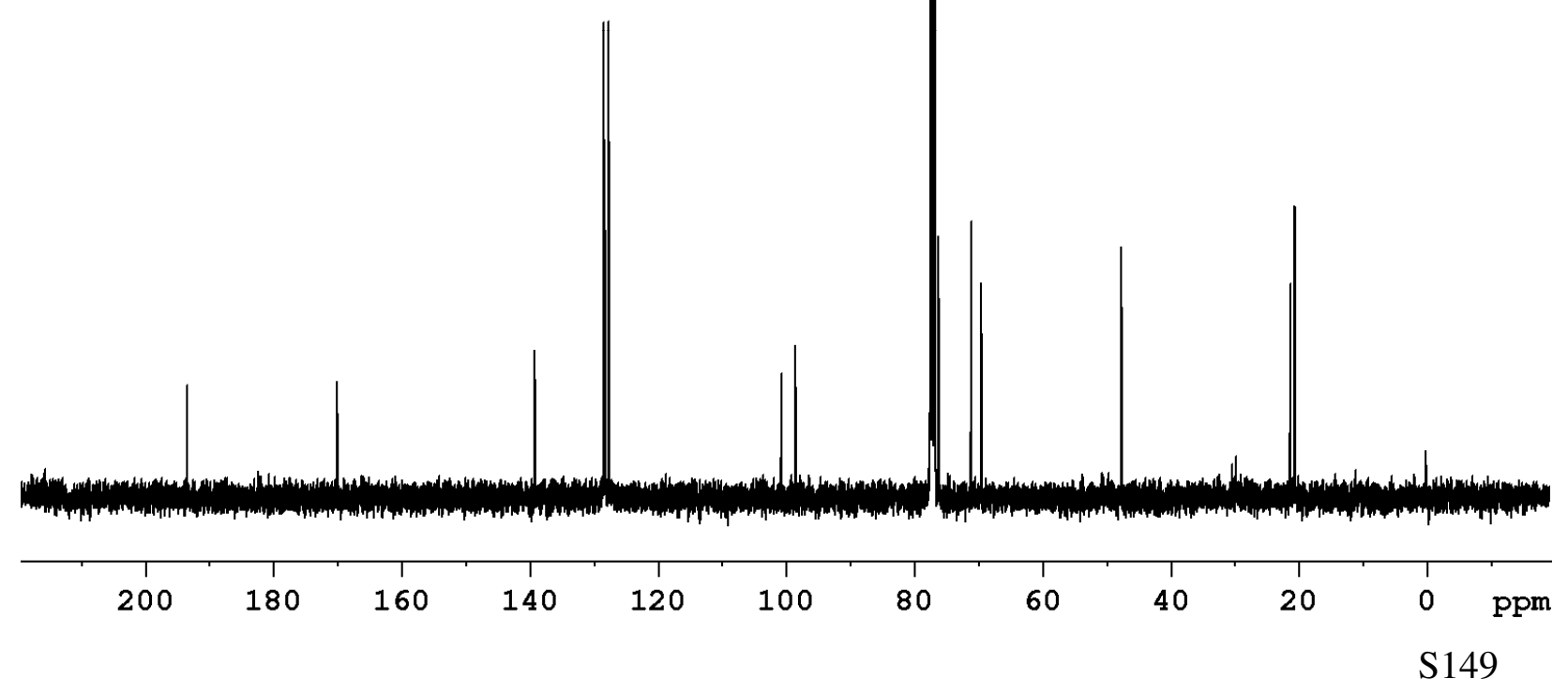


${ }^{1} \mathrm{H}$ - and ${ }^{13} \mathrm{C}$ NMR spectra of $\mathbf{2 7}$

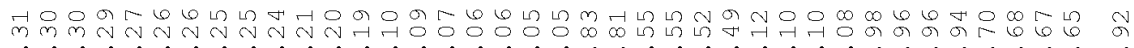

$\therefore$<smiles>CC(=O)O[C@H](c1ccccc1)[C@H]1COC/C1=C\C(=Cc1ccccc1)c1ccccc1</smiles>
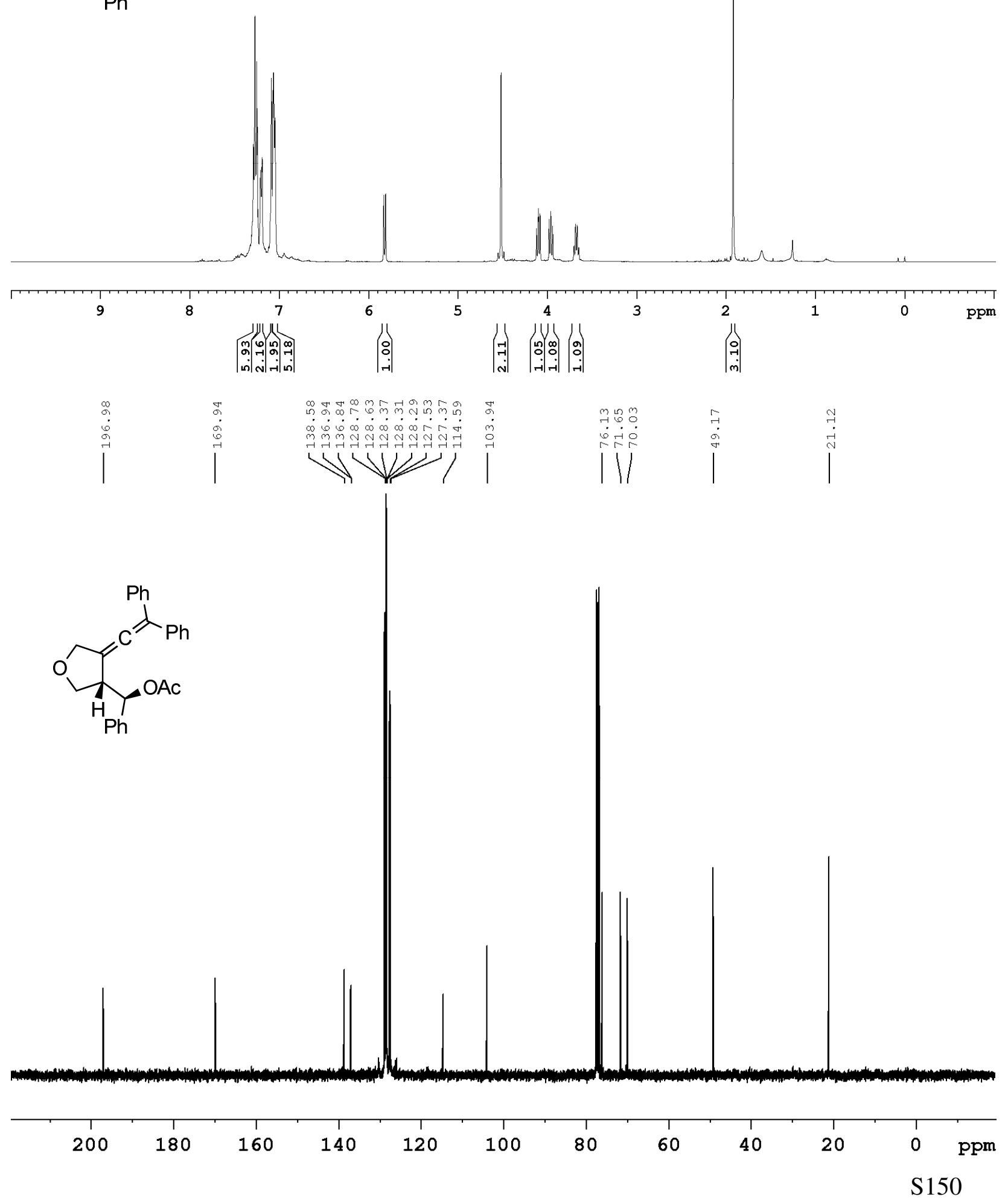
${ }^{1} \mathrm{H}$ - and ${ }^{13} \mathrm{C}$ NMR spectra of $\mathbf{2 8}$
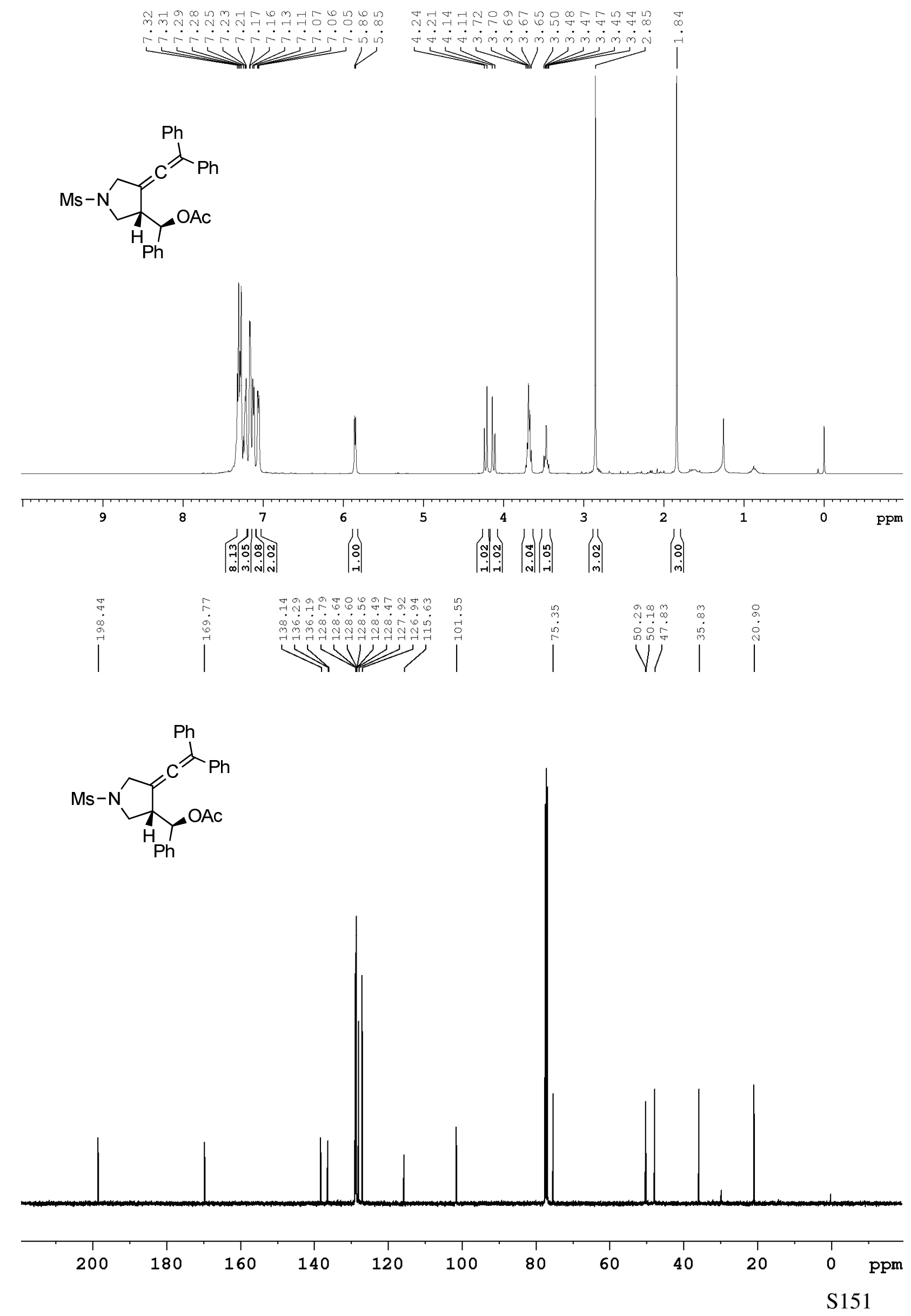
${ }^{1} \mathrm{H}$ - and ${ }^{13} \mathrm{C}$ NMR spectra of 29

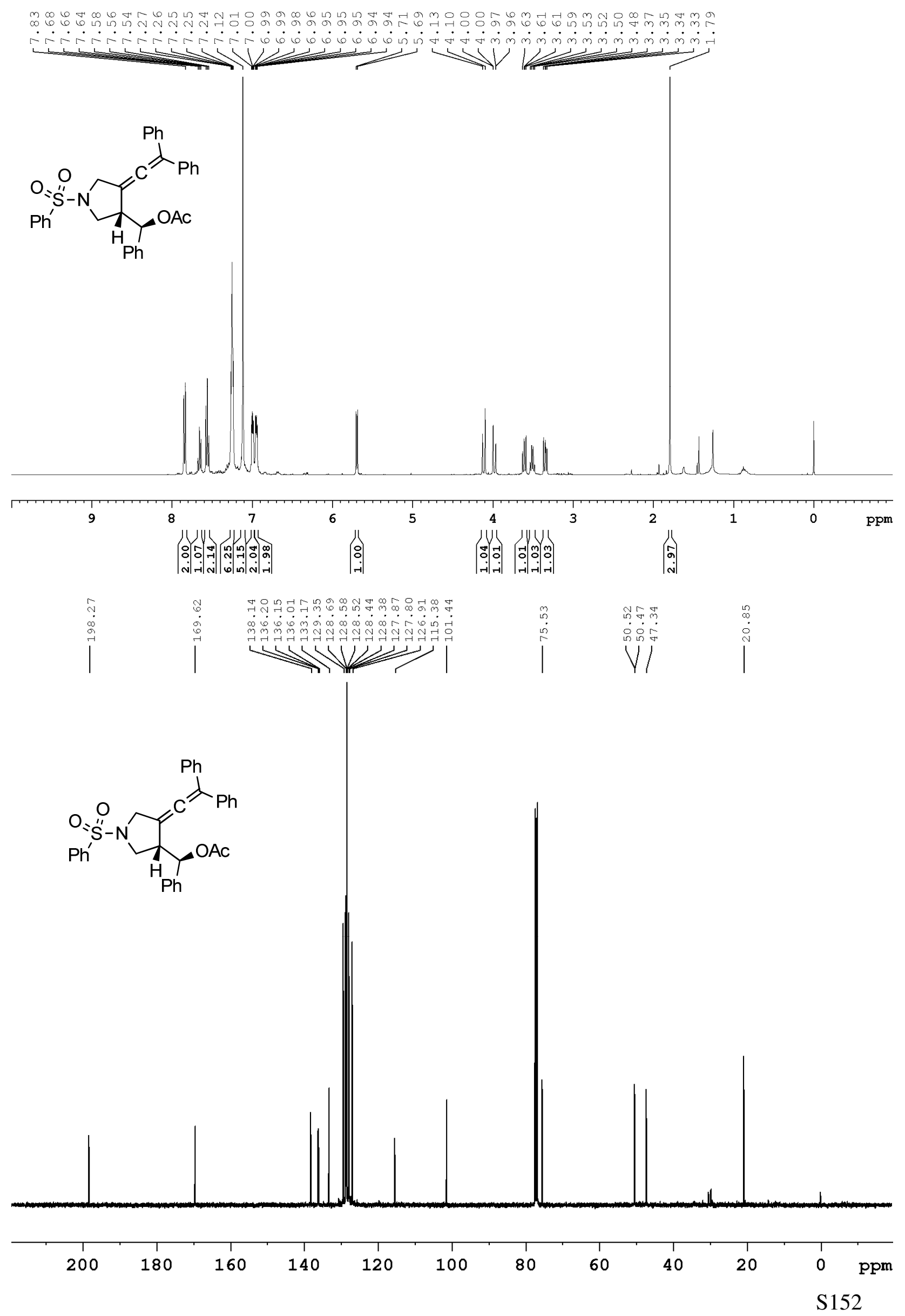


${ }^{1} \mathrm{H}-$ and ${ }^{13} \mathrm{C}$ NMR spectra of 29-D 3

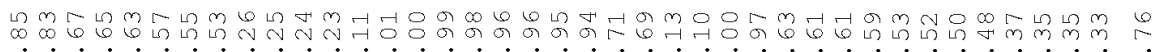

1<smiles>CC(=O)OC(C(=O)c1ccccc1)C1CN(S(=O)(=O)c2ccccc2)CC1=Cc1ccccc1</smiles>
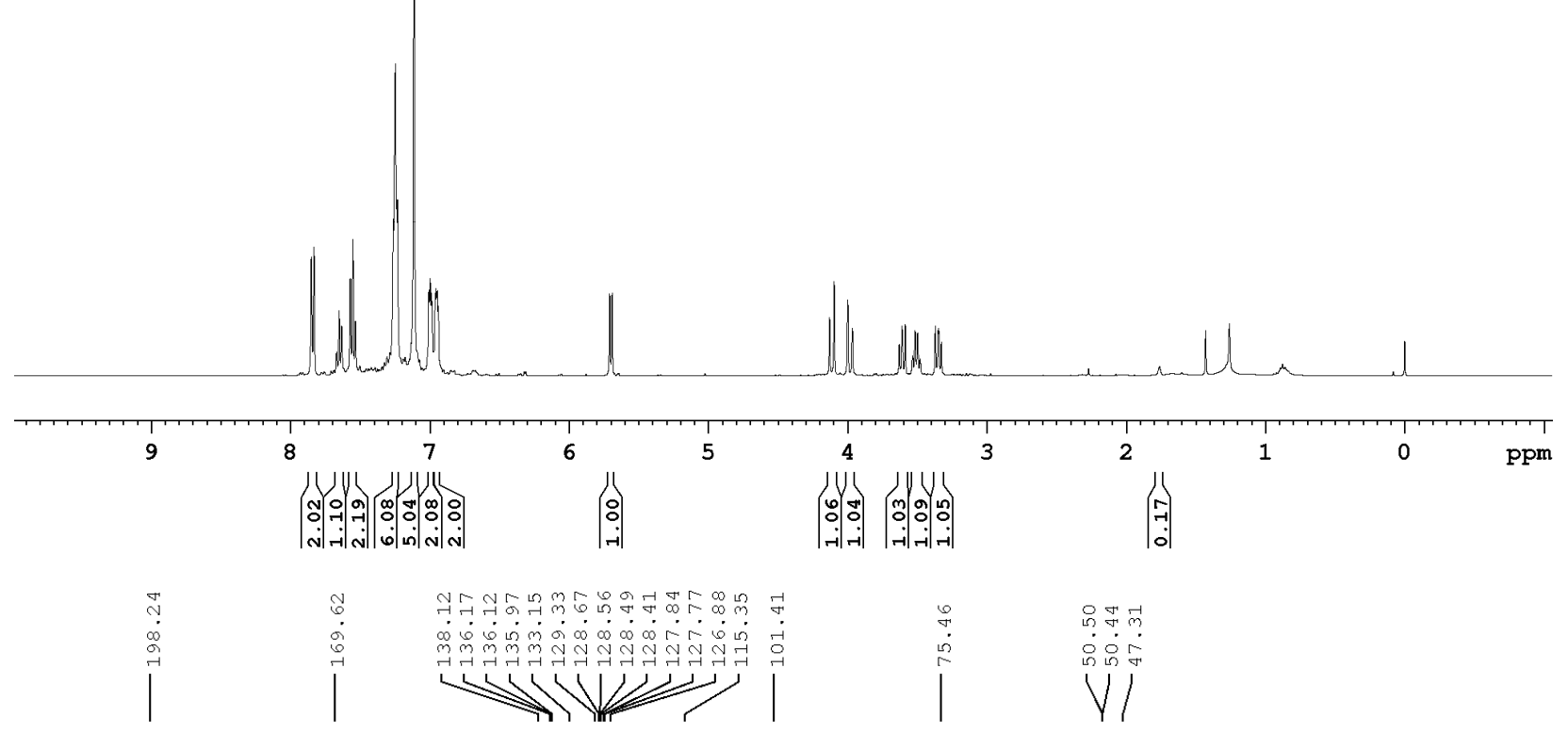<smiles>CC(=O)OC(C(=O)c1ccccc1)C1CN(S(=O)(=O)c2ccccc2)CC1=Cc1ccccc1</smiles>

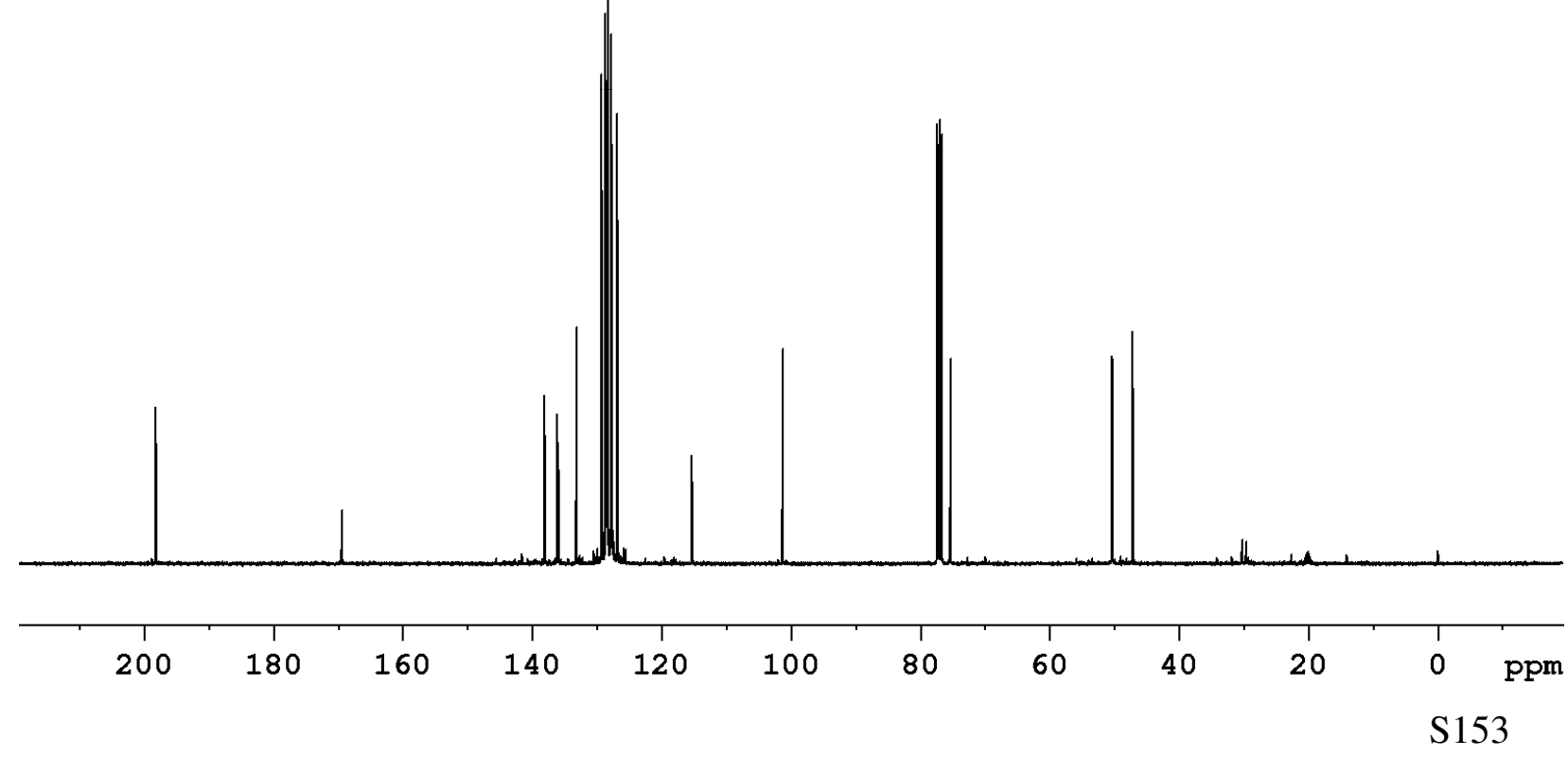


${ }^{1} \mathrm{H}$ - and ${ }^{13} \mathrm{C}$ NMR spectra of $\mathbf{3 0}$

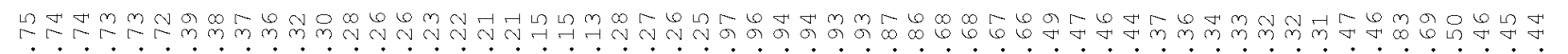<smiles>CC(C)(C)[C@]1(C(=O)O[Na])CN([13CH3])CC1=Cc1ccccc1</smiles>
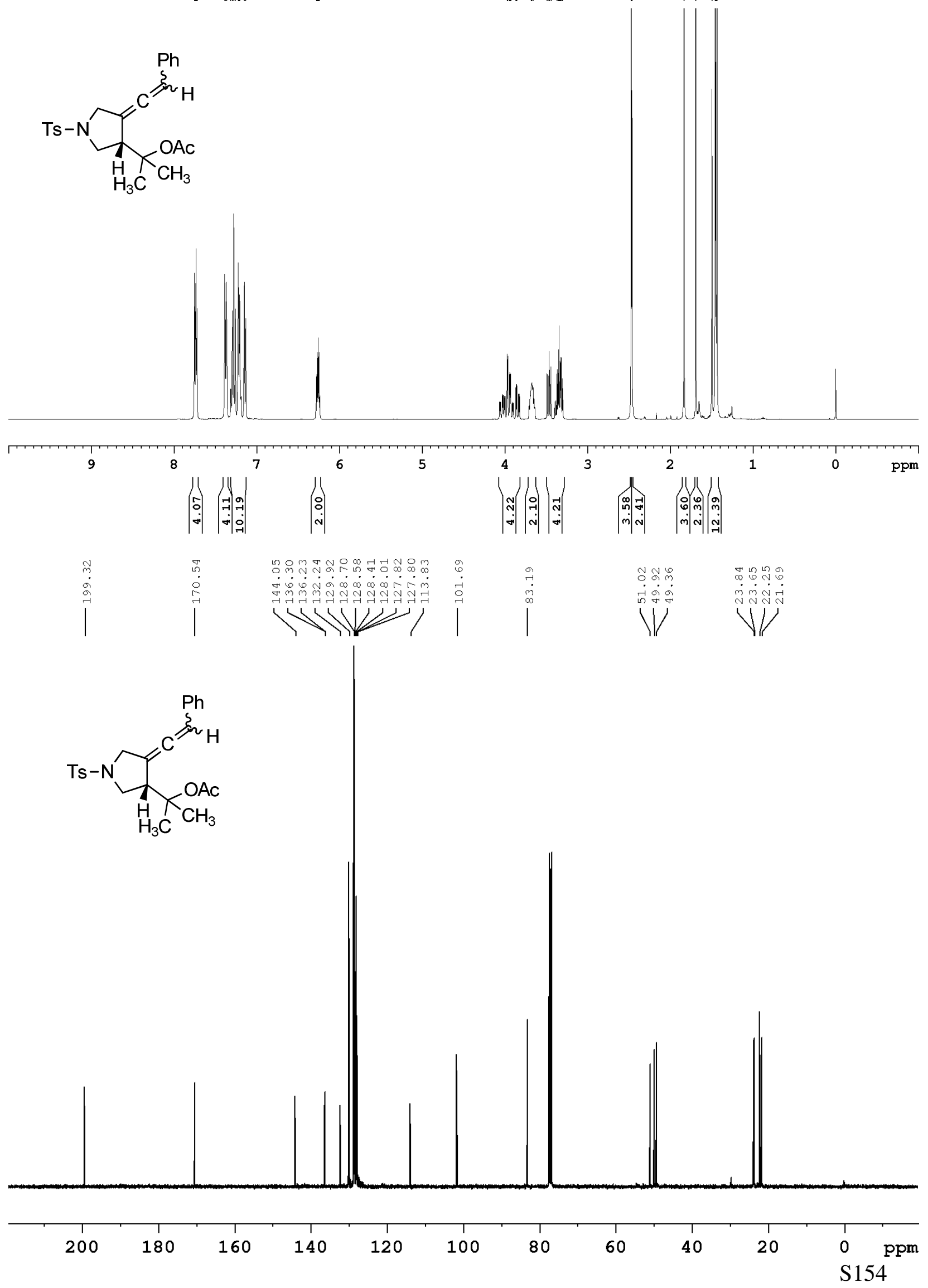
${ }^{1} \mathrm{H}$ - and ${ }^{13} \mathrm{C}$ NMR spectra of $\mathbf{3 1}$

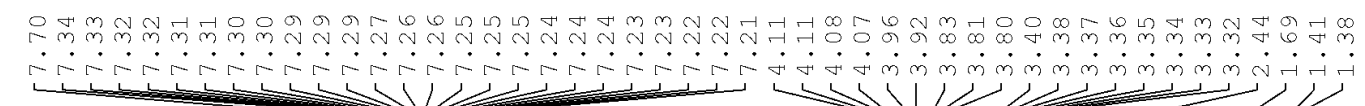<smiles>[Y5]N1CC(=CC(c2ccccc2)c2ccccc2)C(C(C)(C)C)C1</smiles>
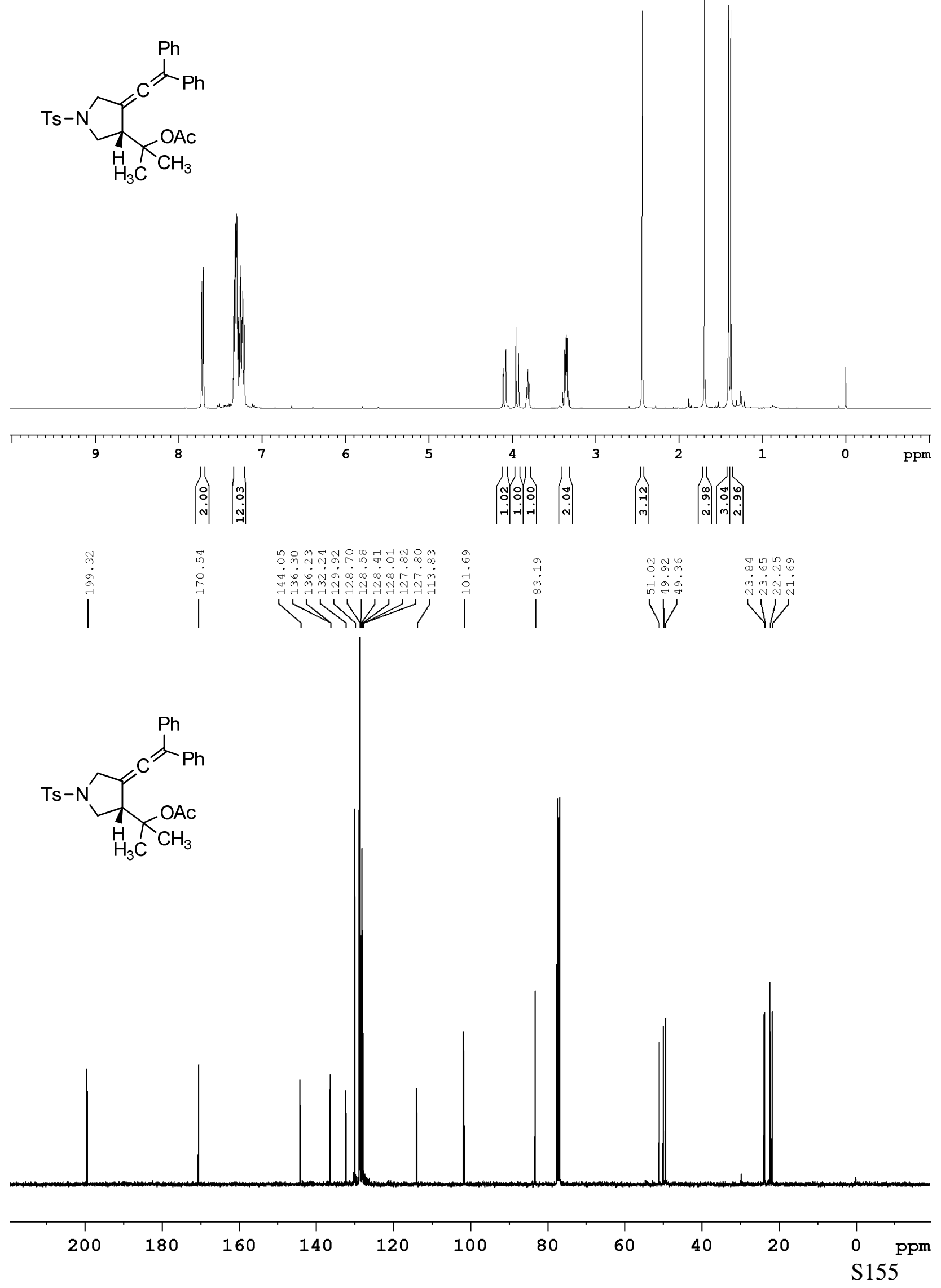
${ }^{1} \mathrm{H}$ - and ${ }^{13} \mathrm{C}$ NMR spectra of $\mathbf{3 2}$

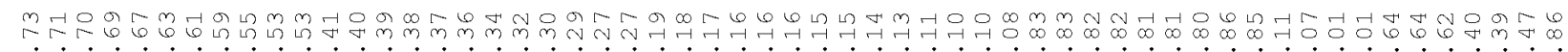

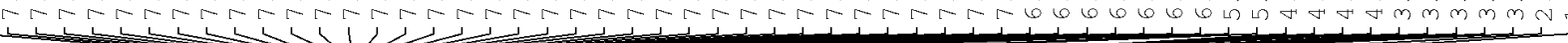<smiles>CN1CC(=Cc2ccccc2)C(C(=O)OC(c2ccccc2)c2ccccc2)C1</smiles>
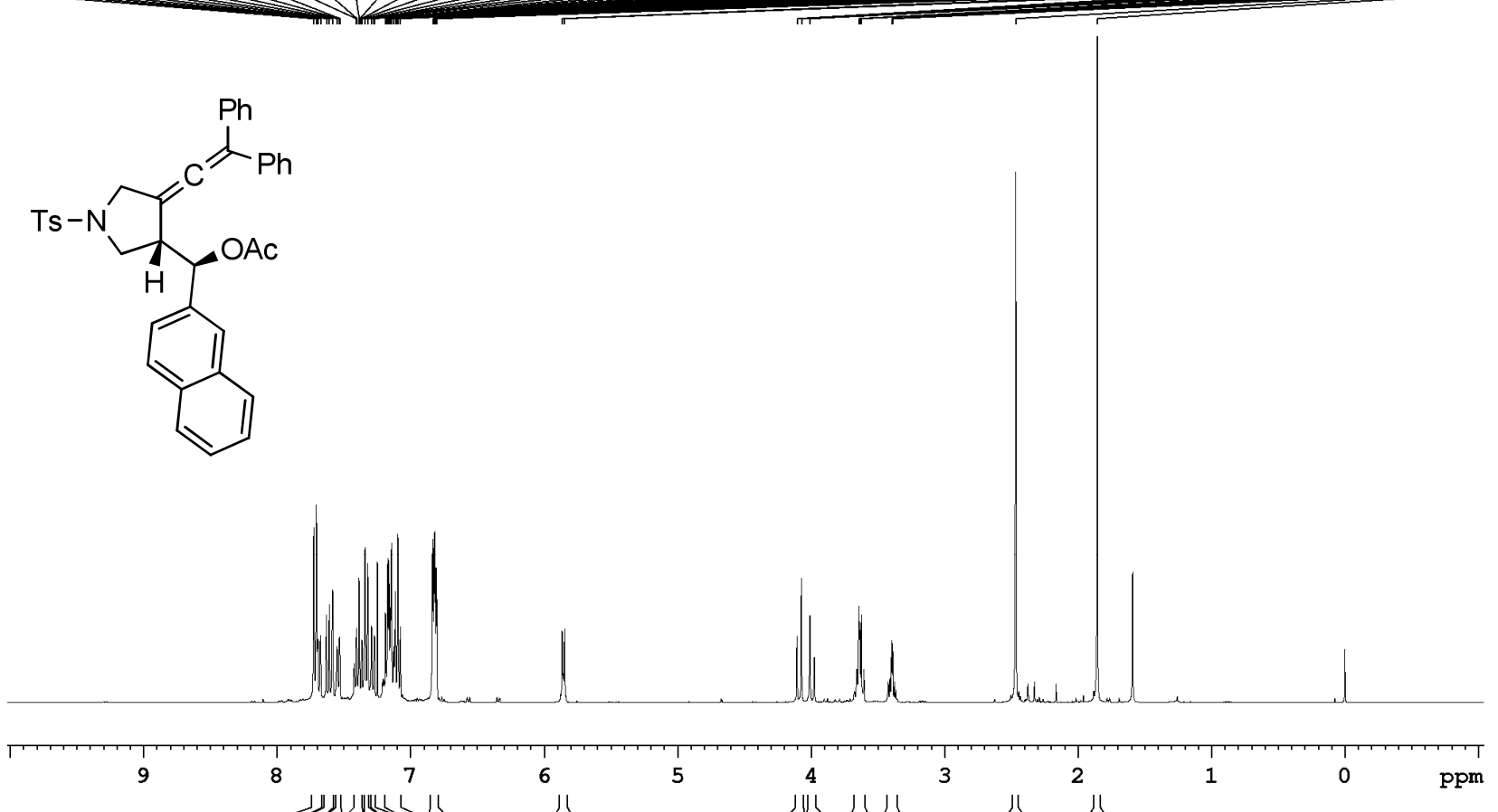

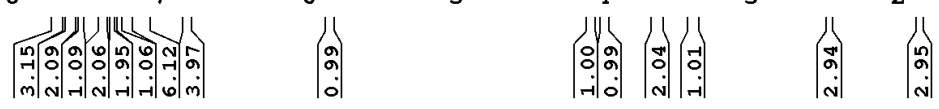

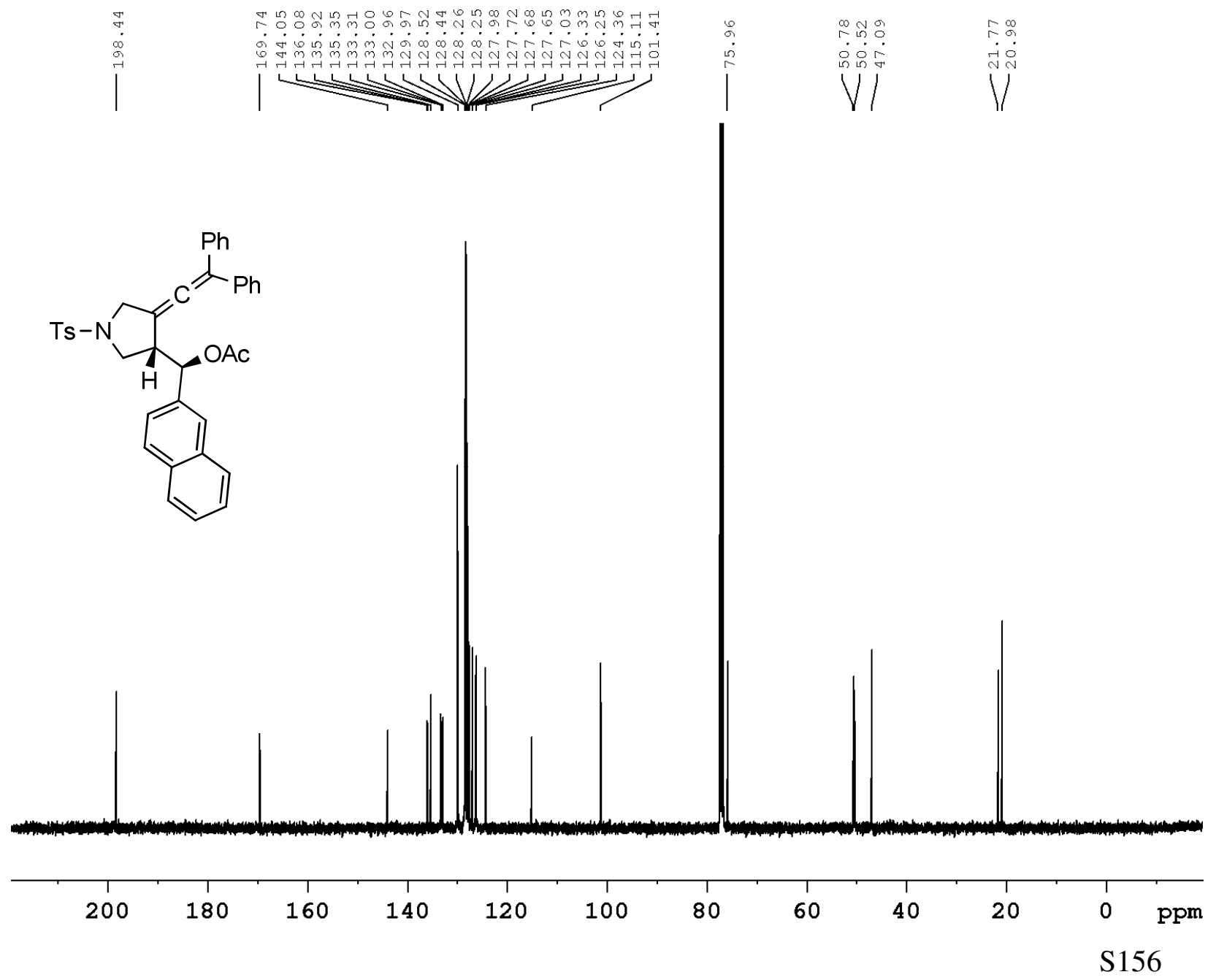


${ }^{1} \mathrm{H}$ - and ${ }^{13} \mathrm{C}$ NMR spectra of $\mathbf{3 3}$
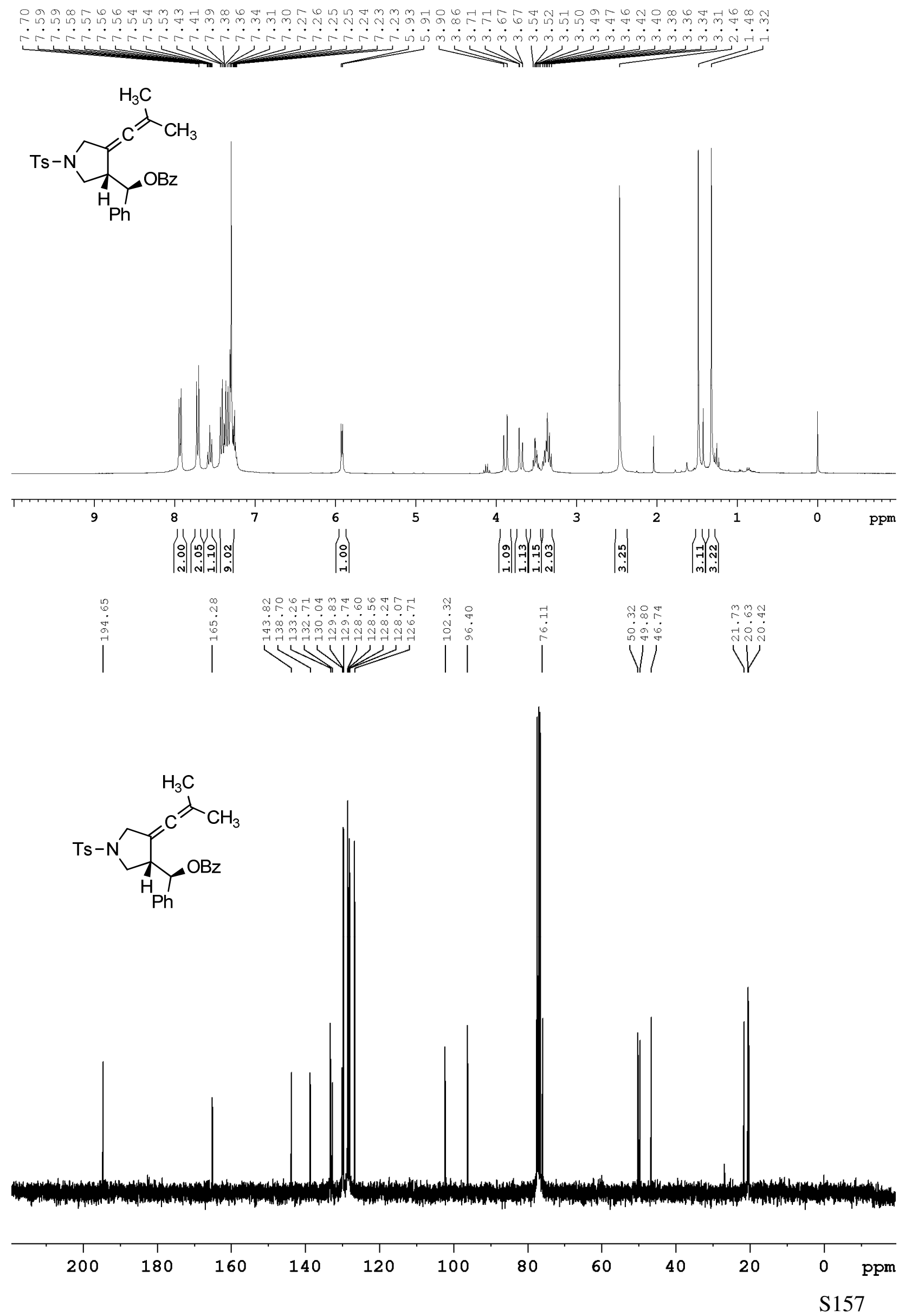
${ }^{1} \mathrm{H}$ - and ${ }^{13} \mathrm{C}$ NMR spectra of 37
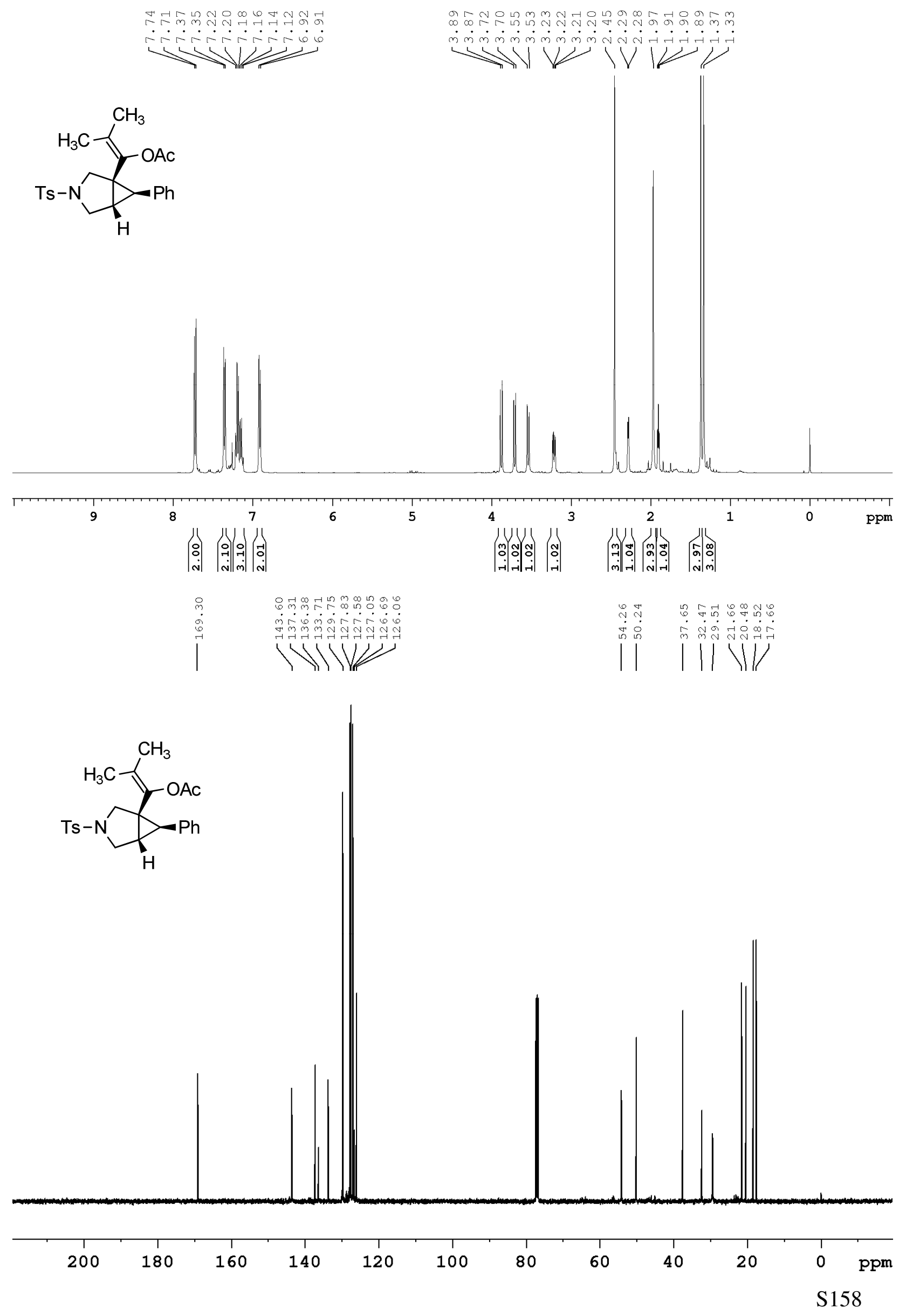
${ }^{1} \mathrm{H}$ - and ${ }^{13} \mathrm{C}$ NMR spectra of $\mathbf{4 1}$

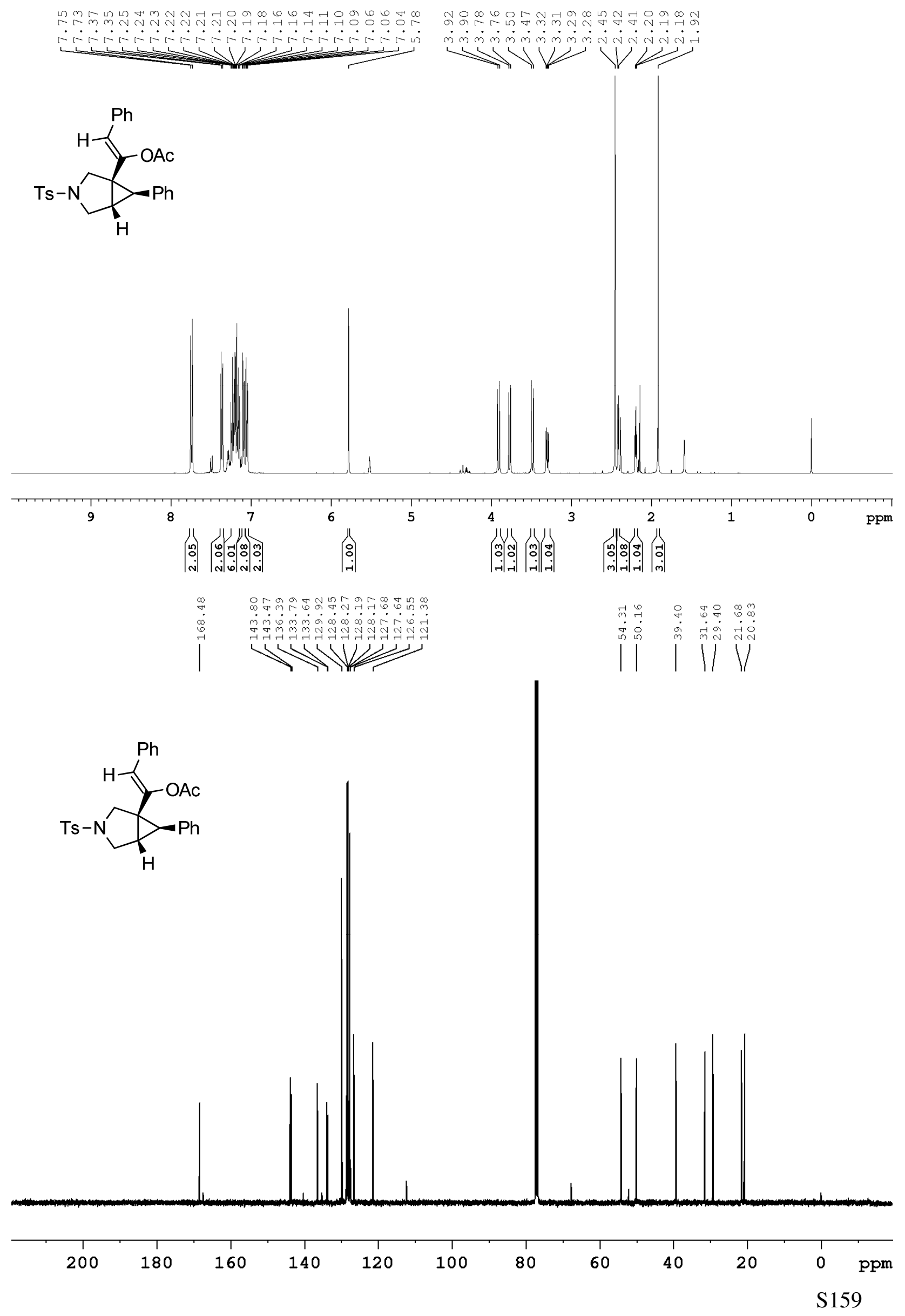




\section{Chiral HPLC chromatograms}

Chiral HPLC chromatogram of racemic s5.

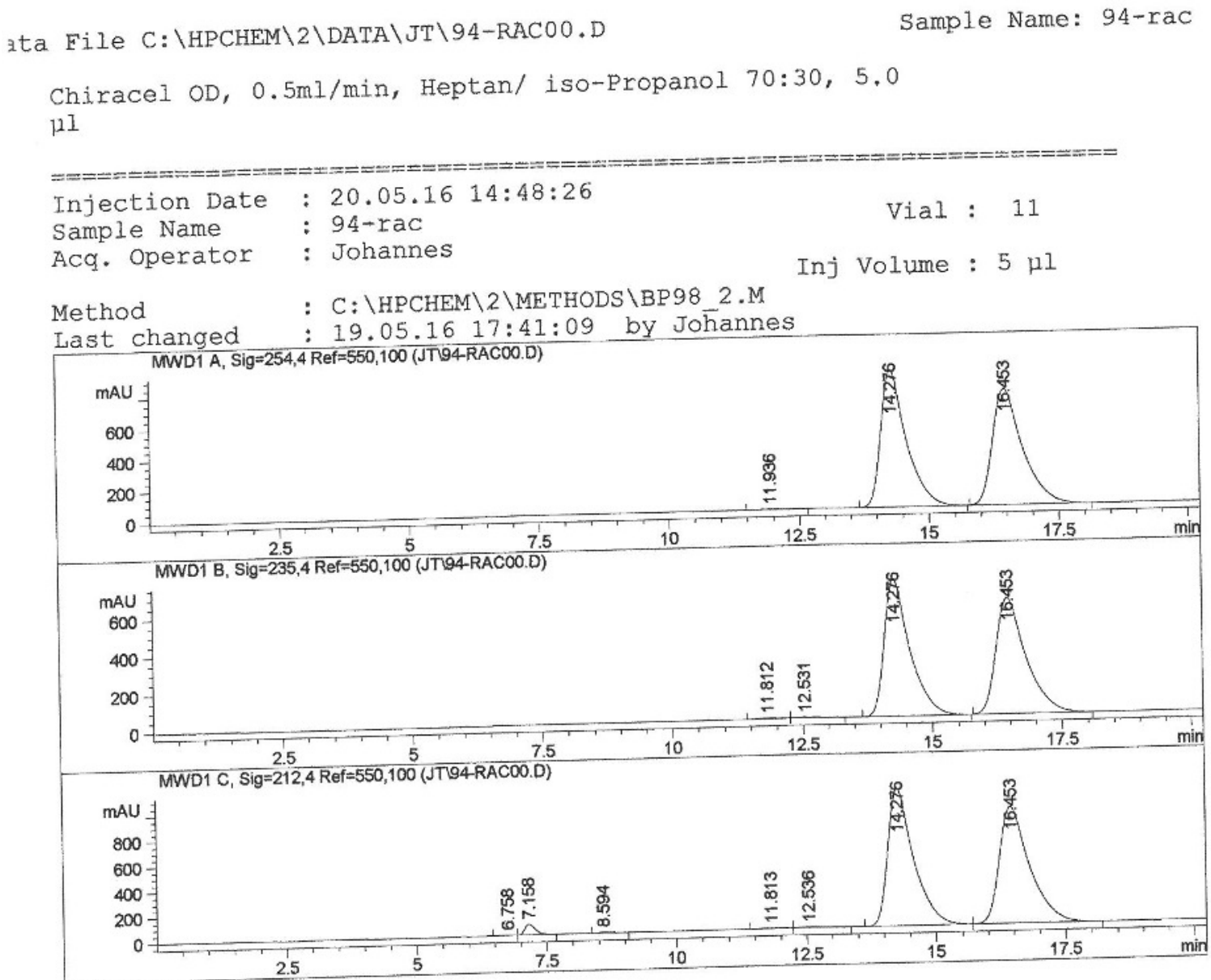

Area Percent Report

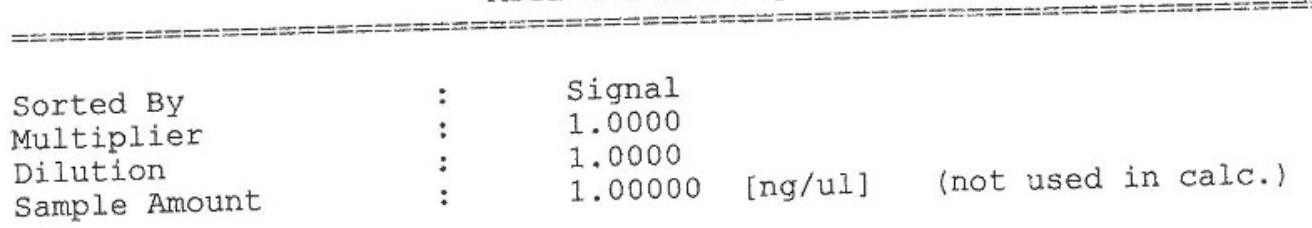

Signal 1: MWD1 A, Sig=254,4 Ref $=550,100$

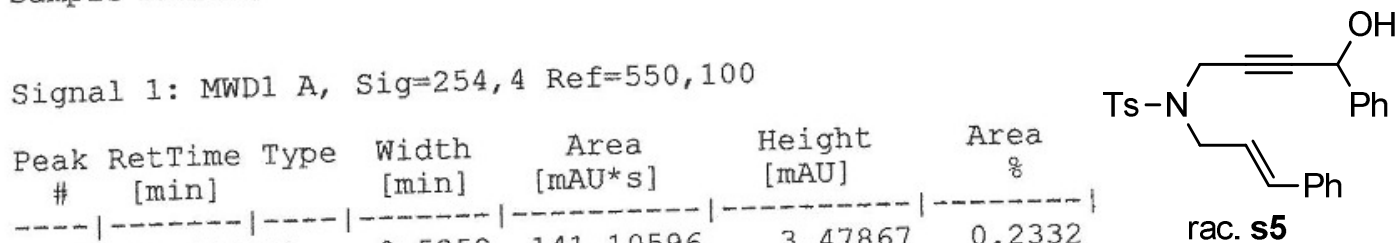

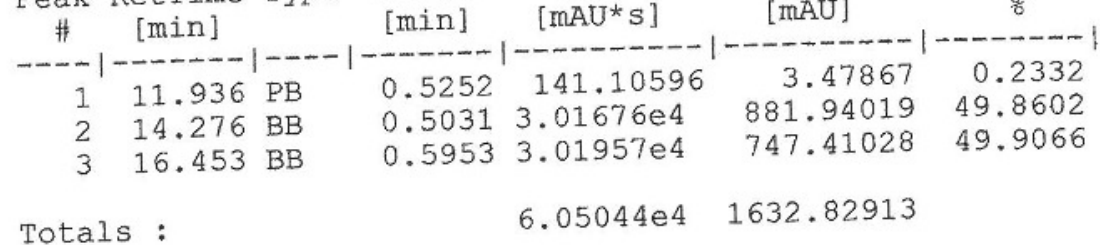

rac. $\mathbf{5 5}$

Results obtained with enhanced integrator!

Instrument 2 14.06.16 08:11:02 Johannes 
Signal 2: MWD1 B, Sig $=235,4$ Ref $=550,100$

\begin{tabular}{|c|c|c|c|c|c|c|}
\hline $\begin{array}{c}\text { Peak } \\
\#\end{array}$ & $\begin{array}{l}\text { RetTime } \\
\text { [min] }\end{array}$ & Type & $\begin{array}{l}\text { Width } \\
\text { [min] }\end{array}$ & $\begin{array}{c}\text { Area } \\
{\left[\mathrm{mAU} U^{\star} \mathrm{S}\right]}\end{array}$ & $\begin{array}{l}\text { Height } \\
\text { [mAU] }\end{array}$ & $\begin{array}{c}\text { Area } \\
8 \\
8\end{array}$ \\
\hline 1 & 11.812 & PV & 0.4694 & 160.65797 & 5.24419 & 196 \\
\hline 2 & 12.531 & $\mathrm{VB}$ & 0.4052 & 138.92909 & 4.77808 & 0.2764 \\
\hline 3 & 14.276 & $\mathrm{BB}$ & 0.5038 & $2.49852 \mathrm{e} 4$ & 729.17847 & 49.7090 \\
\hline 4 & 16.453 & $\mathrm{BB}$ & 0.5956 & $2.49782 \mathrm{e} 4$ & 617.88678 & 49.6950 \\
\hline & & & & $5.02630 \mathrm{e} 4$ & 1357.08752 & \\
\hline
\end{tabular}

Results obtained with enhanced integrator!

Signal 3: MWD1 C, Sig $=212,4$ Ref $=550,100$

\begin{tabular}{|c|c|c|c|c|c|c|}
\hline $\begin{array}{c}\text { Peak } \\
\#\end{array}$ & $\begin{array}{c}\text { RetTime } \\
\text { [min] }\end{array}$ & Type & $\begin{array}{l}\text { Width } \\
\text { [min] }\end{array}$ & $\begin{array}{c}\text { Area } \\
{\left[\mathrm{mAU}^{*} \mathrm{~s}\right]}\end{array}$ & $\begin{array}{l}\text { Height } \\
\text { [MAU] }\end{array}$ & $\begin{array}{c}\text { Area } \\
8\end{array}$ \\
\hline 1 & 6.758 & BV & 0.2333 & 67.18088 & 4.23063 & $0.0876^{\prime}$ \\
\hline 2 & 7.158 & VB & 0.2234 & 1204.95728 & 83.99081 & 1.5708 \\
\hline 3 & 8.594 & $\mathrm{BB}$ & 0.2598 & 167.83302 & 9.51403 & 0.2188 \\
\hline 4 & 11.813 & PV & 0.3575 & 187.83055 & 6.27069 & 0.2449 \\
\hline 5 & 12.536 & VB & 0.3647 & 170.87863 & 5.74953 & 0.2228 \\
\hline 6 & 14.276 & BV & 0.5084 & $3.73995 \mathrm{e} 4$ & 1084.39868 & 48.7543 \\
\hline 7 & 16.453 & VB & 0.5994 & $3.75120 \mathrm{e} 4$ & 920.59729 & 48.9009 \\
\hline & & & & $7.67102 \mathrm{e} 4$ & 2114.75167 & \\
\hline
\end{tabular}

Results obtained with enhanced integrator!

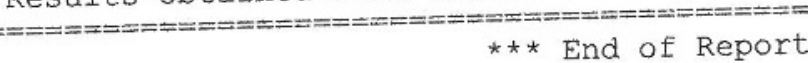




\section{Chiral HPLC chromatogram of (-)-s5.}

ata File C: \HPCHEM $2 \backslash \mathrm{DATA} \backslash J \mathrm{~T} \backslash 94-\mathrm{H} 1000 . \mathrm{D}$

Chiracel OD, 0.5ml/min, Heptan/ iso-Propanol 70:30, 5.0

$\mu 1$

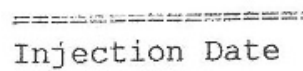

$20.05 .16 \quad 14: 01: 21$

Sample Name : 94-H1

Vial : 12

Acq. Operator

: Johannes

Inj Volume : $5 \mu \mathrm{l}$

Method

: C: $\backslash$ HPCHEM $\backslash 2 \backslash$ METHODS $\backslash$ BP98 $2 . M$

Last changed : 19.05.16 17:41:09 by Johannes
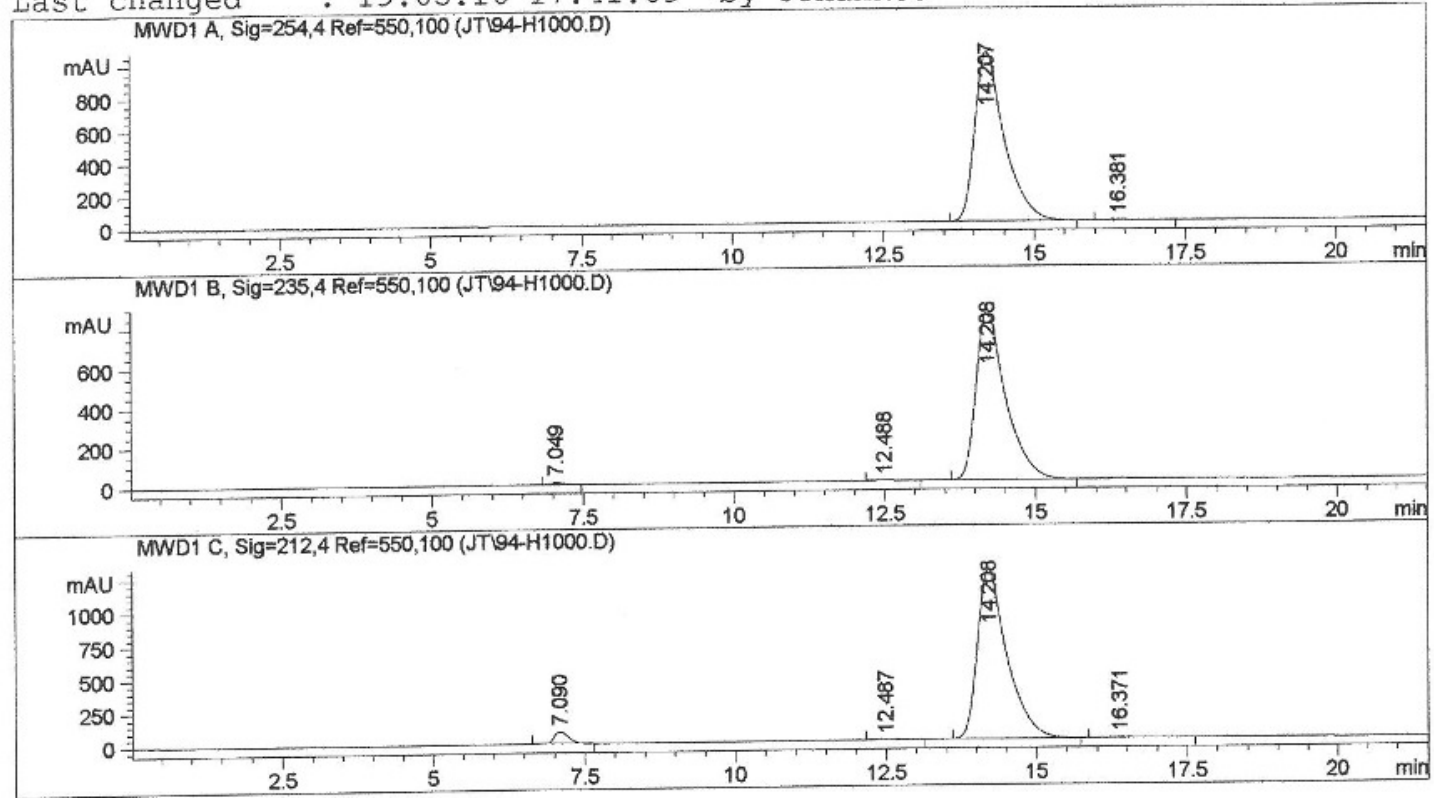

Area Percent Report

\begin{tabular}{|c|c|c|c|}
\hline Sorted By & : & Signal & \\
\hline Multiplier & $:$ & 1.0000 & \\
\hline Dilution & : & 1.0000 & \\
\hline Sample Amount & : & 1.00000 & (not used in calc.) \\
\hline
\end{tabular}

Signal 1: MWD1 A, Sig=254, 4 Ref $=550,100$

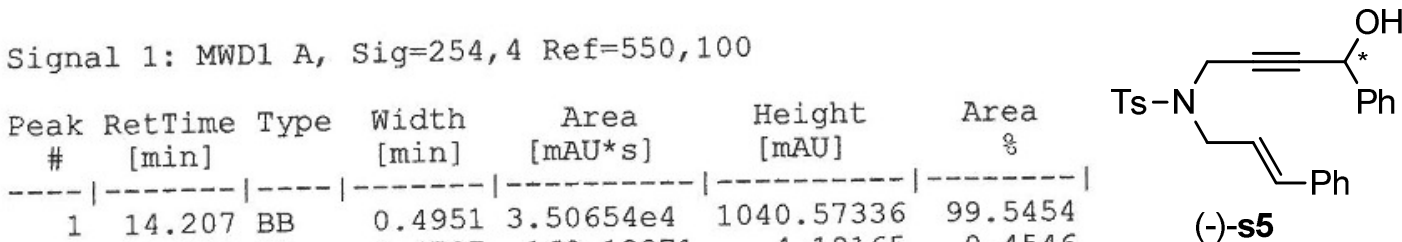

Totals :

$3.52255 \mathrm{e} 4 \quad 1044.76501$

Results obtained with enhanced integrator! 
Signal 2: MWD1 B, Sig=235,4 Ref $=550,100$

\begin{tabular}{|c|c|c|c|c|c|c|}
\hline $\begin{array}{c}\text { Peak } \\
\#\end{array}$ & $\begin{array}{l}\text { RetTime } \\
\text { [min] }\end{array}$ & Type & $\begin{array}{l}\text { Width } \\
\text { [min] }\end{array}$ & $\begin{array}{c}\text { Area } \\
{\left[\mathrm{mAU}{ }^{\star} \mathrm{S}\right]}\end{array}$ & $\begin{array}{l}\text { Height } \\
\text { [mAU] }\end{array}$ & $\begin{array}{c}\text { Area } \\
\frac{8}{0}\end{array}$ \\
\hline 1 & 7.049 & $\mathrm{BB}$ & 0.2199 & 154.03902 & 11.10339 & 0.5274 \\
\hline 2 & 12.488 & PP & 0.3387 & 75.33035 & 3.19906 & 0.2579 \\
\hline 3 & 14.208 & $\mathrm{BB}$ & 0.4952 & 2.89781 e4 & 859.85797 & 99.2147 \\
\hline Cota & : & & & $2.92074 \mathrm{e}^{4}$ & 874.16042 & \\
\hline
\end{tabular}

Results obtained with enhanced integrator!

Signal 3: MWD1 C, Sig $=212,4$ Ref $=550,100$

\begin{tabular}{|c|c|c|c|c|c|c|}
\hline $\begin{array}{c}\text { Peak } \\
\#\end{array}$ & $\begin{array}{l}\text { RetTime } \\
\text { [min] }\end{array}$ & Type & $\begin{array}{l}\text { Width } \\
\text { [min] }\end{array}$ & $\begin{array}{c}\text { Area } \\
{\left[\mathrm{mAU}^{\star} \mathrm{s}\right]}\end{array}$ & $\begin{array}{l}\text { Height } \\
\text { [mAU] }\end{array}$ & $\begin{array}{c}\text { Area } \\
\frac{8}{0}\end{array}$ \\
\hline 1 & 90 & BB & 0.2 & 1487.55676 & 86.81792 & 3.3014 \\
\hline 2 & 12.487 & $\mathrm{PP}$ & 0.3077 & 86.85436 & 3.73013 & 1928 \\
\hline 3 & 14.208 & BB & 0.4969 & $4.32690 \mathrm{e} 4$ & 1271.82080 & 96.0295 \\
\hline 4 & 16.371 & $\mathrm{BB}$ & 0.4920 & 214.62169 & 5.35048 & 0.4763 \\
\hline
\end{tabular}

Totals :

$4.50580 e 4 \quad 1367.71934$

Results obtained with enhanced integrator!

$* \star \star$ End of Report 


\section{Chiral HPLC chromatogram of (+)-s5.}

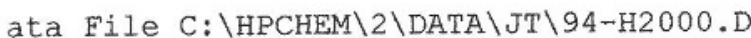

Chiracel OD, 0.5ml/min, Heptan/ iso-Propanol $70: 30,5.0$

$\mu 1$

Injection Date : $20.05 .16 \quad 14: 24: 29$
Sample Name $\quad: 94-H 2$

Sample Name : $94-\mathrm{H} 2$
Acq. Operator : Johannes

Vial : 13

Inj Volume : $5 \mu \mathrm{I}$

Method

: C: \HPCHEM $\backslash 2 \backslash$ METHODS \BP98 2.M

Last changed : 19.05.16 17:41:09 by Johannes
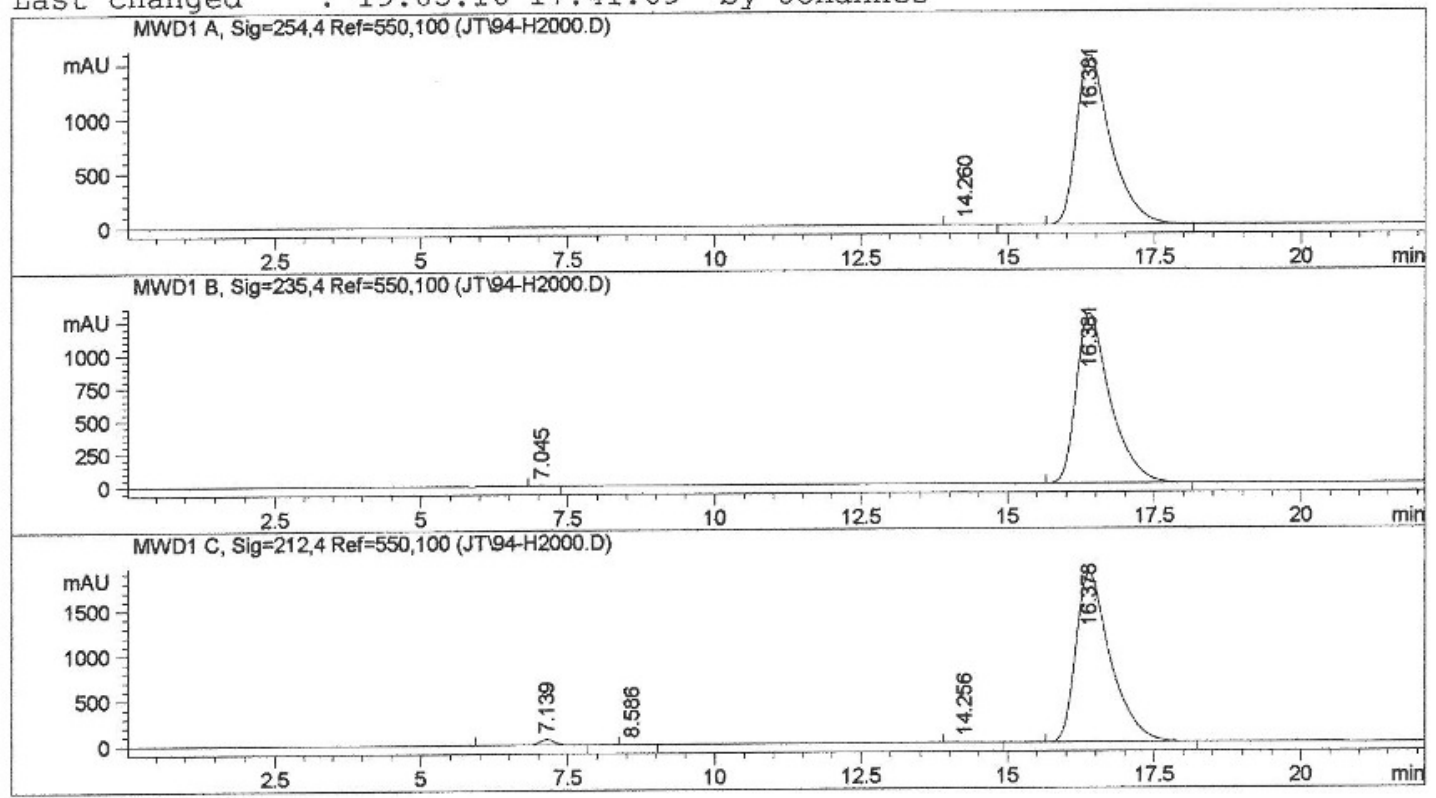

Area Percent Report

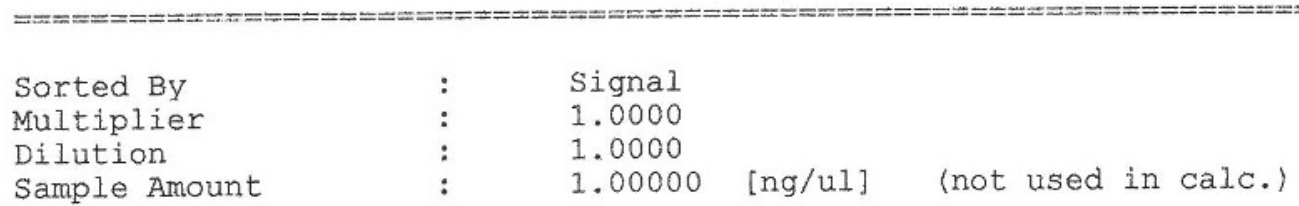

Signal 1: MWD1 A, Sig=254,4 Ref $=550,100$

\begin{tabular}{|c|c|c|c|c|c|c|}
\hline $\begin{array}{c}\text { Peak } \\
\#\end{array}$ & $\begin{array}{l}\text { RetTime } \\
\text { [min] }\end{array}$ & туре & $\begin{array}{l}\text { Width } \\
\text { [min] }\end{array}$ & $\begin{array}{c}\text { Area } \\
{\left[\mathrm{mAU}^{\star} \mathrm{S}\right]}\end{array}$ & $\begin{array}{l}\text { Height } \\
\text { [mAU] }\end{array}$ & $\begin{array}{c}\text { Area } \\
\quad 8\end{array}$ \\
\hline 1 & 14.260 & $\mathrm{BB}$ & 0.4011 & $\begin{array}{l}96.58170 \\
27249 e 4\end{array}$ & $\begin{array}{r}3.22758 \\
1561.05188\end{array}$ & $\begin{array}{r}0.1537 \\
99.8463\end{array}$ \\
\hline ota & : & & & $6.28214 \mathrm{e}^{4}$ & 1564.27946 & \\
\hline
\end{tabular}

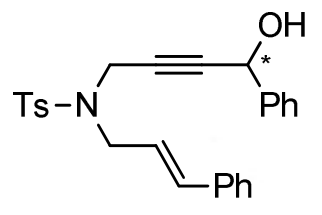

Results obtained with enhanced integrator! 


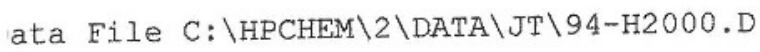

Signal 2: MWD1 B, Sig=235, 4 Ref $=550,100$

\begin{tabular}{|c|c|c|c|c|c|c|}
\hline $\begin{array}{c}\text { Peak } \\
\quad \#\end{array}$ & $\begin{array}{l}\text { RetTime } \\
\text { [min] }\end{array}$ & Type & $\begin{array}{l}\text { Width } \\
\text { [min] }\end{array}$ & $\begin{array}{c}\text { Area } \\
{\left[\mathrm{mAU}^{\star} \mathrm{s}\right]}\end{array}$ & $\begin{array}{l}\text { Height } \\
\text { [mAU] }\end{array}$ & $\begin{array}{c}\text { Area } \\
\frac{8}{8}\end{array}$ \\
\hline $\begin{array}{l}1 \\
2\end{array}$ & $\begin{array}{r}7.045 \\
16.381\end{array}$ & $\begin{array}{l}\mathrm{PB} \\
\mathrm{BB}\end{array}$ & $\begin{array}{l}0.2032 \\
0.5970\end{array}$ & $\begin{array}{l}57.75610 \\
5.18569 \mathrm{e} 4\end{array}$ & $\begin{array}{r}4.23124 \\
1289.89954\end{array}$ & $\begin{array}{r}0.1113 \\
99.8887\end{array}$ \\
\hline ota & i & & & $5.19147 \mathrm{e} 4$ & 1294.13078 & \\
\hline
\end{tabular}

Results obtained with enhanced integrator!

Signal 3: MWD1 C, Sig=212,4 Ref $=550,100$

\begin{tabular}{|c|c|c|c|c|c|c|}
\hline $\begin{array}{c}\text { Peak } \\
\#\end{array}$ & $\begin{array}{l}\text { RetTime } \\
\text { [min] }\end{array}$ & Type & $\begin{array}{c}\text { Width } \\
\text { [min] }\end{array}$ & $\begin{array}{c}\text { Area } \\
{\left[\mathrm{mAU}^{*} \mathrm{~S}\right]}\end{array}$ & $\begin{array}{l}\text { Height } \\
\text { [mAU] }\end{array}$ & $\begin{array}{c}\text { Area } \\
\frac{8}{8}\end{array}$ \\
\hline 1 & 7.139 & PB & 0.2774 & 1211.07166 & 63.23554 & 1.5506 \\
\hline 2 & 8.586 & $\mathrm{~PB}$ & 0.2384 & 68.56026 & 4.07323 & 0.0878 \\
\hline 3 & 14.256 & $\mathrm{BB}$ & 0.3618 & 114.42650 & 3.97535 & 0.1465 \\
\hline 4 & 16.378 & $\mathrm{BB}$ & 0.6019 & $7.67095 e 4$ & 1880.42078 & 98.2151 \\
\hline Tot & : & & & $7.81035 \mathrm{e} 4$ & 1951.70490 & \\
\hline
\end{tabular}

Results obtained with enhanced integrator!

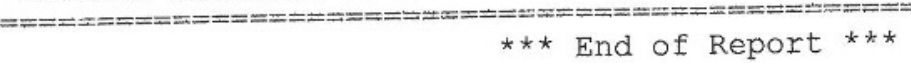




\section{Chiral HPLC chromatogram of racemic $\mathbf{4 0 .}$}

Data File C: \HPCHEM \I\DATA \JT\CHPLC-LO.D

Chiralcel AD-H; Heptan/Isopropanol $90: 10,0.5 \mathrm{~mL} / \mathrm{min} ; 2$ $\mu 1$

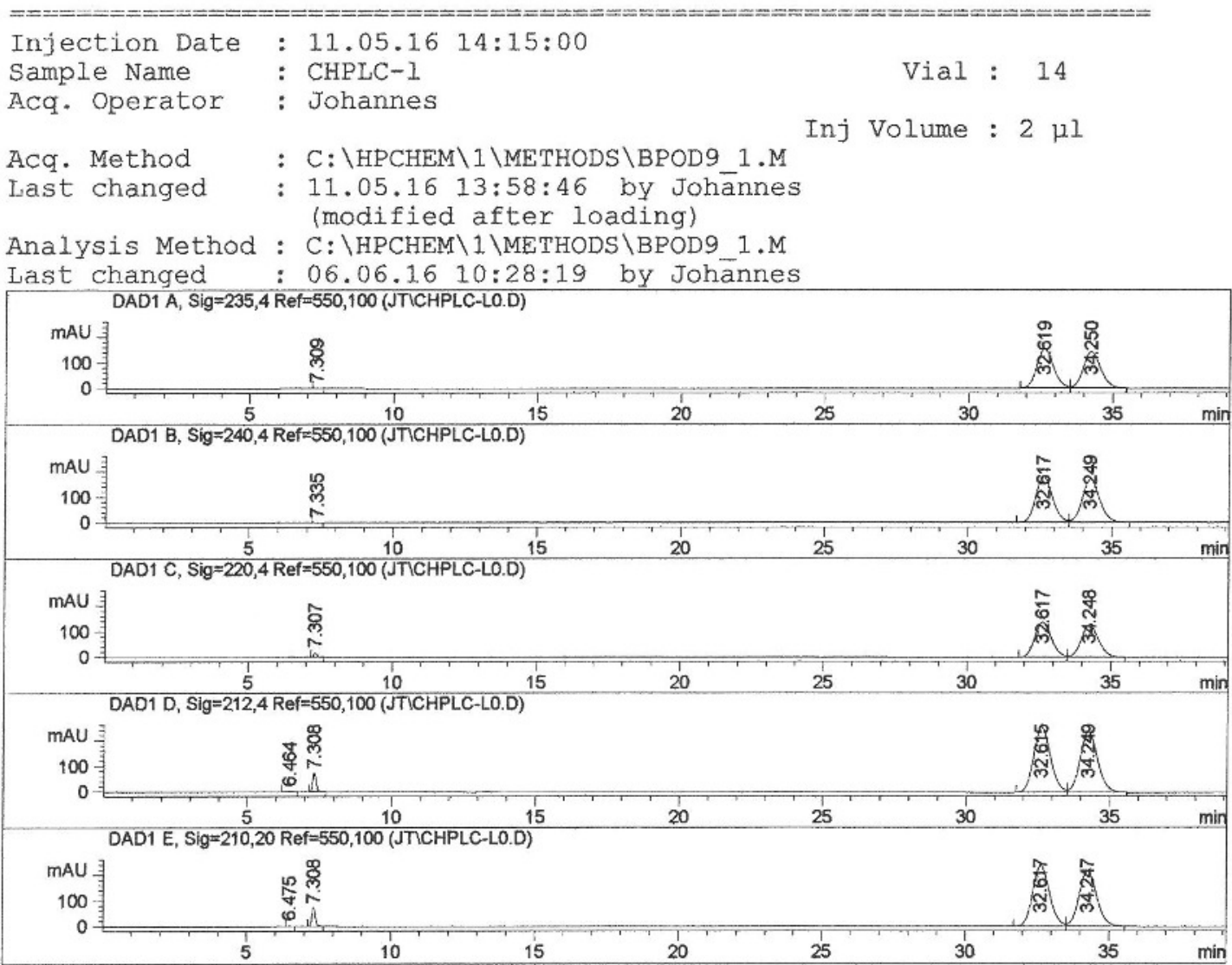

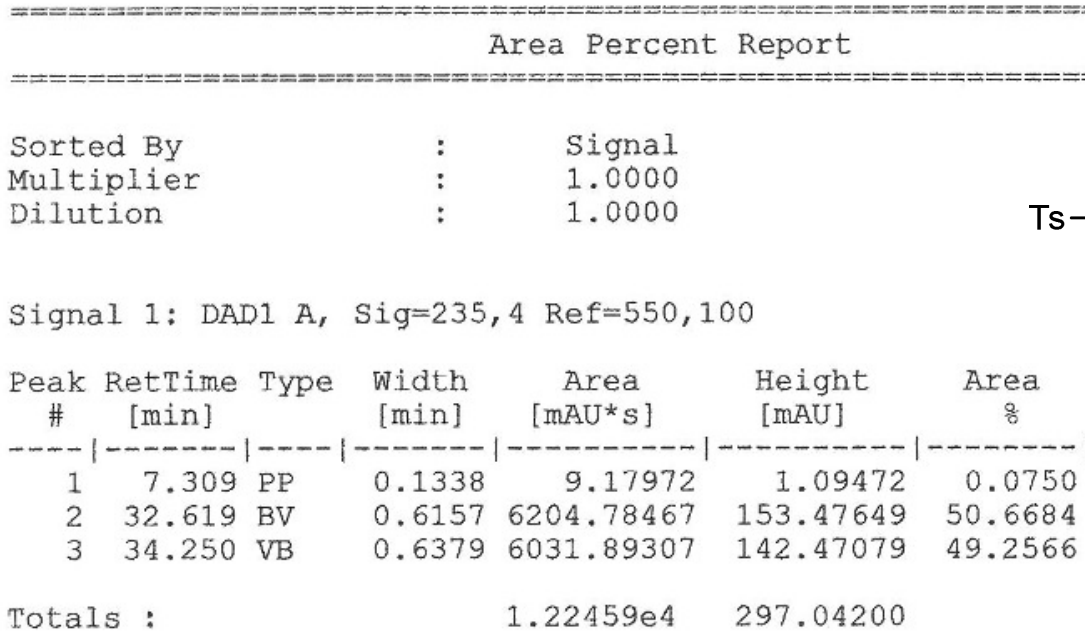


Data File $C: \backslash$ HPCHEM $\backslash 1 \backslash D A T A \backslash J T \backslash C H P L C-L O . D$

Sample Name: CHPLC-1

Results obtained with enhanced integrator!

Signal 2: DAD1 B, Sig=240,4 Ref $=550,100$

\begin{tabular}{|c|c|c|c|c|c|c|}
\hline $\begin{array}{c}\text { Peak } \\
\text { \# }\end{array}$ & $\begin{array}{c}\text { RetTime } \\
\text { [min] }\end{array}$ & Type & $\begin{array}{l}\text { Width } \\
\text { [min] }\end{array}$ & $\begin{array}{c}\text { Area } \\
{\left[\mathrm{mAU}^{*} \mathrm{~s}\right]}\end{array}$ & $\begin{array}{l}\text { Height } \\
\text { [mAU] }\end{array}$ & $\begin{array}{c}\text { Area } \\
\text { \& }\end{array}$ \\
\hline 1 & 7.335 & $\mathrm{BP}$ & 0.1350 & 3.40666 & $3.71576 \mathrm{e}-1$ & 0.0236 \\
\hline 2 & 32.617 & BV & 0.6245 & 7320.46875 & 180.76482 & 50.6284 \\
\hline 3 & 34.249 & VB & 0.6552 & 7135.33936 & 168.11732 & 49.3480 \\
\hline Totá & : & & & $1.44592 \mathrm{e} 4$ & 349.25372 & \\
\hline
\end{tabular}

Results obtained with enhanced integrator!

Signal 3: DAD1 C, Sig=220, 4 Ref $=550,100$

\begin{tabular}{|c|c|c|c|c|c|c|}
\hline $\begin{array}{c}\text { Peak } \\
\#\end{array}$ & $\begin{array}{l}\text { RetTime } \\
\text { [min] }\end{array}$ & Type & $\begin{array}{l}\text { Width } \\
\text { [min] }\end{array}$ & $\begin{array}{c}\text { Area } \\
{\left[\mathrm{mAU}^{*} \mathrm{~s}\right]}\end{array}$ & $\begin{array}{l}\text { Height } \\
\text { [mAU] }\end{array}$ & $\begin{array}{c}\text { Area } \\
\therefore 8\end{array}$ \\
\hline $\begin{array}{l}1 \\
2 \\
3\end{array}$ & $\begin{array}{r}7.307 \\
32.617 \\
34.248\end{array}$ & $\begin{array}{l}\text { BP } \\
\text { BV } \\
\text { VB }\end{array}$ & $\begin{array}{l}0.1245 \\
0.6068 \\
0.6147\end{array}$ & $\begin{array}{r}143.36980 \\
5540.64355 \\
5403.53076\end{array}$ & $\begin{array}{r}17.33884 \\
137.28847 \\
127.44588\end{array}$ & $\begin{array}{r}1.2931 \\
49.9718 \\
48.7351\end{array}$ \\
\hline ot & & & & $1.10875 \mathrm{e} 4$ & 282.07319 & \\
\hline
\end{tabular}

Results obtained with enhanced integrator!

Signal 4: DAD1 D, Sig=212, 4 Ref $=550,100$

\begin{tabular}{|c|c|c|c|c|c|c|}
\hline $\begin{array}{c}\text { Peak } \\
\#\end{array}$ & $\begin{array}{c}\text { RetTime } \\
\text { [min] }\end{array}$ & Type & $\begin{array}{l}\text { Width } \\
\text { [min] }\end{array}$ & $\begin{array}{c}\text { Area } \\
{\left[\mathrm{mAU}{ }^{\star} \mathrm{s}\right]}\end{array}$ & $\begin{array}{l}\text { Height } \\
\text { [mAU] }\end{array}$ & $\begin{array}{c}\text { Area } \\
8 \\
8\end{array}$ \\
\hline 1 & 6.464 & VB & 0.1658 & 20.79175 & 1.57735 & 0.0988 \\
\hline 2 & 7.308 & $\mathrm{BB}$ & 0.1286 & 620.42572 & 3933 & 2.9475 \\
\hline 3 & 32.615 & BV & 0.5818 & $1.03373 \mathrm{e} 4$ & 254.52271 & 49.1108 \\
\hline 4 & 34.249 & VB & 0.6194 & $1.00704 \mathrm{e} 4$ & 237.16504 & 47.8429 \\
\hline & & & & $2.10490 \mathrm{e} 4$ & 568.20443 & \\
\hline
\end{tabular}

Results obtained with enhanced integrator!

Signal 5: DAD1 E, Sig=210,20 Ref=550,100

\begin{tabular}{|c|c|c|c|c|c|c|}
\hline $\begin{array}{c}\text { Peak } \\
\#\end{array}$ & $\begin{array}{c}\text { RetTime } \\
\text { [min] }\end{array}$ & Type & $\begin{array}{l}\text { Width } \\
{[\min ]}\end{array}$ & $\begin{array}{c}\text { Area } \\
\text { [mAU*s] }\end{array}$ & $\begin{array}{l}\text { Height } \\
\text { [MAU] }\end{array}$ & $\begin{array}{c}\text { Area } \\
\frac{8}{8}\end{array}$ \\
\hline 1 & 6.475 & $\mathrm{BB}$ & 0.1247 & 9.96863 & 1.20294 & 0.0508 \\
\hline 2 & 7.308 & $\mathrm{VB}$ & 0.1298 & 623.46643 & 74.38312 & 3.1759 \\
\hline 3 & 32.617 & $\mathrm{BV}$ & 0.6232 & 9619.19141 & 236.14920 & 49.0001 \\
\hline 4 & 34.247 & VB & 0.6465 & 9378.35156 & 219.46964 & 47.7732 \\
\hline Tota] & : & & & $1.96310 \mathrm{e} 4$ & 531.20489 & \\
\hline
\end{tabular}

Results obtained with enhanced integrator!

$\star * *$ End of Report $* * *$ 


\section{Chiral HPLC chromatogram of (-)-40.}

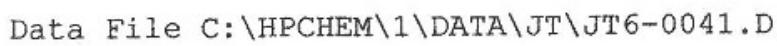

Chiralcel AD-H; Heptan/Isopropanol $90: 10,0.5 \mathrm{~mL} / \mathrm{min}$; 1 $\mu 1$

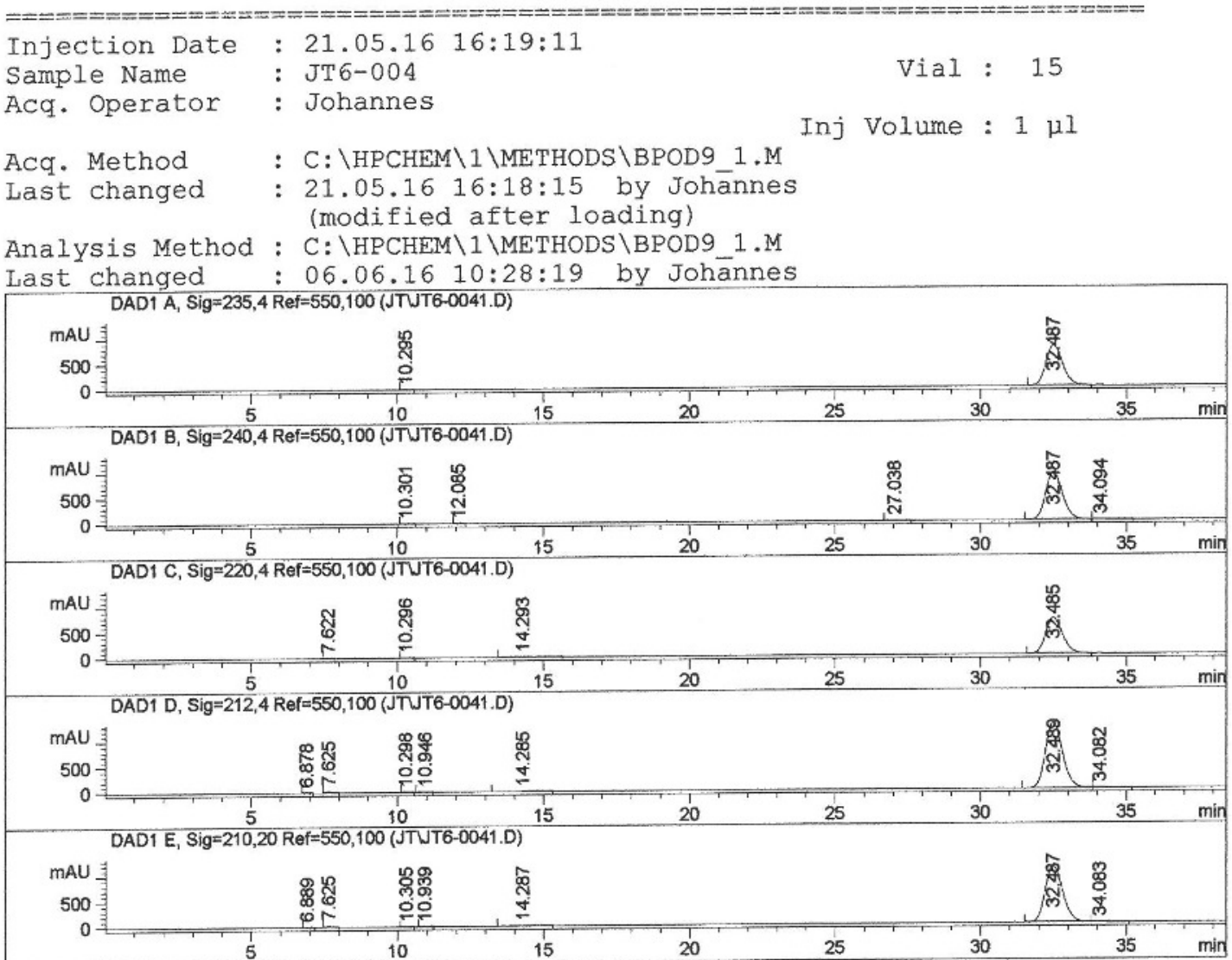

\section{Area Percent Report}

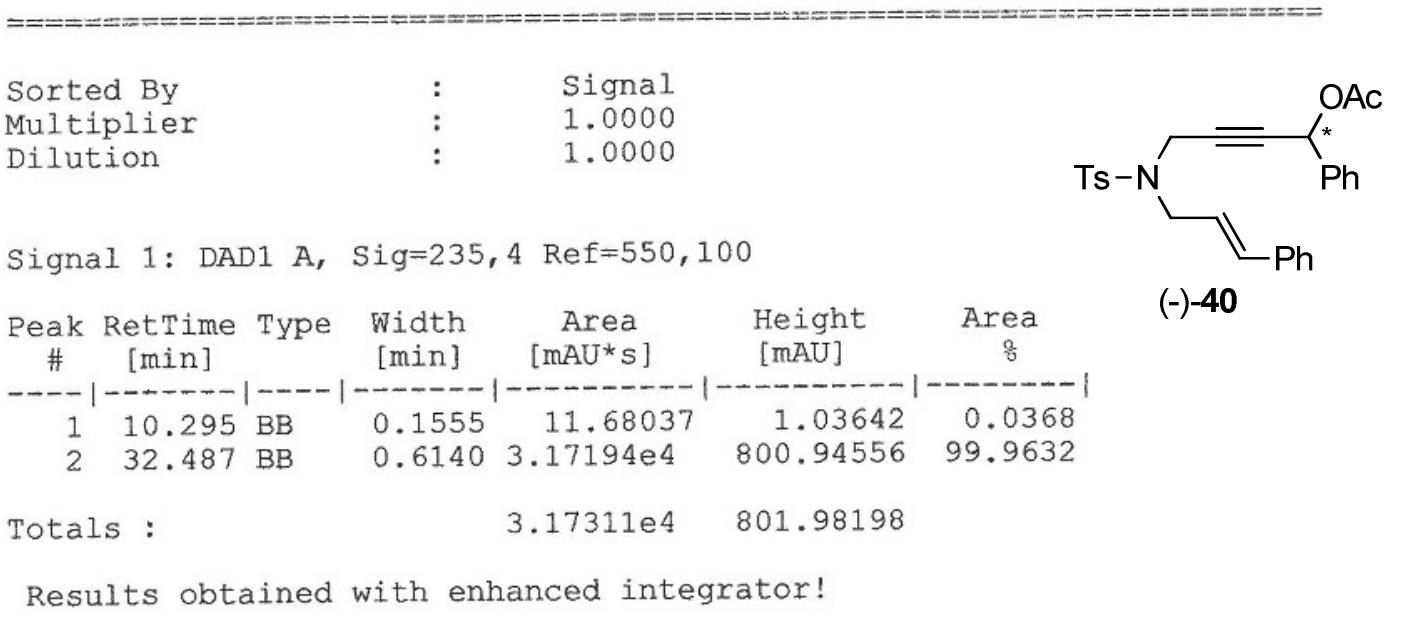

Instrument 1 14.06.16 08:49:13 Johannes

Page 1 of 3 
Signal 2: DAD1 B, Sig=240,4 Ref $=550,100$

\begin{tabular}{|c|c|c|c|c|c|c|}
\hline $\begin{array}{c}\text { Peak } \\
\#\end{array}$ & $\begin{array}{l}\text { RetTime } \\
\text { [min] }\end{array}$ & Type & $\begin{array}{l}\text { Width } \\
\text { [min] }\end{array}$ & $\begin{array}{c}\text { Area } \\
{\left[\mathrm{mAU}^{*} \mathrm{~s}\right]}\end{array}$ & $\begin{array}{l}\text { Height } \\
\text { [mAU] }\end{array}$ & $\begin{array}{c}\text { Area } \\
\frac{0}{\delta}\end{array}$ \\
\hline 1 & 10.301 & PB & 0.1575 & 9.80391 & $9.11089 e-1$ & 0,0259 \\
\hline 2 & 12.085 & $\mathrm{BB}$ & 0.1775 & 5.69989 & $4.01757 e-1$ & 0.0151 \\
\hline 3 & 27.038 & $\mathrm{BP}$ & 0.3577 & 20.17130 & $6.80699 e-1$ & 0.0533 \\
\hline 4 & 32.487 & $\mathrm{BB}$ & 0.6163 & $3.76279 e 4$ & 945.42584 & 99.3848 \\
\hline 5 & 34.094 & BB & 0.4817 & 197.24382 & 5.11675 & 0.5210 \\
\hline
\end{tabular}

Totals :

$3.78609 \mathrm{e} 4 \quad 952.53614$

Results obtained with enhanced integrator!

Signal 3: DAD1 C, Sig $=220,4$ Ref $=550,100$

\begin{tabular}{|c|c|c|c|c|c|c|}
\hline $\begin{array}{c}\text { Peak } \\
\#\end{array}$ & $\begin{array}{c}\text { RetTime } \\
\text { [min] }\end{array}$ & Type & $\begin{array}{l}\text { Width } \\
\text { [min] }\end{array}$ & $\begin{array}{c}\text { Area } \\
{\left[\mathrm{mAU}^{*} \mathrm{~s}\right]}\end{array}$ & $\begin{array}{l}\text { Height } \\
\text { [mAU] }\end{array}$ & $\begin{array}{c}\text { Area } \\
8\end{array}$ \\
\hline 1 & 7.622 & BB & 0.1389 & 50.47483 & 5.72000 & 0.1772 \\
\hline 2 & 10.296 & $\mathrm{PP}$ & 0.1637 & 13.43837 & 1.15512 & 0.0472 \\
\hline 3 & 14.293 & $\mathrm{BB}$ & 0.7140 & 204.22917 & 3.45302 & 0.7172 \\
\hline 4 & 32.485 & $\mathrm{BP}$ & 0.6043 & $2.82095 e 4$ & 708.88312 & 99.0584 \\
\hline ptál & $1 s$ : & & & $2.84776 \mathrm{e} 4$ & 719.21125 & \\
\hline
\end{tabular}

Totals :

$2.84776 \mathrm{e} 4 \quad 719.21125$

Results obtained with enhanced integrator!

Signal 4: $\mathrm{DAD1} D, \mathrm{Sig}=212,4$ Ref $=550,100$

\begin{tabular}{|c|c|c|c|c|c|c|}
\hline $\begin{array}{c}\text { Peak } \\
\#\end{array}$ & $\begin{array}{c}\text { RetTime } \\
\text { [min] }\end{array}$ & Type & $\begin{array}{c}\text { Width } \\
\text { [min] }\end{array}$ & $\begin{array}{c}\text { Area } \\
{\left[\mathrm{mAU}{ }^{*} \mathrm{~s}\right]}\end{array}$ & $\begin{array}{l}\text { Height } \\
\text { [mAU] }\end{array}$ & $\begin{array}{c}\text { Area } \\
\frac{\text { o }}{6}\end{array}$ \\
\hline 1 & 6.878 & $\mathrm{BB}$ & 0.1394 & 15.26829 & 1.59832 & 0.0288 \\
\hline 2 & 7.625 & $\mathrm{BP}$ & 0.1383 & 284.15472 & 32.39476 & 0.5357 \\
\hline 3 & 10.298 & PB & 0.1539 & 11.61285 & 1.01211 & 0.0219 \\
\hline 4 & 10.946 & $\mathrm{PP}$ & 0.1490 & 15.87730 & 1.39293 & 0.0299 \\
\hline 5 & 14.285 & $\mathrm{BB}$ & 0.7759 & 246.22545 & 3.96810 & 0.4642 \\
\hline 6 & 32.489 & $\mathrm{BB}$ & 0.5787 & $5.21842 e 4$ & 1293.29175 & 98.3840 \\
\hline 7 & 34.082 & $\mathrm{BB}$ & 0.4687 & 283.99109 & 7.51823 & 0.5354 \\
\hline Ea & : & & & $5.30413 \mathrm{e} 4$ & 1341.17619 & \\
\hline
\end{tabular}

Results obtained with enhanced integrator!

Signal 5: DAD1 E, Sig=210, 20 Ref $=550,100$

\begin{tabular}{|c|c|c|c|c|c|c|}
\hline $\begin{array}{c}\text { Peak } \\
\#\end{array}$ & $\begin{array}{l}\text { RetTime } \\
\text { [min] }\end{array}$ & Type & $\begin{array}{l}\text { Width } \\
\text { [min] }\end{array}$ & $\begin{array}{c}\text { Area } \\
\text { [mAU*s ] }\end{array}$ & $\begin{array}{l}\text { Height } \\
\text { [mAU] }\end{array}$ & $\begin{array}{c}\text { Area } \\
\frac{8}{8}\end{array}$ \\
\hline 1 & $6.8 \varepsilon r \gamma$ & BB & 0.1564 & 19.44858 & 1.76671 & 0.0414 \\
\hline 2 & & $\mathrm{PP}$ & & 320. & 36.0 & 31 \\
\hline 3 & 10.305 & $\mathrm{BB}$ & 0.1609 & 11.31044 & 1.02402 & 0.0241 \\
\hline 4 & 10.939 & PP & 0.1547 & 13.06187 & 1.20302 & 0.0278 \\
\hline 5 & 14.287 & $\mathrm{BB}$ & 0.7062 & 235.78671 & 3.96245 & 0.5020 \\
\hline 6 & 32.487 & BB & 0.6282 & $4.60853 \mathrm{e} 4$ & 1110.49170 & 98.1119 \\
\hline
\end{tabular}

Instrument 1 14.06.16 08:49:13 Johannes

Page 2 of 3 
Data File C: \HPCHEM \1\DATA \JT\JT6-0041.D

\begin{tabular}{|c|c|c|c|c|c|c|}
\hline $\begin{array}{c}\text { Peak } \\
\#\end{array}$ & $\begin{array}{c}\text { RetTime } \\
\text { [min] }\end{array}$ & Type & $\begin{array}{l}\text { Width } \\
\text { [min] }\end{array}$ & $\begin{array}{c}\text { Area } \\
{\left[m A U^{\star} s\right]}\end{array}$ & $\begin{array}{l}\text { Height } \\
\text { [mAU] }\end{array}$ & $\begin{array}{c}\text { Area } \\
\&\end{array}$ \\
\hline 7 & 34.083 & $B B$ & 0.4792 & 286.38113 & 7.15150 & 0.6097 \\
\hline Tota. & : & & & $4.69722 \mathrm{e} 4$ & 1161.61805 & \\
\hline
\end{tabular}

Results obtained with enhanced integrator!

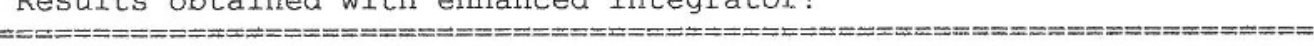

$\star \star \star$ End of Report $* * *$ 


\section{Chiral HPLC chromatogram of (+)-40.}

Jata File C: \HPCHEM $1 \backslash D A T A \backslash J T \backslash J T 6-0050 . D$

Chiralcel AD-H; Heptan/Isopropanol $90: 10,0.5 \mathrm{~mL} / \mathrm{min}$; 1

$\mu 1$
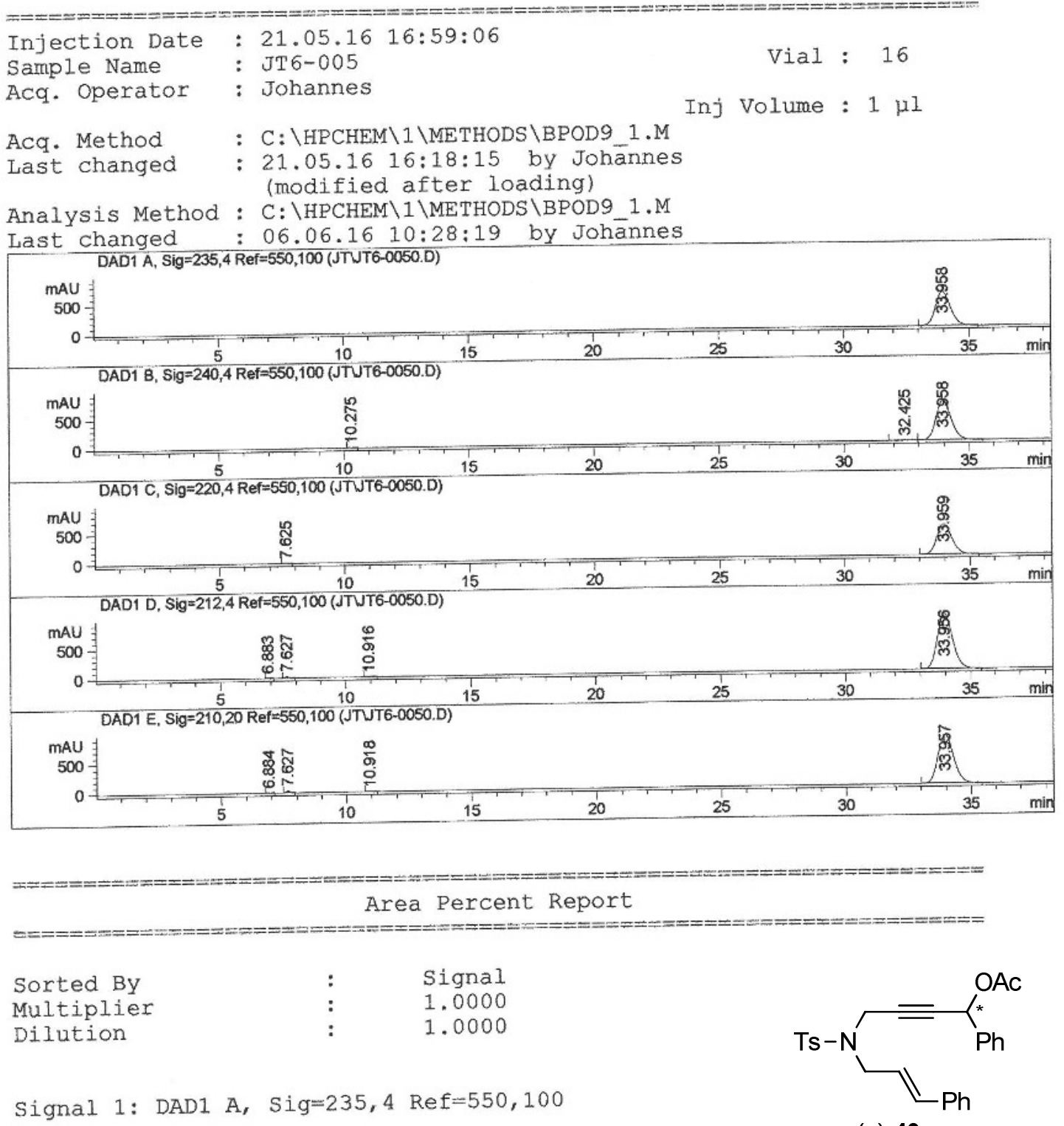

$$
\begin{aligned}
& \begin{array}{ccccc}
\text { Peak RetTime Type } & \text { Width } & \text { Area } & \text { Height } & \text { Area } \\
\text { \# [min] } & {\left[\mathrm{mAU}^{\star} \mathrm{S}\right]} & {[\mathrm{mAU}]} & 8
\end{array}
\end{aligned}
$$

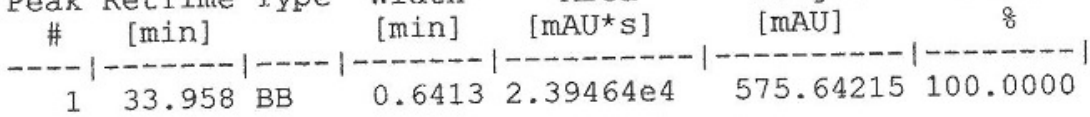

$$
\begin{aligned}
& \text { Totals : } \\
& 2.39464 \mathrm{e}^{4} \quad 575.64215
\end{aligned}
$$

$(+)-40$

Results obtained with enhanced integrator! 
Signal 2: DAD1 B, Sig=240,4 Ref=550,100

\begin{tabular}{|c|c|c|c|c|c|c|}
\hline $\begin{array}{c}\text { Peak } \\
\#\end{array}$ & $\begin{array}{l}\text { RetTime } \\
\text { [min] }\end{array}$ & Type & $\begin{array}{l}\text { Width } \\
\text { [min] }\end{array}$ & $\begin{array}{c}\text { Area } \\
{\left[\mathrm{mAU}^{\star} \mathrm{S}\right]}\end{array}$ & $\begin{array}{l}\text { Height } \\
\text { [mAU] }\end{array}$ & $\begin{array}{c}\text { Area } \\
8 \\
8\end{array}$ \\
\hline 1. & 10.275 & BB & 0.1400 & 6.38028 & $5.81728 e^{-1}$ & 0.0225 \\
\hline 2 & 32.425 & BV & 0.4409 & 45.94538 & 1.29816 & 0.1623 \\
\hline 3 & 33.958 & VB & 0.6456 & $2.82594 \mathrm{e} 4$ & 678.99255 & 99.8152 \\
\hline ot & : & & & $2.83118 \mathrm{e} 4$ & 680.87245 & \\
\hline
\end{tabular}

Results obtained with enhanced integrator!

Signa1 3: DAD1 C, Sig=220, 4 Ref $=550,100$

\begin{tabular}{|c|c|c|c|c|c|c|}
\hline $\begin{array}{c}\text { Peak } \\
\#\end{array}$ & $\begin{array}{l}\text { RetTime } \\
\text { [min] }\end{array}$ & Type & $\begin{array}{l}\text { Width } \\
\text { [min] }\end{array}$ & $\begin{array}{c}\text { Area } \\
{\left[\mathrm{mAU}^{*} \mathrm{~S}\right]}\end{array}$ & $\begin{array}{l}\text { Height } \\
\text { [mAU] }\end{array}$ & $\begin{array}{c}\text { Area } \\
\text { : }\end{array}$ \\
\hline $\begin{array}{l}1 \\
2\end{array}$ & $\begin{array}{r}7.625 \\
33.959\end{array}$ & $\begin{array}{l}\mathrm{BB} \\
\mathrm{PB}\end{array}$ & $\begin{array}{l}0.1343 \\
0.6228\end{array}$ & $\begin{aligned} & 45.03848 \\
& 2.13609 \mathrm{e} 4\end{aligned}$ & $\begin{array}{r}5.13668 \\
511.89813\end{array}$ & $\begin{array}{r}0.2104 \\
99.7896\end{array}$ \\
\hline & & & & $2.14059 \mathrm{e} 4$ & 517.03481 & \\
\hline
\end{tabular}

Results obtained with enhanced integrator!

Signal 4: DAD1 D, Sig=212, 4 Ref $=550,100$

\begin{tabular}{|c|c|c|c|c|c|c|}
\hline $\begin{array}{c}\text { Peak } \\
\#\end{array}$ & $\begin{array}{l}\text { RetTime } \\
\text { [min] }\end{array}$ & Type & $\begin{array}{l}\text { Width } \\
\text { [min] }\end{array}$ & $\begin{array}{c}\text { Area } \\
{\left[\mathrm{mAU}{ }^{*} \mathrm{~s}\right]}\end{array}$ & $\begin{array}{l}\text { Height } \\
\text { [mAU] }\end{array}$ & $\begin{array}{c}\text { Area } \\
\text { 号 }\end{array}$ \\
\hline 1 & 6.883 & PB & 0.1118 & 10.92664 & 1.27776 & 0.0275 \\
\hline 2 & 7.627 & $\mathrm{BB}$ & 0.1391 & 254.59866 & 28.81953 & 0.6402 \\
\hline 3 & 10.916 & $\mathrm{~PB}$ & 0.1442 & 13.08889 & 1.15484 & 0.0329 \\
\hline 4 & 33.956 & BB & 0.6217 & $3.94878 e 4$ & 940.75189 & 99.2994 \\
\hline & & & & $3.97664 \mathrm{e} 4$ & 972.00402 & \\
\hline
\end{tabular}

Results obtained with enhanced integrator!

Signal 5: DAD1 E, Sig $=210,20$ Ref $=550,100$

\begin{tabular}{|c|c|c|c|c|c|c|}
\hline $\begin{array}{c}\text { Peak } \\
\text { \# }\end{array}$ & $\begin{array}{l}\text { RetTime } \\
\text { [min] }\end{array}$ & Type & $\begin{array}{l}\text { Width } \\
\text { [min] }\end{array}$ & $\begin{array}{c}\text { Area } \\
{\left[\mathrm{mAU}^{\star} \mathrm{S}\right]}\end{array}$ & $\begin{array}{l}\text { Height } \\
\text { [mAU] }\end{array}$ & $\begin{array}{c}\text { Area } \\
8\end{array}$ \\
\hline 1 & 6.884 & PP & 0.1272 & 9.68000 & 1.18633 & 0.0270 \\
\hline 2 & 7.627 & $\mathrm{BB}$ & 0.1402 & 287.32141 & 32.15666 & 0.8028 \\
\hline 3 & 10.918 & $\mathrm{~PB}$ & 0.1477 & 12.37264 & 1.13062 & 0.0346 \\
\hline 4 & 33.957 & $\mathrm{BB}$ & 0.6640 & $3.54783 e 4$ & 827.87103 & 99.1355 \\
\hline rota & s : & & & $3.57877 \mathrm{e} 4$ & 862.34464 & \\
\hline
\end{tabular}

Results obtained with enhanced integrator! 


\section{Chiral HPLC chromatogram of racemic $\mathbf{1 1}$ from racemic $\mathbf{4 0 .}$}

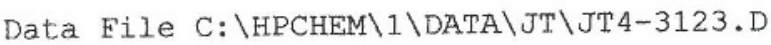
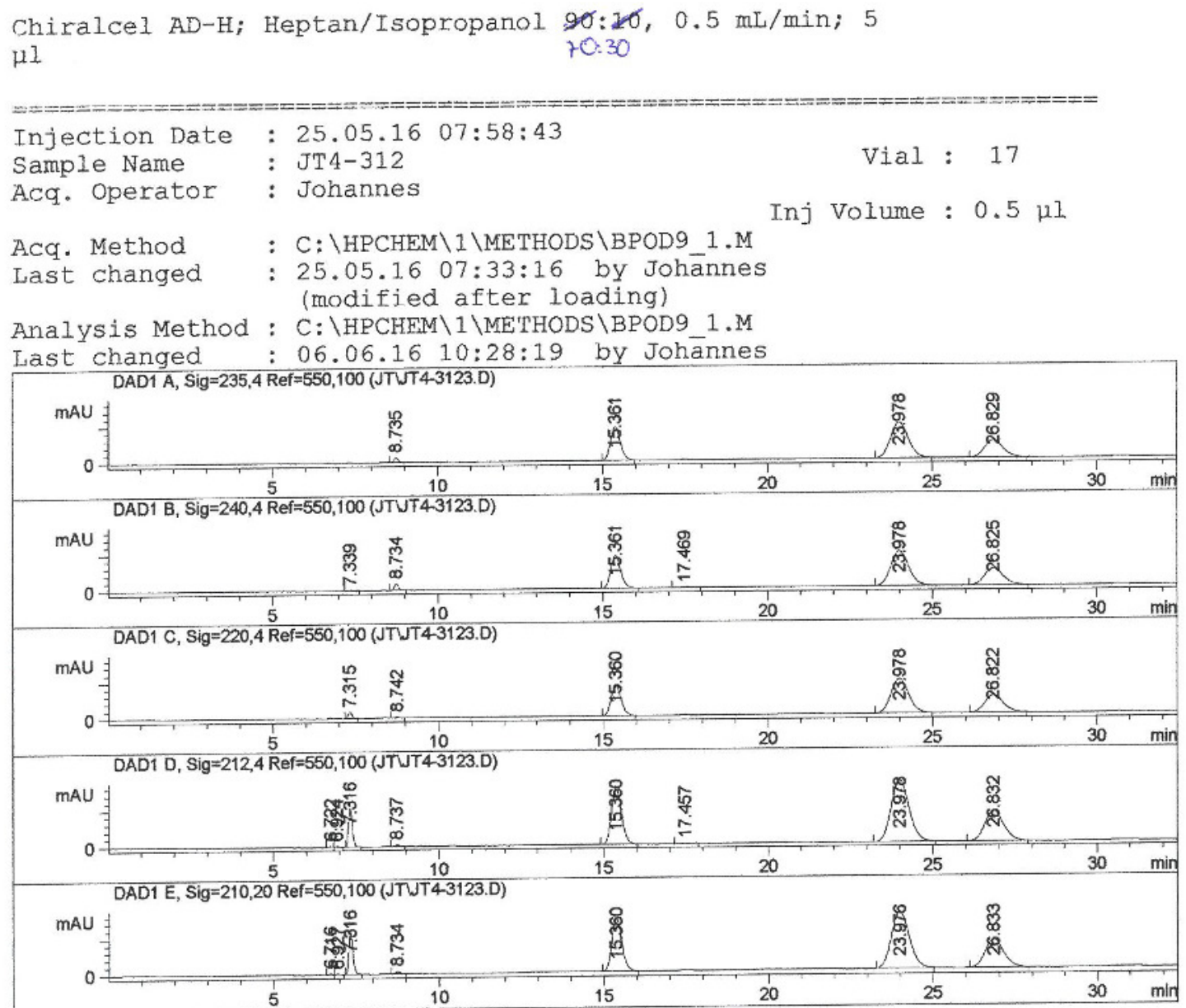

Area Percent Report

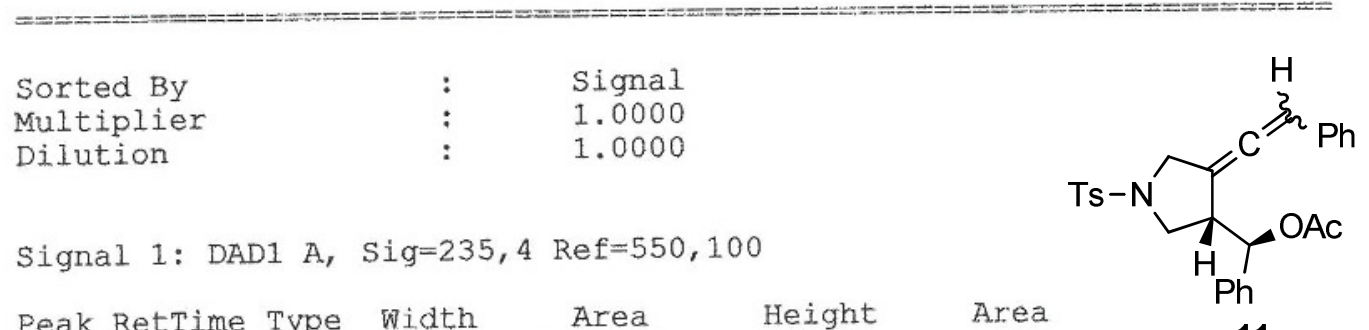

\begin{tabular}{|c|c|c|c|c|c|c|}
\hline $\begin{array}{c}\text { Peak } \\
\#\end{array}$ & $\begin{array}{l}\text { RetTime } \\
\text { [min] }\end{array}$ & Type & $\begin{array}{l}\text { Width } \\
\text { [min] }\end{array}$ & $\begin{array}{c}\text { Area } \\
{\left[\mathrm{mAU} U^{\star} \mathrm{S}\right]}\end{array}$ & $\begin{array}{l}\text { Height } \\
\text { [mAU] }\end{array}$ & $\begin{array}{c}\text { Area } \\
\frac{8}{5}\end{array}$ \\
\hline 1 & 8.735 & $\mathrm{BB}$ & 0.1440 & 59.49535 & 6.42400 & 1.5507 \\
\hline 2 & 15.361 & $\mathrm{BB}$ & 0.3226 & 957.52789 & 46.04716 & 24.9566 \\
\hline 3 & 23.978 & $\mathrm{BB}$ & 0.5567 & 1.866 .95618 & 49.42047 & 48.6595 \\
\hline 4 & 26.829 & $\mathrm{BB}$ & 0.5453 & 952.79694 & 24.30997 & 24.8333 \\
\hline & & & & 3836.77635 & 126.20160 & \\
\hline
\end{tabular}

rac. 11

from rac. 40

Totals:

Page 1 of 3 
Results obtained with enhanced integrator!

Signal 2: DAD1 B, Sig $=240,4$ Ref $=550,100$

\begin{tabular}{|c|c|c|c|c|c|c|}
\hline $\begin{array}{c}\text { Peak } \\
\#\end{array}$ & $\begin{array}{l}\text { RetTime } \\
\text { [min] }\end{array}$ & Type & $\begin{array}{l}\text { Width } \\
\text { [min] }\end{array}$ & $\begin{array}{c}\text { Area } \\
{\left[\mathrm{mAU}^{\star} \mathrm{s}\right]}\end{array}$ & $\begin{array}{l}\text { Height } \\
\text { [mAU] }\end{array}$ & $\begin{array}{c}\text { Area } \\
8\end{array}$ \\
\hline & & & & 4.51900 & $4.06562 e^{-1}$ & \\
\hline $\begin{array}{l}1 \\
2\end{array}$ & $\begin{array}{l}7.339 \\
8.734\end{array}$ & $\begin{array}{l}B P \\
V B\end{array}$ & $\begin{array}{l}0.1417 \\
0.1420\end{array}$ & $\begin{array}{r}4.51900 \\
82.84147\end{array}$ & $\begin{array}{r}4.06562 e^{-1} \\
8.78827\end{array}$ & 2.1899 \\
\hline 3 & 15.361 & $\mathrm{BB}$ & 0.3236 & 933.93945 & 44.72154 & 24.6890 \\
\hline 4 & 17.469 & $\mathrm{~PB}$ & 0.2831 & 19.34198 & $8.20125 e^{-1}$ & 0.5113 \\
\hline 5 & 23.978 & $\mathrm{BB}$ & 0.5779 & 1824.19531 & 48.16427 & 48.2232 \\
\hline 6 & 26.825 & BB & 0.5282 & 917.97662 & 23.27351 & 24.2670 \\
\hline Tota & : & & & 3782.81384 & 126.17428 & \\
\hline
\end{tabular}

Results obtained with enhanced integrator!

Signal 3: DAD1 C, Sig $=220,4$ Ref $=550,100$

\begin{tabular}{|c|c|c|c|c|c|c|}
\hline$\underset{\#}{\text { Peak }}$ & $\begin{array}{c}\text { RetTime } \\
\text { [min] }\end{array}$ & Type & $\begin{array}{l}\text { Width } \\
\text { [min] }\end{array}$ & $\begin{array}{c}\text { Area } \\
{\left[\mathrm{mAU}^{\star} \mathrm{S}\right]}\end{array}$ & $\begin{array}{l}\text { Height } \\
\text { [mAU] }\end{array}$ & $\begin{array}{c}\text { Area } \\
\therefore\end{array}$ \\
\hline 1 & 7.315 & $\mathrm{BB}$ & 0.1307 & 76.34180 & 9.02341 & 2.0401 \\
\hline 2 & 8.742 & $\mathrm{BP}$ & 0.1215 & 12.82998 & 1. 41931 & 0.3429 \\
\hline 3 & 15.360 & $\mathrm{BB}$ & 0.3153 & 897.29565 & 43.01862 & 23.9791 \\
\hline 4 & 23.978 & $\mathrm{BB}$ & 0.5642 & 1802.54517 & 47.76945 & 48.1708 \\
\hline 5 & 26.822 & $\mathrm{BB}$ & 0.4979 & 952.97797 & 24.28164 & 25.4671 \\
\hline & & & & 3741.99056 & 125.51243 & \\
\hline
\end{tabular}

Results obtained with enhanced integrator!

Signal 4: DAD1 D, Sig=212,4 Ref $=550,100$

\begin{tabular}{|c|c|c|c|c|c|c|}
\hline $\begin{array}{c}\text { Peak } \\
\text { \# }\end{array}$ & $\begin{array}{c}\text { RetTime } \\
\text { [min] }\end{array}$ & Type & $\begin{array}{l}\text { Width } \\
\text { [min] }\end{array}$ & $\begin{array}{c}\text { Area } \\
{[m A U * s]}\end{array}$ & $\begin{array}{l}\text { Height } \\
\text { [mAU] }\end{array}$ & $\begin{array}{c}\text { Area } \\
\text { \& }\end{array}$ \\
\hline 1 & 6.722 & BV & 0.1154 & 13.44177 & 1.71508 & 0.1900 \\
\hline 2 & 6.924 & $\mathrm{VP}$ & 0.1230 & 11.12405 & 1.36597 & 0.1572 \\
\hline 3 & 7.316 & $\mathrm{BB}$ & 0.1303 & 445.21219 & 52.83036 & 6.2925 \\
\hline 4 & 8.737 & $\mathrm{BP}$ & 0.1387 & 20.91398 & 2.28679 & 0.2956 \\
\hline 5 & 15.360 & $\mathrm{BB}$ & 0.3129 & 1650.71509 & 78.62199 & 23.3308 \\
\hline 6 & 17.4 .57 & PB & 0.2722 & 29.21442 & 1.29022 & 0.4129 \\
\hline 7 & 23.978 & BB & 0.5513 & 3240.31445 & 85.27641 & 45.7978 \\
\hline 8 & 26.832 & $\mathrm{BB}$ & 0.5209 & 1664.33118 & 42.50045 & 23.5232 \\
\hline & & & & 7075.26713 & 265.88728 & \\
\hline
\end{tabular}

Results obtained with enhanced integrator!

Signal 5: DAD1 E, Sig=210,20 Ref $=550,100$

\begin{tabular}{|c|c|c|c|c|c|c|}
\hline $\begin{array}{c}\text { Peak } \\
\text { \# }\end{array}$ & $\begin{array}{c}\text { RetTime } \\
\text { [min] }\end{array}$ & Type & $\begin{array}{l}\text { Width } \\
\text { [min] }\end{array}$ & $\begin{array}{c}\text { Area } \\
{\left[\mathrm{mAU}^{\star} \mathrm{s}\right]}\end{array}$ & $\begin{array}{l}\text { Height } \\
\text { [mAU] }\end{array}$ & $\begin{array}{c}\text { Area } \\
\text { g }\end{array}$ \\
\hline & & & & 12. & & \\
\hline 2 & 6.927 & & 0.1269 & 9.91562 & 1.16943 & 0.1510 \\
\hline
\end{tabular}

Instrument 1 14.06.16 09:07:42 Johannes 


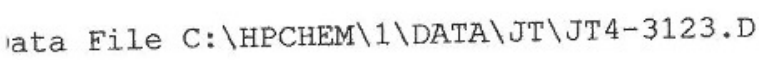

\begin{tabular}{|c|c|c|c|c|c|c|}
\hline $\begin{array}{c}\text { Peak } \\
\#\end{array}$ & $\begin{array}{l}\text { RetTime } \\
\text { [min] }\end{array}$ & Type & $\begin{array}{l}\text { Width } \\
\text { [min] }\end{array}$ & $\begin{array}{c}\text { Area } \\
{\left[\mathrm{mAU}{ }^{\star} \mathrm{s}\right]}\end{array}$ & $\begin{array}{l}\text { Height } \\
\text { [mAU] }\end{array}$ & $\begin{array}{c}\text { Area } \\
\text { : }\end{array}$ \\
\hline 3 & 7.316 & $\mathrm{BB}$ & 0.1360 & 494.23807 & 57.63836 & 7.5262 \\
\hline 4 & 8.734 & $\mathrm{BP}$ & 0.1423 & 30.10466 & 3.30348 & 0.4584 \\
\hline 5 & 15.360 & $\mathrm{BB}$ & 0.3157 & 1509.64648 & 72.25777 & 22.9888 \\
\hline 6 & 23.976 & $\mathrm{BB}$ & 0.5820 & 2974.50952 & 78.52073 & 4.5 .2956 \\
\hline 7 & 26.833 & $\mathrm{BB}$ & 0.5865 & 1535.81787 & 39.07567 & 23.3873 \\
\hline Total & Is : & & & 6566.88171 & 253.65242 & \\
\hline
\end{tabular}

Instrument 1 14.06.16 09:07:42 Johannes

Page 3 of 3 


\section{Chiral HPLC chromatogram of racemic 11 from (-)-40.}

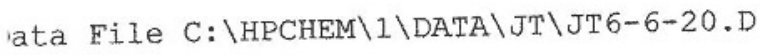

Chiralcel AD-H; Heptan/Isopropanol 70:30, $0.5 \mathrm{~mL} / \mathrm{min}$; 0

$.5 \mu 1$

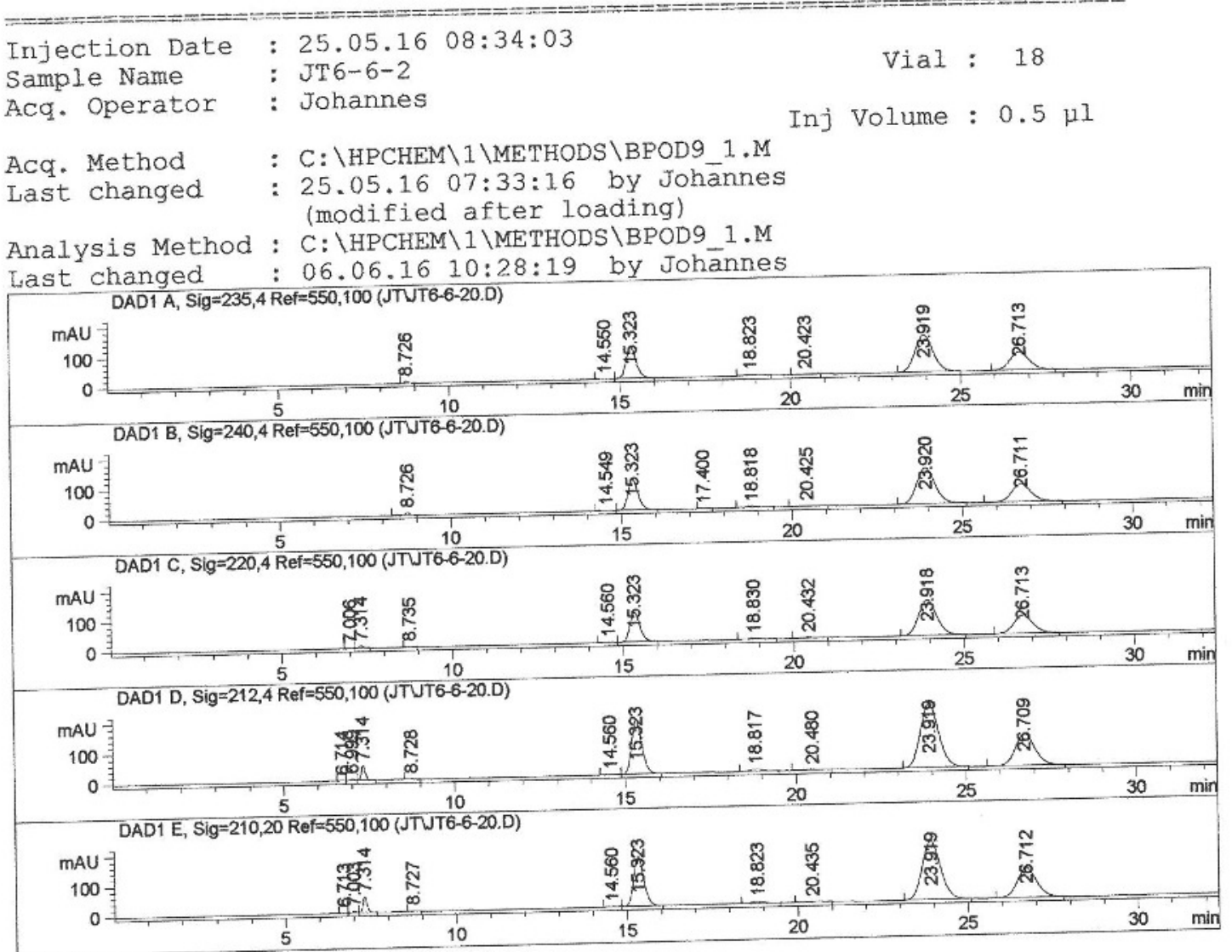

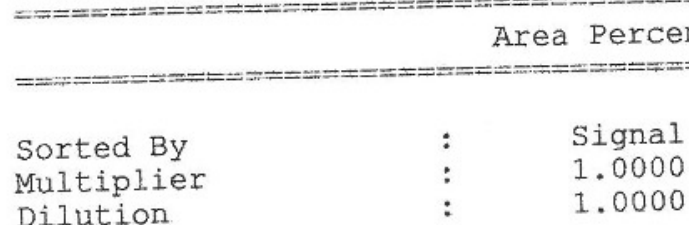

Signal 1: DAD1 A, Sig $=235,4$ Ref $=550,100$

\begin{tabular}{|c|c|c|c|c|c|c|}
\hline $\begin{array}{c}\text { Peak } \\
\#\end{array}$ & $\begin{array}{c}\text { RetTime } \\
\text { [min] }\end{array}$ & Type & $\begin{array}{l}\text { Width } \\
\text { [min] }\end{array}$ & $\begin{array}{c}\text { Area } \\
{\left[\mathrm{mAU}^{*} \mathrm{~S}\right]}\end{array}$ & $\begin{array}{l}\text { Height } \\
\text { [mAU] }\end{array}$ & $\begin{array}{c}\text { Area } \\
8\end{array}$ \\
\hline 1 & 8.726 & BB & 0.1381 & 53.36460 & 5.86628 & 0.5638 \\
\hline 2 & 14.550 & PV & 0.2069 & 23.67114 & 1.50972 & 0.2501 \\
\hline 3 & 15.323 & VB & 0.3179 & 2187.89600 & 105.53717 & 23.1156 \\
\hline 4 & 18.823 & $\mathrm{BB}$ & 0.3611 & 95.33984 & 3.79448 & 1.0073 \\
\hline 5 & 20.423 & BB & 0.3173 & 36.77313 & 1.50363 & 0.3885 \\
\hline 6 & 23.919 & BB & 0.5818 & 4637.79687 & 123.60366 & 48.9993 \\
\hline
\end{tabular}<smiles>[B]N1C/C(=C\C=C/c2ccccc2)[C@@H]([C@H](OC(C)=O)c2ccccc2)C1</smiles>

rac. 11 from $(-)-40$

Page 1 of 3

Instrument 1 14.06.16 09:10:14 Johannes 


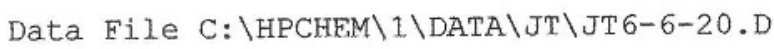

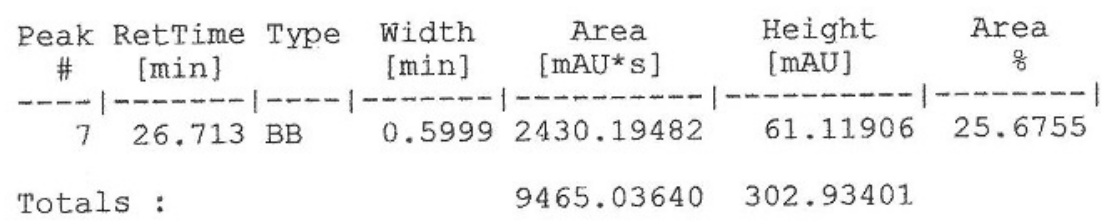

\begin{tabular}{|c|c|c|c|c|c|c|}
\hline $\begin{array}{c}\text { Peak } \\
\#\end{array}$ & $\begin{array}{c}\text { RetTime } \\
\text { [min] }\end{array}$ & Type & $\begin{array}{l}\text { Width } \\
\text { [min] }\end{array}$ & $\begin{array}{c}\text { Area } \\
{\left[\mathrm{mAU}^{*} \mathrm{~S}\right]}\end{array}$ & $\begin{array}{l}\text { Height } \\
\text { [mAU] }\end{array}$ & $\begin{array}{c}\text { Area } \\
8\end{array}$ \\
\hline 1 & 8.726 & BB & 0.1455 & 83.20725 & 8.25825 & 0.8948 \\
\hline 2 & 14.549 & PV & 0.2060 & 23.67996 & 1.45425 & 0.2546 \\
\hline 3 & 15.323 & VB & 0.3224 & 2136.29297 & 102.82585 & 22.9726 \\
\hline 4 & 17.400 & BB & 0.2179 & 7.14280 & $4.12792 e^{-1}$ & 0.0768 \\
\hline 5 & 18.818 & $\mathrm{BB}$ & 0.3504 & 101.42088 & 3.86178 & 1.0906 \\
\hline 6 & 20.425 & $\mathrm{BB}$ & 0.4156 & 39.64080 & 1.14601 & 0.4263 \\
\hline 7 & 23.920 & $\mathrm{BB}$ & 0.5825 & 4521.27734 & 120.29467 & 48.6194 \\
\hline 8 & 26.711 & $\mathrm{BB}$ & 0.5972 & 2386.66553 & 59.33994 & 25.6649 \\
\hline La & & & & 9299.32753 & 297.59354 & \\
\hline
\end{tabular}

Results obtained with enhanced integrator!

Signal 2: DAD1 B, $S i g=240,4$ Ref $=550,100$

\begin{tabular}{|c|c|c|c|c|c|c|}
\hline $\begin{array}{c}\text { Peak } \\
\#\end{array}$ & $\begin{array}{c}\text { RetTime } \\
\text { [min] }\end{array}$ & Type & $\begin{array}{l}\text { Width } \\
\text { [min] }\end{array}$ & $\begin{array}{c}\text { Area } \\
{\left[\mathrm{mAU}^{*} \mathrm{~s}\right]}\end{array}$ & $\begin{array}{l}\text { Height } \\
\text { [mAU] }\end{array}$ & $\begin{array}{c}\text { Area } \\
\&\end{array}$ \\
\hline 1. & 7.006 & VV & 0.1110 & 10.22370 & 1.25572 & 0.1109 \\
\hline 2 & 7.314 & VB & 0.1258 & 70.27075 & 8.38323 & 0.7621 \\
\hline 3 & 8.735 & $\mathrm{BP}$ & 0.1154 & 11.20264 & 1.31521 & 0.1215 \\
\hline 4 & 14.560 & PV & 0.1999 & 27.61477 & 1.75185 & 0.2995 \\
\hline 5 & 15.323 & VB & 0.3222 & 2046.90198 & 98.61362 & 22.1999 \\
\hline 6 & 18.830 & BB & 0.3535 & 88.54707 & 3.47258 & 0.9603 \\
\hline 7 & 20.432 & $\mathrm{BB}$ & 0.3576 & 56.82317 & 2.03628 & 0.6163 \\
\hline 8 & 23.918 & $\mathrm{BB}$ & 0.5832 & 4475.31445 & 118.87106 & 48.5375 \\
\hline 9 & 26.713 & $\mathrm{BB}$ & 0.5738 & 2433.42651 & 60.94784 & 26.3920 \\
\hline & . & & & 9220.32504 & 296.64740 & \\
\hline
\end{tabular}

Results obtained with enhanced integrator!

Signal 3: DAD1 C, Sig=220,4 Ref $=550,100$

\begin{tabular}{|c|c|c|c|c|c|c|}
\hline $\begin{array}{c}\text { Peak } \\
\#\end{array}$ & $\begin{array}{c}\text { RetTime } \\
\text { [min] }\end{array}$ & Type & $\begin{array}{l}\text { Width } \\
\text { [min] }\end{array}$ & $\begin{array}{c}\text { Area } \\
{\left[\mathrm{mAU}^{*} \mathrm{~S}\right]}\end{array}$ & $\begin{array}{l}\text { Height } \\
\text { [mAU] }\end{array}$ & $\begin{array}{c}\text { Area } \\
\&\end{array}$ \\
\hline 1 & 6.71 .4 & BV & 0.1251 & 12.04998 & 1. 38991 & 0.0716 \\
\hline 2 & .999 & VV & & 47.06841 & 5.41814 & 0.2798 \\
\hline 3 & 7.3 & VP & 0. & 408.16278 & 48.74987 & 2.4262 \\
\hline 4 & 8.728 & $\mathrm{~PB}$ & & 6777 & 1.91035 & 0.1020 \\
\hline 5 & 14 & $\mathrm{PP}$ & 0.1978 & 29.83169 & 1.91466 & 0.1773 \\
\hline 6 & & VB & 0.3 & 3747.78638 & 180.14720 & 22.2779 \\
\hline 7 & 18.81 & $\mathrm{BB}$ & 0.3128 & 177.82269 & 6.80584 & 1.0570 \\
\hline
\end{tabular}

Results obtained with enhanced integrator!

Signal 4: DAD1 D, Sig=212, 4 Ref $=550,100$

Instrument $1 \quad 14.06 .16 \quad 09: 10: 14$ Johannes 
Data File C: \HPCHEM \1\DATA \JT\JT6-6-20.D

\begin{tabular}{|c|c|c|c|c|c|c|}
\hline $\begin{array}{c}\text { Peak } \\
\#\end{array}$ & $\begin{array}{c}\text { RetTime } \\
\text { [min] }\end{array}$ & Type & $\begin{array}{l}\text { Width } \\
\text { [min] }\end{array}$ & $\begin{array}{c}\text { Area } \\
{\left[\mathrm{mAU}^{*} \mathrm{~s}\right]}\end{array}$ & $\begin{array}{l}\text { Height } \\
\text { [mAU] }\end{array}$ & $\begin{array}{c}\text { Area } \\
\text { : }\end{array}$ \\
\hline 8 & 20.480 & PB & 0.3837 & 60.23061 & 1.87017 & 0.3580 \\
\hline 9 & 23.919 & $\mathrm{BB}$ & 0.5341 & 8021.96143 & 213.63205 & 47.6847 \\
\hline 10 & 26.709 & $\mathrm{BB}$ & 0.5724 & 4300.83350 & 107.11890 & 25.5653 \\
\hline \multicolumn{4}{|c|}{ Totals : } & $1.68229 \mathrm{e} 4$ & 568.95709 & \\
\hline
\end{tabular}

Results obtained with enhanced integrator!

Signal 5: DAD1 E, Sig=210,20 Ref=550,100

\begin{tabular}{|c|c|c|c|c|c|c|}
\hline $\begin{array}{c}\text { Peak } \\
\#\end{array}$ & $\begin{array}{c}\text { RetTime } \\
\text { [min] }\end{array}$ & Type & $\begin{array}{c}\text { Width } \\
\text { [min] }\end{array}$ & $\begin{array}{c}\text { Area } \\
{\left[\mathrm{mAU}{ }^{\star} \mathrm{S}\right]}\end{array}$ & $\begin{array}{l}\text { Height } \\
\text { [mAU] }\end{array}$ & $\begin{array}{c}\text { Area } \\
88\end{array}$ \\
\hline 1 & 6.713 & BV & 0.1271 & 12.11417 & 1.37017 & 0.0781 \\
\hline 2 & 7.003 & VV & 0.1279 & 46.82285 & 5.46803 & 0.3018 \\
\hline 3 & 7.314 & VP & 0.1316 & 449.52707 & 52.64375 & 2.8978 \\
\hline 4 & 8.727 & $\mathrm{BP}$ & 0.1365 & 26.69574 & 2.98018 & 0.1721 \\
\hline 5 & 14.560 & $\mathrm{BP}$ & 0.1913 & 30.33364 & 1.93081 & 0.1955 \\
\hline 6 & 15.323 & VB & 0.3268 & 3424.72192 & 164.56442 & 22.0768 \\
\hline 7 & 18.823 & $\mathrm{BB}$ & 0.3287 & 161.05367 & 6.16757 & 1.0382 \\
\hline 8 & 20.435 & $\mathrm{BB}$ & 0.3868 & 59.05185 & 1.94196 & 0.3807 \\
\hline 9 & 23.919 & $\mathrm{BB}$ & 0.5642 & 7354.93506 & 194.93289 & 47.4122 \\
\hline 10 & 26.712 & $\mathrm{BB}$ & 0.5786 & 3947.49170 & 98.70076 & 25.4468 \\
\hline ota & & & & $1.55127 \mathrm{e} 4$ & 530.70055 & \\
\hline
\end{tabular}

Results obtained with enhanced integrator!

*** End of Report *** 


\section{Chiral HPLC chromatogram of racemic 11 from (+)-40.}

lata File $C: \backslash H P C H E M \backslash 1 \backslash D A T A \backslash J T \backslash J T 6-0070 . D$

Chiralcel AD-H; Heptan/Isopropanol 70:30, $0.5 \mathrm{~mL} / \mathrm{min} ; 0$

$.5 \mu 1$

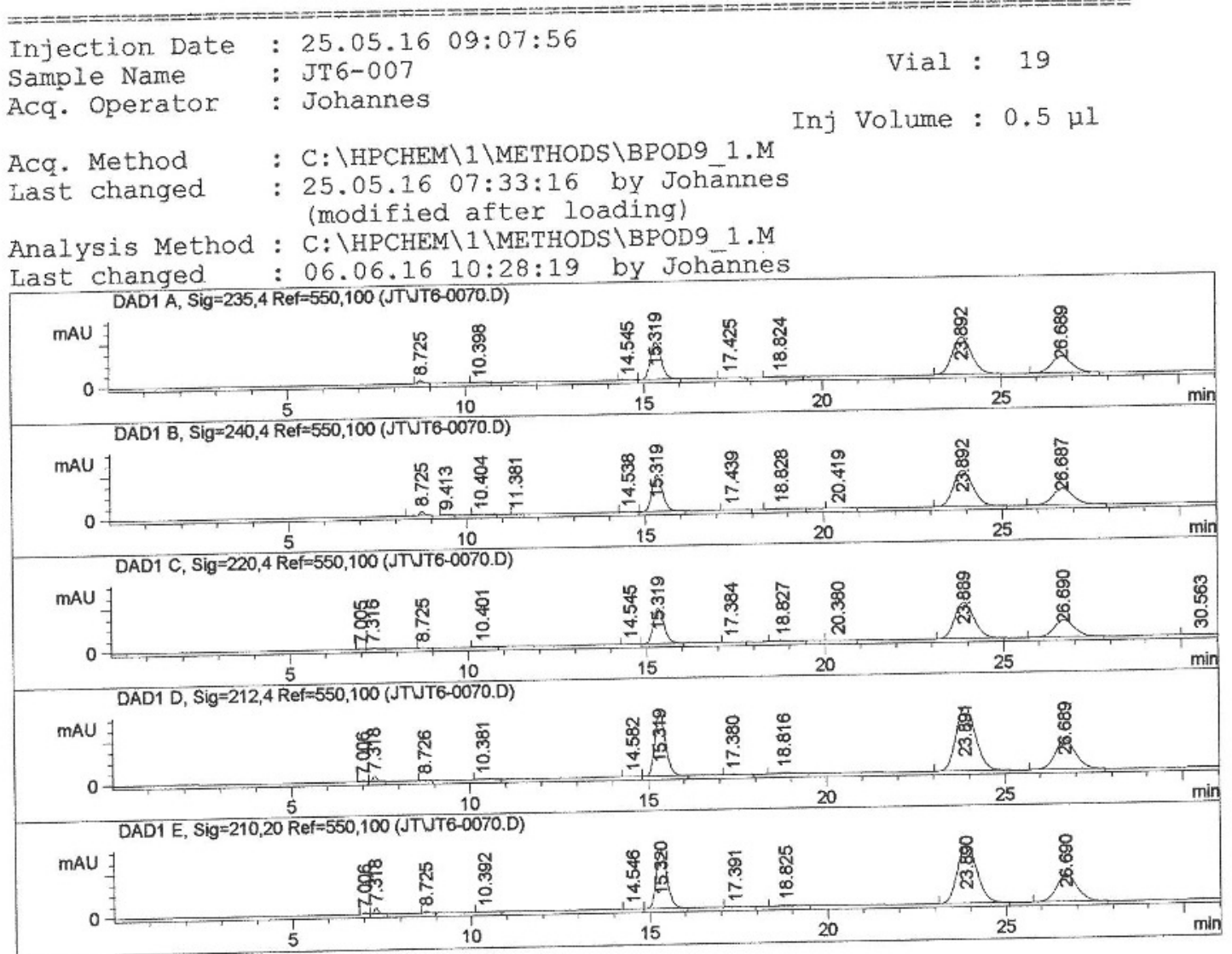

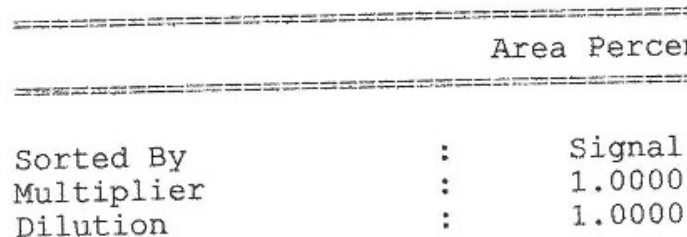

Signal 1: DAD1 A, Sig=235, 4 Ref $=550,100$

\begin{tabular}{|c|c|c|c|c|c|c|}
\hline Peak & $\begin{array}{l}\text { RetTime } \\
\text { [min] }\end{array}$ & Type & $\begin{array}{l}\text { Width } \\
\text { [min] }\end{array}$ & $\begin{array}{c}\text { Area } \\
{\left[\mathrm{mAU}{ }^{*} \mathrm{~s}\right]}\end{array}$ & $\begin{array}{l}\text { Height } \\
\text { [mAU] }\end{array}$ & $\begin{array}{c}\text { Area } \\
\text { \& }\end{array}$ \\
\hline 1 & 725 & $\mathrm{BB}$ & 0.1429 & 1.31 .61082 & 14.36156 & 0.9597 \\
\hline 2 & 10.398 & $\mathrm{~PB}$ & 0.2 & 19.18990 & 1.21862 & 99 \\
\hline 3 & 14.545 & $\mathrm{BP}$ & 0.1949 & 17.94833 & 1.17047 & 0.1309 \\
\hline 4 & 15.319 & $\mathrm{VB}$ & 0.3220 & 3649.50293 & 175.92934 & 26.6133 \\
\hline 5 & 17.425 & $\mathrm{BP}$ & 0.2583 & 27.82711 & 1.33988 & 0.2029 \\
\hline & & $\mathrm{BB}$ & 0.3771 & 107.37460 & 4.16081 & 0.7830 \\
\hline
\end{tabular}<smiles>[B]N1C/C(=C\c2ccccc2)[C@@H]([C@H](OC(C)=O)c2ccccc2)C1</smiles>

rac. 11

from $(+)-40$

Page 1 of 3

Instrument 1 14.06.16 09:12:43 Johannes 
ta File $C: \backslash H P C H E M \backslash 1 \backslash D A T A \backslash J T \backslash J T 6-0070 . D$

\begin{tabular}{|c|c|c|c|c|c|c|}
\hline $\begin{array}{c}\text { Peak } \\
\#\end{array}$ & $\begin{array}{c}\text { RetTime } \\
\text { [min] }\end{array}$ & Type & $\begin{array}{l}\text { width } \\
\text { [min] }\end{array}$ & $\begin{array}{c}\text { Area } \\
{\left[\mathrm{mAU}^{\star} \mathrm{s}\right]}\end{array}$ & $\begin{array}{l}\text { Height } \\
\text { [mAU] }\end{array}$ & $\begin{array}{c}\text { Area } \\
\text { 8 }\end{array}$ \\
\hline---1 & $-\cdots---$ & & & -------- & & \\
\hline 7 & 23.892 & BB & 0.5876 & 6569.02686 & $\begin{array}{r}174.35538 \\
79.70199\end{array}$ & $\begin{array}{l}47.9033 \\
23.2669\end{array}$ \\
\hline 8 & 26.689 & BB & 0.5990 & 3190.61182 & & \\
\hline Total & $s:$ & & & $1.37131 \mathrm{e} 4$ & 452.23804 & \\
\hline
\end{tabular}

Results obtained with enhanced integrator!

Signal 2: DAD1 B, Sig $=240,4$ Ref $=550,100$

\begin{tabular}{|c|c|c|c|c|c|c|}
\hline $\begin{array}{c}\text { Peak } \\
\#\end{array}$ & $\begin{array}{c}\text { RetTime } \\
\text { [min] }\end{array}$ & Type & $\begin{array}{l}\text { width } \\
\text { [min] }\end{array}$ & $\begin{array}{c}\text { Area } \\
{\left[\mathrm{mAU}{ }^{*} \mathrm{~s}\right]}\end{array}$ & $\begin{array}{l}\text { Height } \\
\text { [mAU] }\end{array}$ & $\begin{array}{c}\text { Area } \\
8\end{array}$ \\
\hline 1 & 8.725 & BB & 0.1459 & 183.68872 & 19.50283 & 1.3647 \\
\hline 2 & 9.413 & $\mathrm{BB}$ & 0.1398 & $\begin{array}{r}6.87149 \\
.078\end{array}$ & $6.47387 e^{-1}$ & 0.0511 \\
\hline 3 & 10.404 & $\mathrm{~PB}$ & 0.1990 & 13.98977 & $8.91886 e^{-1}$ & 0.1039 \\
\hline 4 & 11.381 & $\mathrm{BP}$ & 0.1435 & 3.81604 & $3.38687 e-1$ & 0.0284 \\
\hline 5 & 14.538 & $\mathrm{BP}$ & 0.2158 & 18.58493 & 1.15455 & 0.1381 \\
\hline 6 & 15.319 & VB & 0.3225 & 3555.41675 & 171.08620 & 26.4147 \\
\hline 7 & 17.439 & $\mathrm{BP}$ & 0.2892 & 38.07566 & 1.70068 & 0.2829 \\
\hline 8 & 18.828 & $\mathrm{BP}$ & 0.3362 & 112.13548 & 4.24433 & 0.8331 \\
\hline 9 & 20.419 & $\mathrm{~PB}$ & 0.4129 & 28.61126 & $8.58279 e^{-1}$ & 0.2126 \\
\hline 10 & 23.892 & BB & 0.5848 & 6407.03320 & 169.55775 & 47.6006 \\
\hline 11 & 26.687 & BB & 0.6141 & 3091.76343 & 76.73026 & 22.9700 \\
\hline & & & & $1.34600 e^{4}$ & 446.71284 & \\
\hline
\end{tabular}

Results obtained with enhanced integrator!

Signal 3: DAD1 C, Sig=220,4 Ref $=550,100$

\begin{tabular}{|c|c|c|c|c|c|c|}
\hline $\begin{array}{c}\text { Peak } \\
\#\end{array}$ & $\begin{array}{c}\text { RetTime } \\
\text { [min] }\end{array}$ & Type & $\begin{array}{l}\text { Width } \\
\text { [min] }\end{array}$ & $\begin{array}{c}\text { Area } \\
{\left[\mathrm{mAU}{ }^{*} \mathrm{~s}\right]}\end{array}$ & $\begin{array}{l}\text { Height } \\
\text { [mAU] }\end{array}$ & $\begin{array}{c}\text { Area } \\
\text { \& }\end{array}$ \\
\hline 1 & 7.005 & PV & 0.1153 & 13.58169 & 1.59587 & 0.1018 \\
\hline 2 & 7.316 & $\mathrm{VP}$ & 0.1444 & 38.79678 & 4.33553 & 0.2908 \\
\hline 3 & 8.725 & $\mathrm{BB}$ & 0.1384 & 29.44343 & 3.22796 & 0.2207 \\
\hline 4 & 10.401 & $\mathrm{PP}$ & 0.2140 & 32.93599 & 2.02240 & 0.2469 \\
\hline 5 & 14.545 & $\mathrm{PP}$ & 0.1932 & 21.54790 & 1.35744 & 0.1615 \\
\hline 6 & 15.319 & VB & 0.3181 & 3408.79517 & 164.24544 & 25.5526 \\
\hline 7 & 17.384 & BB & 0.2634 & 31.18473 & 1.42454 & 0.2338 \\
\hline 8 & 18.827 & $\mathrm{BB}$ & 0.3515 & 97.29305 & 3.84127 & 0.7293 \\
\hline 9 & 20.380 & PB & 0.3345 & 44.97383 & 1.64743 & 0.3371 \\
\hline 10 & 23.889 & BB & 0.5716 & 6360.47461 & 168.79597 & 47.6787 \\
\hline 11 & 26.690 & $\mathrm{BB}$ & 0.5967 & 3197.15894 & 79,58985 & 23.9662 \\
\hline 12 & 30.563 & $\mathrm{BBA}$ & 0.4355 & 64.09778 & 1.76558 & 0.4805 \\
\hline & & & & $1.33403 \mathrm{e} 4$ & 433.84928 & \\
\hline
\end{tabular}

Results obtained with enhanced integrator! 


\begin{tabular}{|c|c|c|c|c|c|c|}
\hline $\begin{array}{c}\text { Peak } \\
\#\end{array}$ & $\begin{array}{l}\text { RetTime } \\
\text { [min] }\end{array}$ & Type & $\begin{array}{l}\text { Width } \\
\text { [min] }\end{array}$ & $\begin{array}{c}\text { Area } \\
{\left[\mathrm{mAU}^{*} \mathrm{~s}\right]}\end{array}$ & $\begin{array}{l}\text { Height } \\
\text { [mAU] }\end{array}$ & $\begin{array}{c}\text { Area } \\
8\end{array}$ \\
\hline 1 & 7.006 & PV & 0.1196 & 51.89496 & 6.61312 & 0.2183 \\
\hline 2 & 7.318 & VP & 0.1251 & 208.02481 & 24.99872 & 0.8749 \\
\hline 3 & 8.726 & $\mathrm{BP}$ & 0.1377 & 43.39190 & 4.78804 & 0.1825 \\
\hline 4 & 10.381 & $\mathrm{PP}$ & 0.2181 & 42.92411 & 2.42923 & 0.1805 \\
\hline 5 & 14.582 & $\mathrm{BP}$ & 0.2298 & 25.15008 & 1.57901 & 0.1058 \\
\hline 6 & 15.319 & VB & 0.3222 & 6231.04687 & 300.16443 & 26.2059 \\
\hline 7 & 17.380 & $\mathrm{BP}$ & 0.2782 & 46.72343 & 2.11095 & 0.1965 \\
\hline 8 & 18.816 & $\mathrm{BB}$ & 0.3093 & 192.74763 & 7.46246 & 0.8106 \\
\hline 9 & 23.891 & $\mathrm{BB}$ & 0.5564 & $1.13609 \mathrm{e} 4$ & 300.93887 & 47.7805 \\
\hline 10 & 26.689 & $\mathrm{BB}$ & 0.5644 & 5574.48535 & 138.84207 & 23.4446 \\
\hline Tota & 15 & & & $2.37773 \mathrm{e} 4$ & 789.92690 & \\
\hline
\end{tabular}

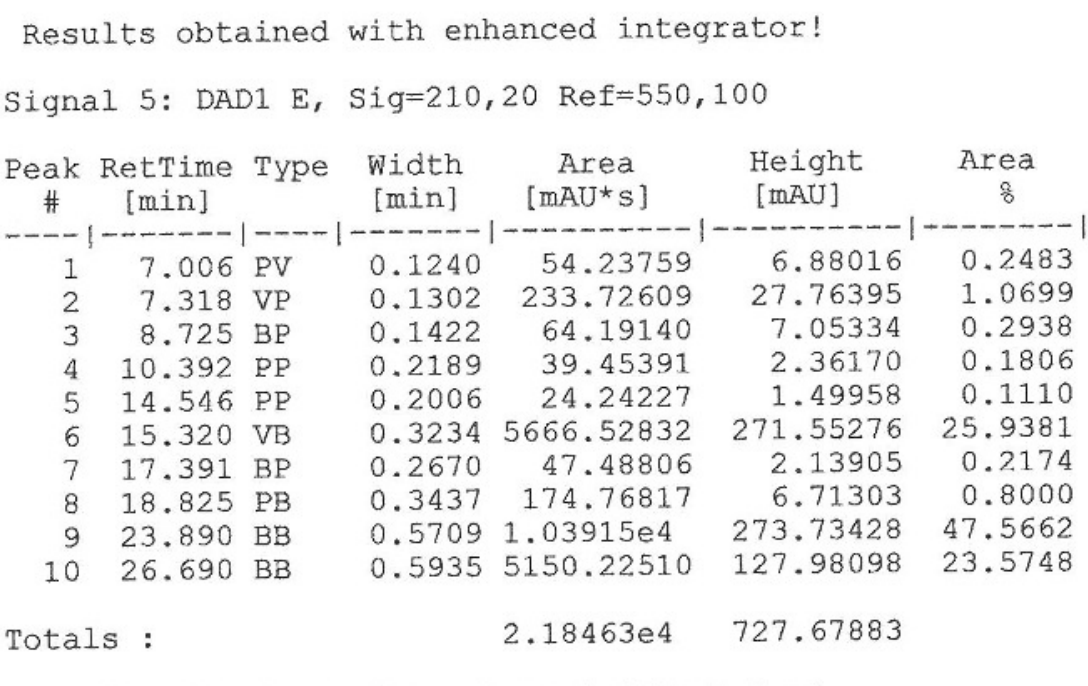

Results obtained with enhanced integrator! 


\section{Chiral HPLC chromatogram of racemic $\mathbf{4 1}$ from racemic $\mathbf{4 0 .}$}

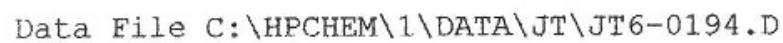

Chiralcel AD-H; Heptan/Isopropanol 90:10, $0.5 \mathrm{~mL} / \mathrm{min} ; 2$ $.5 \mu 1$

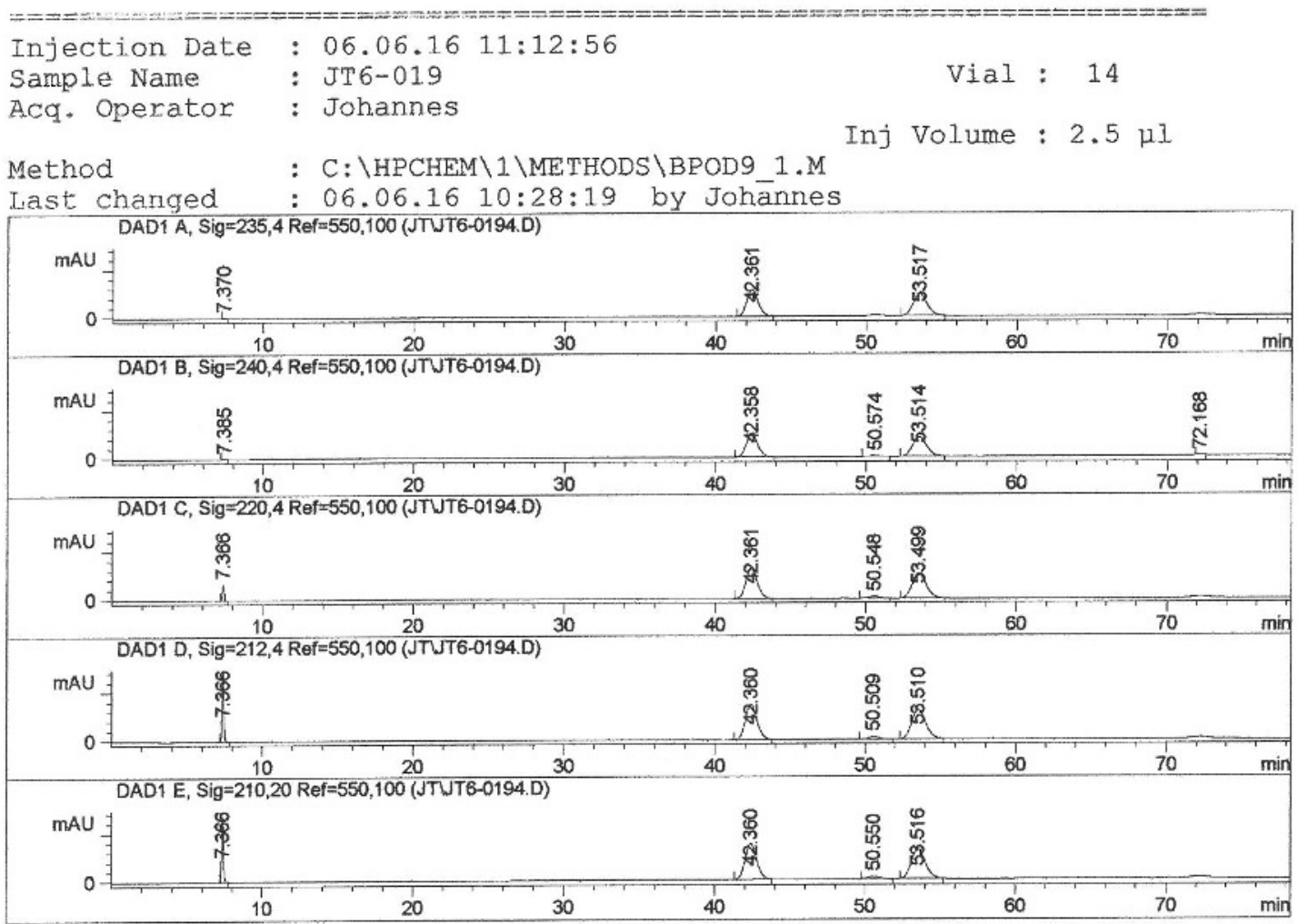

\begin{tabular}{|c|c|c|}
\hline \multicolumn{3}{|c|}{$\begin{array}{l}\text { Area Perce } \\
==m==m=1\end{array}$} \\
\hline Sorted By & : & Signal \\
\hline Multiplier & : & 1.000 \\
\hline Dilution & : & 1.000 \\
\hline
\end{tabular}

Signal 1: DAD1 A, Sig $=235,4$ Ref $=550,100$

\begin{tabular}{|c|c|c|c|c|c|c|c|}
\hline $\begin{array}{c}\text { Peak } \\
\#\end{array}$ & $\begin{array}{c}\text { RetTime } \\
\text { [min] }\end{array}$ & Type & $\begin{array}{l}\text { Width } \\
\text { [min] }\end{array}$ & $\begin{array}{c}\text { Area } \\
{\left[\mathrm{mAU}^{\star} \mathrm{s}\right]}\end{array}$ & $\begin{array}{l}\text { Height } \\
\text { [mAU] }\end{array}$ & $\begin{array}{c}\text { Area } \\
\text { 응 }\end{array}$ & rac. 41 \\
\hline---1 & ------ & & $-\cdots-\cdots$ & $--------\cdots$ & $-\infty-----$ & $----\infty-1$ & from rac. 40 \\
\hline 1 & 7.370 & $\mathrm{BB}$ & 0.1212 & 16.84126 & 2.02059 & 0.2791 & \\
\hline 2 & 42.361 & BB & 0.7582 & 3007.35181 & 56.39302 & 49.8473 & \\
\hline 3 & 53.517 & $\mathrm{BB}$ & 0.9083 & 3008.93262 & 45.92965 & 49.8735 & \\
\hline ota & $s:$ & & & 6033.12568 & 104.34326 & & \\
\hline
\end{tabular}

Results obtained with enhanced integrator! 
Signal 2: DAD1 B, Sig=240,4 Ref $=550,100$

\begin{tabular}{|c|c|c|c|c|c|c|}
\hline $\begin{array}{c}\text { Peak } \\
\#\end{array}$ & $\begin{array}{c}\text { RetTime } \\
{[\text { min] }}\end{array}$ & Type & $\begin{array}{l}\text { Width } \\
\text { [min] }\end{array}$ & $\begin{array}{c}\text { Area } \\
{\left[\mathrm{mAU}^{*} \mathrm{~s}\right]}\end{array}$ & $\begin{array}{l}\text { Height } \\
\text { [mAU] }\end{array}$ & $\begin{array}{c}\text { Area } \\
8\end{array}$ \\
\hline 1 & 7.385 & BB & 0.1286 & 5.37614 & $5.99382 \mathrm{e}-1$ & 0.0990 \\
\hline 2 & 42.358 & $\mathrm{BB}$ & 0.7770 & 2652.63843 & 49.51777 & 48.8589 \\
\hline 3 & 50.574 & BB & 0.6370 & 93.43158 & 1.76671 & 1.7209 \\
\hline 4 & 53.514 & BB & 0.8510 & 2649.38306 & 40.30000 & 48.7989 \\
\hline 5 & 72.168 & BB & 0.4230 & 28.35325 & $8.20867 e-1$ & 0.5222 \\
\hline Tota & . & & & 5429.18245 & 93.00472 & \\
\hline
\end{tabular}

Results obtained with enhanced integrator!

Signal 3: DAD1 C, Sig=220, 4 Ref $=550,100$

\begin{tabular}{|c|c|c|c|c|c|c|}
\hline $\begin{array}{c}\text { Peak } \\
\quad \#\end{array}$ & $\begin{array}{c}\text { RetTime } \\
\text { [min] }\end{array}$ & Type & $\begin{array}{l}\text { Width } \\
\text { [min] }\end{array}$ & $\begin{array}{c}\text { Area } \\
{\left[\mathrm{mAU}{ }^{\star} \mathrm{S}\right]}\end{array}$ & $\begin{array}{l}\text { Height } \\
\text { [mAU] }\end{array}$ & $\begin{array}{c}\text { Area } \\
\text { g }\end{array}$ \\
\hline 1 & 7.366 & BB & 0.1 & 266.38574 & 32.55140 & 3.6564 \\
\hline 2 & 42.361 & $\mathrm{BB}$ & 0.7234 & 3396.5 & 63.97599 & 46.6206 \\
\hline 3 & 50.548 & BB & 0. & 225.92747 & 4.17552 & 3.1011 \\
\hline 4 & 53.499 & $\mathrm{BB}$ & 0.7714 & 3396.59888 & 52.17897 & 46.6219 \\
\hline$o t$ & : & & & 7285.41722 & 152.88188 & \\
\hline
\end{tabular}

Results obtained with enhanced integrator!

Signal 4: DAD1 D, Sig=212,4 Ref $=550,100$

\begin{tabular}{|c|c|c|c|c|c|c|}
\hline $\begin{array}{c}\text { Peak } \\
\#\end{array}$ & $\begin{array}{l}\text { RetTime } \\
\text { [min] }\end{array}$ & Type & $\begin{array}{l}\text { Width } \\
\text { [min] }\end{array}$ & $\begin{array}{c}\text { Area } \\
{\left[\mathrm{mAU}{ }^{*} \mathrm{~S}\right]}\end{array}$ & $\begin{array}{l}\text { Height } \\
\text { [mAU] }\end{array}$ & $\begin{array}{c}\text { Area } \\
\frac{9}{8}\end{array}$ \\
\hline 1 & 7.366 & $B B$ & 0.1290 & 1180.08142 & 141.87401 & 12.4405 \\
\hline 2 & 42.360 & $\mathrm{BB}$ & 0.7756 & 3999.94971 & 74.36214 & 42.1678 \\
\hline 3 & 50.509 & $\mathrm{BB}$ & 0.6498 & 303.01257 & 5.50781 & 3.1944 \\
\hline 4 & 53.510 & $\mathrm{BB}$ & 0.7836 & 4002.74487 & 61.15689 & 42.1973 \\
\hline ot & s : & & & 9485.78857 & 282.90086 & \\
\hline
\end{tabular}

Results obtained with enhanced integrator!

Signal 5: DAD1 E, Sig=210,20 Ref $=550,100$

\begin{tabular}{|c|c|c|c|c|c|c|}
\hline $\begin{array}{c}\text { Peak } \\
\#\end{array}$ & $\begin{array}{c}\text { RetTime } \\
\text { [min] }\end{array}$ & Type & $\begin{array}{l}\text { Width } \\
\text { [min] }\end{array}$ & $\begin{array}{c}\text { Area } \\
{\left[\mathrm{mAU}{ }^{\star} \mathrm{s}\right]}\end{array}$ & $\begin{array}{l}\text { Height } \\
\text { [mAU] }\end{array}$ & $\begin{array}{c}\text { Area } \\
\frac{\circ}{\delta}\end{array}$ \\
\hline 1 & 7.366 & $\mathrm{BB}$ & 13 & 1152.51929 & 135.39398 & 12.2365 \\
\hline 2 & 42.360 & $\mathrm{BB}$ & 0.7882 & 4005.732 & 74.8 & 42.5294 \\
\hline 3 & 50.550 & $\mathrm{BP}$ & 0.6444 & 264.33728 & 4.93926 & 2.8065 \\
\hline 4 & 53.516 & $\mathrm{BB}$ & 0.7807 & 3996.14893 & 61.28865 & 42.4276 \\
\hline t.a] & : & & & 9418.73792 & 276.49566 & \\
\hline
\end{tabular}

Results obtained with enhanced integrator! 


\section{Chiral HPLC chromatogram of racemic $\mathbf{4 1}$ from (-)-40.}

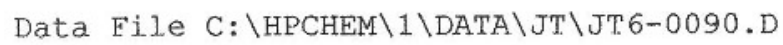

Chiralcel $\mathrm{AD}-\mathrm{H}$; Heptan/Isopropanol 90:10, $0.5 \mathrm{~mL} / \mathrm{min} ; 2$ $.5 \mu 1$

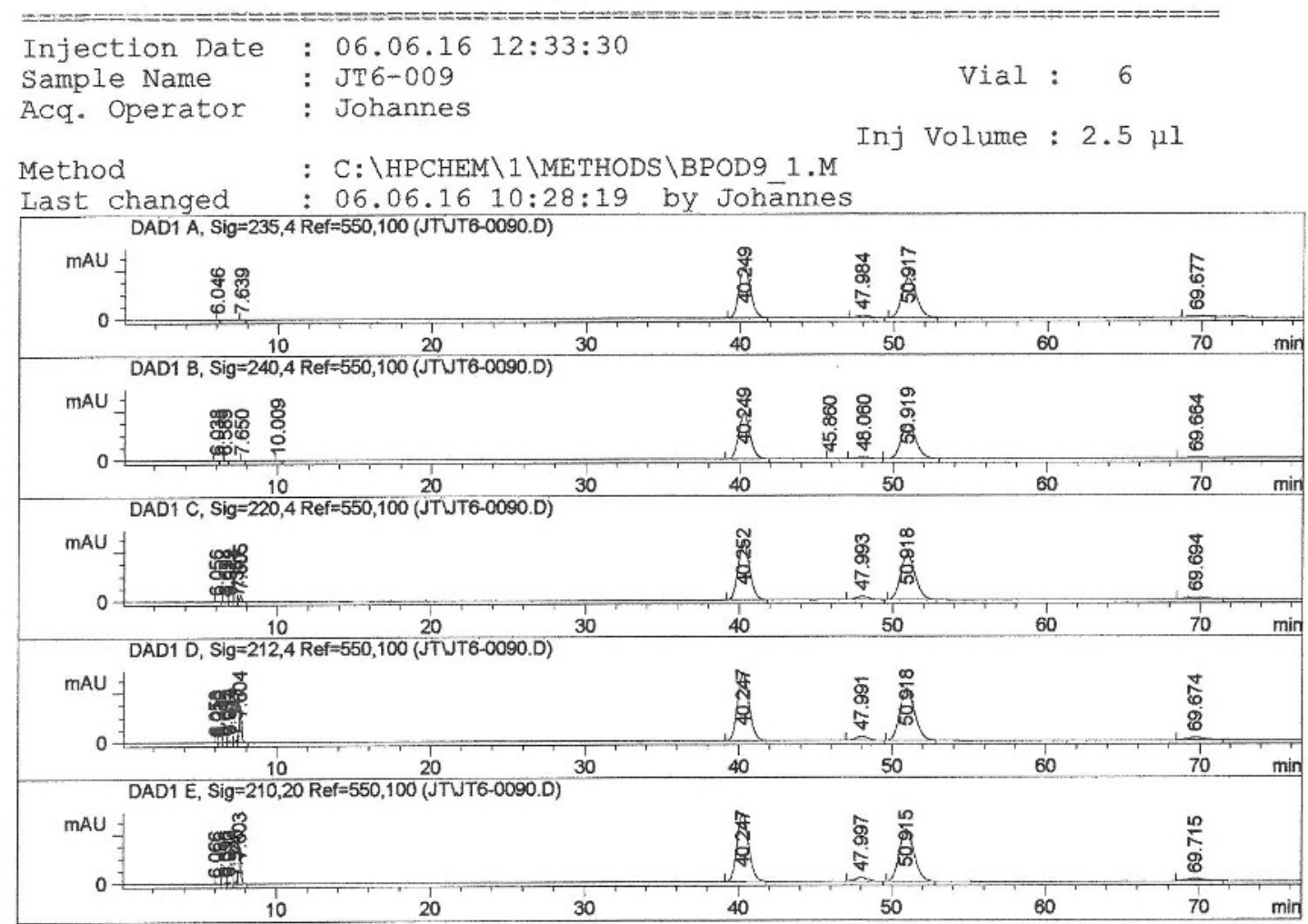

Area Percent Report

\begin{tabular}{|c|c|c|}
\hline Sorted By & : & Signal \\
\hline Multiplier & : & I. 0000 \\
\hline Dilution & : & 1.0000 \\
\hline
\end{tabular}

Signal 1: DAD1 A, Sig $=235,4$ Ref $=550,100$

\begin{tabular}{|c|c|c|c|c|c|c|}
\hline $\begin{array}{c}\text { Peak. } \\
\quad \#\end{array}$ & $\begin{array}{l}\text { RetTime } \\
\text { [min] }\end{array}$ & Type & $\begin{array}{l}\text { Width } \\
\text { [min] }\end{array}$ & $\begin{array}{c}\text { Area } \\
{\left[\mathrm{mAU}^{*} \mathrm{~s}\right]}\end{array}$ & $\begin{array}{l}\text { Height } \\
\text { [mAU] }\end{array}$ & $\begin{array}{c}\text { Area } \\
\text { \&. }\end{array}$ \\
\hline 1 & 6.046 & BB & 0.0844 & 6.51027 & 1.11005 & 0.0291 \\
\hline 2 & 7.639 & $\mathrm{BP}$ & 0.1481 & 9.92897 & 1.03344 & 0.0443 \\
\hline 3 & 40.249 & $B B$ & 0.7901 & $1.07821 \mathrm{e} 4$ & 210.31075 & 48.1521 \\
\hline 4 & 47.984 & PP & 0.6599 & 478.35623 & 8.72136 & 2.1363 \\
\hline 5 & 50.917 & BB & 0.9849 & $1.07567 \mathrm{e} 4$ & 161.52888 & 48.0389 \\
\hline 6 & 69.677 & BB & 0.8396 & 358.09802 & 5.02392 & 1.5992 \\
\hline Tota] & Is: & & & $2.23917 e^{4}$ & 387.72841 & \\
\hline
\end{tabular}<smiles>[3H]N1C[C@H]2[C@H](C1)C2(/C(=C/c1ccccc1)OC(C)=O)c1ccccc1</smiles>

rac. 41

from $(-)-40$ 


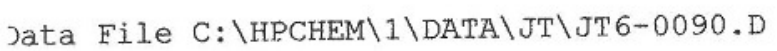

Results obtained with enhanced integrator!

Signal 2: DAD1 B, Sig=240, 4 Ref $=550,100$

\begin{tabular}{|c|c|c|c|c|c|c|}
\hline $\begin{array}{c}\text { Peak } \\
\#\end{array}$ & $\begin{array}{c}\text { RetTime } \\
\text { [min] }\end{array}$ & Type & $\begin{array}{c}\text { Width } \\
\text { [min] }\end{array}$ & $\begin{array}{c}\text { Area } \\
{\left[\mathrm{mAU}{ }^{\star} \mathrm{s}\right]}\end{array}$ & $\begin{array}{l}\text { Height } \\
\text { [mAU] }\end{array}$ & $\begin{array}{c}\text { Area } \\
\frac{0}{3}\end{array}$ \\
\hline 1 & 6.038 & $P P$ & 0.0854 & 5.01595 & $8.43583 e-1$ & 0.0256 \\
\hline 2 & 6.589 & BB & 0.1062 & 2.69266 & $3.82039 e^{-1}$ & 0.0137 \\
\hline 3 & 7.650 & PP & 0.1349 & 5.41680 & $5.69881 e^{-1}$ & 0.0276 \\
\hline 4 & 10,009 & $\mathrm{PP}$ & 0.1618 & 6.16963 & $5.54714 \mathrm{e}^{-1}$ & 0.0315 \\
\hline 5 & 40.249 & $\mathrm{BB}$ & 0.7868 & 9486.54297 & 184.81607 & 48.3697 \\
\hline 6 & 45.860 & $\mathrm{BB}$ & 0.3353 & 13.14154 & $4.68471 \mathrm{e}^{-1}$ & 0.0670 \\
\hline 7 & 48,060 & BP & 0.6539 & 322.76489 & 5.82955 & 1.6457 \\
\hline 8 & 50.919 & VB & 0.9910 & 9481.81348 & 142.01178 & 48.3456 \\
\hline 9 & 69.664 & $\mathrm{~PB}$ & 0.9888 & 289.01950 & 3.45208 & 1.4736 \\
\hline & : & & & $1.96126 e^{4}$ & 338.92817 & \\
\hline
\end{tabular}

Results obtained with enhanced integrator!

Signal 3: DAD1 C, Sig=220,4 Ref $=550,100$

\begin{tabular}{|c|c|c|c|c|c|c|}
\hline $\begin{array}{c}\text { Peak } \\
\#\end{array}$ & $\begin{array}{l}\text { RetTime } \\
\text { [min] }\end{array}$ & Type & $\begin{array}{l}\text { Width } \\
\text { [min] }\end{array}$ & $\begin{array}{c}\text { Area } \\
{\left[\mathrm{mAU}{ }^{*} \mathrm{~s}\right]}\end{array}$ & $\begin{array}{l}\text { Height } \\
\text { [mAU] }\end{array}$ & $\begin{array}{c}\text { Area } \\
8\end{array}$ \\
\hline 1 & 6.056 & $\mathrm{BP}$ & 0.1239 & 15.09715 & 1.91615 & 0.0574 \\
\hline 2 & 6.694 & $\mathrm{BV}$ & 0.1650 & 25.82416 & 2.07732 & 0.0983 \\
\hline 3 & 6.912 & $\mathrm{VB}$ & 0.1451 & 28.61702 & 2.85193 & 0.1089 \\
\hline 4 & 7.351 & BV & 0.1321 & 18.36119 & 2.22997 & 0.0699 \\
\hline 5 & 7.605 & VB & 0.1480 & 258.96463 & 26.97521 & 0.9853 \\
\hline 6 & 40.252 & $B B$ & 0.7291 & $1.22600 \mathrm{e} 4$ & 239.54041 & 46.6447 \\
\hline 7 & 47.993 & BP & 0.6596 & 747.07776 & 13.45777 & 2.8424 \\
\hline 8 & 50.918 & BB & 0.8208 & $1.22429 \mathrm{e} 4$ & 183.86858 & 46.5799 \\
\hline 9 & 69.694 & $\mathrm{BB}$ & 0.9621 & 686.88831 & 8.43472 & 2.6134 \\
\hline
\end{tabular}

Totals :

$2.62837 \mathrm{e} 4 \quad 481.35204$

Results obtained with enhanced integrator!

Signal. 4: DAD1 D, Sig=212,4 Ref=550,100

\begin{tabular}{|c|c|c|c|c|c|c|}
\hline $\begin{array}{c}\text { Peak } \\
\#\end{array}$ & $\begin{array}{c}\text { RetTime } \\
\text { [min] }\end{array}$ & Type & $\begin{array}{l}\text { Width } \\
\text { [min] }\end{array}$ & $\begin{array}{c}\text { Area } \\
{\left[\mathrm{mAU}^{*} \mathrm{~S}\right]}\end{array}$ & $\begin{array}{l}\text { Height } \\
\text { [mAU] }\end{array}$ & $\begin{array}{c}\text { Area } \\
8\end{array}$ \\
\hline 1 & 6.058 & PV & 0.1188 & 24.42590 & 3.13721 & 0.0762 \\
\hline 2 & 6.210 & VB & 0.1237 & 9.12330 & 1.22971 & 0.0285 \\
\hline 3 & 6.695 & BV & 0.1494 & 52.00496 & 4.99872 & 0.1622 \\
\hline 4 & 6.914 & VV & 0.1340 & 77.86956 & 8.25750 & 0.2428 \\
\hline 5 & 7.347 & VV & 0.1245 & 78.86638 & 9.94369 & 0.2460 \\
\hline 6 & 7.604 & VB & 0.1476 & 1481.13831 & 154.86461 & 4.6191 \\
\hline 7 & 40.247 & BB & 0.7343 & $1.43057 \mathrm{e} 4$ & 278.93512 & 44.6139 \\
\hline 8 & 47.991 & $\mathrm{BP}$ & 0.6691 & 918.64337 & 16.51226 & 2.8649 \\
\hline 9 & 50.918 & BB & 0.9233 & $1.42763 \mathrm{e} 4$ & 213.73300 & 44.5222 \\
\hline 10 & 69.674 & $\mathrm{BB}$ & 0.9378 & 841.50342 & 10.65020 & 2.6243 \\
\hline
\end{tabular}

Totals :

$$
3.20656 \mathrm{e} 4 \quad 702.26202
$$

Instrument 1 14.06.16 09:02:15 Johannes

Page 2 of 3 


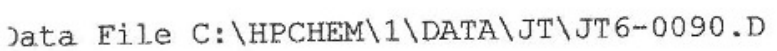

Results obtained with enhanced integrator!

Signa.1 5: DAD1 E, Sig $=210,20 \operatorname{Ref}=550,100$

\begin{tabular}{|c|c|c|c|c|c|c|}
\hline $\begin{array}{c}\text { Peak } \\
\#\end{array}$ & $\begin{array}{l}\text { RetTime } \\
\text { [min] }\end{array}$ & Type & $\begin{array}{l}\text { Width } \\
\text { [min] }\end{array}$ & $\begin{array}{c}\text { Area } \\
{\left[\mathrm{mAU}^{\star} \mathrm{s}\right]}\end{array}$ & $\begin{array}{l}\text { Height } \\
\text { [mAU] }\end{array}$ & $\begin{array}{c}\text { Area } \\
\quad 8\end{array}$ \\
\hline 1 & 6.066 & $\mathrm{~PB}$ & 0.1525 & 35.20809 & 3.40820 & 0.1098 \\
\hline 2 & 6.696 & BV & 0.1487 & 48.30781 & 4.66812 & 0.1506 \\
\hline 3 & 6.913 & VV & 0.1428 & 65.72852 & 6.92062 & 0.2050 \\
\hline 4 & 7.348 & VV & 0.1227 & 77.14208 & 9.92549 & 0.2406 \\
\hline 5 & 7.603 & VB & 0.1572 & 1506.17468 & 149.90187 & 4.6969 \\
\hline 6 & 40.247 & $\mathrm{BB}$ & 0.7676 & $1.43369 \mathrm{e} 4$ & 278.94653 & 44.7085 \\
\hline 7 & 47.997 & $\mathrm{BP}$ & 0.6442 & 873.22107 & 16.01218 & 2.7231 \\
\hline 8 & 50.915 & $\mathrm{BB}$ & 0.9462 & $1.43067 \mathrm{e} 4$ & 214.49744 & 44.6145 \\
\hline 9 & 69.715 & PB & 0.9624 & 818.05066 & 10.21433 & 2.5510 \\
\hline ota & : & & & $3.20674 \mathrm{e}^{4}$ & 694.49478 & \\
\hline
\end{tabular}

Results obtained with enhanced integrator!

$\star * \star$ End of Report 
Chiral HPLC chromatogram of racemic $\mathbf{4 1}$ from (+)-40.

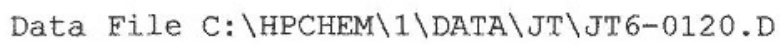

Chiralcel AD-H; Heptan/Isopropanol 90:10, $0.5 \mathrm{~mL} / \mathrm{min} ; 2$ $.5 \mu 1$

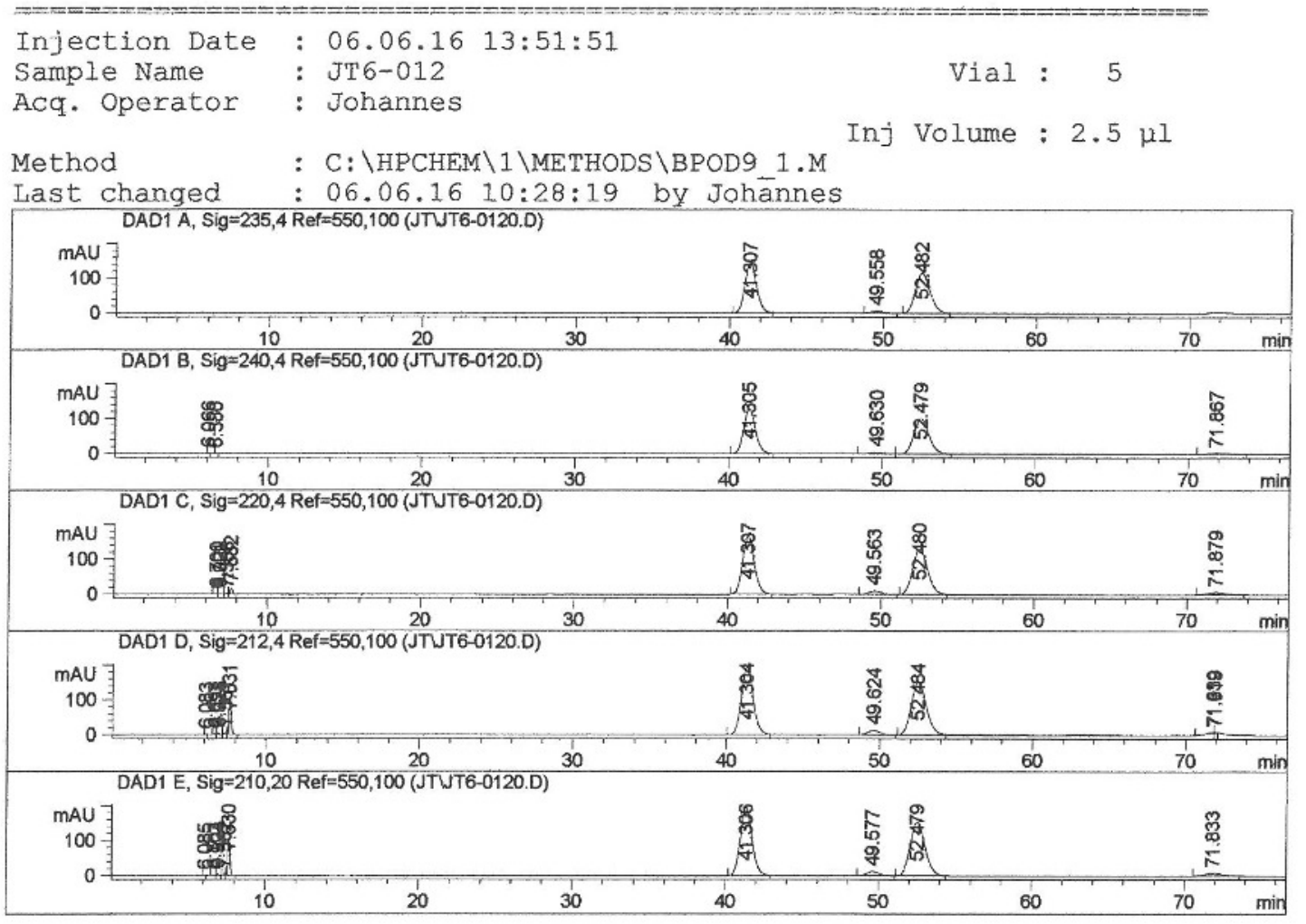

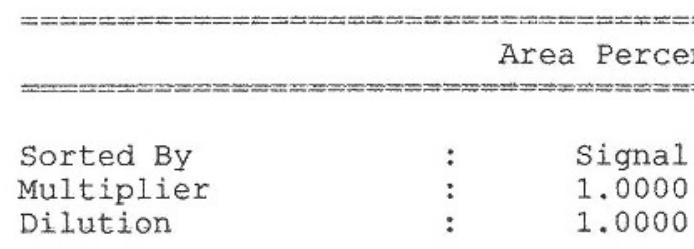

Signal 1: DAD1 A, Sig=235, 4 Ref $=550,100$

\begin{tabular}{|c|c|c|c|c|c|c|}
\hline $\begin{array}{c}\text { Peak } \\
\#\end{array}$ & $\begin{array}{c}\text { RetTime } \\
{[\mathrm{min}]}\end{array}$ & Type & $\begin{array}{l}\text { Width } \\
\text { [min] }\end{array}$ & $\begin{array}{c}\text { Area } \\
{\left[\mathrm{MAU}^{*} \mathrm{~s}\right]}\end{array}$ & $\begin{array}{l}\text { Height } \\
{[\mathrm{mAU}]}\end{array}$ & $\begin{array}{c}\text { Area } \\
\frac{8}{8}\end{array}$ \\
\hline 1 & 41.307 & $\mathrm{BB}$ & 0.8160 & 7886.21484 & 147.43654 & 49.0764 \\
\hline 2 & 49.558 & $\mathrm{BP}$ & 0.6436 & 341.24438 & 6.30344 & 2.1236 \\
\hline 3 & 52.482 & $\mathrm{BB}$ & 0.9415 & 7841.79883 & 115.87350 & 48.8000 \\
\hline Total & s : & & & $1.60693 \mathrm{e} 4$ & 269.61348 & \\
\hline
\end{tabular}<smiles>[3H]N1C[C@@H](c2ccccc2)C(/C(=C/c2ccccc2)OC(C)=O)(c2ccccc2)C1</smiles>

rac. 41 from $(+)-40$

Results obtained with enhanced integrator! 
Signal 2: DAD1 B, Sig=240, 4 Ref $=550,100$

\begin{tabular}{|c|c|c|c|c|c|c|}
\hline $\begin{array}{c}\text { Peak } \\
\#\end{array}$ & $\begin{array}{l}\text { RetTime } \\
\text { [min] }\end{array}$ & Type & $\begin{array}{l}\text { Width } \\
\text { [min] }\end{array}$ & $\begin{array}{c}\text { Area } \\
{\left[\mathrm{mAU} U^{*} \mathrm{~s}\right]}\end{array}$ & $\begin{array}{l}\text { Height } \\
\text { [mAU] }\end{array}$ & $\begin{array}{c}\text { Area } \\
8\end{array}$ \\
\hline 1 & 6.066 & $\mathrm{BB}$ & 0.0787 & 2.7351 .8 & $5.09255 \mathrm{e}-1$ & 0.0190 \\
\hline 2 & 6.580 & $\mathrm{BB}$ & 0.1110 & 2.83891 & $3.34671 \mathrm{e}^{-1}$ & 0.0197 \\
\hline 3 & 41.305 & $\mathrm{BB}$ & 0.8097 & 6947.38574 & 129.53375 & 48.2117 \\
\hline 4 & .630 & PP & 251 & 248.31111 & 4.25322 & 1.7232 \\
\hline 5 & 52 & VB & 0.9828 & 6952.66504 & 102.05565 & 48.2483 \\
\hline 6 & 71.867 & $\mathrm{~PB}$ & 0.9992 & 256.22531 & 3.02796 & 1.7781 \\
\hline
\end{tabular}

Totals :

$$
1.44102 \mathrm{e} 4 \quad 239.71450
$$

Results obtained with enhanced integrator!

Signal 3: DAD1 C, Sig=220,4 Ref $=550,100$

\begin{tabular}{|c|c|c|c|c|c|c|}
\hline $\begin{array}{c}\text { Peak } \\
\#\end{array}$ & $\begin{array}{c}\text { RetTime } \\
\text { [min] }\end{array}$ & Type & $\begin{array}{l}\text { Width } \\
{[\min ]}\end{array}$ & $\begin{array}{c}\text { Area } \\
{\left[\mathrm{mAU}{ }^{*} \mathrm{~S}\right]}\end{array}$ & $\begin{array}{l}\text { Height } \\
\text { [mAU] }\end{array}$ & $\begin{array}{c}\text { Area } \\
\text { \& }\end{array}$ \\
\hline 1 & 6.700 & PV & 0.1590 & 14.38467 & 1.28135 & 0.0745 \\
\hline 2 & 6.921 & VP & 0.1276 & 11.90230 & 1.45309 & 0.0617 \\
\hline 3 & 7.366 & BV & 0.1178 & 12.39170 & 1.68444 & 0.0642 \\
\hline 4 & 7.632 & VB & 0.1442 & 166.09053 & 17.91488 & 0.8607 \\
\hline 5 & 41.307 & $\mathrm{BB}$ & 0.7878 & 8997.89063 & 168.271 .44 & 46.6257 \\
\hline 6 & 49.563 & $\mathrm{BP}$ & 0.6784 & 561.75555 & 9.89352 & 2.9109 \\
\hline 7 & 52.480 & $\mathrm{BB}$ & 0.8517 & 8965.38086 & 132.11217 & 46.4573 \\
\hline 8 & 71.879 & PB & 0.9772 & 568.33307 & 6.92735 & 2.9450 \\
\hline
\end{tabular}

Totals :

$$
1.92981 e^{4} \quad 339.53822
$$

Results obtained with enhanced integrator!

\begin{tabular}{|c|c|c|c|c|c|c|}
\hline $\begin{array}{c}\text { Peak } \\
\#\end{array}$ & $\begin{array}{c}\text { RetTime } \\
\text { [min] }\end{array}$ & Type & $\begin{array}{l}\text { Width } \\
\text { [min] }\end{array}$ & $\begin{array}{c}\text { Area } \\
{\left[\mathrm{mAU}^{\star} \mathrm{s}\right]}\end{array}$ & $\begin{array}{l}\text { Height } \\
\text { [mAU] }\end{array}$ & $\begin{array}{c}\text { Area } \\
\frac{2}{\delta}\end{array}$ \\
\hline 1 & 6.083 & BV & 0.0892 & 9.10819 & 1.45234 & 0.0399 \\
\hline 2 & 6.697 & BV & 0.1484 & 32.56999 & 3.15475 & 0.1427 \\
\hline 3 & 6.928 & VP & 0.1378 & 36.01552 & 4.12803 & 0.1578 \\
\hline 4 & 7.361 & VV & 0.1224 & 56.12460 & 7.24709 & 0.2459 \\
\hline 5 & 7.631 & VB & 0.1445 & 955.03955 & 102.64928 & 4.1837 \\
\hline 6 & 41.304 & $\mathrm{~PB}$ & 0.7240 & $1.04838 \mathrm{e} 4$ & 196.01259 & 45.9261 \\
\hline 7 & 49.624 & $\mathrm{BP}$ & 0.6443 & 667.32166 & 12.31210 & 2.9233 \\
\hline 8 & 52.484 & $\mathrm{BB}$ & 0.8509 & $1.04470 \mathrm{e} 4$ & 154.10020 & 45.7649 \\
\hline 9 & 71.839 & PV & 0.4075 & 109.36835 & 3. 22592 & 0.4791 \\
\hline 10 & 71.919 & $\mathrm{VB}$ & 0.1451 & 31.19264 & 2.90817 & 0.1366 \\
\hline
\end{tabular}

Signal 4: DAD1 D, $\mathrm{Sig}=212,4$ Ref $=550,100$

Totals :

$$
2.28275 \mathrm{e} 4 \quad 487.19047
$$

Results obtained with enhanced integrator! 
Signal 5: DAD1 E, Sig=210,20 Ref $=550,100$

\begin{tabular}{|c|c|c|c|c|c|c|}
\hline $\begin{array}{c}\text { Peak } \\
\#\end{array}$ & $\begin{array}{c}\text { RetTime } \\
\text { [min] }\end{array}$ & Type & $\begin{array}{l}\text { Width } \\
\text { [min] }\end{array}$ & $\begin{array}{c}\text { Area } \\
{\left[\mathrm{mAU}^{\star} \mathrm{s}\right]}\end{array}$ & $\begin{array}{l}\text { Height } \\
\text { [mAU] }\end{array}$ & $\begin{array}{c}\text { Area } \\
\frac{8}{8}\end{array}$ \\
\hline 1. & 6.085 & $\mathrm{BV}$ & 0.1022 & 10.83159 & 1.61631 & 0.0462 \\
\hline 2 & 6.703 & BV & 0.1501 & 31.20285 & 2.98187 & 0.1330 \\
\hline 3 & 6.924 & VP & 0.1372 & 31.04913 & 3.58093 & 0.1323 \\
\hline 4 & 7.362 & BV & 0.1187 & 55.87156 & 7.19051 & 0.2381 \\
\hline 5 & 7.630 & VB & 0.1522 & 1011.95624 & 105.21263 & 4.3119 \\
\hline 6 & 41.306 & BB & 0.7855 & $1.05382 \mathrm{e} 4$ & 196.55139 & 44.9026 \\
\hline 7 & 49.577 & $\mathrm{PP}$ & 0.6531 & 648.12897 & 11.79438 & 2.7616 \\
\hline 8 & 52.479 & BB & 0.9472 & $1.05020 \mathrm{e} 4$ & 154.86108 & 44.7483 \\
\hline 9 & 71.833 & $\mathrm{BB}$ & 0.9639 & 639.76239 & 7.94120 & 2.7260 \\
\hline & & & & $2.34689 \mathrm{e} 4$ & 491.73032 & \\
\hline
\end{tabular}

Results obtained with enhanced integrator!

$\star \star \star$ End of Report $\star * *$ 